\title{
Bois légal \\ Vérification et gouvernance dans le secteur forestier
}

David Brown, Kate Schreckenberg, Neil Bird, Paolo Cerutti, Filippo Del Gatto, Chimere Diaw, Tim Fomété, Cecilia Luttrell, Guillermo Navarro, Rob Oberndorf, Hans Thiel, Adrian Wells

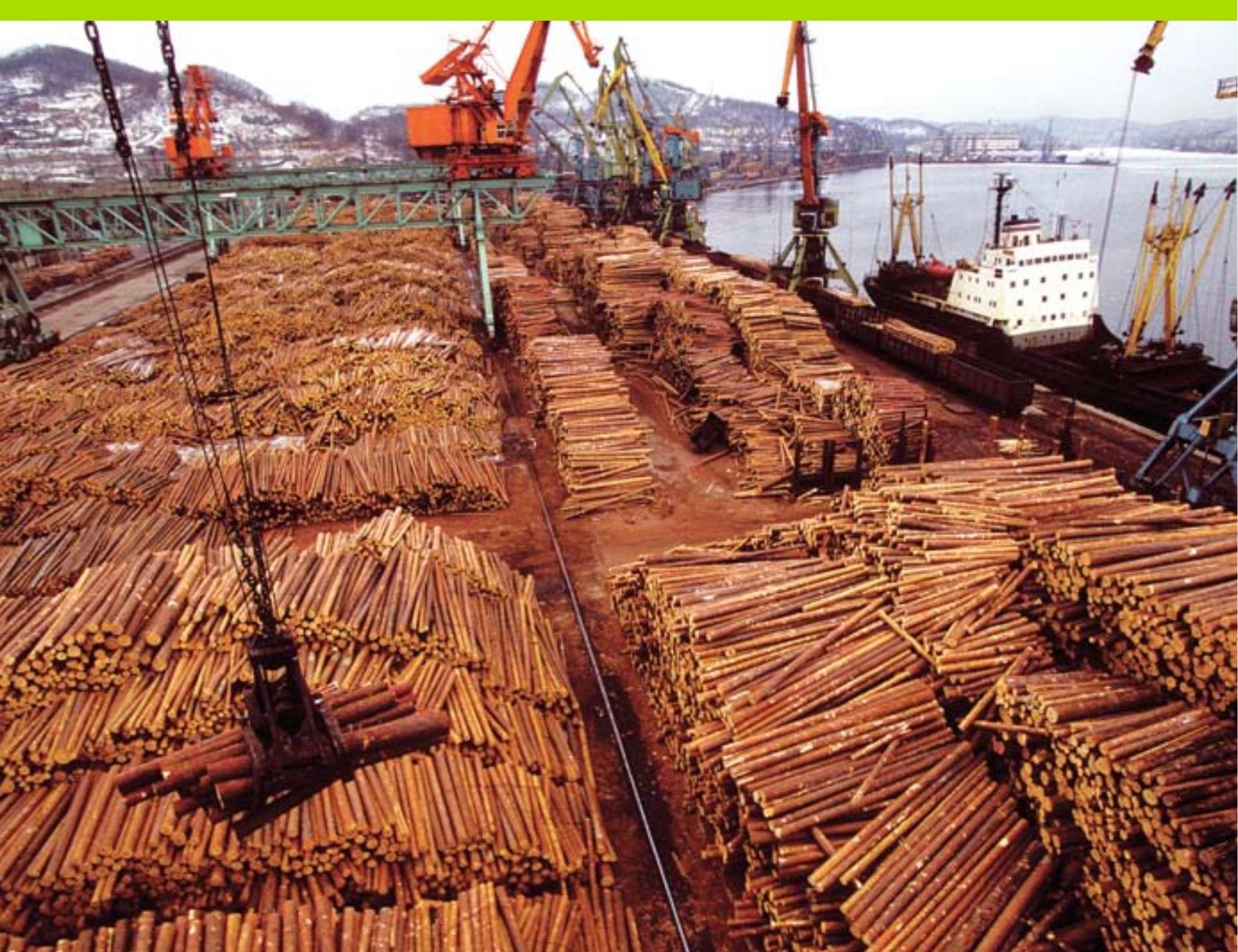





\section{Bois légal \\ Vérification et gouvernance dans le secteur forestier}

David Brown, Kate Schreckenberg, Neil Bird, Paolo Cerutti, Filippo Del Gatto, Chimere Diaw, Tim Fomété, Cecilia Luttrell, Guillermo Navarro,

Rob Oberndorf, Hans Thiel, Adrian Wells
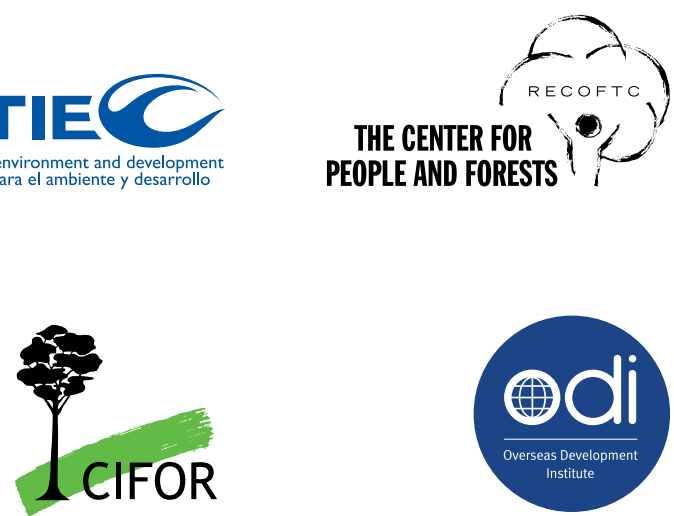
() 2009 par le Center for International Forestry Research et le Overseas Development Institute.

Tous droits réservés.

Imprimé en Indonésie

ISBN : 978-602-8693-11-0

Brown, D., Schreckenberg, K., Bird, N., Cerutti, P., Del Gatto, F., Diaw, C., Fomété, T., Luttrell, C., Navarro, G., Oberndorf, R., Thiel, H. et Wells, A. (éds) 2009 Bois légal : Vérification et gouvernance dans le secteur forestier. CIFOR et ODI, Bogor, Indonésie.

Photographies de couverture :

(c) Panos Pictures/Gerd Ludwig (illustration principale de la première de couverture)

Traduction de : Brown, D., Schreckenberg, K., Bird, N., Cerutti, P., Del Gatto, F., Diaw, C., Fomété, T., Luttrell, C., Navarro, G., Oberndorf, R., Thiel, H. et Wells, A. (eds) 2008 Legal Timber: Verification and governance in the forest sector. ODI, London.

\section{CIFOR}

Jl. CIFOR, Situ Gede

Bogor Barat 16115

Indonésie

$\mathrm{T}+622518622622$

$\mathrm{F}+622518622100$

Renseignements généraux : cifor@cgiar.org

Renseignements sur les publications : cifor-publications@cgiar.org

www.cifor.cgiar.org

Les opinions exprimées dans cet ouvrage sont celles des auteurs. Elles ne représentent pas nécessairement celles des institutions dont les auteurs font partie ni des personnes ou institutions ayant financé la publication du présent ouvrage.

\section{Centre de recherche forestière internationale}

CIFOR défend le bien-être humain, la conservation de l'environnement et l'équité en menant une recherche pour éclairer les politiques et les pratiques qui affectent les forêts dans les pays en développement. CIFOR est l'un des 15 centres au sein du Groupe consultatif sur la recherche agricole internationale (CGIAR). Le siège du CIFOR est situé à Bogor, en Indonésie. CIFOR a également des bureaux en Asie, en Afrique et en Amérique du Sud. 


\section{Table des matières}

Abréviations et acronymes viii

Remerciements $\quad$ xi

Principaux collaborateurs $\quad$ xii

Préface xiv

Avant-propos $\quad$ xvi

\section{Partie A Introduction à la vérification}

1 Evolution de la politique sur l'exploitation forestière illégale 3

2 Le contrôle forestier : une discipline émergente 15

3 Leçons à tirer de l'expérience extra-sectorielle 29

\section{Partie B Études de cas de pays portant sur la vérification forestière}

4 Aperçu des questions examinées dans les études de cas de pays 57

5 Le Forest Practices Board en Colombie-Britannique 85

6 Évolution du système de contrôle des forêts au Costa Rica 97

7 Le système national de contrôle des forêts au Honduras 109

8 Vérification forestière au Nicaragua 121

9 Respect de la loi sur les forêts dans le système brésilien de gouvernance forestière 133

10 Le système national externalisé de contrôle des forêts en Equateur 145

11 Le système de vérification forestière au Cameroun 157

12 Ghana : s'attaquer à la réforme de la gouvernance par la conception $\begin{array}{ll}\text { d'un système } & 169\end{array}$

13 L'expérience de l'observation indépendante des forêts au Cambodge 179

14 Comités multi-sectoriels de protection des forêts aux Philippines 189

15 Vérification de la conformité légale en Indonésie 199

16 Approches multiples pour améliorer le contrôle des forêts en Malaisie 215

\section{Partie C Examen des questions transversales}

17 Présentation des questions transversales 247

18 Appropriation des systèmes de vérification forestiers 253

19 Les fondements juridiques des systèmes de vérification dans le secteur forestier 263

20 Indépendance de la vérification 275 
21 Impact sur le développement des systèmes de vérification du secteur forestier

22 Convergence de la certification et de la vérification à travers la légalisation des activités forestières

23 Nouvelles technologies pour appuyer une gouvernance forestière améliorée

24 Processus à multiples parties prenantes : enseignements tirés du processus de vérification du bois

\section{Partie D Elaborer des systèmes de vérification des forêts efficaces et équitables}

25 Principes et pratique : conception du système de vérification dans le secteur de la forêt

26 Conclusions : vérification de la légalité et de la réforme de la gouvernance de la forêt

Annexe: messages politiques 


\section{Tableau, figures et encadrés}

\section{Tableau}

1.1 Estimations du taux des exportations de bois d'œuvre de certains pays généralement considérées comme illégales (2006) 11

2.1 La certification, comparée à la vérification 25

3.1 Comparaison de certaines expériences extra sectorielles avec la vérification et la situation concernant la vérification du commerce du bois d'œuvre $\quad 32$

4.1 Questions de vérification abordées par les études de cas de pays 57

4.2 Moteurs-clés qui gouvernent la participation des parties prenantes à l'établissement d'un système de vérification

4.3 Destinations des exportations (2006) des pays des études de cas VERIFOR, exprimées en pourcentage des exportations totales

5.1 La Colombie-Britannique : quelques statistiques clés 85

6.1 Le Costa Rica : quelques statistiques clés 97

7.1 Le Honduras : quelques statistiques clés 109

$\begin{array}{lll}8.1 & \text { Nicaragua : quelques statistiques clés } & 121\end{array}$

8.2 Enquêtes menées par le Procureur pour l'environnement du Nicaragua $\begin{array}{ll}\text { en } 2005 & 128\end{array}$

$9.1 \quad$ Brésil : quelques statistiques clés 133

9.2 Contrôle du respect de la législation forestière au Brésil 142

10.1 Equateur : quelques statistiques clés 145

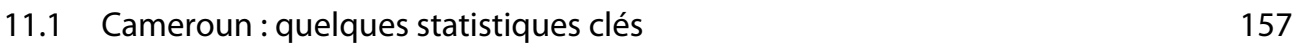

$\begin{array}{lll}12.1 & \text { Ghana : quelques statistiques clés } & 169\end{array}$

13.1 Cambodge : quelques statistiques clés 179

14.1 Les Philippines : quelques statistiques clés 189

15.1 Indonésie : quelques statistiques clés 199

15.2 Éléments clés du système de vérification des forêts en Indonésie 205

$\begin{array}{ll}16.1 & \text { Malaisie : quelques statistiques clés } \\ & 215\end{array}$

16.2 Comparaison des systèmes de vérification forestière en Malaisie 220

17.1 Une matrice modèle pour analyser et vérifier l'architecture du système $\quad 248$

22.1 Conditions imposées en matière de légalité et de durabilité des principaux systèmes de certification forestière 298

22.2 Quelques différences essentielles entre certification et vérification dans le secteur forestier

\section{Figures}

1.1 Zone de forêts certifiées (millions d'hectares) 5

1.2 Zone certifiée en pourcentage de la totalité de la couverture des forêts régionales 
2.1 Cadre stylisé de la vérification nationale et internationale et des systèmes de gouvernance des forêts typiques

4.1 Volumes des exportations de bois feuillus tropicaux pour les pays des études de cas VERIFOR (2006)

4.2 Destinations principales des exportations de bois feuillus tropicaux vers I'UE de certains pays producteurs (données 2006)

5.1 Principales activités du Forest Practices Board en Colombie-Britannique pendant ses dix premières années d'existence (1995-2004)

5.2 Vérification dans le secteur forestier en Colombie-Britannique

6.1 Flux des procédures administratives dans le système de contrôle des forêts au Costa Rica

6.2 Flux des procédures de contrôle dans le système de contrôle des forêts au Costa Rica

6.3 Contre vérification et application de sanctions dans les procédures d'administration et de vérification du Costa Rica

7.1 Procédures administratives pour l'approbation de plans d'aménagement forestier au Honduras

7.2 Procédures administratives pour l'approbation et la mise en œuvre des plans d'opérations forestières au Honduras

7.3 Acteurs internes et externes au secteur intervenant dans la vérification forestière au Honduras

8.1 La vérification et le contrôle des forêts au Nicaragua

8.2 La vérification et le contrôle des forêts externes au secteur au Nicaragua

10.1 Structure de base du SNTCF

10.2 Organisation des services de contrôle des forêts

10.3 Système de recoupements et de contrepoids du SNTCF

11.1 Importance des destinations des exportations camerounaises de bois (2005)

11.2 Acteurs et fonctions de vérification au Cameroun

12.1 Le système actuel de contrôle des forêts au Ghana 174

12.2 Le système de vérification envisagé au Ghana

13.1 Structure du système d'observation indépendante au Cambodge (1999 à 2003)

13.2 Structure du système d'observation indépendante au Cambodge (2003 à 2005)

14.1 Composition des MFPC 192

15.1 Systèmes de vérification des forêts en Indonésie 204

15.2 D'administration des bois (PUHH) en Indonésie 206

16.1 D'administration des bois dans les réserves forestières permanentes en Malaisie péninsulaire

16.2 Suivi, audit et conformité des forêts en Malaisie péninsulaire 226

16.3 D'administration des bois pour les SFMLA au Sabah 229

16.4 Suivi, audit et conformité des forêts à Sabah 231

16.5 Administration standard du bois au Sarawak 235 
16.6 Suivi et audit des forêts au Sarawak

16.7 Surveillance, vérification et suivi des importations de bois

\section{Encadrés}

2.1 Pourquoi le secteur de la forêt est-il enclin à l'illégalité ? 19

2.2 Comment l'illégalité se manifeste-t-elle dans la foresterie ? 21

$3.1 \quad$ Le Système de certification du processus Kimberley 35

$\begin{array}{ll}3.2 & \text { Le Protocole de Montréal } \\ 3.37\end{array}$

3.3 La Convention sur le Commerce international des espèces sauvages de faune et de flore menacées d'extinction (CITES) 38

3.4 La Convention-cadre des Nations Unies sur les changements climatiques (CCNUCC) et son Protocole de Kyoto

3.5 L'agence de normalisation pour les produits alimentaires et la transformation de la viande

3.6 L'Agence internationale de l'énergie atomique 47

3.7 L'observation internationale des élections 50

3.8 Le travail des ombudsmans $\quad 52$

4.1 Résumé du cadre analytique 59

4.2 Éléments d'un système de vérification 80

7.1 Observation indépendante des forêts (OIF) au Honduras $\quad 112$

16.1 Litiges en cours sur les droits aborigènes et coutumiers autochtones $\begin{array}{ll}\text { en Malaisie } & 218\end{array}$

19.1 Adoption d'une approche progressive à la légalisation 267

20.1 Conditions générales requises par I'ISO au titre des organismes
d'accréditation et des systèmes de certification

23.1 Programmes d'appui technologique à la réforme du secteur forestier dans le Mato Grosso, Brésil 


\section{Abréviations et acronymes}

\begin{tabular}{|c|c|}
\hline ACDI & Agence canadienne de développement international \\
\hline АCP & Etats d'Afrique, des Caraïbes et du Pacifique \\
\hline AIEA & Agence internationale de l'énergie atomique \\
\hline ALFA & Application de la loi forestière en Amazonie (Brésil) \\
\hline ARE & Agence de recherche sur l'environnement \\
\hline AusAID & Agence australienne pour le développement international \\
\hline $\mathrm{CB}$ & Colombie Britannique (Canada) \\
\hline BRIK & $\begin{array}{l}\text { Comité pour la revitalisation de l'industrie du bois d'œuvre } \\
\text { (Indonésie) }\end{array}$ \\
\hline BID & Banque interaméricaine de développement \\
\hline CATIE & $\begin{array}{l}\text { Centro Agronómico Tropical de Investigación y Enseñanza (Centre } \\
\text { agronomique tropical de recherche et d'éducation) }\end{array}$ \\
\hline CEDENMA & $\begin{array}{l}\text { Comité équatorien pour la protection de la nature et de } \\
\text { l'environnement }\end{array}$ \\
\hline CIA & Institut professionnel pour les ingénieurs agronomes (Costa Rica) \\
\hline CIFOR & Centre de recherche forestière international \\
\hline CITES & $\begin{array}{l}\text { Convention sur le commerce international des espèces sauvages de } \\
\text { faune et de flore menacées d'extinction }\end{array}$ \\
\hline CONADEH & Commission nationale du Honduras pour les droits humanitaires \\
\hline CONAFLOR & $\begin{array}{l}\text { Commission interinstitutionnelle de coordination du programme } \\
\text { forestier national (Brésil) }\end{array}$ \\
\hline $\mathrm{CdP}$ & Conférence des Parties \\
\hline CLPI & Consentement libre, préalable et informé \\
\hline COVIRENAS & $\begin{array}{l}\text { Comités pour la surveillance des ressources forestières et naturelles } \\
\text { (Costa Rica) }\end{array}$ \\
\hline CPET & Point central d'expertise sur l'approvisionnement en bois \\
\hline Danida & Agence danoise de développement international \\
\hline DENR & $\begin{array}{l}\text { Département de l'environnement et des richesses naturelles } \\
\text { (Philippines) }\end{array}$ \\
\hline DFID & Département britannique du développement international \\
\hline DFO & District Forest Office (Malaisie) \\
\hline DMC & Demande de mesures de correction \\
\hline CCNUCC & Convention-cadre des Nations Unies sur le changement climatique \\
\hline $\mathrm{CE}$ & Commission Européenne \\
\hline CI & Contrôleur indépendant \\
\hline CNUCED & $\begin{array}{l}\text { Conférence des Nations Unies sur l'environnement et le } \\
\text { développement }\end{array}$ \\
\hline CSF & Conseil de soutien de la forêt \\
\hline EIA & Evaluation de l'impact environnemental \\
\hline ENR-SECAL & $\begin{array}{l}\text { (Financé par la Banque Mondiale) Environment and Natural } \\
\text { Resources Sector Adjustment Loan (prêt d'ajustement sectoriel pour } \\
\text { l'environnement et les ressources naturelles) (Philippines) }\end{array}$ \\
\hline RT & Equipe d'examen \\
\hline
\end{tabular}


EURATOM Communauté européenne de l'énergie atomique

FAO Organisation des Nations Unies pour l'alimentation et l'agriculture

FC Commission de foresterie (Ghana)

FCMRP Projet de surveillance et de reportage des crimes forestiers (Cambodge)

FLEG Application des règlements forestiers et gouvernance

FLEGT Application des règlements forestiers, gouvernance et échanges commerciaux

FMI Fonds monétaire international

FMU Unité de gestion forestière

FNUF Forum des Nations Unies sur les forêts

FPB Forest Practices Board (Colombie Britannique, Canada)

FRPA Forest and Range Practices Act (Colombie Britannique, Canada)

GCF Gestion communautaire de la forêt

GDF Gestion durable des forêts

GFW Global Forest Watch

GPTIRID Groupe de travail interministériel permanent visant à réduire le déboisement en Amazonie brésilienne

GTZ Entreprise allemande pour la coopération technique internationale

GW Global Witness

IBAMA Centre brésilien de recherche sur l'environnement et les sources naturelles (Brésil)

ICF Centre national pour la préservation des forêts (Honduras)

IFF Forum intergouvernemental sur les forêts

IFM Contrôleurs/contrôle forestier(s) indépendant(s)

INAFOR Centre national forestier (Nicaragua)

ISO Organisation internationale de normalisation

KPCS Système de certification du processus de Kimberley

LEI Institution indonésienne d'Ecolabel

LGUs Unités locales du gouvernement (Philippines)

LPIs

Instances indépendantes d'évaluation (Indonésie)

MARENA Ministère de l'Environnement et des sources naturelles (Nicaragua)

MDP Mécanisme de développement propre

MINEFI Ministère des finances (Cameroun)

MINFOF Ministère des forêts et de la faune (Cameroun)

MFPCs Comités multisectorielles pour la protection des forêts (Philippines)

MTCC Conseil malaisien pour la certification du bois d'œuvre

ONG Organisation non gouvernementale

ODI Overseas Development Institute

OHL Opération forêts durables (Indonésie)

OI Observateur indépendant

OMC Organisation mondiale du commerce

PA Protocole d'accord

PAS Programme d'ajustement structurel

PEFC Programme de mise en vigueur des plans de certification forestière

PFN Programme forestier national 


\begin{tabular}{|c|c|}
\hline PIF & Panel intergouvernemental sur la forêt \\
\hline PP & Processus plurilatéral \\
\hline PUHH & Système d'administration du bois (Indonésie) \\
\hline RDC & République démocratique du Congo \\
\hline RECOFTC & $\begin{array}{l}\text { Centre de formation de foresterie communautaire régionale pour l'Asie } \\
\text { et le Pacifique }\end{array}$ \\
\hline REM & Contrôle du prélèvement des ressources \\
\hline RGC & Gouvernement royal du Cambodge \\
\hline SIG & Système d'information géographique \\
\hline SFMLAs & Accords de licence sur la gestion durable des forêts (Malaisie) \\
\hline SGS & $\begin{array}{l}\text { Société générale de surveillance (Bureau d'inspection, de vérification, } \\
\text { d'essai et de certification établi en Suisse) }\end{array}$ \\
\hline SIGIF & $\begin{array}{l}\text { Système de gestion informatisée de l'information forestière } \\
\text { (Cameroun) }\end{array}$ \\
\hline SINAC & Système national de zones de conservation (Costa Rica) \\
\hline SISCOM & Système national d'information forestière (Brésil) \\
\hline SKSHH & Permis de transport (Indonésie) \\
\hline SNCFF & $\begin{array}{l}\text { Stratégie d'application des réglementations nationales sur les forêts et } \\
\text { la faune (Cameroun) }\end{array}$ \\
\hline SNTCF & Système national de contrôle forestier externalisé (Equateur) \\
\hline SPG & Système de positionnement géographique \\
\hline STIDC & Conseil de développement de l'industrie du bois de Sarawak \\
\hline TFF & Fondation forêt tropicale \\
\hline THP & Permis d'exploitation du bois d'œuvre \\
\hline TICEN & Traité d’interdiction des essais nucléaires \\
\hline TLAS & Système d'assurance de la légalité du bois d'œuvre \\
\hline TNC & Conservation de la nature \\
\hline $\mathrm{TdR}$ & Termes de référence \\
\hline TRAFFIC & Réseau de surveillance du commerce de la faune et de la flore \\
\hline TTP & Permis de transport du bois \\
\hline TVA & Agence de validation du bois (Ghana) \\
\hline UE & Union Européenne \\
\hline USAID & Agence des Etats-Unis pour le développement international \\
\hline VERIFOR & $\begin{array}{l}\text { Le projet de recherche sur la vérification forestière sur lequel se base } \\
\text { cet ouvrage }\end{array}$ \\
\hline VCL & Vérification de la conformité légale \\
\hline VLO & Vérification de l'origine légale \\
\hline VLTP & Programme de validation du bois d'œuvre légal (Ghana) \\
\hline VPA & Accord sur le partenariat volontaire \\
\hline WALHI & Forum indonésien sur l'environnement \\
\hline WRI & Institut des ressources mondiales \\
\hline WWF & Fonds mondial pour la nature \\
\hline ZFE & Zone de forêt permanente \\
\hline
\end{tabular}




\section{Remerciements}

Ce livre est le fruit d'une collaboration internationale entre chercheurs du Royaume-Uni, du Cameroun, du Costa Rica et de la Thaillande, assistés par de nombreux partenaires et alaborateurs en Europe, en Afrique, en Asie et en Amérique latine. Nous remercions également tous ceux qui nous ont aidés à préparer les études sur lesquelles se fonde l'ouvrage, ainsi que ceux qui nous ont aidés à nous procurer l'information nécessaire d'une autre façon. Nous sommes particulièrement reconnaissants envers les co-auteurs des divers documents et études de cas produits dans le cadre du projet VERIFOR, tous ceux qui ont pris part à la Consultation des experts à Palma de Majorque en 2006, et les participants des divers ateliers et évènements animés dans le cadre du projet VERIFOR en Amérique du Sud, en Asie et en Afrique.

A Londres, Josie Tucker, puis Francesca Iannini ont géré avec beaucoup d'efficacité le projet VERIFOR et elles ont contribué au bon achèvement de ce livre. Elles étaient assistées par une équipe internationale dont faisaient partie Marcela Duran et Victor Madrigal Granados (CATIE), Cecile Effila et Hari Sukmara (CIFOR) et Wallaya Pinprayoon et Sanjiv Ray (RECOFTC). Nous les remercions tous, ainsi que les directeurs et autres membres des quatre instances participantes qui, au fil des ans, ont soutenu le projet. Jennifer Rietbergen-McCracken a été une éditrice particulièrement douée et efficace.

Pour ce qui est des agences de financement, Frank Jacobs et John Bazill (CE), Herman Savenije et Antje van Driel (Pays-Bas), Vincent van den Berk (Pays-Bas/CE), Evy Gräfin von Pfeil (GTZ), Julia Gruber (CIM) et Neil Scotland (un ancien du DFID/CE) nous ont particulièrement aidé avec leurs conseils et leur assistance. Nous remercions également : Chris Beeko (Commission forestière du Ghana), Marvin Centeno et Richard Modley (GTZ), Julia Falconer et Mathieu Bousquet (CE), John Hudson et Hugh Speechly (DFID), Jaime Guillén (Rainforest Alliance), Jader Guzmán (MAG-FOR-Nicaragua), Yanni Vilchez (SNV), Alberto Salas (UICN) et Bernardo Ortiz (TRAFFIC Amérique du Sud).

Ce livre est un produit du projet VERIFOR ; il a été réalisé avec l'aide financière de l'Union Européenne, du gouvernement néerlandais et Allemand, lequel a fourni des fonds supplémentaires pour le programme Amérique latine. Nous tenons à les remercier pour leur contribution. A noter toutefois que la responsabilité du contenu de cette publication incombe uniquement aux bénéficiaires et qu'elle ne pourra en aucune circonstance être considérée comme l'expression de la position des institutions de financement ou d'autres donateurs. 


\section{Principaux collaborateurs}

\section{David Brown}

David Brown est chargé de recherche pour le Programme sur les forêts, l'environnement et le changement climatique de l'Overseas Development Institute (ODI), et directeur du projet VERIFOR. Ce sociologue politique a concentré ses travaux sur la gestion des richesses naturelles en Afrique subsaharienne. M. Brown s'intéresse actuellement à la gouvernance environnementale et aux forêts dans la politique de changement climatique. Email : d.brown@odi.org.uk

\section{Kate Schreckenberg}

Kate Schreckenberg est chargée de recherche auprès de l'ODI. Cette forestière sociale de formation, qui à une spécialisation dans la gestion forestière commune et les chaînes de valeur des produits naturels, s'intéresse principalement à la contribution des forêts à la réduction de la pauvreté et à l'impact des politiques de la réduction des émissions issues de la déforestation et de la dégradation tropicale (REDD) sur les pauvres qui dépendent des forêts. Email : kschreckenberg@gmail.com

\section{Neil Bird}

Neil Bird est chargé de recherche auprès de l'ODI. Il tire son expérience forestière pratique de son travail de conseiller technique auprès des autorités forestières nationales en Guyane, à Belize et au Ghana. Actuellement, M. Bird effectue des recherches sur le développement institutionnel d'agences sur l'environnement et le financement des biens publics.Email : n.bird@odi.org.uk

\section{Paolo Cerutti}

Paolo Omar Cerutti travaille en tant que chercheur au Centre de recherche forestière international (CIFOR) et fait partie de l'équipe de recherche de VERIFOR en Afrique centrale. Les intérêts de ce forestier de formation se concentrent actuellement sur la gouvernance des forêts. Email : p.cerutti@cgiar.org

\section{Filippo Del Gatto}

Filippo Del Gatto est facilitateur d'un programme conjoint IUCN/TRAFFIC et VERIFOR consacré à la gouvernance et la transparence forestières en Amazonie. M. Del Gatto a acquis une riche expérience de terrain dans le domaine des politiques d'exploitation illégale du bois, des politiques foncières et du secteur forestier communautaire en Amérique Central et en Amérique du Sud. Email : fdelgatto@gmail.com

\section{Chimere Diaw}

Chimere Diaw coordonne l'Initiative africaine des forêts modèles (AMFI) et il fait partie du Networking committee du réseau international des forêts modèles. M. Diaw est à la tête du Forest Governance Learning Group (FGLG) au Cameroun. Cet anthropologiste social s'intéresse à la gouvernance à l'échelle du paysage et aux droits de propriété. Email : c.diaw@africanmodelforests.org 


\section{Tim Fomété}

Timothée Fomété Nembot est un conférencier détaché à l'Université de Dschang, au Cameroun. Il est aussi le correspondant de VERIFOR pour l'Afrique. Cet économiste forestier a pour principaux intérêts de recherche : l'économie des ressources en bois d'œuvre, la gestion des richesses naturelles et le financement durable. Email : timfomete@yahoo.fr.

\section{Cecilia Luttrell}

Cecilia Luttrell est chargée de recherche auprès del'ODI et actuellement basée en Indonésie. Ayant suivi une formation de géographe et de forestier, son travail se concentre sur la gouvernance des ressources naturelles et l'analyse institutionnelle de la pauvreté. Deux de ses intérêts sont actuellement les effets de la pauvreté sur la sylviculture communautaire et le débat sur le carbone forestier. Email : cecilia.luttrell@gmail.com

\section{Guillermo Navarro}

Guillermo A. Navarro est Professeur agrégé en économie et politique forestières au sein du "Latin American Chair on Forest Landscape Management " au Centre agronomique de recherche et d'éducation (CATIE). Point focal de VERIFOR pour l'Amérique centrale, il a mené des recherches sur la gouvernance et l'économie forestières dans la région. Email : gnavarro@catie.ac.cr

\section{Rob Oberndorf}

Robert Burton Oberndorf, (Juris Doctor, master en droit) est un conseiller juridique international, spécialisé dans les richesses naturelles et l'environnement. M. Oberndorf est actuellement correspondant de VERIFOR pour la région Asie Pacifique et coordinateur des Projets de régimes foncier, de marchés et de commerce en faveur des pauvres du Centre de formation de foresterie communautaire régionale pour l'Asie et le Pacifique (RECOFTC) à Bangkok (Thaïlande). Email : robert@recoftc.org

\section{Hans Thiel}

Vice-ministre de l'environnement en Equateur, Hans Thiel a surveillé le processus de réforme qui a mené à l'introduction du système équatorien de contrôle forestier externalisé et des normes simplifiées de légalité pour la gestion durable des forêts (GDF). Ancien point focal de VERIFOR pour l'Amérique du Sud, M. Thiel conseille actuellement les Etats membres de l'Organisation du traité de coopération amazonienne (OTCA) afin d'améliorer la mise en vigueur de leurs lois forestières dans le contexte de l'initiative ALFA. Email : hthiel.ec@gmail.com

\section{Adrian Wells}

Adrian Wells est chargé de recherche auprès de l'ODI. Il a une formation en droit de l'environnement et s'occupe de la gouvernance des ressources naturelles et du financement du changement climatique. M. Wells a récemment été détaché au Ministère des affaires étrangères et du Commonwealth de Grande-Bretagne ainsi qu'au Département britannique du développement international (DFID) où il s'occupe des forêts et du changement climatique. Email : a.wells@odi.org.uk 


\section{Préface}

Ce livre traite des liens entre la gestion forestière et la bonne gouvernance. Les thèmes des études de cas qu'il examine se trouvent au cœur de la tension entre la conservation des forêts et le développement économique : les rapports entre les communautés vivant aux alentours de la forêt, l'industrie du bois d'œuvre qui cherche à exploiter ses ressources et les intérêts de la société manifestés pour les normes et les politiques qui règlementent cette interface.

En Amérique latine, nous ne sommes que trop conscients de la capacité des ressources forestières à agir en tant que force, tant dans un sens positif que dans un sens négatif. Les forêts sont une source de capital et il existe de nombreux conflits d'intérêt parmi les parties prenantes pour le contrôle des richesses de la terre et des forêts. Bien trop souvent, les lois forestières ont été conçues par un nombre restreint de privilégiés visant à tirer profit des opportunités qui leur sont offertes, ne laissant que des institutions faibles, à l'origine même de l'illégalité, de la corruption et de la liquidation de biens nationaux de grande valeur. Dès lors, une bonne gouvernance dans le secteur de la forêt se doit de lutter contre cette culture obsolète pour l'amélioration de la société dans son ensemble.

Cette publication tombe à point nommé. Dans un contexte marqué par des droits acquis considérables, Bois légal : Vérification et gouvernance dans le secteur forestier a beaucoup à offrir puisqu'il s'agit d'une évaluation indépendante et objective de cette discipline nouvelle qu'est la vérification de la forêt. L'ouvrage reflète le résultat d'un projet de recherche appliquée de quatre ans dont le but est d'encourager le développement d'options institutionnelles pour la vérification dans le secteur forestier ; des options qui soient propres à chaque pays, nationales, socialement inclusives et tournées vers l'efficacité et la bonne gouvernance. Il n'a d'autre programme à promouvoir que l'aide pour la mise en place de systèmes durables qui se conforment à tous ces impératifs.

Bois légal met l'accent sur les différents types de défis que rencontrent tous ceux qui cherchent à améliorer la qualité de la gouvernance forestière. Il suggère qu'une approche uniforme serait une solution inadéquate, tout en reconnaissant en même temps la nécessité de développer une compréhension commune des forces à l'origine du besoin de vérification dans des environnements divers. Le cadre analytique du projet se révélera utile pour ceux qui travaillent à la conception de systèmes de vérification des forêts. Les sections au milieu du livre passent en revue de nombreuses pistes d'implémentation de la vérification, à l'intérieur comme à l'extérieur du secteur forestier, qui inciteront à réfléchir, ainsi que bon nombre de suggestions pratiques pour aider les décideurs à mettre en place les processus de réforme de la gouvernance forestière. Le livre se termine sur une discussion intéressante sur les principes d'une vérification efficace. Ces principes pourraient bien servir de base à de futures discussions nationales, au fur et à mesure que la pratique de la vérification se développe à l'échelle internationale.

Le projet a été coordonné par l'ODI en Grande-Bretagne, en association avec trois organismes régionaux de recherche : CATIE au Costa Rica, CIFOR au Cameroun et 
RECOFTC en Thaillande. Ces partenaires ont collectivement fourni des perspectives régionales différentes sur la définition d'un défi planétaire. Le résultat est un " tout " bien plus important que ses "éléments". Nous vivons à l'ère de la mondialisation où une meilleure compréhension entre les différentes parties du monde est devenue extrêmement importante. CATIE, qui est l'institution pour laquelle je travaille applaudit cette possibilité de rassembler les résultats de nos recherches et de partager nos expériences avec nos partenaires sur trois continents.

Dès lors, je vous invite à lire ce livre et à découvrir comment le secteur forestier avance, pour devenir une composante centrale des stratégies nationales pour le développement durable.

José Joaquín Campos

Directeur Général

CATIE 


\section{Avant-propos}

\section{Le contenu du livre}

Ce livre se penche sur un sujet d'actualité dans le développement de la politique forestière internationale : Comment vérifier la légalité du bois d'œuvre vendu sur les marchés régionaux et internationaux de façon à satisfaire à la fois les intérêts des Etats producteurs et les demandes des consommateurs ? Cette question, apparemment simple et technique, est en fait complexe et politique. Elle porte sur une interface essentielle dans les relations intergouvernementales, où les droits de propriétés des Etats producteurs sont défendus avec une grande ténacité. Elle comble une fracture géographique et sociale importante : les consommateurs concernés se trouvent essentiellement dans le nord postindustriel, alors que les producteurs sont surtout dans le sud en pleine industrialisation. Elle se réfere par ailleurs au thème de l'exploitation forestière illégale, qui se trouve au centre d'un mouvement international dans lequel les acteurs privés (commerciaux, aussi bien que ceux à but non lucratifs) jouent des rôles de plus en plus importants dans la gouvernance publique et commencent à établir des agendas politiques pour le nord et pour le sud. Dès lors, la spécificité technique du sujet cache sa pertinence, bien plus importante.

Le thème de ce livre est d'un côté spécifique au secteur forestier ; et de l'autre, il renvoie à des problèmes bien plus vastes concernant l'équilibre entre le contrôle de l'état souverain et la gestion internationale des biens publics planétaires, l'illégalité en tant que dimension de mauvaise gouvernance et les mécanismes de responsabilités publiques nationales et internationales. Il lance un défi au " Consensus westphalien » libéral (c.-à-d., le principe de la souveraineté nationale) qui régit les relations internationales depuis le dix-septième siècle. Il soulève des questions concernant le statut des forêts, en tant que ressources, sous un contrôle national sans équivoque, une interprétation légale qui, en dépit des désaccords dans les cercles écologiques, a été reconnu et réaffirmé dans tous les traités et conventions nationaux d'après-guerre, dont les conférences internationales importantes telles que les "Sommets sur la Terre " de Rio de Janeiro (1992) et de Johannesburg (2002). Enfin, il souligne les grandes divergences d'idées entre les nombreux utilisateurs et les bénéficiaires du bois d'œuvre et des produits forestiers, tant à l'échelle nationale que sur le plan international, ainsi que l'importance d'une garantie que toutes les initiatives de politique internationale soient conçues de façon à préserver les besoins des plus défavorisés et des plus vulnérables.

La vérification, telle qu'elle est définie dans cet ouvrage, est une réponse à un doute important sur le fonctionnement du système normal de contrôle forestier. Elle englobe deux des principaux aspects de «l'additionnalité » pour traiter ce doute : pour commencer, le développement et la mise en place d'une série de mesures supplémentaires permettant de vérifier et de valider les revendications sur la conformité légale dans le secteur forestier; et ensuite l'introduction d'un deuxième groupe d'acteurs extérieurs au secteur de la forêt, pour aider à renforcer la crédibilité de ces nouvelles mesures et la responsabilité de ceux qui sont chargés de les mettre en place. 
Le livre est le point culminant de quatre ans de recherches effectuées par deux équipes multinationales et pluridisciplinaires qui, ensemble, ont mené à bien le projet de recherche appliquée de VERIFOR. Ce projet est destiné à contrôler les systèmes de vérification existants dans le secteur forestier et ailleurs, afin de mettre au point une série de principes pour la vérification des forêts. Des partenaires de quatre institutions ont participé aux recherches : l'Overseas Development Institute (ODI) au Royaume Uni, le Centre agronomique de recherche et d'enseignement (CATIE) du Costa Rica (qui couvre toute l'Amérique latine), le bureau d'Afrique centrale du Centre international de recherche forestière (CIFOR) au Cameroun (couvrant toute l'Afrique) et le Centre de formation régional sur la sylviculture communautaire pour l'Asie et le Pacifique (RECOFTC) en Thailande (couvrant l'Asie et le Pacifique). Le projet en tant que tel couvrait les principales régions productrices de bois d'œuvre tropical et surveillait, sans toutefois intervenir, les problèmes connexes en zones tempérées. Pour plus de détails sur l'étude VERIFOR, vous pouvez aller sur le site www.verifor.org.

En rapportant les résultats du projet de recherche VERIFOR, ce livre contribue à la discipline émergeante du contrôle forestier. Il présente une douzaine d'études de cas de pays relatant les expériences nationales de vérification dans le secteur de la forêt, ainsi que huit comptes rendus de cas de vérification extra sectorielles et sept études de problèmes intersectoriels permettant de prendre certains des problèmes communs à tous les types de systèmes de vérification qui pourraient être traités par ceux qui conçoivent les systèmes de vérification des forêts. Le but est d'offrir un travail qui soit analytique, sceptique et interrogateur, tout en apportant des conseils utiles à ceux qui veulent comprendre les limites des dispositions actuelles de gestion de la forêt et mettre en place des réformes sur la gouvernance des forêts. Le livre aborde par ailleurs le souci de créer de nouvelles structures de surveillance publique minutieuse aptes à apporter l'indépendance et l'appropriation, et qui répondra aux besoins d'importants groupes intéressés nationaux et internationaux. D’une façon plus générale, l'ouvrage vise à éclairer les débats de plus en plus nombreux sur la gouvernance réelle des sortes de biens publics hybrides nationaux et mondiaux que représentent les ressources forestières. Il s'interroge sur la meilleure façon, pour les acteurs régionaux, nationaux et internationaux d'agir ensemble dans cet univers mondialisé pour la sauvegarde de la gestion des richesses naturelles qui sont à la fois essentielles au bien-être régional et très convoitées au niveau international.

L'analyse proposée dans ce livre soulève un certain nombre de questions intéressantes et déterminantes, non seulement pour une gestion adéquate du secteur forestier, mais également pour une gouvernance de qualité plus étendue dans certains Etats qui jusqu’à présent sont fracturés et instables. Parmi ces questions :

- Existe-t-il des exigences différentes pour les responsabilités publiques nationales et internationales?

- Les niveaux de responsabilité se garantissent-ils mutuellement?

- Qui doit être responsable envers qui ?

- Dans quelle mesure les différents programmes de sociétés étrangères, d'entreprises locales, de gouvernements donateurs et de gouvernements hôtes, d'ONG régionales et étrangères coïncident-ils? 
Comment apporter un élément d'indépendance dans la gestion des ressources forestières de telle façon qu'il soit culturellement approprié et ne " colporte pas de moralité environnementale " (pour reprendre l'expression imagée de Lipschultz et Fogel 2002, p. 135).

Ce livre essaie d'éclairer ces questions. L'affirmation sous-jacente étant que le secteur forestier est un environnement particulièrement productif dans lequel il s'agit de comprendre les défis de gouvernance qui assaillent le monde moderne, du fait qu'il rassemble et engage un large éventail d'intérêts et d'acteurs aux niveaux planétaire, international, national et régional ; étatique et non étatique ; des secteurs public, privé et de la société civile.

La conclusion générale que l'on voit apparaître progressivement est que pour être effectif dans les situations typiques des états producteurs tropicaux, le contrôle de l'exploitation forestière illégale ne peut être abordé uniquement comme étant un problème de criminalité, pas plus qu'on ne peut laisser des parties extérieures le gérer totalement. Il serait préférable de le positionner au sein d'un processus de réforme de gouvernance plus large et bien ancré.

\section{La structure du livre}

L'ouvrage est divisé en quatre parties. La Partie A, Introduction à la vérification, retrace l'historique de l'évolution de la politique relative à l'exploitation forestière illégale et l'émergence de la vérification dans le secteur de la forêt. Elle examine également l'expérience plus longue de la vérification dans d'autres secteurs et identifie une série de principes généraux de vérification efficace basés sur cette expérience extra sectorielle. La Partie B, Etudes de cas de la vérification des forêts, se penche sur les expériences de vérification de la forêt au Brésil, au Canada (Colombie-Britannique), au Cambodge, au Cameroun, au Costa Rica, en Equateur, au Ghana, au Honduras, en Indonésie, en Malaisie, au Nicaragua et aux Philippines. La Partie C, Analyse des questions transversales, examine sept points généraux qui découlent de ces études. Elle porte sur les forces diverses qui peuvent aboutir à une demande publique de vérification de la légalité et des réponses à cette revendication, et les leçons que l'on peut tirer de ces expériences de bénéfice potentiel pour d'autres pays qui doivent encore se lancer dans la vérification des forêts. La Partie D, intitulée La création de systèmes efficaces et équitables de vérification des forêts, reprend tous ces points pour voir dans quelle mesure les principes généraux identifiés ci-dessus sont manifestes dans le secteur forestier et si leur présence ou absence s'avèreront importants pour l'objectif d'amélioration de la qualité de la gestion des forêts. Cette partie propose quelques conclusions sur les défis présentés par la vérification de la forêt et les témoignages existants sur l'efficacité des diverses initiatives politiques de vérification des forêts à ce jour. Enfin le livre se termine par une annexe dans lequel un certain nombre de stratégies politiques sont proposées pour l'amélioration des normes d'appropriation, d'indépendance et de légalité et les impacts du développement sur les systèmes de vérification des forêts. 


\section{Partie A}

\section{Introduction à la vérification}





\section{Chapitre 1}

\section{Evolution de la politique sur l'exploitation forestière illégale}

\subsection{La politique des biens publics mondiaux et de la forêt nationale}

Pendant longtemps, la politique forestière a été un domaine débattu au niveau international et contesté. Ce haut niveau d'intérêt mondial a, en partie, pour origine le fait que les ressources forestières sont considérées comme des biens publics aussi bien que privés. ${ }^{1}$ Qui plus est, la dimension biens publics est à cheval entre les intérêts nationaux et internationaux. Le problème de base est que les forêts comportent un important volet biens publics mondiaux (lequel prend de plus en plus d'envergure dans les médias internationaux et les débats sur l'environnement), tout en étant gérées, sur pratiquement toute la planète, comme étant des ressources souveraines des états producteurs. Il existe un intérêt particulièrement fort pour les problèmes de déforestation et de dégradation des forêts : ces problèmes sont fortement associés aux producteurs du sud, qui sont essentiellement tropicaux, et s'avèrent très importants dans les économies en voie d'industrialisation. Dans les sociétés déjà industrialisées du nord développé (et qui deviennent de plus en plus "post-industrialisées »), la déforestation a cédé le pas au reboisement, souvent à une échelle importante. Une couverture forestière croissante est une caractéristique de la post-industrialisation, à tel point que l'on a proposé une " courbe environnementale de Kuznets " dans laquelle l'industrialisation s'accompagne d'abord d'une déforestation qui croît jusqu'à atteindre un point critique, avant que la relation ne s'inverse et que la couverture forestière augmente de façon plus régulière (Rudel 1998 ; Persson 2003).

La déforestation est un problème qui concerne donc principalement les pays du sud, même si les voix qui s'élèvent se situent essentiellement dans les pays du nord. Un conflit de programmes politiques accompagne ces changements environnementaux : conscientes de leurs besoins en matière de développement, les sociétés industrialisées et celles qui doivent encore le devenir arguent du fait qu'elles devraient pouvoir disposer d'autant de potentiel pour l'exploitation des ressources naturelles pour constituer un capital que les pays industriels au $19^{\mathrm{e}}$ siècle. Ce à quoi le nord postindustriel riposte que quelle que soit l'extravagance de ses modèles de consommation passés et présent, la poursuite de la

\footnotetext{
1 Le caractère mondial et national des biens publics forestiers trouve son origine dans les avantages de l'écosystème et du climat qu'ils apportent. Une grande partie du débat sur les forêts en tant que biens publics est liée au fait que les acteurs du secteur privé ont les mauvais mobiles pour financer leur approvisionnement et (ou) leur conservation. L'approvisionnement du marché est donc nettement inférieur au niveau socialement optimal et est un échec commercial. C'est ce qui justifie l'intervention non-marchande dans un souci d'approvisionnement ou de protection de ces biens publics forestiers. Dans la mesure où les avantages sont nationaux, la responsabilité incombe au gouvernement concerné. Dans la mesure où les bénéfices sont de niveau mondial, la responsabilité incombe à la communauté internationale.
} 
déforestation est un luxe que la Terre ne peut plus se permettre à l'ère d'un changement climatique menaçant.

L'intérêt écologique mondial a très bien été exprimé par les ONG du nord à la recherche d'un moyen de réaffirmer les valeurs des biens publics des forêts vierges de la Terre. Bon nombre d'ONG écologiques internationales avait espéré une convention contraignante à la Conférence des Nations Unies sur l'environnement et le développement (CNUCED) de Rio de 1992, à laquelle s'est opposé le groupe des 77 pays en développement (G77), et les parties ont seulement conclu une série de principes non exécutoires sur les forêts.

A l'origine, les tentatives pour maintenir la problématique de la déforestation tropicale au programme du traité international passaient par le biais du processus intergouvernemental post-Rio. Le Panel intergouvernemental sur les forêts (PIF) a été suivi du Forum intergouvernemental sur les forêts (FIF) puis, à un niveau politique plus élevé, par le Forum des Nations Unies sur les forêts (FNUF). Le résultat de tout ceci est bien maigre. Il n'y a eu aucun progrès dans les négociations devant aboutir à la réalisation d'un traité international exécutoire sur les forêts, et le FNUF propose à présent un accord non contraignant qui ressemble beaucoup à celui envisagé juste après la CNUCED il y a une quinzaine d'années.

Confrontés à cette impasse au niveau intergouvernemental, les bailleurs de fonds internationaux ont pris l'initiative en mains et ont cherché à améliorer la performance du secteur forestier en faisant pression directement sur les gouvernements producteurs. Dans les années 1990, l'arme de choix principale consistait à imposer des conditions sur l'aide internationale au développement, soit dans le cadre d'une plus longue liste de conditions d'aide, soit en tant que mesures spécifiques au secteur forestier. Il est couramment reconnu que ces conditionnalités n’ont donné de bons résultats dans aucun contexte (Killick 1998), notamment dans le secteur forestier (Ross 2001 ; Seymour et Dubash 2000). A un niveau général, le principal problème est que de telles conditionnalités sont appliquées par la Banque Mondiale et le Fonds monétaire international ; ces institutions bancaires ont pour vocation fondamentale d'accorder des crédits, et non de les retenir. Leur capacité à sanctionner les gouvernements enregistrant de mauvais résultats était aussi très limitée dans le temps, un fait que ces gouvernements ont vite fait de comprendre. En ce qui concerne le secteur de la forêt, force est de constater un problème supplémentaire : l'industrie forestière est souvent lucrative et bien intégrée dans les circuits des politiques nationales. Autrement dit, l'industrie forestière est souvent plus influente dans l'environnement du producteur que ne le sont les donateurs officiels. Sur les deux plans, les conditionnalités n'ont pas su garantir des avantages importants pour la gouvernance des forêts dans de nombreux contextes à haut risque.

\section{La certification dans le secteur privé}

Au cours de la dernière décennie du vingtième siècle, la tendance a été à l'utilisation croissante des instruments du secteur privé pour essayer de mettre fin au problème de la déforestation et de mettre en place une gestion durable de la forêt, essentiellement à 
travers des schémas de certification forestière. Le premier, le Conseil de soutien de la forêt (CSF) a été mis en place en 1993 au lendemain du Sommet de la CNUCED de Rio. Le lancement du CSF représentait le point culminant d'un processus qui était en gestation depuis plusieurs années sous l'égide d'un groupe de négociants en bois d'œuvre et d'ONG. A noter que le WWF jouait un rôle éminent (Nussbaum et Simula 2005, p. 240-249).

La certification émise par le secteur privé a beau avoir des effets bénéfiques, il n’en demeure pas moins que son impact est inégal. Elle n'a pas emporté le succès escompté dans les régions et pays où elle est sans doute le plus nécessaire ; à savoir, les régions tropicales où la majorité de l'exploitation forestière se produit encore dans les forêts vierges (voir les Figures 1.1 et 1.2). Ces forêts sont d'importance capitale, non seulement en raison de leur signification sylvicole, emblématique et de leur capacité de stockage du carbone atmosphérique. Elles se trouvent souvent, d'autre part, dans des pays confrontés à de graves problèmes de gouvernance publique qui touchent à la fois le secteur forestier et l'administration publique. Certains pays sont par convention considérés comme des Etats "à haut risque " au niveau de la gestion forestière et de la qualité générale de la gouvernance (Contreras-Hermosilla et al. 2007). Un grand nombre de ces pays se trouvent face à un problème sérieux : la faiblesse ou l'absence de droits fonciers sur la terre

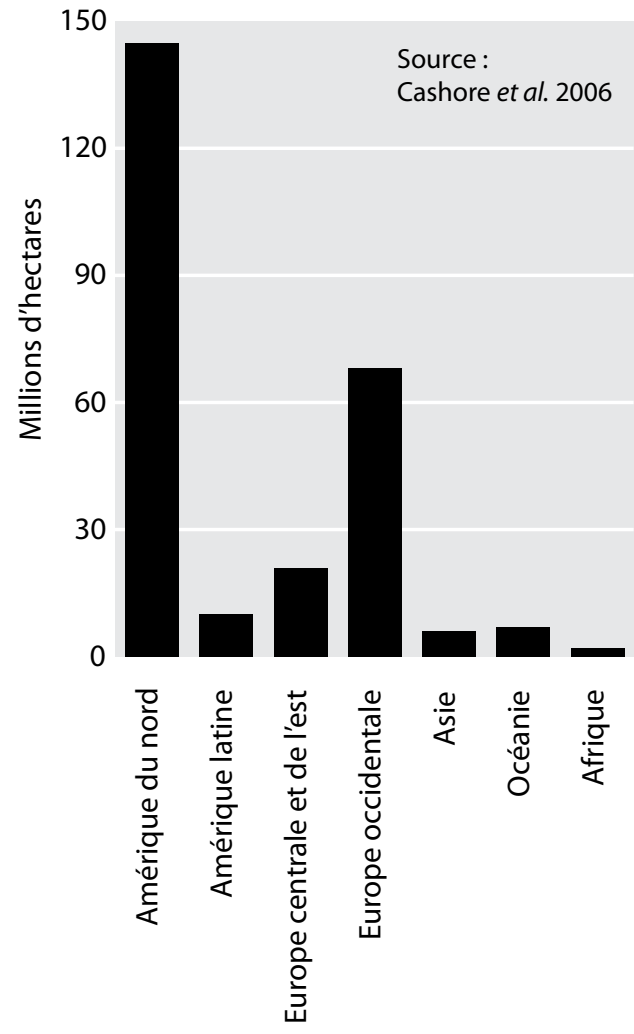

Figure 1.1 Zone de forêts certifiées (millions d'hectares)

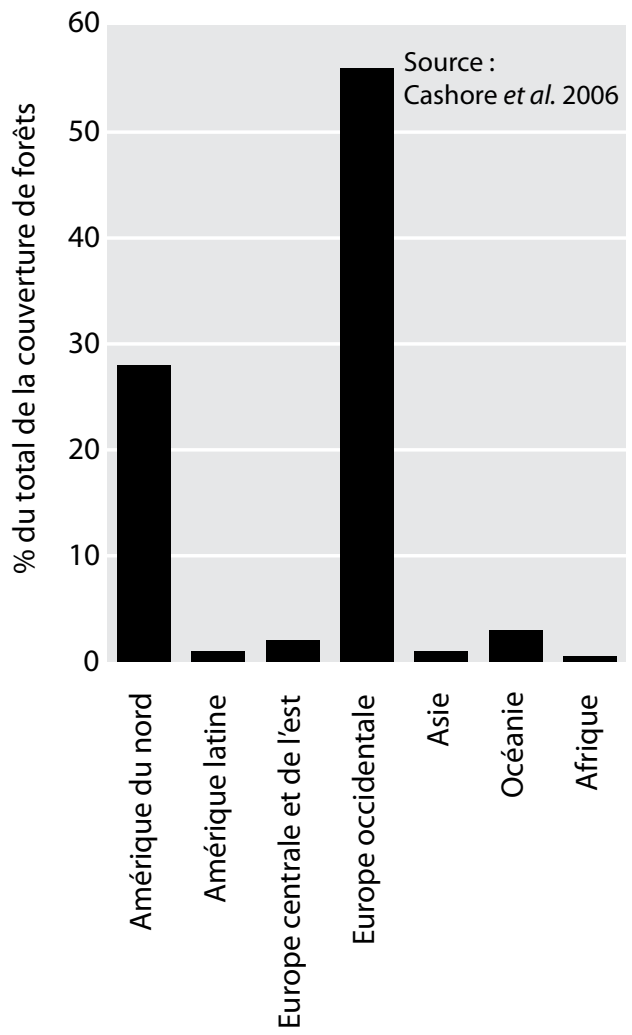

Figure 1.2 Zone certifiée en pourcentage de la totalité de la couverture des forêts régionales 
ou la forêt des populations indigènes et d'autres groupements pauvres et marginalisés de la population. Ces insuffisances remontent souvent aux époques coloniales et l'incapacité à les résoudre reflète à la fois la complexité des problèmes sous-jacents et l'absence de volonté politique à attaquer ces problèmes qui pourraient semer la discorde et perturber la société.

Il est largement admis que la certification a très peu incité ces pays à régler leurs affaires. Les augmentations des prix du bois d'œuvre certifié ont en général été insignifiantes et ne suffisent sans doute pas pour couvrir les coûts de la certification (Fischer et al. 2005). Ces coûts, très élevés parfois, notamment dans les forêts vierges plus complexes du fait des frais élevés liés à la réalisation de contrôles de certification et les bénéfices très réduits que permettent les forêts gérées durablement. Pour une industrie qui historiquement, a toujours été insuffisamment gérée, la " production durable " implique presque toujours une réduction de l'achat de bois d'œuvre rentable. Les principaux bénéficiaires, dans de telles situations, étant des producteurs de forêts bien gérées et ne posant aucun problème, désireux de préserver leurs acheteurs et de protéger leur réputation sur le marché. Ceux qui devaient vraiment améliorer leur performance n’ont dans de très nombreux cas pas été affectés.

\subsection{La montée de l'exploitation forestière illégale}

A la fin des années 1990, la crainte que les conditionnalités d'aide et que la certification ne suffisent pas pour contrer la déforestation rapide ou pour affronter les sérieux problèmes de gouvernance forestière a engendré un intérêt accru pour le problème de l'exploitation illégale des forêts. Un des arguments, entre autres, était qu'un accent mois important sur le caractère " illégal » pourrait aider à surmonter les difficultés conceptuelles de problèmes plus larges, plus difficiles et plus intangibles telles que la "durabilité ", notamment dans les milieux complexes typiques des forêts vierges. L'intérêt sous-jacent était la durabilité, mais l'approche par le biais de la légalité était, de l'avis général, la façon la plus réaliste d'atteindre cet objectif. Parallèlement et du fait que cette approche dépend finalement des demandes du consommateur et non de la volonté du producteur, on estimait que cette stratégie aurait plus de chances de réussite dans des environnements où la gouvernance est faible.

L'exploitation forestière illégale peut se définir, en gros, comme « une exploitation forestière en violation des lois nationales et internationales pertinentes ». Ces pratiques sont répandues dans de nombreuses régions forestières, en particulier (mais pas exclusivement) dans les tropiques. Mais, jusqu’à récemment, elles étaient considérées comme une préoccupation nationale et un problème que, en l'absence de convention internationale, seules les autorités nationales pouvaient traiter. Il n'en demeure pas moins quavec les changements dans les rapports internationaux survenus à la fin de la guerre froide, les limites politiques conventionnelles ont été largement érodées, aide au développement inclus. ${ }^{2}$ Dès la fin des années 1990, l'attention du donateur s'est tournée vers « la loi sur

2 Humphreys présente ce mouvement comme révélateur d'une « fuite des autorités règlementaires du système des Nations Unies » vers un groupe plus réduit d'intérêts du nord, essentiellement (2006, p. xviii). 
l'application des règlements, la gouvernance et le commerce forestiers (FLEGT) en tant que champs d'intérêt potentiels ", et « l'exploitation forestière illicite » est intervenue pour la première fois dans les débats intergouvernementaux. Au départ, les pressions visant à aborder le problème de l'exploitation illégale des forêts venaient principalement du nord postindustriel et riche. Parmi les initiatives de politique internationale importantes, on citera le Programme d'Action du G8 pour les Forêts (1998) et l'Initiative du président des Etats-Unis contre l'exploitation forestière illégale (2002). La cause a été largement défendue par la Banque Mondiale et par l'Union Européenne avec un rôle prépondérant pour le Royaume Uni.

La préoccupation internationale liée à l'exploitation illicite des forêts se concentre sur un certain nombre de problèmes. L'illégalité est considérée en partie comme une évidence tangible de la gouvernance défaillante qui assaille le secteur forestier dans de nombreux pays et se manifeste entre autres par l'indiscipline de l'industrie et par le caractère non durable de ses pratiques. Une autre question, à savoir, les coûts sociétaux élevés de l'illégalité en ce qui concerne les revenus auxquels le producteur se voit obligé de renoncer, a également été soulevée et dans certains cas (comme le Cameroun), elle a jeté la base d'un plus grand intérêt national pour l'exploitation forestière illégale pour inclure, outre les ministères sectoriels, des services importants tels que les finances et la planification nationale (voir Auzel et al. 2003). Les preuves se multiplient, mettant en évidence les effets négatifs sur les moyens de subsistance des populations pauvres qui dépendent des forêts, découlant de la vie à proximité d'une industrie non réglementée, et de la dégradation des forêts et de l'environnement qui en résultent (Samfu 2002).

La compétitivité du commerce international est aussi un facteur, et le peu d'importance de l'industrie forestière tropicale dans le marché international du bois d'œuvre contredit la situation désordonnée de l'industrie en général. En 2000 par exemple, la part de l'Europe occidentale et de l'Amérique du nord représentait $75 \%$ de l'exportation mondiale de produits forestiers ; celle de la région en voie de développement Asie Pacifique $10 \%$. Les autres régions tropicales ne représentaient donc qu'un maximum de $15 \%$; or, c'est la zone où les problèmes en termes d'exploitation forestière illégale sont les plus importants. La part africaine se limitait à $2 \%$ de la valeur ajoutée et des exportations dans le secteur de la forêt en 2000 (Lebedys 2004) et il est vraisemblable que la part de ces producteurs tropicaux primaires continuera à diminuer.

Ces statistiques toutefois ne prennent pas en ligne de compte l'impact que l'illégalité peut avoir sur le comportement du commerce légal. Par exemple, une étude sur les effets sur l'industrie américaine estime que :

- $30 \%$ du bois dur international (bois de charpente et contre plaqué) sont commercialisés illégalement ;

- Cela fait baisser les prix de 7 à $16 \%$ au niveau mondial, et

- Les coûts d'opportunité des exportateurs aux Etats-Unis représentent, en termes de baisse des prix et d'effets négatifs sur le commerce extérieur, une valeur annuelle de 460 millions de dollars américains (Seneca Creek Assoc. 2004). 
La concurrence avec le bois illégal entraîne manifestement des coûts importants pour l'industrie occidentale. A cette époque marquée par les idéologies du libre-échange (mais aussi des contrôles sévères sur la production), dans laquelle de nombreux gouvernements s'inquiètent de plus en plus de la garantie des livraisons futures de matières premières, des voix puissantes s'élèvent de plus en plus, réclamant l'élimination des négociants qui contournent les lois et vendent à des prix inférieurs à ceux du marché réglementé.

\subsection{Les acteurs internationaux et la gouvernance forestière}

La fin de la guerre froide a donné une impulsion à un mouvement d'ONG écologiques qui faisaient de plus en plus parler d'elles et qui revendiquaient une influence sur le développement de la politique, tant aux niveaux national qu'international. A cet égard, le secteur forestier a réfléchi aux changements plus importants du discours sur le développement, bon nombre desquels étaient conformes au "Consensus de Washington ". Cette série de prescriptions de politique largement adoptée met l'accent sur la libéralisation des investissements et du commerce, la privatisation des services publics et le démantèlement des mesures de protection. Les principes du Consensus de Washington, se reflétant dans un esprit de réforme plus large, prônaient des notions élargies de libéralisation et de simplification supprimant ainsi de nombreuses barrières conventionnelles entre les dispositions nationales et privées et la souveraineté nationale et internationale.

Cette libéralisation constitutionnelle s'est renforcée pendant un certain temps comme en témoignent par exemple des événements marquants comme la convention de Cotonou qui fut ratifiée en 2000 (les négociations ayant commencé en 1998) par l'Union Européenne et ses partenaires des Etats d'Afrique, des Caraïbes et du Pacifique (ACP), qui a affirmé la valeur d'une approche large, participative, et a stipulé entres autres, que les acteurs non étatiques, société civile comprise, devraient être impliqués dans les politiques et stratégies de coopération et financés adéquatement pour remplir ces rôles (Article 4, ACP/CE 2000). Dans le secteur forestier, ce changement se notait dans le contexte de la voix, toujours plus forte, des acteurs non étatiques en relation avec la pratique industrielle (par exemple, les objectifs de la certification forestière) et le développement de la politique forestière de la Banque Mondiale (des mouvements tels que l'alliance Banque Mondiale/WWF). Au seuil du $21^{\mathrm{e}}$ siècle, deux courants d'activisme écologique se sont imposés dans le cadre de l'attaque contre l'illégalité. Dans certains pays (le Brésil, l'Equateur, l'Indonésie et le Canada en sont des exemples notoires), les acteurs locaux commencèrent à faire pression pour d'importantes réformes forestières. Dans d'autres Etats (le Cambodge et le Cameroun par exemple), des acteurs extérieurs ont occupé le devant de la scène. Dans les organismes de ce type, le travail d'un certain nombre d'ONG est à l'origine de l'intérêt des bailleurs de fonds ; ces ONG établies pour la plupart en Grande-Bretagne et aux Etats-Unis, défendent les droits environnementaux et ont entamé des recherches sur le secteur forestier et ses liens avec l'ordre politique et elles se manifestent de plus en plus sur la scène internationale. Le travail de Global Witness a été particulièrement important, notamment au Cambodge (dès 1996, bien que le contrat officiel a duré de 1999 à 2003) puis au Cameroun (dès 2000). D’autres agences comme 
l'Agence de recherche sur l'environnement (ARE), qui a joué un rôle prédominant en Indonésie et Greenpeace International, qui a assumé la tâche de surveiller les forêts au niveau planétaire. Tant au Cambodge qu’au Cameroun, Global Witness a été - et c'est ce qui caractérise l'Organisation - pendant d'importantes périodes sous contrat avec l'Etat producteur, bien que financé par les donateurs dans le cadre des conditionnalités du prêt, essentiellement la Banque Mondiale et le département britannique du développement international (DFID), avec quelques contributions supplémentaires de la part d'autres donateurs bilatéraux. Ces contrats ont mis en évidence l'importance rampante de l'illégalité dans le secteur forestier des deux pays et obligea les gouvernements qui jusquelà « étaient dans le déni » à reconnaître l'étendue du problème. Les donateurs détenaient ainsi des armes puissantes leur permettant de justifier la nécessité de prendre des mesures de correction urgentes. Les contrats des ONG révélèrent aussi les faibles niveaux de performance et de compétence des agences de contrôle publiques que les ONG avaient été chargées d'évaluer et de renforcer.

L'exécution de ces contrats était plutôt " en dents de scie " comme le montreront les études de cas dans ce livre, aboutissant dans un cas (Cambodge) à la résiliation. Toutefois, on a pu ainsi dénoncer fermement l'illégalité comme un problème de gouvernance dans les pays où l'intérêt national n'avait pas été fort jusque-là, et ils ont eu un impact très fort sur le cours des réunions à haut niveau sur la l'application des règlements forestiers et la gouvernance (FLEG) organisées par la Banque et les donateurs de l'UE (en 2001) en Asie du Sud-est, puis en Afrique (2002) et plus tard en Europe et dans le Nord de l'Asie (2006).

\subsection{Développements au niveau international}

\section{Le plan d'action de l'UE sur la FLEGT}

A peu près au même moment, et s'inspirant fortement de ces développements, la Commission Européenne (CE) a commencé à mettre au point un Plan d'action sur la l'application des règlements forestiers, la gouvernance et le commerce (FLEGT) qui obtint l'aval du Conseil de l'Europe en octobre 2003 (Commission des Communautés européennes 2003). ${ }^{3}$ Le Plan d'action a instauré une approche innovatrice de la lutte contre l'exploitation illégale des forêts ; il établit un lien entre la réforme de bonne gouvernance dans les pays producteurs et l'effet de levier exercé par le marché intérieur influent de l'UE. Parmi les principales composantes, on retiendra l'appui à la réforme de la gouvernance et un schéma d'octroi de licence pour assurer que seul le bois légal a droit d'entrée sur le territoire de l'UE. Ce dernier devait se concrétiser par le biais de partenariats bilatéraux volontaires (et contraignants) avec des Etats producteurs de bois sélectionnés.

\footnotetext{
3 Les processus régionaux FLEG étaient des arènes intergouvernementales créées pour assurer les engagements politiques pour renforcer la gouvernance des forêts ; ils étaient très encouragés par la Banque Mondiale, alors que l'initiative FLEGT est née du désir européen d'utiliser le marché potentiel considérable des Etats membres de l'UE pour influencer le comportement des producteurs de bois d'œuvre tropical. La différence majeure étant que chaque accord FLEG constituait une déclaration d'intention politique, sans mécanisme de mise en oeuvre ; la FLEGT, elle, propose ce mécanisme.
} 
Une caractéristique importante du Plan d'action est sa cohérence en termes de politique environnementale, mais aussi, d'objectifs de développement social. Le plan stipule que :

"L’on s'efforcera notamment à promouvoir des solutions équitables et justes visant à régler le problème de l'exploitation illégale des forêts, des solutions sans impact négatif sur les pauvres ; à aider les pays partenaires à mettre en place des systèmes permettant de vérifier que le bois a été récolté légalement ; à promouvoir la transparence de l'information ; à renforcer des capacités au profit des gouvernements et de la société civile des pays partenaires, et à promouvoir la réforme politique. "

(Commission des communautés européennes 2003, p. 3)

Ceci a abouti à un accord sur une réglementation du Conseil de l'UE concernant " l'établissement d'un plan d'octroi de licence FLEGT pour l'importation de bois d'œuvre dans la Communauté européenne » en décembre 2005 (2173/2005). Cette réglementation est un important pas en avant, puisqu'elle a fermement déplacé les actions de gouvernance forestière du côté de la demande. Depuis, le groupe Ad Hoc de l'UE (un forum destiné aux conseillers officiels en matière de foresterie venant de pays de l'UE) s'est réuni pour mettre au point la position de négociation de l'UE. L'objectif est de créer des accords de partenariat volontaire (VPA) avec les états producteurs intéressés. Ces derniers offriront une méthode pour garantir la livraison de bois d'œuvre légal sur le marché européen et ils ouvriront la voie à l'UE afin d'aider les pays producteurs à améliorer la qualité de leur gouvernance forestière. L'UE a clairement fait savoir qu'il ne s'agit là que d'un premier pas. D'autres mesures, dont, sans doute, des "options supplémentaires " telles que la législation, pour contrôler les importations de bois et de produits récoltés illégalement dans le territoire de l'Union, pourront être envisagées en temps opportun (Brack 2006). D'autre part, plusieurs états membres modifient leurs règles d'achats pour privilégier le bois d'œuvre légal et certifié.

Bien plus qu'un engagement régional ou mondial, l'engagement de l'UE à une série d'arrangements bilatéraux (UE/Etat producteur) plutôt qu’à un accord régional ou international, a comme le stipulait la note d'information FLEGT de l'UE $\mathrm{n}^{\circ} 8$ (Commission Européenne 2004), été établi par crainte que toute tentative de négociation d'un accord universel entre l'UE et ses partenaires tropicaux ne pose problème avec les réglementations de l'OMC sur les barrières non tarifaires du commerce. Il se peut que cette position ait été prise par excès de prudence. Toujours est-il que l'OMC a outrepassé certaines mesures environnementales, bien que les motifs des décisions doivent être interprétés avec prudence et qu'ils ne défient pas nécessairement le principe d'application de normes aux accords bilatéraux. Dans la fameuse affaire du "thon-dauphin ", par exemple, la controverse relevait sans doute davantage de la discrimination commerciale que de l'admissibilité de normes environnementales (Vogel 1995, Chapitre 4). ${ }^{4}$

4 Dans ce cas précis, les Etats-Unis avaient interdit l'importation de thon du Mexique, du Venezuela et de Vanuatu, invoquant le fait que les méthodes de pêche utilisées violaient l'Acte de protection de la Marine américaine sur la protection des mammifaires (1972), prenant spécifiquement en considération la mort de dauphins pêchés en même temps que le thon. Le Mexique présenta le cas à un groupe spécial de règlement des différends du GATT comme exemple du protectionisme commercial américain. Le groupe a partiellement soutenu la plainte mexicaine : non par rapport aux problèmes de conservation mais pour le motif plus restreint que les Etats-Unis avaient cherché à limiter l'importation en appliquant une méthode non-conforme aux règles du commerce tels que définies par le GATT (Vogel 1995). 
Tableau 1.1 Estimations du taux des exportations de bois d'œuvre de certains pays généralement considérées comme illégales (2006)

\begin{tabular}{|c|c|c|c|c|c|c|}
\hline \multirow[t]{2}{*}{ Pays } & \multicolumn{4}{|c|}{ Principales concessions } & \multirow{2}{*}{$\begin{array}{l}\text { Autres } \\
\text { permis et } \\
\text { ailleurs }\end{array}$} & \multirow[t]{2}{*}{ Autres } \\
\hline & $\begin{array}{l}\text { Attribution/ } \\
\text { réattribution }\end{array}$ & $\begin{array}{l}\text { Plans de } \\
\text { gestion des } \\
\text { forêts }\end{array}$ & $\begin{array}{l}\text { Exploitation } \\
\text { forestière } \\
\text { illégale }\end{array}$ & $\begin{array}{l}\text { Crime } \\
\text { économique }\end{array}$ & & \\
\hline $\begin{array}{l}\text { Brésil } \\
\text { (Amazonie) }\end{array}$ & $40 \%$ & $40 \%$ & $20 \%$ & $30 \%$ & $30 \%$ & - \\
\hline Birmanie & $?$ & $?$ & $?$ & $?$ & $90 \%$ & - \\
\hline Cameroun & $20 \%$ & $30 \%$ & $10 \%$ & $10 \%$ & $30 \%$ & - \\
\hline Chine & $10 \%$ & $?$ & $20 \%$ & $?$ & $10 \%$ & $20 \%$ \\
\hline $\begin{array}{l}\text { République } \\
\text { du Congo }\end{array}$ & $30 \%$ & $60 \%$ & $40 \%$ & $30 \%$ & $0 \%$ & - \\
\hline Côte d'Ivoire & $?$ & $?$ & $?$ & $?$ & $?$ & $70 \%$ \\
\hline $\begin{array}{l}\text { Guinée } \\
\text { Equatoriale }\end{array}$ & $80 \%$ & $80 \%$ & $80 \%$ & $90 \%$ & $0 \%$ & - \\
\hline Gabon & $10 \%$ & $60 \%$ & $10 \%$ & $50 \%$ & $10 \%$ & - \\
\hline Ghana & $30 \%$ & $?$ & $20 \%$ & $30 \%$ & $30 \%$ & - \\
\hline $\begin{array}{l}\text { Indonésie - } \\
\text { bois d'œuvre }\end{array}$ & $30 \%$ & $20 \%$ & $20 \%$ & $30 \%$ & $50 \%$ & - \\
\hline $\begin{array}{l}\text { Indonésie - } \\
\text { pâte }\end{array}$ & $?$ & $0 \%$ & $30 \%$ & $30 \%$ & $50 \%$ & $70 \%$ \\
\hline Malaisie & $10 \%$ & $15 \%$ & $15 \%$ & $10 \%$ & $?$ & $5 \%$ \\
\hline $\begin{array}{l}\text { Papouasie- } \\
\text { Nouvelle- } \\
\text { Guinée }\end{array}$ & $90 \%$ & $90 \%$ & $20 \%$ & $20 \%$ & $0 \%$ & - \\
\hline $\begin{array}{l}\text { Russie } \\
\text { (Orientale) }\end{array}$ & $?$ & $?$ & $10 \%$ & $20 \%$ & $40 \%$ & - \\
\hline $\begin{array}{l}\text { Russie } \\
\text { (Occidentale) }\end{array}$ & $5 \%$ & $?$ & $10 \%$ & $15 \%$ & $5 \%$ & - \\
\hline Iles Salomon & $50 \%$ & $?$ & $50 \%$ & $70 \%$ & $10 \%$ & - \\
\hline
\end{tabular}

Source : www.globaltimber.org.uk/IllegalTimberPercentages.doc 
Une autre approche aurait été que l'UE pousse à conclure un accord mondial. De l'avis général, toutefois, il y a peu de chances qu'un règlement contraignant au niveau international soit approuvé dans la prochaine décennie. A court terme, donc, l'intérêt de l'UE se limite à des négociations au niveau national. Au départ, l'accent est mis sur les pays qui sont importants sur le marché, considérés comme à hauts risques en matière de forêt (et méritent donc une assurance de légalité), et néanmoins susceptibles de signer un $\mathrm{APV}$. Le fait que les négociations se fassent entre l'état producteur et l'UE en tant que bloc, plutôt qu'entre les producteurs et les états membres individuels de l'UE, reflète la compétence de l'Union en matière de commerce dans le territoire de l'Union. ${ }^{5}$

Un résultat de la promotion de FLEGT par l'UE a été l'intérêt international pour un aspect de l'environnement de la forêt qui était déjà implicite dans le discours international, encore que ce soit d'un point de vue impressionniste ; il s'agit de la question du risque au niveau du pays. Depuis longtemps, le commerce international distinguait les pays riches en forêts par niveau de risque, même si le sens de ce terme ne fait l'objet d'une analyse rigoureuse que depuis peu. Des estimations des niveaux d'illégalité dans la production forestière sont en cours, bien que leurs qualités soient variées et qu'il soit difficile de les comparer ; elles ne seront donc pas toujours considérées comme fiables. Ces évaluations se doivent d'être interprétées avec une certaine précaution ; elles reposent souvent sur des chiffres largement répandus, mais pas toujours bien fondés, et elles constituent une interprétation plutôt statique de ce qui est un phénomène dynamique (voir Cerutti et Tacconi 2006). Elles démontrent toutefois que l'illégalité est perçue comme un problème important, mais non exclusif des pays à faible gouvernance et elles tendent à renforcer d'autres évaluations de gouvernance plus importantes tels que l'indice de perception de la corruption (Transparency International) et les évaluations sur la gouvernance de la Banque Mondiale (Kaufmann et al. 2003). Une série d'estimations actuelles largement reconnues est présentée au Tableau 1.1 .

\section{Différences régionales}

Le mouvement FLEGT n'a pas été accueilli partout de la même façon. Les pays d'Amérique latine notamment se sont montrés prudents, alors que le commerce du bois entre cette région du monde et l'Europe est relativement insignifiant. Le Brésil en particulier s'est montré hostile à la FLEGT, instaurant sa propre initiative régionale, l'ALFA (Aplicação da Legislaçâo Florestal na Amazônia). Pour les autorités brésiliennes, la principale menace pour les forêts n'est pas nécessairement l'exploitation illégale de la forêt, mais plutôt la conversion de la terre ; il faudra dès lors d'autres mesures pour lutter contre cette forme de déforestation. Les réactions en Afrique et en Asie ont été plus positives. A la date de la rédaction de cet ouvrage, quatre pays (le Cameroun, le Ghana, l'Indonésie et la Malaisie) ont entamé des négociations officielles avec l'UE pour la conclusion d'APV ; d'autres

5 Chaque Etat membre de l'UE a toutefois compétence sur le commerce dans ses frontières. Si un vendeur de bois d'œuvre voulait vendre du bois illégal à un membre de l'UE, cette transaction relèverait de la police et des autorités environnementales nationales et non de la Commission. Cette pratique a mené certaines ONG environnementales à exercer une pression pour obtenir des actions parallèles au niveau national, pour renforcer les négociations multinationales européennes inévitablement plus lentes à fournir des résultats. 
Etats (comme la République Centrafricaine, la République du Congo et le Liberia) pourraient commencer les négociations prochainement. Dans chaque cas, un membre de l'UE assume le rôle de "mentor » (l'Allemagne pour le Cameroun, le Royaume Uni pour le Ghana, la Commission européenne pour l'Indonésie et les Pays-Bas pour la Malaisie). D'autres suivront sans doute si, comme l'espèrent et l'attendent les consommateurs, le signal fort donné par les coureurs de tête, aboutit à une baisse de la demande en bois d'œuvre légal non vérifié sur les marchés lucratifs européens ainsi qu'un prix avantageux pour le bois "légal et vérifié ». Tel est le contexte dans lequel la vérification de la légalité émerge en tant que dimension importante des processus de gouvernance des forêts.

\subsection{En résumé}

Ce chapitre a placé le souci grandissant du bois illégal dans le contexte de trois séries de relations qui se recoupent, chacune ayant ses facettes contradictoires : (i) la relation entre les biens publics mondiaux et nationaux dans un contexte juridique marqué par la souveraineté nationale ; (ii) la gouvernance du secteur forestier et le rapport entre l'exploitation des forêts et les modèles plus larges de bonne gouvernance et (iii) les formes de responsabilité publique et les rapports entre les acteurs étatiques et non étatiques et les acteurs nationaux et les agents internationaux. Plus loin dans l'ouvrage, il prépare les études et analyses des cas qui illustrent l'approche de l'illégalité dans divers contextes de producteurs ainsi que les enseignements à tirer d'une validité plus étendue. 



\section{Chapitre 2}

\section{Le contrôle forestier : une discipline émergente}

\subsection{Introduction}

Ce livre explore une dimension du domaine de la politique émergente de la l'application des règlements forestiers, la gouvernance et le commerce : la vérification de la légalité. La vérification n'est qu'un pas (ou une série d'actions) dans le processus de la commercialisation du bois d'œuvre légal ; il s'agit néanmoins d'un pas capital, un pas qui englobe nombre de tensions inhérentes à l'examen qui jette un pont entre les contextes nationaux et réunit divers acteurs à travers les frontières internationales.

Pour comprendre l'importance de ce concept, il nous faut tout d'abord examiner certains points de terminologie. La terminologie représente un défi dans le domaine de la vérification des forêts ; cette nouvelle discipline développe sa terminologie au fil du temps et elle est sujette par conséquent à des malentendus. Il pourrait être utile de mettre des jalons pour guider le lecteur à travers le reste de l'ouvrage.

A un degré important, la conceptualisation sur le terrain de la vérification des forêts se trouve structurée par des considérations d'encadrement, et la terminologie émerge en réaction aux pressions qui façonnent le mouvement plutôt qu'à des règles a priori. Les initiatives de lutte contre l'illégalité ont pris des formes diverses dans des situations différentes. Dans certains pays (l'Equateur, les Philippines), les principaux conducteurs ont été internes et les réactions reflètent la nécessité d'une légitimité interne, réunissant de nombreuses parties prenantes locales dans un groupement de gestion collective (voir les Chapitres 10 et 14). Parallèlement, la Malaisie dispose de conducteurs forts, bien que conditionnés ces derniers temps par le besoin des gouvernements nationaux de contrôler l'industrie et de présenter au monde une image très positive. Divers systèmes ont été mis au point ici (voir Chapitre 16). Par contre, les conducteurs sont entièrement externes dans le cas de pays comme le Cameroun et le Ghana. Dans ces situations précises, la réaction nationale a été sérieusement conditionnée par des intérêts extérieurs, notamment la proposition de l'UE pour que le point central de l'APV soit un «système d'assurance de la légalité du bois d'œuvre " (TLAS) (voir Chapitres 11 et 12).

Quels qu'en soient les déclencheurs immédiats, tous ces arrangements ont en commun un élément de doute partagé sur le fonctionnement du "système normal " de contrôle forestier ainsi qu'une reconnaissance manifeste par certaines parties (leur nombre et leur diversité dépendant du contexte) du fait que des mesures exceptionnelles sont requises pour convaincre les sceptiques de la légalité de l'origine du bois d'œuvre. Les situations de ces types constituent le sujet de ce livre et les notions de doute et d'additionnalité sont des principes primordiaux. 


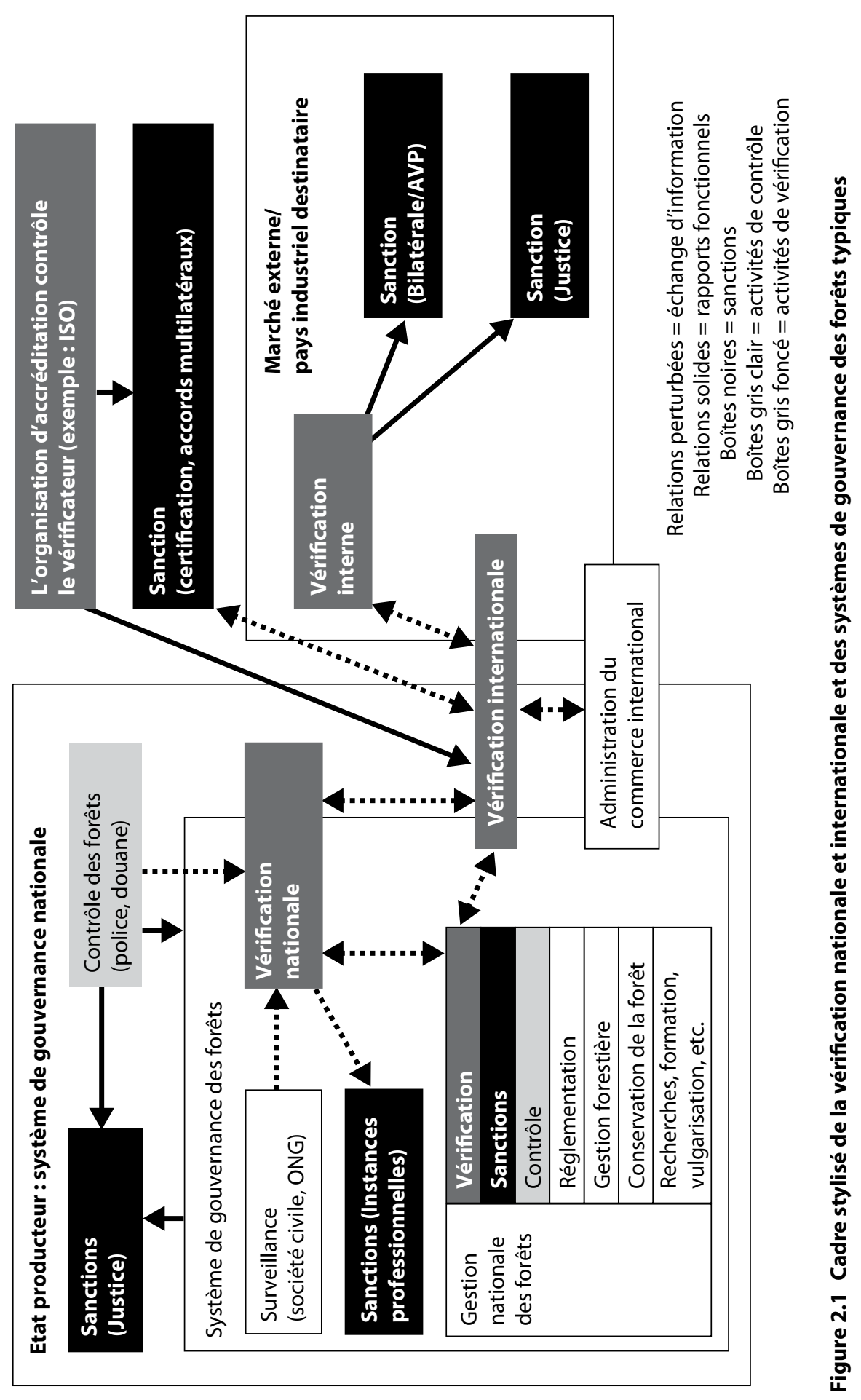


Pour poursuivre le développement de ce point, il nous faut démêler les structures typiques de la gestion forestière et leurs rapports avec les systèmes de gouvernance, lesquels sont conceptualisés dans la Figure 2.1.

\subsection{La terminologie des autorités du secteur forestier}

Nous distinguerons divers niveaux (qui se chevauchent parfois) où l'autorité est exercée dans le secteur de la forêt.

Le premier niveau est le système de gouvernance forestier : il s'agit des cadres politique et légal qui définissent la nature et l'importance des droits détenus par diverses parties prenantes et les instances qui régissent les rôles et les responsabilités de gestion des ressources forestières. Le système de gouvernance des forêts évolue au sein d'un système de gouvernance nationale qui peut être influencé, à un degré plus ou moins fort, par le fonctionnement du système de gouvernance forestier. De même que ce dernier peut être influencé par d'autres forces au sein même du système de gouvernance nationale, en dehors du secteur forestier.

Deuxième niveau est celui de l'administration forestière nationale. Il s'agit de l'institution nationale qui a légalement été mandatée pour gérer, régler, surveiller et contrôler l'utilisation et la conservation des forêts (et ceci comprend aussi le recouvrement des revenus généré par la gestion des ressources forestières). Les fonctions de la gestion forestière pourront être assumées par un ou plusieurs organisme(s), sous des appellations différentes comme ministère des forêts, autorité forestière ou service forestier. Les deux dernières instances peuvent être placées sous l'autorité directe du Ministère impliqué ou gérées par une Commission.

En troisième lieu, nous avons le système national de contrôle des forêts. Ce niveau comporte les éléments d'administration nationale faits pour assurer que les exploitants forestiers se conforment à leurs obligations contractuelles. Dans le contexte de l'application des réglementations forestières, le contrôle est une fonction déléguée par la loi à une autorité légale qui lui concède le droit d'investigation, de rapport, et au sein de sa compétence, assurer la conformité (de saisir les preuves et de sanctionner). Un système de contrôle forestier est donc imbriqué, tout au moins partiellement, dans l'administration forestière ; il comprend des dispositions nationales pour le contrôle de la gestion, de la protection, de la récolte et de l'utilisation des ressources forestières imposées par la Loi. Tout processus administratif qui inclue la " restriction et (ou) le rationnement " d'une ressource nécessitera une certaine forme de contrôle. Une administration forestière qui dispose de toute une diversité de fonctions dans les domaines de la recherche, de la formation et de l'extension, notamment, lesquelles assurent l'existence d'un système de contrôle efficace est vraisemblablement un élément important et sensible de ses responsabilités. D'autres acteurs peuvent être impliqués dans le contrôle : ce seront dans la plupart des cas la police et les autorités douanières.

Le système de vérification des forêts n'est pas un " niveau " d'opérations proprement dit; il s'agirait plutôt d'une activité, ou d'une série d'activités pouvant intervenir à n'importe 
quel niveau ou combinaison de niveaux. Un système de vérification existe et a pour vocation d'assurer la légitimité ou d'accorder une crédibilité aux systèmes de gestion de la forêt lorsqu'il existe des doutes sur leur fonctionnement. Il comprend une disposition de vérification et de validation des revendications de conformité légale (et, plus généralement, la conformité aux normes convenues), avec, à l'appui, les renseignements fournis par la surveillance, les audits, l'observation extérieure et d'autres instruments, ainsi que des procédures permettant d'évaluer cette information et de parvenir à une conclusion sur le niveau réel de conformité - en bref, une proposition de "décision de vérification ". Le besoin de contrôler la légalité implique généralement (mais pas toujours) une classe d'acteurs bien plus étendue que celle qui intervient dans le contrôle des forêts. Comme le montre la Figure 2.1, les sites où l'on entreprend des activités de vérification varient ; ils sont à cheval sur les systèmes de gouvernance nationale et forestière et sur les états producteurs et consommateurs. Le mélange exact d'activités qui inclut le "système de vérification " dans tous les contextes et leurs sites institutionnels sera spécifique à la situation et dépendra des intérêts et des pouvoirs des acteurs impliqués. Tout ceci est approfondi dans les chapitres suivants et en particulier, au Chapitre 4.

La tendance du secteur forestier d'être accusé de mauvaise gestion, voire, d'illégalité, vient d'un certain nombre de caractéristiques typiques à l'exploitation des forêts ; elles prévalent surtout (mais pas uniquement) dans les milieux tropicaux (voir l'encadré 2.1).

Comme l'illustrent les études de cas dans ce livre, les formes prises par une administration des forêts peuvent varier tout comme le peuvent les formes et l'efficacité des fonctions de contrôle et de vérification. Le public se montre assez souvent méfiant vis-à-vis du système de contrôle forestier ordinaire, qui n'est d'ailleurs pas d'avantage pris au sérieux par la politique. Aux niveaux supérieurs, les autorités ne voient pas l'intérêt d'exposer l'industrie forestière à l'examen rigoureux du public ; de leur côté, les bureaucrates aux niveaux inférieurs n'auront pas vraiment envie de mettre en danger leurs propres carrières en défiant les autorités supérieures.

Les doutes des publics extérieurs quant au « fonctionnement normal » sont sans doute liés à des points spécifiques de la séquence de la gestion forestière, ou elles seront générales et concerneront la performance générale du secteur. Quatre domaines principaux sont sujets à préoccupation :

- En premier, la valeur importante que l'on peut obtenir de l'exploitation forestière industrielle signifie que le processus d'attribution de la concession est souvent vulnérable. Ceci est en particulier le cas, lorsque les frontières entre l'entrepreneuriat et les partis politiques sont floues.

- Deuxième préoccupation : l'exploitation forestière en dehors des limites de la concession. Ce souci peut être relié au précédent, surtout lorsque la protection politique autorise les bûcherons à agir en toute impunité. Le fait que les opérations sur le site de l'exploitation s'effectuent dans des situations isolées augmente pour les bûcherons la tentation d'abattre des arbres précieux en dehors des zones autorisées ou de faire des compromis sur des normes contractuelles. 


\section{Encadré 2.1 Pourquoi le secteur de la forêt est-il enclin à l'illégalité ?}

Notons certaines explications typiques:

- L'exploitation du bois d'œuvre s'effectue généralement dans des situations isolées, loin des yeux du public, à des endroits donc où les transgressions passent souvent inaperçues.

- Les ressources en bois d'œuvre ont souvent une valeur extraordinairement élevée pour l'économie locale, de sorte que les avantages de la transgression de la loi seront supérieurs aux peines encourues.

- L'exploitation forestière est une entreprise à forte utilisation de capital ; elle a souvent lieu dans des environnements à haut risque. Ces faits augmentent les taux d'escompte appliqués par les entrepreneurs et les encouragent à continuer à faire fonctionner leurs actifs en dépit des règlements qui ordonnent le contraire.

- L'exploitation du bois d'œuvre a souvent lieu sur des terres publiques, ce qui encourage à conclure des alliances avec les politiques.

- Mêmelorsquel'exploitation alieu sur desterres privées, celles-citendent, théoriquement, à être soumises à une réglementation sévère (c'est dans la nature des arbres en tant que ressources à long terme). Dès lors, les alliances avec ces politiques peuvent être intéressantes pour déstabiliser les contrôles publics.

- L'exploitation du bois d'œuvre est sans doute une des formes les plus lucratives d'entrepreuneriat dans les sociétés riches en forêts. Une forme qui peut être facilement liquider si nécessaire. Ces charactéristiques encouragent aussi les alliances avec la politique (les dons aux partis par exemple, qui aboutissent à des allégations de « capture » par l'industrie).

- Le prix du respect de la légalité peut être élevé.

- Les populations qui vivent dans les forêts font souvent partie des groupes les plus défavorisés et les plus marginalisés de la société (paradoxalement, vue la valeur élevée de la ressource) ; elles n'ont sans doute pas la puissance, voire, l'autorité, d'éviter les abus.

- Les pauvres n'ont que peu de choix ; l'exploitation illégale des forêts n'est qu'une des rares options pour trouver du travail et gagner un salaire.

- Il peut s'avérer difficile de « se comporter en fonction de la Loi », notamment là où les forêts et les arbres sont soumis à des juridictions qui se recoupent (par exemple, en cas de contrôles par différents niveaux de gouvernement).

- Les lois forestières peuvent sembler incohérentes par rapport aux pratiques de gestion de la terre (par exemple, lorsque les arbres font partie du cycle de la jachère ou lorsque les politiques agricoles et forestières sont entre les mains de ministères indépendants), et elles ne pourront dès lors pas être respectées dans certains domaines; ceci risque d'encourager un mépris général de la loi sur le plan de l'utilisation du bois.

- L'exploitation illégale du bois est susceptible d'avoir des conséquences positives, à court terme du moins, comme une augmentation de la production et une réduction du prix du bois (ce qui stimule la concurrence des industries nationales et profite aux consommateurs) (Tacconi 2007). 
- La partie des opérations qui implique le transport de bois bruts en grumes des chantiers d'exploitation vers les dépôts et les scieries, leur traitement sur place et leur transport vers les ports entraîne une troisième série de points vulnérables, dont la surveillance sera couverte par le terme de " contrôle de la chaîne de conservation ». La vulnérabilité est au plus haut point lorsque des grumes de différentes origines sont mélangées dans une seule scierie, surtout lorsque la scierie dispose d'un ensemble d'équipements de transformation.

- Enfin, la relation entre le secteur de la forêt et la société au sens large est souvent problématique, surtout lorsqu'il y a d'importantes différences de pouvoir entre les utilisateurs (industrie forte face aux communautés faibles par exemple) et(ou) d'importantes considérations sur les droits de populations indigènes ou les préoccupations environnementales spécifiques (captage de l'eau et pollution des cours d'eau, inondations, envasement, dégradation du sol, perte de flore charismatique, etc.). Tout ceci peut inquiéter le public et, dès lors, attirer son regard sur la performance de l'administration forestière. Cette dernière est souvent accusée de privilégier l'industrie et de traiter le système de contrôle en conséquence.

L'encadré 2.2 résume les formes typiques de l'illégalité dans le secteur de la forêt et les niveaux auxquels elles surviennent.

Le manque de fonds est quelquefois invoqué pour expliquer la mauvaise performance des autorités de contrôle gouvernementales, bien qu'il s'agisse sans doute là, dans les pays riches en forêts, davantage d'une excuse que d'une véritable motivation, car l'exploitation forestière peut en effet s'avérer très lucrative et la taxation devrait être à même de générer des revenus importants pour l'Etat. On soupçonne que les organismes de contrôle forestier sont souvent privés de fonds pour les rendre inopérables. Quels que soient les problèmes sous-jacents, le contrôle des opérations forestières est souvent un maillon faible de la chaîne de l'autorité forestière ; il contribue considérablement à la faible confiance des observateurs extérieurs (à l'intérieur, tout comme à l'extérieur de la société productrice), dans le sens qu'il s'agit d'un secteur qui n'est pas suffisamment contrôlé par le public.

Dans certaines situations, il existe d'autres moyens, en dehors de la gestion du secteur de la forêt, pour surveiller la performance des opérateurs industriels qui se sont vus accorder un accès privilégié aux ressources publiques. Ainsi, les médias ont tendance à se montrer très actifs dans des pays comme les Philippines et ils dénoncent souvent dans la presse la malhonnêteté des maires et des fonctionnaires. Soulignons toutefois que dans diverses sociétés de pays riches en forêts, la société civile n'a pas vraiment acquis ses "formes modernes " (en termes de presse, de radiodiffusion, d'ONG urbaines qui se font entendre), et les libertés démocratiques ne sont pas généralisées. Les sociétés riches en forêts ont souvent des populations habitant les forêts souvent limitées et sans pouvoir et qui de ce fait et pour d'autres raisons, peuvent bien ne pas avoir les capacités de la société civile pour s'engager auprès d'acteurs et d'agences nationaux.

On a introduit des mesures supplémentaires "pour se donner des marges de sécurité " dans ces environnements pour surveiller l'industrie forestière. Ces mesures constituent le 


\section{Encadré 2.2 Comment l'illégalité se manifeste-t-elle dans la foresterie ?}

Les symptômes typiques de pratiques illicites dans le secteur forestier incluent certains ou l'ensemble des faits suivants :

- L'illégalité dans l'octroi de concessions forestières (exemple : pots de vins pour l'obtention d'une concession);

- La déformation de la capacité et des propriétés de l'entreprise candidate ;

- Le non-respect des conditions contractuelles dans le cadre du développement de l'infrastructure associée (la construction d'une scierie, par exemple) ;

- Le non-respect des droits des communautés indigènes (exemples : droit à la résidence, à la chasse ou à la récolte d'autres produits forestiers que le bois ; droit de commencer une culture itinérante) dans les domaines de la concession ;

- L'exploitation du bois hors des limites des zones spécifiées dans les contrats ;

- La mauvaise identification d'espèces (en général des espèces de grande valeur enregistrées comme étant des variétés de faible valeur) ;

- Le non-respect des conditions des plans de gestion forestière et les cahiers des charges (relatifs, par exemple, aux nombres et aux circonférences des spécimens supprimés) ainsi que les exigences relatives à la consultation du public et au respect des besoins locaux;

- Le non-paiement des droits et des taxes dus ;

- Le bouleversement des intentions de la loi et des cadres réglementaires par le biais de pratiques comme l'abus de l'évaluation des prix des permis de récupération et de transfert ;

- La non-conformité aux normes relatives aux problèmes sociaux et aux questions d'infrastructure (concernant par ex. la qualité des routes, des ponts ou des cliniques construits) ;

- Le non-respet des normes ou des prescriptions environnementales (en matière de pollution, par exemple);

- Le non-respect des normes de travail nationales ou industrielles ;

- La corruption de fonctionnaires ou d'autres individus dans le but d'ignorer les points ci-dessus; et

- L'utilisation de bois bruts de grumes illégaux dans les activités de transformation.

principal sujet de ce livre. Les «systèmes de contrôle des forêts " de routine visent bien sûr (tout comme d'autres éléments de l'administration forestière) à servir les objectifs de vérification, même s'ils n'y répondent pas toujours. L'intérêt actuel de la vérification de la légalité découle de la fréquence de cet échec. Cela implique un niveau de formalisation différent du contrôle routinier en tant que domaine fonctionnel de la gestion de la forêt et l'on peut, généralement le considérer comme complémentaire en termes institutionnels. Ce que de telles mesures supplémentaires impliquent dépendra en grande partie du contexte spécifique. Elles peuvent entraîner des activités par les organismes 
de surveillance agissant au nom de l'Etat ou encore, des tentatives plus systématiques d'établir une légitimité et une confiance dans les travaux de l'administration forestière qui fait participer diverses instances au sein du secteur forestier tout comme à l'extérieur, ou un hybride des deux. Elles peuvent impliquer des missions routinières de surveillance par des catégories spécifiques de personnel, des audits périodiques visant à vérifier le bon fonctionnement des systèmes ou des interventions ponctuelles dont l'objectif sera une évaluation de l'efficacité des contrôles de routine par des observateurs extérieurs, des équipes de spécialistes, ou des groupes de pairs. Dans chaque cas, la caractéristique essentielle est le fait qu'il existe un élément de doute important quant au fonctionnement des structures et systèmes normaux, et la reconnaissance, par des acteurs importants, de la nécessité de prendre des mesures supplémentaires et exceptionnelles pour appréhender ce doute.

\subsection{La vérification et sa terminologie}

Les mesures correctives à adopter dépendent beaucoup du contexte et des intérêts des principaux acteurs. Ces facteurs sont définis plus loin (voir Chapitres 4 et 25). La caractérisation des fonctions de façon adéquate et sans équivoque présente certaines difficultés d'ordre terminologique. Un des problèmes étant qu'il n'existe aucune instance dans le secteur forestier où la conformité légale doit être confirmée par le bais d'une " décision de vérification " et par l'émission d'un " certificat de vérification ". Il existe toutefois un système de vérification exécutable (ou ce système a en tout cas existé pendant un certain temps) dans des situations diverses ; ce système toutefois n'a (ou n'avait) pas le degré de formalisation requis par une telle « décision " de vérification.

La théorie que les systèmes de vérification pourraient fonctionner indépendamment des conditions de la décision de vérification est basée sur la notion selon laquelle la vérification finit par aboutir à la légitimité. La "légitimité ", dans le sens d'une garantie que le contrôle et les autres fonctions qui font l'objet d'un examen minutieux fonctionnement en fait de façon satisfaisante, ou, du moins, de façon assez satisfaisante pour rassurer leurs détracteurs. Plusieurs cas passés en revue dans la Partie B soulignent cette revendication. Le Costa Rica, l'Equateur, les Philippines et peut-être le Cameroun et la Malaisie en sont des illustrations. Comme le montrera le bilan, au Chapitre 3, de certains systèmes de vérification extra sectoriels pour la plupart liés à des traités et des conventions internationaux, cette incertitude terminologique et fonctionnelle est typique du secteur forestier et provient de l'absence d'un accord général et contraignant à l'échelle internationale, voire mondiale. Il s'agit d'une incertitude que l'on pourra résoudre progressivement au fur et à mesure que les systèmes de vérification apparaissent et se renforcent.

Certains cas de très grande envergure ont impliqué des agences extérieures opérant en tant qu'examinateurs indépendants des services nationaux de contrôle et des industries forestières. Toute une terminologie a été utilisée pour caractériser ce rôle, "observateurs indépendants " et "contrôleurs indépendants des forêts" (CIF) étant les deux dénominations les plus courantes. Une première étude des membres de l'équipe de VERIFOR avait 
fait la distinction entre " contrôle forestier indépendant " et " contrôle extérieur ", la distinction étant fondée sur la mesure dans laquelle l'agence en question avait passé un contrat avec l'autorité nationale (Brown et al. 2004). Cette distinction peut être illustrée par les travaux de surveillance des forêts de deux ONG internationales consacrées aux droits environnementaux. Dès lors, on peut considérer Global Witness comme étant un contrôleur indépendant au Cambodge (engagé en tant qu'observateur "officiel » travaillant sous contrat pour le Gouvernement royal du Cambodge, quoique financé par un bailleur de fonds), alors que l'Agence de recherche sur l'environnement (ARE) fait fonction de contrôleur extérieur en Indonésie (agissant sans rôle officiel dans le pays, bien qu'étant, elle aussi, financée par un bailleur de fonds).

En dépit de cet usage, toutefois, le terme d' " observateur indépendant » est sans doute une description plus appropriée que " contrôleur indépendant " dans les exemples où Global Witness a été actif (Cambodge, Cameroun et Honduras) ; peut-être pourra-t-on le retenir comme label pour les opérations spéciales de ce type. Le " contrôle » implique une routine, une permanence et des activités entreprises essentiellement par les cadres (voir par ex. Casley et Kumar 1987). A noter que le contrôle est mené à l'interne et systématiquement par tout le personnel de terrain à des fins de gestion interne. Il est limité à l'estimation des activités de routine et ne dépend pas de connaissances spécialisées, de comparaisons extérieures ou de jugements de valeurs. Ceci, par opposition à "l'évaluation ", qui sera souvent moins fréquente et conçue pour faire des comparaisons lorsque les délais sont longs et les influences extérieures diverses ; l'attribution de la causalité est une opération complexe et il faut faire des jugements de valeur. L'évaluation incarne ce sens de causalité multiple et la complexité du processus d'évaluation.

Le terme d' " auditeur indépendant " sert parfois à caractériser le travail des observateurs indépendants. L'" audit ", toutefois, tend à impliquer une évaluation non continue de la performance. Par ailleurs, les audits sont souvent davantage orientés vers les systèmes plutôt que vers la performance et cherchent à vérifier que les systèmes appropriés sont mis en place et qu'ils fonctionnent plus ou moins convenablement, plutôt que d'analyser des activités ou des violations spécifiques. Dans la littérature financière, d'où vient une grande partie de la terminologie de l'audit, ces vérifications sont divisées en deux catégories principales en fonction du but recherché, les audits internes servant à l'organisation mandatrice, ou " objet de l'audit " et les audits extérieurs pour les objectifs des parties extérieures à l'objet de l'audit (en exemple, les dispositions légales d'une loi nationale sur les sociétés).

Ces genres d'examens rigoureux impliquant les contrôles des droits environnementaux ne sont sans doute pas des types de contrôle ou d'audit. Il s'agirait plutôt d'examens à buts plus précis concernant les capacités des agences de contrôle nationales, avant de plaider (si nécessaire) pour leur réforme. Dans l'exemple de Global Witness, cette dernière démarque impliquait l'annonce des résultats sur le propre site Internet de l'Organisation, où ils étaient repris et utilisés par son équipe d'avocats. Comme le constate van Midwoud, la force acquise par le contrôleur sur l'état producteur dans les situations où il travaille apparemment sur mandat de l'état producteur, alors qu'il 
fait partie en fait d'une série de conditionnalités du bailleur de fonds, signifiait, pour de nombreux observateurs, qu'il s'approchait désagréablement de la surveillance et du contrôle d'un partenariat gouvernemental bilatéral (2006, p. 8). Tout ceci était extérieur à une routine de surveillance ordinaire (même si l'ONG a également commenté cette routine). Mieux vaudrait réserver le label " observateur indépendant » pour de telles dispositions exceptionnelles et garder le terme de " surveillance " pour les activités plus courantes entreprises par l'unité de contrôle interne ; le terme " audit " désignera dès lors les évaluations périodiques à cycle beaucoup plus long, généralement, avec une plus grande inclination à évaluer les fonctions des systèmes de façon identique aux " audits financiers » ou à l'accréditation ISO. Cependant, cette distinction n'est pas courante dans la littérature de la politique, comme en témoignent les sections suivantes.

\subsection{La vérification, comparée à la certification}

Le dernier champ d'ambiguïté terminologique potentielle à considérer afin de pouvoir préparer les arguments qui suivront concerne la différence entre la vérification et la certification. On a noté au Chapitre 1 que la multiplication de la certification dans le secteur privé avait précédé l'intérêt pour la vérification et que les restrictions de la certification aident à expliquer l'intérêt croissant pour la vérification lié, notamment, à la réforme de la gouvernance. La certification prospère surtout là où les problèmes de gouvernance ont déjà été réglés, mais elle ne s’est pas avéré un instrument très fort pour les résoudre elle-même.

La vérification, dans ce livre, renvoie aux activités entreprises par toute une diversité d'agences (secteur public, ONG et secteur privé, souvent ensemble) au nom de l'Etat et dans le but de vérifier et d'évaluer les processus officiels d'utilisation et d'évaluation des ressources. Elle possède un certain nombre de caractéristiques communes avec la certification forestière, notamment le fait qu'elle peut impliquer une vérification par une tierce partie et qu'elle représente une tentative de relier la gouvernance forestière à des normes environnementales ou à d'autres normes. Néanmoins, elle differe de plusieurs façons de la certification (voir Tableau 2.1).

Le Chapitre 22 se penche davantage sur le contraste entre la certification et la vérification et évalue jusqu’à quel point les deux processus peuvent se rapprocher utilement.

\subsection{Les propositions de I'UE pour les Accords de partenariat volontaire}

Les Accords de partenariat volontaire (APV) constituent une importante stimulation en faveur d'une pratique de vérification, chose qu'ils promeuvent aussi avec force. Ils font l'objet de négociations entre l'UE et un certain nombre de pays producteurs. Cette nouvelle évolution sera vraisemblablement une importante innovation dans le cadre des partenariats internationaux. Bien qu'aucun APV n'ait encore été conclu, un élément de «CIF » sera sans doute revendiqué par l'UE. Dans sa Note d'information $n^{\circ} 7$ sur la 
Tableau 2.1 La certification, comparée à la vérification

\begin{tabular}{|c|c|c|}
\hline Attribut & Certification & Vérification \\
\hline Mandat & $\begin{array}{l}\text { Procédure volontaire initiée par } \\
\text { l'industrie. }\end{array}$ & $\begin{array}{l}\text { Services entrepris au nom de } \\
\text { l'Etat pour instaurer une surveillance } \\
\text { de l'industrie obligatoire, instituée } \\
\text { par la loi. }\end{array}$ \\
\hline Instigateur & $\begin{array}{l}\text { Plan du Conseil de soutien de la } \\
\text { forêt (FSC) qui fut lancé par un } \\
\text { groupe de particuliers, d'ONG et } \\
\text { de détaillants en bois redoutant } \\
\text { d'éventuelles interdictions du bois } \\
\text { d'œuvre tropical. Forte autorité des } \\
\text { ONG au niveau international. Autres } \\
\text { plans menés par des ONG et (ou) par } \\
\text { l'industrie. }\end{array}$ & $\begin{array}{l}\text { Lancée par l'Etat (et dans certains } \\
\text { cas, par les bailleurs de fonds) } \\
\text { pour garantir la légalité et certifier } \\
\text { la gouvernance adéquate sur le } \\
\text { territoire national. Forte présence du } \\
\text { bailleur de fonds officiel au niveau } \\
\text { international. }\end{array}$ \\
\hline Objectif actuel & $\begin{array}{l}\text { La gestion durable des forêts sous } \\
\text { condition de gouvernance adéquate. }\end{array}$ & $\begin{array}{l}\text { Conformité légale en cas de } \\
\text { mauvaise gouvernance. }\end{array}$ \\
\hline $\begin{array}{l}\text { Orientation des } \\
\text { systèmes ou de } \\
\text { la performance }\end{array}$ & $\begin{array}{l}\text { Peut varier, bien que disposant } \\
\text { souvent d'une forte dimension } \\
\text { d'audit. }\end{array}$ & $\begin{array}{l}\text { Liée aux systèmes, aux opérateurs et } \\
\text { aux expéditions. }\end{array}$ \\
\hline Additionnalité & $\begin{array}{l}\text { S'ajoute aux exigences du } \\
\text { gouvernement. }\end{array}$ & $\begin{array}{l}\text { Vise à assurer que certaines } \\
\text { conditions légales sont satisfaites par } \\
\text { le gouvernement. }\end{array}$ \\
\hline Coûts & $\begin{array}{l}\text { Les coûts sont supportés par le } \\
\text { secteur (bien que les industriels } \\
\text { reçoivent souvent, dans la pratique, } \\
\text { des subventions des bailleurs de } \\
\text { fonds et des ONG) }\end{array}$ & $\begin{array}{l}\text { Bien que pouvant varier, on peut } \\
\text { anticiper que les coûts peuvent aussi } \\
\text { être en partie supportés par l'Etat ; } \\
\text { les subventions par des acteurs } \\
\text { internationaux et des bailleurs } \\
\text { de fonds bilatéraux seront aussi } \\
\text { justifiées lorsqu'il s'agit de biens } \\
\text { publics internationaux. }\end{array}$ \\
\hline Marque & $\begin{array}{l}\text { Son but, essentiellement } \\
\text { commercial, est d'obtenir un label } \\
\text { vert ou d'aller dans ce sens. }\end{array}$ & $\begin{array}{l}\text { En théorie du moins, il n’a pas la } \\
\text { fonction de marque (bien qu'il puisse } \\
\text { en fin de compte en offrir une). }\end{array}$ \\
\hline $\begin{array}{l}\text { Couverture } \\
\text { géographique }\end{array}$ & $\begin{array}{l}\text { Environ } 96 \% \text { des forêts certifiées } \\
\text { sont tempérées (Poku-Marboah et al. } \\
\text { 2003), avec un fort pourcentage de } \\
\text { plantations forestières. }\end{array}$ & $\begin{array}{l}\text { Les systèmes qui fonctionnent sont } \\
\text { très limités. }\end{array}$ \\
\hline
\end{tabular}

FLEGT (Commission Européenne 2007), l'Union Européenne préconise un « système d'assurance de légalité du bois » comportant les cinq éléments suivants :

1. Une définition du bois d'œuvre produit légalement décrivant les lois à respecter pour qu'un permis soit accordé et que les vérifications requises pour déterminer la conformité soient effectuées; 
2. Un système de traçage du bois d'œuvre, depuis les opérations en forêt jusqu'à l'exportation, système qui exclue de la chaîne d'approvisionnement le bois d'origine inconnue ou illégale ;

3. Un système de vérification de la conformité comportant tous les éléments de la définition de la légalité et le contrôle de la chaîne d'approvisionnement ;

4. L'autorisation d'exporter des produits de bois d'œuvre, sur un marché approuvé ou sur la base d'une expédition individuelle ; et,

5. Une surveillance indépendante pour garantir, à toutes les parties intéressées, que le système fonctionne comme prévu et qu'il garde sa crédibilité.

Ce cinquième élément qu'est la « surveillance indépendante " pourrait s'avérer différent $\mathrm{du}$ CIF entrepris récemment par les contrôleurs environnementaux (décrit ci-dessus en tant qu' " observation indépendante »). Bien qu'il faille déterminer la nature exacte de cette surveillance indépendante au cas par cas, elle sera sans doute beaucoup plus routinière que ne l'est le type de travail d'investigation observé ci-dessus. Le genre de "CIF d'investigation " entrepris par les contrôleurs des droits de l'environnement dans des pays tels que le Cambodge, le Cameroun, Honduras et l'Indonésie revêtait un caractère fondamentalement sceptique, à tel point que les organisations ont répondu en s'attendant à y trouver des problèmes, insistant dans leurs rapports sur de possibles solutions. Les types de CIF commandés dans le cadre des APV auront sans doute un style moins ambitieux et mettront l'accent sur le côté positif. Cela ne signifie pas nécessairement que s'émousse l'aspect critique de la surveillance, mais que l'accent sera davantage mis, sans doute, sur la maîtrise et la gestion des mauvaises nouvelles et sur la mise en place rapide de mesures de redressement pour conserver la confiance dans le secteur, plutôt que sur la dénonciation de systèmes qui fonctionnent mal et de divulguer leurs bases politiques (ce qui est peut-être la méthode privilégiée de certaines ONG de droits environnementaux). Il est possible, aussi, que les gouvernements producteurs imposent certaines conditions pour limiter l'utilisation des données que les agents de surveillance ont découvertes et dont ils pourraient se servir. Dans ce sens, la " surveillance indépendante " se révélera être une bonne méthode pour décrire le type d'activités dans le cadre des APV, et le distinguer du travail de détective international (l'" observation indépendante ") effectuée par des ONG de droits environnementaux.

Un autre aspect des systèmes de vérification de l'UE liés à l'APV, et dont on ne peut pas encore parler avec autorité, concerne la question des systèmes de vérification de l'opérateur, ou des systèmes de vérification basés sur l'expédition. Les systèmes de vérification mis en place à ce jour se concentrent largement sur l'évaluation directe de l'expédition, soit de l'autre côté de la frontière, soit dans des régions sujettes aux problèmes. Pour être sûr de la réussite des systèmes d'assurance de la légalité du type APV, il devra y avoir un effort important dans le sens de l'accréditation de l'opérateur, soutenue par un CIF périodique. Cela s'explique par la forte vocation commerciale des accords en question en contraste avec la tendance plus environnementale de diverses dispositions d'observation existantes. L'accréditation de l'opérateur est plus favorable aux besoins quotidiens du commerce qu'une approche qui repose davantage sur l'expédition. Le paradoxe étant 
que les gouvernements producteurs se verront probablement obligés de passer par la vérification en raison de l'importance du commerce international pour leurs industries forestières ; mais cette vérification risquerait également de compromettre ce commerce du fait qu' elle augmente considérablement les risques d'examen rigoureux de la performance de l'industrie. La licence accordée à l'opérateur est sans doute l'option privilégiée des gouvernements producteurs : en effet, elle sera moins encline à mettre en danger les transactions, et aussi, moins coûteuse en termes de finances et de gestion. Les opérateurs seraient autorisés à exporter le bois d'œuvre puis soumis à des vérifications régulières de leurs systèmes de contrôle et ce, afin de préserver leur statut d'opérateur agréé. Les contrôles en question pourraient impliquer une chaîne complète de contrôles de la conservation " de la forêt à l'exportation " (comme le font actuellement les entreprises finlandaises qui opèrent en Russie) ou, autrement, des contrôles de la production en amont dans le cadre d'un système national de traçage et des contrôles d'exportation distincts au niveau de l'opérateur individuel. De quelle façon que ce soit, l'arrangement perturbera moins les modèles ordinaires de commerce que les contrôles systématiques des expéditions et fonctionnera de telle sorte qu'il ressemblera aux systèmes de certification tels qu'ils fonctionnent actuellement. Les sceptiques, toutefois, répliqueront que l'autorisation de l'opérateur est plus sensible à l'influence politique et particulièrement suspecte dans les conditions de gouvernance faible, là où la vérification s'avère des plus urgentes.

\subsection{En résumé}

Le nouvel accent mis sur la légalité du bois d'œuvre vendu au niveau international en tant que mesure agissant sur la demande reconnaît à la fois le potentiel des marchés de consommateurs à influencer le comportement industriel et l'échec de nombreuses tentatives antérieures du côté de l'offre de réformer la gouvernance forestière. Les connaissances permettant de développer des systèmes de vérification du bois légal sont toujours en processus de développement, mais sont d'ores et déjà disponibles auprès d'une variété de sources, chacune de ces dernières donnant lieu à une terminologie distincte. Aucune d'elles n'est capable de fournir à elle seule un mécanisme d'assurance de légalité qui fonctionne ; en groupe, toutefois, elles permettent de mettre en place les fondements.

Récapitulons : il existe cinq sources d'apprentissage essentielles. Les premières connaissances proviennent de divers systèmes de gestion et de surveillance des forêts qui ont déjà été mis en place dans les pays producteurs du monde entier ; bien qu'ils ne soient pas tous très efficaces, ils sont intéressants et instructifs. Le second groupe de connaissances provient de l'expérience des contrôleurs des droits environnementaux (" observateurs indépendants »), tels que Global Witness et l'Agence de recherche sur l'environnement (ARE). Le troisième volet représente une décennie au moins d'expérience en certification de la forêt. De nombreux obstacles auxquels s'est vu confronter le mouvement de la certification (l'instauration d'autorités de gouvernance, l'interprétation de la conformité avec, dans de nombreux cas, des lois nationales fort complexes et diverses, la sélection de normes de légalité qui abordent les problèmes sociaux tout aussi bien que les contraintes industrielles, mettant en œuvre des systèmes de traçage du bois, s'occupant de conflits 
d'intérêts et la politique concernant les revendications basées sur les taux) sont très similaires à ceux auxquels la vérification fait face à présent. Le quatrième volet provient des processus internationaux qui se développent autour du thème de la vérification, notamment les APV de l'UE. Nous passerons en revue tous ces faits dans la Partie B.

Le dernier groupe, enfin, est celui de l'expérience extra sectorielle. Hors du secteur forestier il existe plusieurs modèles et approches didactiques. Tous n'ont pas été fructueux et ils ne se transferent pas aisément hors de leur cadre d'origine. Ils montrent bien cependant l'importance d'une conception claire des "fonctions régaliennes " que l'Etat doit conserver (règlement, jugement, sanction), et les activités susceptibles d'être externalisées. Ils mettent aussi l'accent sur l'importance d'une plateforme d'engagement public solide comme moyen pour assurer la force et le sens de la propriété qui seront requis dans le cas où ils devront se maintenir dans des environnements politiques très mouvementés. Ils ont tous en commun l'implication de tout un éventail de parties prenantes dans les décisions de vérification, et la crédibilité entre l'Etat, l'industrie et la société civile qui résulte des contrôles et des évaluations. Nous allons nous consacrer à présent à ces expériences extra sectorielles. 


\section{Chapitre 3}

\section{Leçons à tirer de l'expérience extra- sectorielle}

\subsection{La gamme d'expériences de vérification}

Ce chapitre prépare le terrain pour un examen détaillé des études de cas du secteur forestier en passant en revue les expériences extra sectorielles des activités de vérification, afin d'identifier certains principes susceptibles d'avoir des applications intersectorielles.

L'idée de vérifier les activités de l'exploitation forestière remonte sans doute très loin dans l'histoire du commerce du bois d'œuvre; il n'en demeure pas moins que la vérification du secteur de la forêt est un effort relativement récent dans un sens plus conceptualisé et officiel, lorsqu'on la considère comme étant un sujet qui mérite un intérêt sérieux, tant à l'échelle nationale que sur le plan international. Même les systèmes les plus établis de vérification du bois tropical ont moins de dix ans. Aucun, toutefois, ne peut être décrit comme pleinement établi et adulte, tous souffrent encore de défis périodiques qui menacent leur viabilité. Ces expériences représentent une épreuve d'apprentissage utile, bien qu'il faille faire preuve de prudence si l'on veut dériver des principes généraux d'action de systèmes encore en formation.

Il n'en demeure pas moins que la vérification hors du secteur forestier est bien mieux établie et largement étudiée, notamment dans les zones d'activité où la communauté internationale est bien implantée et où elle a le mandat d'interférer dans les affaires des états souverains. Ces exemples offrent des opportunités considérables de tirer des enseignements et de les partager. Ce chapitre vise à tirer certaines de ces leçons. Certains accords de référence : la Convention sur le commerce international des espèces sauvages de faune et de flore menacées d'extinction (CITES) ; le Traité d'interdiction des essais nucléaires (TIEN) ainsi que d'autres traités sur le contrôle des armements ; le Processus Kimberley pour les diamants ; le Protocole de Montréal de la Convention de Vienne pour la protection de la couche d'ozone ; et le Protocole de Kyoto pour la convention cadre des Nations Unies sur le changements climatique (CCNUCC). Parmi d'autres accords importants plus localisés, on citera le travail des ombudsmans dans divers parlements nationaux, les agences de normalisation des produits alimentaires en Europe de l'ouest et les contrôleurs électoraux internationaux à divers endroits. La littérature concernant ces accords étant souvent spécifique aux situations, on est obligé de chercher les similitudes au niveau des caractéristiques analytiques, plutôt que dans les règles courantes.

Les divers systèmes de vérification ont des objectifs différents et souvent multiples dont un ou plusieurs ci-dessous :

- Appuyer l'application de la loi et la «bonne gouvernance "; 
- Instaurer la confiance du marché ;

- Maintien de l'ordre à l'échelle international, contrôles de l'environnement et protection publique ;

- Garantie d'efficacité et de rapport coûts/avantages ;

- Fonctions judiciaires et quasi judiciaires (exemples : l'ombudsman et le rapport judiciaire).

Ces objectifs peuvent être à la fois imbriqués et s'appuyer mutuellement. La conformité au traité international d'interdiction peut donc avoir pour objectif immédiat d'assurer la conformité des parties internationales, étant bien entendu que le but principal est d'assurer le maintien des normes de santé et de sécurité. Ce dernier aide à faire le lien entre les préoccupations nationales et internationales et il assure l'approvisionnement public à large échelle. Dans d'autres cas, les buts sont parfois plus complexes. Exemple : les régulateurs du service public détiennent d'importantes fonctions de vérification liées au caractère non commercial des services impliqués. Le caractère des services offerts et des économies d'échelle dans leur apport signifie que les services publics tels que l'eau, l'énergie et le transport ne peuvent être commercialisés sur une base entièrement compétitive ; dès lors, les régulateurs du service public assument un rôle important, qui est d'assurer non seulement que les services seront livrés comme promis, mais aussi, que le prix et la qualité se trouvent également sous les genres de pression qui seraient autrement garantis par la concurrence du marché. En contraste nous avons le travail des ombudsmans qui est largement impliqué dans le processus judiciaire : les médiateurs vérifient que les procédures convenues sont bien respectées et que ces mêmes procédures servent bien les objectifs visés. Les principaux buts de la vérification du bois d'œuvre comportent trois points au moins de la liste ci-dessus : l'instauration de la confiance du marché, la mise sur pied de contrôles de l'environnement et la promotion d'une bonne gouvernance. La diversité des buts fait une allusion aux défis à venir.

Les instruments de vérification sont d'une grande diversité, dépendant du caractère du produit pris en considération, de la nature des parties de l'accord et des conditions de l'accord qui en régit l'utilisation. Le caractère du produit affecte aussi sa vérifiabilité, dans les sens de sa possibilité de contrôle (c.-à-d. la facilité d'observation de l'activité ou de la substance) et la possibilité d'évaluation (la capacité de comparer la performance observée à un standard) (Greene 1994, p. 4). La vérification des émissions de carbone exige donc des aptitudes et des jugements différents de ceux qui s'avèrent nécessaires à la vérification de diamants commercialisés ; ces deux sont aussi fondamentalement différents en termes de possibilités de contrôle et d'évaluation du commerce du bois d'œuvre.

Les mécanismes de vérification doivent correspondre à leurs objectifs, lesquels reflèteront les intérêts sous-jacents des parties impliquées. Si la nécessité de vérification provient d'une demande de l'industrie, il faudra alors que le mécanisme en soit acceptable ; il en est de même là où les forces motrices viennent de la société civile. Dans le cas où la demande provient du public tout en étant liée au processus démocratique (comme pour les médiateurs), les objectifs devront alors appuyer ce processus et non le miner sous peine de perdre la crédibilité d'un groupe important. Dans le cas du bois d'œuvre, il s'avère 
que la demande provient essentiellement de bailleurs de fonds et de groupes de la société civile (plus particulièrement mais pas uniquement dans les nations consommatrices).

Le Tableau 3.1 met en relief un échantillon d'expériences extra sectorielles qui ont été passées en revue pour cette étude ; il les compare à l'industrie du bois d'œuvre sous l'angle de la facilité et des coûts de vérification.

\subsection{La vérification dans les conventions et les traités internationaux}

Le Chapitre 2 a établi que les notions de doute et de scepticisme jouent un rôle central dans la notion de vérification, et qu'un sens d'additionnalité est implicite dans le concept. Cette additionnalité est liée aux procédures à appliquer et aux publics dont elles servent les intérêts. Cela lui donne plus de sens et de valeur au-delà des mécanismes conventionnels de contrôle forestier, dont les objectifs se limitent aux besoins d'une seule partie (en l'occurrence, le gouvernement hôte). L'approche à partir de la théorie de la vérification du traité institutionnalise considérablement ces éléments d'incertitude et d'additionnalité comme le suggère la définition ci-dessous (reprise d'un ouvrage sur le désarmement et la limitation des armes) :

"La vérification est un processus qui couvre toute les mesures visant à permettre aux parties de conclure un accord afin d'établir que le comportement des autres parties n'est pas incompatible avec les obligations qu'ils assument dans le cadre de cette convention.»

(Sur 1991, p. 13)

L'accord en question peut être multilatéral, régional ou bilatéral ; il sera lié à des évaluations de conformité plus généralisées comportant des standards (par exemple, la loi et le processus légal, comme dans le cas des ombudsmans parlementaires). Une grande partie du débat concerne les accords multilatéraux, même si les principes analytiques s’appliquent plus généralement.

Cette définition a un certain nombre de caractéristiques en commun avec d'autres qui sont standards sur le terrain (voir par ex. Greene 1994). Vue sous cet angle, la vérification doit être considérée comme :

- Un processus d'investigation et de validation; et

- Un processus large et à plusieurs couches.

La théorie de la vérification vue dans le contexte d'un traité se concentrera sur la dimension du processus et sur la vérification en tant que mécanisme complexe plutôt que sur un acte d'inspection technique.

Pourquoi faut-il qu'il y ait cette complexité ? La réponse se trouve dans le fait qu'il existe, pour de nombreux traités et accords, un certain nombre d'éléments dans les situations de production, et les contextes politiques de leur opération qui provoquent des intérêts 


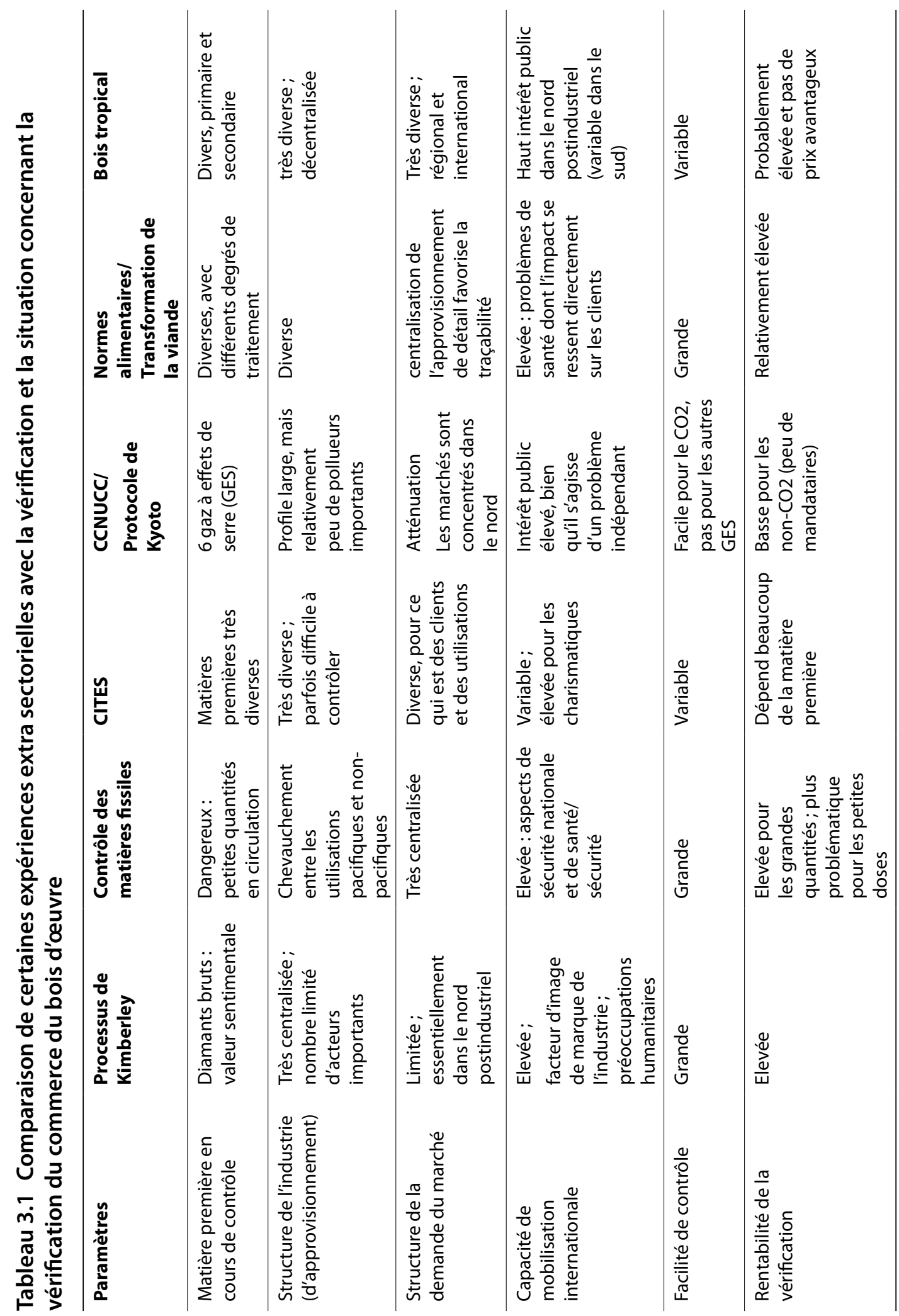


en conflit et requièrent une approche complexe. Les principaux domaines de dispute apparaissant généralement dans la mise en œuvre de tels traités ne sont pas liés aux propositions techniques dont la validité pourra être établie "objectivement "; ils ont davantage rapport aux conflits d'intérêt entre les parties et ils se régleront uniquement par le biais de négociations et de compromis.

La complexité des systèmes de vérification a tendance à être une fonction d'un certain nombre d'influences dont certaines sont liées entre elles, notamment :

- Le nombre de protagonistes ayant un intérêt dans l'accord, et par conséquent, la catégorie d'autorités requises pour communiquer un avis avant de parvenir à une décision de vérification ;

- La variété des intérêts des acteurs et leurs pouvoirs relatifs ;

- Le niveau de confiance mutuel des protagonistes ;

- La mesure dans laquelle la décision de vérification peut être caractérisé en termes absolus ou relatifs ; par exemple, la mesure dans laquelle les décisions sont basées sur des considérations légales et une interprétation stricte de textes légaux ou de points plus subjectifs avec un degré plus élevé d'incertitude, et

- Les limites de tolérance dans le système de diagnostic (c.-à-d. la précision de la vérification des faits du cas).

Dans chacun des cas, la règle opérationnelle veut que : plus la variable est complexe, plus les conditions de vérifications seront sévères. Logiquement, plus il y a d'acteurs et plus leurs points de vue sont divers, plus il leur sera difficile de se mettre d'accord. Parallèlement, une décision basée sur la simple inspection d'un fait observable differre manifestement, du point de vue conditions de vérification, d'une autre qui dépend d'un jugement plus évaluatif de réalités et d'intentions multiples. L'évaluation de la conformité d'une installation pour le traitement de la viande aux normes de production courantes est une tâche moins exigeante que l'évaluation des intentions d'un gouvernement impopulaire qui investit beaucoup d'argent dans la technologie nucléaire à des fins ostensiblement pacifiques. Lorsque la vérification est basée sur un certain nombre de considérations et qu'elle comporte un taux élevé d'incertitude, le processus comportera sans doute une composante fortement politique. De telles considérations peuvent englober, par exemple, non seulement des règles légales, mais aussi les intérêts futurs des parties mêmes, leurs évaluations de ce qu'elles, et d'autres parties puissantes, considèrent comme acceptables et les estimations des divers acteurs en ce qui concerne les coûts et les bénéfices probables. Pour ce qui est des limites de la tolérance, la mesure dans laquelle les principales violations differrent matériellement dans leurs implications des moins importantes conditionnera également la complexité de la réaction à la vérification (les contrôles des matières fissiles constituent un cas de fait évident). Un problème mineur d'étiquetage de produit ou de transgression des limites opérationnelles est d'un ordre autre qu'un problème de violations d'accords systématiques et graves, avec mauvaise intention manifeste et effets potentiellement profonds. 


\subsection{Qu'est-ce qui rend la vérification efficace ?}

La littérature extra sectorielle nous permet d'identifier certains des principes qui aboutissent à une vérification effective. Les principes examinés ci-dessous ne sont pas tous aptes à être respectés de façon égale dans chaque contexte de vérification ; ils ne sont pas non plus tous aussi importants pour la vérification du bois d'œuvre légal. Lorsque ces principes font défaut ou sont faibles, la tâche de la vérification efficace sera plus difficile, augmentant dès lors la nécessité de mesures compensatoires.

L'on pourra identifier dix principes généraux pour une vérification efficace dans la littérature extra sectorielle :

$\mathbf{1}^{\text {er }}$ principe La réciprocité ;

$2^{\text {e }}$ principe La faculté d'éviter la migration vers les non-partis ;

$3^{\text {e }}$ principe Une définition claire des problèmes que le système de vérification est supposé traiter ;

$4^{\mathrm{e}}$ principe L'adoption d'une approche de systèmes et la focalisation sur la distribution et l'équilibre des pouvoirs ;

$5^{\mathrm{e}}$ principe Une large participation dans les processus qui aboutissent à la décision de la vérification ;

$\boldsymbol{6}^{\mathbf{e}}$ principe La précision des normes d'évaluation;

$7^{\mathrm{e}}$ principe L'apport d'incitations au rapport et à la conformité ;

$8^{\text {e }}$ principe Une supervision autonome ;

$\mathbf{9}^{\mathbf{e}}$ principe L'inclusion de tous les maillons de la chaîne de garde et des efforts particuliers pour garantir les phases les plus vulnérables;

$10^{\mathrm{e}}$ principe L'intégration d'approches en faveur des pauvres dans la conception des systèmes de vérification.

Ces dix principes seront passés en revue ci-dessous. Huit études de cas illustrant ces principes en action sont également examinées dans les encadrés 3.1 à 3.8 .

\section{$\mathbf{1}^{\text {er }}$ Principe : La réciprocité}

Lorsqu'un accord est pleinement réciproque, dans le sens qu'il lie toutes les parties de la même façon, sa vérification sera dépolitisée jusqu’à un certain degré. C’est le cas le plus généralement (en principe, mais pas toujours dans la pratique) en ce qui concerne les accords commerciaux ; ça l'est moins fréquemment pour ce qui est des conventions liées aux armements et à l'environnement. Certains traités internationaux importants ne sont pas intégralement réciproques et certains acteurs s'en tiennent à l'écart pour des raisons historiques. Le Traité d'Interdiction des essais nucléaires et la CCNUCC et son Protocole de Kyoto (que certains Etats importants tels que les Etats-Unis n'ont toujours pas ratifié) sont des exemples de traités non réciproques. Cela donne à leur concrétisation et à leur contrôle un caractère hautement politique.

Le Processus de Kimberley illustre de façon intéressante une des voies possibles pour l'établissement de la réciprocité dans une situation de méfiance mutuelle par le biais d'un mécanisme d'évaluation par les pairs (voir Encadré 3.1). Cela implique un 
certain nombre de participants d'Etats producteurs et de représentants de l'industrie et d'ONG. Le Protocole de Montréal relatif à des substances qui appauvrissent la couche d'ozone (Encadré 3.2) marque la reconnaissance de " responsabilités communes, mais différenciées » de tenir compte des besoins particuliers des pays en voie de développement. Une procédure d'ajustement a ensuite autorisé les Etats à varier le rythme de leur abandon progressif sans nécessité d'amendements du traité (Brack 2003).

\section{Encadré 3.1 Le Système de certification du processus Kimberley}

\section{Principes clés : réciprocité ; non-migration ; définition du problème ; participation large ; incitations à la conformité ; impacts sur les pauvres.}

Le Système de certification du processus Kimberley de 2003 (KPCS) pour les diamants bruts est un des résultats du "Processus Kimberley »; il vise à mettre fin au commerce de diamants à la source de conflits armés. Les éléments de base de l'accord sont les suivants : chaque participant s'engage à maintenir les contrôles internes des diamants bruts sur leurs territoires ; il interdit l'importation de diamants bruts, à moins qu'ils ne soient accompagnés d'un certificat du Processus de Kimberley délivré par un autre participant ; il déclare aux autorités d'exportation tous les paquets reçus sur son territoire et il présente régulièrement ses statistiques de commerce.

Le KPCS n'est pas un traité international juridiquement contraignant. II s'agit davantage d'un programme international de certification basé sur un minimum de normes; la décision dépend du consensus des parties. Une innovation dans son système de conformité : le mécanisme d'évaluation par les pairs. Les équipes évaluatrices comprennent des représentants de trois autres gouvernements, un délégué pour les ONG et un autre pour le secteur. Bien que de caractère purement volontaire, 18 évaluations ont été réalisées avant la mi 2005, tous les Etats au sein du KPCS en ayant demandé une. Le KPCS dispose par ailleurs d'une procédure de «plaintes » en vertu de laquelle tout participant ou observateur a la possibilité de communiquer au Groupe de travail du contrôle des informations sur la conformité de tout autre participant.

Malgré certaines appréhensions, la disposition relative à la prise de décision consensuelle s'est révélée être un des points forts du KPCS. Au début du traité, un système de vote aurait eu l'effet d'une « ligue » contre les membres ayant des points de vue opposés. Le risque qu'un membre important quitte la table de conférence était réel et il se serait avéré très destructif. Les conflits importants sont rares, même si certaines décisions constituent parfois le dénominateur commun le moins élevé.

En dépit de l'importance de la production de diamants alluvionnaires comme moyen de subsistance pour les pauvres dans certains pays, cet aspect s'est avéré difficile à traiter dans un processus où le souci majeur est de couper l'approvisionnement des Etats voyous. 


\section{Encadré 3.1 (suite)}

Quelques leçons de base apportées par le KPCS :

- La vulnérabilité des diamants à l'action du consommateur a aidé à regrouper autour de la table de négociation le secteur et plusieurs gouvernements réticents.

- Une forte pression médiatique nourrie par les ONG a contribué à maintenir le dynamisme.

- Un gouvernemement « champion » est important pour l'organisation des réunions, et I'Afrique du Sud a joué ce rôle.

- L'intérêt international (des panels d'experts des Nations Unies, la résolution adoptée l'Assemblée générale des Nations Unies ainsi que des références positives en faveur du Processus de Kimberley lors de deux conférences du G8) a aidé à maintenir le dynamisme et la légitimité.

- Le KPCS n'aurait pas été aussi important sans la forte participation de l'industrie. Si les gouvernements et (ou) les ONG avaient tenté de concevoir un système de certification, le résultat n'aurait sans doute jamais fonctionné. L'industrie avait cerné les problèmes et savait comment les appréhender.

- Le KPCS est « volontaire » ; les pays producteurs et vendeurs de diamants, par contre, devaient devenir membres, rendant l'adhésion pratiquement obligatoire.

Source : Smillie (2005)

\section{$2^{\mathrm{e}}$ Principe : La faculté à empêcher la migration vers les non-partis}

Si les parties, ou les participants, peuvent se rétracter d'un accord lorsque cela leur convient et sans encourir aucun coût important, la convention s'en trouve manifestement affaiblie et ceci a des répercussions sur sa capacité à évaluer la conformité et à amener les parties à honorer les demandes en question. Trait particulier du Protocole de Montréal, qui est un des traités les mieux réussis en matière de conformité et d'impacts : l'étendue de son acceptation internationale a considérablement limité la possibilité, pour les industries, de migrer vers les non parties (voir Encadré 3.2). De même, ce n'est qu'une fois que la CITES a atteint une certaine masse critique que l'on est parvenu à limiter les effets négatifs de l'influence des non parties. Un pas essentiel a été l'adhésion de Singapour à la CITES ; jusque-là, cet Etat avait entravé sérieusement la progression de la Convention en faisant office d'entrepôt important pour le commerce illégal (voir Encadré 3.3).

\section{$3^{\mathrm{e}}$ Principe : Une définition claire des problèmes que le système de vérification est supposé traiter}

Un système de vérification efficace sera vraisemblablement un système où les limites et les objectifs sont clairement définis. Par conséquent, la décision du Processus de Kimberley de se restreindre aux diamants alluvionnaires (une décision qui exclut les gisements des diamants de pipes kimberlitiques) était logique, du point de vue du problème 
(le rôle joué par cette industrie d'extraction à attiser des guerres et des conflits) qu'il devait appréhender. Certains facteurs, dont le rapport élevé poids/valeur, leur facilité d'extraction et l'indiscipline sur le marché mondial du diamant, font déjà des diamants alluvionnaires une véritable cible pour les régimes corrompus. Cette décision a également eu deux autres effets : tout d'abord, elle a permis l'adhésion de gros producteurs de diamants, qui sont particulièrement soucieux de leurs réputations internationales (et qui produisent essentiellement des diamants de gisement kimberlitique) ; ensuite, elle a

\section{Encadré 3.2 Le Protocole de Montréal}

\section{Principes clés : réciprocité ; non-migration ; sécurité à chaque étape ; large participation ; incitations à la conformité.}

Le Protocole de Montréal de 1987, relatif aux substances qui appauvrissent la couche d'ozone, est généralement considéré comme une convention sur l'environnement qui a particulièrement réussi et un modèle riche en leçons importantes pour la création d'autres traités. En vertu du Protocole, les dispositions commerciales ont eu pour effet une dissuasion double en matière de migration vers les non parties : premièrement, en refusant l'accès des non signataires aux substances qui appauvrissent l'ozone (dont l'approvisionnement était entre les mains d'un petit groupe de pays) et deuxièmement, en évitant que les industries ne migrent vers les non parties et accèdent indirectement aux marchés par leur truchement. II n'en demeure pas moins que la pression la plus forte pour l'obtention de la conformité a été les motivations commerciales et autres motivations disponibles, et susceptibles d'inciter les gouvernements et l'industrie à renoncer aux vieilles technologies polluantes pour les remplacer par d'autres, non polluantes cette fois (Barrett 1999).

Brack (2003) attribue le succès du Protocole à des facteurs divers, dont :

- Son aptitude à changer les connaissances et technologies scientifiques (le Protocole a été actualisé cinq fois) ;

- La reconnaissance de « responsabilités communes, mais différenciées » (pour satisfaire aux besoins particuliers des pays en voie de développement) ;

- Une « procédure d'ajustement » permettant aux Etats de modifier le rythme de leur abandon progressif sans avoir à amender le traité ;

- La large participation (gouvernements, industrie, scientifiques, ONG) ;

- Les fortes incitations à la conformité intégrées dans le protocole, alternant la carotte (des aides financières et techniques généreuses) et le bâton (des sanctions commerciales significatives); et

- La vitesse à laquelle l'industrie était en mesure de développer des alternatives pour remplacer les substances appauvrissantes ; elles étaient souvent plus économiques et plus efficaces que les méthodes initiales et constituaient donc une incitation supplémentaire à la conformité.

Source : Brack (2003) 


\section{Encadré 3.3 La Convention sur le Commerce international des espèces sauvages de faune et de flore menacées d'extinction (CITES)}

\section{Principes clés : non migration ; grande participation ; séparation de la vérification et de la mise en vigueur ; autonomie de la surveillance.}

La Convention sur le Commerce international des espèces sauvages de faune et de flore menacées d'extinction (CITES) de 1973 est un accord multilatéral (intergouvernemental) sur l'environnement dont le but est d'assurer que le commerce international en spécimens d'espèces sauvages de faune ou de flore n'en menace pas la survivance.

La CITES règle le commerce de la faune et de la flore essentiellement par le biais d'un système d'autorisations et de certificats distincts à trois niveaux que peuvent délivrer les autorités nationales avant même que les spécimens impliqués dans le commerce international puissent quitter le pays ou y entrer. L'annexe I de la CITES recense toutes les « espèces menacées d'extinction » dont le commerce international n'est autorisé que par exception. L'annexe II inclut les espèces susceptibles d'être menacées si le commerce n'est pas contrôlé et pour lesquelles le commerce est accepté et soumis à des réglementations strictes des les Etats exportateurs et les pays importateurs. L'annexe III inclut les espèces classées spécifiquement par des parties individuelles qui requièrent la coopération d'autres pays pour contrôler le commerce et ce, afin d'éviter la surexploitation à l'intérieur de leurs propres frontières.

Les listes forment l'élément principal du système de conformité de la CITES. Chaque partie se doit de nommer une « Autorité de gestion » nationale qui administre le système de licence, ainsi qu'une ou plusieurs « Autorités scientifiques » pour éclairer l'Autorité de gestion sur les effets du commerce sur l'état de conservation des espèces impliquées. Le système de conformité de la CITES est basé sur le fait que les parties doivent soumettre leurs propres rapports, lesquels seront complétés et contre-vérifiés sur la base des renseignements recueilllis par le Secrétariat et par des ONG militantes. La CITES doit sa capacité de veiller à la conformité en grande partie à la croissance du nombre de ses membres (l'Organisation comptait 160 Parties membres en 2003 réparties sur les cinq continents) et donc, sa capacité d'éviter la migration du commerce vers les non parties. La vente effectuée par une non partie, Singapour en l'occurrence, compliquait, dans certains cas, le contrôle international. Le pays a fait fonction de grand entrepôt pour le trafic de la faune et de la flore sauvages jusqu'à sa ratification de la Convention en 1986.

Les énormes quantités d'espèces et de sous-espèces de la CITES, objets des ventes légales et illégales, sont impressionantes (au moins 33000 espèces) ; il en est de même pour les grandes variations dans la valeur des produits commercialisés. Ces nombres compliquent énormément le contrôle de la conformité et de la vérification : on ne peut, en effet, exiger des douaniers qu'ils reconnaissent plus qu'une petite partie des espèces concernées. 


\section{Encadré 3.3 (suite)}

Le système CITES présente un dilemme fondamental, à savoir que la Convention a une forte vocation de conformité, mais elle ne possède pas de capacité d'exécution centrale. La CITES n'a pas non plus la compétence d'imposer des sanctions; elle pourra tout au plus exercer une pression pour que ces sanctions soient appliquées « comme il se doit » par les autorités nationales, sans spécifier leur contenu (Matthews 1996, p. 422). La vérification au sein de la CITES dépend en grande partie de la circulation de l'information entre les parties. L'absence d'un instrument indépendant de vérification sur place est une faiblesse spécifique. La vérification repose grandement sur le travail de détective des ONG, même si deux d'entre elles seulement (UICN et TRAFFIC) ont un statut officiel au sein du processus de décision de la CITES. Ces dernières années, le secrétariat de la CITES a introduit un certain nombre de nouvelles procédures pour consolider l'aptitude de la Convention à réaliser ses impacts escomptés. On retiendra notamment le processus de " l'Etude du commerce important ", qui se penche sur les espèces couramment vendues de l'Annexe II, et sur la variété de missions d'inspection à entreprendre par le Secrétariat ou par une équipe formé par ce dernier et agissant au nom de la Convention. Les missions d'experts techniques bénéficient souvent d'une large couverture par la presse, à condition toutefois qu'elles se concentrent sur les espèces sérieusement menacées, les tigres par exemple.

La CITES est une des premières conventions environnementales multilatérales; elle a été l'objet, au fil des ans, d'un certain nombre de critiques. Les critiques portant sur ses systèmes de conformité tendaient à focaliser sur son manque d'efficacité et sa dépendance excessive aux mécanismes de « loi souple » pour appréhender la non conformité (Reeve 2002, p. 250). Ses défenseurs, de leur côté, répliquent que la structure présente de la conformité peut, quelles que soient ses imperfections, être un compromis raisonnable, vu les moyens restreints mis à sa disposition et l'invraisemblance d'un changement radical de sa situation financière. Pour eux, le fait que des acteurs internationaux importants investissent une énergie considérable pour essayer de la ralentir indiquerait qu'elle fonctionne assez bien.

Citons, parmi les leçons à retenir de cette étude de cas :

- L'ambition d'un système de vérification est régie par son mandat. Dans le cas de la CITES, le souci majeur est d'éviter le commerce non durable ; sa capacité de réaliser d'autres objectifs sera donc plutôt limitée. Toutefois, certains mécanismes complémentaires (tels que les études du commerce important) peuvent jouer un rôle favorable.

- La conformité et la mise en œuvre doivent être des choses distinctes, mais complémentaires. Il est possible de compenser la faiblesse du mécanisme de conformité par le biais de la prolifération des moyens permettant de générer des réactions à la conformité (dont l'élargissement de la participation en incluant des acteurs non gouvernementaux)

- La réussite de l'exécution repose sur la coopération entre les Parties qui la mettent en place qui relient les niveaux international et national ; ces initiatives peuvent impliquer des investissements importants au niveau du renforcement des capacités. 


\section{Encadré 3.3 (suite)}

- Il faudra adapter le système de vérification à la complexité des produits dans le commerce. Le niveau de complexité est élevé dans le cas de la CITES, ce qui justifiera une approche de classification (le système des listes et de l'autorisation) qui, de façon idéale, reposera sur des organismes forts (la gestion et les autorités scientifiques nationales) ; néanmoins, le talon d'Achille du système d'autorisation est sa vulnérabilité aux abus.

- Tout comme d'autres aspects de loi internationale, la CITES ne dispose pas de pouvoir de police ; il n'en demeure pas moins que certains facteurs portant sur la réputation peuvent se révéler importants pour la conformité (certainement plus importants que des motifs financiers).

- Il faudra faire preuve de prudence pour que les sanctions soient appliquées de façon impartiale, d'autant plus si les parties ont un pouvoir inégal sur la scène internationale.

Source : Brown et Swails (2005)

encouragé une approche collaborative de la vérification internationale ; certains acteurs importants (notamment dans les industries de transformation) la considérant comme un grand soutien pour leurs moyens de subsistance à long terme, ont reconnu en effet qu'il était dans leur intérêt de ratifier le Processus (voir Encadré 3.1).

D’un autre côté, et toujours dans le cas de la CCNUCC, certains prétendent qu'il aurait été plus avisé que la Convention ne contrôle que les émissions de dioxyde de carbone des carburants fossiles (voir Encadré 3.4). Le contrôle aurait été beaucoup plus simple étant donné qu'on avait déjà une possibilité de mesurer le niveau des émissions (quantité de carburants fossiles consommés) et des facteurs mesurables d'émission (quantité d'émission par unité de la catégorie spécifique de carburant). D'autres gaz à effet de serre dans les six catégories qui ont été contrôlées pour la CCNUCC sont beaucoup moins mesurables (le méthane et l'oxyde nitreux par ex., qui n'ont pas encore de mesures approximatives ou de facteurs réguliers d'émissions par classe de polluants). La grande ambition que se donne la CCNUCC comprend donc des processus de vérification compliqués (Victor 2001).

Dans le cas des normes d'hygiène concernant la viande, le système de contrôle prescriptif initial est devenu progressivement un système basé sur les dangers (voir Encadré 3.5). Cela implique de s'éloigner de l'imposition d'un jeu complexe de règles à appliquer universellement pour mieux se concentrer sur le lieu des sites et des activités qui risquent d'être le plus contaminés. La focalisation sur le niveau du risque permet de procéder à une vérification plus rentable que ne le font les systèmes de contrôle qui reposent sur la conformité de l'évaluation avec des mesures largement normatives ; elle permet d'autre part une approche plus réceptive et plus réactive qui convient à certaines circonstances particulières. 


\section{Encadré 3.4 La Convention-cadre des Nations Unies sur les changements climatiques (CCNUCC) et son Protocole de Kyoto}

\section{Principes clés : réciprocité ; non migration ; définition du problème ; large participation ; approche des systèmes ; besoin de clarté des standards ; surveillance indépendante (dans l'infraction).}

La vérification des émissions de gaz est confrontée à des problèmes particuliers d'incertitude du fait de leur intangibilité, de la diversité des facteurs qui les influencent et des désaccords ininterrompus concernant les méthodologies de contrôle. La vérification sous les auspices de la CCNUCC vise à tester la conformité avec les objectifs de réduction des émissions de gaz à effets de serre (GES) identifiés dans le Protocole de Kyoto, qui prévoit la vérification de la performance de réduction nationale ainsi que les effets de trois mécanismes de réduction «flexibles » : (i) le système d'échange des droits d'émissions ; (ii) le Mécanisme du développement propre (impliquant la collaboration entre les Etats industriels et les pays en voie de développement) ; et (iii) la mise en œuvre conjointe (impliquant la collaboration à travers les sociétés industrielles). Cet encadré prend essentiellement en considération la performance nationale des Parties de l'Annexe I (pays industrialisés), bien que la vérification en vertu du MDP soit aussi brièvement examinée.

La vérification des émissions nationales des Parties de l'annexe 1 doit satisfaire aux pressions de la concurrence : la décentralisation du reporting répond à un expédient politique (la souveraineté nationale), mais se heurte rapidement à d'importantes contraintes de capacité (le manque de personnel technique qualifié, notamment en ce qui concerne les Parties dans le sud). La CCNUCC a réagi en proposant des incitations financières et un support technique, bien que les investissements nécessaires soient encore considérables. Le système géré par le Secrétariat international, repose toutefois sur le reporting national. Comme dans le cas d'autres traités, l'essence d'une vérification efficace sous l'égide de la CCNUCC est basée sur l'autoreporting selon un jeu prescrit de formats et soutenu par une série de mécanimes institutionnels permettant de vérifier les constatations par recoupement. La crédibilité de ces dispositions dépend beaucoup de l'efficacité des mesures mises en place pour protéger le système contre toute ingérence politique.

L'entrée en vigueur du Protocole de Kyoto a entraîné la modification des conditions du reporting. Avant même la fin de la première période d'engagement, chaque Partie doit mettre en place un système national permettant d'évaluer les émissions de gaz à effet de serre ainsi qu'un registre national pour enregistrer les transactions d'unités de réduction d'émission générées par divers types d'activités : les unités certifiés de réduction d'émission de projets du MDP, les unités de réduction d'émission de projets de mise en œuvre conjointe et les unités de suppression de projets de puits. Le système national comporte un inventaire exhaustif des émissions. Les parties devront aussi faire des progrès manifestes dans le respect leurs engagements en vertu du Protocole, et utiliseront dans ce cadre un certain nombre de formats de reporting standardisés et techniques.

Le Protocole de Kyoto propose une procédure de conformité innovatrice qui comprend deux parties (englobant la facilitation et l'exécution) ; on reconnaît ainsi le fait que le terrain est 


\section{Encadré 3.4 (suite)}

nouveau pour toutes les parties. La présence d'un processus de facilitation, combiné à un système de sanctionnement clairement défini donne au Protocole une " procédure de nonconformité plus ambitieuse que tout autre traité environnemental existant » (Rapport END 2001, cité dans Stokke et al. 2005).

Le processus d'enquête de la CCNUCC met en jeu des équipes d'examen (ERT) pour les parties de l'Annexe 1. Les ERT ont pour tâche de vérifier les systèmes d'inventaires et les données remises par une partie au Protocole avant et pendant la première période d'engagement (c-à-d., jusqu'en 2012). Elles ont également un rôle de facilitation, offrant un retour d'informations aux parties, pour les aider à améliorer les inventaires de leurs émissions. Le produit du processus d'examen est un rapport qui sera présenté au Comité de Conformité. Certaines dispositions aident à assurer l'impartialité de la sélection et de la composition des ERT. Les équipes sont sélectionnées par le Secrétariat parmi des experts nommés par les parties et par des organisations intergouvernementales, et cette procédure maximise ses contributions à partir de différents groupes d'intérêts au sein du processus. Le Secrétariat, pour sa part, a un degré d'impartialité du fait de sa responsabilité auprès de la Conférence des parties (CdP) et des instances affiliées. Il est aussi financé par les Parties, dont les contributions sont basées sur une échelle d'évaluation des Nations Unies.

La vérification sous l'égide du MDP est une entreprise pleine de défis ; elle implique une collaboration internationale entre divers partenaires du nord et du sud, elle vise la durabilité à long terme et requiert l'additionnalité (c.-à-dired., que les réductions doivent s'ajouter à toutes les réductions d'émissions survenant en l'absence d'une activité du projet). La « durabilité » et « l'additionnalité » sont des critères particulièrement exigeants pour la vérification.

Passons en revue certains points généraux pour résumer la vérification au sein de la CCNUCC :

- La transparence et la rentabilité sont deux critères importantes ; il aurait sans doute été plus sage dans les premières étapes, de limiter le contrôle aux émissions de $\mathrm{CO}_{2}$ du carburant fossile car cela aurait été plus simple, en mesurant la consommation de carburant fossile qui donne une mesure du taux d'émissions de $\mathrm{CO}_{2}$, une des principales causes de la pollution (Victor 2001).

- La transparence et l'efficacité représentent une problématique particulière lorsqu'un système s'écarte de l'enregistrement direct d'un phénomène observable (comme avec le MDP).

- Les protagonistes non-governementaux peuvent avoir des rôles importants à jouer dans les domaines que la vérification conventionnelle risque de ne pas couvrir adéquatement (par exemple, les mécanismes à impact social élevé) ; ils peuvent par ailleurs encourager la conformité là où les contrôles internes sont faibles.

- Dans les cas où un traité impose des coûts importants à ses parties, l'inclusion est une conditions préalable à l'efficacité ; dans cet exemple, la présence de non parties puissantes (les pays de l'Annexe 1 dont les Etats-Unis et l'Australie jusqu'en 2007, ainsi que des pollueurs importants tels la Chine et l'Inde parmi les pays non-Annexe 1) suscite quelques inquiétude. 


\section{Encadré 3.5 L'agence de normalisation pour les produits alimentaires et la transformation de la viande}

\section{Principes clés : chaîne de conservation ; incitations ; surveillance indépendante.}

Les standards en matière de produits alimentaires proposent une étude de cas comparative et intéressante de la vérification du bois d'œuvre et cela, pour un certain nombre de raisons. Premièrement : tout comme le bois et ses fibres, les aliments sont largement commercialisés au niveau international. Deuxièmement : les nations consommatrices imposent dans les deux cas des normes élevées pour les produits en question. Troisièmement : comme pour les forêts, les chiffres relatifs à l'alimentation constituent un motif important pour l'état de l'environnement, surtout dans le nord industriel. Et pour finir : le contrôle des produits alimentaires et de leur sécurité fait l'objet, ces dernières années, au sein de I'Union Européenne, d'une série de réformes institutionnelles importantes : des réformes qui remontent aux crises alimentaires qui ont défrayé la chronique et qui découlent du changement des normes d'acceptabilité publique. Toutefois, la préoccupation essentielle concernant les normes des produits alimentaires (en l'occurrence, la santé et la sécurité de l'être humain) ont des implications immédiates et personnelles pour les consommateurs occidentaux en général, alors que dans le cas du bois et de la fibre, l'intérêt premier se limite à ceux qui sont « écologiquement conscients ». Autre différence entre les produits alimentaires et le bois, surtout dans un contexte commercial : les propriétés intrinsèques des aliments (par exemple, leur capacité à provoquer des maladies) qui en encouragent la règlementation et, dès lors, les mesures nationales ne seront vraisemblablement pas contestées sous le régime de l'OMC.

En Europe, les normes pour les produits alimentaires et plus particulièrement, la viande et les produits qui en dérivent illustrent plusieurs points d'intérêt général pour la vérification. Ces dernières années, la conception du système de contrôle s'est transformée, partant d'une politique normative au sein de laquelle la sécurité de la viande était recherchée par le biais de l'application de directives normatives afin d'assurer la certification adéquate de la viande, pour devenir une politique plus orientée vers le risque basé sur les dangers. Cette nouvelle approche implique l'identification des risques du moment et la mise en place de contrôles efficaces pour les minimiser. La nouvelle approche semble moins rigide ; elle permet de réagir plus rapidement à l'évolution des concepts de risque et à la technologie nouvelle, et est beaucoup plus facile à harmoniser à travers les frontières culturelles. Au Royaume Uni, cette nouvelle approche est appuyée par une série de directives de systèmes concernant la gestion de la sécurité contre les dangers des produits alimentaires (les principes dits " HACCP ») auxquelles tous les fournisseurs sont priés de se conformer. Au niveau européen, la nécessité d'adapter les standards aux conditions locales est de plus en plus reconnue, de telle façon que la conception soit réaliste et apte à une concrétisation efficace dans le contexte donné (par ex., la fumaison artisanale et à petite échelle de la viande dans le sud de l'Europe est un procédé technologiquement simple dans la plupart 


\section{Encadré 3.5 (suite)}

des cas, mais qui implique dans l'ensemble des chaînes d'approvisionnement courtes ; aussi est-elle perçue comme présentant peu de risques).

La vérification dans l'industrie de la viande est liée à deux zones d'activités importantes : l'évaluation de la conformité d'opérateurs privés au sein des règlements des systèmes de production alimentaire et l'évaluation de l'efficacité du contrôle national et des dispositions de mise en œuvre à divers niveaux (en rapport avec la performance des " autorités compétentes » nationales). Avec les règlements actuels, les autorités britanniques sont autorisées à tenir compte des opérateurs qui participent à un régime d'assurances. Des programmes qui certifient que les normes de gestion et de bien-être dans la ferme d'un des membres, ou encore, que les normes de gestion au sein d'une installation de transformation répondent aux niveaux de meilleure pratique convenus à l'échelle nationale peuvent prétendre à certaines exemptions concernant l'audit et le contrôle. Les incitations à la conformité comprennent à la fois la carotte et le bâton. La pire sanction négative étant le retrait de licence dont les conséquences commerciales sont immédiates. Les sanctions positives, elles, sont liées à l'accès au marché préférentiel et également au fait que les hautes normes de traçabilité exigées par les autorités servent à la fois les intérêts du public et ceux du commerce (par ex., elles correspondent bien aux mesures de contrôle du stock dont se servent les supermarchés).

Récemment encore, I'UE a pris des mesures pour élargir l'éventail des organisations susceptibles de se charger de l'inspection de la viande, tout en requérant que tous les praticiens respectent la norme EN 45004 (ISO 17020). Des critères de conflit d'intérêt clairs sont applicables ici : l'organisme en question ne doit être libre de tout intérêt commercial, mais également ne subir aucune pression susceptible d'affecter son jugement ; il doit aussi être libre de tout préjugé pouvant influencer ses conclusions.

Leçons à tirer de cette étude de cas :

- Les avantages du système de contrôle personnalisé adapté aux risques et à la vulnérabilité ;

- Le besoin d'adapter les mécanismes de vérification aux principes du plan (ex., connecter les conditions d'audit à l'évaluation du risque) ;

- Le besoin d'adapter les normes aux conditions locales pour améliorer la probabilité d'une réalisation efficace ;

- L'impact motivant de la liaison entre les nouvelles conditions de contrôle et les normes établies de façon à les renforcer mutuellement (ex., l'accord d'un crédit pour pouvoir participer aux plans d'un régime d'assurances agréé) ; et

- La valeur d'un large concept « d'indépendance » pour veiller à ce que les vérificateurs soient libres de tout préjugé susceptible de miner leur crédibilité.

Source : Brown et van Midwoud (2006) 


\section{$4^{\mathrm{e}}$ Principe : L'adoption d'une approche de systèmes et la focalisation sur la distribution et l'équilibre des pouvoirs}

Que se passerait-il, en termes pratiques, si l'expérience faisait apparaître le besoin d'aller au-delà de la vérification comme étant un moyen direct d'accorder des licences pour une approche d'avantage orientée vers les systèmes ? La littérature sur le traité donne généralement des conseils utiles en ce qui concerne les éléments institutionnels du système décisionnel.

Un certain nombre de composantes sont à ajouter à un régime de vérification sur le plan des obligations des traités et conventions (Sur 1991 ; Cameron et al. 1996 ; Reeve 2002). Pour ce qui est des traités plus complexes (limitation des armements par ex.), le régime de vérification comprendra généralement : des engagements juridiques ; l'échange de données et des arrangements au niveau de la notification; des méthodes de contrôle ; des mécanismes de communication, de consultation et d'éclaircissement ; une méthode de vérification des jugements ; et éventuellement, les mécanismes de conformité (Findlay 2003). Le système de vérification est considéré comme un regroupement de toutes ces caractéristiques bien que certaines définitions excluent les mécanismes de conformité (procédures permettant d'aborder les non conformités supposées et véritables). On a tendance à les considérer comme faisant partie d'un système de mise en application, comme par exemple les divers mécanismes coercitifs susceptibles d'imposer une demande de conformité (à tour de rôle des pressions politiques et économiques et des sanctions telles que le recours à la force), plutôt qu'une simple évaluation de la conformité. Le point litigieux ici ne réside pas dans les précisions de la définition du mot "vérification " par opposition à " mise en application ", mais plutôt dans la nécessité de séparer l'évaluation de la conformité des mesures nécessaires pour l'effectuer. Une bonne pratique serait de garder ces deux éléments séparés pour que la base d'information sur laquelle sont prises les décisions de conformité soit isolée des sanctions susceptibles d'être appliquées pour les imposer, ainsi que les politiques que les sanctions entraîneraient. Lorsque le traité ou la convention est ordonnée par les Nations Unies (comme c'est le cas pour NTBT et Kimberley), la mise en application suppose un caractère profondément politique. Kyoto est un cas intéressant, dans ce sens qu'il fait aussi la distinction entre la facilitation et la mise en application, cette dernière seulement ayant des fonctions judiciaires.

Lang (1996) met en relief la valeur de la séparation des jugements sur la conformité des mesures qu'entraîne l'inobservation au moyen de sanctions et de " mesures d'assistance ". Elle identifie trois éléments institutionnels comme étant des conditions minimales pour un système satisfaisant de contrôle de la conformité, et ces éléments offrent une structure utile pour n'importe quelle forme de vérification :

1. Un organisme chargé de recueillir l'information auprès de toute source disponible ;

2. Un "mécanisme d'examen " pour l'évaluation et l'interprétation de données (ces travaux sont souvent confiés à une ou plusieurs instances différente(s); et

3. Une fonction qui "mesure " et qui sera réservée à un corps politique (l'autorité suprême d'un traité ou d'une convention par exemple, comme la Conférence des Parties), qui agira soit sur les recommandations de l'instance d'examen, soit de sa propre initiative. 
L'accent est mis sur la vérification en tant que processus, dans lequel (idéalement du moins) les fonctions clés sont exécutées séparément par différents organismes. Ce système de gestion de l'information a pour avantage de séparer les niveaux opérationnels entre les mécanismes de vérification et de mise en place, et il préconise par ailleurs la séparation des niveaux de prise de décision entre la politique et la technique. Le fonctionnement de cette instance politique constitue l'élément le plus controversé, notamment dans les situations où le mécanisme se trouve intégré dans un système de gouvernance plus large qui ne se conforme pas aux critères de «bonne gouvernance ». Nous verrons par la suite que ce point prévaut particulièrement dans le secteur forestier où le produit examiné convient à la liquidation politique (par ex., le niveau et le caractère de départ de la ressource répond aux besoins de fonds financiers des acteurs politiques), et où, par conséquent, il peut y avoir des forces puissantes susceptibles d'encourager l'ordre politique à intervenir dans son extraction.

\section{$5^{\mathrm{e}}$ Principe : Une large participation dans les processus qui mènent à la décision de la vérification}

Lorsque des acteurs multiples s'intéressent au problème examiné (fait qui s'accompagne souvent d'une différence de points de vue), les processus décisionnels devraient alors refléter cette divergence. Cela demande des forums institutionnels susceptibles d'accommoder les nombreux acteurs, intérêts et diverses sources d'information, et une définition de la légalité acceptable pour toutes les parties concernées. Un mélange de principe et de pragmatisme se révèle nécessaire. Par exemple, les Etats peuvent se dérober devant l'engagement d'acteurs de la société civile dans des questions qui relèvent de la souveraineté nationale (tel a été le cas avec Kimberley et CITES, par exemple). Ce phénomène peut se justifier en principe dans le fait que l'Etat et la société civile ne sont pas des protagonistes égaux dans le domaine de la politique, et les acteurs de la société civile manquent souvent de légitimité démocratique et de mécanismes pour le renouveau démocratique. Ce dernier s'applique particulièrement à la société civile internationale qui jette des ponts sur des domaines politiques sensibles. Il y a aussi des questions concernant la neutralité d'acteurs de la société civile. Les ONG de conservation par exemple s'orientent de plus en plus sur la mégafaune charismatique comme les pandas, les éléphants et les tigres, au détriment de la biodiversité moins emblématique et cela est dû, dans une large mesure, au pouvoir de levées de fonds plus important des charismatiques (Victor 2001).

Il y a néanmoins de bonnes raisons pour ouvrir le processus aux acteurs de la société civile. Ces derniers peuvent offrir leur expertise, et leur participation est apte à promouvoir la transparence du processus et à aider à engager le grand public. Tout en ayant éventuellement des préjugés dans leur défense (les droits des indigènes, par exemple, sont plus faciles à promouvoir sur la scène internationale que les droits généralisés des pauvres, étant donné qu'une législation internationale les protège déjà) ils sont souvent le dernier recours du pauvre et du marginal dont les intérêts ne sont pas nécessairement bien représentés dans les processus démocratiques. Le processus décisionnel dans des conventions telles que la CITES a sans doute beaucoup gagné de cet élargissement de la participation, bien qu'il subsiste encore des préoccupations quant à la responsabilité en particulier au niveau transnational. 


\section{Principe : La précision des normes d'évaluation}

Convenir d'une définition de la légalité est essentielle pour toute forme de contrôle et constitue un pas particulièrement critique. De nombreux pays admettent la complexité et le caractère contradictoire du contexte légal pour la foresterie, avec ses juridictions qui se chevauchent et ses droits rivaux. Le jugement à partir de l'expérience des traités (et l'extrapolation à un contexte de non réciprocité), l'adoption d'une telle définition peut s'avérer être une affaire exigeante et qui prend du temps.

Le principe de la « limitation du mandat » tend à avoir des significations assez spécifiques dans les négociations internationales sur les armements, en référence àl'autorité du mandat gouvernant. Il peut néanmoins s'appliquer également, et de façon plus idiomatique, pour se concentrer sur la question de savoir s'il faut demander aux vérificateurs de baser leurs décisions de vérification sur le mandat de l'accord, ou s'ils sont autorisés à introduire leurs propres intérêts. Bon nombre de traités internationaux importants sont très précisément " limités par le mandat ", et il est demandé aux vérificateurs de se conformer à une spécification claire et avalisée légalement de leurs tâches, ce qui est logique pour toutes les parties du traité. Si l'on pense qu'ils ne l'ont pas fait, des mesures seront souvent prises pour restreindre leurs tâches. Exemple : des accusations selon lesquelles les inspecteurs que l'Agence internationale de l'énergie atomique étaient trop soumis au préjugé national ont mené à l'affinement du système de recrutement; dès lors, on leur demanda de prêter serment de loyauté à l'ONU, et non pas à leurs Etats parrains comme cela était le cas précédemment (voir Encadré 3.6).

\section{Encadré 3.6 L'Agence internationale de l'énergie atomique}

Principes clés : réciprocité ; non migration ; approche des systèmes ; valeur de la surveillance indépendante.

Le système de sauvegarde de l'Agence internationale de l'énergie atomique (AIEA) englobe les matériaux susceptibles d'être utilisés dans la fabrication d'armements nucléaires. En vertu de son statut, l'AIEA a le droit d'examiner l'équipement et les installations spécialisées des Etats membres pour vérifier de l'absence de tout but militaire, pour exiger le maintien de rapports d'opération et pour demander et recevoir les rapports d'avancement. Pour faciliter la vérification, cette disposition accorde également à l'AIEA le droit d'envoyer des inspecteurs, désignés par I'AIEA, après avoir consulté l'Etat impliqué. La mesure stipule toutefois que les activités de l'AIEA seront menées en respectant les droits souverains des Etats. Les Inspecteurs du Contrôle de sécurité doivent être accompagnés par des représentants de l'Etat, si cela est demandé. Bien que recrutés parmi les Etats membres de I'ONU, les inspecteurs de l'Agence sont des employés des Nations Unies. Ils n'accepteront aucune instruction de leurs propres gouvernements et ils ne violeront pas les règles de confidentialité établies par l'Organisation.

Le « produit » des opérations de vérification de l'AIEA est une déclaration de la différence d'inventaire relevée pour une période spécifique. Cela demande à tirer une conclusion 


\section{Encadré 3.6 (suite)}

concernant l'absence de détournement du matériel nucléaire déclaré et l'absence de matériel et d'activités nucléaires non déclarés dans l'Etat. S'il lui est impossible de tirer une telle conclusion, il le fera savoir.

Les évaluations de conformité et de non-conformité sont menées par le Conseil des gouverneurs de I'AIEA, par son Organe exécutif (constitué de dix membres, désignés par I'Organe sortant) et par vingt membres, élus par la Conférence générale. Dix sièges sont alloués sur la base de la compétence en technologie nucléaire; le choix de vingt autres sièges repose sur une base géographique équitable. Le Conseil des gouverneurs décide, finalement, du degré de conformité par un pays ; il examine également toutes les questions provenant de l'interprétation de (des) accord(s) sur le contrôle de la sécurité. La décision par consensus est privilégiée, mais s'il s'avère impossible d'obtenir un tel consensus, le Conseil détient le droit de décision par vote à la majorité. Le Statut de I'AIEA n'interdit pas à un membre du Conseil d'être impliqué dans la prise de décision concernant sa propre conformité.

L'AIEA et son Conseil de gouverneurs déterminent les actions nécessaires conformément à son mandat. Le mandat actuel de l'AIEA est de sommer l'Etat non conforme à remédier à la non conformité ; de rapporter la non conformité aux membres de l'Agence, au Conseil de Sécurité des Nations Unies et à I'Assemblée générale de I'ONU ; d'écourter ou de suspendre l'assistance nucléaire ; d'ordonner le retour des matériaux et de l'équipement mis à la disposition de l'Etat, et de suspendre ce dernier de l'exercise des droits et privilèges auxquels il avait droit en tant que membre de l'AIEA. II existe une procédure de recours impliquant la Cour internationale de justice.

Les contrôles de I'AIEA se caractérisent donc par une participation large et une structure complexe de surveillance internationale par le biais des structures de l'ONU. Pourtant, son autorité se voit affaiblie par le fait que les cinq Etats dotés d'armes nucléaires (la Chine, la France, la Russie, la Grande-Bretagne et les Etats-Unis) sont indépendantes de l'AIEA et par le fait, aussi, que les Etats européens défendent leur industrie régionale par le biais du système de garantie d'EURATOM.

Leçons à tirer de cette étude de cas :

- La valeur d'organismes forts pour l'établissement des normes, la communication de l'information et le maintien de l'ordre/la conformité ;

- La nécessité de maintenir des limites claires entre le contrôle de routine, la vérification et la mise en vigueur ;

- La valeur d'une participation large dans les décisions de vérification, améliorant leur légitimité et l'autorité internationale ; ainsi que

- La nécessité d'un système d'inspection pour assurer le respect de l'accord et non pas des intérêts individuels des parties.

Source : Persbo et al. (2005) 
La tendance, dans l'environnement, à mettre au point des normes "in situ " soulève d'importantes questions quant à l'identité des vérificateurs et des contrôles qui leur sont imposés. Une condition fondamentale pour les vérificateurs est qu'ils doivent être prêts à donner une recommandation positive pour la décision de vérification une fois que les conditions sont satisfaites, dans le cadre des tolérances convenues ; ils doivent de même se montrer disposés à s'y opposer si les conditions ne sont pas remplies. Cela risque de poser un problème sur lequel les observateurs environnementaux officiels devront se concentrer particulièrement dans le cas où ils décideraient de jouer le rôle de vérificateurs. Comme le constate Haufler (2001), ce domaine représente un défi pour les ONG de plaidoyer que leur mode d'opération ordinaire consiste à insister pour relever les normes, en mettant l'accent sur le côté négatif, et ils peuvent trouver difficile (non sans raison) de travailler principalement en mode positif. D'un côté, une méthode d'opération excessivement positive risque de coopter les ONG et de compromettre leur indépendance ; d'un autre côté, un mode par trop négatif risque d'entraîner des effets très destructeurs. Ceci est particulièrement probable dans un secteur comme la foresterie où il s'est avéré plus difficile pour les ONG de soutenir un marché que de s'y attaquer.

\section{Principe 7 : Fournir des incitations à faire des compte-rendu et à se conformer}

Stratégiquement, les traités forts ont tendance à se concentrer sur les incitations à la conformité, et sur les facteurs aptes à inciter à la non-conformité et à la migration. Les systèmes de vérification fonctionnent au mieux lorsqu'il existe de fortes motivations pour se conformer et peu d'incitations encourageant à quitter le traité. Ces motivations peuvent, à des degrés différents, être financières, stratégiques ou de nature à influencer la réputation. Le Protocole de Montréal est un exemple de traité dont l'efficacité a fortement augmenté grâce à ses qualités " bénéfiques pour tous" (Barrett 1999). Le fait qu'il existait des gains évidents pour les acteurs puissants de l'industrie de la haute technologie a contribué à générer la volonté de soutien politique (voir Encadré 3.2).

Question quelque peu à part, les motivations au rapport. La manière dont est effectué l'établissement des rapports est également importante. Paradoxalement, dans une situation de faiblesse de gouvernance, la demande de rapports efficaces peut elle-même alimenter l'illégalité en raison de la façon selon laquelle les documents prennent une valeur marchande (Brack et al. 2002). La CITES, par exemple, a énormément souffert de documentations frauduleuses, bien que les progrès techniques les rendent plus difficiles. Le secteur forestier est encore plus sujet à de telles fraudes du fait de la nature hautement dispersée de ses activités. Les derniers progrès technologiques avec l'informatisation et la recherche documentaire ( formulaires inviolables", etc.) sont ici d'un intérêt particulier bien qu'ils ne soient pas nécessairement une panacée (voir Chapitre 23).

\section{Principe 8 : Une supervision autonome}

"Le contrôle indépendant ", selon le Plan d'action FLEGT de l'UE, " rend les systèmes de vérification plus crédibles et moins sujets à la corruption " (Commission des Communautés Européennes 2003) : un point de vue que semblent également 
partager les observateurs de nombre d'accords divers. Normalement, cela impliquerait la participation de contrôleurs de parties tierces, lesquels opèreraient indépendamment des premières parties (le commerce) et des secondes parties (sa clientèle), comme dans les cas des observateurs internationaux lors d'élections (Encadré 3.7) et les ombudsmans (Encadré 3.8). Il existe toutefois des problèmes concernant l'équilibre approprié entre l'auto déclaration (par le secteur ou par le gouvernement délivrant le permis) et la supervision extérieure et autonome à toutes les phases du processus de vérification. L'auto déclaration tend à générer le principe de la responsabilité même si elle est faible sur le plan de la légitimité externe, alors que la supervision indépendante tendrait à privilégier le contraire. Nombreux sont les traités internationaux importants qui essaient d'établir un équilibre politique entre les deux, même si cela ne satisfait aucune des parties. L'auto déclaration s'avère très peu satisfaisante pour les affaires stratégiques telles que le Traité d'interdiction totale des essais nucléaires, et l'on constate un scepticisme général lorsqu'il s'agit de " demander à la souris de surveiller le fromage ".

\section{Encadré 3.7 L'observation internationale des élections}

\section{Principes clés : définition claire du problème ; large engagement dans le processus menant à la décision de vérification ; valeur de la supervision indépendante.}

L'observation des élections implique le regroupement délibéré d'informations concernant un processus électoral et la formation d'un jugement informé concernant son honnêteté. Tout comme la vérification de la légalité du bois, l'observation des élections n'est pas une pratique universelle; on l'utilise néanmoins pour alléger les doutes sur le fonctionnement d'un processus de routine. Les observateurs contrôlent la véracité des plaintes pour améliorer la performance des instances directrices et le système général de gouvernance et pour augmenter la légitimité des opérations examinées.

De par sa vulnérabilité face aux accusations de violation de la souveraineté nationale, l'observation internationale des élections requiert une grande prudence. Aucun instrument légal international ne peut garantir le principe de l'observation électorale. Les négociations concernant l'observation des élections revient donc au gouvernement hôte. Les normes internationales autour de la démocratie sont fortes; elles ont aidé à donner une légitimité aux efforts d'observation des élections internationales. Au-delà de ces normes, toutefois, le jugement sur ce qui est « libre et juste » demeure ambigu. La loi électorale n'est pas liée à des normes fixes et universelles, et la complexité du processus électoral rend irréaliste la notion de formule simple. En réponse à ces défis, on éprouve le besoin de se concentrer sur le professionnalisme et l'expertise des observateurs.

Une des principales raisons d'accorder l'acceptation et l'appropriation à l'activité de l'observation est la perception positive des observateurs des élections par les parties prenantes dans le pays en question. L'indépendance et la crédibilité sont des conditions fondamentales; on les associe à la neutralité, aux comportements des attitudes non partisanes et des méthodes impartiales, dont les techniques de reporting. Le caractère 


\section{Encadré 3.7 (suite)}

sporadique et de courte échéance de maints processus d'observateurs internationaux semblerait restreindre leur utilité à cause d'un accent excessif mis sur « le jour de l'élection » et cela, au détriment des événements et des processus menant aux élections. Une des conditions fondamentales pour l'observateur d'élections est la capacité de faire la distinction entre les irrégularités, conséquences d'une faible capacité administrative, et celles qui sont le résultat exprimé de l'intention politique.

En ce qui concerne l'impact et la résonance avec le débat sur la vérification du bois d'œuvre, certains déclarent que l'intervention externe peut être bonne sur le plan de l'exposition des problèmes, mais que cela ne mène pas nécessairement à un changement constructif dans l'arène de la gouvernance démocratique. Pour d'autres, l'observation internationale des élections a contribué au renforcement des normes primaires des administrateurs électoraux ; elle aurait également participé à la croissance des partis politiques et au développement d'une société civile organisée. De savoir si l'observation des élections est un levier important pour les réformes de gouvernance, ou un indicateur trop simple pour influencer la qualité générale de la gouvernance reste à débattre. Dans les démocraties solides, le rôle des observateurs et des contrôleurs électoraux est assumé par des institutions nationales bien enracinées : des commissions électorales indépendantes, les « poll-watchers » (observateurs d'élections) impartiaux, la presse et les tribunaux indépendants. On peut donc se demander s'il est préférable d'investir les efforts et les ressources dans le développement de ces autres organismes horizontaux de démocratie que dans l'observation électorale qui n'est qu'un élément du processus.

Leçons à tirer de cette étude de cas :

- L'observation d'une élection internationale requiert une prise en charge minutieuse car elle est vulnérable à l'accusation de violer la souveraineté de l'Etat. La loi électorale n'est pas associée à des normes fixes et universelles, et la complexité du processus électoral ôte tout réalisme à la notion d'une formule simple.

- L'indépendance et la fiabilité sont des conditions fondamentales pour conférer toute crédibilité au travail des observateurs d'élections. Elles s'associent à la neutralité, aux comportements non partisans des observateurs et de méthodes impartiales, dont des techniques de compte rendus techniques. Le recours à l'observation électorale indépendante suscite des questions de souveraineté nationale et de subjectivité sur ce qui constitue des élections « libres et justes »; dans une telle situation, les observateurs devront maintenir de hauts niveaux de professionnalisme et prouver leur intégrité.

- L'observation indépendante des élections suscite par ailleurs des questions concernant la mesure dans laquelle l'intervention étrangère peut aider à consolider la gouvernance intérieure ; comme dans le domaine de la vérification du bois, on pourrait dire qu'il est plus effectif d'investir dans la consolidation des instances de gouvernance intérieure au lieu de se focaliser sur une perspective extérieure, partielle et potentiellement subjective.

Source : Luttrell et Nash (2008) 


\section{Encadré 3.8 Le travail des ombudsmans}

\section{Principes clés : approche des systèmes ; clarté des normes ; valeur de la surveillance indépendante.}

Généralement, le terme $d^{\prime}$ « ombudsman » se réfère à un fonctionnaire indépendant chargé de recevoir, d'étudier et de traiter les plaintes concernant l'injustice dans l'administration de services publics. Au cours de ces dernières décennies, les ombudsmans ont joué un rôle important dans le processus de transformation de l'Etat. Après un lent démarrage (le premier poste d'ombudsman fut créé au début du XIXe en Scandinavie, bien que le concept sousjacent remonte à la magistrature du Califat ottoman), l'institution s'est propagée sur tous les continents et dans de nombreux pays, avec, cependant, différents niveaux d'engagement politique et de succès. Les postes d'ombudsman sont actuellement répartis sur 120 pays sur quatre continents. L'institution de l'ombudsman fait partie de l'histoire de la transformation de I'Etat au XXe siècle ; son efficacité réside dans le fonctionnement de l'état encapsulant, et les variations dans son mandat et sa performance peuvent être liés à la nature de l'Etat en question (monarchie constitutionnelle par ex., ou république, étendue de la séparation des pouvoirs, etc.)

L'ombudsman travaille au nom du public ; il a généralement le droit de lancer des enquêtes de sa propre initiative. La focalisation est généralement procédurale : l'ombudsman doit s'assurer que les procédures adéquates ont bien été suivies pour obtenir une décision administrative ; la décision elle-même sera moins fréquente au sein du mandat.

La vérification est un principe de travail essentiel que se partagent tous les ombudsmans ; toutefois, la façon dont l'ombudsman a été nommé, son statut légal et la sécurité de sa charge, les organismes envers lesquels il est responsable et la possibilité d'engager du personnel et de contrôler un budget sont toutes des choses qui influencent la capacité d'agir et d'agir de façon indépendante dans un rôle de vérification. De nos jours, il existe des ombudsmans parlementaires dans de nombreux pays ; leur efficacité, toutefois, est limitée par le financement. Les ombudsmans dont le financement dépend trop de l'exécutif se trouvent dans une position vulnérable. Il existe plusieurs moyens pour consolider leur autonomie (des structures institutionnelles par exemple, qui protègent le titulaire du poste de toute interférence politique et des dispositions qui requièrent que les ombudsmans fassent des rapports au grand public).

Tous les ombudsmans ont en commun une série de principes de travail, courants dans les systèmes de vérification. Leçons à tirer :

- Les ombudsmans les plus efficaces sont ceux qui cherchent à améliorer les mécanismes du système administratif, au lieu d'en divulguer ou d'en dénoncer les défauts.

- La mise en place de la légalité en soi n'est pas le motif premier ni le point de ralliement des ombudsmans. Le souci majeur du bon ombudsman sera la justice.

- Des dispositions de financement et de reportage aptes à améliorer les valeurs fondamentales du rôle sont nécessaires (par exemple, des dispositions de financement qui garantissent l'indépendance).

Source : Diaw (2008) 


\section{Principe 9 : L'inclusion de toutes les étapes dans la chaîne de garde et d'efforts particuliers pour garantir les phases les plus vulnérables}

Là aussi, des arguments assez similaires sont applicables. Toute faiblesse dans la chaîne de garde est susceptible d'être exploitée par des opérateurs commerciaux, et pas uniquement par les opérateurs sans scrupules. On a beaucoup écrit sur les maillons vulnérables de la chaîne telle l'inspection avant expédition. Là où (comme c'est le cas pour les normes relatives à la viande) la pratique de la vérification s'est écartée des normes directives pour privilégier une approche basée sur les risques, cela a eu tendance à répondre à des caractéristiques particulières de l'industrie, qui concentrent les risques sur des phases spécifiques (voir Encadré 3.5). Dans ce cas, la plupart des dangers se trouvent à la ferme et non pas à l'abattoir ; il est logique, dès lors, de se concentrer sur la source première du danger plutôt que sur sa manifestation en aval. Dans le secteur de la forêt, l'unité de gestion forestière représente souvent un point faible à cause de son isolement physique, et cela mériterait d'être souligné. Toutefois, la théorie de la conformité devrait une fois de plus mettre en garde contre le danger des effets pervers. Cela pourrait impliquer un mouvement de pratiques illégales vers des points moins problématiques ou, comme dans le cas de la CCNUCC, le risque de fuite au niveau du projet (c.-à-d. les activités de projets qui ont pour effet de reporter la pollution vers des sites hors des limites du projet). Dans le cas du secteur du bois d'œuvre, tout comme ailleurs, il y a de bonnes raisons pour assurer que les activités de conformité sont dotées d'une composante régionale, supranationales basée sur un raisonnement similaire.

\section{Principe 10 : L'intégration d'approches en faveur des pauvres dans la conception des systèmes de vérification}

En général, les études de cas extra sectoriels ne présentent aucune indication forte pour le secteur du bois d'œuvre par rapport à l'équité. Dans les exemples plus marquants, les accords internationaux focalisent sur les avantages internationaux et leurs répercussions sur les pauvres au niveau infranational ne constituent pas un souci majeur ; les effets en faveur des pauvres se positionnent plutôt en bas de la hiérarchie des résultats escomptés. On peut rapprocher ce fait au haut niveau de sophistication technique de la question considérée, de sorte qu'il n'est pas vraisemblable que les pauvres soient engagés en tant que catégorie (dans le cadre du contrôle de sécurité nucléaire, par exemple) ou à la généralité des effets (comme pour la CCNUCC qui contrôle les biens publics mondiaux). Certaines conventions ont bien tenté d'évaluer leurs impacts sur les pauvres. La CITES représente un cas typique, même si les préoccupations à propos de l'écologie et du bienêtre animal ne riment pas trop avec les intérêts sociaux, ce qui complique la mise en place de politiques restrictives de façons légalement cohérentes, mais aussi « en faveur des pauvres ». Parallèlement et alors que les impacts sur les producteurs pauvres préoccupent le Processus de Kimberley, ils ne sont pas automatiquement faciles à défendre si l'on songe à la structure et aux caractéristiques de la chaîne des produits pour les diamants alluvionnaires. S'il est vrai que les pauvres sont très défavorisés par la perte d'emplois dans l'exploitation des diamants, les effets positifs liés au refus de bénéfices exceptionnels aux politiques et aux seigneurs de la guerre pourraient constituer une considération plus exaltante pour les décideurs internationaux. 
Là ou les circonscriptions pauvres ou marginales représentent un souci immédiat, l'évidence privilégie une approche de la législation proportionnée aux besoins. Dans le cas des normes d'hygiène de la viande (Encadré 3.5), on a prévu une disposition spéciale à l'intention des producteurs artisanaux de viande fumée (essentiellement du jambon fumé d'Europe méridionale), pour qui les normes de niveau industriel sont souvent bien audelà de leur capacité technique et leur pouvoir d'achat financier. La forte demande pour de tels produits culturels et leur valeur emblématique ont encouragé l'adoption d'une disposition spéciale, ce qui a été justifié, en termes d'évaluation des risques (ces denrées alimentaires étant souvent le produit de chaînes de production relativement courtes et bien intégrées). De même, plutôt que d'interdire les styles de préparation préférés de certaines minorités ethniques (les "smokies ", ou "fumés " par exemple : des quartiers de chèvre ou de mouton dont les poils et la laine ont été tondus, mais où la peau n'a pas été enlevée, dont sont friands les Antillais et les Africains vivant en Grande-Bretagne) on a investi davantage encore dans la recherche de manières d'assurer que les méthodes de production sont compatibles avec l'intérêt culturel et les besoins des autorités de contrôle, et que les mesures de vérification ont été dûment adaptées. Ces avantages semblent peu importants et périphériques, mais en Europe, en tout cas, la majorité des transformations de la viande sont bien intégrées dans les chaînes d'approvisionnement des supermarchés, ce qui encourage d'importantes économies d'échelle.

\subsection{En résumé}

L'expérience extra sectorielle fournit des indications utiles sur le fonctionnement éventuel des régimes de vérification dans le secteur de la forêt. Des éléments tels que le forage institutionnel, susceptible de satisfaire un grand nombre d'acteurs et d'intérêts ainsi que diverses sources d'information, et une définition de la légalité acceptable pour tous pourraient jouer un rôle proéminent dans tout accord de vérification. De même, la séparation des niveaux décisionnaires entre la politique et la technique, et entre l'évaluation de la conformité et les mécanismes de mise en vigueur au niveau opérationnel pourraient contribuer à dépolitiser les éléments de négociation à exclure du processus politique. Un système séquentiel de gestion de l'information serait recommandable, impliquant trois éléments bien définis (la récolte de l'information, son contrôle et la prise de mesures) avec la démarcation d'une autorité suprême (la fonction de la "prise de mesures ») qui jouit d'une large confiance du public et opère au sein du processus politique. Tout ceci, accompagné de la condition importante que le "processus politique ", dans cette référence, soutienne les principes démocratiques et garantisse une bonne gouvernance dans le sens de l'ouverture, de la transparence et de la responsabilité publique. Une capacité de surveillance indépendante sera de même, associée à des accords internationaux solides, avec cependant une vue équilibrée de ce que cela implique d'acceptable et pour les producteurs et pour les consommateurs, ainsi qu'une grande importance accordée à l'aspect prospectif et constructif plutôt qu'à la dénonciation et aux sanctions.

La deuxième partie de cet ouvrage (B) revient sur le secteur de la forêt et y examine l'expérience de vérification à ce jour, en tant que leçon supplémentaire sur la définition des régimes de vérification viables. 


\section{Partie B}

Études de cas de pays portant sur la vérification forestière 



\section{Chapitre 4}

\section{Aperçu des questions examinées dans les études de cas de pays}

\subsection{Introduction}

Ce chapitre donne un aperçu des diverses études de cas de pays portant sur la vérification dans le secteur forestier, présentées aux Chapitres 5 à 16. Ces études dressent le bilan de la vérification du secteur forestier national dans douze pays : le Brésil, le Canada (Colombie-Britannique), le Cambodge, le Cameroun, le Costa Rica, l'Equateur, le Ghana, le Honduras, l'Indonésie, la Malaisie, le Nicaragua et les Philippines. Ce choix recouvre toute une gamme de contextes, de types de forêts et de relations commerciales différents. Le Tableau 4.1. résume les principales questions de vérification abordées par les études de cas.

\section{Tableau 4.1 Questions de vérification abordées par les études de cas de pays}

\begin{tabular}{ll}
\hline Pays étudiés & Questions de vérification essentielles abordées \\
\hline $\begin{array}{l}\text { Colombie- } \\
\text { Britannique } \\
\text { (Canada) }\end{array}$ & $\begin{array}{l}\text { Ce cas se penche principalement sur le Forest Practices Board, organisme unique } \\
\text { dont la mission est d'obliger l'Etat et l'industrie à rendre des comptes au sujet de } \\
\text { la gestion des forêts. Ce cas présente le rôle de cet élément institutionnel-clé du } \\
\text { système de contrôle et de vérification de la Colombie Britannique (CB) et examine } \\
\text { les modalités de maintien de son indépendance. }\end{array}$ \\
\hline Costa Rica & $\begin{array}{l}\text { Ce cas décrit le système d'administration des forêts du Costa Rica, dans lequel } \\
\text { une variété d'acteurs publics, publics-privés et indépendants intervient pour } \\
\text { assurer le contrôle et la vérification de la production forestière. Ce cas se penche } \\
\text { principalement sur le besoin d'assurer le financement des activités de vérification, } \\
\text { ainsi que sur le rôle des professionnels du secteur forestier. }\end{array}$ \\
\hline Honduras & $\begin{array}{l}\text { Ce cas décrit un système de contrôle national des forêts inchangé dans lequel les } \\
\text { activités de vérification sont réalisées par un observateur indépendant auquel a } \\
\text { fait appel le Commissariat aux droits de l'homme et non la filière forestière. }\end{array}$ \\
\hline Nicaragua & $\begin{array}{l}\text { Ce cas décrit un système de vérification émergent. Il met en évidence le besoin } \\
\text { de poser des limites juridiques claires aux responsabilités de la vérification et } \\
\text { fait le constat qu'un système conçu pour lutter contre l'exploitation forestière } \\
\text { illégale ne permet pas nécessairement de remédier au problème plus général de }\end{array}$ \\
la déforestation.
\end{tabular}


Tableau 4.1 (suite)

\begin{tabular}{|c|c|}
\hline Pays étudiés & Questions de vérification essentielles abordées \\
\hline Equateur & $\begin{array}{l}\text { Ce cas se penche sur le Outsourced National Forest Control System (SNTCF), } \\
\text { système national de contrôle des forêts imparti, innovateur mais éphémère, qui } \\
\text { délègue la responsabilité de l'observation et de l'administration publique des } \\
\text { activités forestières à trois organismes différents, et inclut la participation active } \\
\text { de la société civile et du secteur privé. Ce cas met en évidence que la réussite d'un } \\
\text { système de vérification passe par un soutien public important, des domaines de } \\
\text { compétence juridiques clairement définis et un financement indépendant. }\end{array}$ \\
\hline Cameroun & $\begin{array}{l}\text { Le Cameroun est un des pays pilotes qui se prépare activement à la négociation } \\
\text { d'Accords de partenariat volontaire avec l'UE. Ce cas décrit un programme de } \\
\text { réformes entrainé principalement par les donateurs et ayant conduit à des } \\
\text { améliorations significatives de la gestion des forêts, même si des incertitudes } \\
\text { demeurent sur l'adhésion nationale à long terme. }\end{array}$ \\
\hline Ghana & $\begin{array}{l}\text { Le système national de vérification du Ghana est en phase de création. Ce } \\
\text { cas analyse les éléments de la structure du système et l'importance d'assurer } \\
\text { l'indépendance de celui-ci. Il présente également des informations sur le volume } \\
\text { d'investissements requis pour soumettre un secteur jusque-là très peu contrôlé à } \\
\text { un processus de réforme auquel pourront à la fois se fier la société civile nationale } \\
\text { et le marché international du bois. }\end{array}$ \\
\hline Cambodge & $\begin{array}{l}\text { Ce cas se penche sur l'un des éléments de la vérification (l'observation } \\
\text { indépendante par une tierce partie) au travers de deux prestataires de services } \\
\text { ayant des approches très différentes. Il met en évidence que les programmes } \\
\text { dirigés par des donateurs ont peu de chances d'aboutir sans adhésion nationale } \\
\text { pleine et entière dans le cadre d'une coalition interne pour la réforme. }\end{array}$ \\
\hline Philippines & $\begin{array}{l}\text { Ce cas porte principalement sur les comités multisectoriels de protection de } \\
\text { la forêt aux Philippines, qui montrent comment un processus indépendant à } \\
\text { partenaires multiples peut être mis en place pour jouer le rôle d'un système de } \\
\text { vérification du secteur forestier autorisé par l'Etat. }\end{array}$ \\
\hline Indonésie & $\begin{array}{l}\text { Le système de vérification en Indonésie associe des audits obligatoires et } \\
\text { une autorégulation de la filière. Ce cas décrit les travaux actuels du ministère } \\
\text { des Forêts et de l'institut de l'écolabel indonésien (organisme de certification } \\
\text { volontaire) ayant pour objectifs : (i) de définir et tester un référentiel de légalité } \\
\text { unifié, répondant spécifiquement au FLEGT et anticipant un APV et (ii) de } \\
\text { renforcer les standards de GDF et l'accréditation des auditeurs obligatoires. Des } \\
\text { luttes de pouvoir incessantes entre le gouvernement central et les autorités } \\
\text { provinciales et de districts compliquent la situation. }\end{array}$ \\
\hline Malaisie & $\begin{array}{l}\text { Les trois systèmes (la Malaisie péninsulaire, le Sabah et le Sarawak) se distinguent } \\
\text { par le fait que la vérification de la légalité y est administrée par les pouvoirs } \\
\text { publics et qu'elle s'appuie en autres sur le contrôle régulier des pratiques } \\
\text { d'exploitation, une administration des bois sur papier et des audits périodiques } \\
\text { des offices des forêts au niveau des districts ainsi que des titulaires de permis. Ce } \\
\text { cas fait ressortir l'intérêt de l'association d'audits volontaires (ISO, FSC) et d'audits } \\
\text { obligatoires (sous la tutelle de l'Etat ou d'autres pouvoirs publics) et examine la } \\
\text { question de la convergence de la vérification et de la certification. }\end{array}$ \\
\hline
\end{tabular}


Il a été noté au Chapitre 2 que des systèmes de vérification du secteur forestier liés formellement à des accords commerciaux n'existent pas encore, dans lesquels des dispositifs officiels confirmeraient l'utilisation de la ressource conformément aux dispositions légales et fourniraient une attestation de conformité pour faciliter le commerce international. Ces études de cas sont néanmoins riches en enseignements, ne serait-ce que parce qu'elles fournissent les éléments constitutifs de la création de systèmes futurs. Étant donné la variabilité des conditions initiales dans les pays considérés, le but est d'identifier les principes de conception de systèmes de vérification qui peuvent être appliqués d'une manière générale, et ceux qui sont tellement spécifiques de conditions particulières qu'il faudrait se garder des tentatives de généralisation.

Chaque étude de cas a été préparée par un ou plusieurs membres de l'équipe internationale de recherches VERIFOR, avec l'aide d'un consultant originaire du pays étudié pour certaines d'entre elles. Les recherches initiales ont eu une durée variant de quelques semaines à plusieurs mois. La plupart d'entre elles ont été achevées en 2006. La situation évoluant très rapidement dans la plupart des cas étudiés, il a fallu trouver un compromis entre un descriptif instantané et un processus de mise à jour permanente. ${ }^{1}$ Les recherches étaient constituées d'une série d'entretiens avec des parties prenantes très diverses, d'une analyse bibliographique secondaire et dans la majorité des cas, de réunions de groupes de consultation.

\section{Encadré 4.1 Résumé du cadre analytique}

\section{Moteurs de la vérification :}

- Facteurs qui créent une demande de vérification ; et

- Facteurs qui poussent un pays donné à donner suite à cette demande.

\section{Facteurs contextuels :}

- Nature de l'industrie de la forêt et du bois, et localisation des marchés ;

- Questions de gouvernance ;

- Le cadre politico-administratif, y compris l'administration forestière et l'organisation judiciaire, et des considérations intersectorielles ;

- La structure juridique, notamment les questions de tenure de la ressource forestière ;

- Influences socio-structurelles; et enfin,

- Intérêts extérieurs suscités par le secteur forestier, émanant en particulier de donateurs et de militants environnementalistes internationaux.

\section{Description du système de vérification :}

- Structure organisationnelle ;

- Implantation institutionnelle ;

- Fondation juridique ;

- Indépendance budgétaire ;

- Structures de rapport ;

- Aspects anti-pauvreté ;

- Impacts du système de vérification.

1 Pour chaque étude de cas, la date d'achèvement de l'étude est indiquée dans une note au début du chapitre, date à partir de laquelle aucune mise à jour majeure n’a été réalisée. 
Afin de rendre les comparaisons plus aisées, un cadre analytique commun a été élaboré pour orienter les études. Ce cadre (résumé dans l'encadré 4.1) porte sur trois domaines principaux : les moteurs, le contexte et la description du système, chaque domaine comportant un nombre de variables-clés. Le premier domaine concernant les moteurs s'attache à comprendre ce qui a motivé la création d'un système de vérification et à déterminer le rôle des influences à l'œuvre dans l'optique des différents résultats obtenus. Le second domaine concernant le contexte porte sur les contraintes contextuelles susceptibles d'influer sur la conception des systèmes de vérification. La diversité des systèmes de vérification existants montre l'importance du contexte quand il s'agit de comprendre les pressions qui ont conduit à leur mise en place, ainsi que leur forme définitive. La troisième composante du cadre, la description, présente les caractéristiquesclés de chaque système de vérification. Les parties suivantes donnent plus de précisions sur ces trois dimensions.

\subsection{Les moteurs de la vérification}

Les motivations de la mise en place d'un régime de vérification se répartissent en deux ensembles de points distincts. Le premier groupe de moteurs comprend ceux qui créent la demande de vérification dans certains milieux. Le deuxième groupe de moteurs concerne la volonté du gouvernement et de l'industrie de donner suite à cette demande.

Le Tableau 4.2 traite du premier point et montre les divers intérêts et objectifs que pourraient avoir les différents groupes de parties prenantes par rapport à la vérification. Ce tableau propose une classification en trois groupes constitués par les promoteurs (c'est-à-dire ceux qui sont susceptibles de défendre activement la cause de la vérification), les partisans principaux (qui pourraient fournir un soutien actif même s'ils ont peu de chances de montrer la voie), et ceux qui sont plutôt enclins à rester neutres ou à s'opposer à la vérification. Il ne cherche pas à caractériser le comportement de tous les acteurs d'une catégorie, qu'elle soit individuelle ou collective, mais simplement à préciser les rôles typiquement joués par certaines institutions quand il s'agit de décider d'investir ou non dans la vérification de la légalité. Ainsi, les administrations forestières nationales semblent rarement jouer un rôle prépondérant pour tout ce qui concerne le FLEGT, mais cela ne veut pas dire que tous les collaborateurs de ces administrations sont nécessairement indifférents à cette question sur le plan personnel. De même, les communautés locales ont souvent de bonnes raisons de ne pas soutenir les efforts d'application de la loi, non pas parce qu'elles sont partisanes de l'illégalité, mais parce que leur expérience de ces efforts a souvent été négative. Le tableau n'a pas non plus pour objectif de trouver des coupables. Ainsi, c'est sûrement à juste titre que la grande industrie forestière internationale fortement capitalisée manifeste une adhésion plus forte à la gestion à long terme que les petits producteurs (bien que ce ne soit pas toujours le cas), et que les fonctionnaires des ministères des Forêts éprouvent des difficultés à agir indépendamment des exploitants forestiers, dont ils dépendent à bien des égards. Ce tableau a plutôt pour objectif de mettre en évidence ces dilemmes fréquents et d'aider à comprendre pourquoi les intérêts divergent tant en matière de vérification. 
Ce tableau met en évidence que dans un contexte de pays producteurs, ce sont les donateurs internationaux qui ont été les plus proactifs dans la mise en place de systèmes FLEGT. Les pouvoirs publics nationaux ont rarement pris la tête du mouvement, même si les gouvernements centraux pourraient bien trouver avantageux de prendre le train en marche, surtout dans la mesure où ces systèmes permettraient d'augmenter les recettes fiscales et d'obtenir des marchés à l'étranger. Cependant, les administrations forestières sont susceptibles d'être moins favorables à la vérification parce qu'elles sont plus étroitement impliquées dans les systèmes de favoritisme existants. De la même façon, il est peu probable que les exploitants forestiers aient un rôle actif au début du processus, mais une fois la dynamique engagée, la grande industrie internationale pourrait bien y trouver des avantages, bien plus d'ailleurs que les petits exploitants locaux, craignant d'être marginalisés par les entreprises internationales. La catégorie des «ONG » englobe des réalités très différentes et complexes, difficiles à cerner dans des termes généraux. Certaines ONG internationales et locales sont plutôt favorables à une coopération active avec l'industrie du bois et soutiennent le commerce international si celui-ci est bien géré, tandis que d'autres y sont irrémédiablement hostiles et ne voient dans la vérification qu'un moyen supplémentaire d'exercer des pressions sur l'industrie.

Ce sont les intérêts et influences de parties prenantes très diverses qui doivent être prises en compte conjointement si l'on veut comprendre les forces dynamiques qui génèrent un système de vérification donné. Les intérêts en jeu pourraient avoir trait aux questions directement liées au FLEGT ou à d'autres processus politiques internationaux, dont l'objectif clairement affiché est d'accroître les recettes et d'augmenter l'efficacité de l'application de la législation relative aux forêts. Il peut s'agir également d'intérêts commerciaux (valorisation de la marque), de considérations politiques (le souci d'un gouvernement central de contrôler les activités économiques dans ses provinces) ou de préoccupations liées à l'environnement et aux droits de l'homme. Ce tableau fait clairement ressortir le fait que beaucoup d'éléments moteurs se situent en dehors de la filière forestière, les ONG locales et internationales et d'autres acteurs internationaux jouant un rôle particulièrement important, ce qui se retrouvera probablement dans la plupart des situations décrites.

La nature politique des motivations profondes est clairement mise en évidence dans le cas brésilien. L'hostilité du gouvernement brésilien à l'égard du processus FLEGT s'explique largement par le fait qu'à son avis l'exploitation forestière illégale découle principalement de la conversion illégale des terres et non du commerce illégal. Il soutient que le volume important de bois d'origine illégale sur le marché local est un effet secondaire découlant de l'ampleur de ce phénomène de conversion des terres plutôt que sa cause première. Par conséquent, selon le gouvernement brésilien, la solution n'est pas d'exercer des pressions d'action sur la demande, visant le commerce et en particulier le commerce international (solution qui si elle était valable, justifierait l'intérêt international suscité par les forêts brésiliennes), mais de mettre en place des contrôles d'action sur l'offre, visant les pratiques de gestion foncière (qui relèvent exclusivement de l'Etat brésilien souverain). Dans le cas brésilien, il faut accessoirement tenir compte du fait que les exportations nationales transitent toutes par un nombre réduit de ports (moins d'une vingtaine), qui de ce fait, 


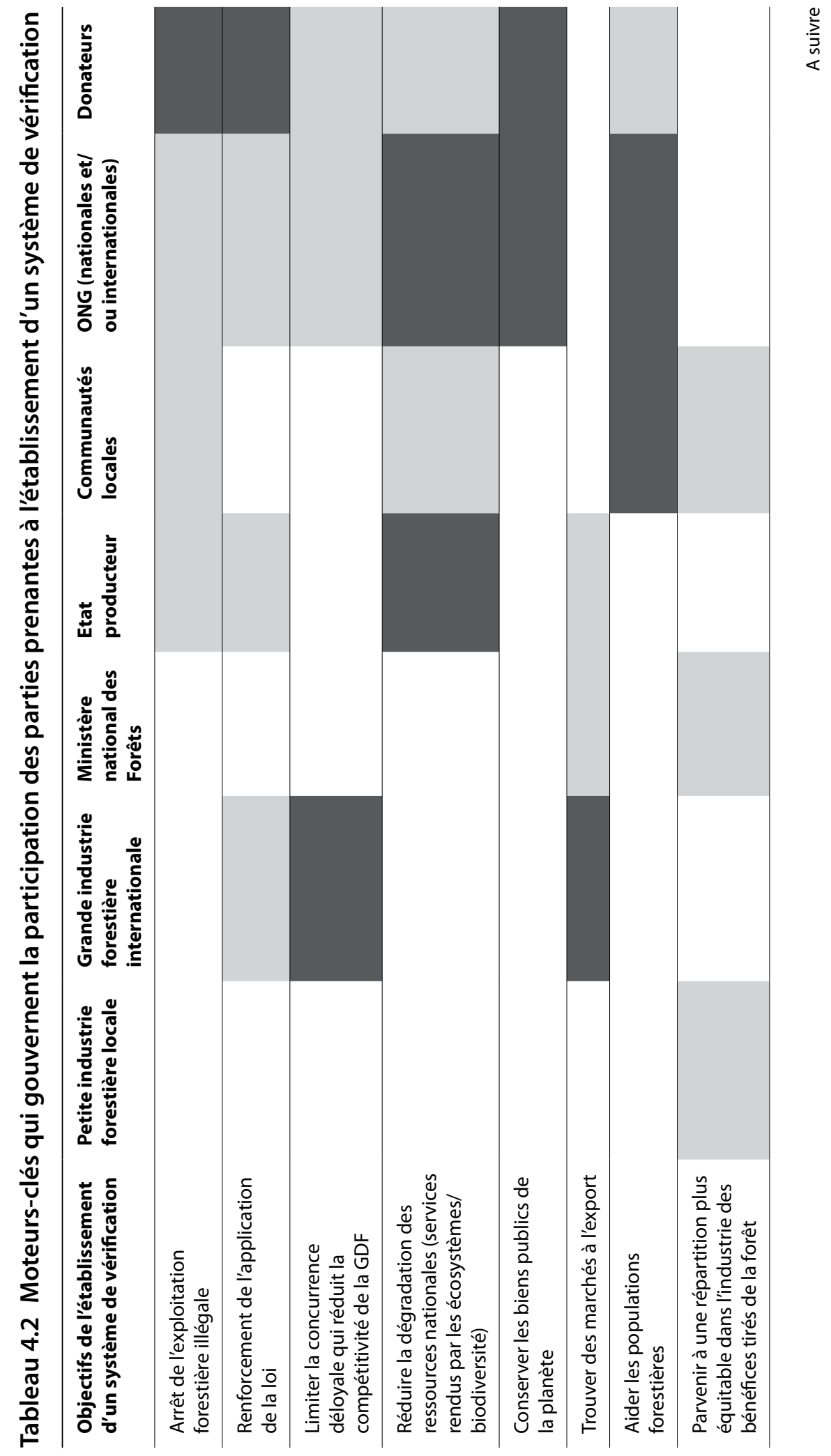




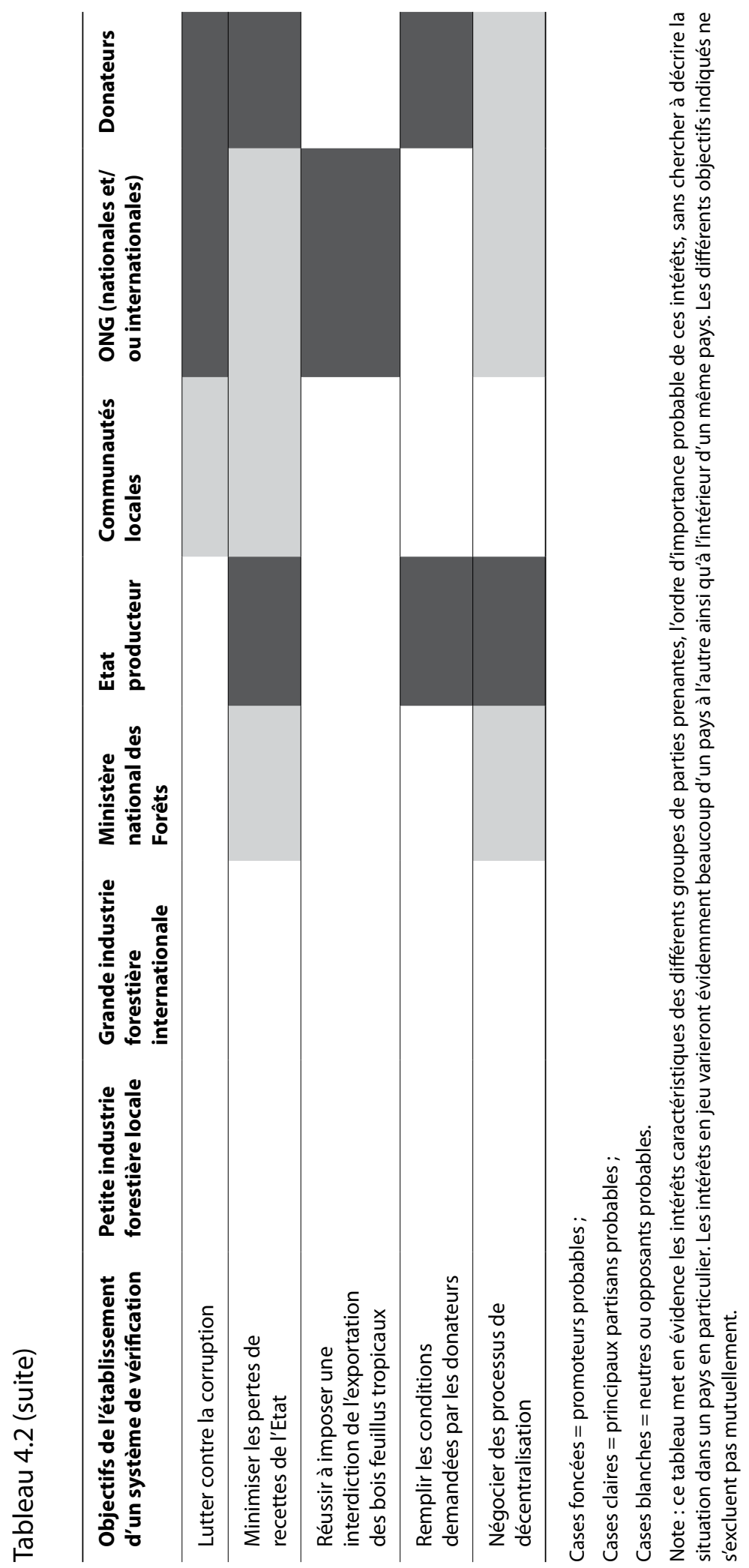


se prête à des contrôles nationaux. Le message que le gouvernement brésilien cherche à faire passer est que ces questions sont du ressort de l'Etat souverain et qu'elles doivent échapper aux influences politiques externes, surtout lorsqu'elles émanent des pays du Nord. Une argumentation similaire peut s'appliquer à l'Indonésie par exemple ou à d'autres pays asiatiques, dans lesquels la conversion des terres pour la production d'huile de palme ou d'autres cultures est en grande partie responsable du déboisement. Dans la plupart des régions d'Afrique, la conversion des terres capitalistique a moins d'influence que les pratiques d'exploitation des forêts (et dans certains cas, la conversion agraire du fait de petits planteurs profitant des coupes forestières), et donc des pressions exercées prioritairement sur l'industrie du bois pourraient être plus efficaces. Il découle clairement de tout de qui précède que la conception d'un système de vérification, quelle que soit la situation, doit commencer par l'évaluation des problèmes de fond qui affectent les régions forestières et par l'analyse des mesures permettant d'y remédier.

La " volonté de mobilisation » est un moteur qui peut être interprété diversement. Cette volonté pourrait indiquer qu'un pays producteur reconnaît la gravité des troubles au sein de la filière forestière et la nécessité d'y remettre de l'ordre. D'un autre côté, elle pourrait n'indiquer qu'une simple mesure d'endiguement sans intention radicale, signifiant seulement que le pays a admis la nécessité d'une réponse précoce, comme meilleure stratégie de contrôle d'une situation sur le point de dégénérer. En ce qui concerne la bonne volonté dont fera preuve un gouvernement donné à l'égard des demandes de vérification qui lui sont faites, il faudrait distinguer les intérêts des gouvernements en général à mettre en place des mécanismes de vérification et les intérêts des ministères des Forêts qui en seront chargés. En général, les ministères des Forêts générateurs de revenus paraissent moins enthousiastes que les ministères distributeurs, tels que les ministères des Finances, quand il s'agit de renforcer les contrôles forestiers. Dans le cas de l'Indonésie par exemple, les pressions en faveur de l'amélioration des pratiques de gestion des forêts sont venues de l'intérieur du gouvernement à la fin des années 90 , après que le ministère des Finances eut constaté l'ampleur massive des pertes dues à l'exploitation forestière illégale (supérieures aux crédits demandés au FMI pour faire face à la crise financière dont était victime le pays). Une fois admis la nécessité d'améliorer les contrôles forestiers et le rôle positif que la vérification de la légalité pourrait jouer dans ce sens, le défi se réduit à l'établissement d'un système de vérification qui soit plausible et légitime aux yeux de tous les publics concernés. Dans le monde complexe de la politique forestière, il est peu probable qu'un système de vérification puisse être mis en place de manière autonome par l'administration forestière. Plusieurs raisons permettent de le penser, dont les suivantes :

- Les coûts élevés de la mise en place d'un système de vérification venant s'ajouter aux structures de contrôle forestier existantes ;

- Cela équivaudrait à supposer que "les choses ne sont pas comme les autorités le prétendent " et qu'il y a des raisons de mettre en doute le fonctionnement normal du système de contrôle forestier ; soit en fait, en termes diplomatiques, la perte de prestige qui en découlerait probablement pour l'administration forestière ; et enfin,

- Les risques élevés pour les autorités politiques et/ou l'industrie forestière de soumettre leurs registres à un examen externe minutieux, et le danger que les forces libérées 
puissent être difficiles à dominer (demandes émanant des populations autochtones ou du «mouvement pour la bonne gouvernance ", par exemple).

Il est beaucoup plus probable que les pressions exercées sur le gouvernement pour qu'il rende plus rigoureux la gestion forestière et le système de contrôle des forêts émanent d'influences externes au secteur forestier, que ce soit de la société (par le biais du militantisme de la société civile par exemple) ou de l'extérieur (par le biais de donateurs internationaux ou d'ONG environnementalistes par exemple), et que les mesures prises résulteront d'un compromis entre les différentes parties concernées.

Cependant, la situation évolue rapidement et des mouvements tels que le FLEGT de l'UE/ la Banque Mondiale et l'initiative ALFA emmenée par le Brésil suscitent des pressions spécifiques que les pays producteurs doivent supporter. Par conséquent, même s'il est peu probable que la majorité des gouvernements producteurs et des administrations forestières mettent en place des systèmes de vérification de leur propre initiative, les pressions auxquels ils sont soumis sont susceptibles d'évoluer. On observerait même le début d'une tendance à l'élargissement de ces influences externes, les forces régionales devenant plus importantes.

Les réponses des pays producteurs à toutes les formes d'incitations externes à la vérification quelles qu'elles soient, seront en toute probabilité fortement conditionnées par leurs intérêts politiques et commerciaux, ainsi que par une série de facteurs liés au contexte.

\subsection{Facteurs contextuels}

Une fois que la nécessité d'une réforme a été admise, ceux dont c'est la mission doivent, soit travailler sur la base des systèmes existants et aller de l'avant par étapes progressives, soit bénéficier d'un soutien, de capacités et de ressources suffisants pour faire table rase du passé et repartir sur de nouvelles bases. Il existe un certain nombre de contraintes structurelles et institutionnelles qui agissent sur les gouvernements producteurs et qui sont susceptibles d'avoir une influence sur leur décision d'agir et sur l'efficacité de leur action. Ces contraintes peuvent être classées en six catégories principales :

- Les caractéristiques du domaine forestier, la nature de l'industrie du bois qui lui est associée et les marchés pour les produits forestiers ;

- Les questions de gouvernance ;

- Le cadre politico-administratif, y compris l'administration forestière et l'organisation judiciaire, et des considérations intersectorielles ;

- La structure juridique, surtout les systèmes de tenure forestière et les formes particulières quils prennent ;

- La structure sociale, tant à l'intérieur qu'à l'extérieur des zones forestières; et enfin,

- Les intérêts externes portés au secteur forestier, émanant en particulier de donateurs et de militants environnementalistes internationaux.

Ces contraintes sont passées en revue ci-après : 


\section{Les caractéristiques de l'industrie de la forêt et du bois, et la localisation des marchés ${ }^{2}$}

Le premier facteur qui influe sur la probabilité d'adopter un système de vérification et qui conditionne également beaucoup le type de système établi est lié au type de domaine forestier, et en particulier à l'abondance, à la valeur et à l'homogénéité de la ressource. Le deuxième facteur est la demande en bois et en produits bois sur les marchés internationaux. Toutes choses étant égales par ailleurs, un pays ayant une ressource en bois importante et de grande valeur ainsi qu'une industrie exportatrice prospère considérée comme un moteur essentiel pour l'économie nationale est plus susceptible d'envisager favorablement la vérification de la légalité comme moyen de valoriser son image internationale et son profil commercial, qu'un pays ayant une ressource appauvrie et une industrie du bois marginale.

Toutefois, d'autres facteurs peuvent également intervenir. Ainsi, la structure contractuelle sur laquelle repose le fonctionnement de l'industrie pourrait avoir une influence sur le plus ou moins grand intérêt porté au développement d'une gestion à long terme qui soit rigoureuse et légale. Les concessions de longue durée (jusqu'à 100 ans dans le cas de la Malaisie) renforceraient cet intérêt, à la condition toutefois que la capacité de transformation locale soit suffisamment compétitive et que les autres acteurs soient contrôlés de manière adéquate. Le fait que les situations diffèrent au sein de l'industrie pourrait également avoir une influence. Même si l'industrie légitime pourrait avoir de bonnes raisons de vouloir apporter la preuve de l'origine légale de sa production dans un pays donné, elle devra faire face à une pléthore de petits producteurs et transformateurs contraints d'acheter leur bois là où ils le peuvent et qui commercialisent leur production sur un marché intérieur moins concerné par la légalité.

On peut également penser que la structure industrielle favorise l'illégalité de par ses conditions opérationnelles. Par exemple, sous l'influence d'une conjonction de pressions internationales et internes, plusieurs des producteurs primaires principaux (l'Indonésie et la Malaisie par exemple) ont renforcé leurs capacités de production bien au-delà des coupes annuelles autorisées dans le domaine national forestier. Dans le cas indonésien, l'écart entre ces deux volumes s'élèverait à environ 46 \% (2005). Pour ces producteurs, les décisions concernant la vérification peuvent non seulement être liées à la volonté de contenir les pressions d'origine interne, mais également au degré de contrôle des importations souhaité, importations dont ces pays dépendent en partie pour assurer une production suffisante dans leurs unités de production. De bonnes intentions sont probablement insuffisantes pour garantir la légalité future de l'industrie, des équipements de coût élevé devant être amortis, ce qui oblige les unités de production à tourner au maximum ou presque de leur capacité.

Les facteurs liés aux échanges commerciaux pourraient peser considérablement en faveur d'une réforme et contribuent à expliquer les réactions à la proposition de l'UE au sujet

2 Nous exprimons notre gratitude à James Hewitt de Global Timber pour l'aide précieuse qu'il a apporté en fournissant la majeure partie des données présentées dans ce chapitre. 
d'Accords de partenariat volontaire. La Figure 4.1 montre le volume des exportations de bois feuillus tropicaux en 2006, par origine. ${ }^{3}$ L'échantillon représenté comprend les onze pays tropicaux des études de cas VERIFOR, qui peuvent être divisés en trois groupes :

1. Les principaux exportateurs de bois feuillus tropicaux, qui exportent environ 10 millions de mètres cubes et plus par an : la Malaisie, l'Indonésie et le Brésil ${ }^{4}$;

2. Un groupe comprenant le Cameroun, les Philippines, le Ghana, l'Equateur et le Cambodge, qui ont tous d'importantes industries exportatrices de bois feuillus, avec des productions annuelles de l'ordre de plusieurs centaines de milliers de mètres cubes.

3. Trois pays d'Amérique centrale (le Costa Rica, le Honduras et le Nicaragua) sans industrie du bois importante au plan international.

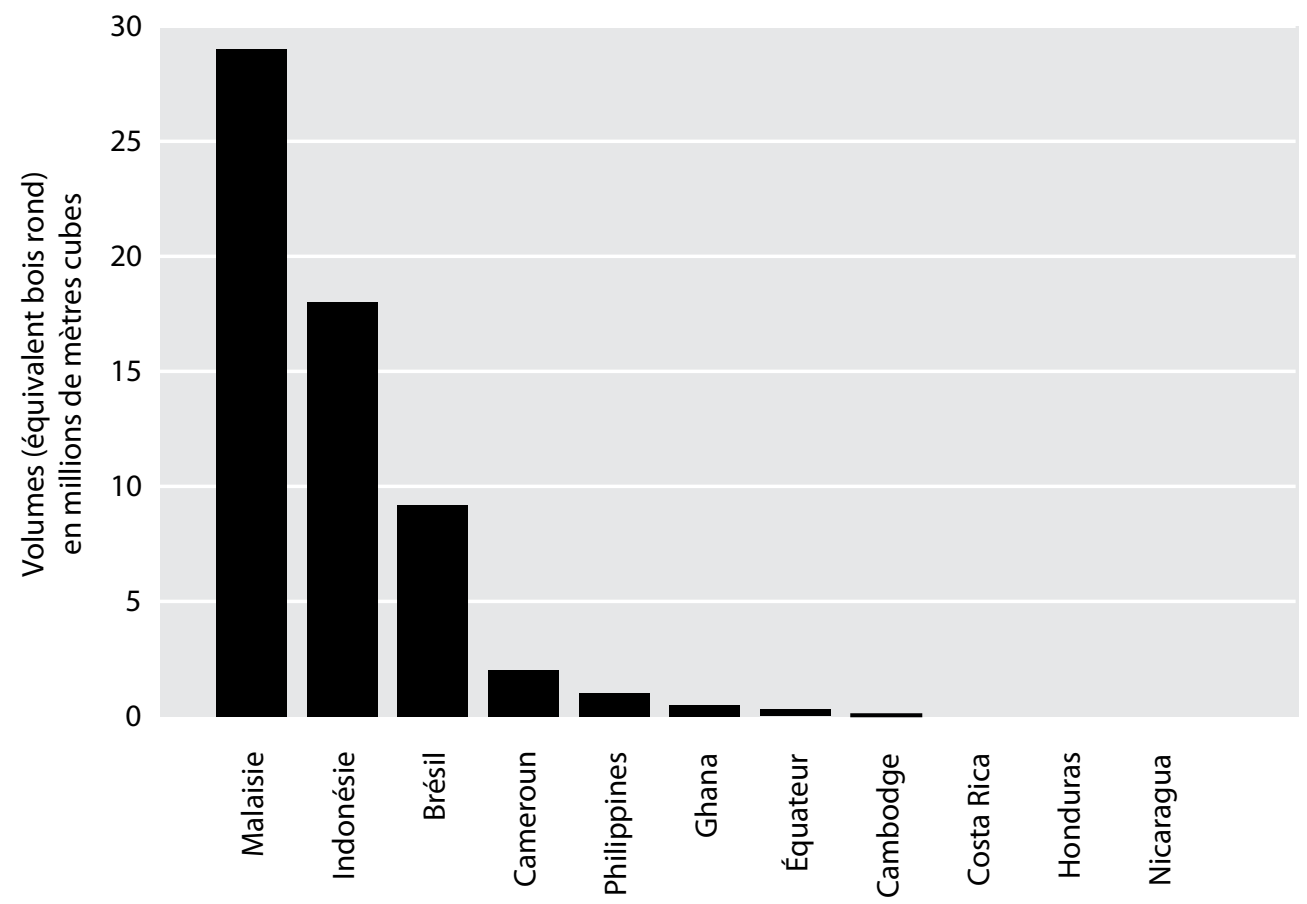

Figure 4.1 Volumes des exportations de bois feuillus tropicaux pour les pays des études de cas VERIFOR (2006)

3 Par définition, les bois feuillus tropicaux incluent ici les meubles en bois et excluent le bois de chauffage, le bois de trituration, ainsi que les pâtes et les papiers.

4 Les exportations de la Malaise atteignent un niveau inégalé à presque 30 millions de mètres cubes annuels, mais il faut noter que ce volume comprend des quantités importantes de bois d'hévéa (Hevea brasiliensis), particulièrement dans les segments des meubles et des panneaux de fibres. Ce bois provient de plantations d'hévéas vieillissantes posant très peu de problèmes de gouvernance et de conservation. 
Si l'on prend 2006 comme année de référence la plus récente, les marchés cibles des produits bois varient considérablement dans l'échantillon de pays VERIFOR. Le Tableau 4.3 donne les parts de marché en pourcentage pour plusieurs des principaux pays consommateurs (l'UE, les US, le Japon et la Chine). De ce point de vue, les pays VERIFOR se classent en trois catégories distinctes :

1. Les pays d'Asie de l'Est (la Malaise, l'Indonésie et les Philippines) pour lesquels le marché à l'export dominant est constitué par le Japon ;

2. Les pays d'Amérique du Nord et du Sud (le Brésil, l'Equateur et la ColombieBritannique canadienne) dont le principal marché à l'export est constitué par les US ; et enfin

3. Les pays d'Afrique occidentale et centrale (le Cameroun et le Ghana), pour lesquels l'UE constitue le marché à l'export dominant.

A la lumière de ces chiffres, il n'est pas surprenant que les APV aient fait plus de progrès avec certains des producteurs africains qu'en Amérique latine. Les marchés européens sont fondamentaux pour la plupart des producteurs africains et leurs chaînes logistiques pour atteindre l'Europe sont relativement courtes. C'est pourquoi l'argument du pouvoir d'achat a plus de prise sur eux. Les caractéristiques des échanges commerciaux de bois en Amérique latine sont différentes, et même si de gros producteurs comme le Brésil entretiennent des échanges commerciaux soutenus avec l'Europe, ces pays sont plus orientés vers les USA et d'autres marchés (dont des marchés internes). Pour les pays

Tableau 4.3 Destinations des exportations (2006) des pays des études de cas VERIFOR, exprimées en pourcentage des exportations totales

\begin{tabular}{lcccccc}
\hline Pays & UE & Japon & USA & Chine & Autres & Total \\
\hline Malaisie & 7 & 27 & 8 & 10 & 48 & 100 \\
\hline Indonésie & 14 & 25 & 10 & 9 & 42 & 100 \\
\hline $\begin{array}{l}\text { Colombie- } \\
\text { Britannique } \\
\text { (Canada) }\end{array}$ & 1 & 11 & 84 & 1 & 3 & 100 \\
\hline Brésil & 26 & 1 & 44 & 7 & 22 & 100 \\
\hline Cameroun & 71 & 0 & 3 & 17 & 9 & 100 \\
\hline Philippines & 2 & 53 & 4 & 19 & 22 & 100 \\
\hline Ghana & 70 & 0 & 20 & 3 & 7 & 100 \\
\hline Équateur & 8 & 0 & 86 & 2 & 4 & 100 \\
\hline Cambodge & - & - & - & 75 & 25 & 100 \\
\hline Costa Rica & - & - & - & - & - & - \\
\hline Honduras & - & - & - & - & - & - \\
\hline Nicaragua & - & - & - & - & - & - \\
\hline
\end{tabular}


d'Amérique centrale, les marchés externes sont très limités et les moteurs externes d'une amélioration de la gouvernance forestière n'existent pratiquement pas. Dans ces pays, le programme de réforme est motivé presque exclusivement par des parties prenantes internes, et le commerce international est rarement un facteur important; des questions d'ordre politique, comme les droits de l'homme et la biodiversité paraissent plus déterminantes. Les producteurs asiatiques sont importants sur le plan international et jouent un rôle prédominant dans les échanges internationaux ; toutefois ils sont proches du marché chinois en plein essor et se caractérisent par des chaînes logistiques complexes dans lesquelles des produits de sources différentes se mélangent. La Figure 4.2. oppose la position dominante de l'UE dans les exportations de bois du Ghana et du Cameroun à la part de marché beaucoup plus réduite de l'UE dans les exportations de bois d'autres pays étudiés.

Étant donné ces facteurs, l'intérêt précoce exprimé par la Malaisie et l'Indonésie dans les APV de l'UE mérite une explication. Le désir de promouvoir un label écologique positif et convaincant est probablement un facteur important : ces deux pays développent tous deux des industries dynamiques dans les secteurs de l'agriculture de plantation et du tourisme et mettent en avant la haute qualité de leurs environnements naturels sur la scène internationale. Les pressions sociales internes ont également une influence, ainsi que le souci de certains segments de l'industrie de défendre leurs propres parts de marché
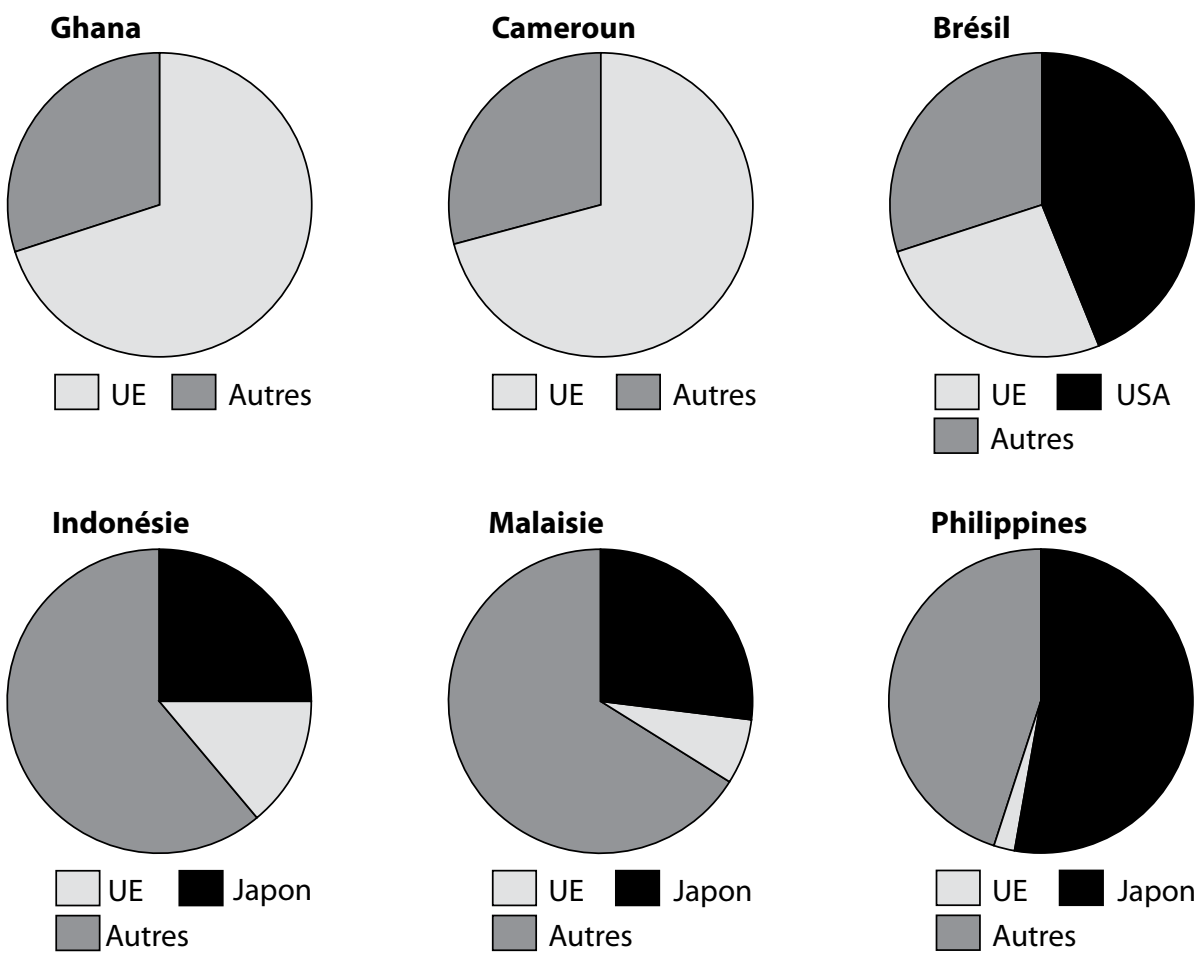

Figure 4.2 Destinations principales des exportations de bois feuillus tropicaux vers l'UE de certains pays producteurs (données 2006) 
sur fond de recul général de celui-ci. Des considérations de prestige (ce sont des acteurs régionaux importants) entrent également en ligne de compte.

Dans les cas où l'intégration del'industrie forestière est développée, aussi bien verticalement (le même acteur industriel contrôlant l'exploitation forestière et la transformation industrielle) qu'horizontalement (production relativement homogène et indifférenciée du point de vue de la taille et de la capacité des acteurs), il existe très probablement des forces favorables à la vérification au sein de l'industrie. Cet intérêt est toujours évident même si l'industrie est en déclin. On peut même dire que c'est dans cette situation qu'il sera le plus vif. Une industrie en déclin peut avoir encore plus besoin de s'assurer un accès au marché qu'une industrie qui se porte mieux, en particulier si elle est dominée par des entreprises à vocation exportatrice, qui ont fait des investissements technologiques considérables et se sont engagés sur des contrats de long terme avec des organismes publics et les propriétaires forestiers. Le Ghana et l'Indonésie sont des exemples typiques. Pour ces producteurs, les pressions externes en faveur d'une légalisation formelle de l'industrie fournissent l'occasion rêvée d'évincer les petits producteurs de la chaîne, situation dont ils pourront tirer partie à long terme. À cet égard, on pourrait établir un parallèle avec le processus Kimberley dans l'industrie du diamant, dans laquelle un petit nombre d'industriels dominants producteurs de kimberlite (mine profonde) sont bien placés pour tirer partie de l'instauration de contrôles plus stricts que les producteurs et les marchés de diamants alluviaux (sédimentaires) plus dispersés et moins rigoureux. À cela s'ajoute le risque que les différences entre classes de producteurs de bois opposées se traduisent en termes nationalistes, facteur supplémentaire dont il faudra tenir compte.

Dans les cas où la réputation de la production est ternie par des accusations d'illégalité alors qu'elle est très orientée vers des marchés " verts " sensibilisés à l'environnement, la vérification peut fournir un moyen très efficace d'exercer des pressions sur l'industrie. Cependant, la capacité d'une industrie à réagir ne doit pas être considérée comme acquise, et divers facteurs peuvent entrer jeu comme la composition de l'industrie de transformation ou la nature du cadre juridique dans laquelle l'industrie opère. Le Vietnam par exemple souhaiterait établir un APV avec l'UE, mais la mise en place d'un tel accord pourrait s'avérer très difficile. La production des scieries et des usines vietnamiennes est assurée principalement par des approvisionnements externes d'origines multiples situées dans toute la sous-région d'Asie du Sud-est. Le suivi de produits fabriqués avec du bois récolté dans divers pays producteurs et transformé dans d'autres sera une opération complexe et difficile, surtout en raison des protocoles diplomatiques entre Etats souverains. Une des faiblesses de l'approche des APV réside dans le fait que, même si l'UE a le droit de limiter les importations sur son territoire provenant d'un pays signataire d'un APV si les marchandises ne satisfont pas aux conditions de cet APV, elle n'a pas de le droit de refuser ces produits s'ils contiennent du bois ou des fibres provenant d'un pays tiers et légalement acquis par l'Etat partenaire de l'EU, intermédiaire dans la chaîne d'approvisionnement, malgré les doutes que l'UE peut éprouver au sujet de leur provenance en début de chaîne. 


\section{Les questions de gouvernance}

D’un point de vue historique, le secteur forestier a toujours été profondément marqué par des questions de gouvernance, surtout dans les pays tropicaux. Il faut remonter à la centralisation du contrôle des ressources, instrument d'administration de colonies réfractaires par les puissances coloniales, pour expliquer l'habitude d'une utilisation abusive des ressources à l'époque postcoloniale, habitude que le mouvement FLEGT se donne pour objectif de combattre. Lorsque se mêlent à la production de bois les intérêts de la politique partisane, que ce soit au sein des éléments dominants de la classe au pouvoir (comme c'est le cas dans la plupart des grands Etats producteurs) ou pour défendre les intérêts d'un régime particulier (comme en Birmanie), les difficultés sont immenses. Parce qu'un système de vérification renforce l'examen minutieux auquel seront soumises les pratiques d'un pays, il y a peu de chances pour que les autorités nationales soient bien disposées à son égard, leur priorité étant la stabilité politique, voire même la survie, plutôt que l'accès aux marchés du bois. Une grande partie des Etats producteurs de bois sont instables politiquement pour des raisons qui dépassent les questions de mauvaise gouvernance interne. Ces Etats sont souvent situés dans des régions mouvementées, prises au piège dans le vide politique de l'après-guerre froide (Baker et al. 2004).

Les intérêts politiques, tout comme l'excès des capacités de production installées, génèrent à eux seuls des pressions susceptibles d'entrâner une utilisation abusive des ressources. Par exemple, les forces armées indonésiennes, auxquelles les crédits affectés par le Trésor public sont insuffisants depuis longtemps, ont été chargées, jusqu'à très récemment, de combler elles-mêmes le déficit de leurs coûts de fonctionnement (qui s'élèveraient à environ $70 \%$ ). Les affirmations qu'elles seraient étroitement impliquées dans la production et le commerce illégal sont fréquentes (Tacconi et al. 2004).

Dans de telles situations, les tentatives de renforcement des capacités de vérification provenant du secteur lui-même se heurtent à des obstacles redoutables. Lorsqu'il existe des groupes de la population importants et déterminés qui se battent pour améliorer la qualité de la gestion des ressources naturelles, il y a des raisons évidentes de rechercher des alliances avec des partenaires extérieurs en vue. Lorsque ce n'est pas le cas, les acteurs extérieurs auront plus de difficultés à trouver à quoi se raccrocher. Chaque situation est distincte et mérite une étude spécifique. D’où que provienne les efforts, il est essentiel qu'ils tiennent compte des rapports de force politiques dans la société et de la place occupée par le secteur forestier dans l'économie politique.

\section{Le cadre politico-administratif national}

L'équilibre des forces et des autorités qui s'établissent entre le ministère des forêts et les autres ministères du gouvernement influe sur la gouvernance des forêts, ainsi que sur la manière dont le gouvernement réagit aux pressions l'incitant à démontrer la légalité de ses pratiques de gestion forestière. 
Des rapports de forces très inégaux entre organes du gouvernement peuvent représenter une menace tout aussi grave pour la bonne gouvernance que le non respect des règles et les activités criminelles au sein de l'industrie, et méritent d'être étudiés. Par exemple, les ministres des forêts ont souvent une influence très grande sur la conduite de l'industrie, y compris sur les questions de discipline (amendes pour les opérateurs récalcitrants), influence qui est susceptibles de dénaturer fondamentalement le fonctionnement de l'administration forestière. Tout d'abord, cela laisse planer un doute sur la gestion de cette dernière, ce qui peut avoir des effets particulièrement pernicieux (par exemple, les sanctions et les amendes que le ministre prétend infliger à l'industrie sont-elles réellement toutes appliquées, ou il y a-t-il un fossé entre les performances proclamées et la réalité ?). Ensuite, dans les cas où des fonctionnaires en début de carrière ont décelé (ou pensent avoir décelé) des abus perpétrés dans les échelons supérieurs, il est peu probable qu'ils mettent leur carrière en danger en s'élevant contre le statu quo. Il ne serait d'ailleurs pas raisonnable d'exiger d'eux qu'ils se mettent en avant pour dénoncer les abus (De Maria 2005). Cette situation crée une culture de résistance (voire même d'opposition systématique dans certains cas) dans toute l'administration forestière aux tentatives de réforme de la gouvernance quelles qu'elles soient. Cette résistance se manifeste entre autres par de l'hostilité à toute mesure visant à établir la vérification de la légalité. Enfin, lorsque le ministre des forêts est étroitement lié au parti au pouvoir, il peut agir dans le secret, ce qui ne fait que renforcer la tendance à utiliser l'industrie comme une vache à lait politique. Cette situation fragilise le fonctionnement démocratique du pays, tout en réduisant l'intérêt que porte l'industrie à tout processus de réforme constructif.

Quand des dispositifs de contrepouvoir existent, ils offrent des possibilités réelles d'amélioration de la gouvernance des forêts, et créent une réelle réceptivité à la vérification et à d'autres mesures visant à améliorer les performances de l'industrie. Le ministère des finances détient un pouvoir important parce qu'il a tout intérêt à améliorer les performances des secteurs productifs et à percevoir efficacement tous les flux de recettes disponibles. Lorsque la mauvaise gouvernance s'étend à l'ensemble du gouvernement, il y a de bonnes chances pour que le ministère des finances ne soit pas non plus au dessus de tout soupçon, mais quoi qu'il en soit, l'évaluation politique des systèmes de vérification passe par une prise en compte de la structure du gouvernement et des freins et contrepoids qui en font partie.

Des considérations similaires s'appliquent aux fonctions judiciaires, en particulier aux rôles joués par l'organisation judiciaire. Pour être efficace, la vérification est fortement tributaire de l'indépendance de l'organisation judiciaire et de sa volonté de fonder ses jugements sur les preuves qui lui sont présentées. Lorsque cette indépendance est compromise, la capacité d'un système de vérification à améliorer la qualité générale de la gouvernance peut être limitée. Il est essentiel de bien prendre conscience de ces réalités politiques si l'on veut comprendre le fonctionnement du système, car les causes des faiblesses de la gouvernance forestière ont de fortes chances de résider ailleurs que dans le secteur forestier lui-même. 


\section{La structure juridique}

Les systèmes du foncier des forêts existants ont une influence tant sur la capacité que sur la volonté des gouvernements de s'engager dans la voie de la vérification forestière. Pour des raisons d'efficacité économique, la vérification dans des forêts presque entièrement publiques et homogènes avec un nombre très réduit de concessions, sera probablement plus aisée, au moins sur le plan technique, que dans des forêts morcelées appartenant à plusieurs propriétaires privés ou à de petites collectivités productrices. La ColombieBritannique est un exemple du premier cas de figure ; la majeure partie de l'Europe, le Sud-est du Canada et certaines parties des Etats-Unis (par exemple l'Etat de Washington) sont des exemples du second cas de figure, tandis que des régions de l'Asie (les Philippines par exemple) sont un exemple du troisième.

Cependant, c'est la plus ou moins grande volonté politique qui déterminera en définitive si ces activités sont effectivement mises en œuvre dans les forêts publiques. Cette volonté politique est plus problématique que la capacité technique. Un cadre juridique perçu comme étant illégitime peut influer négativement sur la volonté des gouvernements d'attirer l'attention du public sur les industries forestières, surtout si les modes de tenure des forêts sont un point sensible. Par exemple, dans les cas où il existe des questions non résolues concernant les droits des populations autochtones (comme c'est le cas dans de nombreux pays asiatiques), le gouvernement est susceptible d'être défavorable à l'initiative de vérification. Cette attitude pourrait s'expliquer par une hostilité non déguisée à l'égard des revendications des populations autochtones, mais aussi simplement par une répugnance à laisser s'exprimer au grand jour un problème qui couve de longue date et qui paraît insoluble. Les autorités estiment souvent qu'offrir des possibilités de discussion publique des questions litigieuses expose à la controverse et qu'il faut absolument décourager toute tentative de ce genre.

La définition du référentiel de la légalité par rapport auquel les entreprises forestières vont être évaluées est également source de conflit. La logique de vouloir synthétiser un référentiel de légalité à partir d'une myriade de lois se comprend assez bien : dans de nombreuses sociétés, les lois sur les forêts sont nombreuses, diverses et contradictoires (l'Indonésie possède par exemple plus de 800 lois relatives à la forêt). Ainsi, d'un point de vue pratique, les lois doivent être condensées suffisamment en un petit nombre de mesures exprimant les intentions principales de la législation. Cependant, le processus de condensation sera probablement hautement sensible et délicat. Les groupes de la société civile (surtout les défenseurs des populations autochtones) profiteront sûrement de l'occasion qui leur est offerte de défendre leur cause. En même temps, les gardiens de la souveraineté nationale sont susceptibles de s'opposer fortement à la présomption selon laquelle ce qui est légal dans une société soit dicté par des intérêts commerciaux, surtout lorsque cette définition du légal demande l'abandon de critères qui leur sont chers. Ces questions sont discutées plus avant dans la partie traitant des référentiels de légalité au Chapitre 19. Certaines questions intéressantes concernant les référentiels de légalité sont soulevées lors de la négociation des accords de partenariat. Par exemple, lorsque le Plan d'Action FLEGT de la Commission Européenne (2005) dit clairement que la question de l'illégalité est seulement un point d'accès vers d'autres considérations de gouvernance 
plus générales, on peut se demander si une telle approche de diversion renforcera la volonté des gouvernements visés de régler la question politique qui était posée au départ, ou si au contraire elle la réduira. Le processus de négociation risque donc de ne satisfaire aucune des parties, ni le gouvernement producteur parce qu'il exige trop de lui, ni les groupes de la société civile et des populations autochtones parce qu'il cède sur trop peu de choses.

\section{Les influences socio-structurelles}

La vérification est influencée par des facteurs liés à l'histoire et à la structure des sociétés de diverses façons. Ces facteurs sont très manifestes quand on considère la faiblesse de la gouvernance dans de nombreuses sociétés riches en forêts. En raison de sa valeur élevée et d'autres caractéristiques, cette ressource extractive a tendance à fragiliser la bonne gouvernance et à peser dans les relations de pouvoir de diverses manières interdépendantes. Ainsi, les gouvernements répugnent souvent à prendre des mesures qui augmenteront le pouvoir des habitants de la forêt sur la gestion de la ressource, si ces mesures limitent leurs possibilités de transiger les bénéfices tirés de la forêt dans le domaine politique. Des acteurs puissants émergent souvent dans ces sociétés (les forces armées dans plusieurs pays par exemple, et des chefs de guerre au Libéria et en République Démocratique du Congo) dont le pouvoir découle ou est renforcé par leur capacité à détourner la haute valeur de la ressource à leur profit. Une mauvaise gouvernance du secteur forestier aboutit à des situations particulièrement instables dans les cas où la majeure partie de la ressource est entre les mains du secteur public (dans l'acceptation la plus étroite du terme) et où les populations forestières les plus proches de la ressource souffrent d'un manque de sécurité foncière, sont pauvres et marginalisées et donc privées de pouvoir (Larson et Ribot 2007). Dans ces situations, les niveaux d'illégalité ont tendance à être particulièrement importants, et les voies vers la réforme particulièrement inextricables.

Le mode de faire-valoir des terres et des arbres est donc une question critique pour la gouvernance forestière, qui a des implications importantes pour l'orientation des efforts de vérification. En plus de la question de principe du pouvoir de l'Etat, les questions principales sont de savoir quels autres acteurs jouissent de la sécurité foncière au regard des terres forestières, et si cette notion s'étend aux arbres sur pied qui se trouvent dessus. Dans certaines parties d'Amérique du Sud, le problème de la propriété est résolu en ce qui concerne les terres et les arbres, les habitants de la forêt pouvant entièrement faire valoir leurs droits de propriété sur ces deux choses. C'est le cas en Equateur et dans certaines parties du Brésil. Dans d'autres cas, le mode de faire-valoir des terres offre une certaine sécurité aux propriétaires fonciers, mais cette sécurité ne s'étend pas nécessairement aux arbres dans tous les cas. Au Ghana par exemple, le contrôle des arbres est dévolu au président, quelle que soit la propriété des terres sur lesquelles ils poussent. Cette disposition était constitutionnelle jusqu'en 1992, et est maintenant englobée dans la loi forestière. Une telle ambivalence peut avoir un effet négatif sur la gérance des forêts parce qu'elle réduit la bonne disposition des propriétaires forestiers envers les exploitants forestiers, et elle affaiblit leur intérêt à observer la loi. À plus long terme, elle 
réduit également leur motivation à reconstituer le stock d'essences indigènes (Danso et Opoku 2005).

Lorsque les revendications foncières traditionnelles ne sont soutenues à aucun niveau, les risques de conflit sont multipliés. L'insécurité foncière transforme les populations forestières en squatteurs de terres publiques, dont la subsistance a été absorbée par la sphère plus large de "l'illégalité ». Dans les zones où l'agriculture itinérante est encore le mode de faire-valoir des terres le plus rationnel d'un point de vue économique pour les populations qui disposent de capitaux limités, ceux-ci ont besoin d'avoir accès à des superficies étendues pour conserver la fertilité des sols. Les populations autochtones pourraient avoir besoin de territoires encore plus vastes si elles veulent conserver leurs modes de vie traditionnels, surtout si elles dépendent de la viande des animaux sauvages pour assurer leurs besoins en protéines et ont besoin d'avoir accès à divers produits forestiers non-ligneux. Ces besoins ne sont pas forcément incompatibles avec l'exploitation des bois, mais leur satisfaction doit être déterminée au cas par cas. La situation la plus fréquente est celle où l'Etat suppose qu'il y a incompatibilité et se sert de cette présomption comme d'une justification pour ne satisfaire que les intérêts d'un groupe de la population, c'est-à-dire de l'industrie du bois.

L'absence de droits de tenure affaiblit gravement le pouvoir de négociation des plus démunis, mais leur capacité à défendre leurs droits est tout aussi importante (Wells et al. 2006). Les plus démunis éprouvent souvent des difficultés à défendre leurs revendications face à des étrangers et sont donc obligés d'adopter des stratégies de survie, valables à court terme, mais qui risquent de compromettre progressivement leurs droits. Leur faible capacité à défendre leurs droits peut être due à leur manque d'autorité face à des actions sanctionnées par l'Etat, ou plus simplement au coût élevé et à la complexité administrative qui seraient associés à une telle tentative. Il n'est pas rare non plus qu'un mode de faire-valoir devienne subordonné à un autre pour des raisons essentiellement politiques, processus surtout susceptible de se produire lorsque la tenure des communautés n'est pas suffisamment soutenue par l'Etat. Par exemple, la tenure des communautés est visiblement déjà très répandue en Papouasie-Nouvelle-Guinée et en République Démocratique du Congo, mais dans ces deux cas, des facteurs politiques renforcent les intérêts politico-industriels et affaiblissent les revendications des plus démunis (voir par exemple, Bird et al. 2007 et Hoare et al. 2007). Au Cameroun, les tentatives de renforcement des droits des communautés dans le secteur artisanal ont fréquemment conduit à l'exploitation de ces opportunités par l'industrie et par une illégalité de plus en plus grande (Oyono, et al. 2006). Cela crée d'immenses difficultés pratiques pour le contrôle et la vérification des forêts. Tous ces facteurs sont susceptibles d'ébranler la volonté des défenseurs de la vérification quand il s'agit de s'attaquer au couple Etatindustrie, sachant que les communautés rurales affectées souffrent d'un manque de droits et de moyens pour défendre leurs intérêts. Lorsque les communautés elles-mêmes n'ont pas d'autre choix que d'agir "illégalement » la plupart du temps, comme dans le cas du Cameroun, la situation pourrait en fait déboucher sur un procès des victimes, plutôt que sur la prise de dispositions plus restrictives visant une industrie rapace. 
Dans les cas où le domaine forestier est divisé entre des concessionnaires industriels et des petites et moyennes entreprises, des considérations économiques passent au premier plan. Des coûts relativement mineurs pour une industrie exigeant des capitaux importants peuvent être considérables pour les petits exploitants, et la façon dont ces coûts sont supportés (par exemple s'ils sont concentrés sur le producteur en début de chaîne) affectera la capacité de ces derniers à respecter les dispositions légales. Il est donc essentiel de comprendre les différentes classes d'opérateurs économiques avant d'évaluer le rapport coût-efficacité d'une initiative de vérification donnée.

Le mode de faire-valoir de la ressource influe également sur l'exécution de la loi. Dans les cas où les droits de tenure des arbres sont faiblement respectés ou absents, l'exécution de la loi pourrait être déviée pour ne viser que ceux qui sont les moins capables de changer leur conduite. Par exemple, les exécuteurs pourraient se concentrer, au moins en partie, sur les activités de petits opérateurs de tronçonneuses, parce que ceux-ci sont une cible facile pour ceux qui chercheraient à générer des rentes de situation (c'est-à-dire des pots-de-vin et de la corruption). Cet état de fait peut masquer les échecs des politiques de fond qui aboutissent à un déclin rapide de la ressource, dont l'absence de mesures d'incitation à la conservation, et non le comportement criminel des plus démunis, est une manifestation. ${ }^{5}$

C'est pourquoi il est important que le système d'assurance de la légalité distingue différentes formes « d'illégalité » et que les efforts se concentrent sur l'aptitude du système à révéler les vraies causes de la " criminalité » et s'attachent à modifier les comportements qui peuvent l'être. Si les énergies du système d'assurance de la légalité sont détournées pour ne viser que les petits opérateurs ayant des capacités très limitées de changement de leurs pratiques, le système aura peu de chances d'atteindre le but recherché, et sera en outre incompatible avec les principes de la " bonne gouvernance ${ }^{6}{ }^{6}$

La force de la société civile et sa capacité à participer au débat sur la légalité sont également la traduction de facteurs socio-structurels solidement établis, dont l'étude a été jugée intéressante pour les recherches menées dans le cadre des études de cas. Un passé récent de révolution ou de guerre civile peut avoir une influence majeure sur l'assurance et la réputation de la société civile, comme l'attestent certains exemples en Asie du Sudest. Des recherches sont nécessaires pour déterminer l'importance du lien entre la structure des classes sociales et le militantisme environnemental. De façon générale, on pourrait s'attendre à ce qu'une sensibilisation et une mobilisation fortes en faveur de l'environnement soient associées à une société diversifiée sur le plan économique et à une classe moyenne développée. Inversement, des pays peu peuplés possédant des

5 Comme l'observe Rackham (1986), l'état de la forêt as plus à voir avec les facteurs qui favorisent la croissance des arbres qu'avec ceux qui encouragent leur coupe.

6 La banque mondiale reconnaît ce danger dans sa stratégie FLEG lorsqu'elle fait l'observation suivante : " Malgré l'ampleur du problème [de la criminalité liée aux forêts], les cas de poursuites et de sanctions sont rares. En fait, lorsqu'il y a des poursuites, elles concernent des pauvres qui cherchent à pourvoir à leur subsistance et qui sont persécutés et envoyés en prison. Les grands opérateurs continuent leurs activités impunément. Sans doute s'agit-il de la pire forme de violation de l'équité et de la justice qui puisse découler de l'échec manifeste de la gouvernance, et c'est à ce problème qu'on doit s'attaquer." (Banque Mondiale 2006, p. xi). 
régions forestières importantes, dont l'économie dépend fortement des rentes provenant d'industries extractives et où les structures sociales sont très polarisées, seraient caractérisés par la faiblesse relative de leur société civile et par un militantisme environnemental peu actif. "La sensibilisation à l'environnement " est une notion plus complexe ; on peut supposer que les petits cultivateurs sont très sensibilisés à l'environnement, mais cela ne se traduit pas forcément par des formes de mobilisation qui permettraient de nouer un dialogue avec le gouvernement ou le milieu humanitaire. Une « société civile forte " n'exprime pas forcément cette force d'une manière qui convienne aux processus politiques modernes, surtout lorsqu'ils ont une dimension internationale. Une société civile faible du point de vue moderne indique plutôt qu'elle a peu de moyens ONG et peu de mouvements associatifs au sens où on les connaît dans le Nord. Cela ne veut pas forcément dire que ces pays sont dépourvus des capacités nécessaires à la mobilisation d'une société civile autochtone ; le problème est plutôt que celle-ci est majoritairement de nature ethnique ou tribale, renforcée par des langues de communication différentes. Une forte dispersion géographique (caractéristique intrinsèque des régions où il subsiste encore de vastes étendues de forêts naturelles) vient s'ajouter à ces difficultés. Tous ces facteurs diminuent la faculté de ces sociétés à nouer le dialogue avec les acteurs internationaux et les "programmes modernes ", ce qui pose également problème pour les gouvernements nationaux.

Il est intéressant de savoir si les mouvements sociaux défenseurs des valeurs de respect de l'environnement sont nécessairement "anti-pauvreté ". En Afrique, le mouvement des ONG est naissant et il entretient des liens satisfaisants avec la société civile dans son ensemble, surtout pour défendre les intérêts des plus démunis. La faculté interne d'autoréglementation des ONG est souvent limitée, ce qui a une incidence sur la capacité du mouvement à s'exprimer d'une seule voix. De la même façon, il est possible que sa capacité à tenir tête aux forces puissantes de l'industrie du bois soit réduite et très dépendante des alliances que formeront ses membres avec des organismes externes, ainsi que des degrés de soutien à l'extérieur du pays. Les ONG environnementalistes ont tendance à être plus puissantes en Amérique latine et en Asie, mais là encore, pas nécessairement " anti-pauvreté ». Certaines ont une préférence marquée pour les problématiques urbaines, ne s'identifient guère aux producteurs paysans indépendants dont ils ne cherchent pas particulièrement à défendre la cause. Même si des ONG environnementalistes axées sur le développement sont actives, elles ont tendance à être plus fortes lorsqu'elles s'appuient sur des groupes de la population clairement définis, comme c'est le cas pour les défenseurs des droits des populations autochtones.

Si l'on peut considérer que la structure sociale rend possible la gouvernance forestière et qu'elle affecte la probabilité que les efforts consentis pour la vérification se traduisent par des effets positifs sur la qualité de la gouvernance, cette influence n'est pas déterminante et la structure sociale et la gouvernance interagissent d'une manière qui n'est pas nécessairement simple. Aussi ces questions doivent-elles être explorées au cas par cas en tenant compte de l'influence de l'histoire. 


\section{Les intérêts externes portés au secteur forestier}

Limportance de l'intérêt externe porté au secteur forestier influe sur la direction de la réforme de celui-ci. Ce soutien peut être particulièrement critique dans les sociétés riches en forêts, dans lesquelles les relations politiques sont déséquilibrées et la gouvernance est faible. Ces alliés externes sont de deux types principaux : les donateurs du Nord d'une part et les militants écologistes et les défenseurs des droits de la personne d'autre part.

Ces dernières années, le groupe des donateurs intéressé par le FLEG comprenait presque exclusivement des institutions de Bretton Woods (la Banque Mondiale et le FMI), et, en ce qui concerne les APV, des bailleurs de fonds du Nord dans le groupe de l'UE (principalement le Danemark, la France, l'Allemagne, les Pays-Bas et le Royaume-Uni). D'autres bailleurs sont actifs dans certaines régions qui reflètent leur situation nationale particulière ; c'est le cas de l'Australie par rapport au Cambodge et d'autres Etats de l'Asie du Sud-est. La présence importante du secteur forestier dans les processus politiques soutenus sur le plan international, tels que les stratégies nationales de réduction de la pauvreté, est susceptible d'accroître l'intérêt des bailleurs, bien que jusqu'à présent les forêts et l'environnement aient toujours été mal représentés dans ces processus (voir Bird et Cabral 2007). Il est intéressant de noter la faible participation jusqu'ici dans le FLEG de grands pays consommateurs tels que la Chine et le Japon. Les forums internationaux comme la réunion FLEG Europe et Asie du Nord qui s'est tenue en novembre 2005 (qui a réuni les pays de l'ancien bloc soviétique) et la réunion du FLEG Chine en octobre 2007 représentent donc une évolution intéressante, qui pourrait aider à élargir la participation de ces acteurs de premier plan.

Les militants écologistes et défenseurs des droits de la personne sont souvent des ONG présents dans les mêmes Etats occidentaux européens que les donateurs humanitaires principaux, mais ils comprennent aussi le puissant groupe de pression de conservation aux USA. Le rôle essentiel joué par les écologistes et les défenseurs des droits de la personne provenant du Nord est une caractéristique intéressante de la scène actuelle du FLEGT et constituent une dimension importante de plusieurs des études de cas traitées dans ce livre. Loin d'être un phénomène isolé, le rôle important joué par ces mouvements dans le secteur forestier est l'aboutissement d'un changement progressif dans la structure de l'autorité publique au cours de la période de l'après-guerre froide, avec plus particulièrement la pénétration d'acteurs privés et non-gouvernementaux dans des domaines jusque-là réservés aux autorités officielles.

Divers commentateurs ont conceptualisé cette scène changeante de plusieurs manières. Pastor (1999) se place du point de vue général de la gouvernance lorsqu'il se penche dans ses écrits sur l'extension de ce qu'il appelle «les dimensions de la responsabilité ». La responsabilité traditionnelle, avance-t-il, a deux dimensions : une dimension horizontale, partagée par les institutions gouvernementales, qui ne devrait pas (en théorie du moins) empiéter sur les domaines de responsabilité légitimes de chacune d'entre elles, et une dimension verticale de responsabilité du gouvernement devant sa population, au travers des élections et d'autres moyens. Pastor propose une troisième dimension de responsabilité, qui renforce les responsabilités verticale et horizontale à la fois, par le biais des actions des 
institutions internationales. Goetz et Jenkin (2004) s'appuient sur deux études de cas en Inde lorsqu'ils étudient les formes d'activisme de la société civile qui remettent en cause la dichotomie verticale-horizontale autour de laquelle la responsabilité a classiquement été envisagée. Les deux organisations considérées ont utilisé des méthodes normalement associées aux institutions d'Etat officielles de la responsabilité horizontale. C'est cette notion qu'ils appellent " une forme de responsabilité hybride ", qui comble l'écart entre les première et deuxième dimensions traditionnelles, sorte d'engagement direct des citoyens (vertical) dans les fonctions de la responsabilité interne à l'Etat (horizontale). Dans le secteur forestier, il existe un facteur de complication qui est le rôle de premier plan joué par des ONG de pression externes et des organisations du secteur privé (la distinction entre les deux n'est pas toujours clairement établie) dans la responsabilité publique. Celles-ci vont au-delà des rôles traditionnellement joués par les organisations non gouvernementales en prenant fait et cause pour l'autoréglementation de l'industrie par des systèmes de certification et des mesures similaires (voir Brosius 1997).

Trois aspects de cette situation sont à souligner dans les études de cas présentées ci-après. Premièrement, les intérêts que les organisations externes ("internationales ») portent à des questions considérées jusqu'ici comme protégées par la souveraineté de l'Etat producteur. L'influence internationale sur l'élaboration des politiques nationales est si forte que l'expression "Etat intermédiaire " a été inventée pour décrire cette relation. L' "Etat intermédiaire " correspond à un Etat qui intègre facilement les attentes externes et les préoccupations planétaires dans les politiques nationales (Kaul et al. 2003). Deuxièmement, il y a les relations entre les organisations externes et divers acteurs nationaux, aussi bien des ONG que des groupes de la société civile. Les rapports de classe qui sous-tendent ces relations sont particulièrement intéressants à étudier (Lipschutz et Fogel 2002). Le troisième point est plus stratégique ; il concerne le degré de contribution d'actions commencées à l'extérieur d'une société donnée à la résolution durable des enjeux de la gouvernance interne. Ce qui s'est passé dans d'autres secteurs ou lors d'initiatives différentes semble indiquer que les pressions externes ont peu de chance d'avoir un effet à long terme, sauf si elles passent par l'intermédiaire d'un programme de réforme dirigé de l'intérieur ; certains grands axes de la politique humanitaire actuelle (par exemple, le soutien financier consenti par plusieurs donateurs associé à des stratégies nationales de réduction de la pauvreté) visent d'ailleurs à renforcer ce lien (voir Booth 2004 et De Renzio et Smith 2005).

\subsection{Eléments-clés et impacts des systèmes de vérification}

\section{Les éléments du système de vérification}

La majeure partie de ce livre est consacrée à la définition des éléments nécessaires à un système de vérification et aux formes de construction les plus à même de soutenir durablement la fermeté des volontés politiques. Du point de vue de la fonctionnalité de l'ensemble, l'analyse des systèmes de vérification doit tenir compte du contexte, même s’il faut également considérer des éléments génériques. 
Les caractéristiques essentielles d'un système de vérification qui ont été retenues lors des recherches VERIFOR comme étant particulièrement intéressantes sont résumées dans l'encadré 4.2. Ce sont les objectifs des systèmes de vérification qui sont d'abord déterminants pour la structure organisationnelle de ceux-ci. Le but d'un système de vérification est-il seulement de contrôler la criminalité et de renforcer l'application de la législation, ou de s'attaquer à des problèmes complexes relatifs à la réglementation de l'industrie ou aux pressions qui s'exercent sur elle? Ou bien y a-t-il une finalité sociale plus générale qui cherche à maximiser le développement et la protection sociale des plus démunis en situation de dépendance par rapport à la forêt ? Lorsque le système cherche à atteindre des objectifs sociaux et des avantages sociétaux plus généraux, il est susceptible d'avoir des effets très positifs sur le degré de participation et "d'adhésion " des acteurs de la société civile. En revanche, lorsqu'il se donne seulement pour objectif d'appliquer la loi, l'intérêt qu'on lui porte sera sans doute moindre. C'est pourquoi la définition de la " criminalité » est une question essentielle, surtout lorsqu'on s'intéresse non plus simplement aux concessionnaires industriels, mais aussi aux petites et moyennes entreprises.

\section{Encadré 4.2 Éléments d'un système de vérification}

\section{Objectifs centraux :}

- Le système a-t-il pour objectif de contrôler la criminalité, de renforcer l'application de la loi et/ou de s'attaquer aux problèmes complexes relatifs à la réglementation de l'industrie et aux pressions qui s'exercent sur cette dernière ?

\section{Implantation institutionnelle :}

- Le système est-il proche des organes officiels du gouvernement ?

- Comment la gouvernance du secteur influe-t-elle sur le fonctionnement du système de vérification?

- Quelles sont les capacités techniques des administrations du secteur?

- Y a-t-il des liens avec d'autres domaines de réforme de la gouvernance et si c'est le cas, le système peut-il tirer partie de ces liens ?

- Le système dépend-il considérablement du soutien de la société civile et/ou des médias pour fonctionner de façon satisfaisante?

- Quels contrôles externes existe-t-il sur le système de vérification et sur la publication des audits?

\section{Fondation juridique :}

- Quel est le fondement juridique du système d'observation des forêts (par exemple, loi sur les forêts, lois de lutte contre la corruption) ; l'exploitation forestière illégale est-elle une infraction d'ordre civil ou une infraction pénale? 


\section{Encadré 4.2 (suite)}

- En quoi consistent les autorités légales ? Qui attribue, qui contrôle et qui sanctionne? Qui sont les autorités légales ? Y a-t-il une séparation entre les fonctions exécutives et judiciaires ? Quelle séparation existe-t-il entre l'attribution de permis et de concessions et l'exercice de la répression criminelle et du contrôle ? Quelles sont les règles qui s'appliquent aux conflits d'intérêt ?

- Quelles sont les conditions juridiques qui doivent être satisfaites pour que le ou les organismes de vérification fonctionnent efficacement ? Les performances de ces organismes sont-elles surveillées et des actions de redressement sont-elles prises au besoin?

- Les infractions à la loi sont-elles évaluées par rapport à des critères pratiques (par exemple, y a-t-il toujours un choix légal qui aurait pu remplacer le comportement jugé illégal, et si ce n'est pas le cas, quelles mesures ont été prises par l'autorité légale pour régler le conflit ?). Même question pour des critères de cohérence.

- Des moyens ont-ils été mis en place pour rendre le système juridique opérant, et si oui, comment?

\section{Structure organisationnelle :}

- Quels sont les degrés de pouvoir et d'influence politiques et industriels sur les nominations et les méthodes de travail de l'organisme de vérification?

- Par quelles méthodes et au moyen de quels dispositifs, si tant est qu'il y en ait, l'indépendance et la transparence sont-elles assurées?

\section{Indépendance budgétaire :}

- Comment le système est-il financé et de quels moyens dispose le montage financier pour protéger le système des ingérences extérieures?

- L'organisme est-il viable en ce qui concerne la fiscalité?

- Voir plus loin pour la suite de l'encadré

\section{Freins et contrepoids entre organismes de contrôle :}

- Quelle est le champ de la surveillance publique et quels liens sont entretenus avec d'autres services publiques tels que la police et les forces armées?

- Comment sont réparties les responsabilités entre divers acteurs au regard de l'application de la loi ?

\section{Structures de rapport :}

- Quels sont les liens avec la société dans son ensemble, et ces liens aident-ils à renforcer la légitimité et l'adhésion nationales ?

- Dans quelle mesure la production de rapports est-elle préservée des pressions particularistes, ou à l'inverse est-elle trop soumise à ce genre de pressions ?

- Les rapports une fois établis sont-ils accessibles et quel est le degré de transparence du processus d'élaboration de rapports? 
Le deuxième facteur à considérer est la localisation institutionnelle, qui influe diversement sur la propension d'un pays à avoir une gouvernance saine, notamment en ce qu' elle reflète les rapports existant entre le ministère des forêts et les autres ministères et administrations concernés (finances, planification, environnement et les collectivités locales). Il faut souligner que les ministères des forêts sont dénués de pouvoir et d'importance dans de nombreux pays producteurs, situation que de nombreux auteurs considèrent comme étant délibérée (voir par exemple, Ross 2001). Cette faiblesse est exacerbée par le fait qu’il y a pléthore d'institutions aux responsabilités imbriquées, ainsi que par la concentration excessive de l'autorité entre les mains d'un petit groupe d'individus. Il est relativement fréquent que ces conflits entre institutions se produisent dans un contexte de pénurie des compétences nécessaires au sein du ministère, de sorte que les possibilités de " redondance » entre prestataires pouvant se remplacer sont limitées, ce qui veut dire qu'il est deux fois plus difficile d'assurer le respect de standards professionnels. L'inaptitude d'un organisme de contrôle forestier à mener à bien sa tâche doit souvent être replacée dans ce contexte institutionnel défavorable pour être bien comprise.

La troisième question intéressante concerne la fondation légale des activités de vérification, y compris l'identité des autorités légales concernées et la base juridique sur laquelle s'appuient les activités d'observation. Toute modification du cadre juridique pour faciliter le bon fonctionnement d'un système de vérification mérite d'être notée. Il peut s'agir de tentatives visant à établir la complémentarité des rôles et des compétences entre les organismes d'application de la loi et l'organisme de vérification. La cohérence du cadre juridique est également importante, dans la mesure où si des incohérences existent, le respect d'une condition légale pourrait entraîner le risque de non-respect d'une autre condition. Cette situation se rencontre surtout dans les cas où l'administration forestière est partiellement décentralisée, mais où cette décentralisation est incomplète, de sorte qu'il y a des dédoublements, voire même des contradictions dans les domaines d'autorité. Dans de tels cas de figure, il est nécessaire d'identifier les procédures mises en place pour régler les conflits qui découlent de cette situation.

Le degré d'attachement à l'équité et à la justice peut se mesurer aux capacités d'adaptation des voies légales aux besoins des utilisateurs de la ressource. Ainsi, dans les cas où les infractions légales sont évaluées en permanence ou périodiquement par rapport à des critères pratiques, le système a les moyens de tenir compte des contraintes auxquels doivent faire face les acteurs, surtout lorsqu'ils n'ont que peu de moyens à leur disposition. Le degré d'attachement des autorités peut également être mesuré par leur volonté de mettre en place des procédures d'appel, que ce soit pour l'industrie ou pour les populations qui sont en relation avec elle.

Les autres dimensions des systèmes de vérification étudiées se rapportent à leur capacité à fonctionner de manière indépendante ainsi qu'au degré de protection dont ils bénéficient par rapport aux pressions politiques mentionnées plus haut. Il est clair que le degré d'indépendance budgétaire est un facteur intéressant, et que les décisions de vérification doivent être objectives, et inchangées quels que soient les intérêts financiers de l'organisme de vérification. L’idéal serait la sécurité financière à long terme, dans 
la mesure où celle-ci offrirait les conditions les plus propices pour que soit préservé l'attachement à l'indépendance. Les situations dans lesquelles l'indépendance budgétaire était compromise ont présenté un intérêt évident pour la recherche.

De même, les structures de rapport doivent aussi être protégées des ingérences extérieures. Les modalités de traitement des informations sont particulièrement révélatrices du degré d'attachement à la véracité des comptes rendus tout comme l'est la netteté de la séparation entre le traitement des informations et les décisions politiques faites sur la base de ces informations. Le fait que les décisions finales soient " politiques » est peut-être secondaire par rapport au fait qu'elles soient reconnues comme telles, et au fait que les autorités encouragent la diffusion des informations sur lesquelles s'appuient ces décisions. Sur ce point il pourrait être judicieux d'étudier le degré de latitude politique présent dans le processus de gestion des informations. En utilisant les bons instruments de gestion, il est possible d'exclure la politique, de rendre automatique la production de rapports et de la mettre à l'abri des pressions particularistes (voir par exemple, Brown et al. 2004). Si des tentatives de ce genre sont constatées, elles indiquent clairement que les autorités ont véritablement l'intention de faire avancer les choses dans ce sens.

\section{Les impacts du système de vérification}

Une fois qu'un organisme de vérification est en place, il faut chercher des preuves de ce qu'il peut effectivement changer les choses. Dans le cadre des recherches, les impacts doivent être évalués à deux niveaux : d'abord, il y a l'ensemble des impacts que l'organisme de vérification cherche à obtenir, et ensuite le degré d'efficacité de ces impacts dans la lutte contre la pauvreté. Dans chaque cas, il faut identifier des indicateurs d'adhésion aux résultats. Ce pourrait être des aspects comme le degré d'expression d'objectifs sociaux et la mise en place initiale de structures de contrôle et d'évaluation de la réalisation de ces objectifs.

Le gouvernement producteur pourrait présenter les objectifs du système de vérification dans une optique de réforme de la gouvernance, de conservation et d'utilisation durable des ressources naturelles, mais il pourrait n'être intéressé que par la rentabilité de l'industrie. Même si le gouvernement attache réellement de l'importance aux questions qui affectent la société toute entière, il se peut que la réforme soit partielle, les priorités pouvant changer. On pourrait s'attendre à ce que plus les objectifs de la vérification admis par le gouvernement sont limités, moins ils ont de chances de comporter des impacts anti-pauvreté. Mais même lorsque c'est le cas, les impacts anti-pauvreté peuvent reposer sur des conceptions différentes de la pauvreté. Prenons quelques exemples :

- Si la pauvreté est considérée essentiellement par rapport à la fourniture de services publics, les efforts devront être concentrés sur le contrôle et le suivi des revenus dans tout le système de vérification, puis sur l'évaluation du taux de fourniture de ces services. Cela pourrait poser problème et relever d'une trop grande subjectivité ; par exemple si un accord de concession exige du concessionnaire qu'il fournisse une infrastructure sociale, comprenant des centres de santé, des écoles, des ponts, comment la fourniture de ces services publiques pourra-t-elle être évaluée et par qui ? 
Il est fort probable que les agents de contrôle n'aient pas les connaissances techniques suffisantes pour évaluer la qualité de ces infrastructures.

- Si la pauvreté est considérée par rapport à la mise en place de systèmes de protection fondamentaux, les efforts devront être concentrés sur l'exécution et le respect du cahier des charges (notamment les spécifications se rapportant à la responsabilité sociale), afin de voir si le système de vérification respecte bien les conditions sociales (exemple : « interdiction de couper une essence recherchée par les populations locales pour ses produits non ligneux dans un rayon de x km autour d'une localité, sans accord formel de la communauté ").

- Sila pauvretéest considérée par rapportà l'intégration économique et au développement pour éradiquer la pauvreté, les efforts devront être concentrés sur des dimensions macroéconomiques de production et d'affectation de revenus, l'impulsion donnée par l'initiative à la société dans son ensemble (par exemple, emplois créés) et les modalités de suivi de ces deux aspects.

Des indicateurs-clés de préoccupation sociale seront la participation des habitants des forêts dans la conception du système, et le droit de regard qui leur a été accordé sur son fonctionnement. Ces préoccupations ont peu de chances d'être isolées; elles sont liées à l'attachement des autorités nationales aux principes de la bonne gouvernance que sont la responsabilité, la transparence et la participation publique.

\subsection{Synthèse}

Les paragraphes qui précèdent ont établi que le processus de mise en place d'un système de vérification comporte trois phases. Dans la première phase, les causes de fond de la mauvaise gouvernance forestière sont évaluées, ainsi que le degré de probabilité que les problèmes identifiés puissent être résolus par des améliorations apportées à la production et au commerce du bois. (Bien sûr, cela ne veut pas dire que cette évaluation sera réalisée dans les faits ; plusieurs des études qui suivent permettent en effet d'en douter). Dans la deuxième phase, le gouvernement décide de donner suite à ce désir de réforme ou de s'y opposer. (L'adhésion à la réforme variera probablement beaucoup entre les différents organes du gouvernement, comme l'attestent les études de cas). La décision du gouvernement tiendra compte d'un certain nombre de facteurs liés aussi bien aux caractéristiques de l'industrie du bois qu'aux attentes de la société et des partenaires externes de l'industrie par rapport à celle-ci. La troisième phase est consacrée à la conception du système de vérification et peut s'avérer encore plus prolongée et politisée que les deux premières.

Les chapitres suivant examinent des exemples importants de conception de systèmes de vérification du secteur forestier dans douze pays. Ces études sont suivies, dans la Partie C, par un examen des thématiques communes qui se dégagent de ces études de cas. 


\section{Chapitre 5}

\section{Le Forest Practices Board en Colombie- Britannique}

Note : cette étude de cas a été achevée en avril 2006 et a été réalisée par Kate Schreckenberg. Elle s'appuie sur l'étude de cas de pays no 2 de VERIFOR par Schreckenberg, K. 2006 Verification in the forest sector of British Columbia. ODI, Londres.

Tableau 5.1 La Colombie-Britannique : quelques statistiques clés

\begin{tabular}{|c|c|c|c|}
\hline Statistiques & & Date & Source des données \\
\hline Population & 4,3 millions & 2006 & $\begin{array}{l}\text { www.bcstats.gov.bc.ca/ } \\
\text { index.asp }\end{array}$ \\
\hline Territoire & 95 millions ha & 2005 & COFI 2005 \\
\hline Couverture forestière & $62 \%$ & 2005 & COFI 2005 \\
\hline Taux de forêts publiques & $95 \%$ & 2004 & MdF 2004 \\
\hline $\begin{array}{l}\text { Production industrielle de } \\
\text { bois ronds }\end{array}$ & $\begin{array}{l}81,5 \text { millions } \\
\text { de } \mathrm{m}^{3}\end{array}$ & 2006 & MFR 2007 \\
\hline $\begin{array}{l}\text { Emplois directs dans le } \\
\text { secteur forestier }\end{array}$ & 87300 & $2002-2003$ & Md 2003 \\
\hline $\begin{array}{l}\text { Part du secteur forestier } \\
\text { dans le PNB }\end{array}$ & $7,7 \%$ & 2004 & $\begin{array}{l}\text { www.bcstats.gov.bc.ca/data/ } \\
\text { bus_stat/bcea/BCEACurr.asp }\end{array}$ \\
\hline $\begin{array}{l}\text { Valeur des exportations de } \\
\text { produits forestiers }\end{array}$ & $\begin{array}{l}9,6 \text { milliards } \\
\text { USD }\end{array}$ & 2004 & $\begin{array}{l}\text { www.bcstats.gov.bc.ca/data/ } \\
\text { bus_stat/bcea/BCEACurr.asp }\end{array}$ \\
\hline $\begin{array}{l}\text { Principaux marchés } \\
\text { internationaux pour le bois }\end{array}$ & USA et Japon & 2006 & MFR 2007 \\
\hline $\begin{array}{l}\text { Indice de perception de } \\
\text { la corruption } \mathrm{TI} \text { (pour le } \\
\text { Canada) }(0-10 \text {, où } 0= \\
\text { corruption maximum) }\end{array}$ & 8,5 & 2006 & $\begin{array}{l}\text { Transparency International } \\
2006\end{array}$ \\
\hline $\begin{array}{l}\text { Indice de développement } \\
\text { humain (pour le Canada) } \\
\text { ( } 0-1 \text {, où } 0=\text { très bas) }\end{array}$ & 0,95 & 2004 & UNDP 2006a \\
\hline
\end{tabular}

\subsection{Introduction}

Cette étude de cas porte essentiellement sur le Forest Practices Board (bureau des pratiques forestières) de la Colombie-Britannique (CB), organisme unique dans le monde dont la 
mission est d'obliger l'Etat et l'industrie forestière à rendre des comptes au public au sujet de leurs pratiques dans les forêts et prairies. D'autres éléments de l'administration et du système de contrôle des forêts de la CB sont abordés, mais non approfondis.

\subsection{Pourquoi le besoin de vérification ?}

La filière forestière du Canada est essentiellement une affaire provinciale puisque la Colombie-Britannique est la province du pays qui dépend le plus fortement des forêts. Celles-ci recouvrent $62 \%$ des 95 millions d'hectares de territoire de la province (COFI 2005) et l'industrie forestière génère 2,1 milliards CAD de recettes annuelles pour le gouvernement de la CB (MdF 2003). ${ }^{1}$ En 2002/2003, elle comptait 87300 travailleurs forestiers (MdF 2003), représentant 4,4 \% des emplois totaux dans la province, mais la totalité des emplois associés au secteur forestier pourrait s'élever à 14 \% (MdF 2004).

La CB est renommée dans le monde entier pour sa flore exceptionnelle et les espèces rares de sa faune, telles que la chouette tachetée et le caribou de montagne. Les côtes de la CB sont les terres d'origine de $20 \%$ des forêts pluviales tempérées (WRI 2001) restant sur la planète. À la fin des années 80 et au début des années 90, l'industrie forestière de la $\mathrm{CB}$ était en butte à de vives critiques d'ONG locales, nationales et internationales, qui pointaient du doigt les dégradations provoquées par une industrie non régulée. Le conflit de longue durée qui s'ensuivit, appelé " guerre dans les bois », culmina en 1993 lorsque des dizaines de milliers de personnes protestèrent contre les coupes rases de forêt pluviale ancienne dans la baie de Clayoquot. Les groupes écologistes s'en prirent délibérément aux exportations de la CB sur les marchés de produits forestiers européens et américains pour essayer de renforcer la réglementation de l'industrie (Cashore et al. 2006), si bien que certains clients européens et américains de la société d'exploitation forestière active dans la région de Clayoquot annulèrent leurs contrats.

La publicité négative tant à l'intérieur du pays qu'à l'international fut l'un des facteurs qui poussa le gouvernement à créer une commission des ressources forestières, regroupant des représentants d'organismes du travail, de l'industrie du bois et des collectivités locales. La commission conclut que « l'incapacité dont a fait preuve le secteur forestier à reconnaître et à gérer adéquatement les valeurs forestières autres que le bois et à gérer plus intensément les valeurs liées au bois a mis en danger l'existence même du secteur économique le plus important de la CB » (citation dans SLDF/FW 2002). Pour remédier à ce qu'elle considérait comme une absence de cohérence dans la gérance des forêts, la commission recommanda de réunir plusieurs lois et réglementations en un Code d'exploitation forestière unique. Ce Code comportait une réglementation des forêts parmi les plus strictes au monde et contribua fortement à renforcer la confiance dans les activités de l'industrie forestière en CB (Cashore et al. 2006). Parallèlement à l'élaboration du Code en 1995, le gouvernement créa un organisme indépendant, le Forest Practices Board (FPB) la même année.

1 CAD fait référence au dollar canadien, 1 CAD étant égal à 0,87 USD (janvier 2006). 


\subsection{Le mission du FPB}

La mission du FPB est de "servir l'intérêt public en étant l'organisme chargé de veiller à la rigueur des pratiques forestières en Colombie-Britannique » et ses responsabilités sont les suivantes :

- Effectuer des audits des pratiques forestières des organismes publics et des titulaires de permis sur les terres publiques ;

- Effectuer des audits de l'application par les organismes publics de la loi sur les pratiques forestières dans les forêts et les prairies (Forest and Range Practices Act, FRPA, voir ci-après) ;

- Enquêter sur les plaintes du public;

- Réaliser des recherches spéciales sur des questions forestières;

- Participer à des procédures administratives d'appel ; et enfin,

- Produire des rapports sur les activités, les résultats des recherches et les recommandations du bureau.

Au travers de ces activités, le FPB peut proposer des améliorations portant sur les pratiques dans les forêts et les prairies et sur les réglementations gouvernementales, lorsque celles-ci se rapportent à l'application de pratiques forestières spécifiques.

Le FPB bénéficie d'un large soutien parmi toutes les parties prenantes essentielles, telles que les organismes publics, les ONG environnementalistes et les groupes des premières nations. Cela est mis en évidence par le fait que sa mission a été reconduite sous l'empire de la nouvelle loi sur les pratiques dans les forêts et les prairies (Forest and Range Practices Act (FRPA), radicalement différente et axée sur les résultats. Cette loi est entrée en vigueur en 2004, suite aux préoccupations de l'industrie concernant la charge très lourde représentée par la gestion prescriptive préconisée par le Code (" gérance forestière en recettes de cuisine $»)$, et dans un cadre général de dérégulation après l'élection d'un nouveau gouvernement libéral en 2001. Certains écologistets, très insatisfaits par la FRPA, considèrent que le bureau ne présente pas tellement d'intérêt pour la cause qu'ils défendent, c'est-à-dire l'amélioration du cadre politique de la gestion durable des forêts et de l'aménagement du territoire. Certains voudraient que la mission du FPB soit élargie de la simple gérance à un rôle de surveillance politique, qui s'étendrait aux domaines actuellement régis par la loi sur les forêts, y compris les décisions concernant la délivrance des permis de routes et voies forestières et des permis de coupes, la pertinence du processus de révision de l'approvisionnement forestier, les décisions concernant les possibilités annuelles de coupe et leur répartition, et les décisions concernant la délivrance et le renouvèlement des accords de tenure (SDLF/FW 2002). Selon certains, qui expriment l'inquiétude ressentie au sujet de l'ampleur géographique d'autres utilisations du sol, par les industries gazières et pétrolières (principalement autorégulées) dans les terres de l'intérieur par exemple, la mission du FPB devrait être élargie à l'arène plus générale de l'utilisation des ressources naturelles et de l'aménagement du territoire. 


\subsection{Conception du système de vérification}

\section{Les activités du FPB}

Au travers de toute une gamme d'activités, le FPB assure des activités d'observation et de surveillance indépendantes des pratiques forestières dans les forêts et prairies en $\mathrm{CB}$. Bien qu'il n'utilise pas le terme de vérification pour aucune de ses activités, toutes ses activités remplissent ensemble la fonction de nombreux vérificateurs, à savoir de contribuer à renforcer la confiance dans le secteur forestier. Le FPB est extérieur au secteur forestier et entreprend une gamme d'activités, dont la fréquence est indiquée à la Figure 5.1. Ces activités sont décrites ci-après.

\section{Audits des pratiques forestières}

Des audits des activités forestières des titulaires de permis et des organismes publics examinent tout ou partie de la planification et des pratiques forestières relatives aux forêts et aux prairies dans la zone de permis des entités à auditer, y compris la planification des activités, la construction des routes et voies forestières, la maintenance et la désactivation, l'exploitation du bois, la sylviculture et la protection contre les incendies. Les audits du FPB sont aléatoires, mais une fois qu'un permis a été soumis à un audit de conformité du bureau, il est retiré du " paquet » d'audits à sélectionner pendant cinq ans, ce qui permet d'assurer que d'autres permis soient audités, réduisant ainsi le fardeau éventuel que peut représenter des audits successifs pour les titulaires de permis. Depuis 2002, année du

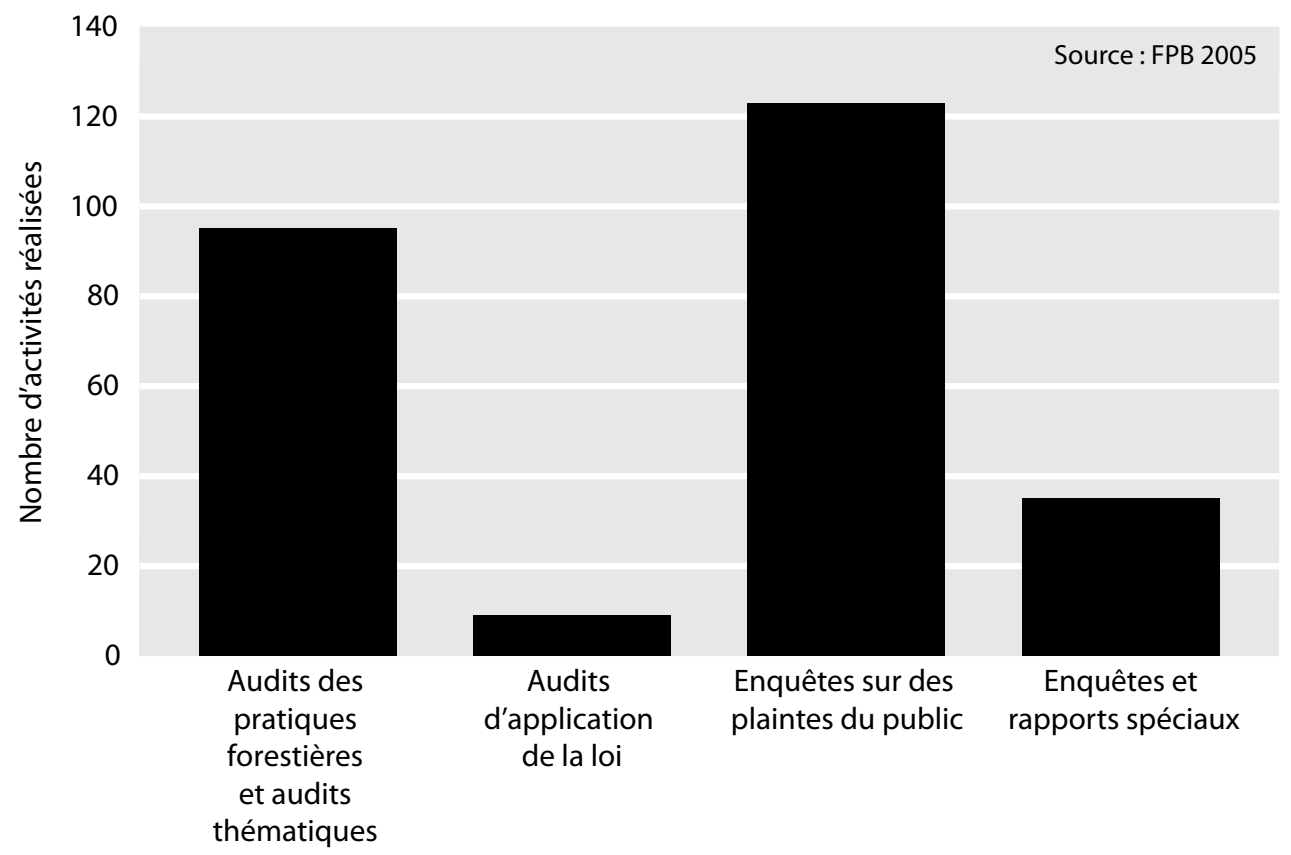

Figure 5.1 Principales activités du Forest Practices Board en Colombie-Britannique pendant ses dix premières années d'existence (1995-2004) 
premier audit par le FPB de société certifiée, il peut utiliser des informations produites lors d'inspections d'organismes de certification. Cela permet de réduire le temps passé sur le terrain et les coûts pour le bureau et le titulaire de permis, si l'entreprise auditée n'a pas d'objections à ce que ces informations soient rendues publiques. Les rapports d'audit sont d'abord remis aux entités auditées, puis communiqués au public et aux administrations gouvernementales.

\section{Audits thématiques}

Un audit thématique examine les pratiques forestières dans les forêts et prairies d'un ou plusieurs titulaires de permis qui se rapportent à une valeur forestière spécifique dans un secteur géographique donné, telle que sa nature riveraine, une qualité de sols ou un aspect visuel.

\section{Audits d'exécution de la loi}

Un audit d'exécution de la loi correspond en quelque sorte à une définition restrictive de la vérification, en ce sens qu'il est vérifie par des contrôles au hasard que les organismes gouvernementaux appliquent correctement la loi FRPA et les réglementations. Cet audit examine entre autres les politiques et procédures relatives à la conformité et au respect de la loi, ainsi que des dossiers, rapports, enquêtes, demandes d'action corrective et prononcés d'infraction spécifiques relatifs à des inspections. Il est souvent réalisé en conjonction avec un audit des pratiques forestières, ce qui permet de vérifier la concordance entre les résultats de l'audit de terrain et les mesures prises par les organismes gouvernementaux pour assurer la conformité et le respect des lois et réglementations.

\section{Enquêtes sur les plaintes}

À côté des audits aléatoires, le FPB exerce une fonction similaire à celle d'un médiateur : il enquête sur toutes sortes de plaintes, y compris des plaintes contre des organismes gouvernementaux, des entreprises et des particuliers. Le bureau intervient en qualité d'enquêteur indépendant et neutre, et non en tant que porte-parole du plaignant. Il peut consulter toutes les parties et tente de régler la plainte. Lorsque l'enquête est terminée, il présente un rapport avec ses recommandations le cas échéant, aux parties concernées, au public et aux ministres compétents.

\section{Rapports spéciaux}

Ce sont les rapports spéciaux produits par le bureau qui présentent le plus d'intérêt pour le public et qui sont susceptibles d'avoir le plus d'influence politique ; ils traitent de questions fréquemment soulevées lors des audits, qui correspondent à des préoccupations du public. Le FPB est totalement libre de choisir les sujets sur lesquels il souhaite enquêter. Les sujets récents étudiés par le bureau ont été par exemple le caribou de montagne, l'exploitation forestière dans les régions de montagne, les pratiques forestières relatives à la gestion de l'épidémie de dendroctones du pin ponderosa, et la réconciliation nécessaire de la gestion des produits forestiers non ligneux avec la planification et les pratiques 
forestières. Lorsqu'il élabore des rapports spéciaux, le FPB se concerte avec le plus grand nombre de parties prenantes possibles.

\section{Application de la loi et sanctions}

Le FPB ne peut pas imposer de sanctions, même lorsqu'une non-conformité est constatée ; il doit en effet se reposer sur la direction pour la conformité et l'exécution de la loi (Compliance and Enforcement, $C \mho E$ ) au sein du ministère des forêts et des prairies (Ministry of Forests and Range, MFR), qui est chargée d'instruire les manquements comme il convient. La direction C\&E peut avoir recours à diverses mesures coercitives en fonction de la gravité de l'infraction, les plus fréquentes étant les amendes et les contraventions (C\&E 2004). Des mesures coercitives plus sévères pourraient également être appliquées, mais elles le sont rarement. Celles-ci comprennent les ordres de suspension de travaux, les demandes d'action corrective, les suspensions ou les annulations de permis, la privation du droit de vendre du bois, ainsi que les avis d'expulsion. Dans des cas extrêmes, les inspecteurs de la $\mathrm{C} \& \mathrm{E}$ peuvent demander des poursuites par le procureur général.

Le bureau peut demander à être informé des suites données à ses recommandations mais il ne dispose pas des pouvoirs qui lui permettraient d'assurer leur mise en œuvre. Cependant, il privilégie fortement l'information du public et met à sa disposition tous les rapports qu'il produit, tant sous leur forme complète qu'abrégée. En fonction des recommandations, une société civile puissante et bien informée peut prendre le relais du FPB en exerçant des pressions sur des groupes cibles (principalement le gouvernement et/ou l'industrie), pour que ces recommandations soient mises en œuvre.

\section{Autres acteurs du système de gouvernance forestière}

Même si le FPB est le seul organisme à avoir une mission spécifique au secteur forestier de la $\mathrm{CB}$, d'autres acteurs jouent des rôles importants pour maintenir la crédibilité de celui-ci (voir la Figure 5.2). Ces acteurs sont décrits ci-après.

La direction C\&E du MFR veille à ce que les lois forestières soient bien respectées dans les forêts publiques de la $\mathrm{CB}$ et intervient en cas de non-conformité. Les membres du personnel de la C\&E évaluent les risques associés aux activités des titulaires de permis et établissent des programmes d'inspection pour les sites prioritaires à risques élevés. Ils effectuent également des inspections en réponse à des informations provenant d'autres membres du personnel, d'organismes, d'opérateurs ou du public, et des inspections de suivi pour remédier aux problèmes qui leur ont été signalés. Les agents responsables de la C\&E effectuent chaque année plus de 16000 inspections d'évaluation de la conformité aux dispositions légales sur les forêts.

L'Association des professionnels de la forêt de CB (ABCFP) est un organisme de réglementation de la profession, auquel doivent adhérer tous les forestiers exerçant leur métier en CB. L'ABCFP établit les standards de compétences et de pratique professionnelles que ses membres doivent respecter, dans le but de préserver l'intérêt du public en matière 


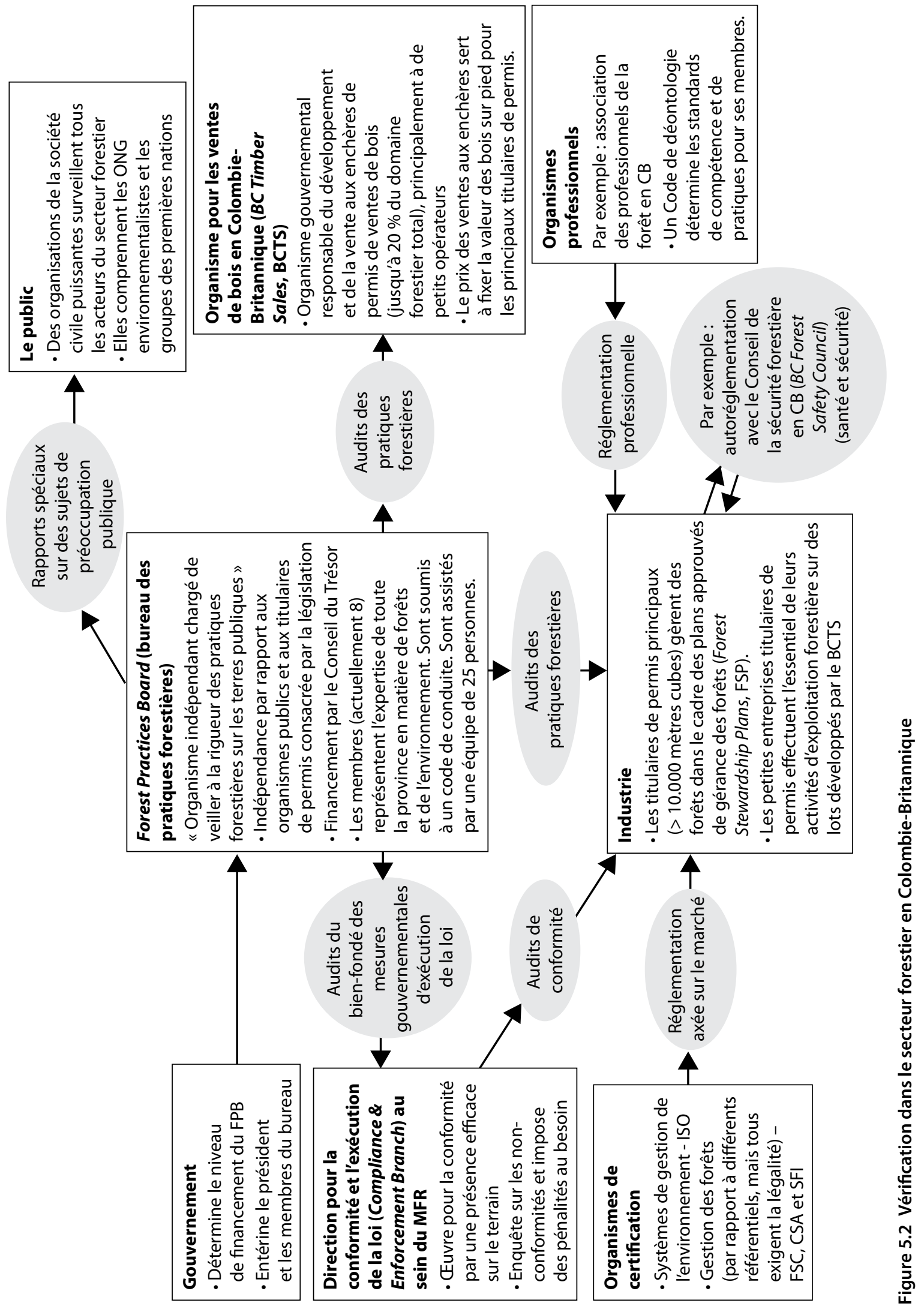


de gérance des ressources et d'obliger ses membres à rendre des comptes au sujet de leurs actions au moyen de programmes disciplinaires et d'assurance de la qualité.

L'industrie forestière a mis en place des initiatives comme celle du British Columbia Forest Safety Council (Conseil de la sécurité forestière en Colombie-Britannique). Créé en septembre 2004, cette association à but non lucratif incorpore toutes les principales organisations du secteur forestier et a pour mission de travailler avec l'industrie forestière pour supprimer les accidents du travail, mortels ou autres, dans l'industrie. Une de ses premières actions a été de mettre en place un médiateur de la sécurité forestière.

La certification par un tiers indépendant est très répandue en CB. La plupart des entreprises forestières ont des systèmes de gestion de l'environnement certifiés par l'International Organization for Standardization (ISO 14001 EMS). En outre, la plupart des grandes entreprises sont membres d'un des trois programmes de certification utilisés au Canada aujourd'hui, qui sont les suivants : Sustainable Forestry Initiative (SFI), Canadian Standards Association (CSA) et Forest Stewardship Council (FSC).

La société civile en CB est bien organisée. Les ONG environnementalistes et les groupes des premières nations jouent un rôle particulièrement important en attirant l'attention sur les pratiques qu'ils jugent incompatibles avec la gestion durable des forêts et/ou l'aménagement du territoire.

\section{Indépendance du FPB}

Dans les limites de sa mission, le FPB sous sa forme actuelle est considéré par toutes les parties comme agissant avec un degré élevé d'indépendance (FPB 2006a). Ses rapports ne mettent en évidence aucun favoritisme, ses recommandations étant adressées aussi bien à l'industrie forestière qu'aux administrations publiques. L'indépendance du bureau par rapport aux titulaires de permis et aux administrations publiques est assurée par la législation, ce qui lui permet également de rendre des comptes au public sans ingérence et sans filtrage. Même si le bureau présente ses rapports au ministre des forêts et des prairies, au ministre de l'environnement, au ministre de l'agriculture et du territoire et au ministre de l'énergie, des mines et des ressources pétrolières, ses rapports et les résultats de ses enquêtes ne sont pas fournis au gouvernement pour révision ou commentaires préalablement à leur diffusion publique. Le bureau a également le pouvoir de rendre obligatoire la présentation de preuves lors de ses enquêtes, et l'autorité requise pour auditer et enquêter sur les pratiques forestières et les mesures d'exécution de la loi des administrations gouvernementales.

Le bureau rend des comptes au gouvernement de la CB. Cela veut dire que l'indépendance du bureau en ce qui concerne son financement et sa composition est susceptible d'être exposée à des manipulations de la part du gouvernement, et indirectement de la part de l'industrie du bois influente, par le biais du soutien qu'elle peut apporter à certains candidats politiques aux élections. Le financement du bureau s'élevait à environ 3,6 millions CAD en 2004-2005 ainsi qu'en 2005-2006 et provient directement du Conseil 
du Trésor. Ce système permet de protéger le bureau des pressions de financement qui pourraient surgir s'il était lié à l'un des quatre ministères chargés de l'administration des forêts. Néanmoins, le bureau n'est pas à l'abri des réductions budgétaires effectuées sur l'ensemble du secteur gouvernemental et a subi récemment une réduction de son budget qui était à l'origine de 5 millions CAD, restreignant ainsi sa capacité d'action. En ce qui concerne sa dotation en personnel, le bureau comporte 8 membres, assistés d'une équipe de 25 forestiers, biologistes, comptables et avocats. Les membres du bureau représentent un large éventail d'expertises et d'expériences en sciences forestières et environnementales, tirées de toute la province. Ils sont nommés à titre personnel, plutôt qu'en qualité de représentants de groupes d'intérêts particuliers. Un Code de conduite a été adopté pour veiller à ce que les membres du bureau remplissent bien leurs obligations légales dans le respect de la justice et de l'impartialité et à l'abri des considérations et des intérêts de personnes. Le président du bureau est choisi au terme d'une procédure de sélection, nécessitant l'aval du gouvernement, tout comme les autres membres du bureau, recommandés par le président.

\section{Règles et procédures de vérification}

\section{Référentiels par rapport auxquels la vérification est effectuée}

La FRPA définit 11 "résultats » relatifs aux forêts et à l'environnement qui doivent être obtenus (par rapport aux bois, aux sols, à l'aspect visuel ou aux espèces sauvages par exemple), mais contrairement au code précédent, elle ne précise pas de critères clairs, transparents et applicables. Les entreprises sont censées soumettre des plans de gérance forestière (Forest Stewardship Plans, FSP), qui doivent indiquer les résultats escomptés pour une « unité forestière d'aménagement » donnée et les stratégies prévues pour parvenir à ces résultats. Les premiers FSP étudiés laissent penser qu'ils varient beaucoup. Certains comprennent des engagements spécifiques, auquel cas le FPB évaluera la conformité à ces engagements. D'autres FSP donnent des résultats mesurables, auquel cas le FPB utilisera des critères et des indicateurs pour évaluer la probabilité de parvenir à ces résultats (FPB 2006b). Le FPB s'est donc engagé dans un processus de collaboration avec toute une gamme de parties prenantes pour développer des indicateurs scientifiquement valides pour chacune des valeurs définie par la FRPA.

\section{Valeurs et principes directeurs du FPB}

En raison des critiques du style très négatif des premiers rapports d'audit, qui centraient principalement leurs efforts sur la mise en évidence des points de "non-conformité ", le FPB s'efforce désormais d'entretenir des rapports plus constructifs avec les entités auditées. Le bureau a élaboré un ensemble de principes directeurs (FPB 2005), reflétant les valeurs-clés de l'organisation, qui sont appliquées au quotidien pour servir de guide aux activités. Ces principes directeurs spécifient que le bureau :

- Agit au service des intérêts du public, et non de ceux d'un groupe unique, quel qu'il soit ;

- A une approche directe ; 
- Met l'accent sur les solutions plutôt que sur l'incrimination ;

- A une attitude non conflictuelle et pondérée ;

- Traite tous les individus d'une façon respectueuse, juste et sensible ;

- Effectue ses activités d'une manière mesurée, sans parti pris ni couleur politique ;

- Remplit sa mission avec intégrité et efficacité ;

- Met à la disposition du public des rapports clairs et concis ;

- Fonde ses actions et ses décisions sur ses connaissances, son expérience et le bon sens ; et enfin

- Est accessible et responsable.

\section{Les appels}

Les entreprises ou les particuliers peuvent faire appel contre une décision de nonconformité devant la Forest Appeals Commission (commission des appels du secteur forestier). Ce tribunal indépendant a le pouvoir conféré par la loi d'instruire des appels contre des décisions administratives sur diverses questions réglementées par la FRPA, la loi sur les forêts, la loi sur les prairies, la loi sur la flore et la faune et la loi sur les forêts privées. Il est composé de membres à temps partiel ayant une variété d'expériences dans des domaines techniques ou des affaires, assistés d'une équipe de sept employés à temps plein. Le FPB peut également faire appel contre des décisions devant la Forest Appeals Commission pour le compte de particuliers.

\subsection{Performances du FPB}

Le FPB n’a pas le pouvoir d'imposer des pénalités ni d'assurer la mise en œuvre de ses recommandations, et il compte uniquement sur la persuasion pour obtenir les résultats qu'il souhaite. Cependant, du fait de son indépendance, les rapports du bureau ont beaucoup d'influence auprès du public. Le suivi de ses propres recommandations a permis au FPB de constater que beaucoup de celles-ci sont en fait appliquées sans ce qu'il ne soit fait mention du fait qu'elles proviennent à l'origine du FPB. Même si les audits représentent un travail important pour les bureaux de district du MFR, les recommandations fréquentes du FPB sur l'utilisation d'outils plus nombreux et plus efficaces pour améliorer l'application de la loi, sont souvent bien accueillies à la direction générale du MFR. Certains des rapports spéciaux du FPB ont eu un impact très évident : le rapport intitulé "Le caribou de montagne en $\mathrm{CB}$ : dernière chance de conservation ?" (FPB 2004), l'un des plus médiatisés, a entraîné la mise en place d'un organe pour la gestion des espèces en voie de disparition à l'échelle de la province. Néanmoins, à peine six mois plus tard, les groupes écologistes ont produit un rapport affirmant que l'industrie du bois n'avait pas changé ses habitudes dans les régions où se trouve l'habitat du caribou de montagne, malgré l'urgence soulignée dans le rapport du bureau du Forest Practices Board (projet pour le caribou de montagne 2005). De la même façon, en 2004 le FPB a rendu public un rapport de réévaluation faisant suite à un rapport précédent sorti en 2003 consacré à la gestion et la conservation des habitats de nidification du guillemot marbré, une des espèces en voie de disparition en CB. Dans ce rapport, le FPB fait état 
des progrès réalisés, mais attire l'attention sur le caractère encore limité et trop lent des efforts de conservation.

Toutes les parties prenantes témoignent une grande confiance au FPB et à ses activités et sont de l'opinion générale qu'il remplit sa mission limitée dans un souci de justice et d'efficacité (FPB 2006a). Cependant, pour certains de ses détracteurs, le fait qu'il ne dispose pas d'instruments légaux pour imposer des amendes ou des poursuites en cas de nonconformité constitue une faiblesse non négligeable, qui amène « tant les administrations gouvernementales que l'industrie forestière a n'en tenir aucun compte [du FPB] » (FPB 2006a). D'autres souhaiteraient voir la mission du FPB englober des aspects de politique forestière plus généraux, voire même les questions plus fondamentales d'aménagement du territoire. On pourrait leur opposer toutefois que la crédibilité du FPB découle justement en partie de ses modalités de fonctionnement dans le cadre de sa mission actuelle : celleci pourrait certes être élargie un tant soit peu, mais les limites actuelles lui permettent de ne pas favoriser les optiques particulières.

\subsection{Les enseignements à tirer}

Cette étude de cas permet de tirer plusieurs enseignements qui sont les suivants :

- Malgré les niveaux élevés de respect de la légalité en $\mathrm{CB}$, il existe un débat intense et parfois très conflictuel sur l'opposition entre la vérification de la légalité et la réalisation de la gestion durable des forêts.

- Le FPB œuvre dans le cadre d'un mandat limité : il ne peut pas imposer de pénalités, et ne peut commenter que les politiques qui concernent directement l'amélioration des pratiques forestières.

- Malgré ces restrictions, ses rapports spéciaux sur des thèmes particuliers permettent au FPB d'offrir un forum neutre où sont débattus de façon constructive les sujets de préoccupation majeure du public, y compris la mise en œuvre de la nouvelle législation forestière.

- Grâce à l'indépendance du FPB, les parties prenantes collaborent avec lui et respectent les résultats de ses travaux.

- La clé de son succès découle notamment de son attitude constructive et professionnelle.

- Le FPB ne peut pas assurer la vérification à lui tout seul. Il œuvre à l'intérieur d'un ensemble d'éléments qui s'influencent les uns les autres et qui comporte des acteurs moins impartiaux comme le gouvernement, le secteur privé et une société civile puissante. 



\section{Chapitre 6}

\section{Évolution du système de contrôle des forêts au Costa Rica}

Note : cette étude de cas a été achevée en mars 2007 et a été réalisée par Guillermo Navarro, Hans Thiel et Joaquín Campos. Elle s'appuie en partie sur l'étude de cas de pays $n^{\circ} .6$ de VERIFOR : Navarro, G. et Thiel, H. 2006 The Costa rican national forest control system. ODI, Londres.

Tableau 6.1 Le Costa Rica : quelques statistiques clés

\begin{tabular}{|c|c|c|c|}
\hline Statistiques & & Date & Source des données \\
\hline Population & 4,3 millions & 2005 & $\begin{array}{l}\text { Division de la population } \\
\text { de I'ONU } 2006\end{array}$ \\
\hline Territoire & 5,1 millions ha & & FAO 2006 \\
\hline Couverture forestière & $47 \%$ & 2005 & $\begin{array}{l}\text { Division des statistiques } \\
\text { de l'ONU, } 2006\end{array}$ \\
\hline Taux de forêts publiques & $24 \%$ & 2000 & FAO 2005 \\
\hline $\begin{array}{l}\text { Production industrielle de } \\
\text { bois ronds }\end{array}$ & $\begin{array}{l}\text { Données } \\
\text { inexistantes }\end{array}$ & $\begin{array}{l}\text { Moyenne } \\
2001-2005\end{array}$ & OIBT, 2005 \\
\hline $\begin{array}{l}\text { Emplois directs dans le } \\
\text { secteur forestier }\end{array}$ & 17000 & $\begin{array}{l}\text { Moyenne } \\
1990-2000\end{array}$ & FAO 2004 \\
\hline $\begin{array}{l}\text { Part du secteur forestier dans } \\
\text { le PNB }\end{array}$ & $1,6 \%$ & $\begin{array}{l}\text { Moyenne } \\
1990-2000\end{array}$ & FAO 2004 \\
\hline $\begin{array}{l}\text { Valeur des exportations de } \\
\text { produits forestiers }\end{array}$ & 18 millions USD & $\begin{array}{l}\text { Moyenne } \\
1990-2000\end{array}$ & FAO 2004 \\
\hline $\begin{array}{l}\text { Principaux marchés } \\
\text { internationaux pour le bois }\end{array}$ & $\begin{array}{l}\text { Données } \\
\text { inexistantes }\end{array}$ & 2004 & Global Timber 2007 \\
\hline $\begin{array}{l}\text { Indice de perception de la } \\
\text { corruption } \mathrm{TI}(0-10 \text {, où } 0= \\
\text { corruption maximum) }\end{array}$ & 4,1 & 2006 & $\begin{array}{l}\text { Transparency } \\
\text { International, } 2006\end{array}$ \\
\hline $\begin{array}{l}\text { Indice de développement } \\
\text { humain }(0-1 \text {, où } 0=\text { très bas) }\end{array}$ & 0,84 & 2004 & UNDP 2006a \\
\hline
\end{tabular}

\subsection{Introduction}

De pays comptant parmi les plus déboisés du monde, le Costa Rica est passé au rang de leader mondial en matière de sylviculture de plantation, de gestion durable des forêts et d'élaboration et de mise en œuvre de politiques forestières innovatrices, visant à la protection et à l'utilisation des ressources forestières et à la promotion du secteur 
forestier. Toutefois, certaines études (CATIE 2001 ; De Camino et al. 2000) indiquent que le déboisement et l'exploitation forestière illégale demeurent des problèmes très importants.

Ce cas décrit la variété d'acteurs publics, publics-privés et indépendants qui interviennent pour assurer le contrôle et la vérification de la production forestière au Costa-Rica. Il analyse la façon dont des forces d'opposition tant à l'intérieur qu'à l'extérieur du secteur forestier ont fragilisé certains éléments fonctionnels importants des systèmes d'administration, de contrôle et de perception des recettes.

\subsection{Contexte de l'analyse}

Le Costa Rica possède une superficie de 5 millions d'hectares dont 62,8 \% sont caractérisés par leur " potentiel forestier» (MINAE 2001). Cependant, la politique de développement agricole agressive menée entre 1950 et 1990 a provoqué une progression du déboisement parmi les plus rapides au monde et une configuration très fragmentée de la couverture forestière. Un point critique a été atteint en 1985 lorsque le couvert forestier ne représentait plus que $24 \%$ de la superficie forestière d'origine (Oficina Nacional Forestal 2001). Cet état de fait a conduit l'Etat à mettre en ouvre un système de mesures incitatives pour créer les conditions propices au reboisement. La loi sur la forêt de 1996 institua un système de paiements pour compenser les propriétaires forestiers pour la préservation de fonctions forestières rendant des services environnementaux à la société (De Camino et al. 2000). Ces mesures incitatives, associées à un changement d'orientation des politiques de développement national en faveur de la promotion des services et de l'écotourisme, ont réussi à faire remonter le couvert forestier, à différents stades de régénération forestière, à $46 \%$ du territoire national (De Camino et al. 2000).

La majeure partie du bois autorisé provient à présent de systèmes agroforestiers et de plantations forestières, $4 \%$ seulement provenant encore de forêts naturelles. Cependant, une part non négligeable (22\%) du total des bois transformés par l'industrie serait encore illégale, et proviendrait d'arbres situés en dehors des forêts, moins soumis aux contrôles de l'administration forestière (Oficina Nacional Forestal 2004).

\subsection{Situation générale du système de contrôle des forêts au Costa Rica}

\section{Structure organisationnelle}

L'autorité forestière nationale du Costa Rica est chargée de la direction de la gestion des forêts. Elle est composée de quatre entités : le système national des aires de conservation (SINAC) et le fonds forestier national, qui dépendent tous deux du ministère de l'Environnement (MINAE), la commission nationale pour la foresterie durable, composée d'institutions universitaires et de recherche qui recommandent des instruments de politique fondés sur des donnés scientifiques, tels que les référentiels de gestion forestière, et enfin l'office national des forêts, organisme participatif pour 
l'élaboration de politiques, comprenant plusieurs parties prenantes du secteur forestier privé, ainsi que des organismes écologiques. ${ }^{1}$

Le SINAC a été fondé en 1998 par la loi sur la biodiversité en tant que système de gestion et de coordination participatif et décentralisé, organisé en 11 aires de conservation. Chacune d'entre elles possède un centre administratif régional, prenant des décisions opérationnelles et financières avec un certain degré d'autonomie. Les 32 bureaux locaux du SINAC sont en contact direct avec les clients et gèrent le processus de " promotion et de contrôle des forêts ", dont :

- La vérification des demandes d'exploitation forestière sur place dans les bureaux et par des inspections de terrain ;

- L'attribution de permis d'exploitation des bois (PEB) aux propriétaires forestiers ;

- La vérification de ce que l'exploitation forestière est bien réalisée conformément aux normes nationales pour la gestion durables des forêts et aux autres dispositions pertinentes ${ }^{2}$;

- La délivrance de permis de transport du bois (PTB), ainsi que d'étiquettes aux propriétaires forestiers ;

- Le contrôle du transport du bois ; et enfin

- L'exécution de la législation relative aux questions administratives, et le signalement des infractions à l'organisation judiciaire.

La fonction de surveillance des activités d'exploitation forestière dans les forêts a été déléguée aux régisseurs forestiers en 1991. Ce sont des forestiers professionnels indépendants agréés par le collège professionnel des ingénieurs agricoles (CIA). Il incombe aux cadres surveillants forestiers du CIA de superviser le travail des régisseurs forestiers. Actuellement, environ 150 régisseurs forestiers sont chargés de 4000 régies forestières par an.

Interviennent aussi dans le système de contrôle des forêts la police nationale, qui vérifie les PTB à des postes de contrôle routier et les comités de surveillance des ressources forestières et naturelles (COVIRENAS). Ces groupes de la société civile ont, conformément à la loi sur les forêts, pour mission de fournir un contrôle social et une surveillance de toutes les activités associées à l'utilisation des forêts naturelles et des espèces sauvages, mais avec la coordination et sous la supervision du SINAC.

\section{L'administration des forêts}

La Figure 6.1 montre le flux des procédures administratives dans le système de contrôle des forêts. Il existe plusieurs options pour demander une autorisation de récolte du bois.

1 La Commission nationale pour la foresterie durable s'appelait autrefois Commission nationale pour la certification des forêts.

2 La loi autorise l'administration à effectuer elle-même la vérification ou à sous-traiter cette tâche à des sociétés d'audit externes, ou à des auditeurs indépendants. 


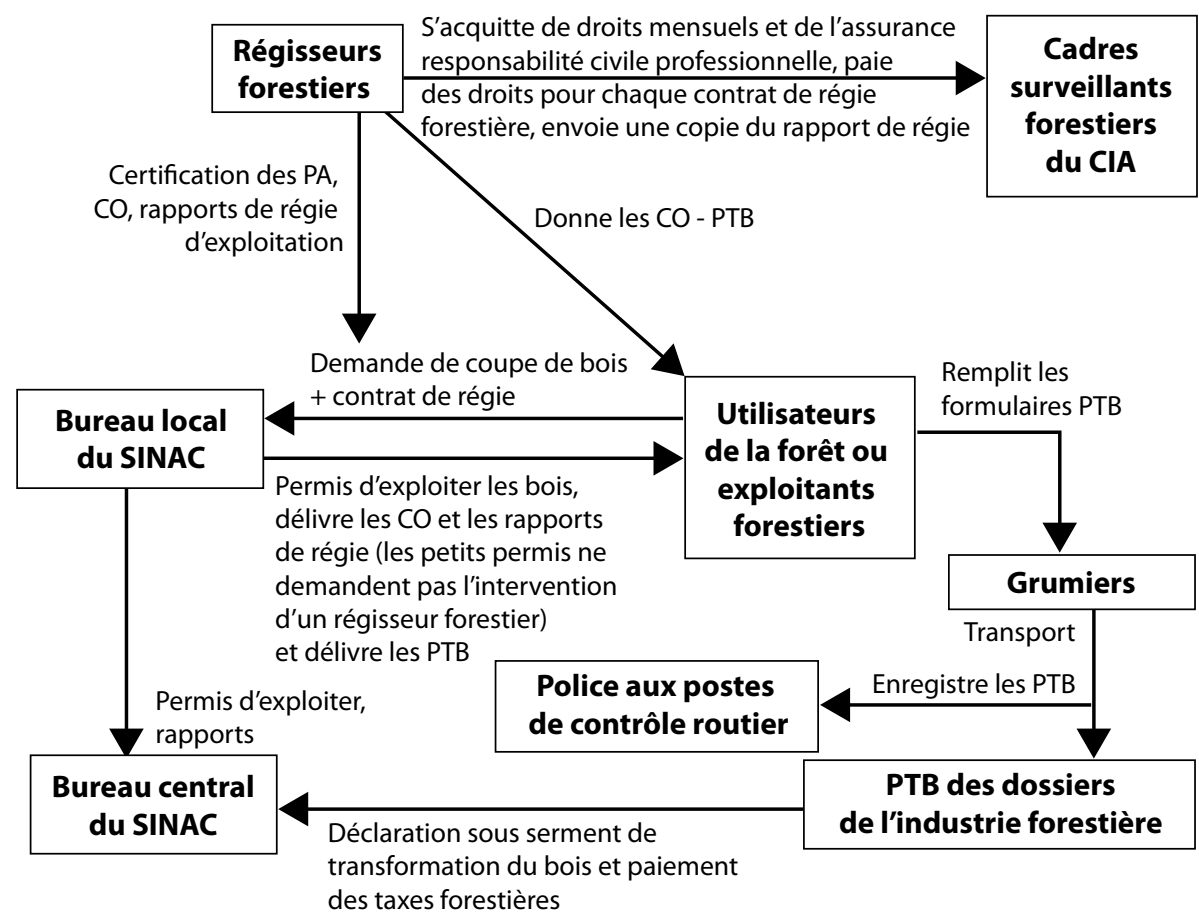

Figure 6.1 Flux des procédures administratives dans le système de contrôle des forêts au Costa Rica

Les régisseurs forestiers doivent superviser la préparation des plans d'aménagement (PA) pour les forêts naturelles et des inventaires de forêts commerciales (IF) pour les arbres situés dans des pâturages. Ils délivrent également des certificats d'origine (CO) pour les arbres issus de plantations forestières et de systèmes agroforestiers, qui peuvent être récoltés et transportés sans restriction. Des «petits permis " (PP) peuvent être préparés par des agents locaux du SINAC pour les propriétaires fonciers souhaitant récolter moins de 20 arbres pour leur utilisation personnelle.

Une fois qu'une demande de coupe a été approuvée par le SINAC, le propriétaire forestier doit engager un régisseur forestier pour superviser la coupe de bois, le contrat étant enregistré auprès du CIA, ainsi que le règlement des droits correspondants. Le SINAC peut alors délivrer le permis d'exploiter (PE). En 2004, les bureaux locaux du SINAC ont délivré $3747 \mathrm{PE}$, dont plus de $50 \%$ étaient de petits permis, $10 \%$ des PA et des IF, et le reste des CO (Arias 2005). Les régisseurs forestiers soumettent des rapports au SINAC au début et pendant l'exploitation. Après vérification de ces rapports, le SINAC délivre aux utilisateurs de la forêt des formulaires d'autorisation de transport du bois (PTB) et des étiquettes en plastique pour marquer les grumes. Une fois les activités d'exploitation terminées, le régisseur forestier adresse un rapport de réception au SINAC, et y joint éventuellement les formulaires PTB et les étiquettes non utilisés. 


\section{Procédures de contrôle}

La Figure 6.2 montre le flux des procédures de contrôle et de vérification. Les bureaux locaux du SINAC effectuent des inspections sur le terrain avant la délivrance des PE. Les régisseurs forestiers sont chargés de contrôler l'exécution des PE ; l'enregistrement de leurs contrats au CIA permet aux cadres surveillants forestiers de superviser leur travail. Le SINAC ne délivre les PTB qu'après la réception d'un rapport de régie forestière satisfaisant, qu'il vérifie parfois sur le terrain. D'après Arias (2005), en 2004, le personnel local du SINAC a effectué 2188 inspections de terrain de PA, d'IF et de PP.

Les PTB peuvent servir de document de la chaîne de contrôle, permettant à l'utilisateur de la forêt et à l'industrie de vérifier la quantité et le type de produits transportés de l'origine jusqu'à la destination finale. Les PTB sont vérifiés aux postes de contrôle routier par la police, souvent assistée de brigades communes SINAC/COVIRENAS. Les agents locaux du SINAC réalisent des inspections de l'industrie pour s'assurer que les piles de grumes et de sciages stockés sont constituées de bois acquis légalement et vérifient pour cela les PTB et les étiquettes. De plus, ils reprennent tous les PTB utilisés et vérifient que les taxes forestières ont été payées pour les volumes et les périodes corrects.

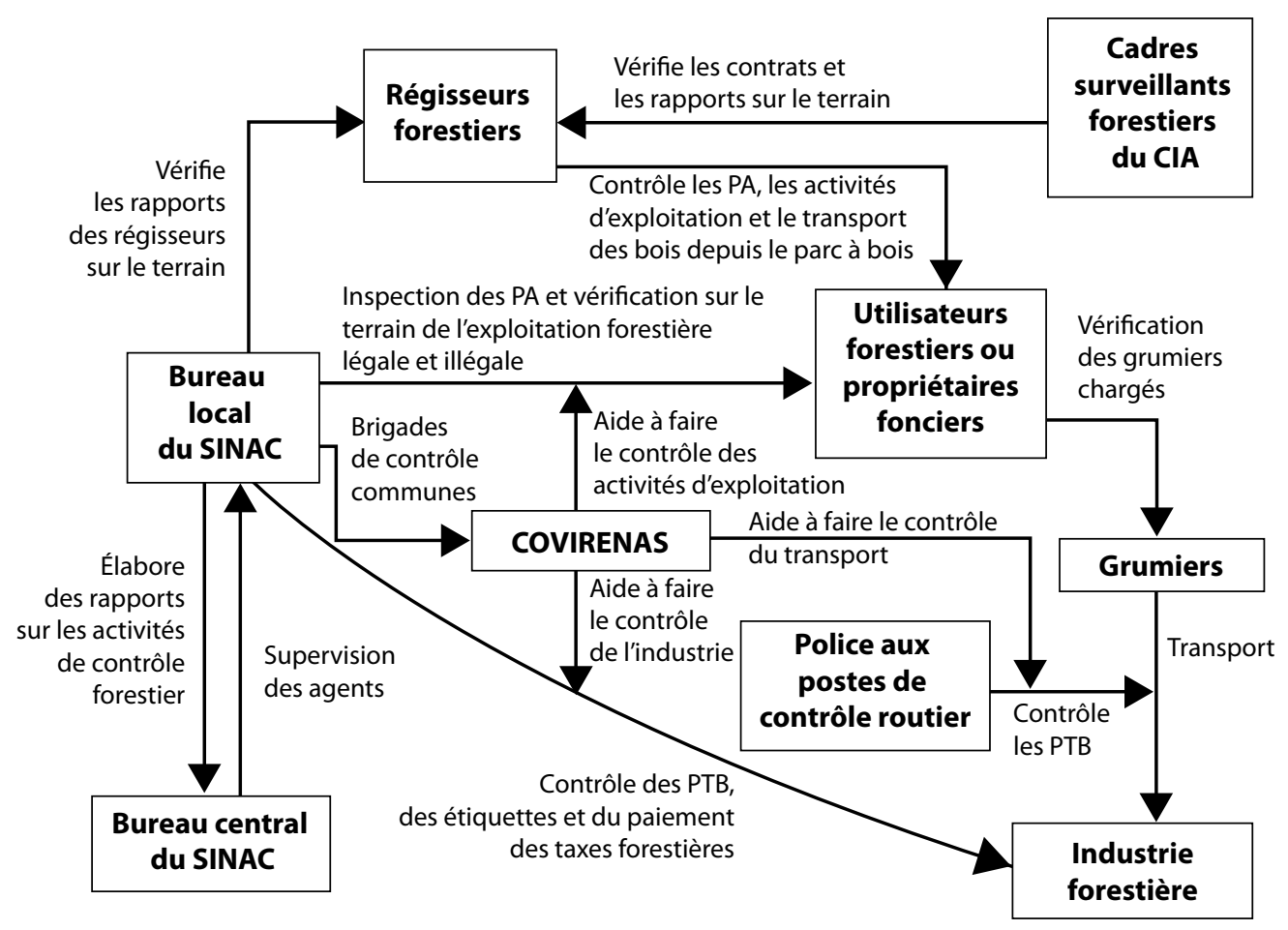

Figure 6.2 Flux des procédures de contrôle dans le système de contrôle des forêts au Costa Rica 


\section{Vérification, contre-vérification et application de la loi}

Le système comporte un certain nombre de points de double contrôle et d'équilibrage intrinsèques (voir la Figure 6.3) :

- Les utilisateurs et les régisseurs forestiers peuvent vérifier la rigueur des fonctions et services administratifs rendus par les agents locaux du SINAC et du CIA, et signaler les irrégularités éventuelles aux instances administratives, professionnelles (CIA) et judiciaires.

- Les agents du SINAC, les COVIRENAS et la police procèdent à des contre vérifications de leurs activités respectives. Tout bois illégalement transporté ou abattu est saisi en attendant une éventuelle sanction judiciaire. Le système judiciaire vend le bois aux enchères et place l'argent dans un compte, dont $50 \%$ est payé à la municipalité et $50 \%$ au fonds forestier si l'accusé est reconnu coupable.

- Le SINAC, la police, les COVIRENAS, l'utilisateur de la forêt ou n'importe quel membre de la société civile peuvent dénoncer le CIA, et le système judiciaire peut dénoncer tout régisseur forestier qui se serait mal conduit.

- Les bureaux centraux du SINAC effectuent des audits internes et des contrôles périodiques des bureaux locaux. Des audits forestiers externes occasionnels par des universités et des organismes de recherche sontorganisés par l'auditeur environnemental du MINAE et le bureau du contrôleur général national.

Constatent et signalent les infractions administratives au SINAC, à l'appareil judiciaire et au CIA

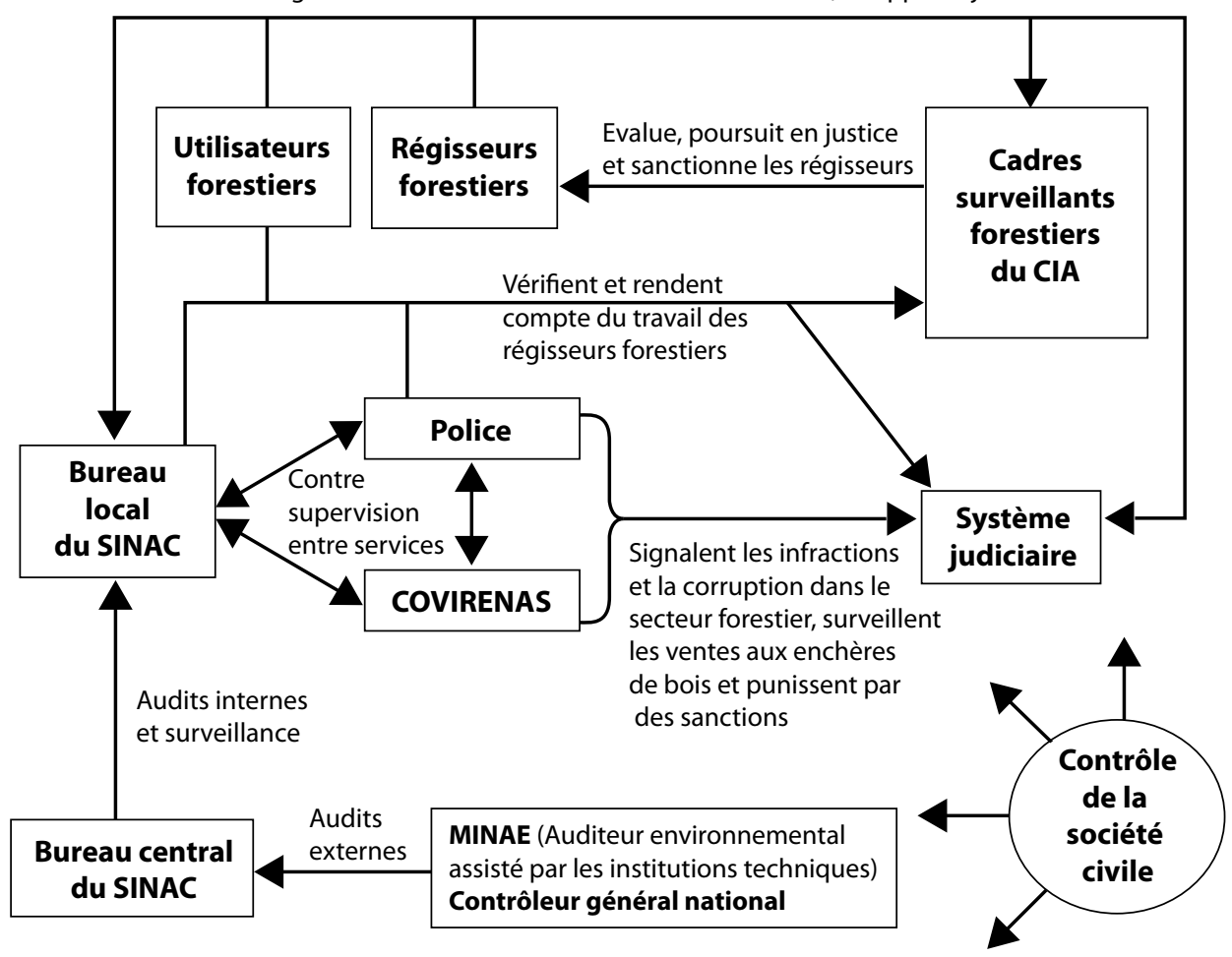

Figure 6.3 Contre vérification et application de sanctions dans les procédures d'administration et de vérification du Costa Rica 


\section{Indépendance financière}

Le système de contrôle et de vérification des forêts est censé être financé par un fonds forestier, établi par la loi sur les forêts ( $\mathrm{n}^{\mathrm{o}}$ 7575) et dont le but est de favoriser le développement, mais aussi l'administration et le contrôle du secteur forestier. Le fonds forestier provient d'une taxe forestière générale, de la vente aux enchères des produits forestiers saisis, ainsi que d'autres sources telles que les dons. La taxe forestière s'élève à $3 \%$ de la valeur cédée des grumes au point de transformation primaire. Cette valeur cédée est déterminée par le SINAC, qui est censé recevoir $23 \%$ de ce montant pour la prestation de ses services d'administration, de contrôle, d'éducation écologique et de promotion du secteur forestier.

Cependant, en raison des imprécisions de la loi, la chambre forestière costaricienne conteste la taxe forestière et soutient qu'il existe des incohérences dans la fixation du montant de base permettant la perception de la taxe. Même si la législation existante permettrait de résoudre ces incohérences, le SINAC se montre peu enclin à le faire, et la perception de la taxe est interrompue. Cela crée par conséquent des difficultés financières pour les diverses parties du système de vérification forestière, dont l'efficacité opérationnelle se trouve largement compromise. Les conséquences de ces difficultés financières sont décrites ci-après.

- Pour combler le déficit, le service de contrôle forestier du SINAC a été financé non seulement par le fonds forestier, mais aussi par le biais du fonds national pour les parcs et du budget central du gouvernement. Cependant, ces difficultés financières ont conduit à une certaine perte d'indépendance, les agents responsables étant parfois forcés d'accepter les offres de transport et de vivres de la part des utilisateurs de la forêt lorsqu'ils effectuent des inspections de terrain. Ce manque d'argent a également eu pour conséquence de réduire le nombre des audits internes et externes externalisés par le SINAC pour évaluer les activités administratives et de contrôle de ses bureaux centraux et locaux, de la police, des régisseurs forestiers et du CIA.

- Le budget del'auditeur environnemental, qui dépend du ministère de l'Environnement est censé provenir en partie de la taxe forestière. Sans celle-ci, l'auditeur ne dispose que de très peu de moyens pour s'acquitter de ses fonctions et on lui reproche sa faiblesse et son inefficacité.

- Pour ce qui est du CIA, la supervision des régisseurs forestiers devrait être financée par les recettes de la taxe forestière à raison de $2 \%$ de celle-ci, montant qui doit désormais provenir des cotisations de ses membres et des droits perçus pour la régie forestière. Par conséquent, les capacités et moyens dont dispose le CIA pour encadrer et effectuer des contrôles systématiques des régisseurs forestiers sont insuffisants (il n'y a que deux cadres surveillants forestiers, alors que 6000 à 8000 rapports de régie sont produits chaque année), son action se limitant donc aux cas de dénonciation de régisseurs forestiers par un tiers.

L'indépendance budgétaire est également problématique pour les régisseurs forestiers, payés directement pour leurs services par les exploitants ou les propriétaires forestiers. Ils ne sont donc pas toujours indifférents aux intérêts économiques de leurs clients, ce qui 
réduit leur performance et leur indépendance dans le système de vérification. D’après certaines sources, pour la plupart des PE, les régisseurs forestiers ne feraient pas de rapport de réception, les propriétaires forestiers se désintéressant de la question après l'enlèvement des bois, et ne souhaitant pas payer les régisseurs pour la dernière inspection.

D'autres services comme la police ont un budget extérieur au secteur forestier, qui leur permet de remplir leur mission avec indépendance. Cependant, le rôle joué par la police dans le système de vérification des forêts est tributaire de la volonté des politiques ; de plus, elle ne contrôle pas toutes les voies de transit des bois, ce qui limite son efficacité. De la même façon, les inspecteurs de la société civile incorporés dans les brigades des COVIRENAS travaillent bénévolement sans contrepartie financière, en étant encadrés par le SINAC, ce qui les rend dépendants et peu efficaces.

\section{Le référentiel de légalité}

Selon la loi sur la forêt, les critères de légalité relatifs à l'utilisation des ressources forestières se rapportent aux deux conditions suivantes : l'observation des interdictions d'exploiter et le respect des procédures nécessaires pour les coupes forestières autorisées. Les sanctions comprennent des peines d'emprisonnement allant de trois mois à trois ans, avec confiscation des machines et du matériel utilisé. Cependant, comme la législation forestière ne spécifie pas les sanctions pour chaque interdiction imposée par la loi, les poursuites deviennent très complexes. Cela entraîne fréquemment la suspension des poursuites criminelles, et revient en pratique à laisser agir les contrevenants en toute impunité.

La Commission nationale pour la foresterie durable est chargée de recommander au SINAC des référentiels de gestion durable des forêts, qui pourront être mis en œuvre dans le cadre d'une norme de légalité obligatoire au Costa Rica. Certains des référentiels recommandés se révélèrent très complexes et difficiles à interpréter, ce qui laissait aux agents du SINAC une grande latitude dans leur application ; cette situation conduisit à l'interdiction administrative d'attribuer des $\mathrm{PE}$ dans les forêts naturelles du fait de la complexité d'évaluation de la légalité de ceux-ci. Il s'ensuit que les propriétaires forestiers préfèrent transformer les forêts en pâturages en supprimant discrètement le couvert forestier, puis en demandant un permis de récolte de tous les arbres à valeur marchande situés sur ces "pâturages ". L'obtention de ce type de permis d'exploiter est plus économique, et offre donc de meilleures perspectives de rentabilité à l'hectare. Selon le FUNDECOR (2003), 30 \% des arbres provenant de pâturages et exploités entre 1999 et 2001 dans la région de Sarapiquí appartenaient en fait à une forêt naturelle dégradée intentionnellement.

\subsection{Les performances du système}

\section{Répartition et coordination des fonctions}

Le système de contrôle des forêts au Costa Rica comporte depuis sa création une série d'acteurs avec des rôles différents, facilitant ainsi la contre vérification entre 
acteurs. Cependant, l'acteur central, le SINAC, est écrasé sous le poids des fonctions supplémentaires qui lui ont été confiées. Parmi celles-ci figure l'administration des "petits permis » qui, selon la loi sur les forêts, devrait être dévolue aux conseils régionaux de l'environnement et aux municipalités. Comme ces conseils n’ont jamais été établis et que le MINAE a suspendu le pouvoir municipal de délivrance des PP, pour des raisons d'expertise technique et de capacités institutionnelles insuffisantes, le SINAC a dû se charger de cette tâche. Parallèlement, l'obligation légale de n'effectuer des inspections préliminaires de terrain pour l'attribution de PEB qu'en cas de motif raisonnable, a été remplacée par un ordre administratif (MINAE Directriz DM-173-2001, 30 janvier 2001) exigeant des inspections de terrain systématiques pour toutes les approbations de PE. Comme les " petits permis " représentent plus de la moitié des PE, cela se traduit par une charge administrative très lourde pour les agents locaux du SINAC et des temps d'attente plus longs, allant d'un à trois mois pour ce type de permis (Arias 2005).

L'absence de coordination à l'intérieur du système est particulièrement problématique pour les brigades de contrôle de la police, qui sont isolées dans la chaine de contrôle forestière et n'ont aucune information du SINAC sur les activités qu'elles sont censées contrôler. Elles n'ont pas non plus les moyens de vérifier les essences de bois, de calculer les volumes ou de recouper les indications d'origine sur les PTB avec l'origine effective dans les chargements des camions. Au lieu de cela, elles se contentent d'enregistrer les informations contenues dans les PTB sur des cahiers, sans notification ni communication aux autres composantes du système de vérification et de contrôle. En outre, la police ne vérifie que les camions qui s'arrêtent volontairement aux postes de contrôle pour montrer leurs PTB. Par conséquent, le transport clandestin de bois en camions ou véhicules fermés qui ne s'arrêtent pas ne pourra être découvert que si des contrôles routiers supplémentaires (non systématiques) sont assurés par le SINAC et les COVIRENAS.

Le pouvoir considérable conféré aux régisseurs forestiers constitue l'un des défauts principaux du système actuel (Ferrouki et Aguilar Schramm 2004). Leur supervision est donc une fonction critique. Le CIA est légalement responsable de l'administration, de la supervision et de la sanction de ses membres, les régisseurs forestiers, sans contrôle extérieur. Le SINAC n'a donc pas la compétence nécessaire au contrôle des régisseurs forestiers et la coordination entre le SINAC et le service des cadres surveillants forestiers du CIA est insuffisante. Cependant, dans le passé, le CIA n'a pas enquêté suffisamment sur les accusations faites contre les régisseurs forestiers, comme l'atteste le petit nombre de suspensions temporaires et le fait qu'il n'y a jamais eu de retrait d'accréditation (Esquivel, E. 2005, comm. pers.). Comme les régisseurs sont mal encadrés, ils ne s'acquittent souvent pas complètement de leur mission de supervision de l'exploitation du début à la fin, et finissent par soumettre des rapports de régie en retard ou pas du tout.

Cette situation s'explique en partie par le fait que les régisseurs forestiers sont surchargés de responsabilités liées au contrôle de l'exploitation et de la gestion forestières et des fonctions techniques et sylvicoles, en plus des tâches administratives dont ils ont la charge, telles que le traitement des demandes de permis de transport du bois. Cette situation pourrait être résolue en repensant la division du travail entre les régisseurs et les 
ingénieurs forestiers, qui pourraient assumer la responsabilité des activités d'exploitation les moins importantes. Le système de contrôle forestier pourrait également définir plus clairement les rôles des parties prenantes importantes, telles que les exploitants forestiers, les routiers et les intermédiaires, dont les rôles dans le système actuel ne sont pas formalisés et qui ne sont pas suffisamment responsabilisés.

\section{Le système de contrôle forestier vise-t-il les bons acteurs ?}

L'accent mis par le SINAC sur le contrôle de ceux qui agissent légalement, associé à l'absence de contrôle systématique des industries de transformation suscitent la critique selon laquelle le système ne s'attaquerait pas suffisamment à l'utilisation de bois illégal et à la conversion des forêts. C'est pourquoi le MINAE a décidé récemment de développer et de mettre en œuvre une stratégie pour le contrôle de l'exploitation forestière illégale (ECTI) dont l'objectif est d'intégrer, de renforcer et de consolider l'administration, le contrôle et la protection de l'utilisation des ressources forestières au plan national. Cette stratégie a pour objectif de réduire l'exploitation forestière illégale et les activités qui lui sont associées, ainsi que leurs conséquences environnementales et sociales négatives, et d'encourager fortement la participation de tous les secteurs de la société civile à ces efforts.

\section{Un système de contrôle peut-il contribuer à la lutte contre la pauvreté ?}

Au fil de l'évolution du système de contrôle forestier, des politiques de lutte contre la pauvreté ont été adoptées. Prenons quelques exemples :

- En ce qui concerne le mode de faire-valoir des terres, les utilisateurs de la forêt disposent de plusieurs moyens pour établir leurs droits de propriété ou de possession des terrains, afin d'obtenir un accès légal à la ressource forestière. ${ }^{3}$

- La taxe forestière doit être payée par les industries forestières, et non par les propriétaires de la ressource, ce qui rend l'exploitation forestière plus aisée pour les utilisateurs des forêts (y compris les petits propriétaires fonciers).

- Le fonds forestier est utilisé pour payer les propriétaires fonciers pour les services environnementaux fournis à la société par leurs forêts naturelles, leurs plantations forestières et leurs systèmes agroforestiers. Le système reconnaît plusieurs modes de faire-valoir des terres, et privilégie les organisations de petits propriétaires et les régions dans lesquelles vivent des communautés à faible indice de développement.

Cependant, le coût élevé de la préparation d'un permis et les formalités bureaucratiques par lesquelles il est nécessaire de passer pour cela limitent l'accès des plus défavorisés à la légalité. De plus, comme certains modes de faire-valoir des terres à la frontière agricole,

3 La propriété des terrains peut être prouvée par une attestation du système judiciaire prouvant que le processus d'attribution des titres suit son cours sans conflits ni difficultés et qu'il existe un plan du domaine inscrit au registre du cadastre. 
où vivent les plus démunis, ne sont toujours pas reconnus par le SINAC, l'exploitation illégale est le seul moyen par lequel ces agriculteurs peuvent accéder au marché.

\subsection{Conclusions}

Létude de cas du Costa-Rica permet de tirer les conclusions suivantes :

- Malgré un développement qui s'étale sur 35 années dans le secteur forestier, le système de contrôle du Costa Rica a eu un succès limité, mais cependant, un certain degré de stabilité. D’une façon générale, il est soutenu par les groupes environnementalistes, des secteurs larges de la société civile, les professionnels de la forêt et les agents administratifs des forêts, auxquels fait contrepoids une opposition active de la part des entités visées par le système de contrôle : les exploitants forestiers, les intermédiaires et les acteurs industriels. Une participation plus large d'acteurs non forestiers au développement du système pourrait réduire sa vulnérabilité aux attaques de parties prenantes puissantes au sein du secteur forestier.

- Un financement adapté de tout instrument de vérification est critique pour obtenir de bons résultats. La taxe forestière n'a pas été efficace ; le système de contrôle a néanmoins réussi à survivre grâce à d'autres sources de financement.

- L’indépendance financière des différentes parties du système est cruciale. La dépendance budgétaire du régisseur forestier par rapport à l'exploitant forestier met en péril son indépendance d'action. En outre, les organismes de surveillance et de vérification tels que le CIA, l'auditeur environnemental et les COVIRENAS manquent des ressources financières nécessaires pour fonctionner de manière efficace.

- Lorsque le cadre juridique impose des restrictions trop lourdes sur l'exploitation des bois dans les forêts naturelles, les utilisateurs des forêts préfèrent contourner l'administration forestière et trouver d'autres stratégies pour accéder au bois qu'ils cherchent à exploiter. Un référentiel de légalité efficace doit également être renforcé par un système de sanctions clairement définies et applicables, sinon les contrevenants continuent d'agir en toute impunité.

- Les compétences publiques ne peuvent être déléguées à des organismes privés ou à la société civile que si un organisme gouvernemental conserve la surveillance et le contrôle des activités déléguées. Lorsque l'administration et la surveillance de la régie des forêts ont été déléguées au CIA, les régisseurs forestiers ont vu leurs fonctions s'accumuler, à tel point qu'ils ont été surchargés de responsabilités étrangères au contrôle forestier. Le contrôle forestier a perdu de son efficacité et sa coordination est devenue difficile pour l'administration forestière.

- Il faut poser une limite claire entre la gestion des forêts et les activités de réglementation. La superposition des rôles de gestion des régisseurs forestiers, lorsqu'ils rédigent des plans d'aménagement, et de réglementation, lorsqu'ils effectuent des inspections et attestent de la bonne mise en œuvre des plans d'aménagement, provoque inévitablement un conflit d'intérêts.

- La collecte des données doit être accompagnée de systèmes de recoupement des informations. La police, les COVIRENAS, les régisseurs forestiers et le SINAC n'ont pas de système d'information pour recouper les données collectées par rapport aux 
$\mathrm{PE}$, ce qui rend un réel contrôle des forêts difficile et inefficace, parce que chaque acteur dans le système de contrôle et de vérification n'influe que sur une toute petite partie de la chaîne de production.

- Les collectivités locales ne jouent pas un rôle actif dans l'administration et le contrôle de l'utilisation des ressources forestières sur leurs territoires. Cela n'empêche pas les politiques nationales et les lois sur les forêts d'être axées sur la décentralisation et la déconcentration de l'administration des ressources forestières. Ce déplacement des responsabilités a un coût qui est rarement pris en compte, des moyens financiers étant nécessaires pour créer les capacités permettant à ces organismes locaux et régionaux de s'approprier pleinement l'administration et le contrôle des ressources forestières qui les concernent. 


\section{Chapitre 7}

\section{Le système national de contrôle des forêts au Honduras}

Note : cette étude de cas a été achevée en mai 2008 et a été réalisée par Mauricio Sánchez, Guillermo Navarro et Carlos Sandoval.

Tableau 7.1 Le Honduras : quelques statistiques clés

\begin{tabular}{|c|c|c|c|}
\hline Statistiques & & Date & Source des données \\
\hline Population & 7,2 millions & 2005 & $\begin{array}{l}\text { Division de la population } \\
\text { de l'ONU } 2006\end{array}$ \\
\hline Territoire & 11,2 millions ha & & FAO 2006 \\
\hline Couverture forestière & $41,5 \%$ & 2005 & $\begin{array}{l}\text { Division des statistiques } \\
\text { de l'ONU } 2006\end{array}$ \\
\hline Taux de forêts publiques & $75 \%$ & 2000 & FAO 2005 \\
\hline $\begin{array}{l}\text { Production industrielle de } \\
\text { bois ronds }\end{array}$ & 1,32 millions $m$ & $\begin{array}{l}\text { Moyenne } \\
2001-2005\end{array}$ & OIBT 2005 \\
\hline $\begin{array}{l}\text { Emplois directs dans le } \\
\text { secteur forestier }\end{array}$ & 18000 & $\begin{array}{l}\text { Moyenne } \\
1990-2000\end{array}$ & FAO 2004 \\
\hline $\begin{array}{l}\text { Part du secteur forestier dans } \\
\text { le PNB }\end{array}$ & $2,8 \%$ & $\begin{array}{l}\text { Moyenne } \\
1990-2000\end{array}$ & FAO 2004 \\
\hline $\begin{array}{l}\text { Valeur des exportations de } \\
\text { produits forestiers }\end{array}$ & 40 millions USD & $\begin{array}{l}\text { Moyenne } \\
1990-2000\end{array}$ & FAO 2004 \\
\hline $\begin{array}{l}\text { Principaux marchés } \\
\text { internationaux pour le bois }\end{array}$ & USA & 2004 & Global Timber 2007 \\
\hline $\begin{array}{l}\text { Indice de perception de la } \\
\text { corruption } \mathrm{TI}(0-10 \text {, où } 0= \\
\text { corruption maximum) }\end{array}$ & 2,5 & 2006 & $\begin{array}{l}\text { Transparency } \\
\text { International } 2006\end{array}$ \\
\hline $\begin{array}{l}\text { Indice de développement } \\
\text { humain }(0-1 \text {, où } 0=\text { très bas) }\end{array}$ & 0,68 & 2004 & UNDP 2006a \\
\hline
\end{tabular}

\subsection{Introduction}

Le cas hondurien montre un système de contrôle forestier national qui n'a pas pratiquement pas été rénové, dans lequel la vérification interne se borne à constater que les critères d'obtention d'un permis de récolte et de transport ont été satisfaits. L'étude de cas souligne le rôle important d'un observateur indépendant, auquel a fait appel la Commission nationale hondurienne des droits de l'homme pour réagir face à l'ampleur de la corruption et des conflits dans le secteur forestier. 


\subsection{Situation générale du contrôle forestier au Honduras}

\section{Recul et dégradation des forêts}

La culture, les traditions et l'économie de l'Honduras reposent depuis longtemps sur l'agriculture et l'élevage, même si $87 \%$ de la superficie du pays (selon les chiffres officiels) se prête à la sylviculture. Cependant, une partie de plus en plus grande de la population a des activités liées à l'exploitation forestière. Pour certains, il s'agit d'une façon d'arrondir les revenus de la famille, tandis que pour d'autres, c'est une source de richesse et de pouvoir considérable.

Les régions boisées du Honduras, où vivent environ 1,5 millions de personnes, ont les taux de pauvreté les plus élevés du pays (UNDP 2006b). Les activités forestières constituent pour les habitants l'un des rares moyens de gagner leur vie, et nombreux sont ceux qui sont mêlés à l'exploitation et au commerce illégaux du bois, en raison de la rareté de l'emploi dans ces régions. Ils fournissent en général la main-d'œuvre pour de puissants acteurs économiques dans l'industrie du bois, ou défrichent des terres de leur propre initiative pour développer les cultures ou l'élevage de bestiaux.

En dépit de l'absence de surveillance systématique de la couverture forestière au Honduras, les données officielles indiquent que la réduction annuelle de celle-ci s'effectue depuis 30 ans à un rythme constant égal $1 \%$ (AFE-COHDEFOR 2006). La part illégale de la production forestière varierait de $75 \%$ à $85 \%$ dans les forêts feuillues et de $30 \%$ à $50 \%$ dans les forêts de pins (FEHCAFOR et al. 2003). Une étude de 2003 basée uniquement sur des estimations de la production clandestine de pin situait les pertes financières directes pour le gouvernement du Honduras entre 55 millions USD et 70 millions USD chaque année (idem).

La mise en œuvre de tout système de vérification forestière au Honduras devra faire face à des difficultés extrêmement graves, telles que la corruption et le copinage politique. Comme le dit le proverbe "Eau trouble fait bonne pêche ", certains industriels de la filière du bois ont profité du chaos qui règne dans les activités de contrôle forestier au Honduras pour augmenter leurs capitaux au détriment de la dégradation des ressources forestières et de l'environnement. Non seulement ces industriels ont exploité la situation, mais en outre ils ont contribué à aggraver et à perpétuer ce chaos, en plaçant des personnes qui servent leurs intérêts à des postes publics importants, et ils sont prêts à distribuer des pots-de-vin aux fonctionnaires qui essaient de stopper la destruction des ressources forestières (EIA 2005).

\section{Le régime foncier des forêts}

La répartition des terres au Honduras remonte à l'époque coloniale espagnole, mais des problèmes demeurent quant à la définition des droits fonciers, principalement dans les départements situés au nord-ouest du pays. Selon la loi sur les forêts (décret no 85-71), les zones forestières sont classées comme étant des aires de forêt publiques (les Ejidales, 
appartenant à l'Etat ou à des collectivités) ou des aires de forêt privées (y compris les terres tribales gérées par l'Etat).

Il est fréquent que les droits de propriété et d'usufruit des terres et des ressources forestières soient partiellement confondus, contestés ou tout simplement bafoués (Del Gatto et al. 2004). Les terres sont soumises à une pression constante, à tel point qu'il existe des mouvements autochtones dont l'objectif principal est la récupération des terres, aujourd'hui en possession d'autres propriétaires fonciers ou qui ont été désignées comme appartenant à l'Etat.

\section{Comment le contrôle forestier a-t-il été envisagé dans le passé ?}

Avant 1971, l'Etat n'exerçait qu'un contrôle très faible sur le secteur forestier au Honduras. L'adoption de la loi forestière de 1971 et la nationalisation des forêts pendant la période 1974-1992 ont changé cette situation. Les forêts ont été rendues aux propriétaires fonciers entre 1992 et 2000 pour tenter d'élaborer de nouveaux systèmes de gestion des forêts. Une nouvelle loi sur les forêts a été approuvée à la fin de 2007. Cette loi est la première à reconnaître la séparation entre le secteur forestier et le secteur agricole et le dote d'un budget propre ; elle fonde l'Institut national de la conservation des forêts (Instituto Nacional de Conservación y Desarrollo Forestal, Áreas Protegidas y Vida Silvestre (ICF)) qui remplace l'autorité forestière (Corporación Hondureña de Desarrollo Forestal (AFE-COHDEFOR)). Cette loi a également entraîné la création d'un fonds pour le réinvestissement forestier, mis en place une répartition plus équitable des bénéfices de la forêt entre les communautés forestières, les collectivités locales et le gouvernement central et institué un règlement environnemental plus strict.

Plusieurs organisations non gouvernementales sont actives dans le domaine environnemental. Une des plus influentes sur le plan politique, Alianza Cívica, soutenue par Cáritas de Honduras, est axée principalement sur la défense de l'environnement contre les activités minières. Le mouvement environnemental d'Olancho (Movimiento Ambientalista de Olancho (MAO)), soutenu par le prêtre catholique Andrés Tamayo, qui privilégie plutôt la protection des forêts, se place à un niveau plus populaire et organise par exemple des marches de protestation et d'autres manifestations pacifiques. L'Olancho est une des régions productrices de bois les plus importantes du pays, avec une forte concentration d'exploitants forestiers (légaux et illégaux). Les pressions exercées par ces mouvements de la société civile, le $\mathrm{MAO}$ en particulier, ont contribué à la formation d'une volonté politique, ayant abouti à l'approbation de la nouvelle loi sur les forêts en septembre 2007.

\subsection{La responsabilité légale du contrôle forestier}

Le cadre légal du système de contrôle forestier au Honduras est très étendu et fragmenté, puisqu'il comprend 21 lois et règlements. L'instrument principal est constitué par la nouvelle loi sur les forêts qui, entre autres choses, considère la dégradation de l'environnement comme un acte criminel et met en vigueur des sanctions beaucoup plus sévères pour l'exploitation forestière illégale. 
C'est l'ICF, institut chargé de la supervision des industries extractives, du transport et de la transformation des produits forestiers, qui est investi légalement de la mission de contrôle des forêts. Mais d'autres entités de l'Etat, telles que la police nationale, le secrétariat aux ressources naturelles et à l'environnement, le procureur public chargé du respect de l'environnement et les municipalités participent également à des activités de contrôle forestier.

Le procureur public chargé du respect de l'environnement rattaché au ministère public (Ministerio Público), ainsi que les forces armées jouent également un rôle très actif dans le contrôle forestier, dans la mesure où ils interviennent directement dans des opérations communes dans les forêts ou sur les routes. En se fondant sur le décret no 153-95, la

\section{Encadré 7.1 Observation indépendante des forêts (OIF) au Honduras}

Le CONADEH est un organisme protecteur du citoyen, fondé dans le but de " garantir les droits et libertés reconnus dans la Constitution de la République » (décret n 153-95), dont le droit à un environnement sain. Afin de se lancer dans l'aspect environnemental de sa mission et de répondre aux préoccupations grandissantes du public au sujet de l'exploitation forestière, le CONADEH a engagé un processus de concertation avec Global Witness en 2004, qui s'est soldé par le lancement d'un projet pilote d'OIF au Honduras, auquel participent les deux organisations. Ce projet a bénéficié dès le début du soutien officiel de l'autorité forestière de l'époque (AFE-COHDEFOR), qui cherchait une occasion d'améliorer son image ternie en faisant la preuve de son adhésion à la transparence et à l'examen du public.

Malgré des fonds très réduits, le projet continue à être opérationnel depuis trois ans et s'est développé en agrandissant son personnel et son rayon d'action géographique. Ses objectifs principaux sont : (i) de produire des informations fiables sur l'exploitation forestière illégale et le commerce qui y est associé ; (ii) d'assurer l'objectivité et la transparence des activités d'observation de l'autorité forestière ; et enfin (iii) de renforcer la capacité opérationnelle de l'autorité forestière en exécutant des missions de terrain communes et en mettant en commun leurs expériences et compétences. Après la phase pilote initiale d'une durée d'un an, le rôle et le soutien de Global Witness se sont progressivement réduits, la conduite de la mise en œuvre de I'OIF ayant été transmise au CONADEH. Le projet est actuellement entièrement réalisé par ce dernier, sans aucune aide de Global Witness. La consolidation du projet a franchi une étape importante lors de la mise en place d'un comité consultatif interinstitutionnel en 2007, qui examine les rapports OIF avant leur publication. Ce comité englobe l'autorité forestière (actuellement ICF), la Commission nationale de lutte contre la corruption et l'accusateur public chargé du respect de l'environnement, le procureur de la République pour l'environnement, les forces armées et les représentants des syndicats professionnels forestiers et des groupes de la société civile. Le nombre élevé de participants au comité entraîne inévitablement une certaine complexité de fonctionnement, mais contribue également à favoriser la reconnaissance du projet et à améliorer la qualité des rapports de suivi. 
Commission nationale hondurienne des droits de l'homme (Comisionado Nacional de los Derechos Humanos (CONADEH)) a également lancé une initiative d'observation indépendante des forêts (OIF) en 2005, dont l'objectif est de renforcer les efforts de lutte contre l'exploitation forestière illégale (voir Encadré 7.1).

Des entités nationales et infranationales de coordination interinstitutionnelle ont récemment été créées pour soutenir la gouvernance et la modernisation du cadre juridique. À l'échelle nationale, le Programme pour les forêts honduriennes est un organisme indépendant, ayant le rôle de forum permanent de coopération entre les parties prenantes du secteur forestier. Celui-ci permet d'assurer la participation à armes égales des institutions publiques, des acteurs privés et des $\mathrm{ONG}$ au processus de planification visant à guider le développement durable des ressources forestières du pays. Dans la région de la côte Nord du Honduras, le Réseau pour la gestion des forêts de feuillus (REMBLAH) est une organisation de la société civile à but non lucratif et non politique, qui œuvre en faveur de la coopération entre parties prenantes dans le secteur forestier.

\subsection{La conception du système de contrôle forestier}

Le Honduras n'a pas de système de vérification forestière consolidé. L'ICF est directement responsable de l'application de la loi sur les forêts et de la direction de l'administration des ressources forestières du pays. ${ }^{1}$ L'ICF a également le pouvoir d'approuver la formulation et la mise en œuvre de plans d'aménagement forestier pour les forêts publiques et privées et d'autoriser la récolte des bois, conformément aux plans d'aménagement approuvés. L'activité principale à faire l'objet d'une vérification est l'attribution des permis de récolte, qui doit satisfaire à certains critères. Cette activité consiste à préparer et à approuver un plan d'aménagement (voir la Figure 7.1), puis à préparer et à approuver un plan annuel d'opérations (voir la Figure 7.2).

Le plan annuel d'opérations (PAO) est l'instrument technique permettant d'exécuter un plan d'aménagement forestier; sa formulation et son approbation font intervenir le propriétaire des forêts, le technicien engagé par celui-ci et des agents régionaux de l'ICF (même si pendant une courte durée en 2006, les plans d'opérations ont été approuvés par les bureaux centraux de l'ICF). La Figure 7.2 montre les étapes et les acteurs intervenant dans le processus d'approbation et de mise en œuvre des plans d'opérations.

Après l'approbation du PAO et l'acquittement des taxes municipales par le propriétaire foncier et l'entrepreneur autorisé, l'ICF est payé pour ses services administratifs et le bureau régional fournit le permis de transport. ${ }^{2}$ La délivrance de ce permis a deux objectifs principaux : de vérifier la légalité des produits transportés et de vérifier la production industrielle de sciages, par rapport au volume de bois ronds acquis.

\footnotetext{
1 Le système mis en œuvre actuellement n'a pas tellement changé par rapport à celui qui était appliqué par le prédécesseur de l'ICF, AFE-COHDEFOR, en attendant l'élaboration de nouvelles dispositions réglementaires et de prescriptions techniques et administratives.

2 D'après la nouvelle Loi forestière, l'ICF est doté d'un budget indépendant. Mais jusqu'à ce qu'une nouvelle régulation ne soit publié pour mettre en application cette loi, le système existant de paiements des propriétaires reste.
} 


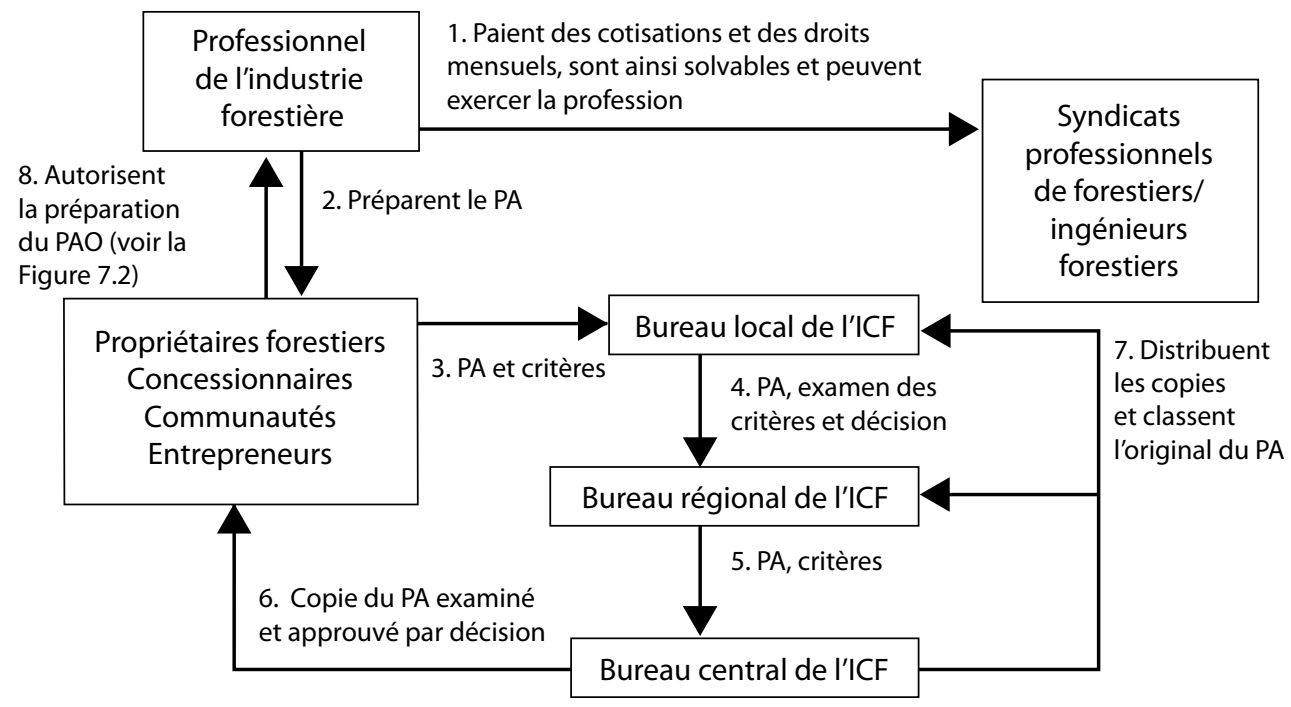

Figure 7.1 Procédures administratives pour l'approbation de plans d'aménagement forestier au Honduras

Les bureaux régionaux de l'ICF attribuent également des permis de récolte non commerciaux, qui interdisent la vente de produits. Cependant, la violation de ces permis est presque une institution, les titulaires de permis récoltant systématiquement plus de bois qu'ils n'y sont autorisés pour leur utilisation personnelle, bois qu'ils commercialisent sur des marchés locaux en sachant que rien n'est fait pour mettre un frein à cette pratique. L'exploitation d'arbres sains en utilisant des permis délivrés pour la récolte d'arbres morts, tombés ou brûlés sur pied est également une des violations de permis pratiquées. De la même façon, les permis délivrés pour abattre des pins atteints par le dentroctone méridional du pin, Dendroctonus frontalis, sont susceptibles d'être utilisés pour couper des arbres sains en dehors des zones affectées par le parasite. Cet état de fait a été corroboré par le projet pilote d'OIF, comme le montrent certains de ses rapports (CONADEH et Global Witness 2005 ; CONADEH et Global Witness 2006).

Au sein de l'ICF, le département d'audit technique (DAT) est chargé de diriger les activités de contrôle et de s'assurer que les normes et les dispositions réglementaires techniques sont bien respectées. Son champ d'intervention comporte quatre domaines :

- L'évaluation des plans d'aménagement à l'échelle nationale,

- La tenue d'un registre des industries forestières,

- La tenue d'un registre des parcs à bois locaux, et enfin

- La conduite d'audits des industries forestières et des lieux de stockage, qui doivent être effectués selon une méthodologie standardisée, également utilisée dans les bureaux régionaux de l'ICF. 


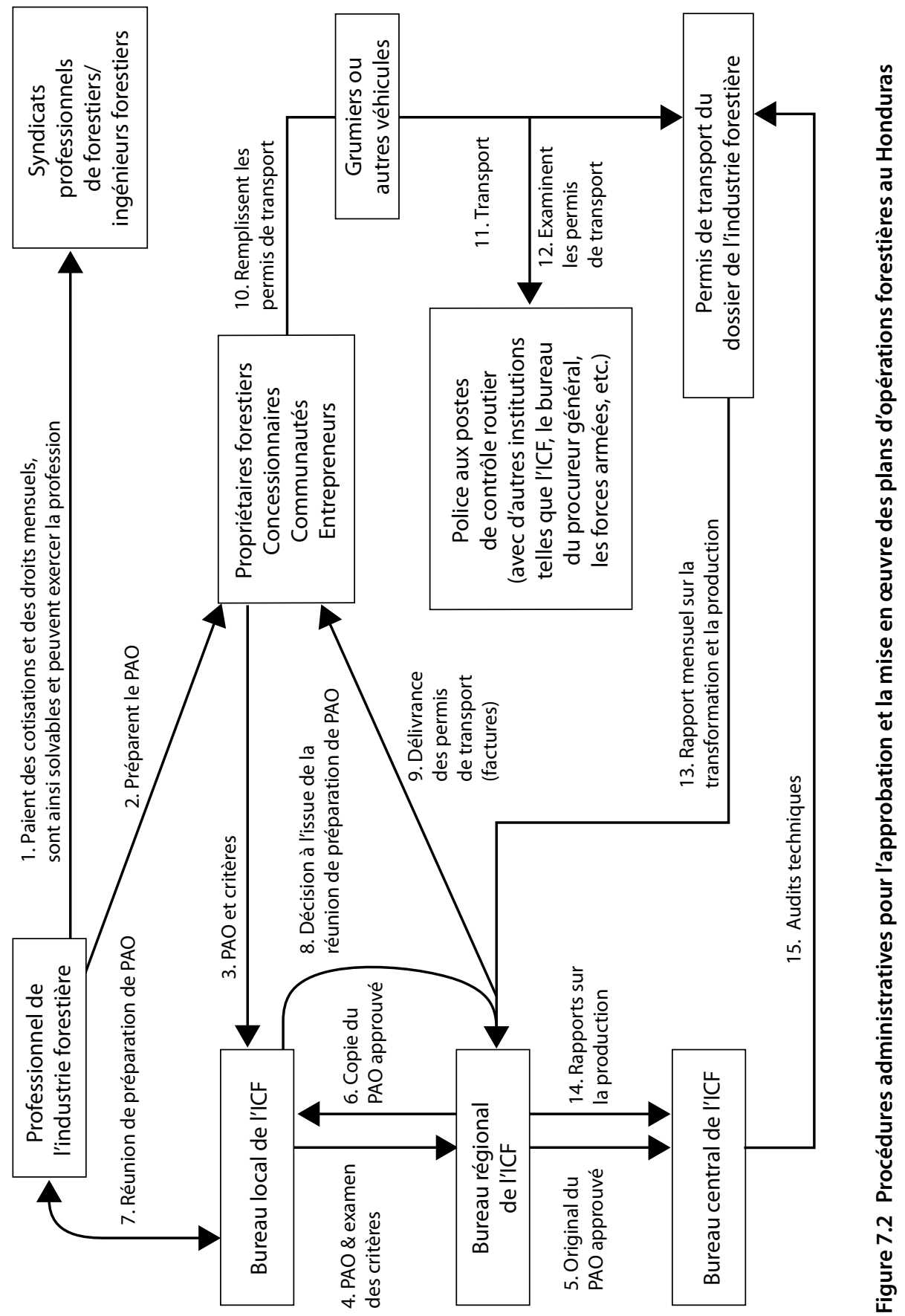




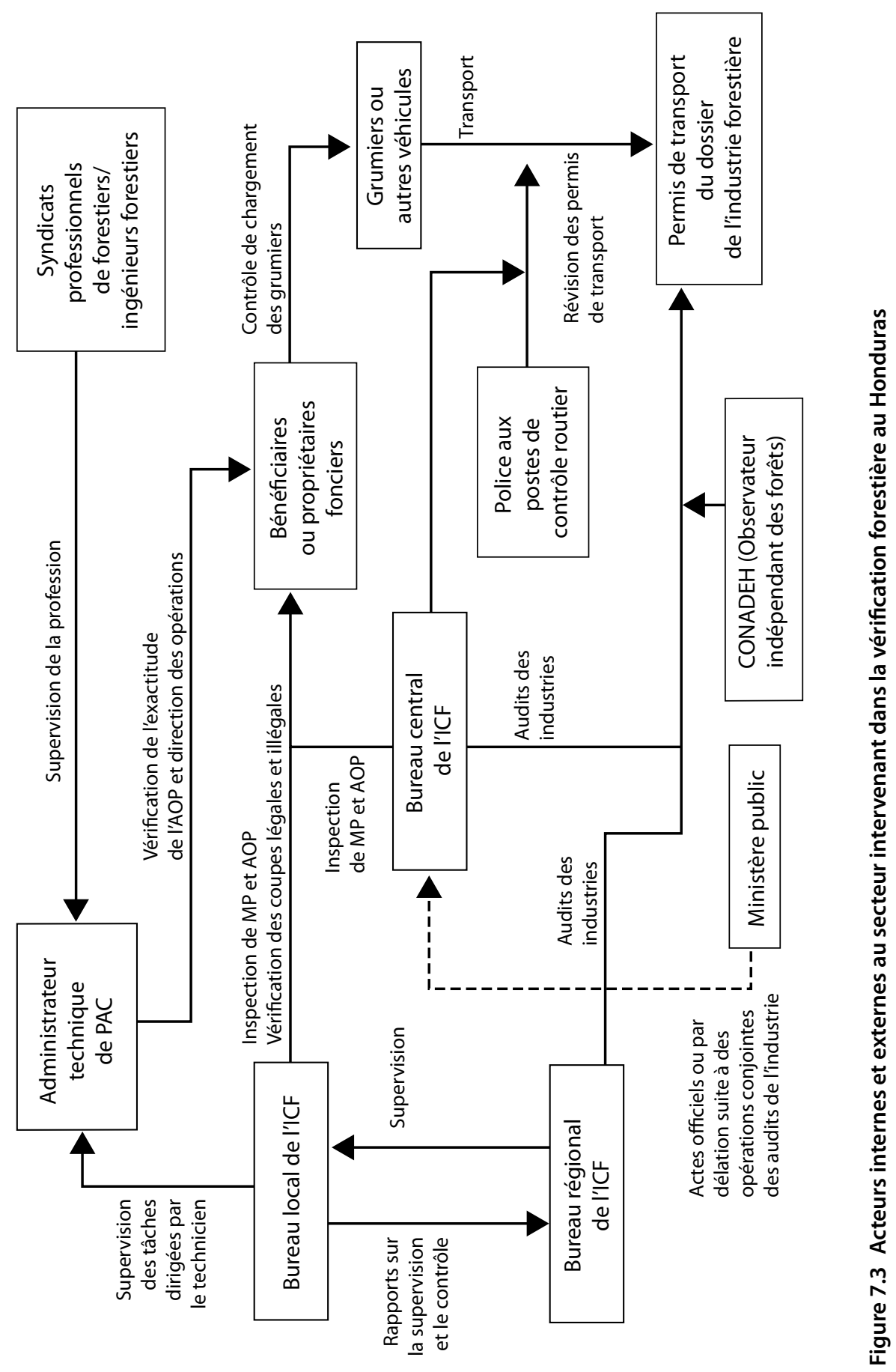


La vérification des PAO dans la forêt comprend les étapes de préparation, d'exécution et de réception finale, qui doivent démontrer, entre autres, que la délimitation sur le terrain, les activités de protection, la construction et la maintenance des routes et voies forestières, les traitements sylvicoles (arbres producteurs de graines, gestion des déchets, éclaircies périodiques etc.), le traitement des arbres restant sur la coupe, l'utilisation des permis de transport et la prévention et la lutte contre l'érosion des sols sont correctement réalisés. Cependant, le manque de moyens matériels et humains signifie que la vérification n'est pas réalisée avec suffisamment de rigueur.

La vérification du transport consiste à examiner les permis de transport pour confirmer la présence des documents originaux et de copies conformes à bord des véhicules et qu'ils sont bien remplis, avec des précisions sur la quantité de bois indiquée et la quantité transportée, la date de délivrance ou de facturation, le numéro de résolution du plan d'opérations, les labels ICF, la confirmation des dimensions des pièces transportées et des informations sur les véhicules indiqués sur les factures.

La Figure 7.3 montre la relation qui existe entre les agents responsables de l'ICF et les autres acteurs de la vérification intervenant aux côtés de l'ICF ou indépendamment. La police a le pouvoir et la charge de demander à ce qu'on lui présente les permis de transport aux postes de contrôle fixes et à n'importe quel point de l'itinéraire de transport des produits entre la forêt et les sites industriels. Le bureau du procureur de district effectue des activités de vérification similaires à celles de l'ICF, mais selon une méthodologie et des normes différentes. Le projet d'OIF mis en œuvre par le CONADEH (voir Encadré 7.1) constitue un intervenant extérieur important.

\subsection{Les performances du système de contrôle forestier}

Jusqu'à très récemment, l'acteur principal du contrôle forestier au Honduras a toujours été l'autorité forestière (AFE-COHDEFOR). Cet organisme, créé en 1974, a été chargé du contrôle de toutes les forêts situées dans des zones boisées publiques et privées, ce qui comprenait la conservation, le reboisement, l'exploitation, la transformation et le commerce des produits issus de celles-ci. En 1992, lorsque les forêts nationalisées ont été rendues aux propriétaires fonciers, l'AFE-COHDEFOR est passé d'un statut de "propriétaire " et d'administrateur de fait des ressources forestières, à un rôle de superviseur, chargé de fonctions non génératrices de revenus, telles que l'approbation de plans d'aménagement forestier sur des domaines privés et de l'organisation de ventes aux enchères de bois issus des forêts nationales.

Selon un rapport d'une commission d'audit (Flores et Eveline 2004), 38 \% des moyens financiers et logistiques de l'AFE-COHDEFOR étaient concentrés à la direction générale à Tegucigalpa. Moins de $24 \%$ des moyens totaux étaient consacrés aux quatre régions du pays produisant plus de $92 \%$ du bois rond national. D'un point de vue financier, l'AFE-COHDEFOR dépendait des ventes de bois et des paiements du secteur privé. Il faisait l'objet de vives critiques émises par les groupes environnementalistes qui lui reprochaient son inefficacité et la corruption qui sévissait dans ses rangs. La création 
de l'ICF, organisme décentralisé rattaché à la présidence, doté de son propre budget et indépendant financièrement, a eu pour but de remédier à la situation en succédant à l'AFE-COHDEFOR. Toutefois, il n'est pas du tout certain que l'ICF parvienne à diminuer l'exploitation forestière illégale au Honduras.

Dans le système de l'AFE-COHDEFOR, c'est le département d'audit technique (DAT) qui assumait les responsabilités de vérification les plus importantes, mais celles-ci étaient centralisées au niveau de la direction générale. Les droits payés sous la forme d'une « taxe forestière " étaient insuffisants pour entretenir un système administratif aussi compliqué et pour en même temps financer les activités de contrôle et de vérification du DAT dans tout le pays.

En 2006, selon le DAT, $90 \%$ des audits mettaient en évidence des anomalies sous une forme ou une autre (dont la gravité était variable), mais seulement $3 \%$ des cas étaient résolus en raison du bourbier juridique dans lequel tout le système était plongé, des irrégularités commises par les divers intervenants, ou de l'incurie ou du manque de volonté existants dans toute l'administration. Même s'il existe un manuel de procédures avec une méthodologie de conduite d'audit, les agents responsables peuvent décider de ne pas en tenir compte si bon leur semble.

Selon l'accusateur public chargé du respect de l'environnement, l'AFE-COHDEFOR n'a pas de protocole de conduite d'audit défini, il n'y a pas de contrôles et le ministère public doit participer à un examen du DAT pour harmoniser les méthodologies de ces deux institutions. Par exemple, l'accusateur public chargé du respect de l'environnement a mis presque trois mois à réaliser un audit que l'AFE-COHDEFOR a terminé en un jour et demi.

Les rapports portant sur des missions OIF de terrain, produits par le CONADEH, sont soumis à l'examen des membres du comité consultatif interinstitutionnel, avant d'être rendus publics sur le site Web du CONADEH. L'OIF représente désormais le mécanisme principal qui permette de mettre en évidence que les activités de terrain ne sont pas mises en œuvre conformément aux permis délivrés. ${ }^{3}$ Cependant, les observateurs signalent l'existence de trois faiblesses principales dans la mise en ouvre actuelle de l'OIF au Honduras :

- L'absence de protocole de sélection de dossiers ou d'opérations d'exploitation forestière à auditer, ce qui veut dire que l'OIF peut être victime de manipulations politiques visant à cibler un certain type de contrevenants ;

- L'absence de standards permettant d'assurer l'objectivité des évaluations, et

- Les menaces proférées par les contrevenants à l'encontre des auditeurs OIF qui craignent pour leur sécurité personnelle.

3 Plus de 50 rapports de mission ont été publiés pendant les trois premières années d'activité, tous peuvent être consultés sur http://www.conadeh.hn/informes_monitoreo_forestal.htm. 


\subsection{Les enseignements à tirer}

Le cas du Honduras montre qu'une réelle adhésion des parties prenantes est essentielle pour que le contrôle et la vérification des forêts aient des chances d'aboutir. L'inclusion de groupes organisés de la société civile, du secteur privé et des collectivités locales dans la formulation des politiques nationales sur le contrôle des forêts a été importante au cours des deux décennies passées. Cependant, le gouvernement central a fait preuve d'une ouverture insuffisante de la formulation des politiques au public, préférant la concertation à une réelle élaboration participative des politiques.

Au Honduras, se soustraire aux règles qui régissent la conduite humaine est un fait culturel courant et profondément ancré à tous les niveaux de la société, et le secteur forestier ne fait pas exception à la règle. Les pouvoirs économique et politique vont de pair et sont tous deux couramment utilisés par certains groupes puissants pour bafouer ou contourner les règlements, que ce soit des procédures très simples, ou plus gravement, des lois. Cependant, les puissants ne sont pas les seuls à tourner la loi. Les permis de récolte non commerciaux constituent une mesure qui devrait favoriser les plus démunis. Or, la violation routinière de ce type de permis ainsi que des autres en toute impunité crée les conditions propices à une culture de transgression des règles établies. Par réaction à cette culture de transgression des règles, on trouve la culture de l'établissement de règles chimériques et de la réglementation excessive des activités de production. Cette prolifération de règlements et d'exigences, associée à la confusion et à l'incertitude qui prévalent au sujet des modes de faire-valoir, rendent impossible la conformité totale (Del Gatto et al. 2004).

Enfin, cette étude de cas nous fournit un enseignement particulièrement intéressant au sujet de l'OIF. Habituellement, l'OIF se définit comme le recours à une tierce partie indépendante pour accomplir des activités d'observation des forêts avec l'accord des autorités gouvernementales (Global Witness 2005). On admet généralement que la tierce partie est une ONG à but non lucratif ou une entreprise privée. Cependant, l'expérience hondurienne indique qu'une institution publique nationale peut aussi jouer un rôle efficace d'observateur indépendant des forêts, comme c'est le cas du CONADEH, qui agit sans nécessairement avoir l'accord des autres autorités gouvernementales. ${ }^{4} \mathrm{Il}$ s'agit là d'une variation intéressante par rapport aux projets précédents d'OIF réalisés dans d'autres pays, laissant entrevoir la possibilité d'une approche plus endogène et institutionnalisée de l'OIF.

4 Même si la loi n'y oblige pas, le projet d'OIF au Honduras a bénéficié de la reconnaissance officielle de l'AFECOHDEFOR. D'après la majorité des parties prenantes, cette reconnaissance a facilité la tâche de l'OIF et a permis d'instaurer une relation de collaboration avec l'AFE-COHDEFOR (ainsi qu'avec son successeur, l'ICF). 



\section{Chapitre 8}

\section{Vérification forestière au Nicaragua}

Note : Cette étude de cas a été réalisée en mars 2007 par Filippo Del Gatto, Guillermo Navarro, Ove Faurby et Armando Argüello.

Tableau 8.1 Nicaragua : quelques statistiques clés

\begin{tabular}{|c|c|c|c|}
\hline Statistiques & & Date & Source des données \\
\hline Population & 5,5 millions & 2005 & $\begin{array}{l}\text { Nations Unies, Division } \\
\text { de la population } 2006\end{array}$ \\
\hline Superficie & $\begin{array}{l}12,1 \text { millions } \\
\text { de ha }\end{array}$ & & FAO 2006 \\
\hline Couverture forestière & $42,7 \%$ & 2005 & $\begin{array}{l}\text { Nations Unies, Division } \\
\text { de la statistique } 2006\end{array}$ \\
\hline Propriété de la forêt publique & Pas de données & 2000 & FAO 2005 \\
\hline $\begin{array}{l}\text { Production de bois rond } \\
\text { industriel }\end{array}$ & Pas de données & $\begin{array}{l}\text { Moyenne } \\
2001-2005\end{array}$ & OIBT 2005 \\
\hline $\begin{array}{l}\text { Emplois officiels dans le } \\
\text { secteur forestier }\end{array}$ & 9000 & $\begin{array}{l}\text { Moyenne } \\
1990-2000\end{array}$ & FAO 2004 \\
\hline $\begin{array}{l}\text { Contribution du secteur } \\
\text { forestier au PIB }\end{array}$ & $0,7 \%$ & $\begin{array}{l}\text { Moyenne } \\
1990-2000\end{array}$ & FAO 2004 \\
\hline $\begin{array}{l}\text { Valeur des exportations de } \\
\text { produits forestiers }\end{array}$ & 12 millions USD & $\begin{array}{l}\text { Moyenne } \\
1990-2000\end{array}$ & FAO 2004 \\
\hline $\begin{array}{l}\text { Principaux marchés } \\
\text { internationaux du bois }\end{array}$ & Pas de données & 2004 & Global Timber 2007 \\
\hline $\begin{array}{l}\text { Indice TI de perception } \\
\text { de la corruption }(0-10,0= \\
\text { corruption la plus forte) }\end{array}$ & 2,6 & 2006 & $\begin{array}{l}\text { Transparency } \\
\text { International } 2006\end{array}$ \\
\hline $\begin{array}{l}\text { Indice du développement } \\
\text { humain }(0-1,0=\text { très bas })\end{array}$ & 0,70 & 2004 & PNUD 2006a \\
\hline
\end{tabular}

\subsection{Introduction}

Le Nicaragua est considéré comme le pays le plus pauvre du continent américain avec un PIB (produit intérieur brut) annuel par habitant de 950 USD (BCN 2006 ; PNUD 2005). Tandis que la contribution du secteur forestier au PIB est minime, de l'ordre de $1 \%$ environ, les forêts sont perçues comme étant la ressource naturelle la plus importante du pays pour le développement économique et la réduction de la pauvreté. Environ $43 \%$ du territoire du Nicaragua est couvert par des forêts (5,2 millions de ha), dont plus de la 
moitié, c'est-à-dire à peu près 3 millions de ha, convient à la gestion forestière tournée vers la production.

Cette étude de cas décrit le système de contrôle des forêts au Nicaragua, en s'attachant particulièrement aux caractéristiques de vérification. Elle illustre quelques aspects clés des systèmes de vérification et de contrôle des forêts, qui peuvent aussi se révéler adaptés à d'autres pays :

- Insistance sur le respect des règles de la part des exploitants. Au Nicaragua, si les activités de vérification et de contrôle portent sur la récolte et la transformation du bois, l'attribution des droits d'exploitation par les instances forestières n'a pas fait l'objet d'un examen très approfondi. On observe, cependant, des signes de changement. Par exemple, des enquêtes menées en 2006 ont permis de découvrir l'attribution frauduleuse de permis d'exploitation à petite échelle à de grandes entreprises d'exploitation forestière.

- Un intérêt limité pour la déforestation. La principale cause de la disparition des forêts au Nicaragua est le changement d'utilisation des sols pour l'élevage des bovins et l'agroindustrie. En dépit de cela, la vérification et le contrôle des forêts se concentrent surtout sur les activités d'exploitation, tandis que la déforestation gagne du terrain partout de façon sauvage, sauf dans les zones protégées où elle est surveillée. Cependant là aussi, des changements en cours semblent aller dans le bon sens, la déforestation ayant été déclarée crime environnemental par une loi récente.

\subsection{Le débat sur la vérification et le contrôle des forêts au Nicaragua}

Le débat national sur la vérification et le contrôle des forêts au Nicaragua se polarise sur l'exploitation illégale et le commerce qui en découle. Ces activités sont perçues par le grand public comme les principales responsables de la déforestation. Cette perception est accentuée par les médias qui décrivent souvent le secteur du bois comme une " mafia " qui dévaste l'environnement et affaiblit l'autorité et la légitimité de l'Etat.

Par conséquent, la plupart des parties prenantes s'accordent sur l'urgence qu'il y à mettre fin à l'exploitation et au commerce du bois s'exerçant dans l'illégalité. Les exploitants forestiers privés font les frais de cette situation : une réglementation excessive, trop de contrôles et une opinion publique trop critique qui les blâme pour un problème, en l'occurrence la déforestation, dans lequel ils portent une responsabilité limitée. En dépit du lien présumé entre l'exploitation illégale et la déforestation, ces deux phénomènes ne sont pas liés au Nicaragua. Si l'exploitation illégale semble être plus importante, en termes de volume, dans les forêts de pins (Richards et al. 2003), le déboisement a lieu presque en totalité dans les forêts de feuillus de la région atlantique, et est surtout accéléré par des investissements dans l'élevage bovin et l'agriculture (Faurby 2005).

Si l'exploitation illégale n'est pas la principale responsable du déboisement, d'où vient ce débat national qui vise l'industrie du bois ? Selon certains observateurs, le pouvoir du lobby de l'élevage bovin est un facteur qui entre en jeu. Les entrepreneurs du secteur 
forestier affirment que ce lobby a tout intérêt à focaliser le public et les efforts du gouvernement sur les activités forestières, détournant ainsi l'attention de la conversion en terre d'élevage. Et au Nicaragua comme ailleurs, la " croisade » contre l'exploitation illégale, grâce à son grand retentissement, permet aux hommes politiques de se présenter en acteurs publics engagés. Lors de la campagne qui a précédé les élections de 2006, on a pu observer d'ailleurs un plus grand nombre de déclarations à propos de l'exploitation illégale et l'approbation de nouveaux textes de loi, actions davantage suscitées par une envie de publicité que par un véritable intérêt pour la recherche de solutions durables.

\subsection{Missions de vérification et de contrôle}

La vérification et le contrôle des forêts au Nicaragua s'inscrivent dans un cadre juridique complexe, composé de multiples textes normatifs :

- Le statut d'autonomie des régions de la côte atlantique de 1987 (Loi n 28) ;

- La loi sur les municipalités de 1988 (Lois nº 40 et 261);

- La loi sur l'environnement de 1996 (Loi n² 217);

- La loi sur les forêts de 2003 (Loi n 462);

- La loi sur les délits environnementaux de 2005 (Loi n 559);

- La loi sur l'interdiction d'abattage de 2006 (Loi n 585).

Ces divers textes de loi posent le principe de l'intervention de plusieurs organismes et acteurs à qui sont dévolues les activités de vérification et de contrôle (voir Figures 8.1 et 8.2). La mission de chaque institution ou acteur est définie par la loi, mais il n'existe pas de mission globale portant sur la totalité du système de vérification des forêts.

Certaines de ces missions jouissent d'un soutien plus grand de la part du public que d'autres. Ce soutien ne semble pas être en rapport ni avec les mérites particuliers de la mission, ni avec le niveau de participation des intervenants dans la définition de celle-ci. En revanche, la perception du public semble plus pertinente quant à l'accomplissement du mandat par rapport au débat ouvert sur l'exploitation illégale. Ainsi, bien que l'on observe une large adhésion du public à la mission de l'Institut National des Forêts (INAFOR) qui contrôle l'usage des forêts, on ne peut pas en dire de même de la façon dont cet Institut remplit sa mission. En revanche, la récente initiative du procureur pour l'environnement en vue d'enquêter sur l'exploitation illégale a été saluée par les médias et une grande partie du public.

\subsection{Vérification forestière au Nicaragua : schéma et pratique}

Comme nous l'avons déjà signalé, les dispositions régissant la vérification et le contrôle des forêts au Nicaragua impliquent une multiplicité d'institutions et d'acteurs. Certaines de ces dispositions peuvent être considérées comme des mécanismes internes au secteur, fixés par la loi sur les forêts et la réglementation afférente. D'autres sont des dispositions externes au secteur définies dans divers textes législatifs, tels que le statut d'autonomie 
des régions de la côte atlantique, la loi sur les municipalités, la loi sur l'environnement et la loi sur les crimes environnementaux. Si ces mécanismes font l'objet d'une description séparée ci-dessous, ils se chevauchent en réalité et certains intervenants (les municipalités, par exemple) jouent un rôle sur les deux plans, interne et externe.

\section{Les mécanismes de contrôle et de vérification fixés par la loi sur les forêts du Nicaragua}

La Figure 8.1 montre le système de vérification et de contrôle interne au secteur, avec ses principaux intervenants et leurs fonctions. Le système met surtout l'accent sur le respect des règles de la part des exploitants (surveillance des propriétaires de forêts ou des entrepreneurs qui détiennent un permis d'exploitation).

L'INAFOR, organe exécutif dépendant du Ministère de l'agriculture et des forêts (MAGFOR), est responsable du suivi et du contrôle courants de l'usage des ressources forestières hors des zones protégées ; l'INAFOR inspecte aussi la transformation et le commerce des produits forestiers. Ces fonctions sont déléguées aux agents municipaux de cet Institut. Leurs principales tâches consistent en :

- L'examen au bureau des plans de gestion forestière et des plans annuels de récolte ;

- Des inspections sur le terrain des sites forestiers ;

- La révision des rapports mensuels transmis par les régisseurs forestiers, qui sont des professionnels agréés ;

- Le contrôle des activités de transport ;

- L'inspection des installations industrielles.

Pour assister l'INAFOR dans ses responsabilités en matière de contrôle, la loi sur les forêts instaure aussi la délégation des obligations de suivi et d'administration des forêts aux régisseurs forestiers dont les missions pour l'Etat sont :

- De vérifier l'élaboration correcte des plans de gestion forestière et des plans annuels de récolte ;

- De superviser les travaux d'exploitation ;

- De contrôler les permis de transport ;

- D'adresser des rapports mensuels au bureau local de l'INAFOR.

Les agents municipaux de l'INAFOR sont en théorie inspectés de façon impromptue par son département de suivi qui est basé dans la capitale. Mais ces inspections étant généralement peu fréquentes et assez superficielles, ces agents locaux peuvent donc travailler dans une grande autonomie (CINCO et CIP 2006).

L'administration locale a le pouvoir de signer des accords de partenariat avec l'INAFOR, en vertu desquels les municipalités peuvent assumer une fonction de contrôle et d'administration des forêts. Un certain nombre d'accords a déjà été signé mais, pour la plupart, ils sont encore en gestation. Certaines municipalités sont aussi impliquées dans 


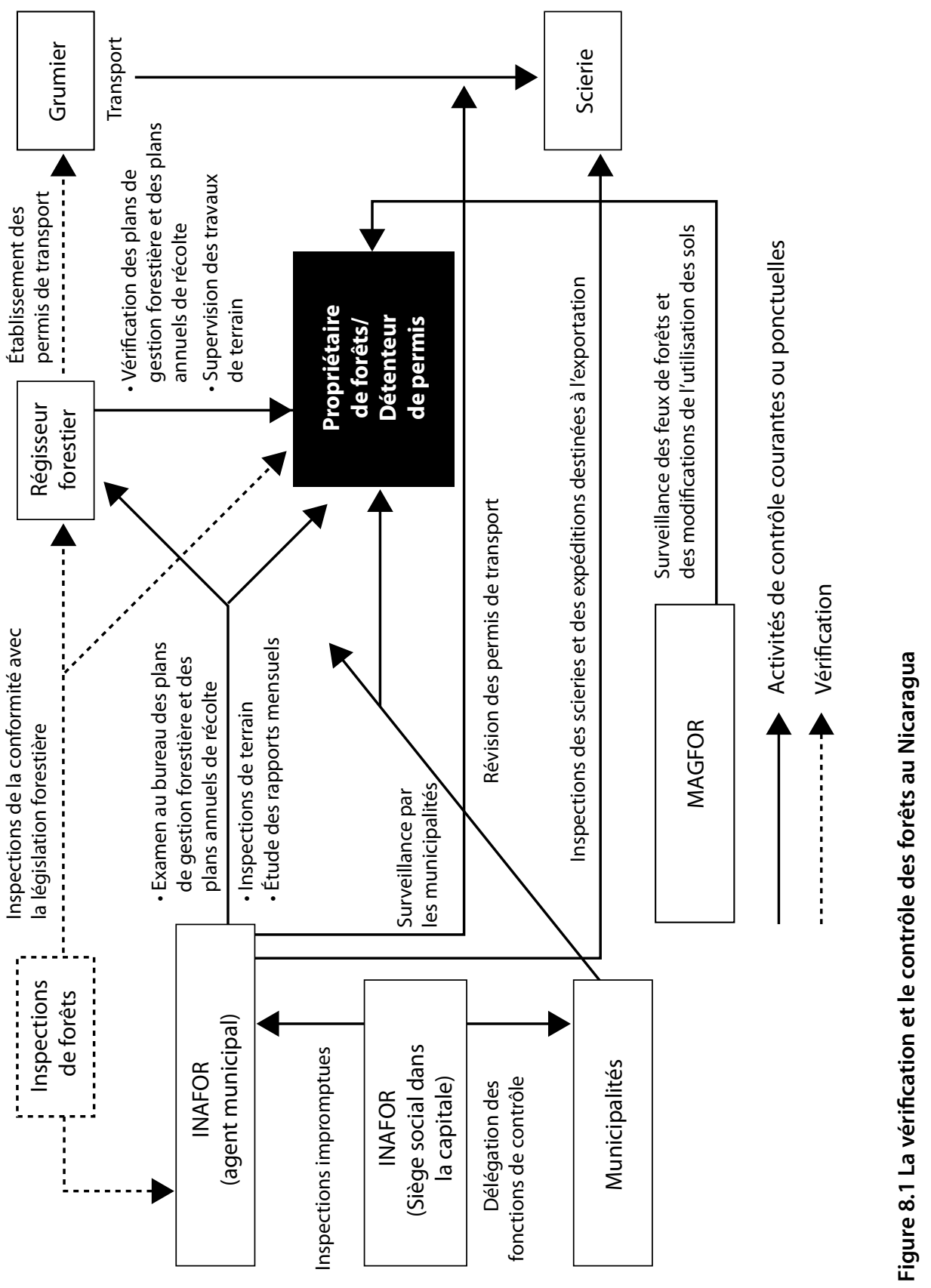


le contrôle des feux de forêts et des modifications d'utilisation des sols. Cependant, en dehors des zones protégées, ceci est du ressort de MAGFOR.

En théorie, les inspections de forêts peuvent être considérées comme la " démarche de vérification » de ce système de contrôle à plusieurs niveaux. Ces inspections par des tiers sont définies par la loi sur les forêts et devraient être réalisées par des personnes ou des entreprises indépendantes et spécialisées, agréées par l'INAFOR. Elles visent à vérifier la mise en œuvre des plans de gestion forestière et des plans annuels de récolte. Grâce à ces mesures, les inspecteurs forestiers peuvent vérifier ce que font les exploitants et les régisseurs forestiers (et aussi indirectement les agents municipaux de l'INAFOR). Mais le tendon d'Achille des inspecteurs forestiers a été la question du financement. Tandis que l'INAFOR recourt à leurs services, la loi sur les forêts ne précise pas l'origine du financement de leurs prestations. C'est pour cette raison que ce mécanisme de vérification n’a pas encore été mis en place.

En juillet 2006, l'INAFOR et Global Witness, organisation non gouvernementale basée au Royaume-Uni, ont signé un accord pour la mise en œuvre d'un projet pilote d'observation indépendante des forêts (Independent Forest Monitoring, IFM) au Nicaragua, dont la mission est de recueillir des informations fiables sur l'exploitation illégale dans le pays et de veiller à l'objectivité et à la transparence des activités de suivi menées par les instances responsables (INAFOR and Global Witness 2006). D'après certains observateurs, la mission attribuée aux inspecteurs forestiers a été effectuée, au moins en partie, par l'observateur indépendant des forêts. Mais la plupart des parties prenantes (y compris l'équipe de l'IFM) ont un point de vue différent et affirment que l'initiative de l'IFM devrait accompagner et observer les inspecteurs forestiers dans leur travail, et ne pas être considérée comme venant en remplacement de ceux-ci.

\section{Vérification forestière par des organismes externes au secteur}

La Figure 8.2 illustre le système de vérification et de contrôle externe au secteur, tel qu'il est défini par les divers textes législatifs. Comme nous l'avons déjà dit, ce système insiste surtout sur le respect des règles par les exploitants.

La loi sur l'interdiction de l'abattage de 2006 a interdit la récolte de bois dans toutes les zones protégées. Avant cette loi, des activités de gestion forestière étaient possibles dans certaines de ces zones. L'INAFOR ne pouvait alors délivrer un permis d'exploitation qu'après l'approbation du plan de gestion forestière par le Bureau régional du ministère de l'environnement et des ressources naturelles (MARENA). Le suivi des travaux forestiers dans les zones protégées était aussi du ressort du Bureau régional du MARENA, comme le contrôle du transport des produits forestiers qui en sortaient. Toutes ces missions ont été supprimées par l'interdiction d'abattage dans les zones protégées, mais le MARENA est toujours chargé d'y enquêter sur le déboisement et les activités illégales d'exploitation.

La loi sur les municipalités accorde aux administrations locales un rôle important en matière de suivi et de surveillance, qui s'exerce en général par l'Unité environnementale 


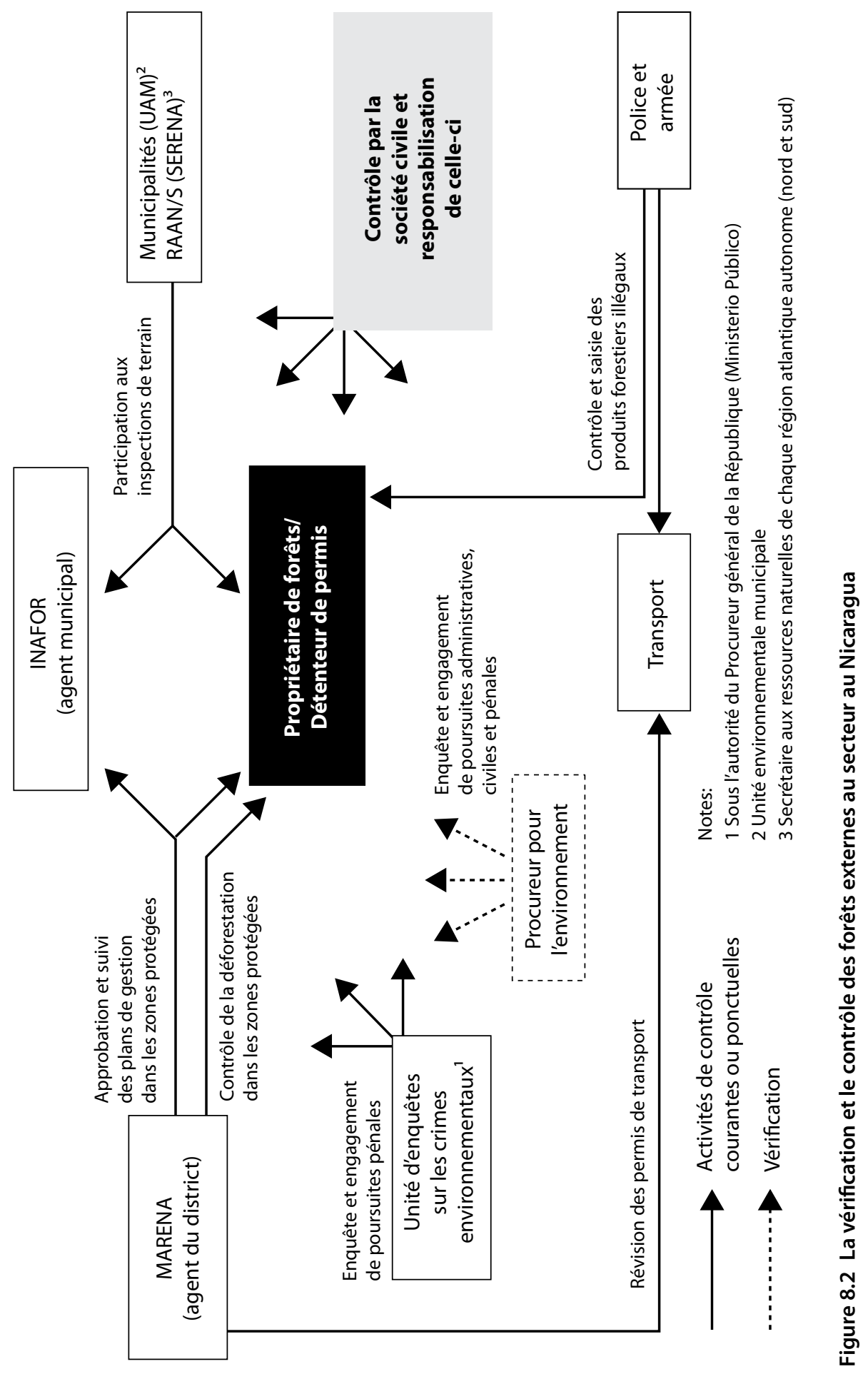


municipale (UAM) par l'intermédiaire d'inspections de terrain en collaboration avec les agents locaux de l'INAFOR et du MARENA. Dans les deux régions autonomes de la côte atlantique (la région autonome nord-atlantique ou RAAN, et la région autonome sud-atlantique ou RAAS), ce rôle est assumé par le Secrétaire aux ressources naturelles (SERENA) du gouvernement régional.

La police nationale et l'armée sont requises pour épauler l'action de l'INAFOR et du MARENA en vue de contrôler le transport des produits forestiers. Depuis le vote de la loi sur l'interdiction d'abattage, ils jouent aussi un rôle clé dans la surveillance de l'application des nouvelles interdictions d'abattage, en particulier (mais pas seulement) le long des frontières.

Dans ce cas, le "vérificateur » du système est le procureur pour l'environnement, institué par la loi sur l'environnement de 1996, qui dépend du Bureau du Procureur général, et qui est en charge de la représentation et de la défense des intérêts du public et de l'Etat pour les questions environnementales. Cette institution a été très active ces dernières années dans le combat contre l'exploitation illégale. Comme le montre le Tableau 8.2, plus de $50 \%$ des enquêtes menées en 2005 ont concerné les activités illégales d'exploitation, de transport et de transformation du bois.

L'unité d'enquêtes sur les crimes environnementaux a été créée en 2006 au sein du Bureau du Procureur public pour contribuer à l'application de la nouvelle loi sur les crimes environnementaux. Cette unité peut mener ses propres enquêtes de terrain sur les violations définies par cette loi. Bien qu'il soit trop tôt pour le dire, elle pourrait finalement devenir un nouvel acteur de vérification de l'ensemble du système de gouvernance forestière du Nicaragua.

Ces deux entités étant financées par le budget alloué tous les ans par l'Assemblée nationale aux institutions qui les accueillent (Bureau du Procureur général et Bureau du Procureur public), leur financement est plutôt indépendant d'éventuels aléas externes. Cependant, au Nicaragua, une partie de l'industrie du bois entretient des liens étroits

\section{Tableau 8.2 Enquêtes menées par le Procureur pour l'environnement du Nicaragua en 2005}

\begin{tabular}{lll}
\hline Crimes & Nombre & Pourcentage \\
\hline $\begin{array}{l}\text { Activités illégales d'exploitation, de transport et de } \\
\text { transformation du bois }\end{array}$ & 310 & 53,2 \\
\hline $\begin{array}{l}\text { Incendies volontaires } \\
\begin{array}{l}\text { Autres (par ex. braconnage, pêche non autorisée, pollution } \\
\text { de l'air/de l'eau/du sol, extraction illégale de matériaux) }\end{array}\end{array}$ & 21 & 97,8 \\
\hline Total & 583 & 37 \\
\hline
\end{tabular}

Source : Procureur pour l'environnement 2006 
avec les principaux partis politiques, qui, pour leur part, ont la mainmise sur la plupart des organismes publics, dont le Bureau du Procureur général et le Bureau du Procureur public, entraînant une grande vulnérabilité en matière de manipulation politique.

\subsection{Quelles missions de vérification peut-on repérer?}

En vertu de la loi sur les forêts, le principal objectif des inspections de forêts (qui n'ont pas encore eu lieu, comme nous l'avons déjà signalé) est d'évaluer le travail des exploitants et des régisseurs forestiers par rapport à un ensemble de normes relatives à la gestion forestière. Par conséquent, leur fonction de suivi est très restreinte et se concentre surtout sur des éléments bien précis du système de gouvernance forestière.

Si l'on considère que c'est l'INAFOR qui octroie l'agrément des inspecteurs forestiers, qui les fait travailler sous contrat et qui les paie, il semble improbable que ces inspecteurs soient attentifs aux modalités d'attribution des ressources de cet institut. D'ailleurs, ceci n’entre pas dans le cadre de leur mission fixée par la loi.

Malgré sa mission bien plus étendue, le Procureur pour l'environnement n'a généralement pas une grande fonction de surveillance, étant essentiellement chargé d'enquêter sur le respect de la réglementation pour chaque exploitant. Cependant, ceci est en train de changer. Ce changement a été suscité par les nombreux abus commis par l'INAFOR concernant les permis relatifs aux petites forêts situés sur les terres agricoles (qui ne font donc pas l'objet de l'élaboration d'un plan de gestion), définis dans la réglementation liée à la loi sur les forêts. L'attribution illicite et à foison de ces permis à de grandes entreprises travaillant dans des zones forestières étendues a incité le procureur pour l'environnement à s'intéresser à la totalité du système (y compris aux décisions d'attribution des ressources et à la performance de l'INAFOR), exerçant par conséquent une fonction d'inspection de plus en plus vaste.

Dans les deux mécanismes de vérification, il n'est pas prévu de fonction officielle de remontée de l'information, par laquelle l'information recueillie par les inspecteurs forestiers ou le procureur pour l'environnement peut être validée et transmise aux organismes concernés afin de contribuer au processus de réforme. De même, il n'existe pas de procédure permettant de mettre l'information à la disposition du public.

En revanche, ces deux mécanismes sont très axés vers la lutte contre les infractions. Les rapports des inspecteurs forestiers, après approbation par l'INAFOR, peuvent servir de document technique pour l'application des sanctions fixées par la loi sur les forêts et la réglementation afférente tandis que le procureur pour l'environnement a le pouvoir de prononcer des condamnations se traduisant au niveau de l'INAFOR par la prise de sanctions administratives et au niveau du système judiciaire par des sanctions civiles et pénales. 


\subsection{Bilan des mécanismes de vérification}

\section{Inspections de forêts}

Il n'est pas possible d'évaluer le fonctionnement des inspections de forêts puisqu'elles n'ont pas encore eu lieu. Néanmoins, la plupart des intervenants sont favorables à ce mécanisme et regrettent qu'il ne soit pas appliqué.

Comme l'indique la Figure 8.1, le contrôle courant des activités forestières est délégué aux agents municipaux de l'INAFOR en collaboration avec les régisseurs forestiers. Ces deux groupes d'acteurs font partie de la communauté forestière locale (c'est-à-dire à peu près les personnes intervenant en relation avec le secteur forestier local). Comme l'on pouvait s'y attendre, les cas de non respect des règles par les exploitants forestiers locaux sont traités dans un esprit de conciliation qui est favorable aux sanctions légères. En général, les violations sont vues comme des erreurs admises (qui se justifient en raison de la difficulté de l'environnement de travail) et les sanctions sont appliquées avec une grande souplesse. Ceci contribue à maintenir la cohésion sociale de la communauté forestière locale (Pendleton 1998). Mais en même temps, cela engendre de la méfiance chez les acteurs externes au secteur.

Dans ce contexte, de nombreux observateurs pensent que les inspections de forêts sont un mécanisme nécessaire (et pas trop contraignant), qui pourrait apporter davantage de transparence et déboucher sur une plus grande obligation de rendre des comptes, tout en étant acceptable pour la communauté forestière locale.

\section{Procureur pour l'environnement}

Les données présentées dans le Tableau 8.2 montrent l'investissement personnel et l'action du Procureur pour l'environnement qui effectue un travail remarquable avec de maigres ressources.

Plus de la moitié $(53,2 \%)$ des enquêtes menées en 2005 ont concerné les activités illégales d'exploitation, de transport et de transformation du bois, tandis qu'aucune n’a porté sur la déforestation. C'est surprenant si l'on considère que le Nicaragua perd entre 50000 et 70000 hectares de forêts chaque année (FAO 2005 ; Faurby 2005). Comme indiqué au début de cette étude de cas, la principale cause de la disparition de forêts au Nicaragua est de loin le changement d'utilisation des sols pour l'élevage des bovins et l'agriculture, bien plus que les activités d'exploitation forestière.

Par conséquent, tandis que l'action du procureur pour l'environnement correspond au débat public sur l'exploitation illégale et dans une certaine mesure au désir du public de voir que des mesures sont prises, on peut soutenir qu'il fait peu d'efforts pour enrayer la disparition des ressources forestières du pays.

De même, les effets en termes de gouvernance forestière globale semblent limités. Les exploitants forestiers ont tendanceà refuser les activités du procureur pour l'environnement. 
D'abord, ils ressentent un parti pris à leur encontre (et c'est bien compréhensible), tandis qu'on néglige la déforestation. Deuxièmement, les mesures prises par le procureur pour l'environnement ont souvent été accompagnées de déclarations assez dures envers les exploitants forestiers. Troisièmement, la démarche juridique est différente. Le procureur pour l'environnement s'appuie sur les textes de la loi sur l'environnement et de la loi sur les crimes environnementaux tandis que l'INAFOR et les exploitants forestiers se réfèrent aux dispositions de la loi sur les forêts et la réglementation afférente. La nouvelle loi sur l'interdiction d'abattage, qui est vague, rajoute du flou au cadre juridique.

Tout ceci a produit une sorte de barrière faite de méfiance et de ressentiment entre les deux côtés. Selon Lang (1996), " le contrôle a lieu dans la mesure où il est accepté par ceux que l'on contrôle ". Si l'on accepte ce point de vue, on pourrait avancer que l'action du procureur pour l'environnement ces dernières années n'est pas allée dans le sens de l'amélioration des conditions de la gouvernance forestière au Nicaragua.

D'après le procureur pour l'environnement, le manque d'intérêt pour la déforestation en 2005 vient du fait que la " coupe rase " et le " changement d'utilisation des sols forestier " n’ont été clairement définis comme crimes que lors de l'entrée en vigueur mi-2006 de la nouvelle loi sur les crimes environnementaux. Par conséquent, en dépit de la confusion qui règne autour des critères de légalité, cette nouvelle loi pourrait finalement avoir une influence sur les activités du procureur pour l'environnement et favoriser une approche plus équitable des délits forestiers. Ce serait certainement une bonne chose pour les forêts du Nicaragua.

\subsection{Enseignements}

Les leçons que l'on peut tirer de cette étude de cas sont les suivantes :

- Le soutien du public pour une mission de vérification n'est pas nécessairement fonction de sa portée ou de la participation des parties prenantes à sa définition. Au Nicaragua, ce soutien semble être plus en rapport avec la perception que le public a de la façon dont l'institut ou l'acteur concerné s'acquitte de ses fonctions.

- En plus des missions des divers instituts, il y a sans doute besoin d'un cadre juridique unique pour la totalité du système de vérification des forêts. Au Nicaragua, divers textes de loi posent le principe de l'intervention de plusieurs institutions et acteurs à qui sont dévolues les activités de vérification et de contrôle. La mission de chaque institution ou acteur est définie par la loi, mais il n'existe pas de mission englobant la totalité du système de vérification des forêts.

- Il est nécessaire d'apporter de la clarté afin de savoir quels textes juridiques s'appliquent. Le procureur pour l'environnement s'appuie sur les textes de la loi sur l'environnement et de la loi sur les crimes environnementaux tandis que l'INAFOR et les exploitants forestiers se réfèrent aux dispositions de la loi sur les forêts et la réglementation afférente. La nouvelle loi sur l'interdiction d'abattage, qui est vague, rajoute au flou juridique. 
- Les sources de financement doivent être clairement définies lors de la conception du système. Au Nicaragua, les inspecteurs forestiers (pivots du système de vérification des forêts) ne sont pas encore opérationnels parce que la loi sur les forêts ne précise pas l'origine du financement de leurs prestations.

- Si les IFMs interviennent, leur rôle doit être clairement précisé par rapport à la mission des autres institutions ou acteurs - viennent-il les épauler ou les remplacer?

- Enfin, cette étude soulève la question importante du but dans lequel sont effectuées les activités de vérification. Le sujet de préoccupation est-il le respect de la loi, ou bien un enjeu public plus élevé tel que la déforestation ? Bien qu'au Nicaragua le changement d'utilisation des sols soit la principale cause de disparition de forêt, les activités de vérification et de contrôle se sont concentrées sur l'exploitation et la transformation du bois. Il faut que le système soit clair en matière d'objectifs visés. 


\section{Chapitre 9}

\section{Respect de la loi sur les forêts dans le système brésilien de gouvernance forestière}

Note : Cette étude de cas a été réalisée en décembre 2007 par Hans Thiel. Elle s’appuie en partie sur Thiel, H. et Viergever, M. 2006 Giants don't leap: Verification in Brazil's process towards sustainable forestry. Étude de cas de pays VERIFOR n ${ }^{\circ} 5$. ODI, Londres.

Tableau 9.1 Brésil : quelques statistiques clés

\begin{tabular}{llll}
\hline Statistiques & & Date & Source des données \\
\hline Population & 186,4 millions & 2005 & $\begin{array}{l}\text { Nations Unies, Division } \\
\text { de la population 2006 }\end{array}$ \\
\hline Superficie & $\begin{array}{l}835,6 \text { millions } \\
\text { de ha }\end{array}$ & & FAO 2006 \\
\hline Couverture forestière & $57,2 \%$ & 2005 & Nations Unies, Division \\
de la statistique 2006
\end{tabular}

\subsection{Introduction}

Cette étude de cas illustre l'importance de l'engagement politique, d'un dirigeant visionnaire et de la coordination de tous les secteurs pour s'attaquer aux causes qui, au 
Brésil, sont à la base de la déforestation, de l'exploitation forestière illégale et abusive et de la corruption qui va de pair avec ces situations. Des réformes juridiques et institutionnelles sans précédent se dessinent qui, associées à des innovations technologiques, vont renforcer l'application des lois qui réglementent l'utilisation des terres et des forêts et la prise de sanctions. Un système décentralisé de contrôle, de vérification et d'administration de la forêt se dégage de ce processus de réforme, système intéressant dans lequel l'autorité et diverses compétences sont exercées conjointement au niveau national et par les administrations forestières des états grâce à l'appui d'un système d'information innovant. ${ }^{1}$

Le Brésil est un pays de la taille d'un continent dont $57 \%$ de la superficie est recouverte de forêt. Le bassin de l'Amazone est brésilien à $60 \%$. La filière bois compte pour $4 \%$ du PIB du Brésil et pour $8 \%$ de ses exportations, générant plus de 1,4 milliard USD de recettes fiscales annuelles et créant environ 2 millions d'emplois directs et indirects. Plus de $85 \%$ de la production de bois provenant des forêts naturelles du pays vient de l'Amazonie brésilienne, où 2570 entreprises d'exploitation forestière travaillant dans 72 régions de production de bois (les " polos madereiros ") génèrent un revenu annuel brut de 2,5 milliards USD. La valeur du bois exporté de l'Amazonie brésilienne atteint presque 1 milliard USD par an, dont $30 \%$ sont destinés aux Etats membres de l'Union Européenne (Thiel and Viergeveer 2006).

Plus des deux tiers des 17 millions $\mathrm{de}^{3}$ de bois rond produit dans l'Amazonie brésilienne en 2003 provenait du dégagement. L'élevage bovin et la culture du soja, responsables de $70 \%$ à $80 \%$ de la déforestation, sont à l'origine d'énormes quantités de bois illégal pour l'industrie. Ceci provient du fait que l'agriculture n'est pas régie par un régime foncier, contrairement à la gestion forestière, balisée par un cadre juridique. La déclaration des terrains fonciers n'est pas bien organisée au Brésil et le fait qu’on ne connaisse pas les propriétaires favorise l'utilisation et l'exploitation abusives de la forêt au détriment d'une gestion et d'une conservation durable de cette ressource. D'où une disparité considérable entre les volumes de bois autorisés et la superficie déboisée par l'exploitation forestière. On estime que $80 \%$ de la production totale de bois de l'Amazonie brésilienne résulte de l'abattage illégal (PAS 2006).

\subsection{Vérification et contrôle des forêts : éléments moteurs et mission}

Le rythme alarmant et préoccupant de la déforestation en Amazonie brésilienne d'une part et la position du Brésil en tant que plus gros producteur et consommateur mondial de bois tropicaux d'autre part, ont conduit le gouvernement brésilien à instaurer le programme forestier national (NFP) en 1998 et à le renforcer en 2000. Ce programme était le résultat d'un large processus participatif, impliquant plus de 600 organismes de différentes branches de la société, dont le but était de promouvoir le développement

1 Exercice conjoint d'une compétence publique : Les autorités publiques nationales et sous-nationales ont les mêmes compétences ou fonctions, chacune selon son niveau hiérarchique et au sein du territoire de son ressort. 
durable et la conservation des forêts d'une manière qui soit compatible avec les autres politiques publiques. Le NFP vise à réduire la déforestation en : (i) doublant la superficie des zones reboisées pour assurer l'approvisionnement de l'industrie du bois et diminuer ainsi la pression exercée sur les forêts naturelles; et (ii) en promouvant la gestion durable de la forêt par le renforcement des compétences. Lélément central du programme est la régularisation du régime foncier et des conditions de gestion complexes des terres boisées, surtout dans les forêts publiques fédérales. En 2003, la Commission interinstitutionnelle de coordination du programme forestier national (CONAFLOR), composée de 37 institutions appartenant à différents secteurs, a été créée par décret présidentiel pour mettre en œuvre ces politiques.

Grâce à son programme de gestion des ressources forestières ProManejo, l'Institut brésilien de l'environnement et des ressources naturelles renouvelables (IBAMA) constituait un autre moteur de la réforme. L'objectif principal de ProManejo était de soutenir le développement et l'adoption d'une gestion durable de la forêt en Amazonie brésilienne. Ce programme comprenait l'évaluation des systèmes d'administration et de vérification des forêts en place et l'élaboration de solutions plus efficaces et transparentes. En vigueur depuis 1999, ProManejo a été mis en œuvre dans le cadre du programme pilote de préservation de la forêt tropicale humide du Brésil (PPG7) qui est financé par les membres du G7, l'Union Européenne et les Pays-Bas. Bien que le PPG7 ait apporté un soutien financier et technique, le projet a été conduit par le gouvernement brésilien en collaboration avec la société civile et le secteur privé.

L'année 2003 a également vu la création du groupe de travail interministériel permanent en vue de réduire la déforestation de l'Amazonie brésilienne (GPTIRID), coordonné par le Cabinet (Casa Civil) de la Présidence de la république. ${ }^{2}$ Le GPTIRID regroupe 13 ministères qui sont concernés d'une façon ou d'une autre par l'Amazonie brésilienne, ainsi que des instances chargées de faire appliquer les lois (le Ministério Público et la police fédérale) et des organismes scientifiques tels que l'Agence spatiale brésilienne (INPE). ${ }^{3}$ Son plan d'action soutient le suivi et le contrôle, l'aménagement du territoire, et le développement durable dans le domaine de l'économie et des infrastructures (GPTIRID 2004).

Entre juin 2003 et mai 2006, la Casa Civil, les ministères de l'Environnement (MoE), de l'Intégration nationale, de la planification, du budget et de la gestion, ainsi que sept états d'Amazonie ont élaboré un nouveau cadre de développement pour la région, le Plano Amazonía Sustentavel (Plan pour une Amazonie durable) (PAS 2006). L'objectif de ce plan est de coordonner les actions fédérales et celles des états concernant les territoires et les secteurs, en encourageant la participation active de la société civile, du secteur privé, des états et des municipalités. Grâce à la décentralisation et à des politiques régionales élaborées dans un souci de rassemblement visant le consensus et le dialogue sociétal, le

2 La Casa Civil est un organe très puissant, intermédiaire entre les pouvoirs exécutif et législatif, dont la mission comprend l'examen de tous les projets de loi.

3 Le rôle du Ministério Público est d'enquêter sur les actes criminels et de les dénoncer au pouvoir judiciaire. Le Ministério Público est indépendant du pouvoir exécutif. 
PAS espère mettre fin aux inégalités régionales, à la concentration des rentes foncières, à l'exclusion sociale et aux schémas de croissance prédateurs. Les grandes orientations du PAS sont la promotion de l'aménagement du territoire, la clarification du régime foncier et la réduction du déboisement illégal par la surveillance, les sanctions et l'encouragement à utiliser le sol d'une manière plus intensive. Il met l'accent sur une bonne gouvernance, le respect de la législation et la présence active de tous les niveaux de l'Etat dans la région, comme conditions préalables à une croissance durable. Le PAS souligne aussi le besoin d'une intégration économique régionale au sens large, en se cantonnant pas aux frontières brésiliennes mais englobant toute l'Amazonie d'Amérique du Sud.

Tous ces processus bénéficient d'une volonté politique très forte impliquant les plus hauts niveaux du gouvernement et les principaux acteurs de la société civile. Bien que le débat ouvert se concentre sur les préoccupations concernant la déforestation, la faiblesse de la gouvernance et les inégalités en Amazonie brésilienne, la question sensible de la souveraineté de la forêt tropicale en tant que richesse de l'humanité et "l'internationalisation de l'Amazonie " sont aussi vues comme des menaces et sont par conséquent des éléments moteurs incitant à l'action.

\subsection{Architecture des institutions}

Le cadre actuel dans lequel évoluent toutes les grandes institutions environnementales publiques est le Système Environnemental National (SISNAMA), dont l'organisme principal, le Conseil national de l'environnement (CONAMA), comprend 103 représentants de toutes les branches de la société. Le SISNAMA conseille le gouvernement, encourage les études, propose des directives et établit également des normes et des procédures dans le domaine de l'environnement. Le pilier de ce système est le ministère de l'environnement, qui est responsable de la planification, de la coordination et de la supervision des politiques environnementales. Au niveau national, l'organe exécutif est l'IBAMA. Au-dessous, les agences environnementales des états sont responsables de la mise en ouvre des programmes et des projets ainsi que du suivi et du contrôle environnementaux sur les territoires de leur ressort. Elles peuvent élaborer leurs propres normes et procédures du moment qu'elles n'entrent pas en conflit avec la législation fédérale. Lorsque les agences des états n'ont pas la capacité nécessaire, l'IBAMA est censé intervenir.

La mission pour l'exercice décentralisé et conjoint de l'autorité en matière de forêt par le gouvernement central, les états et les municipalités a été introduite dans la constitution brésilienne en 1998. Ce n'est cependant qu'en mars 2006 que la nouvelle loi sur les forêts publiques a réformé le code de la forêt de 1965, en conférant aux états et aux municipalités la responsabilité de délivrer les permis et de suivre les activités forestières dans les forêts privées, municipales et des états et dans les réserves de leurs compétences respectives. Conjointement avec les états, l'organe fédéral, l'IBAMA, surveille l'exercice de ces compétences et l'application de la loi en mode décentralisé. Cette administration décentralisée de la forêt doit être mise en œuvre grâce à un système d'information innovant et transparent qui permet aux instances forestières nationales de surveiller et 
de superviser les états. La nouvelle loi a aussi créé le Service brésilien des forêts pour réglementer la gestion des forêts publiques fédérales, promouvoir la gestion durable de la forêt et gérer le Fonds national pour le développement de la forêt. D’autre part, la loi de 2006 encourageait une exploitation forestière légale en permettant, pour la première fois, de récolter du bois légalement dans les forêts publiques grâce à des concessions.

Les états avaient jusqu'à octobre 2007 pour prendre des responsabilités en matière de forêt dans le cadre du SISNAMA, notamment pour établir leurs propres systèmes d'administration et de contrôle des forêts. Ces systèmes doivent être reliés au système national d'information forestière (SISCOM) et aussi au système national d'information environnementale (SISNIMA). Bien que les états aient la liberté de mettre en place leurs propres systèmes d'administration et de vérification des forêts, ils peuvent aussi demander la collaboration de l'IBAMA. Les conditions de cette collaboration sont fixées par les autorités fédérales et comprennent un accord concernant le recours aux systèmes électroniques et une commission de suivi regroupant divers intervenants. L'IBAMA peut fournir aux états le système d'administration forestière nouvellement élaboré, le DOF (Documento de Origen Florestal), et la formation correspondante.

L'application de la loi est séparée verticalement entre les Ministérios Públicos fédéraux et ceux des états, les deux agissant de façon conjointe pour poursuivre les contrevenants et les traduire devant la cour d'assises. Les sanctions administratives, telles que la saisie de bois ou les amendes, sont gérées en même temps par l'IBAMA et les organismes environnementaux des états fédéraux.

Le rôle de la société civile brésilienne, agissant comme observateur indépendant dans ce processus, ne doit pas être ignoré : la capacité des ONG environnementales, des médias, de l'industrie et du monde universitaire à exercer un contrôle sociétal et un examen attentif des acteurs publics est relativement élevée.

\subsection{Administration et vérification des modifications de I'utilisation des sols et de la récolte de bois}

\section{Utilisation des sols et modifications dans ce domaine}

En ce qui concerne les propriétés dans l' "Amazonie légale ", seulement $20 \%$ de cellesci peuvent être déboisées, les $80 \%$ restants étant conservés à titre de " réserve garantie par la loi ». ${ }^{4}$ Pour assister les organismes environnementaux des états dans la mise en œuvre de cette réglementation, l'IBAMA a élaboré, par l'intermédiaire du PPG7, un plan d'aménagement du territoire, le système de délivrance des permis environnementaux dans les propriétés rurales (SLAPR), qui associe cette délivrance de permis avec le suivi et le contrôle des modifications d'utilisation des sols. Pour obtenir un permis environnemental (LUA), les coordonnées géographiques de la propriété, la réserve garantie par la loi, les

4 L'«Amazonie légale » du Brésil telle que définie par la loi comprend les états suivants : Acre, Amapá, Amazonas, Pará, Rondônia, Roraima, Mato Grosso, Maranhão et Tocantins. 
zones protégées sur les pentes raides et le long des cours d'eau doivent être saisies dans le système, afin que les instances environnementales puissent surveiller par les images satellites les changements intervenant dans la couverture forestière. L'utilisation du SLAPR dans les états fédéraux est variable : certains, comme le Mato Grosso, s'en servent depuis des années, tandis que d'autres, tels que le Roraima, ne l'ont pas encore mis en application.

\section{La récolte de bois}

Avant de soumettre un plan de gestion forestière, une entreprise d'exploitation forestière doit obtenir un permis environnemental (LUA) et un document d'" approbation préalable" (APAT) (MMA 2006a). LAPAT permet de vérifier la légalité de la propriété foncière, le statut juridique des personnes ou des entreprises concernées, ainsi que la preuve de l'existence de la forêt en question grâce à une carte géographique associée à une image satellite. Ces paramètres ont été les caractéristiques les plus fréquentes des activités frauduleuses de "blanchiment " de bois illégal qui impliquent des entreprises fictives, ou des forêts qui n'existent pas en faisant état de faux crédits de volumes de bois.

Dès que ces conditions juridiques sont vérifiées, un plan de gestion forestière ou une demande de modification de l'utilisation des sols peuvent être soumis à l'approbation de l'organisme environnemental de l'état concerné. ${ }^{5}$ Les plans de gestion de la forêt naturelle doivent être conformes aux critères de gestion durable de la forêt exposés par le gouvernement fédéral dans la Norme $n^{\circ} 5$. Ces plans doivent être préparés par un ingénieur forestier ou un agronome inscrit au Conseil régional de l'ingénierie, de l'architecture et de l'agronomie (CREA), et exécutés sous sa responsabilité technique. Dès l'approbation du plan de gestion par l'administration, celle-ci délivre une autorisation d'exploitation (AUTEX) qui génère un crédit de volume de bois dans le système de déclaration d'origine (DOF). Les exploitants forestiers enregistrés dans le système et dans un nouveau cadastre technique peuvent ensuite accéder en ligne au système DOF, de la même façon que les clients d'une banque utilisent les guichets automatiques pour retirer de l'argent sur leurs comptes.

Le système permet aux exploitants forestiers de gérer leurs propres volumes de bois et de proposer ces volumes en ligne à des acheteurs industriels inscrits. Dès que l'acheteur a accepté en ligne la vente de bois proposée, l'opération est enregistrée par le système et les volumes convenus de bois par espèces sont automatiquement débités du crédit de volume. L'exploitant imprime alors le permis de transport de bois portant un code-barres (aussi appelé DOF). Quand le bois est livré à l'industriel, les informations figurant sur le permis sont saisies dans le système DOF, qui la recoupe automatiquement avec celles de la base de données et qui génère ensuite un crédit de volume pour cet industriel. Ceci permet à l'industriel d'émettre son propre permis DOF pour le transport de ses produits vers ses clients.

5 Dans les états qui n'ont pas encore pris en charge l'administration forestière, celle-ci est toujours gérée par les bureaux locaux et régionaux de l'IBAMA. 
En conférant à l'exploitant la responsabilité d'imprimer le permis DOF et de faire un rapport à l'administration, ce nouveau système réduit les coûts, la bureaucratie et le risque que les volumes de bois soient utilisés de façon frauduleuse par des tiers. Le lien créé entre les exploitants et les acheteurs par un processus administratif est une innovation intéressante. Ceci ouvre la voie à la mise en place d'un marché électronique du bois légalement récolté.

\section{Vérification et suivi}

La vérification du respect des lois dans la forêt est effectuée conjointement par les organismes environnementaux des états et par l'IBAMA. Tous les plans de gestion doivent être suivis au moins une fois tous les trois ans à l'aide de directives détaillées (avec des indicateurs et des listes de points à vérifier), en vue de s'assurer que les lois sont respectées par l'exploitant (IBAMA 2004). L'exécution des plans de gestion est censée être suivie par le professionnel qui en a la responsabilité technique, mais c'est rarement le cas. Sabogal et al. (2005) recommandent que les responsabilités de ces professionnels forestiers soient mieux précisées et que l'IBAMA et le CREA poursuivent en justice les contrevenants avec plus d'efficacité. L'indépendance des forestiers professionnels est aussi un sujet de préoccupation car leurs services sont payés directement par ceux qui exécutent le plan de gestion, c'est-à-dire les exploitants.

Les autorités environnementales fédérales et celles des états réalisent des inspections impromptues sur les routes pour vérifier queles expéditions de bois sont bien accompagnées de leur permis de transport. À l'aide du code-barres, ils peuvent comparer les informations figurant sur le permis avec celles de la base de données en ligne DOF. Les recoupements électroniques inhérents au système DOF déclenchent aussi des alertes qui permettent aux autorités (le MoE, l'IBAMA, la police fédérale et le procureur fédéral) d'optimiser et de cibler leurs activités de vérification et de lutte contre les infractions. D'autre part, celles-ci sont aidées dans la détection du déboisement illégal par le programme DETER, système de surveillance par satellite extrêmement efficace, mis au point par l'INPE, qui est capable de repérer une modification de l'utilisation des sols en Amazonie brésilienne tous les 15 jours (voir www.obt.inpe.br/deter).

\section{Remontée de l'information}

Conformément à la politique du gouvernement relative à l'accès du public à l'information, toutes les données concernant le déboisement et la délivrance de permis d'exploitation forestière doivent être accessibles sur internet. Les états avaient jusqu'à septembre 2007 pour adopter le système DOF ou pour mettre en place leur propre système équivalent, en donnant à l'IBAMA un accès gratuit aux informations pour qu'elles puissent être intégrées au système national d'information forestière (SISCOM), qui recoupe les données avec celles du programme DETER accessible au public et avec celles du cadastre technique fédéral (Chavez, J.H. 2006 comm. pers., septembre). À l'avenir, tous les plans de gestion de la forêt approuvés devront aussi être enregistrés dans le cadastre national des plans 
de gestion durable de la forêt, qui sera accessible au public sur internet et sera géré par l'IBAMA (MMA 2006b).

\subsection{Pouvoir et indépendance}

La volonté et les orientations politiques comme la coordination interinstitutionnelle aux plus hauts niveaux du gouvernement font que le système de gouvernance forestière qui se dégage du processus de réforme au Brésil est très solide comparativement aux processus semblables observés dans les autre pays d'Amérique latine. Les instances environnementales n'agissent pas isolément mais au sein d'un ensemble d'organismes publics qui se coordonnent pour œuvrer d'une manière complémentaire en vue de faire appliquer la législation sur la forêt et d'améliorer la gouvernance du secteur forestier.

Bien que toujours en cours d'élaboration, le système brésilien décentralisé de gouvernance forestière crée deux niveaux indépendants d'institutions, celles situées à l'échelon fédéral et celles qui sont à l'échelon des états. L'indépendance horizontale entre les niveaux est renforcée par une indépendance verticale, puisque la plupart des acteurs du système tels que les instances environnementales, les Ministérios Públicos, la police, l'INPE ou le CREA sont complètement indépendants les uns des autres. Le Tableau 9.2 donne un bref aperçu des relations entre les divers acteurs et systèmes.

\subsection{Bilan et incidences du système}

Entre la création du GPTIRID en 2004 et mars 2007, des opérations de lutte contre les infractions ont été menées: 18 de grande envergure et environ 400 de moindre importance. Les résultats communiqués par le MoE sont impressionnants (MMA 2007) : 460 peines d'emprisonnement ont été prononcées et les amendes infligées aux auteurs d'infraction ont dépassé 1,3 milliard USD. ${ }^{6}$ Les rapports font état de la saisie d'environ $814000 \mathrm{~m}^{3}$ de bois rond, de 471 tracteurs, de 171 camions et de 643 tronçonneuses. En raison de la lenteur du processus judiciaire, le nombre véritable des personnes emprisonnées et des amendes payées est bien plus bas. Néanmoins, ces résultats ont eu un impact énorme sur les références en matière de respect de la loi et de tolérance de la corruption dans le secteur forestier.

L'une des actions les plus spectaculaires qui illustre les effets positifs de la coordination de la lutte contre les infractions est l' "Operação Ananias " à Altamira dans l'état de Pará en février 2007, qui a impliqué 150 agents, 39 voitures, un hélicoptère et un avion. Cette opération a débouché sur le démantèlement d'un réseau criminel constitué de fonctionnaires et d'exploitants forestiers et sur 37 peines d'emprisonnement. Le ministre de l'Environnement a déclaré que les délais de détection des crimes avaient été réduits de moitié grâce au nouveau système DOF.

6 Ont été emprisonnés 333 personnes du secteur privé, 113 fonctionnaires fédéraux, 17 fonctionnaires d'État et 3 policiers. 
Il a poursuivi en affirmant que le déboisement avait diminué de $52 \%$ au cours des années 2005 à 2006 (MMA 2007). Les données de l'INPE montrent également que, de 2004 à 2005, le déboisement a baissé de $31 \%$ en passant de 27429 à 18793 km² (INPE 2007).

Il est difficile de faire la part entre l'influence des politiques forestières et du contrôle de l'application de la loi sur ces chiffres et celle d'autres facteurs macroéconomiques et externes au secteur, tels que la faiblesse des cours des denrées agricoles sur le marché mondial. Néanmoins, l'expérience brésilienne montre que le contrôle du respect de la législation relative aux forêts doit s'ancrer dans un système de gouvernance forestière de grande ampleur dans lequel les facteurs politiques, normatifs, institutionnels et sociétaux interagissent d'une façon cohérente et durable en vue d'atteindre les résultats souhaités.

\subsection{Enseignements}

Le cas du Brésil illustre comment la collaboration et la coordination interministérielles - impliquant des branches du gouvernement qui ne participent pas habituellement au débat politique sur le développement forestier, telles que les ministères de la justice, de la défense, et de la science et de la technologie - ont renforcé les mesures prises au plan national pour s'attaquer au problème de l'illégalité. Canalisée par un programme forestier national largement soutenu associé à une influence politique au plus niveau et un encadrement fort, cette démarche semble être la clé du succès de la réforme.

La réforme juridique a aussi été importante, l'évolution la plus significative étant la loi de 2006 sur la gestion des forêts publiques, qui va dans le sens d'une clarification du régime foncier. Elle a également posé le principe de la vérification et instauré l'exigence d'inspections indépendantes, tous les trois ans, des concessions accordées dans les forêts publiques.

L'indépendance est assurée par l'architecture du système. Les instances environnementales n'agissent pas isolément mais au sein d'un ensemble d'organismes publics qui se coordonnent pour œuvrer d'une manière complémentaire en vue de faire appliquer la législation sur la forêt et d'améliorer la gouvernance du secteur forestier. La décentralisation des fonctions de réglementation vers les états pose le problème de la définition claire de la division des responsabilités entre les différents niveaux de gouvernement, chacun agissant de même au sein de sa hiérarchie et du territoire de son ressort. Selon le principe de subsidiarité, les instances nationales peuvent néanmoins prendre en charge l'application de la loi et la surveillance dans les forêts dans certains états d'Amazonie à la couverture forestière importante, dans lesquels les organismes environnementaux manquent sérieusement de ressources et ont besoin de renforcer leurs capacités. 


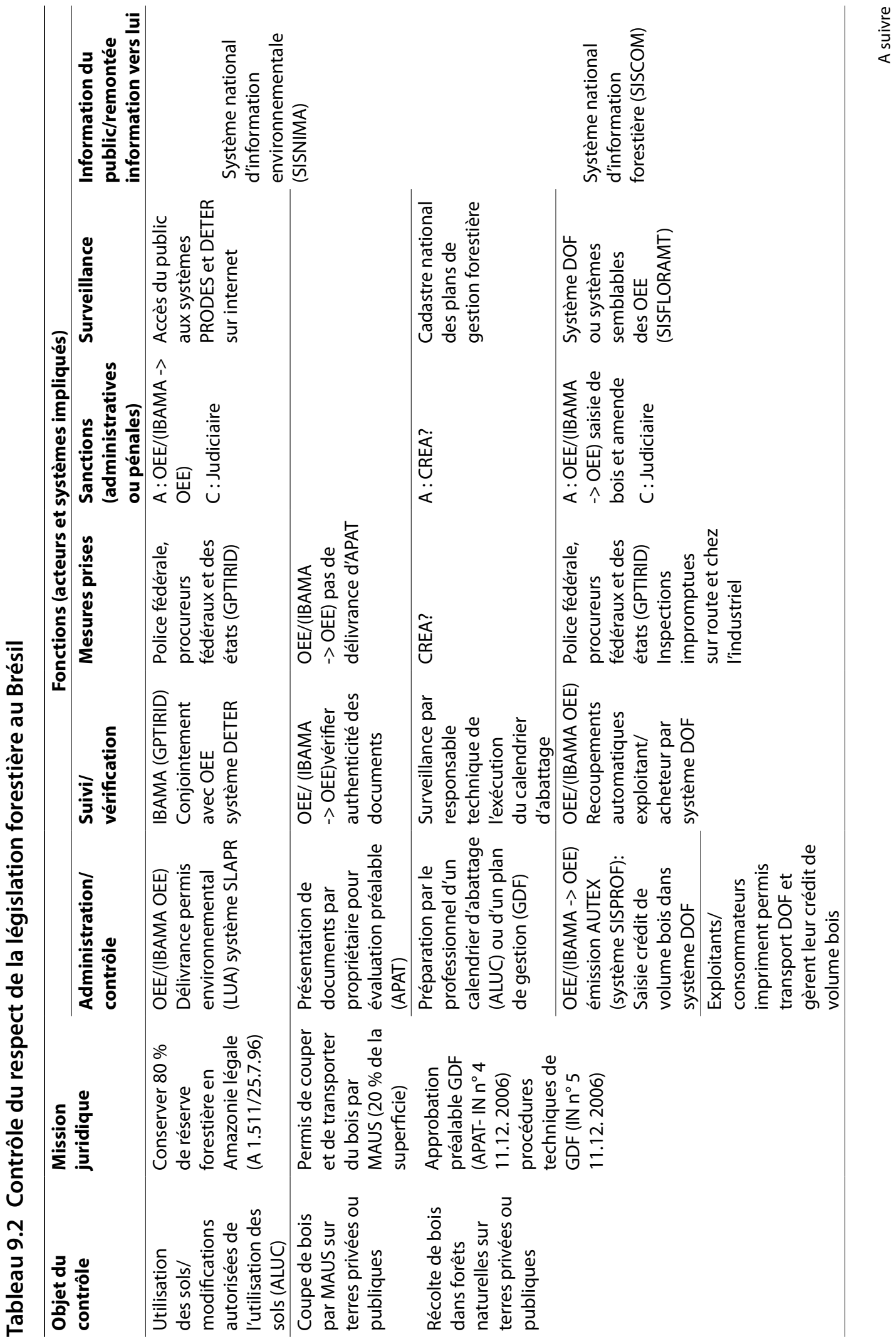




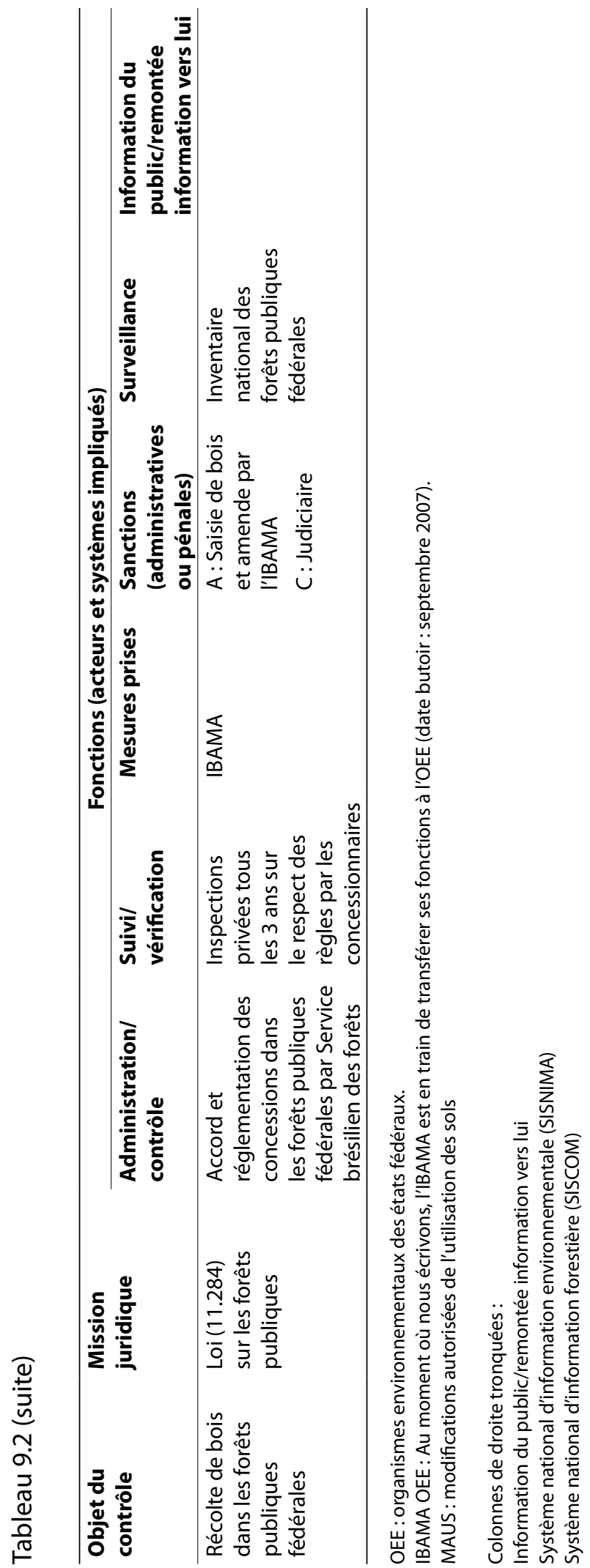


Enfin, cette étude illustre comment le recours aux technologies innovantes (par exemple les images par satellite pour suivre la modification d'utilisation des sols) peut offrir de nouvelles possibilités en matière de contrôle de l'application de la réglementation (Bird and Thiel 2007). L'investissement dans un certain nombre de technologies a été considérable, notamment dans les bases de données en ligne, afin de répondre au défi que pose le contrôle d'un secteur si dispersé. Ce système d'information global permet aux autorités nationales et fédérales d'inspecter et de vérifier l'action d'un réseau décentralisé de contrôle et d'administration de la forêt. L'accès du public à ces informations donne la possibilité à la société civile de surveiller la situation et de réduire ainsi le nombre de décisions arbitraires et la corruption. 


\section{Chapitre 10}

\section{Le système national externalisé de contrôle des forêts en Equateur}

Note : Cette étude de cas a été réalisée en décembre 2007 par Guillermo Navarro, Filippo Del Gatto et Martin Schroeder. Elle s'appuie en partie sur Navarro, G., Del Gatto, F. et Schroeder, M. (2006) "Le système national externalisé de contrôle des forêts en Equateur ", Étude de cas de pays VERIFOR n³. Londres : ODI.

Tableau 10.1 Equateur : quelques statistiques clés

\begin{tabular}{llll}
\hline Statistiques & \multicolumn{1}{c}{ Date } & Source des données \\
\hline Population & 13,2 millions & 2005 & $\begin{array}{l}\text { Nations Unies, Division } \\
\text { de la population 2006 }\end{array}$ \\
\hline Superficie & $\begin{array}{l}27,7 \text { millions } \\
\text { de ha }\end{array}$ & & FAO 2006 \\
\hline Couverture forestière & $39,2 \%$ & 2005 & Nations Unies, Division \\
de la statistique 2006
\end{tabular}

\subsection{Introduction}

En 2003, le lancement par l'Equateur du système national externalisé de contrôle des forêts (Sistema Nacional Tercerizado de Control Forestal ou SNTCF) a attiré l'attention du monde entier. Cette innovation déléguait la responsabilité du suivi et de l'administration 
des opérations forestières à trois organismes différents dont le rôle était le suivant : contrôle du transport des produits forestiers et des animaux sauvages, suivi des activités ayant lieu dans la forêt et exercice d'une fonction administrative et de vérification. Cette étude de cas analyse l'élaboration de ce système de contrôle des forêts, intégrant des activités de vérification, qui a été en vigueur pendant quelques mois seulement avant que son succès ne soit la cible d'une violente opposition dans le pays, ce qui a finalement entraîné sa suspension partielle et l'a nettement affaibli.

\subsection{Les politiques à l'origine du SNTCF}

Avec la disparition annuelle de 137000 hectares de forêts, l'Equateur possède le plus fort taux de déforestation (1,2\%) de l'Amérique du Sud (FAO 2000). Celle-ci est entretenue en partie par une exploitation illégale massive, dont les estimations varient de $50 \%$ à $75 \%$ (Álvaro 2003 ; Echeverría 2004 ; The Economist 2003). Le SNTCF a été conçu dans le cadre de la stratégie forestière mise en place en 1999 par l'Equateur dans l'intention de combattre cette exploitation illégale à grande échelle. Bien que la stratégie forestière ait résulté d'un processus largement participatif, le SNTCF lui-même a été mis au point par un groupe relativement restreint de personnes et d'institutions.

- Ceci s'est fait sous la conduite de quelques hauts fonctionnaires du ministère de l'Environnement (MoE) très qualifiés, dévoués et charismatiques.

- Groupe de défense de l'environnement le plus actif et techniquement compétent en Equateur, le CEDENMA (Comité équatorien pour la protection de la nature et de l'environnement) se faisait l'écho de la société civile urbaine.

- L'industrie du bois a conclu des alliances avec les petits négociants en bois, principalement par l'intermédiaire de l'AIMA et de COMAFORS. ${ }^{1}$ Cependant, en dépit de sa position officielle en faveur du contrôle de l'exploitation illégale, elle ne l'a jamais véritablement soutenu, devenant même son principal opposant lorsque l'administration et le suivi ont été délégués à la succursale équatorienne de la société suisse SGS (Société Générale de Surveillance) (The Economist, 2003 ; Álvaro, 2003 ; Thiel, 2004).

- Quelques bailleurs de fonds bilatéraux et multilatéraux ont apporté une assistance technique et financière à la mise en œuvre de la politique forestière, surtout pour aider la société civile dans son rôle d'observateur. Il s'agissait de l'organisme de coopération internationale allemande GTZ, de l'Agence américaine pour le développement international (USAID), de la Banque de développement interaméricaine (IDB) et de l'Organisation des Nations Unies pour l'alimentation et l'agriculture (FAO).

- Les forestiers professionnels et leurs associations étaient aussi impliqués, mais avec une voix limitée au chapitre.

\footnotetext{
1 AIMA (Association équatorienne des industriels du bois) est une grande association des industries de transformation du bois en Equateur, qui comprend les plus gros producteurs de contreplaqué. COMAFORS (Association pour la gestion durable de la forêt) est une organisation sans but lucratif, liée à l'AIMA, qui se consacre à la promotion de la gestion durable de la forêt. C'est aussi l'un des membres fondateurs de Vigilancia Verde, l'organisme public/privé chargé de contrôler le transport des produits forestiers et des animaux sauvages. Par ailleurs, Price Waterhouse Coopers avait fait appel à l'administrateur de COMAFORS à l'époque pour travailler sur les documents de l'appel d'offres pour l'externalisation des services d'administration et de vérification.
} 
En dehors du MoE, les autres institutions gouvernementales ont brillé par leur absence. ${ }^{2}$ Ceci a posé un problème car une bonne partie de l'opposition au système provenait du sein du MoE, et de ses centaines d'employés soucieux de perdre les avantages officieux maintenus par le statu quo. Si d'autres ministères, dont les employés n'avaient pas d'enjeu dans le système en place, s'étaient investis, une volonté politique forte aurait pu être mobilisée, capable de contrebalancer la résistance interne au MoE.

Deux parties prenantes directement concernées manquaient également à l'appel : les populations autochtones et les propriétaires de forêts privées, qui ensemble contrôlent la majorité des forêts du pays et représentent des centaines de milliers de personnes dépendant de la forêt pour vivre. ${ }^{3}$ Bien que les organismes de la société civile aient joué un rôle politique important dans l'établissement du SNTCF, les groupes de la société civile représentant ou non la communauté autochtone comme les propriétaires de forêts des zones rurales, étaient pratiquement absents. Les participants étaient pour l'essentiel des personnes habitant Quito, ayant fait des études universitaires et soucieuses de l'environnement, appartenant à la classe moyenne urbaine du pays.

\subsection{Le cadre juridique du SNTCF}

La délégation de missions publiques à des entités privées est prévue dans la Constitution politique de l'Equateur et dans la loi de modernisation de l'Etat de 1993 (Echeverría 2004), mais c'est la loi sur les forêts et la conservation de 1981 et la réglementation afférente qui portent création du SNTCF. Cependant, la complexité du système nécessitait l'élaboration d'un cadre législatif spécifique pour sa mise en œuvre :

- Le décret ministériel $n^{\circ} 86$ du MoE (décembre 2000) reconnaissait le rôle de contrôle de Vigilancia Verde, un organisme à la fois public et privé regroupant le MoE, la police, l'armée et cinq ONG environnementales locales. ${ }^{4}$

- Le décret ministériel n ${ }^{\circ} 131$ du MoE (janvier 2001) a institué de nouvelles normes importantes dans le domaine de la gestion forestière et a défini le rôle, les fonctions et les responsabilités des régisseurs forestiers, qui sont des professionnels reconnus par le MoE (voir Figure 10.1).

- En autorisant le MoE à déléguer des responsabilités administratives et de supervision à une entreprise privée, le décret exécutif $\mathrm{n}^{\circ} 2609$ (mai 2002) a permis à ce ministère de promulguer le décret ministériel n ${ }^{\circ} 50$ (juillet 2000), qui déterminait la base juridique et les procédures administratives de mise en œuvre du processus de délégation. En mai 2000, un contrat a été attribué à la succursale équatorienne de la société suisse SGS suite à un appel d'offres.

- En juin 2004, plusieurs autres décrets sont venus compléter les normes et procédures administratives relatives à la gestion forestière et aux récoltes dans les forêts naturelles,

\footnotetext{
2 Bien que la participation du Conseil national de modernisation de l'État ait été limitée.

3 Les propriétaires forestiers privés ont participé cependant comme opposants au système, souvent mobilisés et soutenus par les magnats du bois locaux.

4 Les ONG regroupées dans Vigilancia Verde sont Fundación Ecuatoriana Populorum Progressio (FEPP), Fundación Natura, CARE Ecuador, Fundación Maquipucuna et COMAFORS.
} 
dans les plantations et les domaines agroforestiers d'une part, et aux responsabilités et aux obligations des régisseurs forestiers d'autre part.

Malgré ces tentatives de clarification du statut juridique du système, la constitutionnalité et la validité du contrat entre le MoE et SGS ont été remises en question par les professionnels du secteur forestier. En mai 2003, le procureur général de l'Etat a confirmé la constitutionnalité du contrat. Cependant, en octobre 2003, le tribunal constitutionnel équatorien s'est prononcé contre l'externalisation de services à SGS, jugeant que la loi administrative plaçant l'administration et la supervision forestières aux mains du privé était contraire à la constitution. Le tribunal a aussi déclaré que la mesure administrative autorisant SGS à recevoir des honoraires pour ses services était aussi contraire à la constitution.

Le jugement ne règlait pas directement la question de la validité du contrat SGS, incitant le CEDENMA et d'autres groupes à croire que le contrat était toujours valable. Cependant, le $\mathrm{MoE}$ a récupéré unilatéralement tous les droits d'administration et de supervision précédemment délégués à SGS, semant la confusion à propos du contrat avec cette société, et au sein de celle-ci, troisième composante clé du SNTCF. Selon de nombreux observateurs, l'opposition interne au $\mathrm{MoE}$ constitue actuellement le principal obstacle à la signature du nouvel addendum négocié par tous les principaux acteurs mi2004, qui pourrait permettre le rétablissement du système.

\begin{tabular}{|l|}
\hline \multicolumn{1}{|c}{$\begin{array}{c}\text { Ministère de I } \\
\text { Délègue }\end{array}$} \\
\hline $\begin{array}{l}\text { Vigilancia Verde } \\
\text { (MoE, police, armée et cinq } \\
\text { ONG environnementales } \\
\text { locales) }\end{array}$ \\
$\begin{array}{l}\text { Fonctions : } \\
\text { - Contrôle le transport } \\
\text { de produits } \\
\text { - Permet un contrôle par } \\
\text { la société civile et la } \\
\text { responsabilisation de } \\
\text { celle-ci } \\
\text { - Gère un fonds fiduciaire }\end{array}$ \\
\hline
\end{tabular}

Source : d'après Thiel (2005)

Régisseurs forestiers
(Forestiers professionnels
reconnus par le MoE)
Fonctions:
- Vérifier l'élaboration des
plans de gestion forestière
avant leur approbation
- Contrôler la mise en
œuvre des plans de
gestion et des opérations
d'exploitation
- Établissement des permis
de transport dans la forêt

Régisseurs forestiers Forestiers professionnels

Fonctions :

垈ifier l'élaboration des avant leur approbation Contrôler la mise en gestion et des opérations d'exploitation de transport dans la forêt
Services externalisés de vérification et d'administration (confié à SGS après appel d'offres)

Fonctions :

- Élaboration d'un système d'informations et de statistiques forestières

- Vérification des rapports des régisseurs

- Approuver et surveiller les plans de gestion

- Délivrer les permis d'exploitation aux exploitants

- Délivrer les permis de transport aux régisseurs

- Collecter pour le compte de l'état la taxe sur la valeur du bois sur pied 


\subsection{Contexte du SNTCF}

\section{Organisation du système}

Le SNTCF représentait une approche intégrée à l'application de la loi en matière de forêt, visant à améliorer la transparence, à réduire la bureaucratie et à s'attaquer aux pratiques illégales et à la corruption. La structure de base (indiquée à la Figure 10.1) repose sur l'externalisation des responsabilités de suivi et d'administration des opérations forestières à trois entités différentes : la première étant un organisme mi-public/mi-privé (Vigilancia Verde) contrôlant le transport des produits forestiers et des animaux sauvages, le deuxième étant des acteurs privés (les régisseurs forestiers) investis de la mission publique de suivi dans la forêt, et le troisième étant une entreprise privée (SGS) à qui ont été confiés les services d'administration et de vérification des forêts. L'externalisation de services d'administration et de vérification vers le SNTCF était censée libérer les ressources du MoE pour qu'il puisse s'attaquer au problème central de la déforestation et de l'exploitation clandestine.

Dans le cadre des travaux de la SGS étaient prévues la création et la mise en œuvre d'une base de données et de statistiques forestières fournissant des services multiples pour le compte du MoE, tels que l'élaboration et l'application de plans de gestion forestière, la délivrance de permis d'exploitation et de transport, et la collecte, pour le compte de l'Etat de la taxe sur la valeur du bois sur pied. Afin de financer ses travaux, la SGS était autorisée à demander un droit de 2,50 USD directement aux exploitants forestiers par mètre cube de bois récolté dans les forêts naturelles (en sus de la taxe sur la valeur du bois sur pied de 3 USD pour le $\mathrm{MoE}$ ) et un droit de 0,10 USD par mètre cube coupé dans les plantations forestières (exempt de la taxe sur la valeur du bois sur pied).

\section{Administration et contrôle}

Le but principal du SNTCF était d'assurer deux fonctions complémentaires : (i) des services d'administration performants pour réduire les coûts liés à la bureaucratie et pour inciter à rester dans la légalité et (ii) des services de contrôle efficaces permettant de mieux détecter et mieux punir les activités illégales.

Le pivot de ce nouveau système administratif était la base de données et de statistiques qui devait relier toutes les unités administratives au niveau local et régional ainsi que les points de contrôle routier de Vigilancia Verde. Cette nouvelle organisation accélérait grandement les procédures administratives. En vertu du contrat passé avec le MoE, la SGS n'avait que huit heures pour approuver (ou rejeter) un plan de gestion et délivrer, en cas d'accord, pour le compte du Ministère, les permis d'exploitation et de transport correspondants à l'exploitant et au régisseur forestiers. Pour assurer ces prestations et faciliter l'accès à la légalité aux petits exploitants, le contrat de la SGS stipulait la mise en place d'un réseau d'unités administratives mobiles, locales et régionales. Cependant, en dépit de ce réseau, l'éloignement de la plupart des forêts faisait que la SGS ne pouvait effectuer ses inspections de terrain qu'après l'approbation des plans de 
gestion et le début de la récolte. De toute évidence, ceci ne favorisait pas la prévention d'irrégularités éventuelles.

Les services de contrôle étaient aussi partagés entre trois différentes entités du système externalisé (voir Figure 10.2). La SGS supervisait les exploitants et les régisseurs forestiers. Ces derniers, pour leur part, étaient en charge de la supervision de tous les sites d'exploitation faisant l'objet d'un permis : inspection et établissement de rapports sur la conformité et l'exactitude des plans de gestion forestière, sur la récolte et sur les opérations à la suite de celle-ci. Le fait que les régisseurs forestiers inspectaient et certifiaient les plans de gestion qu'ils avaient eux-mêmes élaborés constituait une faille dans le système. Ils étaient aussi responsables de la vérification des volumes chargés, des espèces et de toute autre information pertinente relative à chaque camion sortant de la forêt. Les infractions ou irrégularités étaient notées dans un rapport d'infraction sur la base duquel le $\mathrm{MoE}$ pouvait prendre les mesures nécessaires pour lutter contre la fraude. Cependant, bien que le code pénal équatorien considère que l'exploitation illégale soit un crime passible de quatre ans d'emprisonnement, le MoE subit une pression politique pour appliquer seulement des sanctions administratives fondées sur la législation forestière (par exemple des amendes et la saisie de produits illégaux), qui ne correspondent pas à la gravité et à l'ampleur des actes commis.

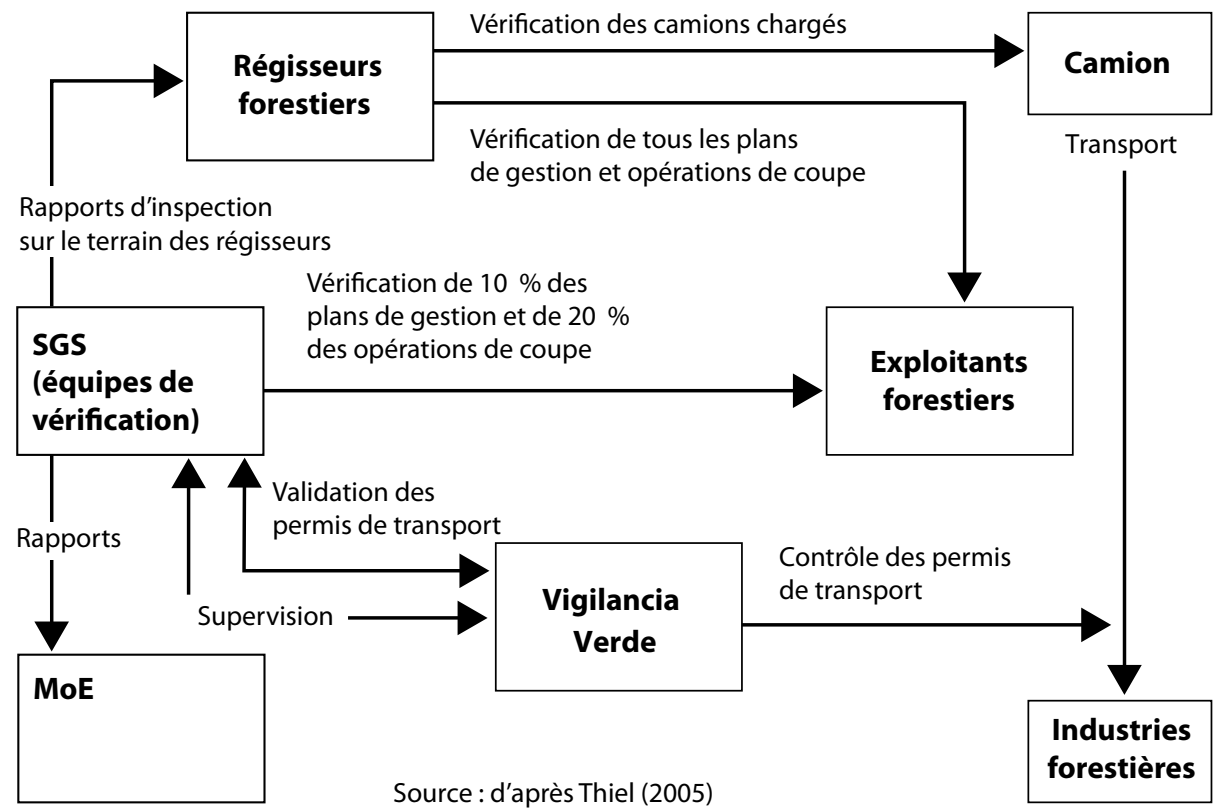

Figure 10.2 Organisation des services de contrôle des forêts 
Vigilancia Verde contrôlait le transport de tous les produits forestiers et animaux sauvages. Au plus fort de ses activités, elle gérait un réseau de douze points de contrôle routier et de six patrouilles mobiles, chacun comprenant des représentants des instances forestières, de la société civile, de la police et de l'armée. Ces équipes travaillaient 24 heures sur 24 et leurs membres étaient réaffectés à un point de contrôle différent tous les 15 jours. Quant au MoE, il vérifiait que la confiscation de tout produit par Vigilancia Verde s'effectuait dans les règles. Le MoE devait superviser et inspecter la SGS de façon continue et sanctionner tout manquement au contrat.

\section{Recoupements et contrepoids}

À part les vérifications officielles réalisées par les différentes entités, le système intégrait des recoupements et contrepoids qui permettaient à tous les participants de suivre les activités des autres et de rapporter toute irrégularité éventuelle (voir Figure 10.3).

Parallèlement au contrôle du transport routier, Vigilancia Verde surveillait aussi de près les autres entités du système :

- Elle vérifiait les permis de transport que les régisseurs forestiers recevaient et remplissaient pour le compte des instances forestières.

- Elle avait un intérêt direct dans la supervision des ventes aux enchères du bois confisqué, effectuées par les bureaux régionaux du MoE, puisqu'elle recevait $50 \%$ de la valeur des ventes.

- Grâce à ses groupes de défense de la société civile, Vigilancia Verde remplissait le rôle d'inspection du MoE par la société civile aux niveaux local et national.

- De plus, les membres de Vigilancia Verde assuraient la surveillance de la part de la société civile des missions de la SGS aux points de contrôle routier et dans les patrouilles mobiles.

La SGS a joué un rôle en validant directement dans le système les informations sur le transport des produits, et en contrôlant et rapportant toute irrégularité dans le travail des membres de Vigilancia Verde.

Dans le cadre des recoupements et contrepoids, on observe un Comité de régisseurs forestiers, chargé de traiter le signalement de toute irrégularité ou infraction commises par un régisseur forestier, et de recommander d'éventuelles sanctions au MoE et à l'assocition des ingénieurs forestiers à laquelle adhère le régisseur en question.

Jusqu'à la suspension des travaux de la SGS, les régisseurs forestiers et les exploitants étaient très bien placés pour examiner la qualité des services administratifs assurés par la SGS. En outre, le MoE surveillait les services confiés à la SGS et avait pouvoir de sanctionner tout manquement au contrat. L'accès libre à la base de données et de statistiques grâce à un site internet était censé améliorer la transparence globale de tout le système. 


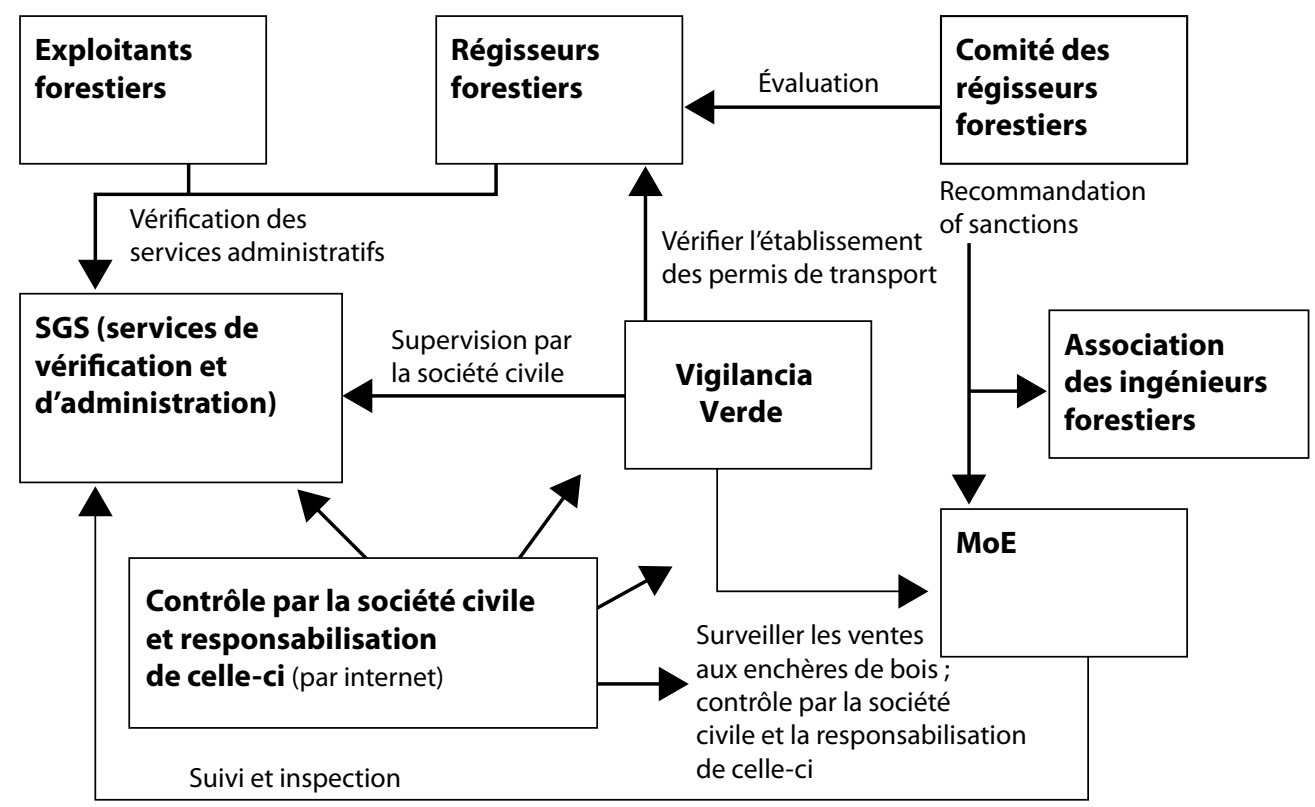

Figure 10.3 Système de recoupements et de contrepoids du SNTCF

\section{Dimension de lutte contre la pauvreté}

La conception et l'élaboration du SNTCF ont été dominées, au moins en partie, par le souci de lutter contre la pauvreté. Un des objectifs fondamentaux était d'apporter plus de transparence au processus de mise sur le marché comme préalable à une négociation optimisée et plus équitable entre les concessionnaires et les négociants en bois. La création d'unités administratives mobiles, prévues dans le contrat de la SGS, répondait à cet objectif. Leur fonction était d'amener les services administratifs directement auprès des communautés, afin que les petits exploitants puissent réduire leur dépendance vis-àvis des intermédiaires du commerce du bois, respecter la législation en diminuant leurs coûts, et enfin en retirer des bénéfices plus importants.

La législation découlant du SNTCF prévoyait des règles de fonctionnement simplifiées pour la gestion forestière à petite échelle dans les forêts naturelles, pour l'exploitation du bois en dehors des zones forestières, et pour la conversion dans la limite de $30 \%$ de propriétés privées en terre non boisée. Des permis spéciaux ont été mis en place pour faciliter l'exploitation de certaines espèce de bois des systèmes agroforestiers. De même, l'exploitation du bois dans les plantations et dans les autres zones forestières plantées nécessitait peu de bureaucratie. ${ }^{5}$ De plus, le décret exécutif n ${ }^{\circ} 346$ permettait aux concessionnaires pauvres sans titre foncier officiel d'obtenir des permis d'exploitation

5 Même si ces coûts de fonctionnement restent plus élevés que ceux des activités agricoles. 
s'ils prouvaient qu'ils occupaient la terre par un certificat indiquant que le processus d'obtention du titre foncier était en cours ou par la déclaration de trois témoins attestant qu’ils étaient propriétaires de cette terre.

Le SNTCF n'est pas allé jusqu'à mettre en place différentes normes de vérification et taxes pour les petits propriétaires forestiers. Ces derniers sont en fait désavantagés en devant payer plus par mètre cube pour les services des régisseurs forestiers parce que ces derniers comptent des droits plus élevés pour les petits volumes en raison de leurs frais fixes (tels que le transport).

\subsection{Effets}

Il est difficile d'évaluer les répercussions du SNTCF parce que les trois entités n'ont travaillé ensemble que pendant quelques mois au cours du second semestre 2003. ${ }^{6}$ En octobre 2003, le MoE suspendait les services de la SGS, déclenchant l'effondrement de tout le système. Cependant, la séparation des responsabilités en matière de délivrance et de contrôle des permis et de punition des infractions encouragée par le SNCTF, ainsi que l'implication de multiples vérifications et acteurs se sont traduites par une bien meilleure détection des irrégularités et délits forestiers. En 2002, Vigilancia Verde a saisi $5000 \mathrm{~m}^{3}$ de bois illégal, soit cinq fois plus que l'Etat agissant seul en 1999. Au cours des quelques mois de 2003 quand Vigilancia Verde et la SGS travaillaient ensemble, le volume de bois confisqué a nettement augmenté, indiquant que si la SGS avait poursuivi ses activités, les saisies auraient pu presque doubler par rapport à celles de l'année précédente. Au cours de ces mêmes mois, la SGS a recommandé au MoE la suspension de 42 permis d'exploitation, pour abattage en dehors des zones attribuées, abattage de volumes supérieurs et d'espèces non autorisées, pour fraude sur les permis de transport, etc. Certains observateurs affirment que ce succès est à l'origine des violences dirigées contre la SGS (menaces envers le personnel, attaques de bureaux et vols d'ordinateurs), venant de personnes résidant localement mais à l'instigation de groupes ayant des intérêts en jeu.

\subsection{Enseignements tirés de la mise en place et de l'arrêt du SNTCF}

La mise en place et l'arrêt du SNTCF nous donnent des leçons importantes concernant l'élaboration et la conception d'un système de contrôle et de vérification de la forêt. La conception et l'acceptation sociale du SNTCF ont été favorisées par une période de stabilité politique en termes de lignes de conduite et de mission nationales. C'était aussi le résultat d'une stratégie forestière perçue comme répondant par des actions concrètes aux problèmes de la déforestation, des activités illégales dans le domaine de l'exploitation et du commerce du bois. La mise en œuvre du SNTCF était soutenue par l'existence

6 Les activités de la SGS ont démarré mi-juin 2003 dans la province d'Esmeraldas et se sont étendues au niveau national à partir de début août. 
de deux alliés influents, le mouvement environnemental urbain et la communauté des bailleurs de fonds, et la participation d'acteurs forts et actifs au plan politique par le truchement de Vigilancia Verde.

En même temps, un certain nombre de facteurs ont entraîné un sentiment négatif vis-àvis de la légitimité du SNTCF et sa disgrâce. Il s'agissait principalement de l'incapacité des parties prenantes environnementales à contrebalancer une faible gouvernance, des intérêts industriels influents en faveur du statu quo et le désengagement d'acteurs ruraux clés en raison du fait que le SNTCF n'était pas suffisamment axé sur la lutte contre la pauvreté. Le SNTCF aurait pu être soutenu contre ses détracteurs si l'on avait reconnu le besoin d'obtenir l'adhésion de ceux qui avaient bénéficié de l'ancien système et mis en place un programme de transition clair pour les acteurs tels que les fonctionnaires du MoE, dont la fonction était bouleversée par le SNTCF. Sa popularité aurait pu être plus grande s'il avait introduit un mécanisme de compensation et d'incitation destiné aux exploitants forestiers, afin de contrebalancer le poids de l'augmentation du contrôle des forêts. Cette fragilité était aggravée par le sentiment que le droit de vérification perçu par la SGS dans le cadre du contrat d'externalisation, en sus de la taxe sur la valeur du bois sur pied, augmentait le coût de l'accès à la légalité, et qu'il fallait payer deux fois pour la même chose. Enfin, l'incertitude législative provoquée par l'absence d'évaluation rigoureuse des incompatibilités constitutionnelles et institutionnelles, en particulier concernant la délégation de pouvoirs de l'Etat, a procuré aux opposants du SNTCF l'occasion de remettre en cause sa légitimité.

En termes de performance au titre du SNTCF, la SGS était efficace et transparente en fournissant dans les délais des informations en ligne au $\mathrm{MoE}$, au public et aux groupes d'intérêt. Cependant, la SGS s'est trouvée confrontée à un conflit d'intérêts en raison de ses fonctions d'administration et de contrôle, situation héritée du précédent (et actuel) système de contrôle des forêts appliqué par le MoE.

L'expérience du SNTCF met aussi en évidence l'importance du financement de chaque partie du système de façon à se prémunir contre toute interférence extérieure. Le financement de Vigilancia Verde provenait en partie de bailleurs de fonds et d'entreprises, les uns et les autres pouvant compromettre l'indépendance et la durabilité du système, et à $50 \%$ de la vente aux enchères de bois, ce qui pouvait inciter à l'illégalité de façon perverse (car la détection de bois illégal devenait plus profitable que la prévention). Le fait que les services de la SGS aient été payés directement par les usagers était l'un des aspects les plus controversés du système ayant suscité le plus d'opposition. Cependant, si elle avaient été payée par le MoE, la SGS aurait été bien plus vulnérable aux pressions des hautes sphères politiques, qui auraient pu se traduire par des retards de paiement. Enfin, le paiement des régisseurs forestiers par les propriétaires de forêts ou les négociants en bois sensibilisait ces derniers aux intérêts économiques de leurs clients.

Pendant la courte durée de son application, le SNTCF a réussi à contrôler divers types d'illégalités dans le secteur forestier, tels que le transport illégal des produits forestiers et les faux permis d'exploitation. Il a corrigé de mauvaises habitudes institutionnelles telles 
que la corruption, l'influence et le " clientélisme politique ». Cependant, le fait que le SNTCF se concentre seulement sur ceux qui cherchent à travailler dans la légalité est une préoccupation persistante, surtout du secteur forestier privé équatorien. La récolte clandestine, la déforestation, les industries du bois et les marchés du bois restaient aux mains du MoE, en dépit de sa capacité discutable en matière de contrôle. 



\section{Chapitre 11}

\section{Le système de vérification forestière au Cameroun}

Note : Cette étude de cas a été réalisée en mars 2008 par Paolo Cerutti et Timothée Fomété.

Tableau 11.1 Cameroun : quelques statistiques clés

\begin{tabular}{|c|c|c|c|}
\hline Statistiques & & Date & Source des données \\
\hline Population & 15,5 millions & 2002 & MINEFI 2002 \\
\hline Superficie & 47,3 millions de ha & & $\begin{array}{l}\text { République du } \\
\text { Cameroun } 2005\end{array}$ \\
\hline Couverture forestière & $45 \%$ & 2005 & MINFOF et FAO 2005 \\
\hline Propriété de la forêt publique & $86 \%$ & 2005 & MINFOF et FAO 2005 \\
\hline $\begin{array}{l}\text { Production de bois rond } \\
\text { industriel }\end{array}$ & 2,3 millions de $\mathrm{m}^{3}$ & 2006 & MINFOF 2007a \\
\hline $\begin{array}{l}\text { Emplois officiels dans le } \\
\text { secteur forestier }\end{array}$ & $12-13000$ & 2006 & MINEFI 2006 \\
\hline $\begin{array}{l}\text { Contribution du secteur } \\
\text { forestier au PIB }\end{array}$ & $6 \%$ & 2004 & CBFP 2006 \\
\hline $\begin{array}{l}\text { Valeur des exportations de } \\
\text { produits forestiers }\end{array}$ & $\begin{array}{l}244 \text { milliards de } \\
\text { CFA }\end{array}$ & 2006 & INS 2006 \\
\hline $\begin{array}{l}\text { Principaux marchés } \\
\text { internationaux du bois }\end{array}$ & $\begin{array}{l}\text { Chine, Italie, } \\
\text { Espagne }\end{array}$ & 2006 & MINFOF 2007b \\
\hline $\begin{array}{l}\text { Indice } \mathrm{TI} \text { de perception } \\
\text { de la corruption }(0-10,0= \\
\text { corruption la plus forte) }\end{array}$ & 2,3 & 2006 & $\begin{array}{l}\text { Transparency } \\
\text { International } 2006\end{array}$ \\
\hline $\begin{array}{l}\text { Indice du développement } \\
\text { humain }(0-1,0=\text { très bas })\end{array}$ & 0,51 & 2004 & PNUD 2006 \\
\hline
\end{tabular}

1 CFA $1=0,002$ USD (Mars 2008).

\subsection{Introduction}

Le Cameroun est au centre d'une préoccupation mondiale à propos de l'exploitation illégale. Le système de vérification forestière du pays livre des enseignements précieux et des pistes pour l'amélioration de la gouvernance forestière aux niveaux régional et national. Le recours à des observateurs indépendants pour l'attribution des titres d'exploitation forestière et pour le contrôle des forêts a été instructif en matière de 
appropriation et d'indépendance des processus de vérification, ainsi qu'à propos des effets éventuels sur la pratique de gestion forestière.

En novembre 2007, le Cameroun a entamé des négociations avec l'Union Européenne en vue d'un accord de partenariat volontaire (APV).

\subsection{Le secteur forestier national}

$\mathrm{Au}$ Cameroun, les forêts denses productives sur terrain drainé couvrent environ 17,5 millions d'hectares (Eba'a Atyi 1998). Un plan de zonage élaboré en 1993 a divisé le territoire national en deux parties, la forêt permanente et la forêt non permanente. La production annuelle de bois provient d'une centaine d'unités forestières d'aménagement situées au sein de la forêt permanente.

Le secteur forestier constitue la deuxième exportation de l'économie camerounaise après le pétrole, représentant $16 \%$ des exportations de 2003 (s'élevant à environ 380 millions USD) et environ $6 \%$ du PIB (CBFP 2006). L'exploitation et la transformation du bois sont très concentrées, plus de $80 \%$ de la production nationale de bois étant générée par moins de 20 grandes entreprises surtout européennes. La capacité de transformation du secteur forestier officiel est estimée à environ 1,9 million $\mathrm{de}^{3}$ tandis que celle du secteur forestier officieux a récemment été évaluée à environ 0,3 million de $\mathrm{m}^{3}$ (MINEFI 2006), bien qu'une étude exhaustive de ce dernier fasse défaut.

La crise économique qui a affecté le Cameroun à la fin des années 1980 a eu un impact important sur l'industrie du bois. Elle a conduit à une augmentation rapide du nombre d'entreprises d'exploitation forestière agréées, notamment des entreprises nationales (Eba'a Atyi 1998). L'adoption en 1994 d'une nouvelle loi sur la forêt a provoqué un sérieux déclin de la disponibilité de bois légal. S'ajoutant à la capacité limitée du ministère des Forêts et de la Faune (MINFOF) en matière de contrôle des activités forestières, ceci a contribué à laisser le champ libre à l'illégalité dans le secteur forestier. Pour illustrer la situation, les chiffres pour 1998/1999 indiquaient un total d'environ 2,9 millions de $\mathrm{m}^{3}$ pour les exportations officielles de bois et seulement 1,9 million $\mathrm{de}^{3}$ pour la production officielle (MINFOF 2004).

Les activités forestières illégales repérées ont incité la communauté de bailleurs de fonds à demander instamment plus d'efficacité dans le domaine de l'application des lois et de la vérification forestières au Cameroun. La pression sur les pouvoirs publics pour qu'ils respectent les conditions des bailleurs de fonds et les objectifs de politique sectorielle est arrivée à un moment où l'image internationale du pays était aussi sérieusement menacée. En 1998 et en 1999, par exemple, le Cameroun a été "perçu comme le pays le plus corrompu » par Transparency International dans ses études internationales. ${ }^{1}$

1 Le Cameroun était $85^{\mathrm{e}}$ sur 85 pays en 1998; et $99^{\mathrm{e}}$ sur 99 en 1999. En 2005, il avait progressé à la $137^{\mathrm{e}}$ place sur 158, et en 2006 il était $138^{\mathrm{e}}$ sur 163. 
L'accès au marché européen est essentiel au secteur national du bois carl'Europe est toujours la destination dominante des exportations camerounaises de bois (voir Figure 11.1). Ceci explique pourquoi le plan d'action de l'Union Européenne relatif à l'application des réglementations forestières, à la gouvernance et aux échanges commerciaux (EU FLEGT) a pris rapidement de l'ampleur non seulement dans la sphère politique mais aussi au sein du secteur industriel privé et de la société civile. Dans le cadre de ce plan, le Cameroun a entamé des négociations avec l'Union Européenne en vue d'un accord de partenariat volontaire (APV) en novembre 2007.

\subsection{Suivi indépendant et contrôle des forêts : mission et cadre juridique}

En vertu de la loi de 1994, les unités forestières d'aménagement devaient être attribuées par vente aux enchères et exploitées conformément aux plans de gestion approuvés. Cependant, en 1996 et 1997, le ministère attribuait toujours de nombreuses unités forestières d'aménagement de façon administrative, enfreignant ainsi la loi (Global Forest Watch 2000). Suite à ces irrégularités, la Banque Mondiale a appelé au changement en le posant comme condition de la troisième phase du programme d'adaptation structurelle (SAP III) (Cerutti and Tacconi 2006), et entre autres, à la nomination d'un observateur indépendant du processus d'attribution des concessions.

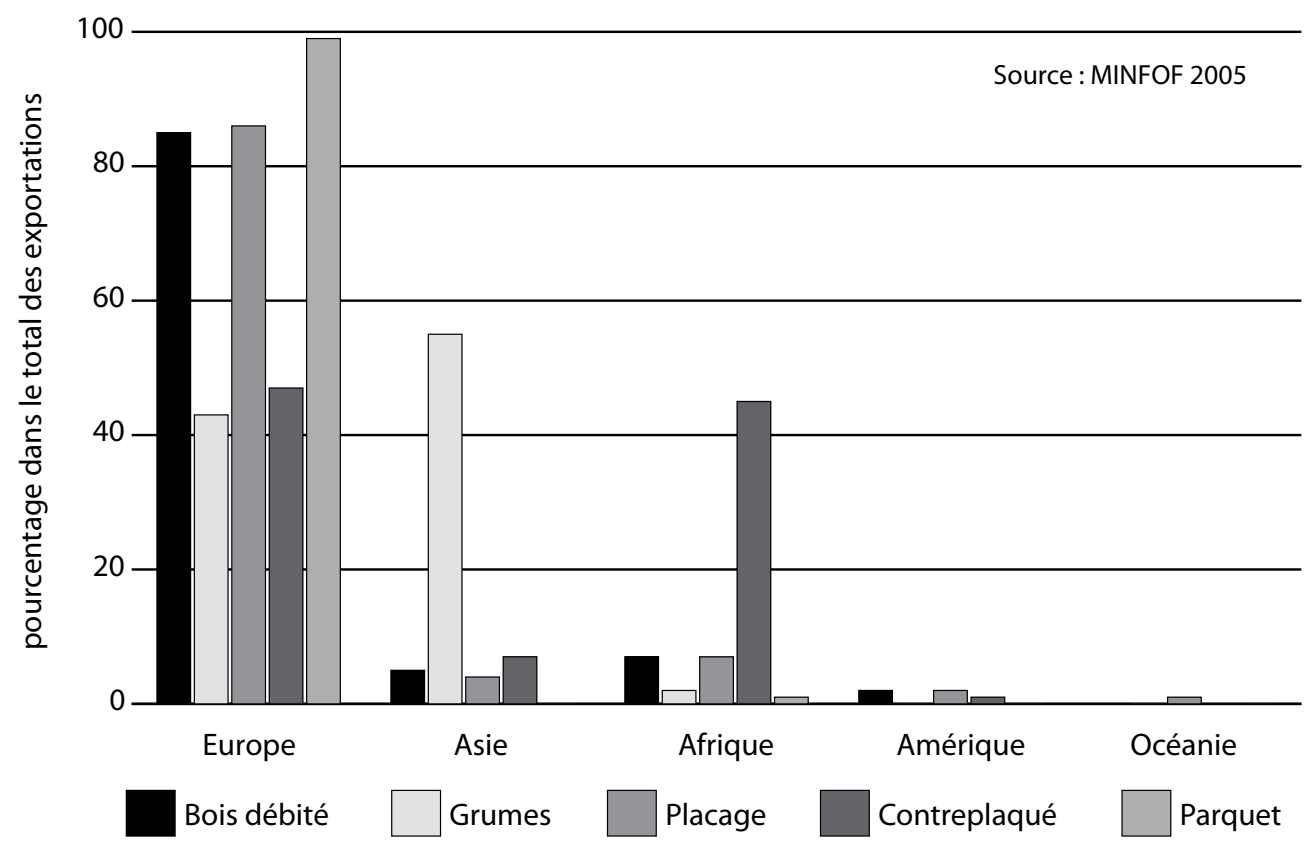

Figure 11.1 Importance des destinations des exportations camerounaises de bois (2005) 
En 2000, conformément à l'article 124 du décret d'application de 1995, le MINFOF publia un document instaurant l'application du système de contrôle au sein de l'administration forestière. Le système, qui s'articulait autour d'une unité centrale de contrôle (UCC) et des brigades provinciales de contrôle (BPC), était conçu pour veiller : (i) à ce que les réglementations relatives aux activités d'exploitation soient appliquées ; (ii) à ce que des données et statistiques concernant la taxation soient recueillies et analysées ; et (iii) à permettre la détection de l'exploitation illégale pour entamer des poursuites.

En fait, seulement cinq forestiers ont été affectés a l'UCC, mal équipés pour les missions de recherche sur terrain, sous-payés et sans lien avec l'administration centrale. Ceci a encouragé le personnel à voir le contrôle des forêts d'abord comme une occasion de gagner de l'argent et non pas comme un moyen de gérer efficacement le domaine forestier.

Suite aux grandes préoccupations, en particulier dans la communauté des bailleurs de fonds, face à cet échec du système de contrôle traditionnel à assurer une bonne gouvernance, un certain nombre d'observateurs indépendants ont été appelés pour remplir des fonctions de contrôle et de vérification. Il en existait trois, exposées ci-dessous :

\section{Processus d'attribution des titres d'exploitation forestière}

Suivant les exigences de la Banque Mondiale, le gouvernement camerounais a mis en place au sein du comité interministériel un observateur indépendant qui surveillait l'attribution des concessions. Une entreprise de juristes et de comptables du secteur privé (le cabinet Behle de Douala) a été retenue pour remplir ce rôle dépendant du ministère des Forêts, qui est "l'autorité suprême " en matière de délivrance de titres. Le cabinet Behle s'est retiré à la fin de la première phase pour être remplacé par le cabinet BlochKolle en 2003.

\section{Respect des règles de la part des exploitants forestiers}

S'inspirant de rapports de bailleurs de fonds faisant état que le Cambodge avait été confronté à des problèmes semblables d'exploitation illégale et que Global Witness, ONG britannique œuvrant pour les droits environnementaux, avait contribué à les résoudre, le gouvernement camerounais invita cette organisation à effectuer deux missions d'évaluation de la situation en 2000, financées par le Department for international development (DFID) britannique, la Banque Mondiale et l'Agence canadienne de développement international (ACDI). Après que les missions d'évaluation aient eu lieu, la présence d'un observateur indépendant (OI) devint une condition de SAP III, et Global Witness a obtenu un contrat en 2002 pour prolonger sa mission de surveillance indépendante des activités forestières. Le fait que le contrat soit payé directement par divers bailleurs de fonds, que sa signature n'ait pas fait suite à un appel d'offres, et que la présence de l'OI soit liée à une condition, n'a pas suscité l'adhésion, ni la participation nationale au processus de décision. 
En 2005, Global Witness a été remplacé par une autre ONG britannique, Resource Extraction Monitoring (REM) avec un nouveau contrat financé par la Commission Européenne. Depuis, REM a mis en œuvre un projet relatif à l' "observation indépendante des systèmes de mise en application de la loi et de la gouvernance relatives à la forêt " (IM-FLEG). Le principal objectif de la mission actuelle de REM est de " contribuer à l'application des principes de bonne gouvernance dans le secteur forestier afin que les réglementations forestières soient mieux appliquées " (Resource Extraction Monitoring 2006). Ce contrat devait expirer en mars 2008, mais au moment où nous écrivons, il est prorogé pour une période initiale de trois mois. Ensuite, le financement de l'IM FLEG sera assuré dans le cadre du Programme Sectoriel Forêt Environnement (PFSE) qui a été adopté en 2004 comme instrument d'harmonisation des financements des bailleurs de fonds avec les priorités du gouvernement camerounais pour une meilleure gestion du secteur forestier et environnemental.

\section{Suivi des concessions forestières}

En 2000, Global Forest Watch (GFW), initiative du World Resources Institute (WRI) ayant démarré en 1997, a présenté un "Aperçu de la situation de l'exploitation forestière au Cameroun» (Global Forest Watch 2000). Cette étude rassemblait des analyses fondées sur des images satellite, des techniques SIG et des données relatives au secteur forestier du Cameroun. Suite à cet aperçu publié en 2000, l'Observatoire mondial des forêts/WRI a signé un contrat avec le MINFOF pour jouer le rôle de troisième niveau de vérification, afin de suivre les activités forestières à l'aide de techniques de télédétection.

Le contrôle des exportations et le développement de la chaîne de garde des produits forestiers ont été gérés au cours de cette période dans le cadre du contrat de la Societé Générale de Surveillance (SGS), entreprise suisse du secteur privé, à qui l'on avait fait appel en 1994 pour assurer le contrôle des exportations de bois non transformés (c'est-àdire des grumes) et la collecte des taxes d'exportation correspondantes.

Le rôle de ces observateurs/contrôleurs dans l'organisation institutionnelle a été officiellement reconnu en 2005 lors de l'adoption d'une stratégie nationale de contrôle des forêts et de la faune (SNCFF). La mise en ouvre de la SNCFF s'est accompagnée d'une hausse importante du nombre de contrôleurs forestiers et de matériels mis à leur disposition. L'UCC a été remplacée en 2005 par une brigade nationale de contrôle, initialement composée de six membres mais qui sont passés à douze en 2006.

En raison du processus de réforme d'adaptation structurelle et du besoin d'augmenter la contribution du secteur forestier au revenu national, le ministère des Finances (MINEFI) s'est aussi plus impliqué dans ce domaine. Le décret 08/009/PM du 23 janvier 1998 transféra au ministère des Finances toutes les compétences fiscales précédemment exercées par le ministère des forêts. De plus, en mars 1999, le Programme de sécurisation des recettes forestières (PSRF) a été créé au sein du MINEFI afin de veiller au suivi rigoureux des recettes fiscales provenant du secteur forestier et d'augmenter la contribution de celuici dans le budget de l'Etat. Le PSRF s'inscrivait dans un cadre de collaboration entre le 
MINEFI et le MINFOF, ces deux ministères étant censés échanger des informations, pour une démarche plus efficace et rationnelle dans le domaine du recueil de données et de détection des infractions (Cerutti and Assembe 2005). À ce jour cependant, aucun de ces ministères n'a considéré que la collaboration par le PSRF n'était prioritaire, et le recoupement des données et des informations est toujours très limité.

\subsection{Organisation du contrôle et de la vérification des forêts}

La Figure 11.2 donne un aperçu du cadre dans lequel évoluent les différents acteurs impliqués dans le contrôle et la vérification des forêts au Cameroun et des fonctions qui leur incombent. Le personnel affecté au contrôle vérifie la légalité de toutes les

\section{Le circuit \\ du bois \\ Contrôles \\ Vérification}

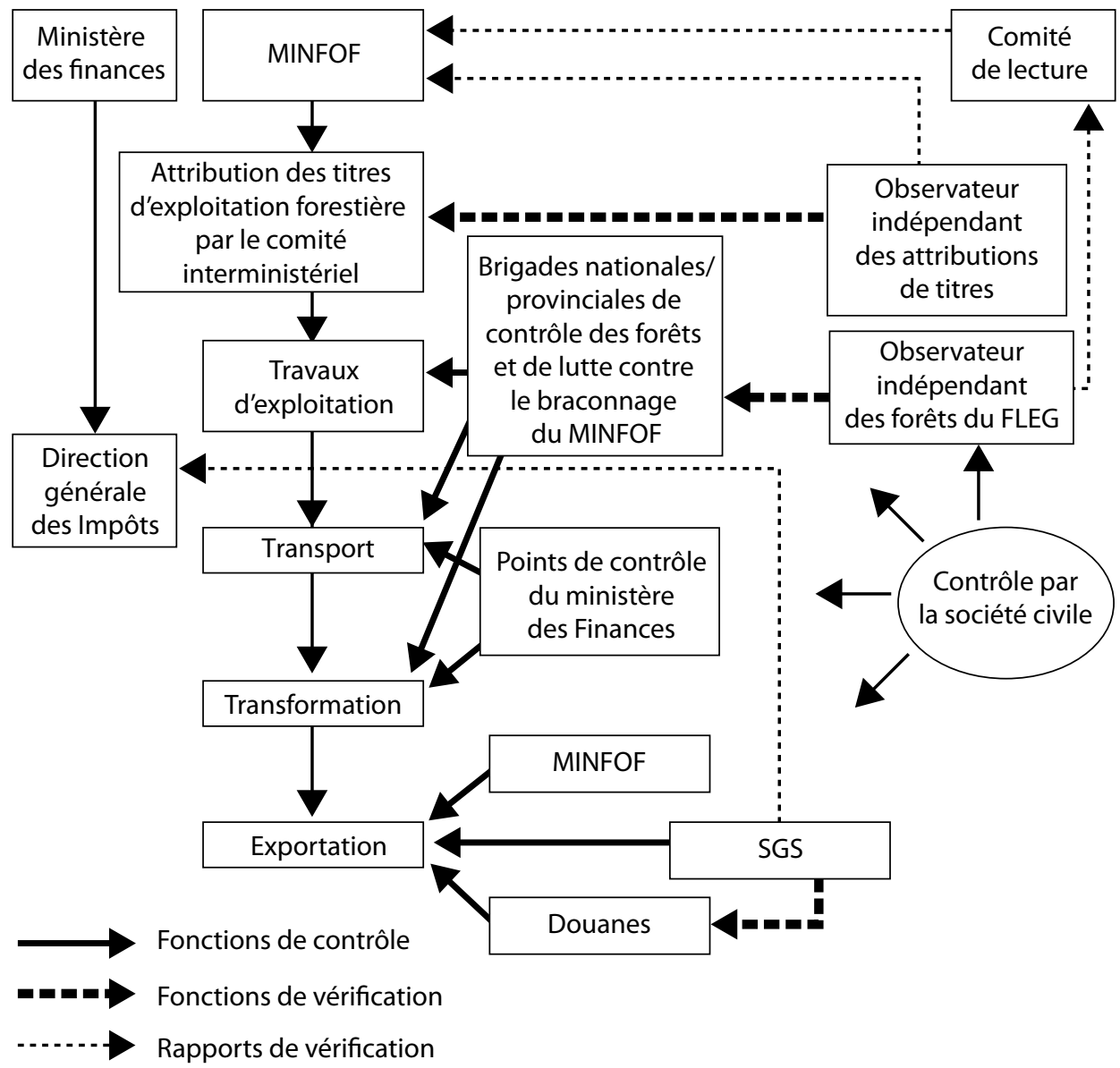

Figure 11.2 Acteurs et fonctions de vérification au Cameroun 
activités forestières (récolte, transport, transformation et exportation) exercées par les entreprises privées, les particuliers ou les groupes de villageois, et leur conformité avec les réglementations dans le domaine. Le suivi sur le terrain est à la fois régulier et ponctuel, à la demande d'acteurs concernés : particuliers, organisations de la société civile ou secteur privé. En cas de découverte d'activités illégales, les agents du contrôle sont censés : (i) exposer la liste des délits correspondants dans le rapport de mission ; (ii) établir un procès-verbal de constat d'infraction, document de base en vue d'intenter un procès; et (iii) saisir les grumes, le bois ou tout produit et matériel forestiers à titre de preuve.

Le fonctionnement du système de contrôle et son efficacité sont censés être évalués par une inspection annuelle interne réalisée par l'Inspecteur général du MINFOF. Cependant, aucune inspection n'a encore été effectuée au moment où nous écrivons ce chapitre.

La vérification des activités de terrain a lieu lorsque l'observateur indépendant (OI) procède à des missions communes avec les brigades nationales de contrôle. L'OI a innové récemment en appliquant une approche thématique aux missions de terrain, certaines d'entre elles ayant été consacrées à une catégorie particulière de permis d'exploitation forestière.

La mise en place d'une institution pour traiter les informations recueillies par l'OI a constitué une innovation intéressante dans le cas du Cameroun. Comme les rapports de missions de contrôle sur le terrain exposaient des faits et des conclusions n'ayant pas reçu l'approbation des parties concernées, un Comité de lecture a été créé pour valider leurs observations. Ce comité est constitué d'agents chargés de l'application de la loi sur la forêt, de l'OI et des représentants des bailleurs de fonds. Le Comité examine les rapports de missions de contrôle avant qu'ils soient soumis à l'approbation du ministre des forêts et de la faune, pour être mis ensuite à la disposition du public grâce au site internet de l'observateur et à la presse nationale. Les sanctions s'appuient sur les procès-verbaux de constat d'infraction et sur les rapports des missions de terrain communes à l'OI et au MINFOF, ainsi qu'aux recommandations faites au ministre. Les procédures d'appel et d'arbitrage sont traitées par le Comité de lecture. Le Comité est censé contribuer à accroître la transparence, bien que les sanctions et les paiements effectifs correspondant aux infractions découvertes soient toujours très difficiles à vérifier, d'autant plus en raison de la collaboration limitée, évoquée plus haut, entre le MINFOF et le MINEFI. De surcroît, la totalité du processus de prise de décision est subordonné aux intérêts du ministre des forêts et de la faune. Les réunions sont organisées par lui, quand bon lui semble, le nombre de réunions ayant tendance récemment à être en chute libre (Resource Extraction Monitoring 2007). Les décisions relatives au montant des amendes et aux sanctions sont aussi prises par le ministre, à huis clos. Il en résulte que le processus est vulnérable à la politisation.

Pour soutenir le suivi des activités relatives à l'application de la loi sur la forêt, les bailleurs de fonds ont financé la création de trois bases de données informatisées. D’abord, le Système Informatique de Gestion d'Informations Forestières (SIGIF), installé au MINFOF depuis 1998, a été développé par des consultants financés par l'ACDI en vue 
de gérer la production de bois et les domaines connexes, tels que la superficie des titres d'exploitation, les impôts dus sur la superficie, les permis actifs au cours d'un exercice fiscal ainsi que diverses informations techniques. Dans le cadre des négociations sur l'accord de partenariat volontaire (APV) et de l'émission d'un "document de légalité ", une version plus récente du SIGIF est en cours d'élaboration et devrait suivre la totalité de la chaîne de la valeur du bois sur pied au port d'embarquement, en garantissant la légalité des activités forestières. Une seconde base de données, version spéciale du SIGIF (TRINITE II Forêts), a été mise sur pied pour aider le PSRF à gérer les montants de taxes forestières à payer par chaque entreprise. Troisièmement, le Système Informatique de Gestion des Infractions et du Contentieux Forestier (SIGICOF), conçu par Global Witness en 2005, devrait comporter des données relatives aux missions entreprises pour faire appliquer la législation forestière, permettant la gestion quotidienne des procès portant sur des infractions forestières. A ce jour, cependant, le SIGICOF n'est pas utilisé par l'administration, conduisant à une duplication et à la confusion des fonctions parmi les ministères concernés (forêts, finances, justice), qui continuent à travailler chacun de leur côté (Resource Extraction Monitoring, 2007). L'absence d'harmonie entre ces trois systèmes limite leur efficacité et empêche le système de tourner à plein régime. ${ }^{2}$

\subsection{Le système de vérification : évaluation provisoire}

De nombreuses personnes reconnaissent que l'incidence et l'étendue des activités forestières illégales dans les unités de gestion forestière camerounaises a progressivement diminué depuis 2001. La situation structurelle a probablement joué un certain rôle, notamment la nouvelle attribution des unités forestières d'aménagement qui a permis aux entreprises d'exploitation forestière d'avoir un accès légal au bois. Ce processus a repris en 2000 après presque trois ans de suspensions dues aux irrégularités de 19961997. Cependant, la performance du système de contrôle et de vérification a aussi été un facteur de réduction de l'exploitation illégale, en incitant à un meilleur respect des lois dans les unités forestières d'aménagement et en favorisant la disponibilité et la transparence des informations. On a aussi observé une reconnaissance de plus en plus grande du rôle positif que les organisations de la société civile peuvent jouer en matière d'amélioration de la gouvernance forestière, comme en atteste leur implication dans le processus de préparation de l'APV. Cependant, une évaluation plus approfondie du cadre du contrôle et de la vérification révèle que la responsabilité du fonctionnement et de l'entretien des systèmes de contrôle et de vérification semble toujours reposer de façon excessive sur des bailleurs de fonds externes, et que l'adhésion des ministères concernés continue à être très limitée.

\footnotetext{
2 En fait, l'échec de l'harmonisation des systèmes d'information est aussi dû à un quatrième système, au départ PAGODE, maintenant remplacé par SYDONIA. Le système PAGODE (Procédures Automatisées de Gestion des Opérations Douanières et de Commerce Extérieur) permettait à toute marchandise arrivant à Douala d'être dédouanée avant que l'exportation ait lieu. Depuis janvier 2007, un nouveau système douanier informatisé, SYDONIA (Système Douanier Informatisé) a été lancé à Douala, remplaçant PAGODE. SYDONIA s'inscrit dans un réseau d'échange d'informations avec d'autres bases de données, notamment celles qui concernent le secteur du bois, mais l'interconnexion n'est pas encore fonctionnelle.
} 
En effet, même après presque six ans de surveillance indépendante, les missions d'application de la législation forestière souffrent de graves problèmes, nombre d'entre eux provenant de ce manque d'adhésion. Cette situation renforce l'argument que l'adhésion totale à un système d'application de la législation forestière par les ministères concernés se fait difficilement si ces ministères doivent consentir à l'arrivée conditionnelle d'un observateur indépendant. En fait, la liste des problèmes exposés dans le dernier rapport annuel de l'observateur indépendant décrit une situation sur le terrain qui n'est pas très différente de celle observée à la fin des années 1990 (Resource Extraction Monitoring 2007). Ces problèmes sont les suivants :

- Les missions de contrôle sur le terrain, planifiées centralement, sont devenues la règle, et les brigades provinciales (sans doute le principal maillon dans la chaîne du contrôle des forêts) ont été plutôt marginalisées, jouant un rôle secondaire. Les financements qui leur sont attribués sont insuffisants pour réaliser les missions de contrôle. Certaines brigades provinciales dépendent des entreprises d'exploitation forestière pour couvrir certains coûts liés à leurs missions, ce qui met leur objectivité en question.

- Les missions de terrain ont été rares à cause du manque de personnel et de matériel, y compris pour le transport. Il en résulte une moindre efficacité des contrôles car les agents du MINFOF n'ont souvent pas accès aux données fondamentales, telles que les états de production, les rapports précédents relatifs à la lutte contre les infractions ou les cartes originales indiquant les limites des permis d'exploitation. Ils ne disposent pas non plus de budget pour louer des véhicules afin de procéder à l'enlèvement du bois qu'ils saisissent.

- Il n'existe aucun suivi effectif du travail réalisé par les agents de lutte contre les infractions, ni de système de sanctions contre ceux qui ne respectent pas les procédures requises. Les agents de lutte contre les infractions forestières mettent rarement en doute les mesure prises, ou les documents signés ou émis par l'administration centrale.

La coordination des actions visant à réprimer les activités illégales est faible car de nombreux acteurs clés, tels que la police, l'armée et les magistrats sont encore très peu informés de l'existence d'une stratégie de contrôle. Par conséquent, les divers systèmes de contrôle (forêts, finances et douanes) ne couvrent pas encore toutes les activités forestières.

Sur le papier, le processus FLEGT a été un bon catalyseur pour développer une norme nationale de légalité pour le bois, et un document " projet zéro " a déjà été préparé pour entamer les négociations de l'APV. Bien que la consultation de la société civile ait été beaucoup négligée au départ dans le processus de mise en place de cette norme, les organisations de cette société civile ont récemment été davantage associées et ont été en fait très actives lors de la préparation du document stratégique.

L'expérience du Comité de lecture, qui analyse les rapports de contrôle del'OI et des brigades nationales de contrôle, est unique en Afrique centrale ; elle a joué un rôle particulièrement important en permettant l'adhésion du ministère au système de vérification. Bien que ce soit une avancée intéressante et largement positive, la performance de ce comité a 
été limitée par le fait que le ministre reste l'autorité suprême et a pratiquement tout pouvoir pour punir ou non un cas d'infraction et pour fixer le montant des amendes relatives aux sanctions imposées. L'efficacité du système a été limitée par les longs retards dans l'organisation des réunions du Comité de lecture et l'absence de mécanismes pour confirmer les sanctions prises à l'égard des contrevenants. On notera que, bien que l'on ne puisse dire que le Comité de lecture fonctionne efficacement, ses problèmes se trouvent sans doute en dehors du système de vérification, et plus dans la structure globale de la gouvernance forestière qui est pour le moment peu réformée.

Comme la base de données SIGICOF n'est pas encore mise en service par les responsables de la lutte contre les infractions forestières, la procédure judiciaire forestière continue à être une mosaïque de mesures multiples prises par des personnes travaillant dans des bureaux différents, sans souci de coordination des informations (Resource Extraction Monitoring 2007). L'inefficacité des méthodes d'application de la loi qui en résulte ainsi que les faibles amendes infligées aux contrevenants signifient que l'illégalité continue à être profitable, même lorsque des infractions sont repérées et punies (Resource Extraction Monitoring 2007).

\subsection{Enseignements}

Si l'intérêt international pour la vérification est venu principalement des ONG et d'organisations spécialisées, surtout internationales, certains acteurs appartenant au secteur privé (par exemple observateurs du processus de vente aux enchères, et la SGS) ont aussi joué un rôle important dans la situation camerounaise. En ce qui concerne les observateurs du processus d'attribution des concessions, les conflits d'intérêt ont été évités en choisissant ces observateurs par une mise en concurrence de plusieurs consultants tout à fait reconnus dans le domaine de l'audit juridique, comptable et financier.

Le recours aux ONG internationales pour la surveillance indépendante des forêts a été fondé sur l'affirmation que ces organisations, contrairement aux secteurs public et privé, n'auraient aucun intérêt direct dans ce domaine et auraient des idéaux à défendre en termes de bonne gouvernance. Elles ont aussi une réputation à maintenir et sont généralement considérées plus crédibles par la communauté internationale. Cependant, la nécessité de choisir parmi le très petit nombre d'ONG pouvant assurer ce service révèle les limites du marché. Dans le cas du Cameroun, les contrats de Global Witness ont été octroyés de façon administrative (sans concurrence) sous la pression des bailleurs de fonds, et REM a été la seule organisation à soumissionner pour la poursuite du contrat en 2005. De plus, il y a eu plusieurs déclarations, surtout de la part des entreprises d'exploitation forestière et du ministère des forêts et de la faune, sur le fait que le choix d'une ONG qui fait campagne a conduit à une utilisation abusive des informations et au non-respect de ses conditions contractuelles. Ces accusations restent difficiles à apprécier puisqu'il n'existe pas de système d'agrément professionnel des observateurs indépendants des forêts, ni de mécanisme institutionnel par lequel une évaluation pourrait avoir lieu. 
Tout en œuvrant dans le même but (l'amélioration de la gouvernance du secteur forestier), les trois observateurs travaillant au Cameroun ont rencontré des difficultés différentes et leur action n'a pas eu les mêmes effets. On pourrait avancer que dans les cas où des critères objectifs étaient établis (comme la liste de points à vérifier avant l'octroi d'une concession), le travail de l'observateur était facilité et son impact plus grand que dans les cas où les sanctions étaient examinées à huis clos et laissées à l'appréciation du ministre. Ces exemples ne montrent pas une amélioration de la transparence.

Une leçon que l'on peut tirer de l'expérience camerounaise est que les systèmes de contrôle et de vérification doivent s'appuyer sur des procédures objectives assorties de dispositions juridiques claires en matière de prise de décisions en des points clés du système. En l'absence de critères objectifs (par exemple, pour le niveau des sanctions à infliger aux exploitants en infraction), on observe une énorme frustration chez les fonctionnaires du MINFOF, ceci pouvant compromettre l'efficacité de l'ensemble du système de vérification. La subjectivité concernant ces questions d'importance peut se traduire par un traitement peu équitable des entreprises d'exploitation forestière et, en fait, par une corruption.

Un autre enseignement important est que, si le ministère n'adhère pas au processus de réforme ou si les réformes sont réalisées en posant des conditions, même l'application de critères objectifs ne débouche pas nécessairement sur de véritables améliorations sur le plan de la gouvernance. Par exemple, si les rapports des deux observateurs sur l'attribution des concessions lors de la période 2000-2005 font état de nombreuses pratiques douteuses et suspectes, il ne semble pas que leurs préoccupations soient prises au sérieux ou que les habitudes en matière d'appels d'offres soient en voie de changer. On aurait pu s'y attendre si la gouvernance s'était véritablement améliorée et si le ministère avait vraiment soutenu les réformes qu'il était censé mettre en œuvre. En fait, l'audit économique de 2006 du secteur forestier remarquait que la concurrence loyale avait baissé au fil du temps dans le processus d'enchères et que l'observateur indépendant rapportait que l'équité envers les soumissionnaires n'était pas toujours respectée (MINEFI 2006).

Bien que non directement impliqué dans des actions très visibles, telles que les missions de terrain menées par l'observateur des activités forestières, GFW, avait, et a toujours, un rôle important à long terme en apportant des informations crédibles sur l'état global du secteur et en développant les capacités du ministère à utiliser les nouvelles technologies de cartographie et des méthodes de planification récentes. Tandis que les travaux de cet observateur n'a pas attiré autant l'attention internationale que ceux de l'observateur des concessions, son rôle discret a toutefois été important et complémentaire de celuici. Cependant, dans ce cas aussi, l'adhésion du ministère reste une question essentielle pour l'adoption, le développement et la mise en œuvre des capacités acquises lorsque l'observateur quittera le pays. 
Ceci étant dit, l'action des observateurs de la société civile a permis au public de prendre mieux conscience de certains problèmes tels que l'exploitation illégale, et à fourni aux bailleurs de fonds les informations nécessaires pour presser le gouvernement de prendre des mesures correctives. L'engagement de la société civile en faveur de la vérification de la légalité des activités forestières peut être vu comme une étape essentielle vers une plus grande adhésion du pays au système de vérification, ainsi que vers un renforcement de la crédibilité de l'ensemble des mécanismes de gouvernance forestière.

\subsection{Conclusion}

Le Cameroun a acquis une expérience considérable de vérification dans le domaine de la gestion du domaine forestier permanent, en employant une palette d'intervenants venant des $\mathrm{ONG}$ et du secteur privé et comprenant des spécialistes des secteurs forestier, juridique et financier. Tandis que le cas du Cameroun montre l'importance d'un oil extérieur dans l'amélioration de la diffusion et de la transparence des informations, il prévient contre toute interprétation trop simpliste des conditions institutionnelles dans le domaine de la vérification forestière.

L'expérience du Cameroun se distingue - en particulier par rapport à celle du Cambodge - par la souplesse de la démarche qui permet la mise en place de nouvelles institutions et de nouveaux mécanismes pour intégrer les divers éléments du système de vérification, et pour valider les informations qu'ils fournissent. Même s'ils ne sont pas encore complètement opérationnels, les points suivants sont intéressants :

- Lélaboration de systèmes d'information numérique et de structures institutionnelles enregistrant la production de bois et permettant l'exécution de tâches fiscales importantes, telles que la collecte de taxes;

- Une séparation des pouvoirs (administration, suivi et inspection, et l'imposition de sanctions) comme première étape pour assurer l'indépendance, avec des observateurs indépendants comme surveillants n'ayant pas capacité à sanctionner ; et le Comité de lecture veillant à ce que des consultations aient lieu et agissant comme arbitre en cas de désaccord entre l'observateur et les brigades nationales de contrôle.

Dans l'ensemble, le cas du Cameroun montre l'importance du soutien et de la pression venant de l'extérieur (notamment des conditions imposées par les bailleurs de fonds) dans la mise en place de systèmes de vérification et de contrôle des forêts. Cependant, l'adhésion nationale et un mécanisme de financement sûr sont essentiels pour l'indépendance et l'efficacité du système. D’aucuns pensent que, au Cameroun, la trop grande dépendance des financements provenant des bailleurs de fonds pour la mise en œuvre du système a fragilisé sa pérennité, le rendant plus vulnérable lors de périodes de baisse de l'aide. 


\section{Chapitre 12}

\section{Ghana : s'attaquer à la réforme de la gouvernance par la conception d'un système}

Note : Cette étude de cas a été réalisée en septembre 2007 par Neil Bird. Elle s'appuie en partie sur Bird, N., Fomété, T. et Birikorang, G. 2006 Ghana's experience in timber verification system design. Étude de cas de pays VERIFOR ${ }^{\circ} 1$. ODI, Londres.

Tableau 12.1 Ghana : quelques statistiques clés

\begin{tabular}{|c|c|c|c|}
\hline Statistiques & & Date & Source des données \\
\hline Population & 22,1 millions & 2005 & $\begin{array}{l}\text { Nations Unies, Division } \\
\text { de la population } 2006\end{array}$ \\
\hline Superficie & 22,8 millions de ha & & FAO 2006 \\
\hline Couverture forestière & $24,2 \%$ & 2005 & $\begin{array}{l}\text { Nations Unies, Division } \\
\text { de la statistique } 2006\end{array}$ \\
\hline Propriété de la forêt publique & $100 \%$ & 2000 & FAO 2005 \\
\hline $\begin{array}{l}\text { Production de bois rond } \\
\text { industriel }\end{array}$ & 2,16 millions de $\mathrm{m}^{3}$ & $\begin{array}{l}\text { Moyenne } \\
2001-2005\end{array}$ & OIBT 2005 \\
\hline $\begin{array}{l}\text { Emplois officiels dans le } \\
\text { secteur forestier }\end{array}$ & 30000 & $\begin{array}{l}\text { Moyenne } \\
1990-2000\end{array}$ & FAO 2004 \\
\hline $\begin{array}{l}\text { Contribution du secteur } \\
\text { forestier au PIB }\end{array}$ & $4,9 \%$ & $\begin{array}{l}\text { Moyenne } \\
1990-2000\end{array}$ & FAO 2004 \\
\hline $\begin{array}{l}\text { Valeur des exportations de } \\
\text { produits forestiers }\end{array}$ & 110 millions USD & $\begin{array}{l}\text { Moyenne } \\
1990-2000\end{array}$ & FAO 2004 \\
\hline $\begin{array}{l}\text { Principaux marchés } \\
\text { internationaux du bois }\end{array}$ & $\begin{array}{l}\text { Italie, Allemagne, } \\
\text { Etats-Unis }\end{array}$ & 2004 & Global Timber 2007 \\
\hline $\begin{array}{l}\text { Indice } \mathrm{TI} \text { de perception } \\
\text { de la corruption }(0-10,0= \\
\text { corruption la plus forte })\end{array}$ & 3,3 & 2006 & $\begin{array}{l}\text { Transparency } \\
\text { International } 2006\end{array}$ \\
\hline $\begin{array}{l}\text { Indice du développement } \\
\text { humain }(0-1,0=\text { très bas })\end{array}$ & 0,53 & 2004 & PNUD 2006a \\
\hline
\end{tabular}

\subsection{Introduction}

L'établissement d'un système de vérification du bois fait l'objet d'une grande attention au Ghana depuis 2005. D'importants sommes ont été investies dans un modèle original qui s'appuie sur les contrôles réglementaires existant dans le secteur forestier. À ce jour, 
cependant, le système envisagé reste incomplet, en dépit d'un grand intérêt et d'un soutien au plan international pour encourager son démarrage. Cette étude de cas est axée par conséquent sur les préoccupations liées à la conception du système et indique le niveau d'investissement nécessaire pour transformer un secteur précédemment sous contrôle limité par une réforme qui a la confiance de la société civile nationale comme du marché international du bois.

\section{2 Éléments moteurs du système de vérification en voie de formation}

Avec environ $5 \%$ du PIB, la production de bois compte pour beaucoup dans l'économie du Ghana. Elle occupe la quatrième place pour les opérations de change, soit environ $12 \%$ des opérations de change du pays entre 1990 et 2000. Le bois est donc un secteur clé de l'économie nationale et a une forte dimension politique en raison de l'implication des élites dans la filière. Il est prouvé que la réalisation de très gros profits est très répandue depuis quelque temps (Birikorang and Rhein 2005), l'industrie du bois s'étant énormément développée depuis les vingt dernières années. Ceci a été engendré par trois principaux facteurs : (i) prêts préférentiels de la Banque Mondiale aux industriels qui investissaient dans des activités forestières, et principalement la transformation ; (ii) une interdiction sur les exportations de grumes ; et (iii) le prix fixé par le gouvernement ghanéen qui était trop bas. Ceci a conduit au doublement de la capacité des installations de transformation du bois au cours des années 1990, celle des scieries atteignant environ 3,4 millions de $\mathrm{m}^{3}$ en 2001, ce qui représentait dix fois le rendement durable estimé du domaine forestier permanent (Banque Mondiale 2007). On observe une préoccupation grandissante vis-à-vis de la diminution rapide de l'offre nationale de bois résultant de cette stratégie de croissance industrielle. Cette préoccupation s'accompagne d'une prise de conscience qu'un plus grand contrôle est maintenant nécessaire pour apporter une lueur d'espoir au secteur forestier, même s'il faut délaisser le bois des forêts naturelles pour exploiter celui des plantations forestières.

Les débouchés très lucratifs de la filière se trouvent surtout sur le marché international, les pays de l'Union Européenne étant les principaux partenaires du Ghana pour le commerce du bois. Ces pays représentaient un peu plus de la moitié des exportations de bois en 2004, avec comme principaux marchés l'Allemagne, l'Italie, la France, le Royaume-Uni et l'Espagne. La dimension internationale de ce commerce du bois exige que le Ghana soit sensible à l'évolution de la demande et l'a conduit à s'intéresser de façon précoce à l'Accord de Partenariat Volontaire de l'Union Européenne. Les incitations financières éventuelles proposées par l'UE sont aussi jugées importantes. Un échange de vues au plan international a par conséquent fortement influencé le cadre du projet de programme de vérification, l'organisme de développement britannique - pays qui est l'un des grands partenaires commerciaux du Ghana - ayant été très impliqué dans son élaboration.

La participation du Ghana au processus d'application des législations forestières et la gouvernance en Afrique (AFLEG), aboutissant à la réunion ministérielle de Yaoundé 
d'octobre 2003, a aussi donné une forte impulsion politique pour remédier aux déficiences nationales observées au sein du secteur forestier.

\subsection{Mission du système de vérification}

Le secteur du bois au Ghana se caractérise par une gouvernance faible et un mépris fréquent de la législation forestière. La Commission forestière (FC), institution nationale responsable du contrôle des forêts, n'a pas été performante dans le domaine du suivi et du contrôle du respect des lois (Banque Mondiale 2007). La FC assume également un certain nombre de fonctions potentiellement incompatibles (par exemple, application de la loi, suivi, gestion forestière, et collecte de l'impôt). Le système national de vérification qui se dessine cherche à surmonter ces deux difficultés : d'une part un secteur qui fonctionne sans tenir compte de la législation et d'autre part des instances forestières incapables d'assurer leur mission de sauvegarde du patrimoine forestier national.

\section{Législation forestière}

La loi sur les concessions $n^{\circ} 124$ de 1962 a constitué une grande étape de la législation forestière nationale. En vertu de l'article 16 de cette loi, tous les terrains forestiers étaient placés sous l'autorité du Président, habilitant l'Etat ghanéen à exercer une tutelle sur la protection, la gestion et le développement. Dans le cadre de ces mesures de contrôle, la première avancée vers la création d'une chaîne de garde du bois coupé a été le décret sur les arbres et le bois de 1974 (NRDC 273), qui demandait au ministère des Forêts de diviser le Ghana en districts et d'attribuer à chacun de ceux-ci une marque d'origine. Il exigeait également des entreprises exportatrices de bois de faire enregistrer une " marque de propriété » au ministère des Forêts.

La loi sur la gestion des ressources en bois $n^{\circ} 547$ de 1997 (TRMA) vise à assurer que la récolte de bois est dans la logique de la gestion et de l'utilisation durables des ressources en bois du Ghana. Cette loi a établi une nouvelle catégorie de droit relatif au bois - le contrat d'utilisation de bois (TUC) - toute la récolte de bois devant s'effectuer dans le cadre de ces contrats, à part quelques exceptions. En vertu de l'article 19 (1) du TRMA, "Tout droit relatif au bois, concession ou bail accordés conformément à des textes réglementaires, et valables immédiatement avant l'entrée en vigueur de cette loi, se poursuivront pour une période ne dépassant pas six mois à partir de la date d'entrée en vigueur de ladite loi. » Bien que la loi TRMA soit entrée en vigueur le 17 mars 1998, les droits relatifs au bois qui existaient au préalable ont continué d'exister, jetant un grave discrédit sur la législation forestière.

Un amendement à la loi sur la gestion des ressources en bois (TRMA) (Loi 617) et un amendement aux réglementations liées à cette loi (LI 1721) ont été adoptés en 2002.

Ces modifications juridiques ont été introduites pour permettre :

- Les appels d'offres pour l'attribution et l'utilisation des ressources en bois ; 
- La mise en œuvre d'accords de responsabilité sociale qui exigent que les concessionnaires aident les communautés se trouvant sur la zone couverte par leur contrat par la réalisation d'équipements sociaux.

En dépit de l'évolution du cadre législatif de la production de bois, le secteur forestier au Ghana souffre énormément des pratiques illégales. Une grande partie de l'approvisionnement du pays en bois de sciage provient du sciage illégal à la tronçonneuse. L'étude sur l'industrie du bois au Ghana et sur l'interdiction d'exporter des grumes (Birikorang et al. 2001) estimait qu'en 1999, sur les 3,7 millions de $\mathrm{m}^{3}$ de bois coupé, les activités illégales de sciage comptaient pour $46 \%\left(1,7\right.$ million de $\left.\mathrm{m}^{3}\right)$ tandis que, d'autre part, l'exploitation industrielle illégale représentait $24 \%\left(0,9\right.$ million $\left.\mathrm{de}^{3}\right)$. Le nombre de personnes impliquées dans le sciage à la tronçonneuse est considérable, les estimations s'élevant à 50000 (Otoo 2003). Cette activité est très répandue parce qu'elle comporte sans aucun doute des avantages pour les pauvres. De transporter du bois sur la tête est payé cinq fois plus que le salaire minimum journalier. Les agriculteurs préfèrent souvent un paiement immédiat des arbres par les scieurs illégaux aux promesses des institutions du secteur forestier concernant des avantages qui sont finalement distribués d'une façon non transparente. Surtout, les chefs, comme les propriétaires terrains, ont été évincés de la prise de décisions par le système de l'administration locale et la présence de la FC au niveau du district. Par conséquent, ils ferment les yeux sur l'exploitation illégale (Birikorang et al. 2001).

Des tentatives récentes de contrôle de la récolte illégale de bois, surtout en dehors des réserves forestières, ont consisté en une série de mesures appropriées, comprenant l'enregistrement des tronçonneurs, la création d'unités mobiles pour la protection de la forêt et des contrôles stricts de la coupe. Parmi les autres dispositions prises, on peut noter des actions coordonnées de l'armée et de la police pour lutter contre ceux qui exploitent dans l'illégalité et la confiscation du bois, de leur matériel et de leurs véhicules. Cependant, ces mesures n'ont pas eu l'impact désiré, principalement en raison de la forte demande de bois et de la faiblesse des sanctions. La capacité des institutions au niveau du district est telle qu'elle ne permettait pas la détection des activités illégales ou bien (comme on le présume) laissait place à une connivence répandue du personnel forestier avec les exploitants illégaux.

On voit que la législation mise en place pour contrôler la récolte et protéger les ressources forestières a été soit inadéquate, soit mal appliquée. Cette situation s'explique par l'économie politique du pays et par l'influence politique exercée par les grandes scieries, qui est de très loin supérieure aux raisons techniques souvent invoquées. Il faudra surmonter ces obstacles si le système de vérification doit être un instrument efficace au service de la légalité.

\section{Principales institutions du secteur forestier}

Le ministère de l'Agriculture, des Forêts et des Mines (MLFM) est chargé de l'élaboration des politiques tandis que l'organisme exécutif est la Commission forestière 
(FC). Cependant, les institutions de l'Etat liées au secteur forestier sont en mutation permanente depuis de nombreuses années. Sur une période de 17 ans, le vote des lois, suivi de leur abrogation et de leur remise en vigueur a placé la FC dans une position de conseiller auprès du ministère, tandis que le département des forêts et d'autres organismes du secteur ont conservé leur fonction d'organes d'application. En vertu de la dernière réforme législative - la loi sur la Commission forestière $n^{\circ} 571$ de 1999 - les quatre organismes publics et services ministériels impliqués dans la réglementation relative aux forêts et à la faune, auparavant séparés, sont devenus des divisions de la FC. La création de la Commission forestière en vertu de cette loi a résolu le conflit entre une Autorité forestière nationale envisagée pour remplacer l'ancien département des forêts (qui aurait eu pour mission de réglementer et de gérer les ressources forestières du Ghana), et la constitution ghanéenne de 1992 qui confiait à une Commission forestière la protection, la gestion et le développement des ressources forestières et en animaux sauvages de la nation. ${ }^{1}$

\subsection{Schéma du système de vérification}

Une importante initiative a eu lieu en janvier 2005 avec le démarrage du programme de validation du bois légal (Validation of Legal Timber Programme ou VLTP). À l'origine de cette initiative se trouve la gestion commune des programmes des réformes forestières, adoptées par le gouvernement et ses partenaires pour le développement au début des années 2000. Une proposition de suivi des grumes a été mise au point par la Commission forestière, qui inclut une nouvelle organisation institutionnelle et des processus de suivi du bois pour améliorer les règles relatives aux ressources forestières et pour contrôler les activités illégales. Les expériences équatorienne et camerounaise de la SGS, partenaire technique du secteur privé sous contrat avec la FC, a permis aux responsables du programme de porter une attention particulière à la réforme institutionnelle, au soutien juridique de cette réforme et aux impacts financiers probables sur le secteur forestier.

Le but du VLTP est de mettre en place un système performant et peu coûteux pour prouver juridiquement l'origine du bois et, par conséquent, le respect de la loi en matière de gestion forestière. Le gouvernement du Ghana s'est engagé à investir 2 millions de USD de ses ressources propres pour la mise au point du nouveau système. Le VLTP a quatre grands objectifs :

- Améliorer le suivi de l'utilisation des ressources forestières ;

- Augmenter l'apport des recettes provenant de la récolte de bois ;

- Maintenir l'accès à une importante destination des exportations (c'est-à-dire l'UE) ;

- Faire le premier pas vers la durabilité.

\section{L'Agence de validation du bois}

Le rôle de la Commission forestière serait totalement redéfini selon le système envisagé qui est en cours d'étude par le VLTP (voir Figures 12.1 et 12.2). La vérification des

1 La solution juridique retenue reflétait le point de vue des services du procureur général et du ministère de la Justice. 


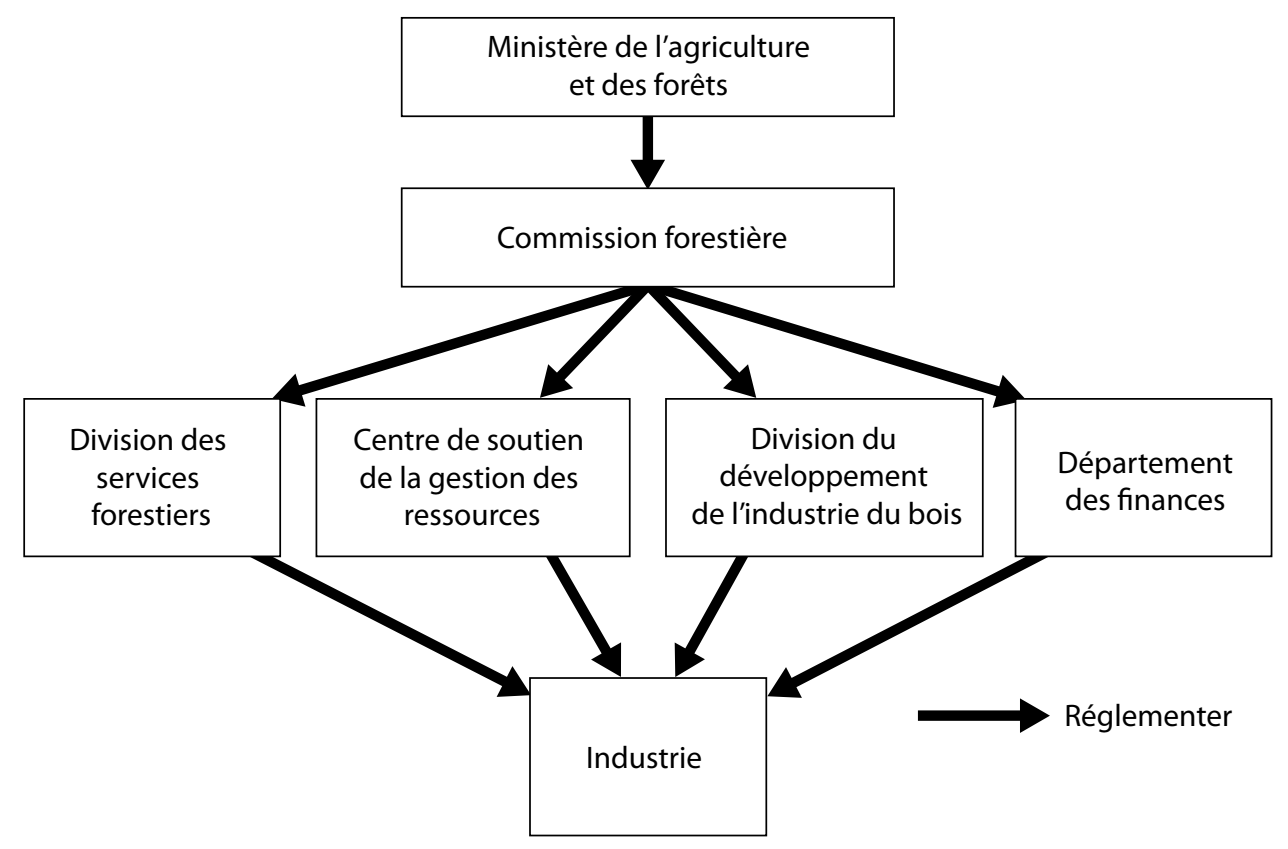

Figure 12.1 Le système actuel de contrôle des forêts au Ghana

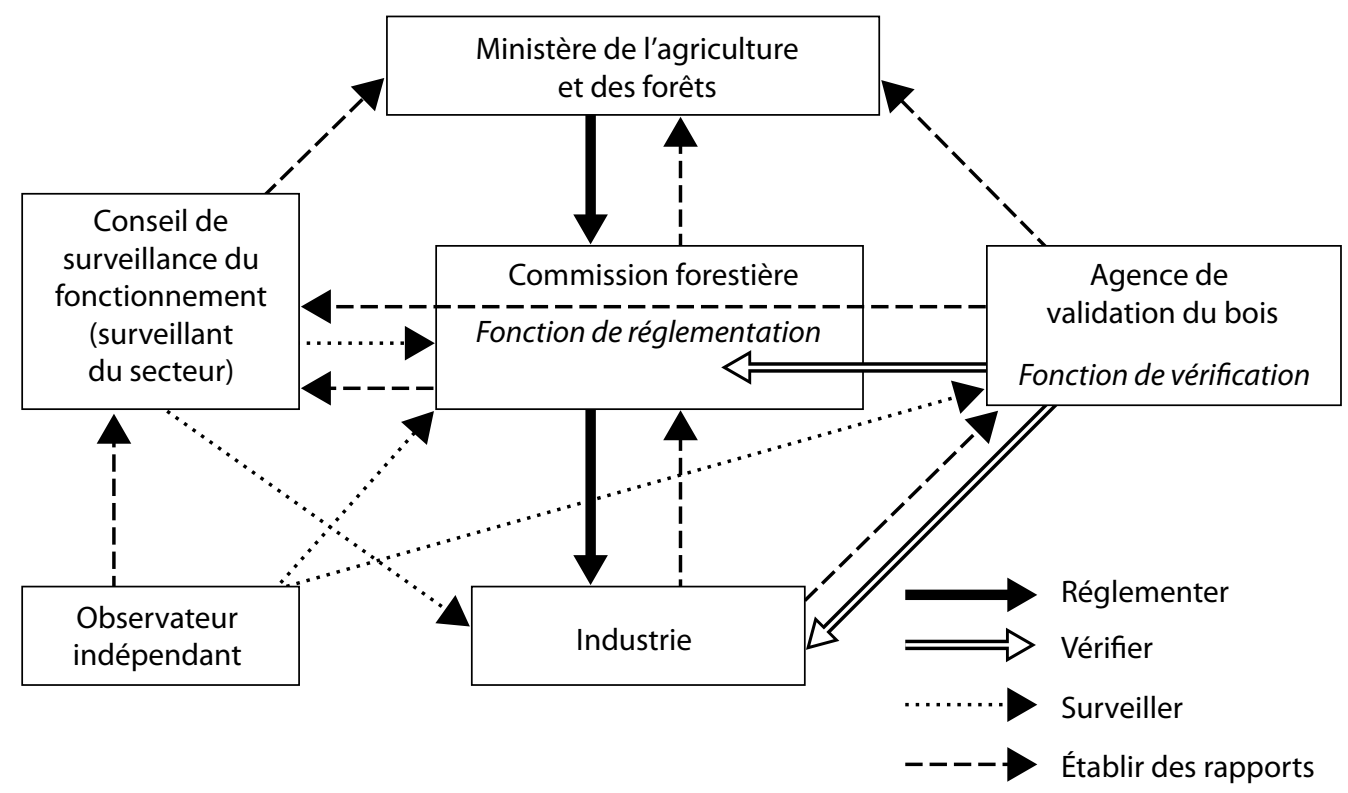

Figure 12.2 Le système de vérification envisagé au Ghana 
activités de réglementation de la Division des services forestiers (Forest Services Division ou FSD) et de la Division du développement de l'industrie du bois (Timber Industry Development Division ou TIDD) incomberait à une nouvelle institution : l'Agence de validation du bois (TVA). Avec la création de l'Agence de validation du bois, le FSD pourrait se concentrer sur ses responsabilités essentielles - l'octroi de droits relatifs à l'exploitation du bois, le suivi et l'application de la loi - en cédant éventuellement la gestion forestière au secteur privé. La TIDD de la FC poursuivrait son rôle d'inspection des arrivées de grumes aux usines de transformation, des analyses des flux entrant et sortant de ces usines, et de classification et d'inspection des exportations de bois. Le défi réside surtout dans une définition claire du rôle et des responsabilités de l'Agence de validation du bois - processus qui n'est pas encore terminé malgré deux années d'efforts suivis.

Le degré de séparation entre l'Agence de validation du bois et la FC est un aspect essentiel de la conception du système. Il reste à voir si cette conception actuelle - qui envisage une agence autonome créée sous l'égide du MLFM - induira le niveau d'indépendance nécessaire, afin que les principales parties prenantes aient confiance dans ce système. Cette nouvelle structure institutionnelle est sans précédent au Ghana et n'a donc pas été testée. La création de l'Agence de validation du bois est une vaste entreprise et une législation sera peut-être nécessaire pour définir sa mission juridique. Le délai relatifà cette mise en place n'est pas fixé, il constitue cependant un obstacle important au lancement rapide du système de vérification envisagé. Il est prévu que l'Agence de validation du bois s'autofinance au bout de trois ans grâce aux paiements effectués par l'industrie du bois en contrepartie de ses services. Cette stratégie dépend dans une large mesure du soutien que lui donnera le secteur forestier, et semble tout à fait ambitieuse étant donné les différences de longue date entre la Commission forestière et l'industrie du bois.

L'industrie du bois a été opposée aux nombreux contrôles de la FC concernant le commerce et l'industrie et elle pense que la Commission ne présente pas un bon rapport coût/performance. Le point de vue de la FC est que l'industrie du bois a tendance à faire pression pour maintenir ses bénéfices au même niveau et à perpétuer le statu quo qui lui garantit un accès préférentiel aux ressources. Cette position est illustrée par le fait que cette industrie vient d'exprimer le souhait de ne pas aller trop vite dans l'adoption d'un système comptable électronique des ressources. En 2004, après avoir commandé une étude de son propre fonctionnement, l'industrie du bois a conclu qu'elle devait augmenter sa production pour ne pas être en déficit (Bruks Associates 2004).

Cependant, les partisans des réformes fiscales relatives aux forêts du Ghana pensent que la conclusion de l'étude est le problème plus que la solution (Birikorang and Rhein 2005).

L'industrie se plaint depuis longtemps du coût élevé du fonctionnement à cause de la bureaucratie gouvernementale. Fondé sur un nombre moins élevé de contrôles et accompagné d'un mécanisme de suivi des grumes plus efficace (permettant des rapprochements plus rapides de données), le nouveau programme de vérification envisagé 
devrait résoudre un certain nombre de ces questions. De toutes façons, l'impact du projet de suivi des grumes sur les volumes de bois traités par l'industrie sera probablement moins important que la raréfaction croissante des ressources à laquelle sont déjà confrontés les industriels, que le projet se fasse ou non. Les objectifs du VLTP, pour leur part, s'harmonisent bien avec les réformes fiscales et institutionnelles prévues qui chercheraient aussi à déréglementer l'industrie, par exemple dans le domaine des prix et de la commercialisation du bois, ainsi que par le transfert de la future gestion forestière au secteur privé.

Les autres parties prenantes du pays qui participent aux négociations sont les propriétaires forestiers, les instances traditionnelles, les assemblées de district, les communautés vivant en lisière de forêt, les agriculteurs, les comités forestiers des communautés et les organisations de jeunes qui considèrent que leur sort est lié au degré de transparence des opérations forestières et de la répartition des recettes tirées des forêts. Leur voix est actuellement retransmise par la société civile dans le processus de consultation de l'accord de partenariat volontaire.

\section{L'observateur indépendant}

La deuxième grande caractéristique du système de vérification envisagé est l'observateur indépendant. La principale fonction de celui-ci est d'accroître la crédibilité et la transparence du système. Le schéma du système prévoit une séparation des fonctions entre l'Agence de validation du bois et l'observateur indépendant. La mise en œuvre du système de vérification serait du ressort de l'Agence de validation du bois, son aboutissement étant le certificat de légalité. Le rôle de l'observateur indépendant serait de tester les critères du système. Certains témoins ont suggéré qu'un partenariat entre une organisation internationale digne de confiance et des ONG locales pourrait permettre de progresser vers l'installation rapide d'un observateur. Cependant, ceci est une autre stratégie non testée, qui soulève des problèmes de légitimité. Un équilibre doit être trouvé entre l'instauration rapide d'un tel organe et sa pérennité probable.

\section{Le Conseil de surveillance du fonctionnement}

Le troisième et dernier élément nouveau du système de vérification est le Conseil de surveillance du fonctionnement qui est envisagé pour servir de surveillant au secteur et qui comprendrait des représentants des principales institutions et parties prenantes. Le conseil surveillerait le fonctionnement de la totalité du programme de contrôle des forêts, suivrait l'application de la loi en général, et comporterait un mécanisme de résolution des conflits.

\subsection{Conclusion}

Des investissements considérables ont été réalisés en vue de créer un système de vérification national au Ghana. Le VLTP, qui se trouve au sein de la FC, est l'initiative phare visant à mettre en place les institutions, les processus et la structure nécessaires pour répondre 
au besoin d'une vérification indépendante, et en particulier, aux exigences de l'accord de partenariat volontaire de l'UE. Le gouvernement semble s'être attaché au départ à un aspect technique : mettre en place la technologie relative au contrôle des forêts (suivi du bois) qui permettrait la validation du bois légal. Le défi maintenant est de réussir le bon dosage institutionnel pour garantir un système de vérification crédible et en déterminer les fondements juridiques.

La question importante est de savoir qui aura le dernier mot en matière de respect de la loi. Avec la culture de la procédure judiciaire qui existe actuellement au Ghana, il y a le danger que le système de contrôle soit émasculé par des poursuites civiles au tribunal. D'où la grande priorité que l'on doit donner à la détermination du statut juridique de tous les composants du système.

Quatre leçons clés se sont déjà dégagées de cette expérience, qui peuvent intéresser les autres pays envisageant de se lancer dans la vérification du bois. La première est que le monopole d'Etat actuel sur le système de contrôle des forêts a présenté de nombreuses difficultés freinant une plus grande obligation de rendre des comptes et une transparence accrue du secteur. Deuxièmement, l'engagement du gouvernement est essentiel pour fixer un juste prix du bois et pour garantir la distribution équitable des recettes, sur lesquelles repose finalement la pérennité du secteur. Troisièmement, un processus acceptable par toutes les principales parties prenantes nécessitera probablement une mise en œuvre échelonnée du système de vérification du bois. L'instauration d'un système crédible n'est pas une mesure à court terme et ne se comptera certainement pas en mois mais en années. Enfin, la priorité du gouvernement qui était d'améliorer les aspects techniques du système de contrôle doit laisser place à une approche plus nuancée reconnaissant et tenant compte de la dimension politique de la réforme au sein du secteur du bois. 



\section{Chapitre 13}

\section{L'expérience de l'observation indépendante des forêts au Cambodge}

Note : Cette étude de cas a été réalisée en juin 2007 par Cecilia Luttrell. Elle se base en partie sur Luttrell, C. et Brown, D. 2006 The Experience of Independent Forest Monitoring in Cambodia. Etude de cas $n^{\circ} 4$ de VERIFOR. ODI, Londres.

Tableau 13.1 Cambodge : quelques statistiques clés

\begin{tabular}{|c|c|c|c|}
\hline Statistiques & & Date & $\begin{array}{l}\text { Source des } \\
\text { données }\end{array}$ \\
\hline Population & 14,1 millions & 2005 & $\begin{array}{l}\text { Division de la } \\
\text { population des } \\
\text { Nations Unies } \\
2006\end{array}$ \\
\hline Superficie & 17,7 millions ha & & FAO 2006 \\
\hline Couverture forestière & $59,2 \%$ & 2005 & $\begin{array}{l}\text { Division de la } \\
\text { statistique des } \\
\text { Nations Unies } \\
2006\end{array}$ \\
\hline $\begin{array}{l}\text { Propriété publique des } \\
\text { forêts }\end{array}$ & $100 \%$ & 2000 & FAO 2005 \\
\hline $\begin{array}{l}\text { Production de bois rond } \\
\text { industriel }\end{array}$ & $166000 \mathrm{~m}^{3}$ & Moyenne 2001-2005 & OIBT 2005 \\
\hline $\begin{array}{l}\text { Emplois dans le secteur } \\
\text { forestier formel }\end{array}$ & 15000 & Moyenne 1990-2000 & FAO 2004 \\
\hline $\begin{array}{l}\text { Contribution du secteur } \\
\text { forestier au PIB }\end{array}$ & $\begin{array}{l}6,6 \% \\
0,5 \%\end{array}$ & $\begin{array}{l}\text { 1990-2000; } \\
\text { après l'interdiction } \\
\text { d'exploitation en } 2002\end{array}$ & $\begin{array}{l}\text { FAO 2004; } \\
\text { IFSR } 2004\end{array}$ \\
\hline $\begin{array}{l}\text { Valeur des exportations de } \\
\text { produits forestiers }\end{array}$ & 84 millions USD & Moyenne 1990-2000 & FAO 2004 \\
\hline $\begin{array}{l}\text { Principaux marchés } \\
\text { internationaux du bois }\end{array}$ & $\begin{array}{l}\text { Chine, Taiwan, } \\
\text { Japon et Thaillande }\end{array}$ & 2004 & $\begin{array}{l}\text { Miller et Boscolo } \\
2004\end{array}$ \\
\hline $\begin{array}{l}\text { Indice de perception de la } \\
\text { corruption de } \mathrm{Tl} \text { (de } 0 \text { à } 10 \text {, } \\
\text { avec } 0=\text { le plus corrompu) }\end{array}$ & 2,1 & 2006 & $\begin{array}{l}\text { Transparency } \\
\text { International } \\
2006\end{array}$ \\
\hline $\begin{array}{l}\text { Indice de développement } \\
\text { humain ( } 0 \text { ou } 1 \text {, avec } 0 \\
=\text { très bas) }\end{array}$ & 0,58 & 2004 & PNUD 2006a \\
\hline
\end{tabular}




\subsection{Introduction}

Cette étude de cas compare l'expérience et l'impact de deux exemples différents d'observation indépendante dans le secteur forestier au Cambodge. Ce faisant, elle se concentre sur un élément spécifique de la vérification.

Le Cambodge est l'un des pays les plus pauvres d'Asie du Sud-est, victime d'une longue histoire de conflits. Le secteur forestier a joué un rôle primordial dans le développement récent du pays. Entre 1991 et 1998, le pays a exporté 2,5 milliards USD de bois, selon les estimations. Dans les années 1980 et 1990, l'exploitation du bois était minée par une mauvaise gestion et une corruption généralisée, ayant entraîné les troubles et des violences. En 1995, la communauté internationale a commencé à se pencher sur la mise en œuvre d'une législation et d'une réforme forestières afin de remédier aux échecs de l'Etat dans ce secteur. En 1999, la Banque Mondiale et d'autres donateurs ont créé le Projet cambodgien d'observation et de signalement des infractions forestières (Forest Crimes Monitoring and Reporting Project ou FCMRP). Il s'agissait de créer un poste d'observateur indépendant $(\mathrm{OI})$ chargé de contrôler l'efficacité des deux ministères impliqués, le ministère de l'agriculture, des forêts et de la pêche (MAFP) et le ministère de l'environnement (MdE). En 1999, le contrat d'OI fut attribué à Global Witness (GW), une ONG britannique de défense des droits environnementaux qui mène une campagne active contre les violations des lois forestières au Cambodge. Suite à l'identification d'affaires controversées et à l'absence de réponse, les relations entre l'OI et le gouvernement cambodgien se sont détériorées. En 2003, le gouvernement a mis fin au contrat avec GW et attribué un contrat similaire à SGS (Société générale de surveillance), une société suisse privée. Faute de financement, les opérations de SGS ont été suspendues en février 2006 et depuis, la fonction d'OI est en suspens.

\subsection{Appropriation}

\section{Facteurs externes}

La mise en place d'un OI fut associée à la priorité donnée à l'application de la législation forestière afin de répondre aux défauts de gouvernance, qui préoccupent aussi bien les donateurs que les ONG. Dans les années 1990, les violences dans le secteur forestier, la politique du pouvoir menée par l'élite cambodgienne et l'agenda environnemental chargé de la communauté internationale ont conduit à une mise en avant de l'exploitation illégale sur la scène politique. Depuis 1995, l'application de la législation forestière et la question de la performance de l'Etat sont devenues une préoccupation majeure des donateurs. ${ }^{1}$ En 1995, une mission conjointe de la Banque Mondiale, du PNUD et de la $\mathrm{FAO}$ a suggéré qu'un meilleur contrôle des zones forestières pourrait accroître les recettes publiques de plus de 100 millions de dollars par an (McKenny 2001, p. 2). ${ }^{2}$

1 Cependant, cette focalisation sur la législation contredisait l'opinion de Le Billon (2002), selon laquelle les problèmes étaient moins le résultat d'une faible capacité de réglementation de la part de l'administration que d'un système parallèle d'" État fantôme " qui permettait aux acteurs non étatiques d'acquérir des pouvoirs et qui a entraîné une situation de " warlordism " (de l'anglais " warlord ", seigneur de guerre).

2 Ces estimations ont par la suite été révisées dans une fourchette entre 40 millions et 80 millions de dollars (Banque Mondiale 1999). 
Les principales recommandations de la mission pour y parvenir étaient d'améliorer la supervision technique et politique du secteur et d'augmenter les redevances sur le bois. La même année, Global Witness a commencé à publier des rapports sans complaisance sur les exportations et les activités illégales dans le secteur forestier. Avec le temps, l'ONG a élargi sa campagne afin d'intégrer un programme de " bonne gouvernance ».

À l'époque de la mise en place de l'OI, le gouvernement royal du Cambodge (GRC) cherchait à améliorer son image sur la scène internationale. Mais dans un pays dépendant fortement de l'aide étrangère, les initiatives étaient en grande partie financées par donateurs et l'OI est devenu la conditionnalité d'un prêt d'ajustement structurel de 30 millions de dollars de la Banque Mondiale. Il n'existait aucune coalition nationale de poids pour exiger la responsabilité dans le secteur et aucun engagement réel du GRC en matière de réforme.

\section{Le mandat de l'OI}

Le " mandat " officiel de l'OI a pris la forme d'un contrat avec le GRC. Cependant, les Termes de référence (TDR) étaient excessivement larges et généraux. Les rôles et responsabilités des agences partenaires étaient souvent obscurs et/ou non avalisées. Ainsi, nombre d'agences censées rendre compte à GW, selon ses TDR, n'étaient pas au courant de ces exigences et aucun protocole d'accord ne fut signé avec elles. L'expérience de SGS fut similaire. Le manque de clarté quant au mandat légal, aux objectifs, à ce qui devait être contrôlé et aux résultats attendus constituait un défaut de conception dans les deux cas et résulta sur une baisse de confiance (Malayang et al. 2002).

\subsection{Conception du système de vérification}

Le FCMRP a instauré une unité de surveillance des infractions forestières composée de trois éléments. Les deux premiers, le Bureau d'observation des infractions forestières (FCMO) du département des Forêts et de la Vie sauvage (DFVS), et le département d'Inspection (DI), situé au ministère de l'Environnement, devaient gérer des « systèmes de suivi des dossiers " (SSD) parallèles pour suivre les infractions commises respectivement dans les exploitations et les zones protégées, grâce à un système informatique permettant de rapprocher les données et d'élaborer des rapports nationaux. Les bureaux provinciaux et de district devaient fournir des informations aux unités d'observation sur une base mensuelle. Le troisième élément (clé) de vérification était l'OI (voir Figure 13.1). Son rôle consistait à examiner les registres du GRC et en déduire si le FCMO et le DI exécutaient réellement leur mandat respectif, ainsi qu’à vérifier que toute infraction était correctement signalée et que toutes les actions officielles déclarées avaient effectivement été entreprises. Durant le contrat de GW, un « point focal » (organe représentatif du Conseil des ministres, principal organe de délibération du GRC) était chargé de recevoir les comptes rendus d'infractions de GW (ainsi que les rapports périodiques sur l'évaluation par l'observateur de la performance des agences du FMCRP) et de les transmettre aux ministères, au Premier ministre, aux donateurs et à la presse. 


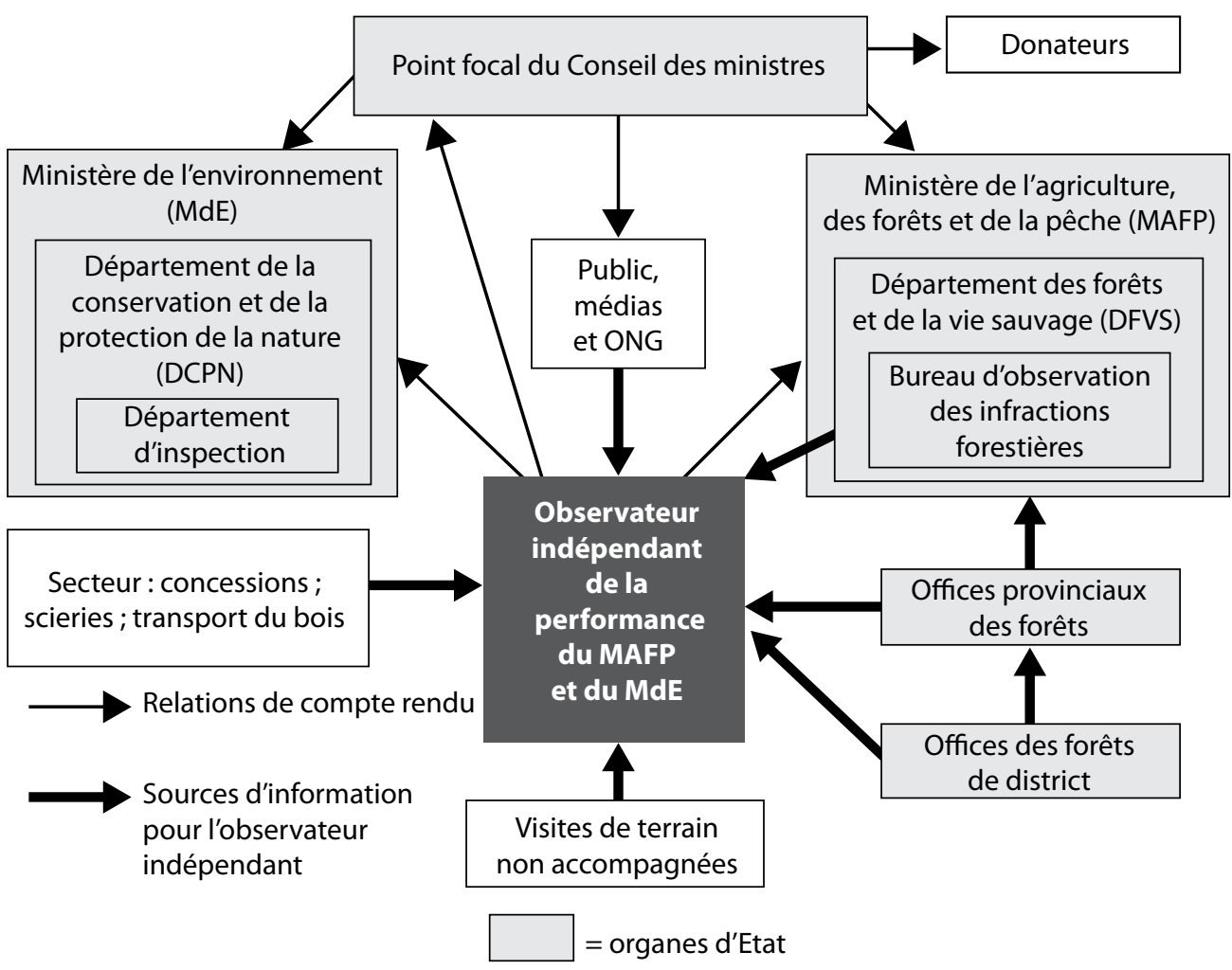

Figure 13.1 Structure du système d'observation indépendante au Cambodge (1999 à 2003)

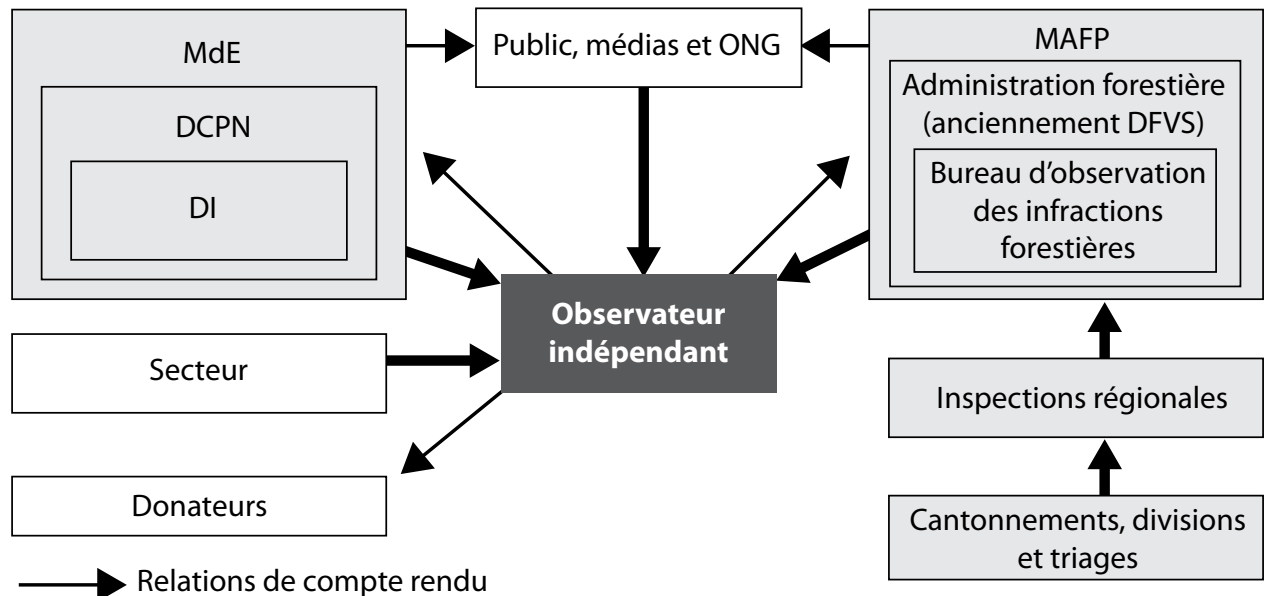

Cette figure reflète également la refonte du Sources d'information pour l'observateur indépendant département des forêts et de la vie sauvage $=$ organes $\mathrm{d}^{\prime}$ Etat en Administration forestière.

Figure 13.2 Structure du système d'observation indépendante au Cambodge (2003 à 2005) 
Les TDR de SGS étaient très similaires à ceux de GW. Cependant, comme l'illustre la Figure 13.2, il existait quelques différences, certes limitées, mais indéniablement importantes. Celles-ci incluaient la stipulation claire que l'observateur ne pourrait être " chargé d'entreprendre une quelconque activité d'observation ou d'inspection dans l'objectif premier de détecter et d'enquêter sur de tels incidents ». Bien au contraire, l'observateur avait pour mission de confirmer que toutes les infractions étaient signalées. Par ailleurs, l'expression «surveillance indépendante " ne figurait pas dans les nouveaux TDR et il n'existait aucun organe tiers (c.-à-d. de point focal) auquel SGS aurait pu rendre compte ou qui aurait agi comme un mécanisme de supervision.

GW et d'autres observateurs ont fortement critiqué ces changements, suggérant qu'ils impliquaient une augmentation des pouvoirs du gouvernement pour influencer l'observateur (Global Witness 2005). Dans la mesure où, selon certains, GW avait outrepassé son mandat, le DFVS et les donateurs espéraient que le travail du nouvel observateur suivrait une approche plus régulière et restreinte - plus en ligne avec une fonction habituelle d'audit. SGS devait agir comme vérificateur du SSD existant (un rôle non considéré comme prioritaire par $\mathrm{GW}$ ) et non enquêter sur les infractions forestières. Dans la pratique, SGS a presque exclusivement limité son rôle au suivi de l'utilisation du SSD.

\section{Assurer l'indépendance : points de désaccord}

\section{Chevauchement entre plaidoyer et observation}

Un point de désaccord concernant «l'indépendance » de l'observateur dans le cas de GW était le chevauchement de ses fonctions d'observateur et de défenseur. GW fut accusé d'avoir collecté des informations dans le cadre de sa mission d'observation indépendante à des fins de plaidoyer. Avant ce rôle d'observateur, GW était en effet spécialisé dans le plaidoyer au Cambodge depuis 1995. Le GRC a estimé que le GW disposait de son propre agenda et que cela compromettait fortement son aptitude à assurer le rôle d'observateur indépendant. Des problèmes sont aussi apparus autour de la question $\mathrm{du}$ personnel. Depuis 2001, le responsable de contrat de GW entretenait des liens étroits avec l'opposition politique cambodgienne et possédait davantage d'expérience dans la gouvernance et les droits humains qu'en foresterie technique. Ces deux facteurs ont renforcé les doutes au sein du GRC et de ses partisans quant aux intentions de l'observateur. À partir de début 2001, on notait une rupture quasi-totale des relations et de la coopération entre l'observateur et les autorités forestières, jusqu'à ce que le contrat soit retiré en 2003.

\section{Gestion insuffisante}

Le fait que le point focal (l'organe tiers de coordination) se trouvait au sein du Conseil des ministres était problématique. Ce point focal estimait qu'il ne disposait pas de ressources suffisantes ; aucune ressource financière ni aucun personnel technique n'étaient fournis par le projet pour étudier les comptes rendus de l'observateur indépendant. Surtout, il n'existait aucune incitation pour que les personnes du point focal se penchent sur des 
questions souvent très controversées ayant des ramifications politiques. Il était largement perçu que l'objectif du point focal consistait davantage à limiter les dégâts pour le GRC qu’à poursuivre l'amélioration de la gouvernance forestière.

L'incapacité du point focal à jouer son rôle tel que spécifié par les TDR de GW a pu conduire à la décision de supprimer cette fonction tampon des TDR de SGS. Ce qui signifiait qu'il n'existait aucune capacité d'arbitrage en cas de désaccord entre l'observateur et les ministères, et aucun organe garantissant que l'observateur se conformait aux TDR (en matière, par exemple, d'enregistrement en bonne et due forme des infractions signalées).

\section{Absence de supervision par la société civile}

La société civile cambodgienne a peu de poids. Au début des années 1990, pourtant, la communauté internationale a soutenu le développement d'une société civile au Cambodge et un certain nombre de grandes ONG professionnelles sont nées. Celles-ci se sont révélées efficaces dans des domaines tels que la sensibilisation et la participation du public, mais elles ont moins réussi à s'attaquer à des questions impliquant plus de risques politiques, comme la corruption. Le grand public n'a pas beaucoup l'occasion de contribuer directement à l'élaboration des politiques et il existe peu de preuves que le gouvernement s'adresse ou répond aux demandes de la société civile. La faible capacité des ONG locales signifie que leurs homologues internationaux disposent souvent d'une voix plus forte.

Avec le recrutement de SGS en tant qu'observateur indépendant, les donateurs ont estimé que pour la crédibilité et l'acceptation du système au niveau national qu'au niveau international, l'implication du grand public et de la société civile dans le processus de vérification était nécessaire. En outre, les conflits générés durant le mandat de GW avaient eu des conséquences négatives sur l'intérêt des donateurs - tant en matière de soutien à une observation indépendante qu'en termes d'intérêt plus large dans le secteur forestier cambodgien. Les donateurs hésitent désormais à réagir aux inquiétudes des ONG ou à compromettre leurs relations avec le GRC sur ces questions. C'est ainsi qu'à part la Banque Mondiale, peu d'entre eux ont exprimé un intérêt dans les activités de SGS. Compte tenu de cette baisse d'intérêt, il était improbable que le GRC réponde directement aux pressions et préoccupations publiques, c'est pourquoi les inquiétudes soulevées par ces " observateurs externes" ont rarement été suivies de réponse. Par exemple, ni le GRC ni les donateurs n'ont répondu aux inquiétudes des ONG concernant les deux premiers rapports de SGS (voir Global Witness 2004 et Forest Certification Watch 2004).

\section{Source de financement}

GW était au départ financé par des subsides octroyés par le ministère britannique du Développement international (DFID) et l'Agence australienne pour le développement international (AusAID), canalisés vers la FAO et gérés par un fonds fiduciaire du PNUD. À partir de 2000, l'Agence danoise pour le développement international (Danida) 
a également fourni des fonds bilatéraux pour financer l'observation indépendante au Cambodge. L'une des limites du modèle de SGS, toutefois, était que la rémunération de l'observateur n'était pas prise en charge par le "prêt Apprentissage et innovation " dans le cadre du Projet pilote de gestion et de surveillance des concessions forestières, mais par l'Administration forestière, l'institution qu'il était justement censé contrôler. Le transport de grumes (interdit depuis le moratoire de 2001 sur la coupe et le transport de bois) a repris en janvier 2005. À la demande du gouvernement, SGS a assumé le rôle de suivi du transport du bois et de validation des volumes de grumes. Certains donateurs et $\mathrm{ONG}$ ont reproché à SGS de ne pas vérifier l'origine légale de ces grumes (Bernsen et Cochrane 2005). Cet exemple suggère qu'il existe un besoin d'isoler l'OI de toute situation où ses résultats pourraient être biaisés par le souci d'assurer le renouvellement de son contrat.

\section{Règles et procédures}

\section{Normes de vérification}

Le cas du Cambodge se caractérisait par l'absence de normes claires et incontestées permettant de vérifier la légalité. Cette situation a toujours cours aujourd'hui. Si l'on prend l'exemple de la légalité de l'origine du bois, il n'existe aucune délimitation officielle du domaine forestier permanent et le droit à la propriété foncière des autochtones est peu clair. Le droit forestier cambodgien fait la distinction entre les terres publiques et privées et entre les forêts de production, communautaires, de protection, inondées et de conversion, mais ces distinctions ne sont pas toujours clairement employées dans la pratique. Conjugué à la situation actuelle de confusion et de systèmes parallèles d'enregistrement des terres et d'octroi des titres de propriété, cela signifie qu'il est possible, par exemple, d'enregistrer la propriété privée d'un terrain auprès d'un ministère, alors que ce terrain a auparavant été classé comme réserve forestière "permanente " par un autre.

\section{Droits et obligations}

L'accès aux informations s'est avéré problématique pour les observateurs indépendants. Les TDR de GW accordaient à l'ONG la liberté d'entreprendre des visites de terrain non accompagnées et incluaient une clause assurant à l'OI " l'accès direct aux registres et aux dossiers du GRC en matière de concessions, de parcs, de zones protégées et autres terrains forestiers publics " ainsi qu'aux registres des douanes concernant les informations " relevant de la détection, la signalisation, le suivi et la suppression d'activités forestières illégales ou non autorisées ». Cependant, aucun mécanisme n’a été mis en place pour concrétiser un tel accès et la question est rapidement devenue un problème récurrent pour l'observateur (Global Witness 2002, p. 3).

Dans le cas des TDR de SGS, toutes les inspections devaient être "facilitées " par l'Administration forestière et l'OI se voyait refuser le droit d'effectuer des " contrôles aléatoires d'actions illégales signalées, mais pas encore transmises à l'agence compétence pour qu' elle intervienne ». Le rapport du Panel d'inspection de la Banque Mondiale (Panel 
d'inspection 2006) a fait part d'inquiétudes quant au fait que ces TDR empêchaient SGS d'initier des enquêtes de terrain de manière indépendante.

Le manque de clarté sur la signalisation constituait un autre défaut de conception clair. En 2001, GW a publié un rapport controversé prévu pour coïncider avec une réunion du groupe consultatif des principaux donateurs du Cambodge et du GRC. Cette publication fut considérée comme peu opportune par le DFVS. Elle a débouché sur l'officialisation de protocoles de signalisation dans l'optique de soumettre l'observateur à un contrôle gouvernemental plus étroit. Les nouveaux protocoles convenus en juin 2001 exigeaient que les rapports d'infraction soient transmis aux directeurs du DFSV et du département d'Inspection, avec copie aux ministres du MAPF et du MdE et aux coordinateurs du point focal. Les temps de réponse furent convenus mais il y eut des désaccords quant à savoir si, dans le cas où le gouvernement ne répondrait pas dans le délai imparti, GW serait libre de diffuser l'information dans le domaine public. Les TDR accordaient à l'OI ce droit de diffusion, mais aucune clause des protocoles de signalisation ne prévoyait qui procéderait aux arbitrages si le gouvernement ne répondait pas dans les temps. L'OI devait en définitive juger par lui-même.

En vertu des conditions de la nomination de SGS, les comptes rendus trimestriels ne devaient pas être diffusés au public avant d'avoir été vérifiés par le GRC. Le GRC et ses agences disposaient de 30 jours ouvrés à compter de la réception d'un compte rendu trimestriel pour en vérifier le contenu. Après quoi, l'observateur était libre de publier ses conclusions, mêmes si elles n'avaient pas été vérifiées. Toutefois, en comparaison avec les TDR de GW, le type d'information pouvant être publié était moins clair.

\subsection{Performance du système}

La première année, le FCMRP s'est concentré sur la formation des personnes chargées de la signalisation et des systèmes informatiques ; un certain nombre de réalisations ont été observées. Mais suite à cela, les relations entre les experts techniques du FCMRP et d'une part GW, d'autre part le GRC, ont commencé à se détériorer. Les conseillers techniques affirmaient qu'on les gênait délibérément dans leur travail et que tous les dossiers enregistrés n'étaient pas entrés dans le SSD (Miller 2004, p. 6). Plus important, il devint clair que le FCMO ne pouvait ou ne voulait pas signaler les infractions commises par des " puissants" (ibid. 2004, p. 3). Suite à une évaluation de projet, le FCMRP fut suspendu début octobre 2002 (Malayang et al. 2002).

Les résultats de l'évaluation de projet étaient positifs, dans la mesure où il était considéré que le FCMRP renforçait les capacités du GRC à traiter les données sur les infractions et augmentait l'intérêt du public, de la société civile et des donateurs dans la réforme forestière. Dans l'ensemble, cependant, l'évaluation concluait que le projet n'avait pas atteint ses objectifs. De plus, il était perçu que la conception avait tenté d'adopter une approche reposant sur la "fixation technologique " qui n'était pas suffisamment claire dans les réponses apportées aux problèmes du faible engagement politique et de la gouvernance insuffisante qui avaient sous-tendu la performance médiocre du secteur. 
Par exemple, le projet n'a pas mis fin aux rigidités administratives ayant empêché les contreparties nationales d'exécuter ce qui était attendu d'elles. L'étroitesse de la conception fut également accusée de limiter les occasions pour les donateurs de s'engager davantage et de manière plus positive dans le secteur (ibid.).

\section{Impacts sur la gouvernance au sens large}

Evaluer l'impact d'une forme quelconque d'observation indépendante qui constitue un élément d'un processus de réforme forestière pose certaines difficultés, compte tenu des autres forces potentiellement influentes telles que, dans ce cas, le FCMRP élargi, le moratoire sur la coupe de bois et la suspension des concessions. Cependant, les observateurs au Cambodge estiment en règle générale que l'observation par GW a eu un effet désincitatif sur les exploitants illégaux et a augmenté la discipline de l'agence officielle chargée de faire appliquer la loi et du secteur. L'effet le plus remarquable et le plus médiatisé a cependant porté sur l'information et la compréhension mises à disposition des communautés nationale et internationale.

GW a affirmé qu'il avait eu un impact sur deux grands événements politiques dans le secteur forestier : le moratoire sur la coupe de bois introduit en 2002 et l'annulation de concessions (Global Witness 2003). L'ONG britannique a certes pu jouer un rôle dans ces événements, mais d'autres facteurs comme les conditionnalités associées à l'octroi de prêt de la Banque Mondiale et la peur que ceux-ci puissent être reportés à cause de la qualité des plans de gestion ont également pu contribuer. Beaucoup s'accordent à dire que GW a considérablement sensibilisé à la question de l'exploitation illégale au Cambodge. Toutefois, des questions demeurent sur l'impact à long terme de son travail. Malgré son succès dans la mise en avant de l'économie politique du secteur forestier, l'organisme n'est pas parvenu à obtenir un changement à long terme et n'a pas fait grandchose pour encourager le ralliement du côté cambodgien.

Le rôle de GW en tant qu'OI doit être envisagé parallèlement à son rôle d'organisation de plaidoyer. GW estime en effet qu'il n'aurait pas autant d'impact sans la réputation que lui a conféré sa mission de plaidoyer. Cela suggère que les impacts réels de l'OI n'ont pas tant été causés par la conception de l'observation indépendante que par la manière dont GW a utilisé cette opportunité aux fins du plaidoyer.

\section{Impact sur le comportement des donateurs}

Un des impacts non prévus de la première phase d'observation fut un changement important du comportement de nombreux donateurs du secteur forestier au Cambodge. On pourrait affirmer que les conflits générés durant le mandat de GW ont eu des conséquences négatives sur le degré d'intérêt des donateurs - tant en matière de soutien à une observation indépendante qu'en termes d'intérêt plus large pour le secteur forestier cambodgien. La volonté d'engagement politique dans le secteur a fortement décliné et les donateurs hésitent à réagir aux inquiétudes des $\mathrm{ONG}$ ou à compromettre leurs relations avec le GRC sur ces questions. Les donateurs qui restent engagés dans le secteur forestier 
concentrent leur attention sur des questions peu risquées telles que les zones protégées ou les forêts communautaires. L'attention des autres s'est déplacée vers le secteur foncier.

\subsection{Enseignements}

Les deux types d'observation indépendante évoqués dans cette étude de cas sont très différents. Dans sa tentative de traiter des questions de gouvernance plus profondes, GW a adopté une approche d'investigation, souvent en conflit avec les agences qui font l'objet de l'observation, tandis que SGS a joué un rôle d'audit, avec le soutien de l'Administration forestière. Il est manifestement nécessaire de faire la distinction entre les deux, tant au niveau de leurs activités que de leurs objectifs. GW a été accusé d'interpréter ses TDR de manière trop laxiste et de ne pas être en mesure de dépasser son rôle de plaidoyer préexistant, tandis que SGS a été accusé d'interpréter ses TDR de manière trop étroite et de ne pas regarder au-delà de ses compétences. La performance de SGS doit toutefois être comprise dans le contexte d'un repli marqué de l'intérêt des donateurs pour les questions forestières du Cambodge.

Les enseignements généraux devant être tirés de cette étude de cas incluent l'importance de la conception du système, notamment la précision des rôles et des responsabilités de l'observateur indépendant, un mécanisme de gestion qui intègre l'OI dans le reste du système de contrôle et la clarification des normes de régularité devant être vérifiées. Plus important, les questions associées à la conception des systèmes de vérification dans un contexte de faible volonté politique et de société civile sous-développée restent sans réponse.

On peut en conclure que différents mécanismes et approches sont appropriés dans diverses situations et périodes au cours de l'évolution du secteur, mais que c'est la constellation des acteurs et non les compétences propres à chacun qui détermine l'efficacité de toute approche. En termes d'impact, l'approche la plus productive peut consister à nommer à la fonction d'OI une organisation autre qu'une association de défense, en veillant à ce que des ONG de surveillance existent et soient en mesure de maintenir la pression sur l'observateur indépendant et de le superviser. Le faire d'une manière durable nécessite que l'attention soit portée au développement d'une coalition nationale forte, en plus d'un certain engagement du gouvernement en faveur de la réforme. L'un des moyens d'y parvenir est de renforcer les capacités qui permettent de progressivement développer l'aptitude et la volonté locales de s'engager auprès de l'observateur. 


\section{Chapitre 14}

\section{Comités multi-sectoriels de protection des forêts aux Philippines}

Note : Cette étude de cas a été réalisée en juin 2008 par Robert B. Oberndorf. Elle se base en partie sur Cruz, R.V.O. et Pulhin, J.-M. 2006 Review of Multisectoral Forest Protection Committees in the Philippines. Etude de cas $n^{\circ} 7$ de VERIFOR. ODI, Londres.

Tableau 14.1 Les Philippines : quelques statistiques clés

\begin{tabular}{|c|c|c|c|}
\hline Statistiques & & Date & Source des données \\
\hline Population & 83,1 millions & 2005 & $\begin{array}{l}\text { Division de la population } \\
\text { des Nations Unies } 2006\end{array}$ \\
\hline Superficie & 29,8 millions ha & & FAO 2006 \\
\hline Couverture forestière & $24,0 \%$ & 2005 & $\begin{array}{l}\text { Division de la statistique } \\
\text { des Nations Unies } 2006\end{array}$ \\
\hline Propriété publique des forêts & $90 \%$ & 2000 & FAO 2005 \\
\hline $\begin{array}{l}\text { Production de bois rond } \\
\text { industriel }\end{array}$ & 1,5 million $\mathrm{m}^{3}$ & $\begin{array}{l}\text { Moyenne } \\
2001-2005\end{array}$ & OIBT 2005 \\
\hline $\begin{array}{l}\text { Emplois dans le secteur forestier } \\
\text { formel }\end{array}$ & 60000 & $\begin{array}{l}\text { Moyenne } \\
1990-2000\end{array}$ & FAO 2004 \\
\hline $\begin{array}{l}\text { Contribution du secteur forestier } \\
\text { au PIB }\end{array}$ & $1,0 \%$ & $\begin{array}{l}\text { Moyenne } \\
1990-2000\end{array}$ & FAO 2004 \\
\hline $\begin{array}{l}\text { Valeur des exportations de } \\
\text { produits forestiers }\end{array}$ & 83 millions USD & $\begin{array}{l}\text { Moyenne } \\
1990-2000\end{array}$ & FAO 2004 \\
\hline $\begin{array}{l}\text { Principaux marchés } \\
\text { internationaux du bois }\end{array}$ & Pas de données & 2004 & Global Timber 2007 \\
\hline $\begin{array}{l}\text { Indice de perception de la } \\
\text { corruption de } \mathrm{TI} \text { (de } 0 \text { à } 10 \text {, avec } \\
0=\text { le plus corrompu) }\end{array}$ & 2,5 & 2006 & $\begin{array}{l}\text { Transparency } \\
\text { International } 2006\end{array}$ \\
\hline $\begin{array}{l}\text { Indice de développement } \\
\text { humain ( } 0 \text { ou } 1 \text {, avec } 0=\text { très bas) }\end{array}$ & 0,76 & 2004 & PNUD 2006a \\
\hline
\end{tabular}

\subsection{Introduction}

L'accélération de la déforestation aux Philippines a entraîné, durant l'ère post-Marcos, de grandes réformes de la gouvernance et de la gestion forestières. Un élément particulièrement intéressant de la conception du système de vérification a consisté à adopter une approche participative pour observer l'utilisation des ressources forestières, 
une approche qui inclut la création de comités multisectoriels de protection des forêts (Multisectoral Forest Protection Committees, ou MFPC). Le recours aux MFPC montre comment un processus indépendant multi-acteurs peut être conçu et utilisé comme un élément structurel majeur qui bénéficie d'un haut niveau d'appropriation, de transparence et de crédibilité dans un système de vérification forestière autorisé par l'Etat. Toutefois, l'expérience des Philippines illustre également les pièges de l'institutionnalisation des MFPC, par exemple la réticence de certaines parties prenantes à recourir aux acteurs non gouvernementaux pour gérer les ressources forestières, ce qui a résulté sur un manque de soutien opérationnel aux MFCP dans certaines régions.

\subsection{Histoire et première mise en place des MFPC}

Au début du $\mathrm{XX}^{\mathrm{e}}$ siècle, environ $50 \%$ de la superficie des Philippines étaient recouverts de forêts (soit 27 millions d'hectares) ; au début des années 1980, il n’en restait plus que 10 millions. Ce recul rapide de la forêt a résulté de divers facteurs, notamment l'exploitation de concession, l'exploitation illégale, la réaffectation des sols et la culture itinérante, ou " kaingin ». Le fait que le gouvernement et le grand public reconnaissent qu'il fallait faire quelque chose contre la perte de ressources forestières a coïncidé avec l'instauration de formes plus participatives et décentralisées de gouvernance des ressources naturelles, en particulier après la révolution de 1986. Le département de l'Environnement et des ressources naturelles (DENR) a ainsi dévolu certains pouvoirs en matière de gestion et de planification des ressources forestières aux unités locales de gouvernement (Local Government Units ou LGU) des provinces, des municipalités et des communes (Cruz and Tapia 2005).

En 1975 déjà, le décret présidentiel n ${ }^{\circ} 705$, également appelé Code de la réforme forestière, lançait une initiative multisectorielle de protection des forêts. A cette époque cependant, peu d'initiatives étaient réellement de nature multisectorielle (Banque Mondiale 2008). Même si certaines impliquaient des acteurs non gouvernementaux, dont des membres du clergé catholique (FAO 2008), la plupart consistaient à créer des comités ad hoc chargés des mesures d'exécution et composés d'autorités militaires ou policières au niveau national et local. En 1992, le Crédit d'ajustement du secteur de l'environnement et des ressources naturelles (ENR-SECAL) a fourni au DENR l'occasion de piloter un modèle MFPC basé sur une large participation des parties prenantes. Dans le cadre de ce programme, 15 premiers MFPC ont été organisés, cinq au niveau régional, cinq au niveau provincial et cinq au niveau municipal.

Ces comités avaient vocation à fournir au DENR un réseau d'organisations publiques, privées et non gouvernementales pour l'appuyer dans ses activités de protection et de gouvernance des forêts. On espérait qu'il serait plus facile de menacer ou de soudoyer un groupe mixte de défenseurs de l'environnement (responsables d'ONG, prêtres et responsables civils), qu'une personne individuelle (Ganapin 2001).

Le programme ENR-SECAL a pris en charge la rémunération des consultants et les dépenses du personnel du DENR ayant fourni la plus grande partie du travail initial 
de facilitation et de mobilisation ; il a en outre financé la préparation du Manuel de procédures pour les MFCP, en 1994. Il a également contribué au premier congrès national des MFPC en 1995, à l'évaluation nationale des MFPC en 1997, aux coûts d'assistance technique, à l'acquisition d'un avion et de bateaux de patrouille et de surveillance, ainsi qu'au développement des moyens de subsistance dans certaines communautés vivant en altitude.

En 1994, Fidel V. Ramos, président de l'époque, a reconnu l'utilité du travail des MFPC et ordonné leur institutionnalisation au sein du DENR. D’autres MFPC furent alors créés par le DENR dans tout le pays, en collaboration avec diverses organisations sectorielles. Au plus fort du programme financé par la Banque Mondiale, plus de 300 MFPC furent créés aux Philippines.

\subsection{Mandat et structure}

Le rôle des MFPC dans la gestion des ressources forestières aux Philippines consistait et consiste (lorsqu'ils sont encore opérationnels) à assurer la surveillance et l'observation des concessions industrielles et des activités de gestion forestière par les communautés. Les conclusions en matière de conformité sont transmises aux autorités du DENR chargées des mesures d'exécution. L'autorité nécessaire à cette fin a été conférée par le décret présidentiel original de 1975 et les réglementations et directives opérationnelles subséquentes promulguées et rédigées par le DENR dans les années 1990.

À l'origine, les MFPC devaient se composer de représentants du gouvernement national, du personnel du DENR, des LGU, des forces militaires et policières, de la société civile, des médias et d'ONG (voir Figure 14.1). Les consultants du programme ENR-SECAL ont donc nommé des représentants au sein des diverses entités qui composent les MFPC. Lesquels représentants devaient ensuite rendre compte à leur propre organisation. Au plus fort du programme, à la fin des années 1990, et avec la création de la Fédération nationale des MFPC (au sein de laquelle les comités pouvaient partager leurs problèmes et les enseignements tirés), la structure des MFPC opérait essentiellement sur quatre niveaux (national, régional, provincial et municipal).

Malgré la diversité de leurs tâches, selon leur échelle opérationnelle, tous les MFCP avaient des fonctions de base communes. Celles-ci allaient de l'observation en réseau des activités illégales d'exploitation forestière à la promotion de la sensibilisation aux activités environnementales/de protection des forêts et à la fourniture de nouveaux moyens de subsistance, en particulier pour les habitants des communautés d'altitude qui dépendaient de l'exploitation illégale et autres activités illicites liées au secteur forestier. Les opérations du programme des MFPC, telles que décrites dans le Manuel des procédures d'exploitation (MPE) de la Banque Mondiale, incluaient les tâches suivantes : l'examen et la mise en œuvre des politiques; l'évaluation des opérations et des activités de protection des forêts existantes ; l'identification des zones critiques ; la surveillance et l'observation des forêts, la saisie et la confiscation des produits illégaux ; les campagnes d'information et d'éducation ; le développement de moyens de subsistance (Cruz et Tapia 2005). 


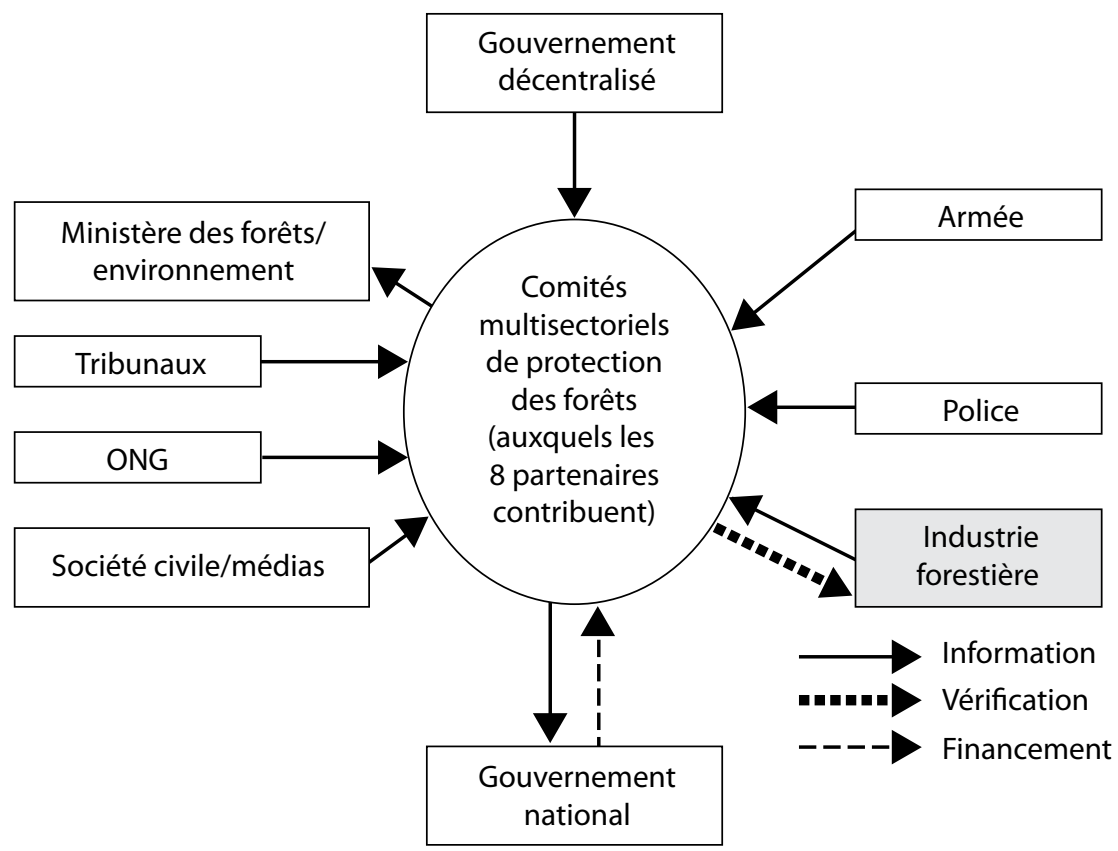

Figure 14.1 Composition des MFPC

Alors que les MFPC en tant que tels n'ont jamais disposé d'un pouvoir d'exécution légalement mandaté, les organisations qui y étaient représentées l'ont exercé, entre autres le DENR, les LGU, diverses unités militaires et la police. Les représentants de ces entités transmettaient à leur organisation respective les informations collectées lors des activités coordonnées du MFPC et informaient également le reste des membres du MFPC des activités et initiatives de ces organisations. Ainsi, les efforts des différents groupes pouvaient, en théorie, être coordonnés de manière plus efficace et les synergies maximisées. Divers MFPC ont mis sur pied des mécanismes d'exécution via la coopération de leurs organisations respectives au niveau local, ce qui consistait par exemple à effectuer des contrôles routiers pour mettre un terme au transport illégal de bois ou à organiser des missions de terrain pour stopper les activités d'exploitation illégale.

\subsection{Rapport avec le DENR et les LGU}

Les MFPC ont toujours eu vocation à soutenir, plutôt qu'à remplacer, les systèmes officiels de contrôle du DENR et de son Bureau de gestion forestière mandatés par le gouvernement. Le DENR est l'agence publique principalement responsable de la gestion des forêts aux Philippines, y compris de l'exécution et de l'émission de tous les contrats et permis liés à l'utilisation des ressources en bois dans le pays. Ayant remplacé l'Accord de licence sur le bois (TLA) auparavant accordé aux concessions industrielles, ils incluent désormais l'Accord de gestion des forêts par les communautés (CBFMA), l'Accord sur l'exploitation industrielle des forêts (IFMA), l'IFMA socialisé (SIFMA) et l'Accord de gestion par la communauté des ressources des zones protégées (PCBRMA). 
Se trouvant sous le niveau national, la structure du DENR est appuyée par les Bureaux provinciaux des ressources naturelles (PENRO) et leurs pendants au niveau communautaire (CENRO). Dans les années 1990, lorsque le modèle MPFC fut déployé, les bureaux PENRO et PENCO et le personnel des organismes d'exécution furent directement chargés de la mise en œuvre des politiques, des programmes, des projets et des activités du DENR ainsi que de l'application des lois et règlements sur l'environnement et les ressources naturelles aux niveaux provincial et communautaire. Dans ce cadre de gouvernance forestière, les LGU partagent avec le DENR la responsabilité de la gestion durable et du développement des ressources forestières dans leur juridiction territoriale (DENR 1998).

\subsection{Performance}

\section{Impacts sur l'exploitation illégale}

Les expériences des quelque 300 MFPC créés durant le programme ENR-SECAL (1992_ 1999 environ) ont varié en matière de protection des forêts et de mesures d'exécution. Certains comités ont eu plus de succès que d'autres, en grande partie en fonction des ressources financières et logistiques à disposition, de la masse critique des individus et des organisations préoccupés par la protection des forêts et souhaitant participer aux activités des MFPC, et du niveau de soutien de la part des LGU. C'est au plus fort du programme ENR-SECAL, lorsque le soutien financier et technique était abondant, que les MFPC furent les plus efficaces dans la signalisation et le démantèlement des opérations d'exploitation interdites, à grande échelle comme à petite échelle. Lorsque le programme a pris fin, nombre de MFPC ont eu du mal à exécuter les activités nécessitant des dépenses (déplacements, communication et participation à des ateliers), surtout au niveau fédéral.

Même si certaines parties prenantes avaient au départ hésité à accepter l'implication d'acteurs non gouvernementaux dans les activités de gestion forestière, cet avis changea lorsque l'avantage de recourir à de telles personnes devint évident et que la culture gouvernementale évolua. Dans les régions où les MFPC réussissaient le mieux dans leurs campagnes contre l'exploitation illégale, on observait souvent une réelle détermination de la part des autorités exécutives locales chargées de faire appliquer les lois et les réglementations forestières, conjuguée à la présence d'ONG, de médias et de groupes religieux actifs.

Certains MFPC, comme ceux de la région 2 (nord-est de Luçon) et de la région 11 (sud de Mindanao) sont parvenus à poursuivre leurs activités après la fin du programme ENR-SECAL, grâce au soutien d'agences membres (gouvernementales et non gouvernementales) et de particuliers. Ces régions se caractérisent par l'appui politique et financier d'une société civile dynamique, des médias solides, un engagement actif de l'Église et un fort soutien de la part des dirigeants de LGU (Cruz et Tapia 2005). Dans les régions où le soutien de la société civile et des LGU est atone, les quelques MFPC qui ont survécu n'ont pas accompli de grandes choses. 


\section{Impacts en faveur des pauvres}

Il était communément admis qu'il fallait compléter les mesures d'exécution par le développement de nouveaux moyens de subsistance, tant pour maintenir hors des forêts les communautés dépendantes de la forêt que pour amortir les effets provoqués par le renforcement des activités de répression sur les exploitants illégaux opérant à petite échelle. Certains MFPC ont été en mesure d'aider les communautés à entrer en contact avec des donateurs et des services d'assistance technique afin d'adopter des moyens de subsistance non basés sur la foresterie. Mais dans la plupart des cas, les MFPC ne disposaient même pas de ressources pour proposer un plan d'amélioration des moyens de subsistance des communautés locales.

Les gains tirés de l'arrêt des opérations d'exploitation illégale et de transport de produits forestiers illégaux sont devenus difficiles à maintenir, car les MFPC étaient tout simplement incapables de fournir une assistance digne de ce nom aux communautés dépendantes de la forêt pour qu'elles développent de nouveaux moyens de subsistance viables.

\section{Faiblesses de conception et de mise en œuvre du cadre}

De multiples facteurs expliquent le succès et la pérennité mitigés du modèle MFPC :

- Manque de soutien de la part de certains membres du personnel de terrain du DENR. Malgré la promotion du modèle MFPC par les hauts responsables du DENR, il existait une culture institutionnelle qui voulait que la gestion des ressources forestières du pays soit de la responsabilité du ministère et que le public n'y joue aucun rôle. Labsence de soutien qui a en résulté a créé des tensions, notamment au regard du rôle réel des MFPC. Avec le recul, la direction du DENR reconnaît qu'il aurait fallu en faire plus pour éduquer le personnel de terrain et expliquer pourquoi le modèle MFPC avait été adopté, afin de s’assurer de son soutien.

- Expansion rapide sans formation adéquate. Le déploiement rapide du modèle MFPC, sans formation suffisante sur les rôles, responsabilités et normes opérationnelles de ces comités a conduit à une interprétation incohérente au niveau local, par les LGU, le personnel du DENR et les autres participants, ainsi qu'à une certaine confusion lors de leur mise en œuvre.

- Perte d'indépendance opérationnelle. Il a été signalé que certains MFPC avaient été "pris en otage " et contrôlés par divers groupes d'intérêt dans le pays, tombant sous l'influence de patrons belliqueux d'exploitations illégales et se retrouvant même directement impliqués dans des activités illégales (Acosta 2008).

- Impacts négatifs non souhaités sur les communautés dépendantes des forêts. Les impacts négatifs de certains MFPC sur les acteurs fragiles du secteur doivent être appréhendés dans le contexte historique. Depuis la période coloniale jusqu'au début des années 1980, les politiques forestières des Philippines avaient encouragé une approche de gestion des forêts très régulée, contrôlée au niveau central et biaisée vers l'industrie. Ces politiques plaçaient l'accès et le contrôle des ressources forestières du pays aux mains de l'élite au pouvoir, laquelle disposait des moyens et des relations politiques 
permettant d'exploiter ces ressources de manière commerciale pour son propre bénéfice. La majorité illettrée et moins privilégiée - notamment la plupart des communautés d'altitude - n'avait en général pas le droit de profiter des avantages potentiels des ressources forestières (De Guzman 1993). Dans ce contexte, il n'est pas surprenant que les MFPC aient eu une influence injustifiée sur les petits opérateurs et les communautés indigentes ; en matière de respect des lois, ces groupes étaient des cibles plus faciles que les concessions plus grandes contrôlées par les puissantes élites, lesquelles bénéficiaient souvent d'une immunité grâce aux moyens dont elles disposaient déjà, tant en termes d'argent que de pouvoir.

\subsection{Statut actuel et futurs rôles des MFPC aux Philippines}

La suppression progressive de grandes concessions forestières (entamée au début des années 1990), associée à l'institutionnalisation des Accords de gestion des forêts par les communautés (CBFMA) et autres instruments fonciers similaires a considérablement modifié le paysage de la gestion et de l'utilisation des forêts aux Philippines. La gestion communautaire et les approches comparables sont désormais plus courantes que la gestion par concession. Par conséquent, l'application des lois forestières est désormais principalement axée sur l'observation des activités d'utilisation de la forêt par les communautés, bien que la surveillance des quelques grandes concessions restantes se poursuive.

Malgré ces changements de priorité dans l'application des lois forestières, les principales contraintes à une application efficace de la réglementation demeurent les mêmes que par le passé. Ces contraintes concernent la capacité inadaptée du gouvernement à réellement appliquer les lois existantes, un manque de programmes permettant de créer un environnement socio-économique propice à une gestion durable de la forêt, et une population accrue demeurant largement dépendante des ressources forestières pour s'en sortir au jour le jour. L'écart entre les ressources disponibles (humaines et financières) et les actions nécessaires est simplement trop grand pour permettre une application réelle des lois. Le personnel dont dispose actuellement le DENR (environ un garde forestier pour 4000 hectares) est handicapé par un appui logistique et juridique insuffisant pour le motiver et lui permettre d'appliquer les lois et réglementations forestières. Un plan en cours de rationalisation du gouvernement, décidé par le décret $n^{\circ} 366$ du 4 octobre 2004, devrait réduire d'environ $40 \%$ l'emploi dans le secteur public lorsqu'il sera entièrement mis en œuvre (Acosta 2008). De telles compressions de personnel ne feront que réduire des ressources humaines déjà limitées allouées aux mesures de conformité et d'exécution des lois forestières.

Dans de telles conditions, les personnes responsables de la surveillance et de la conformité dans le secteur forestier peuvent facilement succomber à la corruption et aux abus de pouvoirs. Ceci est compliqué par des conditions économiques difficiles dans les zones rurales, où les communautés locales ne peuvent se défaire d'une forte dépendance aux ressources forestières pour vivre. 
L'absence de moyens alternatifs de génération de revenus, conjuguée à une hausse continue de la population, n'a fait que renforcer cette dépendance, alors que la collecte de ressources forestières est souvent effectuée en violation des lois et réglementations existantes.

Surmonter les obstacles à l'application véritable de la loi aux Philippines nécessitera par conséquent une approche duale qui améliore les mécanismes de gouvernance pour la conformité et l'application des lois forestières, tout en facilitant l'amélioration des conditions économiques des communautés dépendantes de la forêt, grâce au développement de nouveaux moyens de subsistance. C'est dans ce contexte complexe et difficile que le retour de l'approche MFPC et son institutionnalisation sont envisagés.

Le nombre de MFPC toujours opérationnels aujourd'hui est incertain du fait de l'inactivité de la Fédération nationale des MFPC, pourtant censée faciliter la coordination de tous les MFPC du pays. Cependant, le potentiel des MFPC en tant que mécanisme de soutien efficace en matière de conformité au droit forestier est suggéré par l'existence de certains d'entre eux dans des régions du pays où il existe localement un appui financier, social et politique à leurs activités, des variantes du modèle, comme par exemple l'équipe spéciale sur l'exploitation illégale, créée à Davao del Norte avec l'appui de la collectivité locale, et les brefs, mais non moins importants succès de nombreux MFPC aujourd'hui disparus. Dès lors qu'il existe un soutien de la part des groupes de la société civile et des collectivités locales, cette stratégie se révèle suffisamment viable pour convaincre le DENR de réactiver les MFPC dans des régions choisies.

Le DENR a réaffirmé son engagement à adopter le MFPC comme stratégie de protection et de développement. Ceci est concrétisé dans le Plan directeur révisé pour le développement de la foresterie (FMB-DENR 2005) et dans le Programme général d'action 2005-2010 du DENR pour le secteur forestier. Entre 2005 et 2010, le DENR a pour objectif de soutenir la réactivation et la consolidation de $97 \mathrm{MFPC}$ dans le pays. Il a proposé d'allouer environ 15000 dollars par an et par comité pour aider à financer une partie du coût de leurs opérations.

Le DENR prévoit également de soutenir d'autres initiatives de protection des forêts, notamment une réponse rapide des équipes de travail contre l'exploitation illégale, des conseils de protection multipartites et des équipes d'observation multisectorielles, afin de veiller à ce que les responsables de la mise en ouvre du projet respectent les termes et conditions du contrat y afférant et les autres lois ou réglementations pertinentes (Amaro 2006). Certaines initiatives ont déjà été mises en œuvre, par exemple la création en 2004 de l'équipe spéciale Sagip Kalikasan ("sauvons la nature ") par le décret spécial $\mathrm{n}^{\circ}$ 2004-888 du DENR. Cette équipe spéciale opère 24 heures sur 24 sous la supervision directe du secrétaire du DENR et a la capacité d'intervenir n'importe où dans le pays (Acosta 2008).

La manière dont le retour du modèle MFCP se fera doit encore être entièrement décidée. Le DENR travaille actuellement avec la Banque Mondiale afin qu'une étude approfondie 
soit menée sur l'expérience des MFPC aux Philippines ; elle pourrait débuter au second semestre 2008. Les conclusions seront discutées durant une série d'ateliers multiacteurs et serviront de base à un atelier national au cours duquel un plan d'action pour l'institutionnalisation des MFPC sera élaboré, y compris l'identification des ressources humaines et des équipements financiers nécessaires à long terme.

\subsection{Enseignements}

Pour résumer, les points forts du modèle MFPC incluent une large participation nationale dans la conception et la mise en œuvre du modèle gouvernemental, un niveau élevé d'appropriation et de légitimité du système par le grand public et le gouvernement, des impacts directs sur l'endiguement des activités illégales dans le secteur forestier, l'implication d'une diversité d'acteurs clés au sein du système et un niveau général d'indépendance élevé dans le fonctionnement des MFPC.

Concernant la participation publique dans les activités de vérification, l'étude de cas illustre la nécessité d'un mandat clair des rôles et responsabilités de toutes les parties prenantes et d'un renforcement adéquat des capacités pour assurer une compréhension cohérente de la conception du système dans diverses régions. Il est particulièrement important d'obtenir le ralliement du personnel du DENR à tous les niveaux.

L'efficacité de ce qui est essentiellement un modèle décentralisé de vérification du secteur forestier dépend d'un soutien local appuyé, surtout si le soutien technique, administratif et financier au niveau national est limité. Ainsi, la volonté et l'engagement des ONG et autres groupes civils locaux seront déterminants pour la réussite future des MFPC. C'est également vrai pour les LGU, qui seront les plus susceptibles de fournir les moyens humains et de jouer un rôle de stabilisateur du modèle MFPC, tout en étant une source de financement pour les activités locales.

Toutefois, le soutien local ne suffit pas et un mécanisme de soutien financier à long terme par le gouvernement doit également être défini pour assurer la pérennité des MFPC et leur capacité à agir indépendamment de différents groupes d'intérêt.

Enfin, les procédures opérationnelles du modèle doivent être révisées afin de minimiser les impacts négatifs sur les petits exploitants ou les communautés les plus pauvres. À cet égard, l'expérience suggère également que la mise en ouvre simultanée des programmes de développement dans les régions où opèrent les MFPC augmentera la probabilité d'une meilleure conformité au droit forestier à long terme. 



\section{Chapitre 15}

\section{Vérification de la conformité légale en Indonésie}

Note : Cette étude de cas a été réalisée en août 2008 par Adrian Wells. Elle se base sur Wells, A., Ngadiono et Asycarya, D. 2008 Systems for Verification of Legality in the Indonesian Forest Sector. Etude de cas nationale ${ }^{\circ} 9$ de VERIFOR. ODI, Londres.

Tableau 15.1 Indonésie : quelques statistiques clés

\begin{tabular}{|c|c|c|c|}
\hline Statistiques & & Date & Source des données \\
\hline Population & 222,8 millions & 2006 & $\begin{array}{l}\text { Division de la population } \\
\text { des Nations Unies } 2006\end{array}$ \\
\hline Superficie & 181,2 millions ha & & FAO 2006 \\
\hline Couverture forestière & $48,8 \%$ & 2005 & $\begin{array}{l}\text { Division de la statistique } \\
\text { des Nations Unies } 2006\end{array}$ \\
\hline Propriété publique des forêts ${ }^{1}$ & $99 \%$ & 2000 & FAO 2005 \\
\hline $\begin{array}{l}\text { Production de bois rond } \\
\text { industriel }\end{array}$ & 36,7 millions $\mathrm{m}^{3}$ & $\begin{array}{l}\text { Moyenne } \\
2001-2005\end{array}$ & OIBT 2005 \\
\hline $\begin{array}{l}\text { Emplois dans le secteur forestier } \\
\text { formel }\end{array}$ & 549000 & $\begin{array}{l}\text { Moyenne } \\
1990-2000\end{array}$ & FAO 2004 \\
\hline $\begin{array}{l}\text { Contribution du secteur forestier } \\
\text { au PIB }\end{array}$ & $3,4 \%$ & $\begin{array}{l}\text { Moyenne } \\
1990-2000\end{array}$ & FAO 2004 \\
\hline $\begin{array}{l}\text { Valeur des exportations de } \\
\text { produits forestiers }\end{array}$ & 4,2 milliards USD & $\begin{array}{l}\text { Moyenne } \\
1990-2000\end{array}$ & FAO 2004 \\
\hline $\begin{array}{l}\text { Principaux marchés } \\
\text { internationaux du bois }\end{array}$ & $\begin{array}{l}\text { Japon, Chine, } \\
\text { Europe, Etats- } \\
\text { Unis, Taiwan, } \\
\text { Corée du Sud }\end{array}$ & 2005 & OIBT 2005 \\
\hline $\begin{array}{l}\text { Indice de perception de la } \\
\text { corruption de } \mathrm{TI} \text { (de } 0 \text { à 10, avec } 0 \\
=\text { le plus corrompu) }\end{array}$ & 2,4 & 2006 & $\begin{array}{l}\text { Transparency } \\
\text { International } 2006\end{array}$ \\
\hline $\begin{array}{l}\text { Indice de développement } \\
\text { humain ( } 0 \text { ou } 1 \text {, avec } 0=\text { très bas) }\end{array}$ & 0,71 & 2004 & PNUD 2006 \\
\hline
\end{tabular}

1 Ce chiffre inclut les régions du domaine forestier national qui sont soumises à des droits coutumiers, bien que le droit forestier existant ne les reconnaisse pas comme des droits privés. Le $1 \%$ de propriété privée est probablement sous-estimé, car il existe de nombreuses plantations forestières, détenues par des petits exploitants, et de zones agroforestières privées, en dehors du domaine forestier national. 


\subsection{Introduction}

L'Indonésie compte pour un tiers des exportations totales de bois tropical-deuxième pays au monde après la Malaisie (www.globaltimber.org.uk). Avec un degré élevé d'illégalité, le secteur forestier du pays est devenu la priorité d'un grand nombre d'initiatives de donateurs, d'ONG et du secteur privé visant à promouvoir le commerce légal et durable. Ce chapitre décrit l'influence de ces initiatives sur le système de vérification qui évolue en Indonésie, notamment l'introduction d'une norme de légalité et les réformes en cours des audits externalisés des concessions de bois industriel, dans une tentative de s'attaquer à l'illégalité et d'augmenter la viabilité.

L'Indonésie a été le premier pays à formuler une norme de vérification du bois légal : un pas vers une approche durable. Il est également intéressant de noter que le pays tente d'introduire une plus grande indépendance dans ses systèmes de vérification obligatoire. Cela montre que des réformes de la gouvernance élargie (qui impliquent la définition des droits légaux sur les terres forestières, une application plus efficace des lois et des mesures de régulation des capacités de transformation existantes) sont essentielles à une vérification efficace. L'Indonésie illustre aussi les défis d'une décentralisation politique et administrative rapide pour la vérification forestière. Contrairement au système fédéral malaisien, l'Indonésie conserve une approche uniforme de la vérification des activités forestières, principalement axée sur l'octroi par le gouvernement central de licences aux concessions et aux industries du bois.

Après une brève description du secteur forestier, des facteurs et des mandats légaux de la vérification en Indonésie, ce chapitre évoquera les divers composants du système de vérification dans ce pays (couvrant l'administration forestière, la certification de la conformité obligatoire et l'enregistrement des exportations). S'en suivra une discussion sur les points forts et les points faibles actuels ainsi que sur les possibles réformes d'appui. Un résumé des enseignements tirés sera donné en guise de conclusion.

\subsection{Contexte}

Environ $70 \%$ des terres indonésiennes constituent le domaine forestier national, lequel est directement administré par l'Etat. La foresterie industrielle domine la politique et la pratique depuis des décennies. Cependant, bien que dans les années 1990, le secteur forestier ait généré chaque année entre 7 et 8 milliards de dollars de recettes en devises, la grande pauvreté existe toujours dans les régions forestières. Selon le Centre d'études économiques et sociales du pays (CESS), la proportion de foyers et de villages pauvres dans et autour des régions forestières est $10 \%$ plus élevée que dans les régions non forestières (CESS-ODI 2005).

Depuis la chute du régime de Suharto en 1998, la contribution du secteur forestier industriel à la croissance et à la génération de revenus a décliné. Cela s'explique par une réduction de la possibilité annuelle de coupe, due à la baisse de la ressource, par une récente vague d'opérations de répression contre l'exploitation illégale et par une concurrence de marché accrue avec d'autres pays transformateurs comme la Malaisie 
et la Chine. Cela a également entraîné une importante surcapacité dans le secteur de la transformation.

Depuis, le gouvernement indonésien a fait de la foresterie l'un des trois secteurs prioritaires, avec la pêche et l'agriculture, de la revitalisation économique. Les priorités actuelles incluent une croissance dans les plantations d'arbres industrielles et communautaires afin de combler le considérable déficit d'approvisionnement autrement comblé par l'exploitation illégale. Toutefois, cela se confronte à un certain nombre de grands défis politiques.

En premier lieu, de grandes régions du domaine forestier national sont à présent fortement dégradées ou déboisées. Parallèlement, environ 1,5 million d'hectares de forêts situés sur des terrains privés ou titrés en dehors du domaine de l'Etat produisent désormais plus de 6 millions de $\mathrm{m}^{3}$ de bois par an. Ce chiffre est à comparer avec la possibilité de coupe en $2006: 8,15$ millions $\mathrm{m}^{3}$ pour les 170 concessions commerciales, qui couvraient 6,7 millions d'hectares, dont seule une faible proportion est toujours active. Cela a entraîné des visites aux populations locales, du moins dans les zones du domaine forestier qui ne sont plus productives, afin de leur octroyer des droits privés pour planter et gérer des arbres. D’après les estimations, modifier de $10 \%$ une région allouée aux cultures d'arbres de petits propriétaires générerait environ 850 millions de dollars de valeur économique et quelque 500000 emplois (Brown et al. 2006).

Deuxièmement, seuls $10 \%$ du domaine forestier national ont été entièrement classés selon les procédures (qui comprennent la notification aux communautés locales). Ces exigences représentent un sérieux défi aux actuels détenteurs de licence. En l'absence de données cadastrales complètes, l'étendue exacte des droits de propriété en forêt demeure floue. Dans le même temps, la loi 41/1999 sur les forêts place des régions sous le contrôle des communautés de droit coutumier (hutan adat), non pas comme des forêts privées (hutan hak), mais simplement comme une catégorie du domaine forestier national. Cela reflète le fait qu'il n'existe actuellement aucun mécanisme d'enregistrement des droits collectifs, malgré la reconnaissance des systèmes de droit traditionnel dans la loi agraire de 1960 (Contreras-Hermosilla et Fay 2005). En conséquence, d'importantes portions du territoire forestier national sont disputées par les populations locales.

Troisièmement, la répartition relative de l'autorité entre le gouvernement central et les autorités locales est fortement contestée. L'introduction de la décentralisation politique et administrative en 1999 a fortement accru l'autorité des gouvernements de district et des provinces bénéficiant d'une autonomie spéciale (Aceh et Papouasie) en matière de ressources naturelles. Toutefois, des inquiétudes selon lesquelles les licences accordées aux gouvernements locaux accéléraient la conversion des terres et l'exploitation illégale ont entraîné une recentralisation du contrôle, le plus récemment en vertu de la régulation PP3 de 2008. La plupart des catégories de licence relèvent désormais du ministère des forêts et les autorités locales ne peuvent plus octroyer de licences de terrain qu'à des fins de reforestation communautaire ou de services environnementaux. 


\subsection{Facteurs sous-tendant les systèmes de contrôle et de vérification des forêts}

Il existe plusieurs grands facteurs derrière la vérification du secteur forestier en Indonésie.

L'une des principales préoccupations du gouvernement a été de redynamiser la filière bois au lendemain de la crise financière de 1997. Cela s'est traduit par la suppression des concessions industrielles sous-productives ou inactives, suite à des audits obligatoires de conformité menés par des organismes d'évaluation indépendants (LPI), en vertu des normes et procédures établies par le mécanisme de certification volontaire de la gestion durable des forêts (GDF) mis en place par l'Institut indonésien de l'écolabel (LEI).

Un autre facteur clé a été l'impact des campagnes menées à l'étranger par la société civile pour s'attaquer à l'exploitation illégale galopante sur les marchés indonésiens, qui aggrave la baisse de productivité et la guerre des prix avec les producteurs comme la Chine. Les campagnes les plus remarquées ont inclus le suivi sur le terrain par l'alliance des ONG EIA et Telapak ainsi que les efforts de Greenpeace pour faire pression sur les consommateurs européens ( complices de crimes ") et des Amis de la Terre (les "Dirty Five »). Ces campagnes ont permis d'améliorer les processus d'approvisionnement des acheteurs et de réformer des politiques d'approvisionnement public de certains des principaux clients européens de l'Indonésie, notamment le RU.

Les campagnes menées par la société civile ont également débouché sur :

- Un protocole d'accord avec le RU sur les mesures à prendre contre l'exploitation illégale. Cette initiative a inauguré le travail de formulation d'une norme de conformité légale.

- Des protocoles équivalents avec d'autres clients, notamment les Etats-Unis, ainsi qu'un travail politique facilité par la Banque Mondiale sur les " 10 mesures " pour la prévention, la détection et la suppression efficaces des exploitations illégales. Ces mesures sont les suivantes : (i) créer la responsabilité, (ii) définir la légalité, (iii) améliorer le suivi, (iv) archiver les données, (v) assurer la transparence, (vi) s'attaquer à la surcapacité de la filière de transformation du bois, (vii) promouvoir le commerce légal du bois, (viii) créer une capacité d'application de lois, (ix) amender les lois et (x) poursuivre et juger les criminels forestiers.

- Un ensemble d'initiatives commerciales privées assorties de leurs propres normes de vérification de l'origine légale (VOL) et de la conformité légale (VCL), notamment la Tropical Forest Foundation (TFF) et le Global Forest Trade Network (GFTN) du WWF.

- Une pression sociétale plus importante pour agir. À son tour, cela a résulté en 2004 sur une décision présidentielle ordonnant des mesures répressives coordonnées dans toute l'Indonésie (Operasi Hutan Lestari ou OHL). 
Le facteur de changement le plus récent est une décision de l'Union Européenne d'entamer le dialogue avec le gouvernement indonésien autour d'un possible Accord volontaire de partenariat (AVP) sur l'application des réglementations forestières, la gouvernance et les échanges commerciaux (FLEGT en anglais). Entre autres effets, cela a entraîné la décision d'adopter un système d'assurance de la légalité du bois couvrant une norme de légalité, la chaîne de traçabilité, la vérification et le suivi indépendant, en tant que politique nationale.

\subsection{Mandats de vérification}

La loi 41/1999 sur les forêts et la loi 32/2004 sur la décentralisation fournissent le mandat légal global de suivi et de vérification dans le secteur forestier. L'entrée en vigueur d'une réglementation en vertu de chacune de ces lois prévoit la supervision et le contrôle de la mise en œuvre des politiques forestières. Ces politiques incluent la loi PP6/2007 sur la gestion des forêts (telle qu'amendée par la PP3/2008), la PP38/07 sur la répartition de l'autorité entre le gouvernement central et les autorités locales et la PP41/2008 sur la structure du gouvernement local.

Spécifiquement, la loi PP6/2007 (article 125) prévoit le suivi (collecte de données de routine) et l'évaluation (évaluation de la planification de la gestion et de l'utilisation des forêts). La loi PP41/2008 ajoute qu'il s'agit d'une responsabilité simultanée du gouvernement central et des autorités provinciales et de district, le premier étant également chargé de définir la norme.

Un ensemble d'ordres administratifs (décrets ministériels) fournit également une orientation sur la répartition des rôles et des responsabilités en matière de suivi et d'évaluation, dans trois domaines :

- Le système d'administration des bois (PUHH), notamment la collecte des droits de coupe (PSDH) et des taxes de reboisement (DR). Ces décrets incluent des recommandations détaillées sur les responsabilités du gouvernement local en matière d'inventaire avant exploitation, l'approbation des quantités de coupes annuelles, le rapprochement des statistiques de production avec les inventaires avant exploitation et la gestion des autorisations de transport.

- La certification de conformité, qui requiert l'audit périodique des concessions de forêts naturelles et artificielles, ainsi que des industries primaires liées au bois, par rapport aux critères obligatoires de GDF et aux directives d'évaluation. La responsabilité des audits est partagée entre un groupe de travail (Pokja) du ministère des Forêts et 16 organismes d'évaluation indépendants (LPI) agréés par le ministère.

- L'enregistrement obligatoire des exportations (ETPIK), qui exige la confirmation préalable par le Bureau indonésien de revitalisation de l'industrie forestière (BRIK) qu'un exportateur obtient son bois de sources légales. 


\subsection{Systèmes de vérification}

\section{Vue d'ensemble}

Les trois composants du système indonésien de vérification des forêts (administration du bois, certification obligatoire de conformité et enregistrement des exportations) fonctionnent tous sous l'autorité déléguée du ministère des Forêts et sont résumés Figure 15.1. Dans ce système, les audits de conformité de chaque titulaire de licence et les autorisations d'exporter accordées par le BRIK examineront les données d'observation produites par l'administration des bois afin d'en vérifier la conformité.

Le Tableau 15.2 compare le champ d'application et le contenu de ses trois composants. Manifestement, aucune politique ou réglementation seule ne rassemble ces éléments dans un système unique d'assurance de la légalité, ce qui crée certaines lacunes et incohérences. Entre autres, les LPI demeurent de la compétence spécifique du gouvernement central, même si tous les niveaux du gouvernement sont responsables du suivi comme de l'évaluation. Des structures équivalentes d'audit externalisé n'existent pas au niveau du gouvernement local, par exemple pour faciliter la supervision des offices des forêts de districts par les provinces, ou des licences accordées par les gouverneurs ou les chefs de district.

En outre, le système ne fournit pas de supervision tierce qui soit indépendante du ministère ou du secteur. Par exemple, les LPI sont directement accrédités par le ministère des Forêts, lequel évalue ensuite les comptes rendus d'audit. Seules les synthèses de ces comptes rendus sont mises à disposition du public.

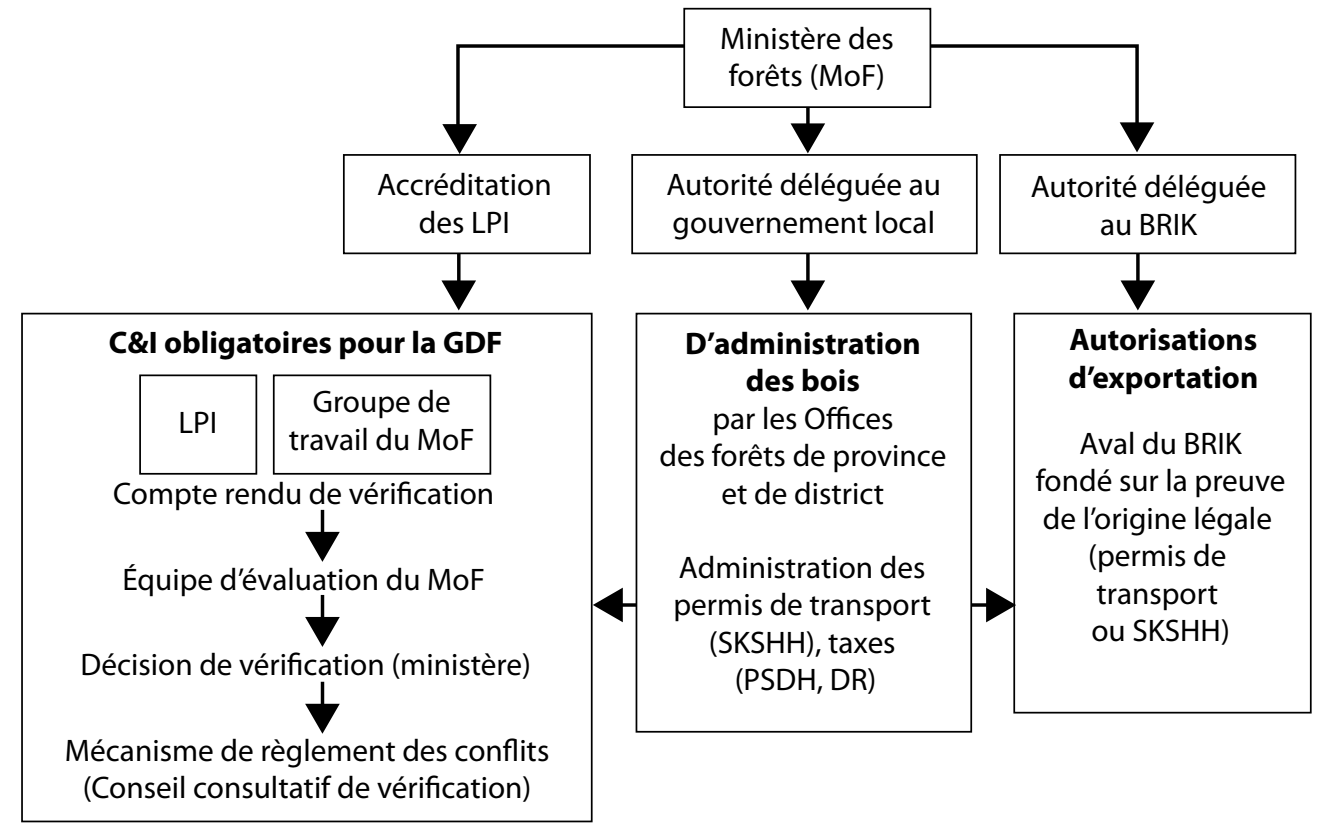

Figure 15.1 Systèmes de vérification des forêts en Indonésie 
Tableau 15.2 Éléments clés du système de vérification des forêts en Indonésie

\begin{tabular}{llll}
\hline 1. Objectif & $\begin{array}{l}\text { Administration du } \\
\text { bois d'œuvre }\end{array}$ & $\begin{array}{l}\text { Autorisations } \\
\text { d'exportation }\end{array}$ & $\begin{array}{l}\text { Audit des titulaires } \\
\text { de licence }\end{array}$ \\
\hline & $\begin{array}{l}\text { Recettes, origine } \\
\text { légale }\end{array}$ & Origine légale & $\begin{array}{l}\text { Recettes, gestion } \\
\text { durable de la forêt, } \\
\text { revitalisation de } \\
\text { l'industrie }\end{array}$ \\
\hline 2. Champ d'application & & Non & Non \\
\hline $\begin{array}{l}\text { Allocation des ressources, } \\
\text { licences }\end{array}$ & Non & Oui \\
\hline Gestion forestière & Non & Non & Non \\
\hline Chaîne de traçabilité & Oui & Oui & \\
\hline
\end{tabular}

\section{Composants}

\begin{tabular}{|c|c|c|c|}
\hline Norme de légalité & $\begin{array}{l}\text { D'administration } \\
\text { des bois (PUHH), } \\
\text { collecte des taxes } \\
\text { et des redevances } \\
\text { (PSDH, DR) }\end{array}$ & $\begin{array}{l}\text { Origine légale } \\
\text { basée sur les } \\
\text { permis de } \\
\text { transport (SKSHH) }\end{array}$ & $\begin{array}{l}\text { Normes obligatoires } \\
\text { de GDF }\end{array}$ \\
\hline Systèmes d'information & $\begin{array}{l}\text { Inventaires avant } \\
\text { exploitation, } \\
\text { données de } \\
\text { production, } \\
\text { données spatiales } \\
\text { (délimitations, } \\
\text { blocs de coupe) }\end{array}$ & $\begin{array}{l}\text { Données de } \\
\text { production (base } \\
\text { de données } \\
\text { interne) }\end{array}$ & $\begin{array}{l}\text { Données provenant } \\
\text { de la surveillance de } \\
\text { routine }\end{array}$ \\
\hline Suivi et exécution & Oui & Oui & Non \\
\hline Audit & $\begin{array}{l}\text { Oui, par le } \\
\text { ministère des forêts }\end{array}$ & Non & $\begin{array}{l}\text { Oui, par des } \\
\text { auditeurs agréés } \\
\text { par le ministère des } \\
\text { forêts }\end{array}$ \\
\hline Supervision externe & Non & Non & Non \\
\hline Décision de vérification & Ministère des forêts & BRIK & Ministère des forêts \\
\hline Règlement des conflits & Non & Non & $\begin{array}{l}\text { Oui (Conseil de } \\
\text { vérification) }\end{array}$ \\
\hline \multicolumn{4}{|l|}{ 4. Indépendance } \\
\hline $\begin{array}{l}\text { Séparation des pouvoirs } \\
\text { (tiers parti, indépendant) }\end{array}$ & Non & Non & Non \\
\hline Sous-traitance & Non & Oui & Oui \\
\hline Financement & $\begin{array}{l}\text { Producteurs } \\
\text { (droits) }\end{array}$ & $\begin{array}{l}\text { Exportateurs } \\
\text { (taxes) }\end{array}$ & Ministère des forêts \\
\hline
\end{tabular}




\section{Administration des bois}

Ladministration routinière des bois (et en particulier l'administration des permis de transport SKSHH) par le gouvernement local alimente les audits de conformité du ministère des forêts et des LPI, ainsi que les autorisations d'exporter octroyées par le BRIK. L'administration des bois reste une activité papier et comprend cinq grandes étapes résumées à la Figure 15.2.

\section{Certification obligatoire de la conformité}

La certification obligatoire de la conformité a été introduite dans une tentative de revitaliser les filières du bois en berne - victimes depuis la crise financière de 1997 de l'endettement, d'une guerre des prix accrue et d'une baisse des ressources. En 2002, le ministère des forêts de l'époque a dû se prononcer sur le retrait ou le prolongement d'une centaine de licences d'exploitation du bois. Il fut décidé de ne prolonger que les licences capables de satisfaire aux normes de GDF.

Plusieurs décrets furent ensuite émis, introduisant des normes obligatoires de conformité et des directives d'évaluation pour :

- Les concessions de forêts naturelles (décrets 4795/Kpts-II/2002 et 208/Kpts-II/2003 sur la GDF au niveau des unités de gestion) ;

- Les concessions de forêts plantées (décrets 177 et 178/Kpts-II/2003 sur la GDF au niveau des unités de gestion);

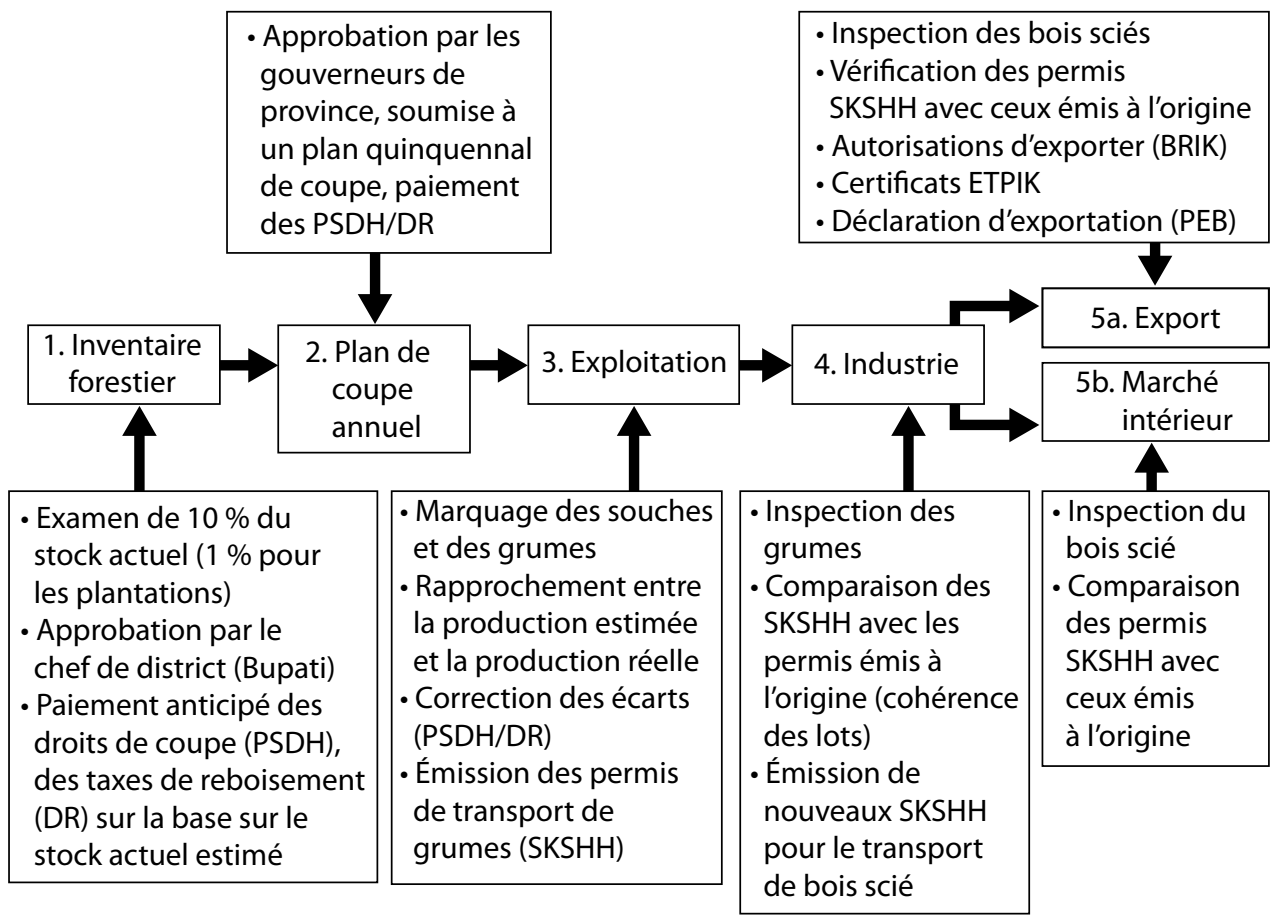

Figure 15.2 D'administration des bois (PUHH) en Indonésie 
- Les industries de transformation du bois ayant une capacité établie de plus de $6000 \mathrm{~m}^{3}$ /an (décrets 6884/KPTS-II/2002 et 325/Kpts-II/2003 sur la légalité de l'approvisionnement en bois, entre autres).

Le ministère des Forêts a également introduit un système d'audit externalisé en vertu du décret $(\mathrm{SK}) \mathrm{n}^{\circ}$ 6420/Kpts-II. Cela a encouragé et permis d'adapter les processus indépendants de certification des forêts établis par l'Institut indonésien de l'écolabel (LEI). Ce système d'audit externalisé se compose :

- D’organismes d'évaluation indépendants (LPI) agréés par le ministère des Forêts pour réaliser l'audit des concessions et des industries forestières. Chaque LPI se compose d'une équipe d'évaluation de terrain et d'un comité technique qui évalue les données. Les LPI sont agréés sur la base de leur capacité administrative et technique et de leur indépendance. En particulier, ces organismes ne peuvent pas posséder de parts dans les sociétés forestières évaluées, que ce soit au niveau individuel ou institutionnel. Ils ne doivent pas non plus avoir fourni une forme quelconque de services d'expertise aux sociétés évaluées, au niveau institutionnel ou individuel. Le coût des audits menés par les LPI est pris en charge par le ministère des forêts les trois premières années, après quoi, c'est aux sociétés évaluées d'assumer ces coûts.

- D’une équipe d'évaluation du ministère des forêts chargée d'évaluer les comptes rendus d'audit des LPI et de conseiller le ministère sur une décision de vérification appropriée en matière de performance des titulaires de licence. Ayant également la responsabilité de sélectionner les membres du Conseil consultatif de vérification (voir ci-après), cette équipe d'évaluation contribue aussi de manière considérable au processus d'audit.

- D’un Conseil consultatif de vérification, c'est-à-dire un conseil de parties prenantes créé par l'équipe d'évaluation pour traiter des conflits relatifs aux rapports d'audit et aux décisions de vérification.

Le ministère des forêts a déclaré 16 LPI " aptes " à auditer les industries forestières en matière de normes obligatoires et de directives d'évaluation. En fait, l'ampleur de la tâche signifiait que les LPI devaient partager leur charge de travail avec un Groupe de travail (Pokja) équivalent du ministère des forêts. Sur les 27 concessions forestières auditées durant la première session d'évaluation de 2003, 13 d'entre elles ne respectaient pas les normes exigées.

\section{Autorisations d'exportation}

Dans une nouvelle tentative de revitaliser un secteur morose, les ministères du commerce, de l'industrie et des forêts ont signé le 13 décembre 2002 un décret conjoint (SKB $\mathrm{n}^{\circ} 803 / \mathrm{MPP} / \mathrm{Kep} / 12 / 2002$ et $\left.\mathrm{n}^{\circ} 10267 / \mathrm{Kpts}-\mathrm{II} / 2002\right)$ portant création du Bureau de revitalisation de l'industrie forestière (BRIK). Ce décret stipule que les membres du BRIK doivent être des industries forestières et investit le BRIK de la responsabilité exclusive de vérifier la validité et la cohérence de la documentation relative aux permis de transport (SKSHH) qui attestent de l'approvisionnement légal des filières bois, condition préalable à l'enregistrement des exportations (ETPIK). Le BRIK verse à ses membres une commission mensuelle, en fonction de la taille et du type d'industrie. 


\subsection{Atouts du système et contraintes de vérification}

L'intention politique qui sous-tend le système indonésien de vérification ne peut être discutée. L'introduction de normes obligatoires et de directives d'évaluation, ainsi que l'agrément des auditeurs externalisés ont été nécessaires pour améliorer la gouvernance dans le secteur forestier et discipliner l'industrie. Les audits réalisés par les LPI offrent notamment un moyen de s'attaquer à l'illégalité et de soutenir les titulaires de licence dans leur progression vers une approche durable. Le BRIK aussi constitue une innovation potentiellement importante pour encourager l'industrie à s'autoréguler là ou les ressources gouvernementales de vérification de la chaine de traçabilité sont limitées. Toutefois, les systèmes de vérification existants en Indonésie sont également confrontés à d'importantes contraintes.

\section{Administration papier et ressources limitées pour la supervision}

Le système d'administration des bois (PUHH) actuel est probablement plus un mécanisme de comptabilité des bois qu'un moyen de garantir l'origine légale. Les permis de transport ne fournissent pas toujours d'information sur l'origine ultime d'un arrivage de bois. Il est donc plus difficile de garantir la chaîne de traçabilité pour les transformateurs qui s’approvisionnent auprès de différents intermédiaires. La décision ministérielle (Permen) 55/2006 a instauré la révision du processus d'administration du bois, auparavant régie par le décret ministériel (SK) 126/Kpts-II/2003. L'accent est désormais placé sur l'autorégulation par les sociétés afin de réduire les coûts de transaction. Pour autant, la réglementation ne s'adresse pas aux problèmes de l'insuffisance de la coordination et de la supervision entre les niveaux du gouvernement. Les provinces ne réalisent pas d'audit régulier de la gestion des forêts et de l'administration des bois au niveau des districts. Les districts manquent à leur tour de ressources pour superviser les agents de terrain. Peu d'incitations sont offertes à ces agents; nombre d'entre eux ont des perspectives de promotion limitées et manquent de ressources pour exécuter leurs responsabilités élémentaires.

\section{Manque d'indépendance et de transparence dans les audits du secteur forestier}

Des mesures ont été prises pour garantir l'indépendance des LPI agréés par rapport aux sociétés évaluées. Toutefois, le ministère des Forêts conserve l'entière autorité d'accréditer les auditeurs, d'évaluer les comptes rendus et d'émettre les décisions de vérification. Cela a soulevé des inquiétudes sur la vulnérabilité du système d'audit des LPI à de possibles interférences politiques. À aucun moment le public ne peut accéder au processus d'évaluation ou aux comptes rendus d'audit. Même le Conseil consultatif de vérification, qui joue le rôle de mécanisme de règlement des conflits, demeure sous le contrôle du ministère, puisqu'il est nommé par l'équipe d'évaluation du ministère. Le fait que ce conseil ne se soit guère réuni depuis sa création est peut-être une indication de son manque d'importance perçu. La période de soumission de plaintes est limitée, et celles-ci restent également inaccessibles au public tant qu'une décision n’a pas été rendue. 


\section{Inspections physiques insuffisantes et possibles conflits d'intérêt dans les autorisations d'exporter}

Le BRIK n'est pas parvenu à rassurer certains marchés comme le RU en matière d'approvisionnement légal, principalement parce quaucune inspection physique de grumes ou de scierie n'est prévue dans ses systèmes. Le BRIK s'est contenté de vérifier que les quotas de grumes fixés pour chaque scierie correspondaient aux volumes indiqués sur les permis de transport (SKSHH). Le fait que le BRIK soit lui-même un organisme industriel a fait craindre de possibles conflits d'intérêt. Ainsi, des allégations selon lesquelles le bureau avait agi pour consolider son propre cartel d'exportation via l'imposition de charges illégales a conduit le ministère du commerce à ne plus soutenir l'organisation. Les ministères du commerce, de l'industrie et des forêts ont toutefois accepté de prolonger son mandat actuel jusqu'à fin 2008, dans l'attente d'une décision sur le futur régime d'autorisation d'exporter. En attendant, le BRIK continue de gérer une précieuse base de données sur l'approvisionnement en bois des industries de la filière.

\section{Mesures répressives communes : un frein aux processus de vérification?}

En 2004, le président indonésien a lancé une opération forêts durables (OHL), consistant en une mesure coercitive impliquant des interventions répressives menées conjointement par le $\mathrm{MoF}$ et la police. Cette opération destinée à contrer l'exploitation illégale était en partie une réponse aux données générées par les observateurs de la société civile, comme EIA et Telapak. Toutefois, le manque de référence aux processus de vérification existants par les agences chargées de l'application des lois a sans doute annulé la valeur de ceux-ci en tant que mécanisme de lutte contre l'illégalité dans le secteur.

\section{Impacts sur les pauvres}

Les systèmes de vérification actuels ne réduiront probablement pas la pauvreté qui touche les communautés dépendantes des forêts, du fait des coûts de transaction associés à la conformité aux règles d'exploitation forestière et du manque criant de clarté quant aux droits de la population locale sur les terres et les ressources forestières.

Les gérants de forêts communautaires doivent obtenir de multiples permis pour pouvoir gérer et exploiter le bois. Cela inclut un permis de transport pour chaque expédition de bois. Pour les arbres plantés sur des terrains privés, ce permis doit s'accompagner d'un certificat de propriété - que la plupart des communautés locales n’ont pas. Les coûts sont en outre alourdis par l'imposition de taxes par les autorités locales, en plus des taxes, des commissions et des droits de licence forestiers prélevés par le ministère des Forêts (Multistakeholder Forestry Programme 2006a).

En second lieu, le fait que les communautés locales ne disposent peut-être d'aucune certitude juridique signifie qu'elles sont souvent la cible des opérations de répression. En 2005, les licences d'exploitation par les communautés coutumières (IPKMA) en 
Papouasie ont fait l'objet d'un examen particulier dans le cadre de l'OHL. Ces licences avaient été accordées par le gouvernement provincial de Papouasie, mais furent par la suite considérées illégales par le ministère des forêts. D'un côté, l'OHL est parvenu à empêcher l'utilisation abusive, par des syndicats contrebandiers, de permis IPKMA pour accéder au bois et l'exploiter illégalement. D’un autre côté, les IPKMA constituaient pour les communautés locales le seul moyen légal de tirer un revenu du bois sur les terres coutumières. L'OHL a supprimé toute perspective de gestion communautaire directe du bois provenant des forêts naturelles - une situation qui perdure encore trois ans après (Multi-stakeholder Forestry Programme 2006b).

\subsection{Efforts de consolidation des systèmes de vérification}

Les contraintes rencontrées par l'architecture de vérification indonésienne existante sont officiellement reconnues. Le politique devra également exister pour réformer et renforcer ses divers composants en un seul Système d'assurance de la légalité du bois (TLAS) - en réponse aux négociations autour d'un possible AVP avec l'UE.

Les réformes actuelles et proposées incluent :

- Le développement d'une norme consolidée de vérification de la légalité, qui rassemble l'administration des bois (PUHH), et de la conformité légale par les concessions forestières et les industries de transformation ;

- Le développement de systèmes d'information forestière et de protocoles de transparence associés ;

- Des propositions d'introduction d'une plus grande indépendance dans les systèmes de vérification, y compris l'accréditation indépendante des auditeurs ;

- Des propositions d'institutionnalisation de l'observation indépendante par la société civile ; malgré une contribution cruciale à la lutte contre l'exploitation illégale, cela n’a jusqu'alors pas été intégré dans la conception formelle de vérification.

\section{Développement d'une norme consolidée de vérification de la légalité}

Le cadre réglementaire qui régit le secteur forestier en Indonésie est très complexe. Actuellement, l'administration du bois, la certification de la conformité et les autorisations d'exporter traitent chacune des éléments différents de ce cadre. Les efforts en matière d'examen et de rassemblement des principales conditions nécessaires pour déterminer la conformité légale ont été entamés en 2003, en vertu du protocole RU-Indonésie sur l'exploitation illégale. Ce processus a désormais établi un précédent pour les efforts équivalents entrepris ailleurs dans le monde. La norme actuellement acceptée devra, toutefois, demeurer active si elle veut intégrer des réformes plus récentes de gestion des forêts par les communautés (CBFM), en vertu du règlement PP6/2007.

Le travail d'élaboration de cette norme fut entamé en 2003 par une équipe conjointe au protocole RU-Indonésie/Conservatoire de la Nature (TNC). Les premières normes incluaient des provisions pour le classement ; l'impact social et environnemental ; les 
relations communautaires et les droits des travailleurs ; les lois et règlements relatifs à l'exploitation du bois ; les taxes forestières ; l'identification, le transfert et la livraison des grumes; le traitement et l'expédition du bois.

La consultation des parties prenantes sur cette première version a permis de révéler d'importantes différences d'opinion quant à sa légitimité et son aspect pratique. Tout d'abord, elle ne traitait pas correctement du consentement préalable libre et éclairé (CPLE) et de la classification légale des délimitations de concession (de grandes zones du domaine forestier national font l'objet de débats). Deuxièmement, elle n'a pas encore été testée en matière de forêts plantées gérées par la communauté. Les parties prenantes ont recommandé d'œuvrer davantage à l'harmonisation de la norme avec les lois et règlements concernés. Il fut également convenu que l'Institut indonésien de l'écolabel (LEI) ferait avancer la facilitation de la norme, compte tenu du nombre important de ses membres et de sa forte crédibilité technique.

Le système d'administration des bois (PUHH) du ministère des forêts fut par la suite incorporé à la norme. Le LEI a facilité les efforts de différenciation de la norme entre trois régimes de gestion forestière :

- Les unités de gestion des forêts naturelles, des forêts plantées et des forêts communautaires au sein du domaine forestier national ;

- Les licences accordées pour l'exploitation du bois dans le domaine forestier national, mais non basées sur les unités de gestion;

- Les bois provenant de terrains privés et autres zones en dehors du domaine forestier national.

Une quatrième partie de la norme a trait au transport et à la chaîne de traçabilité depuis la source jusqu'à la première transformation, puis jusqu'à la seconde transformation et/ ou l'exportation.

La norme fut de nouveau testée sur le terrain pour les forêts plantées, les forêts naturelles, les forêts communautaires privées (hutan rakyat) et les industries de transformation primaires et secondaires. Ces essais de terrain ont contribué à ébaucher des directives de vérification. Le consensus sur la version finale de la norme fut obtenu le 22 janvier 2007. Des équipes ad hoc ont depuis été mises sur pied pour le développement de procédures et d'un organe de direction chargé de superviser la norme.

Il reste toutefois de grands défis à relever. L'ONG environnementale indonésienne WALHI s'inquiète du fait que la version actuelle minimise les dispositions sur le classement des forêts et le CPLE. Cela se traduit par le glissement du CPLE vers une simple consultation des communautés locales. WALHI a également souligné l'absence de mécanismes permettant de vérifier la crédibilité de la documentation et réitéré ses critiques quant au fait que la norme ne formule qu'un statu quo, malgré un appel par la chambre haute du Parlement indonésien en faveur de réformes radicales du régime foncier et de la gestion des ressources naturelles (TAP MPR IX/1999). Parallèlement, l'industrie 
craint toujours qu'une norme complexe entraîne une hausse des coûts de production et une perte d'avantage concurrentiel face à des pays dont les normes foncières et relatives aux droits des autochtones peuvent être bien plus laxistes (voir Wells et al. 2006).

\section{Systèmes d'information, transparence du secteur forestier}

D'importantes mesures sont prises pour améliorer les systèmes d'information forestière et servir de base pour vérifier la conformité légale. Ces mesures visent à :

- Établir un Système d'inventaire des ressources forestières (FRIS) avec l'appui technique de l'université du Dakota du Sud et du World Resources Institute (WRI) qui fournissent dans un format exploitable un accès public à des informations fiables, exactes et à jour sur les forêts et les ressources en bois, ainsi que sur les décisions s'y rapportant ;

- Mettre en place des protocoles de transparence régissant l'accès aux informations sur le secteur forestier (une équipe spéciale dédiée à la transparence a depuis été mise sur pied pour développer une politique de divulgation du ministère des forêts) ;

- Relier les données spatiales sur les concessions et les blocs de découpe avec les données sur la consommation des scieries (RPBBI), afin de contribuer à la vérification de la chaîne de traçabilité. Par exemple, le projet de soutien FLEGT de l'UE a passé un contrat avec Helveta (une société privée spécialisée dans les technologies de traçabilité et de contrôle de la chaîne d'approvisionnement) pour aider les ministères des forêts et des finances à intégrer quatre bases de données disparates contenant des informations sur l'inventaire, l'industrie, la cartographie et les recettes du secteur forestier.

\section{Renforcer l'indépendance}

Dans un effort pour renforcer la crédibilité, le ministère des forêts et le LEI ont signé un protocole d'accord pour développer un système d'accréditation indépendante des auditeurs. Il complète les propositions visant à créer un organe de gouvernance distinct pour superviser la norme de légalité. Tous deux sont cruciaux si le système de vérification doit aller au-delà de la performance de chaque titulaire de licence, afin également d'examiner de près l'administration du secteur forestier - y compris l'allocation de ressources et l'octroi de licences par le ministère lui-même. Le degré d'indépendance véritable que peut introduire cet accord dépend toutefois de la représentation réelle des différentes parties prenantes dans un tel organe de gouvernance.

Il est également indispensable de superviser les décisions de vérification du ministère, avec provision adéquate pour un procès équitable en cas de litige. Dans ce domaine, le nouveau Conseil national des forêts $(\mathrm{DKN})$ a le pouvoir d'enquêter et de réaliser des auditions fournissant la base d'un mécanisme crédible de règlement des conflits, conformément à l'organe d'appel du LEI. Cependant, le DKN dépend à l'heure actuelle du MoF en matière de financement. Pour être impartial, il lui faudrait être financé indépendamment du MoF (voir Wells et al. 2006). 


\section{Institutionnalisation de l'observation indépendante par la société civile}

La société civile a contribué de manière cruciale à la lutte contre l'exploitation illégale en Indonésie. Pourtant, elle n'occupe aucune place officielle dans les structures existantes de vérification. Dans le cadre d'un futur TLAS, les propositions incluent l'observation indépendante par des comités ad hoc créés par le DKN et/ou ses divisions régionales $(\mathrm{DKD})$.

\section{8 Élargir l'impact}

Si les systèmes de vérification doivent avoir un impact significatif, un éventail de réformes plus larges pourrait également être nécessaire, au-delà des efforts visant à établir une norme de légalité et à renforcer l'indépendance et la supervision par la société civile. Certaines de ces mesures de réforme sont présentées ci-dessous :

- Une solution légitime qui répond aux préoccupations des populations locales et indigènes sur les terres déclarées domaine forestier national doit encore être trouvée dans le cadre de la norme de légalité. Le règlement PP6/2007 a introduit un ensemble de licences limitées dans le temps pour la gestion communautaire des forêts, mais qui ne traitent pas des conflits autour de la propriété foncière. Les alliances de la société civile travaillent à décrire les approches au CPLE, mais celles-ci doivent encore être intégrées dans des processus officiels de classement et d'allocation de zones forestières.

- Les mécanismes de vérification ne sont pas encore en place pour la gestion communautaire des forêts (community-based forest management, CBFM). Pourtant, en termes de volumes de bois produit, la CBFM peut s'avérer bien plus productive et aussi importante que les concessions industrielles. En l'absence de systèmes de vérification sur mesure et de données en résultant, les titulaires de licence communautaire sont vulnérables au retrait arbitraire de leurs droits par des agences chargées d'appliquer la loi. Les systèmes de vérification existants sont également susceptibles de rendre les initiatives de CBFM non viables à cause des coûts de transaction. Des approches plus « légères " peuvent impliquer la vérification par des organisations de terrain intégrées dans la communauté.

- Il reste un argument pour transférer davantage d'autorité aux régions afin de vérifier la conformité des opérateurs, conformément aux lois actuelles sur la décentralisation et les régions disposant d'une autonomie spéciale. Là où le ministère des forêts s'est efforcé d'auditer des centaines de titulaires de licence, cela pourrait restreindre son propre rôle de supervision de la vérification par ses homologues des gouvernements locaux. Cependant, dès lors que les responsabilités du gouvernement local ne s'accompagnent pas de l'autorité nécessaire, elles n’ont pas de rôle très incitatif en matière de suivi et de contrôle.

- Une vérification efficace dépend de l'application effective des lois. Cela implique (i) d'augmenter les efforts pour systématiquement documenter la gestion des procédures juridiques, en cas d'absence de jugements écrits et face à une intégrité limitée dans 
le système judiciaire ; (ii) de renforcer la légalité des poursuites contre les délits d'exploitation illégale - p. ex. le pouvoir du Centre indonésien de rapport et d'analyse des transactions financières (PPATK) de poursuivre les crimes financiers liés au secteur forestier ; (iii) de veiller à ce que les opérations répressives d'application de la loi tiennent correctement compte des précédents comptes rendus d'audit au moment de se prononcer sur l'illégalité.

- Enfin, les réformes des systèmes de vérification ne réussiront probablement pas si les principaux facteurs qui favorisent l'exploitation illégale ne sont pas traités, en particulier la surcapacité existante des filières forestières. Une feuille de route pour la restructuration industrielle a été élaborée, mais elle doit encore être officiellement avalisée par le gouvernement.

\subsection{Enseignements}

En Indonésie, les systèmes de vérification existants bénéficient d'une forte mainmise du gouvernement. Comme en Malaisie, leur gouvernance est considérablement influencée par les processus de certification volontaire des forêts. Cela implique de recourir à des audits externalisés afin d'améliorer l'efficacité et la crédibilité. Mais cela met fin à la supervision indépendante par des tiers, ce qui entraîne des allégations d'interférences politiques et de conflits d'intérêt.

On observe toutefois une volonté d'améliorer de telles dispositions, en se basant sur l'expérience des initiatives de certification indépendante, comme le LEI. Les priorités de réforme actuelles incluent :

- Des systèmes d'information forestière améliorés, en particulier les protocoles de transparence ;

- L'accréditation indépendante des vérificateurs tiers et le développement d'un organe de gouvernance distinct pour superviser la norme de légalité, et ainsi retirer au ministère des forêts le monopole du processus d'audit.

Cependant, l'Indonésie prouve également à quel point l'efficacité des systèmes de vérification dépendent d'une réforme plus large. En particulier, un consensus doit être trouvé sur les moyens légitimes et évolutifs d'identifier et de répondre aux droits des populations locales et indigènes sur les terres forestières ; les gouvernements locaux ont besoin d'une incitation plus forte pour investir dans les processus de vérification ; les opérations d'application de la loi doivent renforcer, et non passer outre, les processus de vérification au moment d'évaluer la conformité légale; enfin, des efforts doivent être entrepris pour concrétiser les recommandations existantes relatives à la surcapacité industrielle, qui encourage l'exploitation illégale. 


\section{Chapitre 16}

\section{Approches multiples pour améliorer le contrôle des forêts en Malaisie}

Note : Cette étude de cas a été réalisée en juin 2008 par Adrian Wells. Elle se base sur Wells, A., Thang, H.C. and Chen, H.K. 2008 Systems for verification of legality in the forest sector, Malaysia: domestic timber production and timber imports. Etude de cas nationale $n^{\circ} 8$ de VERIFOR. ODI, Londres.

Tableau 16.1 Malaisie : quelques statistiques clés

\begin{tabular}{|c|c|c|c|}
\hline Statistiques & & Date & Source des données \\
\hline Population & 25,3 millions & 2005 & $\begin{array}{l}\text { Division de la population } \\
\text { des Nations Unies } 2006\end{array}$ \\
\hline Superficie & 32,9 millions ha & & FAO 2006 \\
\hline Couverture forestière & $63,6 \%$ & 2005 & $\begin{array}{l}\text { Division de la statistique } \\
\text { des Nations Unies } 2006\end{array}$ \\
\hline Propriété publique des forêts & $93 \%$ & 2000 & FAO 2005 \\
\hline $\begin{array}{l}\text { Production de bois rond } \\
\text { industriel }\end{array}$ & 30,8 millions $\mathrm{m}^{3}$ & $\begin{array}{l}\text { Moyenne } \\
2001-2005\end{array}$ & OIBT 2005 \\
\hline $\begin{array}{l}\text { Emplois dans le secteur } \\
\text { forestier formel }\end{array}$ & 208000 & $\begin{array}{l}\text { Moyenne } \\
1990-2000\end{array}$ & FAO 2004 \\
\hline $\begin{array}{l}\text { Contribution du secteur } \\
\text { forestier au PIB }\end{array}$ & $6,7 \%$ & $\begin{array}{l}\text { Moyenne } \\
1990-2000\end{array}$ & FAO 2004 \\
\hline $\begin{array}{l}\text { Valeur des exportations de } \\
\text { produits forestiers }\end{array}$ & 3,3 milliards USD & $\begin{array}{l}\text { Moyenne } \\
1990-2000\end{array}$ & FAO 2004 \\
\hline $\begin{array}{l}\text { Principaux marchés } \\
\text { internationaux du bois }\end{array}$ & $\begin{array}{l}\text { Japon, Chine, } \\
\text { Taiwan }\end{array}$ & 2004 & Global Timber 2007 \\
\hline $\begin{array}{l}\text { Indice de perception de la } \\
\text { corruption de } \mathrm{Tl} \text { (de } 0 \text { à 10, } \\
\text { avec } 0=\text { le plus corrompu) }\end{array}$ & 5,0 & 2006 & $\begin{array}{l}\text { Transparency } \\
\text { Internationa } 2006\end{array}$ \\
\hline $\begin{array}{l}\text { Indice de développement } \\
\text { humain ( } 0 \text { ou } 1 \text {, avec } \\
0=\text { très bas) }\end{array}$ & 0,8 & 2004 & PNUD 2006a \\
\hline
\end{tabular}

\subsection{Introduction}

Ce chapitre présente la fascinante diversité des structures de vérification ayant évolué dans le système fédéral de la Malaisie, dans lequel les 13 Etats disposent de leur propre juridiction en matière de domaine forestier. Alors que l'homogénéité de pratique a été 
atteinte dans les huit Etats producteurs de bois de la Malaisie péninsulaire, les systèmes de vérification au Sabah et au Sarawak (sur l'île de Bornéo) ${ }^{1}$ ont évolué séparément. Cela reflète des différences dans les dispositions institutionnelles en matière de gestion forestière, ainsi que des différences cruciales en matière d'octroi de licences et d'administration du bois, selon la nature et l'étendue du domaine forestier. Et cela a une résonance pour les autres grands pays décentralisés comme l'Indonésie.

Le cas de la Malaisie est particulièrement intéressant du fait des structures composites multi-agences chargées du contrôle des forêts. La vérification est généralement assurée via l'audit des systèmes de gestion forestière, lesquels incluent :

- Les audits obligatoires de gestion durable de la forêt (GDF) des titulaires de licence, menés par les autorités forestières d'Etat;

- La réalisation par les gouvernements locaux d'audits de GDF obligatoires des autorités forestières d'Etat ;

- Les audits réalisés par les organes de certification agréés par rapport aux normes de l'Organisation internationale de normalisation (ISO) volontairement adoptées par les autorités forestières du gouvernement fédéral et des Etats ;

- Les audits réalisés par les organes de certification agréés, sur les normes de GDF volontairement adoptées par les autorités forestières de l'Etat et les titulaires de licence individuels.

Compte tenu du rôle de la Malaisie en tant que grand transformateur et re-exportateur de bois provenant d'ailleurs dans la région, ce cas souligne les mesures prises pour restreindre et administrer certaines catégories d'importation sous pression, afin d'empêcher le commerce illégal.

Après une brève description du contexte forestier, des facteurs et des mandats de vérification en Malaisie, ce chapitre analysera les approches de vérification adoptées en Malaisie péninsulaire, au Sabah et au Sarawak, et au regard des importations de bois. Une discussion de l'impact de la vérification sera suivie d'une section de conclusion énumérant les enseignements pouvant intéresser d'autres pays.

\subsection{Contexte}

Les forêts malaisiennes couvrent 19,5 millions d'hectares, soit environ $60 \%$ de la superficie du pays. Sur ce total, 14,9 millions d'hectares ont été qualifiés de domaine forestier permanent (DFP), dont 11,2 millions d'hectares de forêts de production. ${ }^{2}$ Le reste se compose principalement de réserves foncières destinées à la conversion et au développement. Le DFP comme les réserves foncières de l'Etat demeurent propriété publique. Seule une petite proportion de la couverture forestière $(7 \%)$ se trouve sur des terres privées, des terrains coutumiers autochtones ou des réserves aborigènes.

1 Ces huits États sont : Pahang, Selangor, Terengganu, Johor, Kedah, Perak, Negeri Sembilan et Kelantan.

2 Dans les États péninsulaires, on ne parle pas de DFP, mais de "réserves forestières permanentes " (RFP). 
Environ 200000 personnes travaillent dans le secteur forestier formel (FAO 2004). Sur la période 2001-2005, la production annuelle de bois rond industriel fut en moyenne de $30766000 \mathrm{~m}^{3}$ (OIBT 2005). En 2003, l'industrie du bois a représenté 3,4\% du PIB de Malaisie et 4,3\% de l'ensemble des recettes d'exportation. Cette année-là, le pays fut également le troisième exportateur mondial de grumes après la Russie et les Etats-Unis, le deuxième exportateur de contreplaqués après l'Indonésie et le huitième exportateur de bois scié. Alors que l'offre de bois national décline et que le pays compte un millier de scieries opérationnelles, une part croissante de la production malaisienne consiste à ré-exporter du bois provenant à l'origine de divers pays voisins, dont l'Indonésie, la Papouasie-Nouvelle-Guinée et la Birmanie. Les principaux marchés incluent le Japon, la Chine et Taiwan. L'Union Européenne (pour les producteurs de Malaisie péninsulaire), l'Inde et le Moyen-Orient sont également des grands marchés.

\subsection{Facteurs sous-tendant les systèmes de contrôle et de vérification des forêts}

Les évolutions liées à la vérification dans le secteur forestier malaisien sont principalement motivées par le désir de rester compétitif. Elles incluent :

- La mise en place d'une gestion reposant sur des normes, en vertu des Critères, indicateurs, activités et normes de performance pour la certification de l'aménagement forestier (les MC\&I) ; un dispositif de certification national, le Conseil de certification des bois ; des contrats de gestion durable de la forêt (SFMLA : Sustainable Forest Management License Agreement), d'une durée de 100 ans au Sabah ;

- L'introduction de normes ISO dans de nombreux aspects del'administration forestière, sous l'impulsion du Premier ministre afin d'améliorer l'efficacité ;

- D'importants investissements dans la capacité de production existante afin d'augmenter la valeur ajoutée ; des mesures de soutien incluant des interdictions et des quotas d'exportation de grumes spécifiques aux régions.

Les systèmes de contrôle forestier existants reflètent également les efforts du gouvernement fédéral et de certaines organisations de la société civile pour s'attaquer à la surexploitation et à l'exploitation illégale. Les gouvernements d'Etat ont toujours eu un intérêt à maximiser la production de recettes. Mais au début des années 1990, le gouvernement fédéral est intervenu pour réduire l'exploitation illégale et s'attaquer à l'ouverture excessive des zones forestières et à la surexploitation par les Etats de la péninsule. Il en a résulté l'organisation d'audits obligatoires des départements forestiers d'Etat en matière de MC\&I pour la GDF.

Une diminution des ressources nationales en bois a provoqué une dépendance accrue aux importations et au développement de plantations pour répondre aux pénuries de production. La Malaisie a par conséquent dû introduire une série de mesures pour garantir la légalité des importations (en particulier celles provenant d'Indonésie), sous l'œil très attentif de groupes de la société civile, tels que TRAFFIC Asie du Sud et l'Environmental Investigation Agency (EIA). 
La perspective d'un Accord volontaire de partenariat (AVP) entre l'Union Européenne et la Malaisie sur l'application des réglementations forestières, la gouvernance et les échanges commerciaux (FLEGT) se traduit par une nouvelle pression du marché en faveur de l'amélioration des normes. Les négociations ont débouché sur un débat approfondi autour des thèmes suivants :

- Les niveaux d'indépendance des organes de supervision, puisque les entités chargées du suivi et des audits sont en majorité financées ou détenues par le gouvernement ;

- Les niveaux de participation et de supervision de la société civile dans la conception et la mise en œuvre des systèmes de vérification ;

- Les pressions exercées pour que les droits fonciers des peuples indigènes et leur compensation soient reconnus, là où $93 \%$ des forêts malaisiennes demeurent propriété publique (FAO 2005).

La question des droits indigènes a suivi le développement et la mise en œuvre des MC\&I, ainsi que les négociations actuelles sur l'AVP. Des litiges sont en cours concernant les

\section{Encadré 16.1 Litiges en cours sur les droits aborigènes et coutumiers autochtones en Malaisie}

La Cour d'appel de Malaisie a confirmé le devoir fiduciaire des Etats de la péninsule de classer les zones dans lesquelles les aborigènes sont susceptibles d'avoir un intérêt foncier et de dédommager ceux-ci ainsi que les droits d'usufruit s'ils devaient leur être retirés. Cela souligne qu'il est nécessaire de disposer de directives et de normes claires en matière d'avis public et d'arbitrage, d'indemnisation et/ou d'intégration des droits aborigènes/autochtones dans les processus de classement des terres forestières ou d'aléniation de celles-ci à des fins de conversion.

Il est également fait état que les indicateurs et les moyens d'évaluer la conformité aux MC\&l ne sont pas suffisamment spécifiques ou axés sur la performance pour résoudre les litiges avec les communautés locales. Parmi les problèmes, il n'existe pas encore de mécanisme pour la gestion et le versement de compensations aux communautés aborigènes ou autochtones.

Enfin, à part les chefs de district ou le département des affaires aborigènes, il n'existe à l'heure actuelle aucun arbitre tiers, au sein des parties, qui supervise et se prononce de manière crédible sur les droits coutumiers aborigènes/autochtones.

L'absence de normes et de mécanismes adéquats pour résoudre les conflits peut être l'une des raisons pour laquelle les parties ont souvent eu à recourir au tribunal civil, alors que ces conflits auraient pu être réglés par la négociation ou l'arbitrage. Non seulement ceci est très insuffisant et implique des coûts de transaction élevés pour toutes les parties impliquées, mais l'absence de financement d'un système d'assistance juridique signifie également que les communautés doivent se reposer sur une assistance juridique bénévole. 
droits des groupes aborigènes/autochtones sur le domaine forestier. Cette question est compliquée par l'absence de normes claires permettant de comparer la conformité administrative avec les exigences légales afin de définir, d'annuler ou d'admettre ces droits aborigènes/autochtones. Ces deux catégories de problèmes se retrouvent devant les tribunaux fédéraux suite à des affaires dans la péninsule et au Sarawak (voir Encadré 16.1).

\subsection{Mandats de vérification}

Les systèmes malaisiens de vérification légale comprennent des structures multi-agences complexes de supervision de la production nationale et des importations de bois. Ils ont évolué en suivant différentes trajectoires dans la péninsule, au Sabah et au Sarawak. Conformément à l'article 74 (2) de la Constitution fédérale, les 13 Etats de Malaisie disposent chacun de leur propre juridiction sur les terres et le classement des forêts, leur gestion et les licences. Ce qui signifie que la Malaisie ne constitue pas une entité unique en matière de gestion forestière. Dans la péninsule, tous les Etats ont à présent adopté la Loi nationale sur les forêts (1984).

Conformément à leurs conditions d'accession à la Fédération de Malaisie, le Sabah et le Sarawak gèrent leur secteur forestier selon leurs propres lois et ordonnances. Cellesci incluent le Sabah Forest Enactment de 1968 (amendé en 1992) et les Forest Rules (1969), ainsi que le Sarawak Forest Ordinance de 1954 (amendé en 1999).

Un Conseil national des forêts (NFC) a été créé en 1971 afin de coordonner les politiques entre le gouvernement fédéral et les gouvernements d'Etat. Il a supervisé le développement de la Politique nationale des forêts, à laquelle tous les Etats adhèrent. Le NFC décide également de la possibilité annuelle de coupe (PAC) de chaque Etat, sur une base quinquennale. Toutefois, l'autorité exécutive du gouvernement fédéral (le département forestier fédéral) ne s'étend qu’à la fourniture de conseils et d'une assistance technique aux Etats, ainsi qu'à la formation, la recherche et l'entretien des stations expérimentales et de démonstration.

Dans les trois juridictions (péninsule, Sabah et Sarawak), tous les produits forestiers provenant du domaine forestier permanent (DFP) ou des terres d'Etat en dehors du DFP est considéré propriété de l'Etat. Toute exploitation de produit forestier doit faire l'objet d'une licence et être administrée par l'autorité de l'Etat. Les titulaires de licence opérant dans les réserves forestières permanentes ont l'obligation de développer et de mettre en place des plans de gestion, d'exploitation et de reboisement. Les agents forestiers ont le pouvoir d'arrestation, de recherche, de saisie et d'investigation. Les directeurs forestiers d'Etat décident des amendes et/ou de la poursuite des contrevenants.

Il existe d'importantes différences juridictionnelles entre les trois systèmes (telles que résumées dans le Tableau 16.2), ce qui n’est pas sans conséquences sur la conception de la vérification : 


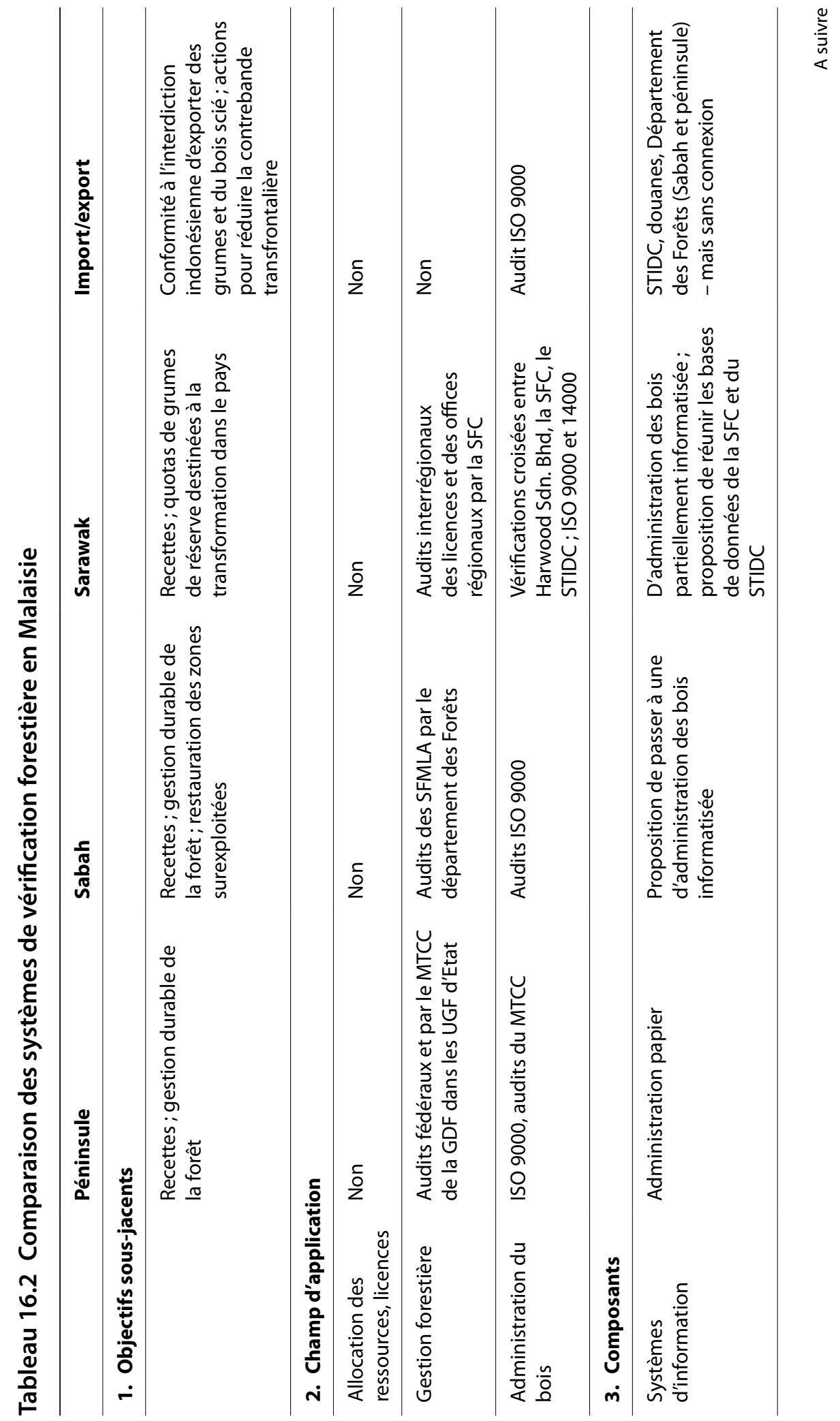




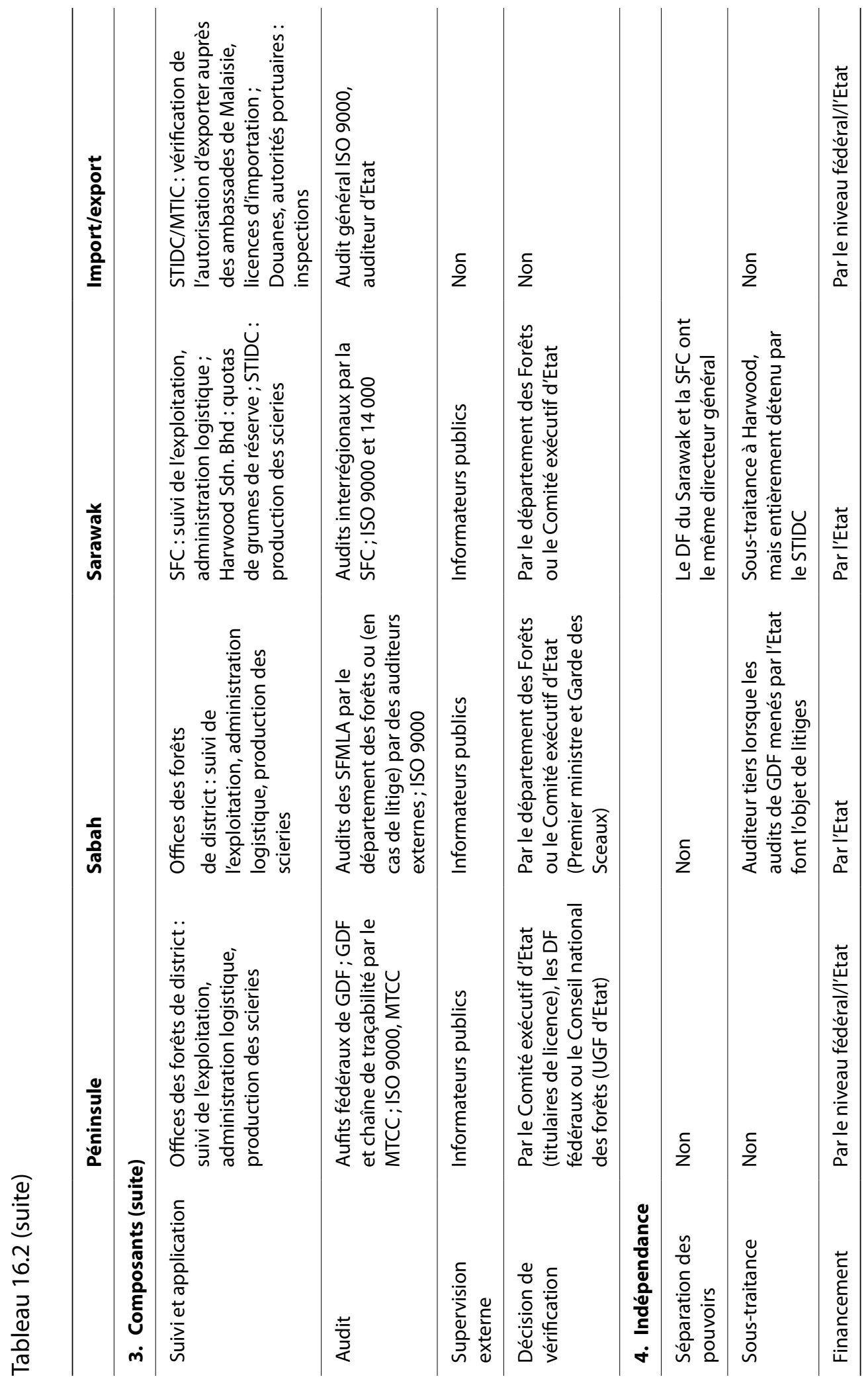


- Des objectifs différents. Par exemple, le système du Sarawak est conçu en partie pour garantir la conformité aux quotas de grumes de réserve destinés à la transformation dans le pays et en partie pour contrôler les importations en provenance d'Indonésie. Le système au Sabah est en revanche conçu pour que les concessionnaires se conforment à la norme afin d'obtenir la certification de gestion durable de la forêt par le Forest Stewardship Council (FSC).

- La structure organisationnelle des agences forestières. Alors que dans la péninsule et au Sabah, la responsabilité de la gestion, du développement et de la réglementation $\mathrm{du}$ secteur forestier incombe aux directeurs forestiers, le Sarawak a pris l'unique mesure de sous-traiter ces tâches à une entreprise d'Etat, la Sarawak Forestry Corporation (SFC).

- La responsabilité de la gestion des forêts et la durée des licences de concession. Dans les Etats de la péninsule, les licences d'exploitation à court terme (en général 12 mois) sont généralement émises pour les Unités de gestion forestière (UGF) directement gérées par les autorités forestières nationales. Le Sabah a en revanche opté pour des contrats de licence de gestion durable de la forêt (SFMLA) d'une durée de 100 ans, en vertu desquels les titulaires de licence assument l'entière responsabilité de la gestion des UGF. C'est également le cas au Sarawak, bien que les contrats de concession forestière n'y soient en général que de 25 ans renouvelables.

- Le rôle du gouvernement fédéral dans le contrôle des forêts. Dans la péninsule (c.à-d. Sabah et Sarawak exclus), le département fédéral des Forêts effectue des audits routiniers de GDF dans les UGF gérées par l'Etat. Toutefois, l'administration des importations de bois reste de la responsabilité d'un organe fédéral statutaire, le Conseil de l'industrie du bois de Malaisie (MTIB) pour la péninsule et le Sabah, et le Conseil du développement de l'industrie du bois de Sarawak (STIDC) pour le Sarawak.

- Disposition légale pour les droits aborigènes et autochtones. Les lois actuelles dans les trois juridictions empêchent tout intérêt foncier aborigène ou autochtone dans les zones classées DFP. Mais contrairement à ce qui se passe dans la péninsule, les ordonnances au Sabah et au Sarawak prévoient l'identification, la suppression ou l'abolition des droits coutumiers en échange d'une compensation.

\subsection{Vérification dans les Etats péninsulaires}

Dans la péninsule, chaque Etat se définit comme une UGF à part entière chargée de préparer et de mettre en œuvre des plans de gestion en vertu desquels les licences de coupe à court terme sont octroyées. Dans les Etats, les départements des forêts sont soumis à des audits de conformité de deux types :

- Des audits obligatoires des UGF d'Etat par le département fédéral des forêts, par rapport aux MC\&I pour la gestion durable de la forêt (GDF) ;

- Des audits volontaires des UGF d'Etat par le MTCC et des certificateurs ISO agréés.

Dans le cas spécial du site Perak Integrated Timber Complex, des audits volontaires des titulaires de licence à long terme sont également menés par le FSC. 


\section{Suivi de routine et application}

Les accords existants de planification et d'administration des bois au sein des UGF d'Etat sont décrits à la Figure 16.1. Le suivi quotidien de la conformité légale des titulaires de licence relève principalement de la responsabilité des Offices des forêts de district (OFD). Les gardes forestiers en charge de chaque zone sous licence réalisent des inspections mensuelles durant l'exploitation afin de veiller à ce que les délimitations, les zones tampons et les limites de coupe soient respectées. Des inspections après exploitation sont menées pour évaluer les dommages aux arbres restants et calculer les redevances, suivies d'un inventaire post-abattage permettant de déterminer la proportion de surface occupée restante et le traitement sylvicole approprié.

Des unités spéciales peuvent être déployées par les OFD pour l'inspection régulière des délimitations de parcelles et pour effectuer deux fois par mois des contrôles routiers et des inspections de parcs à grumes. Ces unités peuvent également être déployées en cas d'allégations par le public d'exploitation ou de transport de grumes illégaux. Une telle participation du public est encouragée par un système de récompense. L'assistance de la police et des forces armées peut être demandée, notamment pour les barrages routiers. Les OFD soumettent des comptes rendus mensuels au département des Forêts, lequel peut réaliser des contrôles surprises.

\section{Audits obligatoires des UGF d'Etat}

Une " équipe interne d'auditeurs » au sein du département fédéral des Forêts réalise des audits annuels de GDF dans les UGF d'Etat de la péninsule, en suivant les procédures d'évaluation du MTCC. L'audit examine les données de suivi, par exemple les comptes rendus mensuels, et inclut des contrôles aléatoires sur les pratiques de gestion dans certaines zones forestières choisies. La procédure évalue également les mesures prises par les UGF d'Etat pour traiter les « demandes d'actions correctives » (DAC) qui sont identifiées dans les évaluations du MTCC pour la certification forestière. Ainsi, les audits obligatoires du département fédéral des Forêts et les audits volontaires du MTTC se renforcent mutuellement.

\section{Audits volontaires des UGF d'Etat}

Outre les audits internes de GDF par le niveau fédéral, il existe à l'heure actuelle deux audits volontaires complémentaires, mais non liés, relatifs à la gestion forestière et à l'administration des bois dans les Etats de la péninsule. Nous les décrivons ci-après.

\section{Conseil malaisien de certification des bois}

Le MTTC a été créé en 1998 pour superviser la mise en place d'un dispositif de certification volontaire, par rapport aux MC\&I pour la gestion durable des forêts. Bien qu'il s'agisse en théorie d'une organisation indépendante établie en vertu de la loi sur les entreprises (1965), le MTTC reste sous l'autorité du ministère fédéral des Industries et des Produits des plantations. Le MTTC a d'abord reçu un financement par ce ministère 
Marquage de $100 \%$ des arbres par l'Office des forêts de district (OFD) ; étiquettes imprimées à utiliser sur les souches et les grumes :

- Le personnel du DF consigne les espèces, le numéro des marques et le nombre de marques laissées sur les arbres (en fonction du potentiel de rendement des grumes);

- Les numéros de marque sont compilés dans un registre devant servir aux stations de vérification.

Abattage des arbres par les opérateurs ; fixation de code-barres du DF sur les souches et les grumes ; marquage de la catégorie de classification indiquant l'origine/source apposée sur les grumes; remplissage de «formulaires de contrôle d'abattage » quotidiens.

\begin{tabular}{|c|}
\hline \\
L'opérateur émet des ordres de livraison des grumes \\
devant être transportées aux stations de vérification.
\end{tabular}

Figure 16.1 D’administration des bois dans les réserves forestières permanentes en Malaisie péninsulaire 
pour couvrir les coûts de ses opérations durant les premières années de sa création. Il est aujourd'hui financé par les intérêts générés par une dotation fournie par le ministère à partir de la collecte des taxes d'exportation sur le bois et les produits du bois.

Fin 2005, le MTCC avait certifié 4,67 millions d'hectares de réserves forestières permanentes couvrant les huit Etats producteurs de bois de la Malaisie péninsulaire, chacun de ces Etats étant considéré comme une UGF distincte en matière de certification MTCC. Le Conseil a également certifié 55949 hectares de l'UGF Sela'an Linau à Sarawak, qui est gérée par Samling Plywood (Baramas) Sdn. Bhd. À partir de 2006, la certification a évolué vers la conformité à la norme MC\&I 2002. En outre, le MTTC a instauré des "procédures d'évaluation liées aux exigences pour la chaîne de traçabilité ". Les évaluations sont menées par des organisations ou des entreprises agréées et nommées par le MTTC.

\section{Normes ISO 9001 (2000) de gestion de la qualité}

Dans le milieu des années 1990, le département fédéral des forêts a introduit des normes ISO de gestion de la qualité (aujourd'hui version ISO 9001 : 2000) afin de garantir la conformité aux procédures administratives pour une " production durable de bois issu des réserves forestières permanentes naturelles ». Elles s'appliquent désormais aux huit Etats de la péninsule qui produisent du bois. La procédure d'évaluation est entièrement basée sur la production et nécessite de remplir des formulaires et autres documents concernant la conformité à la planification de l'exploitation et aux mesures de contrôle. Les audits sont menés tous les ans par l'organe agréé SIRIM QAS, une entreprise malaisienne spécialisée dans la certification, l'inspection et les essais.

La Figure 16.2 décrit les mesures complémentaires de suivi, d'audit et de conformité utilisées en Malaisie péninsulaire.

\section{Points forts du système de vérification en péninsule}

Il existe potentiellement une forte complémentarité entre les audits obligatoires et les audits volontaires en péninsule :

- Les audits volontaires (du MTCC) et obligatoires (du département fédéral des Forêts) de la gestion durable des forêts se soutiennent mutuellement, car ils sont tous menés sur la base des MC\&I. Les audits fédéraux offrent un moyen de suivre et de prendre en charge les DAC identifiées par les évaluateurs du MTCC.

- Les exigences du MTTC en matière de chaîne de traçabilité fournissent un aperçu crucial du traitement en scierie, où les systèmes obligatoires reposent autrement sur l'auto-déclaration, par les opérateurs de scierie, des taux de production et de récupération.

- Les audits selon les normes de performance ISO 9001 : 2000 assurent la cohérence des procédures administratives, notamment du suivi et des comptes rendus par les départements d'Etat des Forêts. 


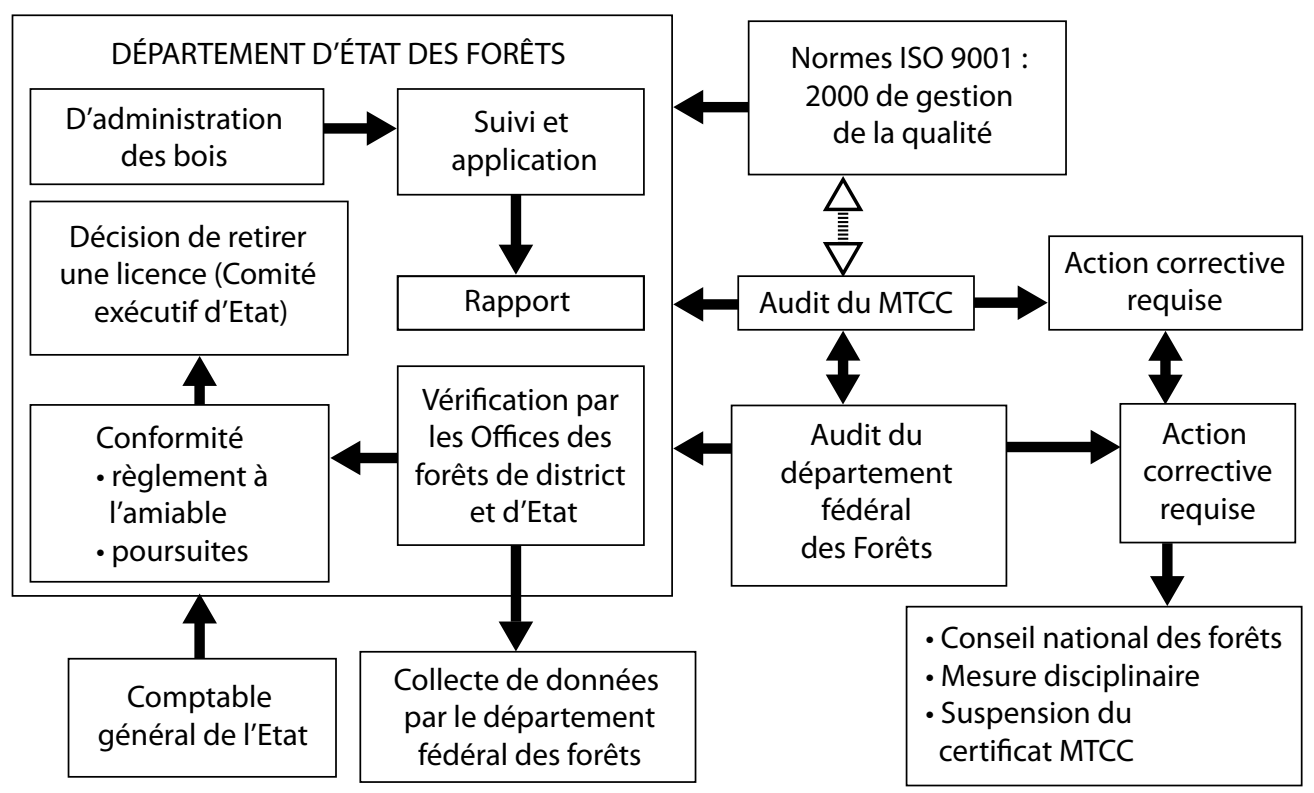

Figure 16.2 Suivi, audit et conformité des forêts en Malaisie péninsulaire

\section{Points faibles du système de vérification en péninsule}

On dénombre quatre domaines de préoccupation dans les activités de vérification en Malaisie péninsulaire.

\section{Problèmes de la chaîne de traçabilité}

Malgré le marquage d'un numéro de série unique sur tous les arbres exploitables, la gestion des autorisations d'enlèvement se fait de manière entièrement manuelle. L'absence d'un système informatique centralisé rend difficile le suivi des consignations individuelles, notamment la confirmation que les autorisations d'enlèvement sont annulées une fois arrivées à destination. Une autre difficulté résulte du fait que les exigences pour la chaîne de traçabilité du MTCC nécessitent que les fournisseurs signent une auto-déclaration stipulant que les matières premières ou produits non certifiés ne contiennent pas de bois issu de sources incertaines. L'absence de garde forestier permanent dans les scieries pour surveiller les taux de production et de récupération constitue une faille potentielle qui pourrait permettre l'entrée de bois originaire de sources controversées (nationales ou importées) dans la chaîne de production.

\section{Champ d'application et fréquence des audits}

Les audits posent problème à plusieurs égards. Tout d'abord, les audits du MTTC, les audits fédéraux sur la GDF et les audits ISO 9001 : 2000 sont limités aux réserves forestières permanentes. Aucun audit obligatoire ou volontaire équivalent n'existe pour l'extraction et l'administration des bois réalisées sur les terres de l'Etat voisin ou aliénées pour être converties. Ensuite, il n'existe aucun lien explicite entre les audits ISO et GDF, comme il existe entre les audits du MTTC et les audits fédéraux sur la GDF. Les audits 
ISO actuels de l'administration forestière se basent uniquement sur la documentation et ne tiennent pas compte des résultats en matière de gestion forestière. Enfin, la taille de certaines grandes UGF permet difficilement aux auditeurs d'assurer une couverture suffisante. L'Etat de Pahang en est un bel exemple (puisqu'il représente une seule UGF de plus d'un million d'hectares de réserves forestières permanentes). Les audits seraient plus efficaces si les grands Etats étaient divisés en plusieurs UGF.

\section{Indépendance}

Bien que le MTCC fonctionne grâce aux intérêts générés par son propre fonds de dotation, il reste sous l'autorité du ministère fédéral des industries et des produits des plantations. Son indépendance perçue serait améliorée s'il existait un système d'accréditation. Les quelques organes de certification actuellement agréés par le MTCC pour mener les audits des UGF (p. ex. SGS, SIRIM QAS) devraient ainsi s'enregistrer auprès du département des Normes afin d'être en mesure d'émettre directement des certificats MTCC. De cette manière, le MTCC pourrait ne plus émettre de certificats de conformité et se concentrer à la place sur la supervision de la norme MC\&I.

\section{Mesure de la conformité}

La décision de retirer une licence en cas d'infraction grave reste aux mains du Comité exécutif d'Etat (en réalité cabinet d'Etat). Cependant, aucune directive n'a été publiée sur les critères de retrait de licence, ni sur l'utilisation des comptes rendus d'audit pour parvenir aux décisions de sanction. Aucune synthèse des décisions du Comité n'est rendue publique.

\subsection{Vérification au Sabah}

Aux termes de la Constitution fédérale, le Sabah constitue une juridiction légale distincte en matière de foresterie. Son département des forêts n'est par conséquent pas soumis aux audits de GDF menées par le département fédéral des forêts. Les mesures de suivi des forêts et d'administration des bois sont en grande partie similaires à celles adoptées dans la péninsule. Toutefois, contrairement au système péninsulaire de licence d'exploitation à court terme, le Sabah a introduit en 1997 un nouveau système de contrats de licence de gestion durable de la forêt (SFMLA) d'une durée de 100 ans. Chaque SFMLA représente une Unité de gestion forestière (UGF) à part entière. Cela permet de placer la priorité sur le respect de la conformité par les titulaires de licence, qui sont responsables de toute la gestion, par opposition à la conformité que doivent respecter les autorités d'Etat de gestion forestière.

\section{Suivi de routine et exécution des SFMLA}

Comme en péninsule, les OFD de Sabah sont chargés de l'administration routinière du bois. Leurs responsabilités incluent la préparation de "plans complets d'exploitation » et la gestion des plaintes et des autorisations d'enlèvement. Afin de permettre la traçabilité jusqu'à la souche, le département des forêts de Sabah est en train de mettre au point des 
mesures de suivi informatique des grumes incluant le marquage par code-barres, des permis de mobilisation des bois et des autorisations d'enlèvement. Les accords actuels de planification et d'administration des bois sont décrits à la Figure 16.3.

Les OFD effectuent également le suivi de la conformitéà des normes de gestion équivalentes à celles du FSC et établies dans le cadre des SFMLA en matière de conservation des forêts, d'impact réduit de l'exploitation, de traitement sylvicole, de protection des forêts, de forêts communautaires et de développement des communautés.

L'une des conséquences de la politique des SFMLA de 100 ans est que le retrait de licence ne peut avoir lieu qu'en cas de circonstances exceptionnelles, avec preuves d'une absence de production à grande échelle. Lorsque les délits sont moins graves, l'OFD travaille directement avec les titulaires de licence pour les amener à se conformer entièrement aux normes établies en vertu des SFMLA. Les OFD aident ces exploitants à élaborer des plans de travail annuels et des comptes rendus trimestriels de progrès. Les « rapports annuels de conformité " des titulaires de licences réalisés sur le terrain par les OFD servent également aux audits de conformité menés par la direction du département des Forêts.

En août 2002, le département des forêts du Sabah a créé une nouvelle division Exécution et Investigation. Celle-ci collabore avec l'Unité de lutte contre l'exploitation illégale du Bureau du Premier ministre du Sabah, ainsi qu'avec la police et les forces armées. Grâce à la formation en interne à l'investigation et au suivi, au contrôle et à l'application effectués par les unités opérationnelles et les OFD, le travail de la division a entraîné une forte hausse des condamnations pour exploitation illégale (de 12 en 2001 à 194 en 2004).

\section{Audits obligatoires des opérations sous licence}

\section{Audits des SFMLA par le département des Forêts de Sabah}

Le département des Forêts du Sabah a introduit une " procédure générale d'audit de la GDF » des 125 clauses du SFMLA standard. Celles-ci sont formulées autour des 10 principes du FSC. En tant que tels, les audits veillent à assurer la conformité et à ce que les titulaires de licence se conforment à la norme à des fins de la certification.

La procédure générale d'audit de la GDF est mise en place par une équipe d'audit désignée au sein du département des forêts du Sabah. Cette équipe transmet une évaluation globale de la viabilité au directeur du département des forêts du Sabah. Lequel émet ensuite un certificat de conformité (pouvant être soumis à des demandes d'actions correctives). Lorsqu'un titulaire de licence a continuellement échoué à respecter son contrat, le directeur peut émettre une recommandation de suspension au Comité exécutif d'Etat. La décision de retirer ou de maintenir une licence relève du Comité exécutif, qui inclut le Premier ministre et le Garde des Sceaux. Depuis 1997, deux licences (sur 18) ont été retirées pour non-exécution et une autre est en suspens. Dans un quatrième cas, la licence a été maintenue suite à l'intervention d'auditeurs externes indépendants. 
Titulaire de licence : inventaire de $100 \%$ des arbres exploitables (plan annuel) ; liste et cartographie des arbres exploités (plan annuel complet d'exploitation des arbres, parcelle d'exploitation).

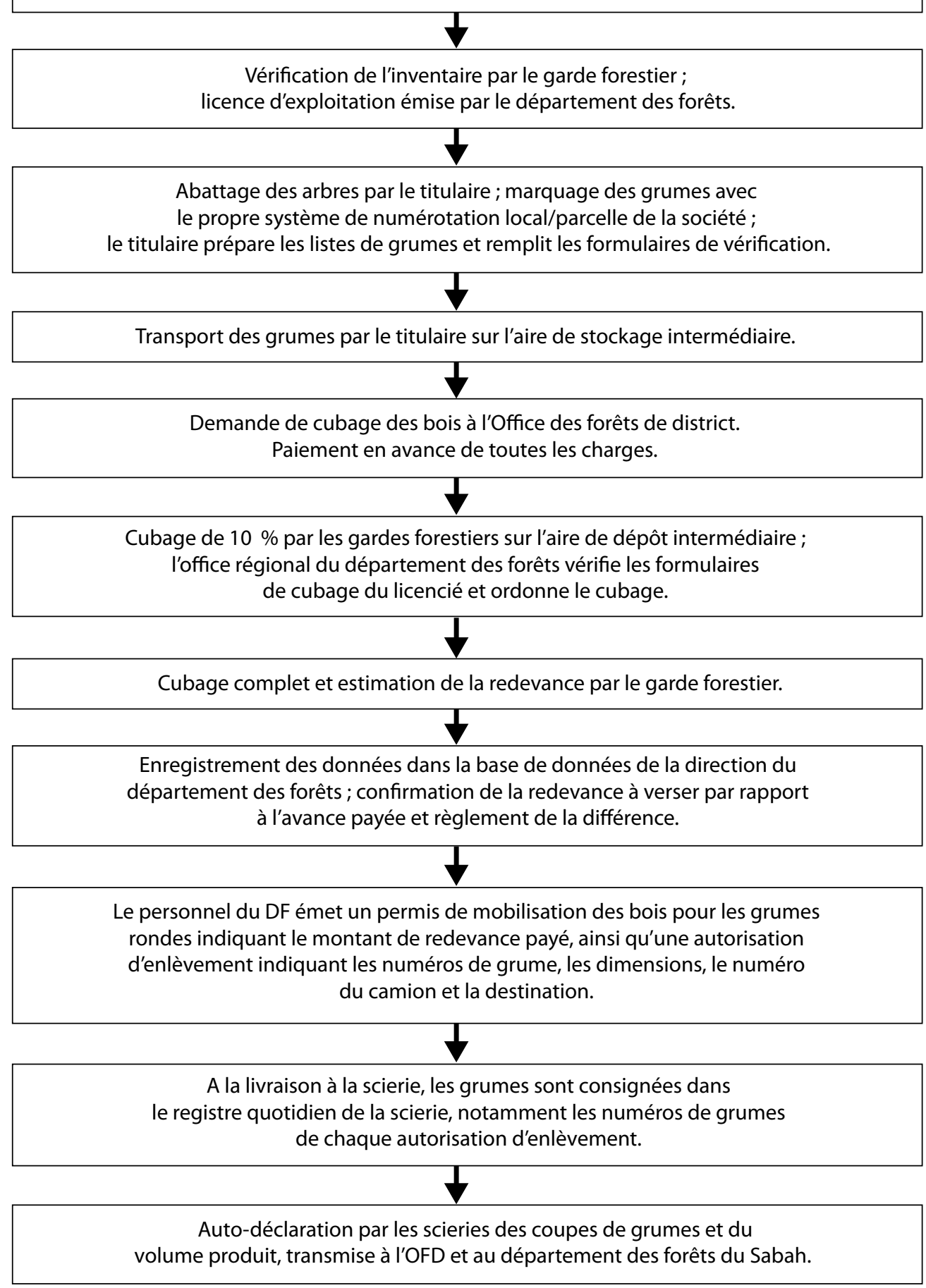

Figure 16.3 D'administration des bois pour les SFMLA au Sabah 
Les comptes rendus d'audit ne sont pas mis à la disposition du public, sauf si une demande spécifique d'accès est effectuée. En l'absence de directives établies sur la confidentialité du processus d'audit, le département des forêts du Sabah détermine lui-même le mérite de telles demandes.

\section{Audits externes ad hoc des opérations sous licence}

Les titulaires d'un SFMLA ne sont actuellement impliqués dans aucun dispositif de certification volontaire. Le département des forêts du Sabah a néanmoins recouru à des auditeurs tiers indépendants en complément de sa propre "procédure générale d'audit de la GDF ». Dans un cas, cela a été instauré lorsque des incohérences significatives ont été identifiées entre le rapport de conformité d'un titulaire de licence et l'évaluation de l'OFD.

Le recours à un auditeur externe traduit également les pressions exercées par l'industrie en faveur d'une plus grande transparence dans le processus de décision d'un audit et d'une vérification. Aucune procédure n'a encore été établie pour déterminer lorsque des auditeurs externes doivent intervenir.

\section{Audits volontaires du département des forêts du Sabah}

Le Sabah n'est pas soumis aux audits de GDF réalisés par le niveau fédéral. Il fait toutefois l'objet d'audits conduits par des organes accrédités en matière de procédures administratives certifiées ISO. À ce jour, le département des forêts du Sabah a identifié plusieurs processus à évaluer selon les normes de performance ISO 9001 : 2000. Cependant, leur champ d'application est moins complet que dans la péninsule, puisqu'il se limite jusqu'à présent aux procédures de collecte des redevances et à la préparation des plans complets d'exploitation. Ils ne s'appliquent pas encore à la " procédure générale d'audit de la GDF ».

Un cas spécial au Sabah est le modèle de concession certifiée FSC à Deramakot, qui reste sous la gestion directe du département des forêts. La concession de 55000 hectares vise à proposer aux SFMLA de long terme un modèle pour évoluer vers la certification. En tant que concession gérée par l'Etat, les systèmes de suivi et d'audit diffèrent de ceux qui sont appliqués aux SFMLA. Les pratiques de gestion sont examinées par Global Forest Systems (GFS), un vérificateur tiers agréé par le FSC. À mesure que les SFMLA se conforment à la norme, il est probable que certains chercheront également la certification FSC. La Figure 16.4 illustre le système de suivi, d'audit et de conformité des forêts au Sabah.

\section{Points forts du système de vérification à Sabah}

Avec les audits obligatoires qui se concentrent sur les titulaires de licences (SFMLA), le département des forêts du Sabah a bien plus de pouvoir pour sanctionner les gérants de forêts que dans la péninsule. Un autre grand atout du Sabah est la priorité donnée à la gestion de la conformité, au moyen d'audits de routine qui fournissent une base pour renforcer les capacités et améliorer la performance dans le temps. Dans d'autres Etats, le 


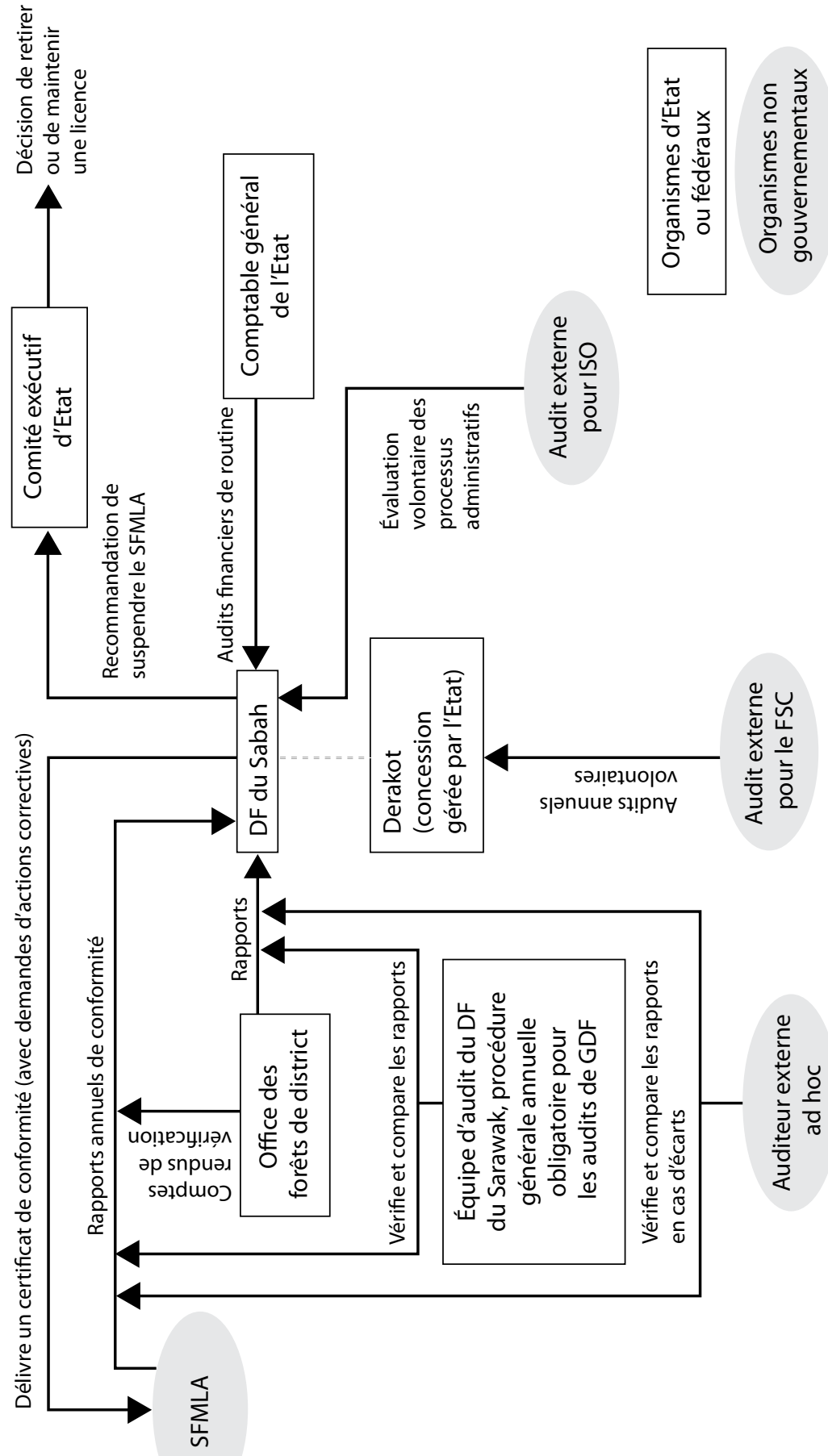

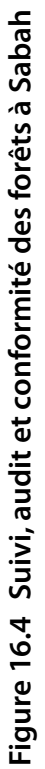


contrôle forestier met davantage l'accent sur le respect de la conformité, y compris sur les inspections post-exploitation, que sur l'accompagnement des titulaires de licence pour les faire se conformer aux normes.

\section{Points faibles du système de vérification au Sabah}

\section{Problèmes dans la chaîne de traçabilité}

Le Sabah envisage de mettre en place un système informatisé d'administration du bois, y compris le marquage à $100 \%$ des souches. Tant que ce système n'existera pas, le lien entre l'administration des bois et le contrôle de l'exploitation sera plus faible qu'en péninsule.

\section{Champ d'application et complémentarité des audits}

À l'heure actuelle, les audits de GDF et ISO ne se complètent pas. Leur complémentarité doit donc être renforcée en étendant le champ d'application des audits ISO, afin qu'ils couvrent l'ensemble de la chaîne de production, depuis la planification de l'exploitation jusqu’à la production de la scierie (incluant également la "procédure générale d'audit de la GDF »). Les procédures du département des forêts pourraient également être mises à jour, en ligne avec les normes ISO 14000 et ISO 19000 de gestion environnementale et de suivi des systèmes.

\section{Indépendance et transparence}

Il n'existe aucune procédure permettant au public d'accéder aux comptes rendus d'audit et aux décisions de vérification du département des forêts ou du Comité exécutif d'Etat. Il n'existe pas non plus de directive claire quant au recours à des auditeurs externes indépendants en cas de désaccord sur l'évaluation du département des forêts. Or, ces deux éléments sont largement considérés comme indispensables pour garantir la crédibilité de la "procédure générale d'audit de la GDF » aux yeux de l'industrie et de la société en général.

\subsection{Vérification au Sarawak}

Comme au Sabah, Sarawak a développé ses propres systèmes de vérification de la conformité légale dans le secteur forestier. La principale différence au Sarawak réside dans sa structure composite multi-agences pour la définition de politiques, la réglementation, le suivi et l'application sous l'autorité globale du ministre de la planification et de la gestion des ressources (MPRM) de l'Etat, qui en est également le Premier ministre. Avec le regroupement en six grands groupes industriels des opérations en amont et en aval, cette structure permet un contrôle étroit de la filière bois par le gouvernement de l'Etat.

Contrairement à la Malaisie péninsulaire, il n'existe aucun audit fédéral obligatoire. Et contrairement au Sabah, les audits d'Etat obligatoires ne recourent pas à des tiers. Le Sarawak a toutefois utilisé les normes ISO de manière extensive. 


\section{Suivi de routine et application}

Si les mesures de suivi de l'exploitation et l'administration des bois sont largement similaires à celles de la péninsule et du Sabah, les structures y afférant sont différentes au Sarawak.

Tout d'abord, le suivi de l'exploitation et l'administration des bois relèvent à présent de la juridiction de la Sarawak Forestry Corporation Sdn. Bhd. (SFC), une entreprise privée détenue par le gouvernement de l'Etat. La SFC a été créée afin de permettre l'externalisation des fonctions clés du département des forêts du Sarawak et de rendre le système plus efficace. Depuis, la SFC a créé un certain nombre de divisions dédiées. Lesquelles incluent l'unité Foresterie durable et conformité (SF\&C) chargée de planifier et de suivre l'exploitation, et l'unité Sécurité et protection des biens (SAPU) chargée de l'application des lois. Le département des forêts se concentre désormais sur la politique, la réglementation et les licences.

En second lieu, le Sarawak a sous-traité à Harwood Timber Sdn. Bhd. le suivi de $60 \%$ de ses quotas de grumes de réserve destinées à la transformation dans le pays. Cette société est une filiale entièrement détenue par le Conseil du développement de l'industrie du bois de Sarawak (STIDC), un organe statutaire chargé de promouvoir la filière bois au Sarawak. Les $40 \%$ de grumes rondes restantes peuvent être exportées. Harwood gère également les dépôts dans lesquels sont entreposées les importations en provenance d'Indonésie en attente de l'inspection et du dédouanement.

\section{Planification et suivi de l'exploitation}

Le directeur du département des Forêts approuve d'abord un Plan de gestion forestière (PGF) qui comprend le tracé et la construction de routes ainsi que des directives sur les opérations d'exploitation. Un plan général d'exploitation est préparé avant le début des opérations sur le terrain, suivi d'un plan détaillé d'exploitation pour chaque bloc. Des autorisations pour le développement d'infrastructures et l'exploitation sont accordées aux titulaires de licence en fonction de leurs progrès opérationnels. Si les mesures requises ne sont pas prises, l'unité SF\&C envoie une requête de mesure corrective avant la poursuite des travaux. L'unité SF\&C a instauré des procédures pour l'inspection des exploitations et l'émission de certificats d'autorisation de coupe, mais aussi pour le suivi et le compte rendu des progrès en matière d'exploitation et de fermeture de blocs. Ces procédures se concentrent sur une inspection post-exploitation de $100 \%$ plutôt que sur le suivi des opérations en cours.

\section{D'administration des bois}

Comme dans la péninsule et au Sabah, l'administration des bois au Sarawak comprend trois grandes étapes : identification des grumes, calcul et règlement des redevances et gestion des autorisations d'enlèvement (telles que résumées à la Figure 16.5). Le système est actuellement administré de deux manières. D'un côté, par le système standard, qui 
s'applique à environ $70 \%$ de la production. D'un autre côté, par un système informatisé qui couvre le reste. Dans les deux cas, les sociétés sont chargées de marquer les grumes.

Harwood travaille en tandem avec l'administration des bois du Sarawak pour veiller à ce que les titulaires de licence se conforment au quota de réserve de $60 \%$ des grumes destinées aux scieries nationales. Harwood soumet des rapports mensuels au ministère de la planification et de la gestion des ressources, au directeur des forêts, au STIDC et à la SFC. Ces rapports retracent les mouvements de grumes dans l'Etat, ainsi que le respect des quotas de réserve et d'exportation par les détenteurs de licence. Les scieries doivent remettre au STIDC des rapports mensuels qui évaluent la production par rapport aux chiffres de la SFC et de Harwood, ainsi qu'aux permis d'exportation du STIDC pour les grumes et les bois sciés.

\section{Application}

Des brigades mobiles de police effectuent régulièrement des barrages routiers et contrôlent les zones frontalières à la recherche d'éventuels mouvements de bois illégal. La police maritime et fluviale est également active. L'unité Sécurité et protection des biens (SAPU) de la SFC et les officiers chargés de l'application des lois du STIDC procèdent à des contrôles aléatoires de scieries et d'expéditions de bois. La SFC renvoie les cas appropriés au département des forêts afin qu'il engage des poursuites. Le STIDC, toutefois, peut ordonner la cessation des activités de scieries non enregistrées ou de scieries ayant commis une infraction.

\section{Audits obligatoires des titulaires de licence et des systèmes de contrôle forestier}

Le Sarawak est actuellement en train de mettre en place un ensemble d'audits obligatoires du système de contrôle forestier et de la conformité des titulaires de licence. Cela inclut les audits interrégionaux par l'unité SF\&C. Ils ont commencé en 2005 et sont toujours à l'essai. Ces audits se focalisent sur les opérations des bureaux régionaux de la SFC et sur le respect de la conformité par les grands groupes industriels. Une structure et des procédures d'audit complètes sont toujours en développement. En outre, le département d'audit interne d'Etat au sein du bureau du Premier ministre effectue des audits de l'organisation et des finances de l'administration du bois, notamment la surveillance de la facturation des redevances. Comme au Sabah, il n'existe pas d'audit fédéral de la GDF. Mais contrairement à son voisin, le Sarawak ne recourt pas à des vérificateurs tiers lorsque les résultats des audits obligatoires sont remis en question.

\section{Audits volontaires des titulaires de licence}

Des éléments du système de contrôle forestier du Sarawak sont certifiés ISO et régulièrement audités en termes de cohérence et de transparence dans l'administration et la gestion de l'information. Tous les processus des unités SF\&C et SAPU sont certifiés ISO 9001 : 2000 et ISO 14001 : 1996. Les systèmes Harwood de suivi des grumes sont 
Plan détaillé d'exploitation, inventaire avant exploitation

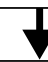

Premier dépôt : marquage et étiquetage (y compris numéro de coupe et de bloc)

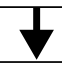

Zone de transition : cubage, classement et tri, apposition du tampon de la société

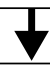

Bassin de flottage : recubage et classement, tri entre grumes destinées à l'export et grumes destinées aux scieries; attribution d'un numéro de série, inscription dans le formulaire de spécification des grumes; demande de marquage par le titulaire, prouvant que la redevance a été payée.

L'unité SF\&C surveille le volume d'exploitation accumulé pour chaque détenteur de licence

\section{$\downarrow$}

L'unité SF\&C vérifie et mesure à nouveau les grumes, le volume entré dans la base de données électronique pour la facturation; apposition du tampon de redevance et émission de l'autorisation d'enlèvement

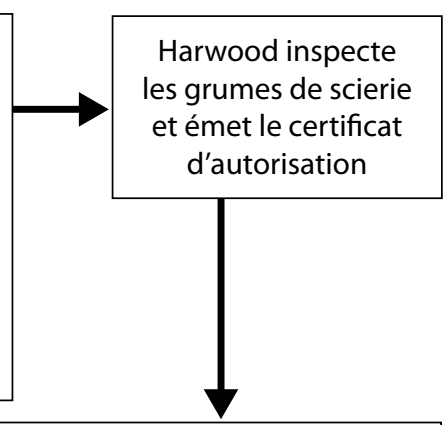

SFC inspecte les grumes avant enlèvement ; émission de l'autorisation d'enlèvement pour chaque lot de grumes portant le sceau de redevance.
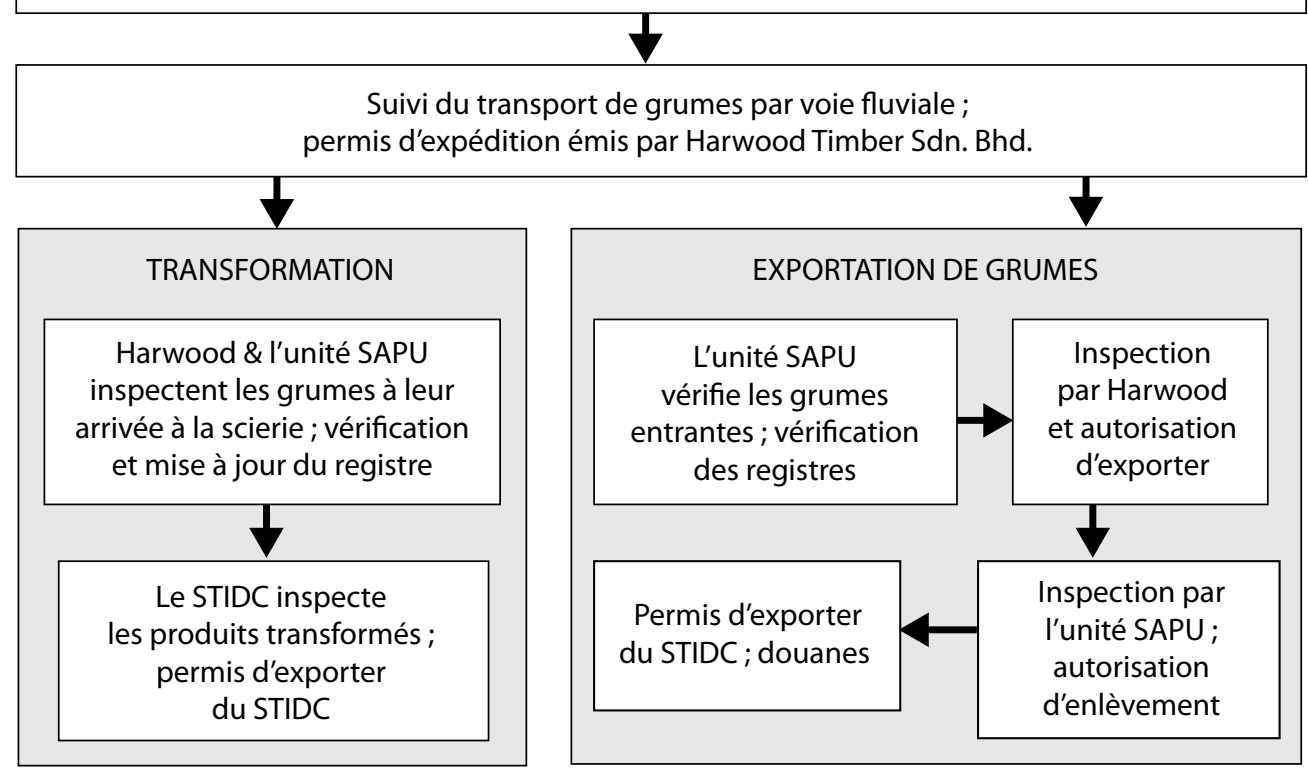

Sources: Global Forestry Services 2004, p. 9 ; Harwood Timber Sdn. Bhd 2005, et al. 27 septembre ; département des forêts de Sarawak et Sarawak Forestry Corporation, 2006, et al. 24 juillet.

Figure 16.5 Administration standard du bois au Sarawak 
également soumis à des audits de routine selon la norme ISO 9001 : 2000. La Figure 16.4 présente le système de suivi, d'audit et de conformité des forêts au Sarawak.

\section{Points forts du système de vérification au Sarawak}

Dans l'attente de la mise en place des audits interrégionaux, la vérification est largement effectuée via un système de vérifications croisées au sein du système d'administration des bois - entre la SFC, Harwood et le STIDC. Si par exemple le système de suivi informatisé de Harwood détecte des écarts en matière de consignation individuelle de grumes, ceux-ci sont automatiquement rapportés à la SFC afin qu'une enquête soit menée. Le développement prévu d'un système de rapprochement en ligne des importations, de la production nationale, de la production des scieries et des exportations renforcerait davantage ce système. Le suivi des quotas nationaux de réserve et d'exportation par Harwood présente l'avantage d'introduire une plus grande efficacité dans la gestion des quotas de réserve ainsi que dans les propres chaînes d'approvisionnement de la société.

\section{Points faibles du système de vérification au Sarawak}

\section{Problèmes dans la chaîne de traçabilité}

Sans marquage à la souche, le système actuel d'administration des bois fonctionne davantage comme un mécanisme de comptabilité des bois qu'un moyen de garantir l'origine légale. Dans le bassin de Rajang, la mise au point d'un marquage informatisé et d'un système de suivi des grumes a constitué un pas important dans la résolution de ces questions, mais des mesures doivent encore être prises pour appliquer cela ailleurs.

\section{Suivi de l'exploitation}

Le suivi et le contrôle actuel des opérations d'exploitation sont principalement effectués au moyen de la planification avant exploitation et d'inspections post-exploitation. Le contrôle préventif doit être amélioré en renforçant le suivi durant les opérations d'exploitation. Toutefois, ce suivi est limité par les restrictions budgétaires du gouvernement. Une solution pourrait être d'accroître l'utilisation de la télédétection. Une attention accrue est également nécessaire en matière d'allocation des fonctions clés entre le département des forêts du Sarawak et la SFC et les options de réallocation ou d'externalisation de celles-ci pour veiller à ce que le suivi et l'application soient correctement financés.

\section{Indépendance}

Au-delà de l'utilisation des normes ISO (et d'une seule concession certifiée MTCC à Sela'an Linau), les systèmes de certification existants ne prévoient pas d'auditeurs externes. En tant que filiale entièrement détenue par le STIDC, le rôle de Harwood dans le suivi des quotas de réserve consiste probablement en une délégation de fonctions au sein de la structure administrative de l'Etat, et non à une véritable sous-traitance à un tiers. Harwood affirme cependant que ses activités d'autorisation de grumes sont totalement indépendantes d'organes statutaires tels que le STIDC. 


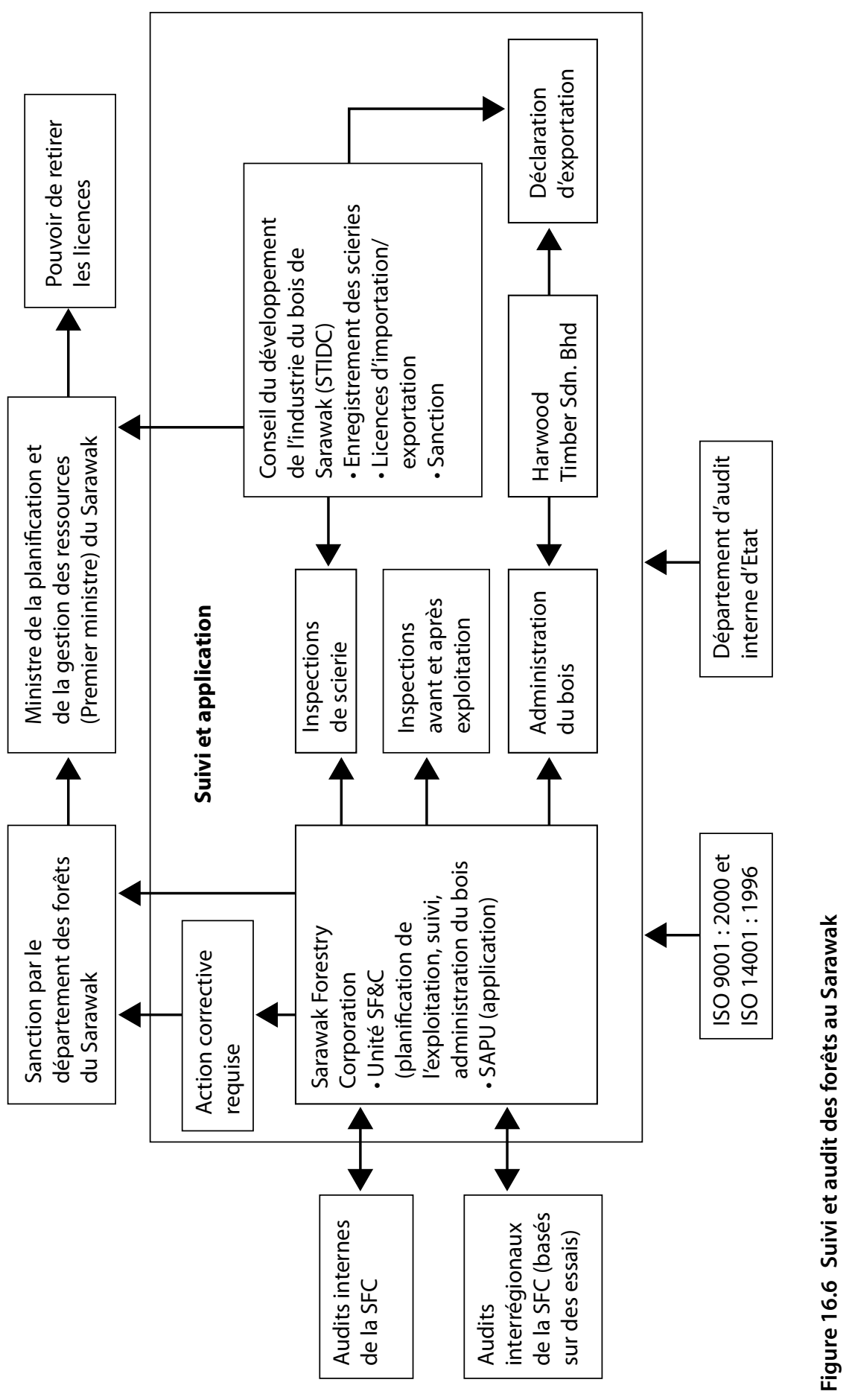




\subsection{Vérification des importations de bois}

Des systèmes de contrôle et de gestion des forêts sans doute assez solides sont en place pour les bois cultivés localement. Néanmoins, alors que la gestion de la ressource nationale s'est progressivement améliorée, l'attention s'est de plus en plus portée sur les importations de bois, qui sont depuis longtemps considérées comme une faille potentielle dans le système.

En vertu de la loi sur les douanes de 1967, tous les bois importés sont soumis à des procédures de dédouanement standard, incluant la soumission et la vérification des déclarations écrites de la valeur, de la quantité et du pays d'origine. Un certain nombre de mesures additionnelles ont été prises afin de réglementer les importations de bois en réponse à des signalements d'exploitation illégale par la société civile et à des restrictions d'exporter par le pays d'origine. Cependant, la vérification à base de recoupements, soit entre différentes agences chargées de l'administration du bois, soit entre différentes formes d'audit, est moins développée que la supervision de la production intérieure.

Toutes les importations de bois ronds, ainsi que les manufactures de base, doivent obtenir une autorisation préalable du MTIB (en péninsule et au Sabah), ou du STIDC au Sarawak. Ceux-ci n'autoriseront ces importations que si la source est authentiquement prouvée et vérifiée par l'ambassade de Malaisie dans le pays d'origine. L'ambassade en question doit avaliser l'autorité d'émission du pays d'origine (par exemple, Myanmar Timber Enterprise) pour chaque convoi.

Le 25 juin 2002, le gouvernement de Malaisie a interdit les importations de bois ronds indonésiens, en réponse à une interdiction d'exportation similaire décidée par l'Indonésie, et a par la suite étendu l'éventail des produits concernés.

Avec des craintes accrues quant à la provenance des bois indonésiens de petite taille, le STIDC a également décidé d'administrer les importations de ces bois dans le cadre de contrats transfrontaliers avec le territoire voisin du Kalimantan occidental. Les importations de bois sciées sont désormais restreintes à cinq points d'entrée précis au Sarawak. ${ }^{3}$ L'autorisation du STIDC est soumise à la présentation d'un permis de transport indonésien $(\mathrm{SKSHH})$ et de déclarations de douanes (PEB) valides.

Les douanes sont dotées du pouvoir de saisir et d'engager des poursuites lorsque les marchandises prévues sont importées sans autorisation préalable du MTIB/STIDC. La loi sur les douanes confère également au MTIB et au STIDC le pouvoir de pénétrer dans les locaux, d'effectuer des inspections et des examens, et d'interdire la vente ou l'exportation de bois. Le MTIB et le STIDC effectuent régulièrement des inspections/ visites aux points d'entrée, y compris aux ports et appontements privés, en plus de celles réalisées par les douanes royales et les autorités portuaires de Malaisie. La Figure 16.7 présente le système de surveillance, de vérification et de suivi des importations de bois.

3 Sematan (port maritime), Biawak, Tebedu, Batu Lintang et Lubok Antu. 


\section{Points forts du système de vérification des importations de bois}

Le MTIB/STIDC est investi de pouvoirs étendus en matière de contrôle des importations de bois. La décision par le Sarawak d'octroyer des licences et des autorisations d'enlèvement sur les arrivages de bois sciés de petite dimension en provenance d'Indonésie met peutêtre fin à une importante faille ayant permis l'entrée de bois illicite dans la chaîne d'approvisionnement.

\section{Points faibles du système de vérification des importations de bois}

Il reste quelques grandes inquiétudes quant à l'efficacité du système :

\section{Aval des ambassades malaisiennes}

Alors que les ambassades de Malaisie situées dans les pays sources doivent vérifier la réputation des fournisseurs si ceux-ci veulent obtenir une licence d'importation du MTIB/STIDC, cela ne suffit pas à garantir la légalité de chaque convoi.

\section{Importations de bois scié de petite dimension}

Une autorisation d'enlèvement n'étant pas nécessaire pour l'expédition de bois de petite dimension, il est difficile de connaître leur destination dans le pays. Cela n'exclut pas que des bois d'origine potentiellement illégale soient introduits dans la chaîne de production. La seule exception est le Sarawak, où le STIDC autorise et marque les importations de bois de petite dimension en provenance du Kalimantan. Mais même au Sarawak, il n'existe à l'heure actuelle aucun mécanisme permettant de vérifier l'authenticité des documents d'exportation indonésiens. Les chiffres des importations malaisiennes ne reflètent pas l'expansion, à toutes les catégories de bois scié, de l'interdiction d'exporter indonésienne de 2004.

\section{Systèmes d'information}

Alors que les statistiques d'importation sont collectées par le MTIB, le STIDC et les douanes, il n'existe pas encore de système permettant le rapprochement en ligne de ces données avec la production intérieure et la production des scieries déclarée ainsi que l'évaluation des quantités probables de bois de contrebande qui entrent dans les flux de production et d'exportation de Malaisie. Le STIDC a commencé à réfléchir à la question, mais les progrès sont plus lents que dans la péninsule et au Sabah. Dans les trois juridictions, le suivi informatisé des grumes aiderait considérablement à effectuer un rapprochement en ligne.

\section{Recours limité à l'audit}

Les audits d'Etat ou fédéraux n'ont pas encore été introduits en matière de mesures des importations, sauf pour les audits internes des opérations des agences chargées de l'application de la loi. Les normes ISO 9001 : 2000 s'appliquent désormais à certaines 


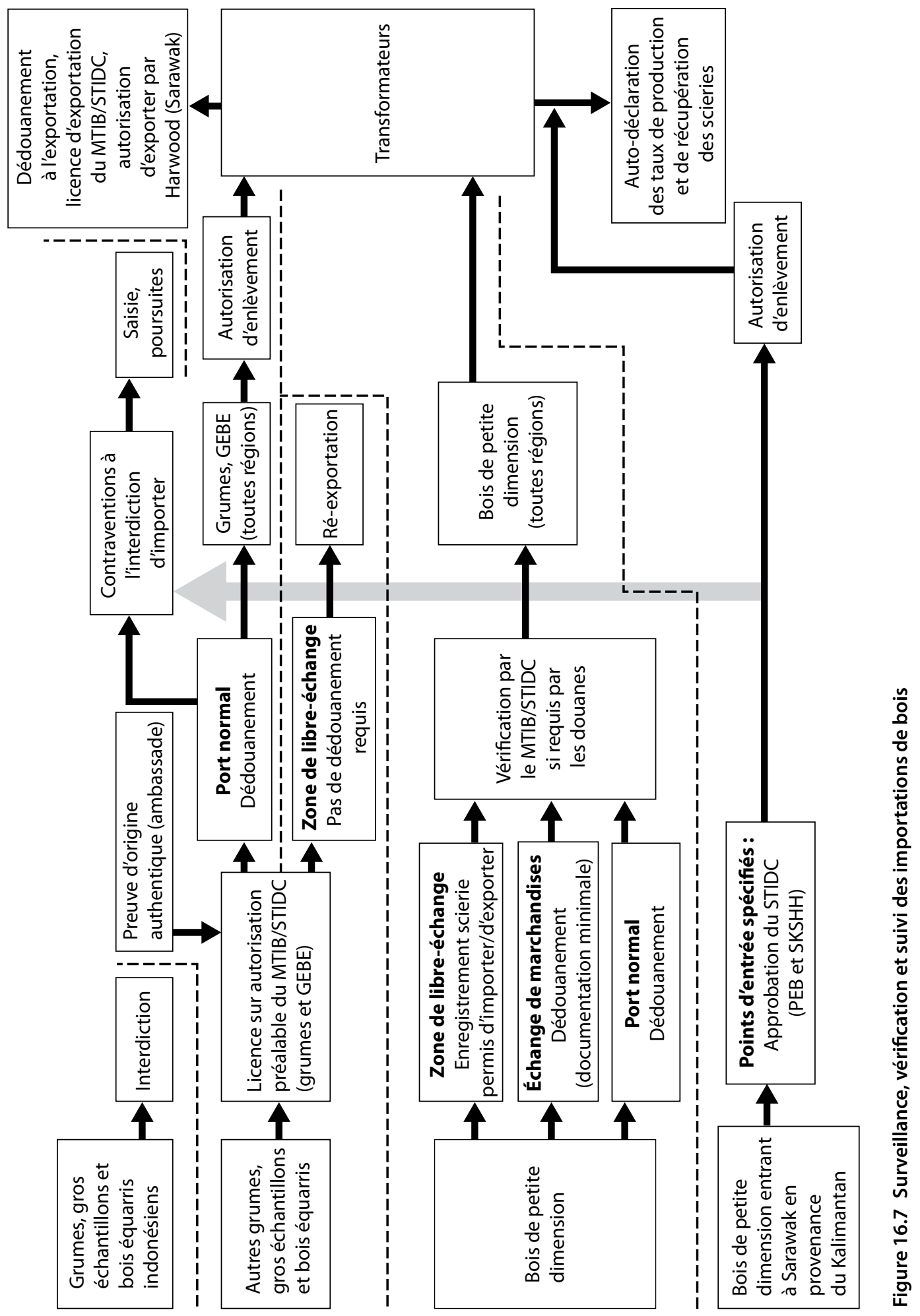


mesures d'importation, telles que les inspections du MTIB, mais celles-ci ne se basent que sur les résultats (papiers) et ne concourent pas à renforcer une quelconque autre forme d'audit.

\subsection{Efficacité du contrôle et de la vérification des forêts}

Les systèmes de contrôle forestier existants en Malaisie bénéficient d'un fort soutien gouvernemental, ce qui prouve l'importance du secteur en matière de recettes et de réputation du pays sur le plan international.

Un ensemble de mesures de vérification a progressivement été introduit ces récentes années afin d'améliorer l'efficacité du système. Au début des années 1990, l'action du gouvernement fédéral dans la péninsule visant à relever le niveau des sanctions et à introduire des directives plus strictes en matière d'octroi de licences et de concessions a eu un impact considérable sur le taux d'exploitation illégale et de surexploitation dans les Etats.

Les audits ont également commencé à jouer un rôle de plus en plus important. En Malaise péninsulaire, les Etats qui ne satisfont pas aux demandes d'actions correctives dans le cadre des audits fédéraux de la GDF doivent rendre des comptes au Conseil national des forêts. L'absence de réponse aux demandes d'actions correctives entraîne également le risque d'une suspension de la certification MTCC - ce qui s'est produit pour l'Etat de Terengganu en 2002.

La participation active des informateurs publics dans la signalisation de cas d'exploitation illégale a également joué un rôle ; cela suggère que les systèmes bénéficient d'un soutien de l'opinion relativement étendu. Il existe cependant quelques domaines d'inquiétude.

Tout d'abord, il existe des failles possibles dans la chaîne de traçabilité, bien que cela varie selon le degré avec lequel chaque Etat: (i) dépend d'une administration papier, (ii) pratique la traçabilité à la souche, (iii) gère les bois en dehors du domaine forestier permanent, (iv) marque et suit les importations de bois (en particulier les bois de petite dimension en provenance d'Indonésie). Les systèmes d'information ne sont pas encore reliés pour permettre un rapprochement rapide de la production de scierie déclarée avec la production et les importations légales.

Ensuite, l'application des lois est entravée par des ressources limitées pour suivre les opérations d'exploitation - manque de personnel et contraintes de coûts pour les autorités forestières.

Troisièmement, malgré l'éventail d'audits obligatoires et volontaires, il existe des craintes sur les questions suivantes :

- Si le champ d'application et la profondeur des audits existants sont suffisants, surtout lorsqu'un Etat entier est considéré comme une seule Unité de gestion forestière, comme c'est le cas des Etats de la péninsule. 
- Si les audits existants sont réellement complémentaires en termes de champ d'application et de méthode. Par exemple, bien que les audits de GDF examinent les résultats de l'administration des bois et de la gestion forestière, les audits ISO ne peuvent examiner que les premiers.

- Si les organes obligatoires sont suffisamment indépendants, étant donné que la responsabilité du suivi et de l'audit obligatoires se limite aux organismes d'Etat et aux organismes détenus ou financés par l'Etat, avec recours limité à des auditeurs externes.

- Si les systèmes sont suffisamment ouverts et transparents ; l'absence d'accès public garanti aux procédures d'audit obligatoire et aux décisions de vérification prises par les autorités forestières ou les fonctionnaires d'Etat est un inconvénient d'un point de vue de gouvernance.

- L'absence de mécanismes bien développés de résolution des recours et des litiges pour garantir l'intégrité perçue de certaines procédures d'audit obligatoire.

- Les pouvoirs relativement limités du gouvernement fédéral en matière d'audits obligatoires des autorités forestières des Etats de la péninsule.

Cependant, le plus grand défi de la vérification concerne les droits légaux des groupes aborigènes/autochtones coutumiers sur le domaine forestier permanent. Aucun système de vérification forestière ne répond à ce problème, à l'exception peut-être des SFMLA au Sabah, qui prévoient des dispositions sur les forêts communautaires et le développement des communautés.

\subsection{Enseignements}

Le cas de la Malaisie illustre à quelles point différentes approches de contrôle et de vérification des forêts peuvent se développer au sein d'une même structure fédérale, en réponse à un contexte forestier différent dans chaque Etat. Dans les trois juridictions, (Malaisie péninsulaire, Sabah et Sarawak), une approche progressive a été adoptée afin d'augmenter la crédibilité, basée sur le renforcement des systèmes de suivi et de vérification existants.

Ce cas montre que les structures composites qui conjuguent audits volontaires (ISO et FSC) et obligatoires (par l'Etat ou en rapport avec l'Etat) peuvent potentiellement réduire la nécessité d'une observation externe ad hoc et intrusive des activités de gestion forestière du pays. Toutefois, l'efficacité des dispositifs dépend de plusieurs facteurs. En particulier :

- Les procédures existantes doivent être complémentaires les unes des autres en termes de champ d'application, d'échantillonnage et de fréquence, afin de permettre un véritable système de contrôle mutuel équilibré, notamment des protocoles d'évaluation comparative des résultats d'audit.

- Les fonctions de suivi et de vérification sous-traitées et les processus d'accréditation connexes doivent être à l'abri de toute influence gouvernementale. 
Le fait que certains éléments du contrôle forestier soient sous-traités à des entreprises détenues par l'Etat (p. ex. Harwood au Sarawak et les régulateurs de l'industrie du bois dans les trois juridictions) signifie que la vérification entièrement indépendante attendue par le marché peut ne pas être assurée. L'absence de procédures établies permettant au public d'avoir accès aux audits d'Etat et aux décisions de vérification connexes constitue un autre problème qui réduit la confiance du grand public et soulève des inquiétudes quant à une possible interférence politique. Le Sabah répond à cette nécessité d'indépendance garantie en travaillant à une certification FSC complète pour tous les titulaires de licence. Ailleurs, le processus d'accréditation des auditeurs est un élément crucial de l'indépendance. L'expérience avec le MTCC indique également que la représentation des organismes de normalisation/accréditation est essentielle pour maintenir la crédibilité, en particulier lorsque ceux-ci sont semi-privés.

Ce cas est également intéressant en ce sens qu'il utilise les normes ISO, ce qui traduit un mouvement général d'amélioration de l'efficacité administrative. Cependant, la plupart des audits ISO (principalement ISO 9000) sont axés sur les réalisations, à savoir la conformité aux procédures administratives, et non sur les résultats (légalité, GDF). Ceci pourrait exiger de passer à ISO 14000 et/ou 19000.

Dans la mesure où la majorité de la production de bois nationale est légale sur le plan des licences et du transport, les commentateurs se préoccupent désormais plus de la nature des normes juridiques (en particulier dans les domaines fonciers et de GDF) qui servent à évaluer la conformité. Bien que les tribunaux se soient prononcés en faveur des droits autochtones, ceci doit encore être traduit dans la réforme des règlements sur le classement, la gestion des forêts et les compensations. Il existe tout un ensemble de règlements qui régissent les licences, la planification de l'exploitation et l'administration du bois. C'est seulement maintenant qu'ils sont rassemblés dans une unique définition de la légalité, dans le cadre du développement d'un AVP entre la Malaisie et l'UE. Jusqu’à présent, les équivalents les plus proches d'une définition de la légalité étaient les normes de GDF établies pour les audits du MTTC et les audits d'Etat de la GDF, ainsi que les audits ISO de conformité aux procédures administratives. Ceux-ci ont remplacé la vérification de la légalité, en l'absence d'une procédure dédiée.

En termes de production de bois nationale, le suivi informatisé est considéré utile pour garantir l'origine légale et améliorer le contrôle de l'exploitation dans les essais au Sarawak. Il est donc envisagé de l'adopter à plus grande échelle. Toutefois, en tant que grand importateur de bois, la Malaisie doit également s'occuper de la vérification de la légalité dans les pays d'origine comme sur son territoire. Ce qui soulève deux types de défis :

- La vérification aux points d'entrée : les ambassades peuvent avoir à vérifier la bonne réputation des opérateurs dans les pays sources, alors que la présentation de la documentation officielle du pays source est considérée comme suffisante aux points d'entrée. Une coopération douanière efficace est donc essentielle pour empêcher les fraudes, mais un système régional d'audit transnational doit encore être créé.

- La séparation des documents importés et nationaux dans les flux de transformation et d'exportation, afin de rassurer les acheteurs. Cela exige, entre autres, que tous les 
arrivages importés (y compris les bois sciés) reçoivent une autorisation d'enlèvement et soient suivis jusqu'au lieu de transformation.

Enfin, l'expérience du Sabah avec ses licences de 100 ans montre que la vérification n'est pas qu'une question d'application des lois. Elle peut également représenter un important outil pour amener les systèmes de gestion des forêts à une conformité légale complète 


\section{Partie C}

\section{Examen des questions transversales}





\section{Chapitre 17}

\section{Présentation des questions transversales}

\subsection{Introduction}

Les études de cas par pays présentées dans la Partie B illustrent la grande diversité des contextes et des facteurs qui prévalent en matière de systèmes de vérification et en déterminent l'architecture. Si certains pays ont adopté une approche progressive en se fondant sur des systèmes de suivi existants, d'autres ont privilégié des réformes plus radicales, souvent facilitées par des intérêts extérieurs. Dans quelques cas, la vérification a été effectuée par une seule et même institution alors que partout ailleurs la responsabilité en est revenue à de multiples intervenants. Les effets de la vérification ont varié en intensité s'étageant d'un impact négligeable à un impact substantiel, voire parfois inattendu.

Ce court chapitre est un exposé succinct des questions qui nous sont apparues à la fois comme constituant des thèmes communs aux études de cas nationales et comme présentant le plus grand intérêt pour l'élaboration d'un système de vérification. Ces questions sont étudiées d'une façon plus détaillée dans les chapitres qui suivent, notamment les aspects qui concernent plus spécifiquement les systèmes de vérification pour déterminer à la fois les facteurs critiques présidant à l'élaboration de ces systèmes et les répercussions (impacts) que ces systèmes pourraient avoir.

\subsection{Une matrice conceptuelle pour analyser le modèle du système de vérification}

Malgré la grande diversité des études de cas et leurs différences évidentes, il est clairement ressorti de l'analyse des résultats que quatre grandes fonctions devaient être incluses dans tout processus de contrôle et vérification, à savoir :

- Le mandat politique précédant la vérification;

- Les fondements juridiques et institutionnels de ce mandat;

- L’architecture du système de vérification ; et

- Les résultats attendus du système.

Ces fonctions ont été précédemment étudiées dans le Chapitre 4 et sont résumées sous forme de matrice modèle dans le Tableau 17.1 ci-après qui en présente les multiples objectifs. Tous les éléments du système de vérification sont d'abord regroupés en une suite logique et reliés au mandat politique au titre duquel le système a été établi, à son contexte juridique et institutionnel et à l'impact sur le terrain. Suivent les thèmes clé qui doivent être analysés pour évaluer la faisabilité du système - il s’agira vraisemblablement 


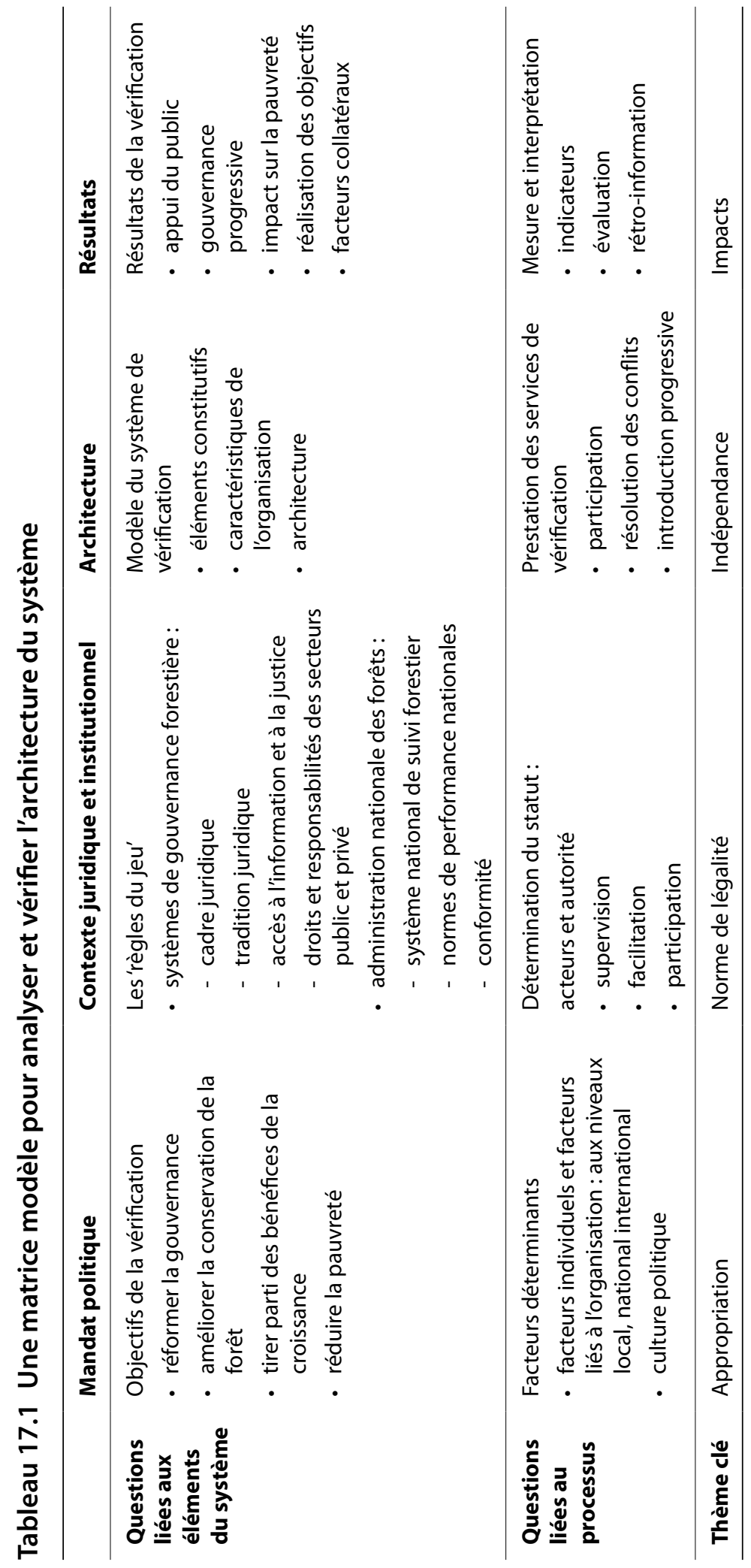


de l'appropriation dans le cas du mandat, de la norme de légalité, de la mesure dans laquelle le système peut préserver son indépendance en dehors de toute influence politique dans le contexte juridique et institutionnel, et de l'impact du système en aval. Enfin, est relevée la nécessité d'établir un lien effectif entre les facteurs qui déterminent et créent la demande de vérification et la façon dont les jugements concernant la vérification sont rendus et la stratégie est déterminée.

S'agissant du thème clé de l'appropriation qui fait l'objet du Chapitre 18, il est clair que l'appropriation du processus par le gouvernement est une condition nécessaire mais non suffisante de la crédibilité de la vérification. Pour que le système de vérification soit crédible, il est indispensable qu'il soit adopté par d'autres intervenants qui s'en approprient les objectifs sous-jacents. De grands partenariats peuvent être conclus entre les divers acteurs concernés, en partie à travers leur participation active à la conception et à la mise en œuvre du système. Différents acteurs ont cependant des aspirations et des objectifs qui diffèrent et il sera donc difficile de trouver un juste équilibre qui variera vraisemblablement d'une situation à l'autre. Les dispositions prévues par les gouvernements devront être assez souples pour tenir compte des opinions exprimées par le grand public et il faudra sans cesse rechercher la juste mesure entre les responsabilités du gouvernement et celles du public en procédant à des analyses au cas par cas.

Un ensemble de règles claires et sans ambiguïté est indispensable pour déterminer que les dispositions édictées en matière de vérification sont bien respectées. Il y a cependant lieu de noter que les cadres juridiques et réglementaires du secteur forestier sont souvent extrêmement complexes et ne constituent pas une référence suffisamment claire pour la procédure d'audit. Pour pallier cette difficulté, il conviendra donc de prendre deux types de mesures à court terme : (i) définir les priorités des lois et réglementations existantes en fonction de l'objectif et du mandat du système de vérification ; et (ii) doter les cadres réglementaires de normes précises en matière de performance. Dans les deux cas, il sera nécessaire (d'élaborer une norme de légalité nationale (voir description au Chapitre 19).

S'agissant du champ d'application de la norme, le registre des options est plutôt vaste allant d'une approche minimaliste (par exemple, seules les règles concernant l'exploitation du bois d'œuvre sont prises en compte) à une approche beaucoup plus globale comportant notamment des objectifs de développement et à visée sociale. Comme le pouvoir de légiférer relève du droit souverain des Etats, il n'est possible que d'anticiper les différentes façons dont la question peut être abordée. Toute définition du champ d'application de la norme doit comporter un énoncé clair de l'objectif (ou des objectifs) du système de vérification qui, à son tour, dépendra du contexte politique et du cadre juridique spécifiques du pays concerné. Il importe aussi de consulter la plus large gamme possible de parties prenantes de sorte que les opinions et avis des différents groupes d'intérêts puissent être pris en compte. Une autre question importante concerne la façon dont les normes une fois établies seront mises en œuvre - soit sous forme de codes de bonnes pratiques soit en tant que dispositions législatives ayant force de loi. La première option présente le double avantage du maintien d'un lien fort avec l'administration des forêts et de la facilité de sa mise en application. L'avantage potentiel de la seconde tient à ce que 
la décision ultime, au stade du règlement des différends, sera prise par les tribunaux en dehors du secteur forestier.

Le maintien d'un fort degré d'indépendance est au cœur de la crédibilité de tout système de vérification et il en sera question au Chapitre 20. Cette indépendance peut être garantie par la diversité des origines et des associations des différents acteurs chargés de la vérification; à travers différentes organisations qui interviennent dans le processus de vérification (ONG, sociétés et entreprises du secteur privé) ou à travers l'adhésion à des normes professionnelles. L'autre possibilité est que les structures institutionnelles plutôt que les caractéristiques des différents acteurs concernés soient mieux à même de préserver cette indépendance. Nombreuses sont les inquiétudes exprimées à propos de l'insuffisance de garantie que présente - pour l'indépendance de cette opération - une vérification effectuée par un seul intervenant. Il est indispensable de mettre en place des mécanismes qui garantissent la responsabilité, la transparence, l'objectivité, la compétence et l'acceptabilité du système, et d'assurer aussi qu'ils comportent suffisamment de procédures de vérification (cross-checks and balances). L'indépendance de tout mécanisme public de reddition des comptes (public reporting) est un élément essentiel de tout système de vérification.

Le Chapitre 21 porte sur le thème final du cadre conceptuel, celui des effets. Notre expérience en matière d'évaluation de l'impact formel des systèmes de vérification mis en place dans le secteur forestier est très limitée, voire inexistante, à ce jour. Les raisons en sont multiples : (i) un grand nombre de ces systèmes sont encore trop récents pour avoir déjà fait l'objet d'évaluations ; (ii) l'évaluation de l'impact est complexe et requiert des ressources considérables ; (iii) les effets à attribuer au système de vérification n'apparaissent pas toujours très clairement ; et (iv) dans les cas de faible adhésion au système (degré d'appropriation insuffisant), évaluer l'impact peut se révéler sans grand intérêt. L'impact sur l'environnement, en particulier, reste encore mal compris. On peut toutefois observer qu'au plus haut niveau politique, de nombreux pays ont explicitement élevé la gestion équitable des forêts au rang d'objectif politique - ce qui implique une cascade de décisions politiques et de textes législatifs pour assurer un impact de l'utilisation des forêts sur le développement. En renforçant la conformité, la vérification devrait logiquement concourir à la réalisation de cet objectif politique.

L'examen des quatre thèmes de la matrice conceptuelle est suivi de deux chapitres (22 et 23) dans lesquels sont étudiés les agendas à venir : tout d'abord, la tendance à la convergence entre le système de vérification et l'opération de certification effectuée par le secteur privé - tendance qui est de plus en plus nette à mesure que les gouvernements cherchent à répondre aux demandes extérieures selon des modes acceptables par l'industrie ; et, ensuite, le rôle des technologies de l'information, notamment la télédétection et les bases de données en ligne, particulièrement marqué au Brésil.

Le dernier chapitre de cette troisième partie porte sur la performance et l'historique des processus suivis par les multiples parties prenantes pour faire avancer leurs stratégies dans des contextes très différents du point de vue des intérêts en jeu ainsi que des autorités 
et des pouvoirs en cause. Cette analyse est essentielle à la conception du système de vérification étant donné que le soutien que pourrait apporter la société civile à ce système aux niveaux national et international devrait dépendre dans une très large mesure et, selon toute vraisemblance, de la capacité non seulement de rassembler les multiples parties prenantes mais aussi de conserver leur appui à long terme. Il s'agit d'arriver à concilier tous ces intérêts de façon à permettre le bon déroulement et le succès final du processus de négociation, malgré les visées divergentes, voire souvent conflictuelles, des parties en cause. 



\section{Chapitre 18}

\section{Appropriation des systèmes de vérification forestiers}

Note : Cecilia Luttrell est l'auteure de ce chapitre, extrait de l'article de Luttrell, C. 2007 Ownership in relation to the design of verification systems. VERIFOR, Briefing Paper No. 5. ODI, Londres.

\subsection{Introduction}

Une grande partie des débats actuels sur la vérification dans le secteur forestier mettent l'accent sur le concept «d'appropriation » en tant que condition préalable de la durabilité à long terme du système et de son degré d'adaptabilité (robustesse) au changement. Dans les dictionnaires, les définitions concernant l'appropriation relèvent surtout que l'un des éléments importants en est la "faculté de maîtriser et d'utiliser à des fins personnelles ". Dans ce sens, l'appropriation ne se réfere pas seulement à des notions de "participation " ou de "soutien " à quelque chose. De Renzio et al. (2006) propose une définition dans le contexte d'une étude sur l'harmonisation de l'aide, à savoir que l'appropriation résulte "du consensus social qui s'est dégagé à propos de la légitimité des institutions régissant les processus décisionnels et de leur capacité à y participer de façon substantielle ». Aux fins de ce chapitre, le terme appropriation s'entend du "soutien actif » apporté aux objectifs, processus et résultats du système de vérification et de la "capacité de maîtriser " lesdits objectifs, processus et résultats. Il en ressort que les parties prenantes s'intéressent aux enjeux du système et aux processus dont elles sont disposées à appuyer le déroulement et qu'elles seront liées par les résultats. Nous étudierons dans ce chapitre les stratégies susceptibles d'étoffer les systèmes de vérification et, dans le même temps, d'en garantir ou, tout au moins, d'en renforcer l'appropriation.

Les descriptions et définitions données dans d'autres contextes laissent entendre que tout processus d'appropriation devra respecter les conditions suivantes :

- Déterminer les objectifs et les motivations au niveau national ;

- Refléter les perspectives nationales (par exemple, en les rattachant à des plans et programmes sectoriels spécifiques) ;

- Compléter les structures des institutions et processus existants au niveau national ;

- Être en adéquation avec les spécialisations, capacités et ressources nationales ;

- Servir les intérêts d'une vaste gamme d'acteurs du secteur public à travers des processus participatifs globaux de sources variées; et

- Inscrire les principes de responsabilité et de transparence dans la mise en œuvre.

Le débat ci-après s'articule autour de six grands thèmes liés à l'appropriation. 


\subsection{Objectifs}

Dans le contexte de l'appropriation, il s'agira avant tout de déterminer la raison pour laquelle un système de vérification a été mis en place et les principaux facteurs sousjacents à cette instauration. Cela aidera à mieux percevoir quels sont les différents intérêts et acteurs redevables du système. Les différences relevées d'une étude de cas national à l'autre dans cet ouvrage montrent que l'établissement d'un système de vérification peut prendre ancrage dans toute une série d'objectifs qui seront déterminés par des causes internes ou externes et pourraient notamment inclure :

- Les modalités et conditions imposées par les bailleurs d'aide ;

- Le souhait exprimé par le secteur privé d'accéder aux marchés internationaux ;

- Le souhait exprimé par le gouvernement national d'augmenter les recettes fiscales ; ou

- Le souhait manifeste du gouvernement national de répondre aux demandes de l'opinion publique ou des ONG pour assurer une gestion durable des forêts ou la protection des droits.

Par exemple, l'élan donné au renforcement des systèmes de vérification en Malaisie est en partie dû aux pressions exercées par l'industrie qui cherche à établir sa crédibilité sur le marché international et en partie attribuable à l'Etat dont l'objectif est de s'assurer des prébendes régulières et de faire droit aux inquiétudes de l'opinion publique en matière d'environnement (voir Chapitre 16). Dans le cas de l'Equateur, l'instauration d'un système de vérification a résulté de pressions sociales internes (voir Chapitre 10), tandis qu'au Cambodge et au Cameroun la création d'un mécanisme de suivi et de contrôle indépendant faisait partie des conditions requises par les bailleurs de fonds (voir Chapitres 13 et 11 ).

Ces pressions peuvent survenir simultanément et se renforcer mutuellement - prendre dûment en compte, par exemple, les demandes pour plus de transparence peut aider à redonner du lustre à la réputation d'un pays au niveau mondial. D'autres facteurs déterminants peuvent cependant être contradictoires comme dans le cas de systèmes de vérification assortis de conditions imposées par les bailleurs et animés par le souhait d'accéder aux marchés internationaux. Par exemple, les bailleurs peuvent être motivés par différentes raisons lorsqu'ils prônent le développement de systèmes de vérification, allant d'une meilleure reddition des comptes concernant l'utilisation des fonds alloués au titre de l'aide à l'encouragement des partisans des réformes. Le règlement de l'UE relatif à la délivrance de licences (CE, 2005a) fait allusion à la diversité des objectifs inscrits dans l'initiative FLEGT : "Le Plan d'Action met l'accent sur les réformes de gouvernance et de renforcement des capacités ; il est appuyé par des actions visant à appuyer une coopération multilatérale et des mesures complémentaires sur le plan de la demande afin d'aider à utiliser le bois légalement exploité et récolté et de contribuer à la promotion de l'objectif plus vaste recherché, à savoir la GDF dans les pays producteurs de bois.» À différents objectifs correspondent aussi divers enjeux et diverses opportunités pour renforcer l'appropriation. 
Le terme d'appropriation doit être utilisé avec circonspection. Il sert le plus souvent à désigner des situations dans lesquelles il ne se produira vraisemblablement pas et s'emploie le plus souvent dans les débats relatifs aux systèmes liés aux bailleurs pour indiquer le degré d'acceptation requis des acteurs nationaux en matière d'idées apportées de l'extérieur. Une question récurrente dans les études de cas VERIFOR porte sur la mesure dans laquelle les systèmes de vérification qui se fondent sur l'imposition de conditions à remplir favorisent l'apparition d'un fort sentiment d'appropriation. L'une des thèses fondatrices de la formule des prêts accordés sous conditions est que l'acceptation de ces conditions par l'Etat emprunteur témoigne de la volonté de cet Etat de prendre des engagements fermes. Cette thèse néglige pourtant l'importance de la realpolitik, des modalités de fonctionnement et des raisons pour lesquelles les conditions imposées à l'octroi de l'aide sont acceptées. Si on entend par appropriation "le soutien actif apporté aux objectifs, processus et résultats d'un système de vérification et la capacité de les maîtriser ", il se pourrait qu'il y ait contradiction entre l'appropriation et l'imposition de conditions. Létude de cas effectuée au Cambodge souligne les dangers inhérents au remplacement de l'idéal d'appropriation par la fixation de conditions. L'échec du mécanisme reposant sur un énoncé de conditions est attribuable à la fois à l'absence d'une coalition nationale forte et à l'absence totale d'engagement du gouvernement en faveur d'une réforme.

Dans l'ensemble, les bailleurs se sont désintéressés de l'énoncé des conditions auxquelles l'aide sera accordée. La nouvelle architecture de l'aide internationale est axée sur la promotion, l'appropriation et la volonté d'engagement au niveau national, les conditions jouant un rôle désormais bien moindre. La coercition imposée par le biais de conditions explicites imposées par les donateurs est considérée comme un instrument peu efficace pour favoriser l'instauration d'un climat propice à l'appropriation, étant donné qu'il s'agit d'un moyen totalement incapable de susciter des vocations et des engagements sur le plan national et qu'il n'a donc pas d'avenir.

S'agissant des accords de partenariat volontaires (APV), force est de constater l'existence de réalités géopolitiques inhérentes aux relations existant entre les pays partenaires et l'UE. En outre, les pays producteurs sont incités à signer de tels accords pour toutes sortes de raisons qui auront des répercussions sur le degré d'appropriation, la notion d'appropriation pouvant aussi contredire celle du partenariat au sens de l'APV. Par définition, en effet, tout partenariat suppose un moindre degré d'appropriation puisqu'il a pour résultat un partage de pouvoir et d'influence entre partenaires et qu'il nécessite une entente pour prendre en compte des intérêts et normes pouvant être divergents ou disparates.

Comme dans le cas des mécanismes liés par les conditions imposées par les bailleurs, les systèmes de vérification qui dépendent du marché international impliquent aussi une certaine érosion du sentiment d'appropriation au niveau national. Prenons les APV, par exemple, qui impliquent une influence de parties prenantes basées en UE - ce qui pourrait diminuer le degré d'appropriation de ces accords au niveau national. 
Dans cet ordre d'idées, on peut aussi se demander comment éviter que les procédures requises au titre du suivi international de l'aide octroyée puissent être invoquées dans le cas de ressources relevant ostensiblement de la souveraineté nationale. Les agents indépendants chargés de ce suivi au Cambodge et au Cameroun ont, dans les deux cas, tenté d'imposer l'influence d'organismes internationaux sur des ressources relevant du droit souverain des Etats - ce qui a soulevé des questions concernant le régime de propriété applicable à ces ressources. Nombreux étaient ceux, en revanche, qui considéraient comme précieuse la protection assurée par les associations internationales dont les audits/vérificateurs indépendants pouvaient exercer leurs fonctions avec une liberté déniée aux ressortissants nationaux. On a cependant pu estimer par ailleurs que le processus d'appropriation au niveau national se trouvait affaibli par cette influence internationale. Le défi opposé dans ces deux cas au processus d'appropriation nationale pourrait avoir été lié non seulement au caractère international de la fonction de vérification mais aussi au type d'organisation assumant cette fonction - ce qui semblerait dénoter que des observateurs ( chiens de garde ") relevant d'ONG (internationales ou nationales) pourraient être mieux acceptés sur le plan national que des vérificateurs indépendants travaillant sous contrat pour l'Etat.

$\mathrm{Au}$ Ghana, les premières remises en cause concernant la vérification et la validation du Programme d'exploitation légale du bois d'œuvre (Validation of Legal Timber Programme) avaient été inspirées par les bailleurs qui cherchaient à contrecarrer les échecs de la gouvernance dans le secteur forestier. Depuis lors, il y a eu de nombreuses tentatives pour impliquer davantage l'industrie, la société civile et divers éléments du gouvernement. Un indicateur manifeste d'appropriation nationale accrue pourrait être la mesure dans laquelle une autorité nationale est disposée à contribuer financièrement à l'initiative. Dans le cas du Ghana, le gouvernement est prêt à investir 2 millions de dollars US de ressources propres dans un système d'audit (voir Chapitre 12).

Il est vraisemblable que les systèmes de vérification qui bénéficieront de la meilleure appropriation nationale seront ceux qui ont été mis en place pour être au service de politiques et causes nationales plutôt que ceux qui visent l'accès aux marchés internationaux ou le respect des conditions fixées par les bailleurs. Quel que soit l'objectif initial cependant, l'appropriation au niveau national peut être renforcée par l'accentuation des complémentarités existant entre les institutions et procédures existantes. Au nombre de ces complémentarités pourraient figurer notamment les procédures en vigueur pour le suivi et l'audit du secteur forestier. L'une des raisons sous-jacentes à la réussite du système de vérification de la CITES en est l'ancrage dans les systèmes nationaux de reddition des comptes et dans le principe de la souveraineté de l'Etat en tant que fondements du système de contrôle (voir Chapitre 3).

\subsection{Participation d'entités gouvernementales extra- sectorielles et décentralisées}

L'appropriation peut être influencée par la mesure dans laquelle les institutions, autres que celles du secteur forestier, participent à la mise en place du système de vérification. 
L'un des enseignements des études de cas concerne la nécessité de coopter des parties extérieures au secteur pour assurer une large acceptation au niveau gouvernemental. Dans l'étude de cas de l'Equateur, le manque de participation d'autres institutions gouvernementales a abouti à la situation particulièrement délicate de l'isolement du ministère chargé de la mise en œuvre, à savoir le ministère de l'environnement. Une grande partie de l'opposition à l'introduction du système de vérification est venue de l'intérieur même de ce ministère, sans qu'aucune volonté politique ne contrebalance cette résistance interne. De même, l'étude de cas réalisée en Indonésie montre que le dernier mot pour statuer sur les normes de légalité pourrait ne pas revenir au ministère des forêts étant donné que certaines questions concernent l'administration des terres. Une autre option pourrait être de placer le système de vérification sous le contrôle d'une autre institution ou d'une commission parlementaire, suggérant ainsi qu'il y aurait des avantages à s'assurer que les décisions concernant le système de vérification soient prises aux échelons hiérarchiques les plus élevés du gouvernement.

Certaines entités nationales au Brésil comme le Programme national des forêts, le Comité interministériel de lutte contre la déforestation en Amazonie et le PPG-7 (voir Chapitre 9) se sont fortement engagées dans les processus de vérification qui leur sont rattachés. Une appropriation aussi étendue et aussi marquée de ces systèmes de vérification est en partie le reflet d'un vaste processus de participation entre de nombreuses administrations étatiques importantes mais s'explique également par la volonté du Brésil de faire valoir son droit souverain à gérer le bassin de l'Amazonie.

Un thème récurrent des études de cas est celui de la nécessité de prendre en compte les échelons décentralisés du gouvernement. En Indonésie, le ministère des forêts a dévolu ses pouvoirs en matière d'administration de l'exploitation forestière au niveau du district mais cela ne recouvre pas la répartition des rôles et responsabilités stipulés dans les lois sur la décentralisation administrative (Wells et al. 2006). Il en est résulté une certaine confusion et une surcharge de travail pour les administrations de district dont les capacités de mise en œuvre sont limitées (voir Chapitre 15).

Les questions abordées ici englobent aussi bien différents degrés de volonté et d'engagement des organismes relevant de l'administration centrale ou décentralisée que les différences de capacités disponibles pour la mise en œuvre du système. L'existence de capacités adéquates dans les institutions responsables est un élément crucial de tout bon système de vérification. Le modèle prévu pour le projet SECAL aux Philippines, par exemple, était trop ambitieux au vu des capacités existantes du ministère de l'Environnement et des Ressources naturelles (Cruz et Pulhin 2006) et, en dehors du secteur forestier, des initiatives comme le processus de Kimberley et l'introduction de sauvegardes nucléaires au niveau international ont été confrontées à de semblables problèmes de capacités dus à la façon dont le système de vérification avait été élaboré (Smillie 2005; Persbo et al. 2005).

Se pose alors une autre question qui est celle de savoir si le renforcement des capacités est essentiel à l'appropriation. Au Cambodge, un organisme de suivi national " extérieur » 
appelé "Court Watch » collabore de façon constructive avec le système judiciaire et alimente le processus de réforme en faisant part de ses constatations - et ce sont précisément ces activités qui lui donnent accès au processus et lui permettent d'en suivre le déroulement (Luttrell et Brown 2006). L'élaboration d'un mécanisme de suivi sans prise de mesures correspondantes pour renforcer les capacités du système pourrait aboutir à un défaut d'appropriation. Cependant, c'est précisément le renforcement des capacités qui pourrait compromettre l'indépendance du système au cas où le bon déroulement du processus serait suivi par l'organisme chargé de la vérification.

L'hypothèse de base d'une grande partie des discussions concernant l'appropriation est que le gouvernement sera l'un des acteurs clé de tout système de vérification lié au secteur forestier et que l'aide extérieure devra toujours être acheminée par les voies gouvernementales pour qu'il y ait appropriation. Faut-il qu'il en soit nécessairement ainsi ? Une idée exprimée en Indonésie est que l'APV aurait tout intérêt à suivre une voie purement commerciale - celle d'un accord conclu directement entre deux entreprises plutôt qu'à travers la mise en place de systèmes de vérification nationaux en veillant à renforcer leurs capacités et à en garantir la crédibilité. Ceci soulève d'autres questions sur la façon de procéder pour que le processus d'appropriation s'appuie sur le public ou sur les citoyens.

\subsection{Participation et appropriation}

Dans toute discussion concernant l'appropriation, il importera de distinguer entre une appropriation nationale - souvent entendue au sens de la seule participation du gouvernement - et une appropriation plus large qui englobe tous les citoyens.

Dans le cas des Etats démocratiques, on peut avancer que l'appropriation par le gouvernement ne recouvre pas l'appropriation par le public. Cependant, cet argument pourrait ne pas s'appliquer dans de nombreuses situations et, en particulier, dans celles où les systèmes étatiques n'ont qu'un faible degré de légitimité et où risque de se poser avec le plus d'acuité la question de l'appropriation interprétée au sens le plus restreint du terme. Le risque le plus évident d'une définition étroite de l'appropriation au niveau national serait de biaiser la vérification pour favoriser les intérêts du gouvernement au détriment des groupes qui seraient exclus de ce système de contrôle. Promouvoir l'appropriation dans un pays qui doute de la capacité des structures officielles à gérer les affaires nationales reposera sur des méthodes très différentes de celles qui seront adoptées dans des pays dont les citoyens font confiance aux institutions étatiques.

On pourrait prétendre que l'appropriation d'un système par le gouvernement et l'appropriation de ce même système par le public ne se rejoignent que dans les situations où des représentants dûment reconnus ont effectivement participé aux consultations et engagements inhérents au processus décisionnel. Peuvent témoigner de cette jonction au niveau national l'adoption manifeste d'attitudes de meilleure écoute et de plus grande souplesse pour incorporer les idées nouvelles préconisées par la société civile, ou encore la poursuite du dialogue pour garantir le maintien du consentement initial 
et l'élaboration d'un plan bien structuré fixant des délais de mise en œuvre. L'accès du public à l'information constitue un élément critique de ce processus.

Une opinion communément exprimée est que l'augmentation du nombre d'intervenants et de la diversification des points de vue assurera une meilleure appropriation. Aux Philippines, par exemple, l'installation d'un forum à parties prenantes multiples a, dans certains cas, favorisé l'appropriation à un niveau infranational. Le document d'information FLEGT No. 9 de la CE souligne la nécessité d'élargir la participation dans le cadre de la définition des normes de légalité étant donné que le préjudice causé par la non-conformité avec la loi affecte une multitude de parties prenantes (CE 2005b). Reste cependant la question soulevée dans les études de cas concernant la mesure dans laquelle la présentation d'une grande diversité de points de vue augmente « l'appropriation par le public " ou ne sert qu'à relayer l'opinion d'un certain nombre de groupes d'intérêts. Le cas de l'Equateur, par exemple, donne à penser que l'introduction de réformes ne peut réussir si elle n'est appuyée et encouragée que par la classe moyenne et l'élite urbaine, en l'absence d'une participation élargie. Le concept sous-jacent au système équatorien externalisé de contrôle des forêts (SNTCF) est celui d'un large processus participatif à multiples consultations; s'agissant cependant de la consultation concernant l'architecture du modèle, elle s'est limitée au secteur forestier au niveau national sans quasiment impliquer de groupes de propriétaires de forêts et autochtones autres que nationaux. D'autres groupes comme ceux relevant des échelons intermédiaires ministériels et de centres d'enseignement professionnel liés à l'industrie du bois ont aussi été exclus du processus de mise en œuvre. Le système SNTCF n'a été en vigueur que pendant une brève période en 2003 avant la suspension de l'externalisation des fonctions d'administration et de supervision.

Accroître l'interaction des ONG dans le processus n'élargit pas nécessairement la portée de l'appropriation. La façon dont des organisations de défense des citoyens ont été recrutées comme observateurs indépendants au Cambodge et au Cameroun n'a pas suscité de mouvement de soutien important en faveur du changement ni d'appropriation de la part du public. De même, il faut reconnaître que la domination exercée par certains groupes d'intérêt environnementaux (essentiellement axés sur les classes moyennes) sur certains comités à multiples parties prenantes chargés de la protection des forêts aux Philippines a rétréci le champ d'action de ces comités en excluant le point de vue d'autres communautés. Une critique semblable peut être énoncée dans le cas de l'Equateur (voir Navarro et al. 2006).

L'appropriation va au delà de la participation et ne sera pas forcément renforcée par la consultation. Il sera nécessaire de garder une certaine souplesse en choisissant les options qui permettront aux résultats des processus de consultation d'influencer les décisions. En outre, comme l'ont montré les ateliers consultatifs qui se sont tenus sur les normes applicables en Indonésie, la consultation ne débouche pas nécessairement sur un consensus. La question fait partie de celles qui n'ont qu'une très faible probabilité de connaître un résultat franchement "gagnant sur tous les plans " permettant à tous les acteurs de s'approprier le système. Les éléments importants qui influenceront le degré 
d'appropriation seront tout à la fois les processus de suivi de ces consultations et les mesures prises pour résoudre les conflits d'intérêt et inclure les acteurs qui auraient été exclus. Il faut aussi relever que des consultations au cours desquelles seraient abordées des questions qui n'ont pas été prises en compte dans l'élaboration du système de vérification pourraient entraîner l'aliénation de certains intérêts.

\subsection{Renforcement de la responsabilité et de la transparence}

Il semble raisonnable de défendre la thèse selon laquelle une plus grande attention devrait être accordée à l'établissement de liens avec des mécanismes visant à garantir la fiabilité et la transparence du système de vérification, l'absence de tels mécanismes pouvant en effet compromettre l'efficacité de la vérification. Au nombre des actions susceptibles de promouvoir la fiabilité et la transparence, peuvent figurer notamment :

- Le resserrement des liens avec des procédures d'examen judiciaire et des commissions parlementaires ;

- L'augmentation de la participation directe du public et de la société civile par voie de presse ou à travers des pages interactives (internet) ; et

- La facilitation de l'accès aux arbitrages et aux procédures de règlement des différends.

En Indonésie, le faible niveau de fiabilité et de transparence de la reddition des comptes publics en matière de prise de décision et de gestion des recettes forestières est en partie attribuable à l'absence d'une loi sur la transparence et l'accès à l'information. Les possibilités de recours à l'assistance juridique et judiciaire ne sont que peu utilisées mais une commission d'arbitrage a été mise en place et les comités parlementaires locaux chargés de suivre ce qui se passe au niveau de l'exécutif assurent leurs fonctions avec une diligence raisonnable.

Il ne suffit pas d'étoffer les structures destinées à appuyer les activités de reddition des comptes pour encourager les simples citoyens ou employés des administrations à participer activement à la responsabilisation des institutions en les amenant à devoir rendre des comptes pour comportement corrompu. Ceux qui dénoncent de tels comportements font bien souvent l'objet de menaces ou de subtiles discriminations. Pour que la participation du public au bon fonctionnement des mécanismes de supervision devienne réalité, il semble donc que s'impose l'instauration de voies de transmission à caractère confidentiel comme de sauvegardes afin de protéger les individus de toute espèce de représailles.

\subsection{Phases d'introduction/Stades de progression du système de vérification}

La mise en place et le fonctionnement d'un système de vérification comportent plusieurs phases qui pourront aussi correspondre au déroulement progressif du processus d'appropriation du système au niveau national : 
- Une phase de planification, correspondant au lancement du processus d'appropriation en prêtant particulièrement attention au choix des acteurs sollicités pour prendre part au processus et aux raisons de ce choix ;

- Une phase d'élaboration, pendant laquelle l'inclusion et l'omission de diverses structures et processus peuvent avoir une influence sur le niveau d'appropriation ;

- Une phase de mise en œuvre et de supervision du système de vérification ; et

- Une phase d'évaluation de l'impact du système de vérification.

Il ressort des observations faites dans le cadre des études de cas que le processus d'appropriation tend à prendre plus d'importance pendant certaines phases de la mise en place du système de vérification et qu'il convient de privilégier différentes formes d'appropriation selon le stade de progression du système. Il peut ne pas être nécessaire d'encourager une forme d'appropriation élargie pendant toutes les phases d'installation du système et certaines expériences portant sur des réformes conduites en dehors de tout processus d'appropriation - aux premiers stades de leur introduction seulement - ont été couronnées de succès.

Tenter d'élargir un processus d'appropriation dès le début d'un programme de réformes risque d'être une gageure puisque les résultats et l'impact de ces réformes n'apparaissent encore pas nettement. Dans certains cas, le processus n'a été enclenché qu'après une longue période de négociation et d'étude sur la rentabilité des investissements déterminante pour la réussite de l'opération. Certaines parties n'ont pas accepté, par exemple, d'adhérer au processus de Kimberley aux premiers stades de son instauration mais les obstacles ont pu être surmontés par la suite. Menée en Indonésie, une autre expérience a montré que la mise en œuvre du système pouvait être bloquée quand toutes les parties intéressées tentaient de s'approprier pleinement l'un des éléments clé du processus (en l'occurrence, il s'agissait de la norme à instituer en matière de légalité). Dans le cas de l'Equateur, la réforme a été rondement menée grâce à l'action décisive d'un petit groupe de réformateurs. La rapidité de l'introduction a cependant entraîné le rejet de ces réformes par certaines parties et il a fallu élargir la participation aux stades ultérieurs pour en assurer la réussite au niveau de l'application. On constate actuellement un renouveau d'intérêt pour un système plus inclusif qui serait mis en œuvre à la fois au niveau national et avec la participation des administrations locales.

\subsection{Appropriation et impact}

L'un des moyens les plus évidents de promouvoir l'appropriation est de veiller à ce que le système de vérification ait un impact positif. L'appropriation est l'aboutissement d'un processus qui n'implique pas seulement une participation et un engagement mais qui donne aussi des résultats nettement bénéfiques. Cette assertion peut sans doute être controversée puisque très peu de systèmes de vérification ont à ce jour fait la preuve de telles retombées positives. Certaines études tendraient même à montrer qu'une très grande partie des coûts de transaction seront plus particulièrement à la charge des plus petits entrepreneurs. L'Equateur fait partie de ces cas d'école. 
Dans le contexte des APV, il y a lieu de signaler que l'incertitude des résultats auxquels pourrait aboutir la conclusion de ces accords ainsi que les risques de fermeture du marché inhérents à l'incapacité de se conformer aux normes requises constituent de très réelles forces de dissuasion qui pourraient compromettre l'appropriation des initiatives engagées. Il importe donc de faire une étude attentive de la répartition des coûts et bénéfices pour préciser quels seront les postes budgétaires les plus sollicités. Davantage de certitude en ce qui concerne la répartition probable des charges financières pourrait en effet inciter ceux dont les comptes font l'objet d'une vérification à s'approprier le système. La mise en place de mécanismes tampons ou de garanties pourrait aussi se révéler nécessaire pour atténuer les risques encourus par les producteurs. Au nombre de ces mécanismes, on pourrait envisager d'imposer à ceux qui sont chargés de superviser les initiatives un accord au titre duquel ils accepteraient de ne pas modifier les normes fixées en matière de légalité ou, dans le cas d'une approche progressive, un accord portant sur un barème fixant les limites des variations des normes dans le temps. Dans le cas des mécanismes dépendant de la loi du marché, il pourrait être plus réaliste de considérer l'appropriation comme une question intéressant davantage les pays consommateurs que les pays producteurs.

\subsection{Résumé}

Nous avons pu montrer que plusieurs facteurs influencent l'appropriation d'un système de vérification. Tout d'abord, les objectifs du système sont l'un des éléments déterminants du degré d'appropriation. Il s'ensuit que les systèmes de vérification qui ciblent les priorités nationales et complètent la structure des institutions nationales bénéficieront vraisemblablement d'une meilleure appropriation. Si le gouvernement s'est approprié le système, il pourra le renforcer par toute une kyrielle d'institutions à divers échelons de mise en œuvre décentralisés mais force est de constater que l'appropriation par le gouvernement ne reflète pas nécessairement l'opinion publique ou l'adhésion des citoyens. Une participation accrue et une meilleure prise en compte de la diversité des opinions, sans oublier le renforcement de la fiabilité et de la transparence (par une meilleure reddition des comptes), pourraient inciter le public à se sentir plus concerné et à s'approprier le système. Il faut aussi reconnaître l'importance des phases de déroulement du processus dans l'appropriation du système de vérification, l'adhésion à celui-ci par le plus grand nombre étant moins important pendant la phase d'élaboration que pendant les phases de mise en œuvre et de supervision. En outre et selon toute probabilité, l'appropriation sera d'autant plus authentique que les effets du système de vérification seront perçus comme étant bénéfiques. 


\section{Chapitre 19}

\section{Les fondements juridiques des systèmes de vérification dans le secteur forestier}

Note : Adrian Wells est l'auteur de ce chapitre, extrait de l'étude de Wells, A. 2006 The legal basis for verification systems - standard setting for legal compliance. VERIFOR, Concepts Paper No. 1. ODI, Londres.

\subsection{Introduction}

Toute vérification portant sur la légalité doit comporter une série de normes claires pour définir la conformité. Sans cet ensemble de normes préétablies, le processus de vérification manquera d'objectivité et sera vulnérable à des accusations qui pourraient lui être portées comme celle de servir des intérêts politiques. Dans le secteur forestier, les normes de légalité varient en fonction du contexte, selon qu'elles visent l'intérêt national, les accords commerciaux bilatéraux, les contrats de marchés publics ou des initiatives de certification volontaires.

Quel que soit l'objectif et indépendamment de celui-ci, la formulation de normes de légalité fait souvent l'objet de très longues négociations. Les cadres juridiques et réglementaires régissant le secteur forestier sont souvent complexes dans de nombreux pays qui doivent en outre veiller à maintenir, dans tout processus d'élaboration de normes, un équilibre fonctionnel entre :

- Le côté pratique - les procédures d'application de la plupart des cadres juridiques sont coûteuses et ne permettent pas d'évaluer avec précision la mesure dans laquelle les obligations ont été respectées; et

- La légitimité telle qu'elle est perçue - les cadres juridiques existants peuvent ne pas être suffisamment contraignants pour garantir les droits des populations locales et des peuples autochtones.

Il sera question dans ce chapitre des nombreuses initiatives prises en matière de fixation des normes de légalité aussi bien par les pays producteurs et consommateurs que par le secteur privé et les ONG. Les cinq aspects suivants ont été pris en compte :

- Les efforts existants pour fixer les normes de légalité applicables au secteur forestier ;

- Les échecs ou lacunes des lois et règlements existants ;

- Les mesures visant le côté pratique et la légitimité des normes de légalité ;

- Le processus à appliquer pour élaborer une norme efficace ; et

- La mise en œuvre de la norme fixée, y compris les délégations de pouvoir nécessaires pour publier la norme au journal officiel et délivrer les certificats de conformité. 


\subsection{Efforts de normalisation dans le secteur forestier}

\section{Elaboration des normes au niveau national dans un contexte de gestion durable des forêts}

Un certain nombre de pays producteurs ont élaboré des normes de légalité applicables à travers des critères et indicateurs (C\&I) à caractère obligatoire pour la gestion durable des forêts (GDF), notamment dans les pays suivants :

- En Equateur (Chapitre 10), où le ministère de l'environnement a contribué à l'établissement de C\&I comme fondements d'un système de suivi et de vérification à parties prenantes multiples au titre du système de contrôle forestier externalisé (SNTCF) ;

- Au Costa Rica (Chapitre 6), qui a mis en place des C\&I équivalents à caractère obligatoire applicables à la GDF au titre de sa National Forestry Certification Commission (Commission nationale de certification forestière) ${ }^{1}$; c'est cette commission qui constitue désormais la base de référence pour la vérification de la conformité dans le cas des propriétaires de forêts privées ;

- En Indonésie (Chapitre 15), où le ministère des forêts a institué des C\&I à caractère obligatoire applicables à la gestion durable des forêts pour les mesurer à l'aune des évaluations effectuées par des auditeurs nommés par le gouvernement pour vérifier la conformité des concessions d'exploitation forestière et des scieries ;

- En Malaisie (Chapitre 16), qui a aussi élaboré des C\&I pour la gestion durable des forêts afin de garder sa part de marché in an effort to retain market standing et où les C\&I constituent dorénavant la base de référence des audits publics à caractère obligatoire ainsi que du Conseil malaisien de certification du bois (Malaysian Timber Certification Council) pour la certification des administrations et concessions forestières étatiques.

\section{Elaboration des normes dans le cadre des accords commerciaux bilatéraux}

Les processus régionaux et bilatéraux concernant l'application des législations forestières et la gouvernance [Forest Law Enforcement and Governance] (FLEG) ont aussi servi à promouvoir les processus de normalisation à caractère obligatoire. Même si leur domaine de compétence est moins vaste que celui des C\&I applicables à la GDF puisqu'il ne recouvre explicitement que les législations et règlements existants, ces processus peuvent concerner une plus large gamme d'activités que celles de la plupart des C\&I nationaux, s'étendant non seulement à la gestion des forêts mais aussi à a transformation du bois et à la délivrance de permis d'exportation. Le " protocole d'accord et de coopération entre le Royaume-Uni et l'Indonésie » signé en 2002 " pour améliorer l'application des législations forestières et la gouvernance " constitue, en particulier, un précédent important pour toute action ultérieure qui pourrait être engagée au titre de l'élaboration de normes applicables au commerce du bois légalement exploité.

1 En 2007, cette commission a pris le nom de National Sustainable Forestry Commission (Commission nationale de foresterie durable). 
Plus récemment, les accords (accords de partenariat volontaire ou APV) concernant les permis d'importation dans l'UE du bois légalement exploité ont été négociés dans le cadre du Plan d'action de la CE pour l'application des législations forestières, la gouvernance et le commerce (FLEGT) en vertu du règlement du Conseil 2173/2005. Au vu des contraintes exercées sur la fixation unilatérale de normes à caractère obligatoire dans le cadre des négociations avec l'OMC-GATT, la CE envisage, au titre de sa politique FLEGT, de laisser le soin à chaque pays producteur d'élaborer sa propre définition concernant le bois exploité légalement. Cela permettrait d'aboutir à une norme "claire, objectivement vérifiable et utilisable dans la pratique ", susceptible aussi d'être modifiée en cas de réforme des règlements (EC 2005b).

Les normes applicables en Indonésie devraient être publiées au journal officiel dès la signature de l'APV envisagé avec l'UE. Des processus de normalisation équivalents ont aussi été engagés au titre d'accords de partenariat volontaire (APV) avec le Cameroun, le Ghana et la Malaisie, dans le cadre de l'application des législations forestières et de la gouvernance (FLEGT). Le récent amendement apporté à la loi Lacey (Etats-Unis) relative à l'interdiction d'importer du bois illégalement abattu, en violation du droit national et international donne encore plus de poids à ces initiatives.

Il n'est cependant pas facile de s'appuyer entièrement sur les législations nationales. Les lois et règlements édictés au niveau national sont souvent complexes, peu clairs et l'objet de fréquentes controverses juridiques. En Indonésie, malgré plusieurs sessions de consultation du public réparties sur une période de cinq ans, force est de constater la persistance de préoccupations concernant le fait de savoir si la norme est, soit : (i) suffisamment robuste pour garantir le respect des conditions stipulées (publication au journal officiel du bornage des concessions), et le consentement libre et éclairé des populations locales et des peuples autochtones ; soit (ii) trop complexe, entraînant des coûts de production plus élevés et la perte d'un avantage concurrentiel par rapport à d'autres producteurs moins chers appliquant des normes moins rigides (Wells et al. 2006).

\section{Politique des marchés publics}

Certains pays importateurs ont mis en place des politiques concernant les marchés publics du bois provenant de l'exploitation à la fois légale et durable des forêts. Le texte d'orientation du Central Point of Expertise (CPET) pour les marchés publics du bois au Royaume-Uni notamment relève que les gestionnaires des forêts devraient avoir un droit légal sur l'exploitation du bois et que les gestionnaires et entrepreneurs du secteur forestier devraient respecter les législations nationales édictées en matière de gestion des forêts, d'environnement, de conditions de travail et de bien-être, de santé et de sécurité ainsi que la Convention sur le commerce international des espèces de faune et de flore sauvages menacées d'extinction (CITES) et le paiement de taxes et redevances. Cependant et précisément parce que les lois des pays peuvent être imprécises et contradictoires, le CPET poursuit en signalant «... qu'il faudra donner une définition pratique du terme " légal » si elle n'existe pas ou respecter un ensemble de lois fondamentales ayant reçu le 
soutien des plus grands groupes de parties prenantes. Cet objectif pourrait être réalisé à travers un processus visant à élaborer des normes au niveau national ou par tout autre moyen approprié. » (CPET 2006, p. 3)

\section{Initiatives de certification volontaire}

Des normes et protocoles non contraignants ont aussi été établis dans le cadre d'initiatives de certification volontaire du bois pour respecter les règlements des pays producteurs dans le but de promouvoir le commerce du bois exploité légalement selon un mode de gestion durable. Des exemples de cette façon de procéder incluent ceux du label de légalité et de traçabilité (ou chaîne de contrôle) délivré par la Fondation des forêts tropicales ou encore de la directive du réseau du commerce mondial des produits forestiers [Global Forest Trade Network] (GFTN) du WWF concernant l'achat responsable de bois d'œuvre et la conformité avec la loi. Assorties d'une demande concernant l'origine légale du produit et le respect de la législation dans un souci de gestion durable des forêts (voir Encadré 19.1), ces initiatives correspondent à une approche à la fois pragmatique et progressive (procédant par étapes) à la mise en œuvre. Elles témoignent aussi du souci de ne pas focaliser l'attention sur la légalité au détriment du développement durable, sans oublier les difficultés de nombreux propriétaires et gestionnaires de forêts à se conformer d'emblée à toutes les conditions requises en matière de légalité et de développement durable.

\subsection{Echecs des lois et règlements existants}

Traduire des lois et règlements nationaux en normes de légalité - qui soient aussi considérées comme à la fois pratiques et légitimes par la plupart des parties prenantes représente souvent un défi difficile à relever.

\section{Aspects pratiques}

Lespopulations pauvres, en particulier, sontconfrontéesà desobstaclesquasiinsurmontables en matière de conformité, même dans le cas où un droit d'usage des ressources de la forêt leur a été conféré. Cette situation reflète la complexité des règlements en vigueur et le coût élevé des transactions visant l'obtention de permis d'exploitation. Au Honduras, ces difficultés ont forcé les membres des coopératives forestières communautaires à adopter des pratiques illégales quand la rentabilité économique de l'exploitation du bois d'œuvre sur le marché intérieur s'est effritée jusqu'à l'insignifiance. Au Costa Rica où tous les C\&I stipulés pour une gestion durable des forêts sont scrupuleusement appliqués, le déficit atteint 200 dollars par hectare - ce qui nécessite le versement de subventions à travers le mécanisme des paiements pour services environnementaux (Navarro, G., 2006 et al. avril).

Les cadres juridiques et réglementaires n’ont généralement pas été conçus pour être utilisés à des fins de vérification des comptes ou d'audit. Les dispositions figurant dans les règlements, en particulier, pourraient ne pas être suffisamment spécifiques pour constituer des normes de performance efficaces. Les lois peuvent aussi être très imprécises pour tout ce qui touche au régime foncier et aux droits des communautés locales. 


\section{Encadré 19.1 Adoption d'une approche progressive à la légalisation}

S'agissant de conformité, le Protocole pour la validation des réclamations, élaboré par l'Institut de normalisation néerlandais Keurhout en association avec l'Association néerlandaise pour le commerce du bois d'œuvre [Netherlands Timber Trade Association] (NTTA) « abaisse la barre » mais seulement dans le contexte du processus en trois étapes qui aboutit à une certification GDF (gestion durable des forêts). La première étape correspond à la détermination du caractère légal de l'origine du bois d'œuvre - notamment pour déterminer si l'exploitation a été effectuée conformément aux lois et règlements en vigueur dans le pays d'origine et aux dispositions régissant les permis d'exploitation, le respect des zones protégées, les espèces, les dimensions des arbres et les volumes récoltés. La deuxième étape concerne l'obligation d'appliquer toutes les autres lois et réglementations relatives à la gestion des forêts, y compris les normes requises en matière de conditions de travail et d'environnement. Un bois d'œuvre satisfaisant aux conditions de légalité en matière d'origine ainsi qu'aux conditions imposées au titre de la deuxième étape est considéré comme ayant atteint le stade de «bois de transition » progressant vers l'obtention d'une certification GDF - correspondant à la troisième étape et au plus haut niveau de performance en matière de gestion. Les titulaires d'un permis de «transition » sont ensuite appelés à suivre un programme supervisé par une tierce partie pour se conformer progressivement à toutes les normes requises en matière de GDF dans des délais déterminés convenus.

Une approche similaire a été adoptée par une société de vérification du secteur privé, la SGS, dans le cadre de son programme de validation indépendante du bois d'œuvre exploité légalement. Dans ce contexte, la vérification de l'origine légale [Verification of Legality] (VOL) - ciblant les droits d'exploitation, la légalité des titres de propriété et les paiements effectués au titre des redevances fiscales - est distinguée de la vérification de la conformité [Verification of Legal Compliance] (VLC) au titre de la planification, de l'exploitation et de la gestion des forêts. Les règles relatives à ces deux types de vérification (VOL et VLC) peuvent être progressivement renforcées, permettant de les améliorer de façon continue au fil du temps.

Une autre société du secteur privé, la Proforest, a élaboré un système qu'elle a appelé le système modulaire de vérification et de mise en œuvre [the Modular Implementation and Verification System] (MIV) pour appuyer les approches permettant d'aboutir à la certification de façon progressive (Nussbaum et al. 2003). Adopté par des projets comme le réseau GFTN du WWF, le MIV distingue trois niveaux de légalité qui correspondent aux fonctions suivantes :

- Respect des droits en matière de ressources : droits à l'extraction ; protection des droits législatifs et coutumiers des populations locales ; et paiement des contributions, redevances et taxes applicables ;

- Légalité des opérations : respect de toutes les lois et réglementations locales, nationales et internationales relatives à l'environnement, au régime foncier, aux droits des travailleurs et des populations autochtones, au développement des communautés, à la santé et à la sécurité, au commerce, etc. ; et mise en place d'un système visant à assurer le suivi des modifications requises en matière d'obligations légales et de conformité ; et

- Contrôle des activités non autorisées : notamment l'identification de menaces possibles, la mise en place de systèmes de prévention et de contrôle avec d'autres parties prenantes, et le suivi des progrès réalisés en matière de contrôle de ces activités. 


\section{Légitimité}

Les cadres juridiques régissant le secteur forestier font fréquemment l'objet de vives contestations. En Indonésie, la loi 41/1999 sur les forêts reconnaît les titres privés de propriété sur les forêts et exclut ces domaines privés du domaine forestier national (National Forest Estate). Un traitement équivalent n'est toutefois pas accordé aux droits coutumiers - malgré des dispositions légales reconnaissant leur existence et leur inscription au cadastre (Contreras-Hermosilla et Fay 2005). Il en résulte que l'aire de délimitation du domaine forestier national empiète souvent sur celle des systèmes d'utilisation des terres et outrepasse les droits coutumiers. La norme de légalité élaborée au titre du protocole d'accord entre le Royaume-Uni et l'Indonésie a donc été critiquée comme légitimant le maintien du statu quo, voire comme marginalisant encore plus les propriétaires fonciers coutumiers et les petits producteurs.

\subsection{Renforcer l'utilisation pratique et la légitimité des normes de légalité}

\section{Restriction du champ d'application des normes de légalité}

Etant donné la complexité des cadres juridiques existants, un certain nombre d'initiatives ont été retenues afin de limiter les normes de légalité à un sous-ensemble de lois et réglementations essentielles. En conséquence et en fonction du contexte national et des objectifs de la vérification, une norme de cette catégorie pourrait inclure une partie ou la totalité des éléments suivants :

- L'origine légale définie simplement comme le droit d'extraction, ou incluant aussi la détermination préalable et le règlement de litiges fonciers, la publication au journal officiel des limites des concessions ainsi que le consentement libre, préalable et éclairé des communautés locales aux opérations d'abattage conduites sur leurs terres communautaires ;

- L'extraction légale du bois, y compris le respect des conditions stipulées dans le permis d'exploitation, l'acquittement des redevances, l'application des règlements édictés en matière de gestion forestière - notamment les C\&I à caractère obligatoire pour la GDF - et des lois relatives à l'environnement, au travail et au bien-être ainsi qu'à la santé et à la sécurité ;

- Les procédures légales, y compris le respect des contingentements assignés aux produits intérieurs transformés et des garanties mises en place pour lutter contre l'inclusion de produits issus de sources non-légales dans les contingents, ainsi que le paiement des taxes prélevées sur les produits transformés; et

- Les transactions commerciales et les exportations à caractère légal, y compris les licences d'exportation, l'obtention des autorisations nécessaires au titre de la CITES et les formalités de dédouanement.

Tout ce qui concerne l'inclusion ou non de lois et règlements dans une norme donnée peut cependant être extrêmement controversée à plusieurs titres, notamment pour déterminer : (i) la hiérarchie des droits applicables au processus (quels sont ceux qui sont 
subordonnés à d'autres) ; et/ou (ii) la partie à laquelle il incombera d'acquitter les coûts de transaction relatifs aux opérations de mise en conformité avec la norme.

En Indonésie, par exemple, un certain nombre d'ONG et d'entreprises commerciales du secteur privé ont avancé l'argument qu'une norme de légalité qui adopterait une interprétation au sens large des lois nationales en vigueur serait vraisemblablement inapplicable dans un contexte de faible gouvernance. Dans les situations où $12 \%$ seulement de la superficie totale du domaine forestier national a fait l'objet d'une publication au journal officiel (Contreras-Hermosilla et Fay 2005), les conditions requises pour obtenir le consentement préalable, libre et éclairé (FPIC) de communautés locales et pour définir les limites des concessions constituent un véritable défi pour la plupart des détenteurs de permis d'exploitation. Il y a cependant lieu de relever que les groupes de défense des droits des peuples autochtones considèrent ces mesures comme le strict minimum pour répondre aux doléances des groupes coutumiers dont les droits ont été bafoués par les affectations et les bornages de terres.

Des tensions similaires concernant le champ d'application d'une norme de légalité ont aussi pu être observées en Equateur. Alors que les normes concernant le respect des obligations légales encourues au titre du SNTCF se limitaient initialement à la gestion des forêts, l'industrie a soutenu que le système ciblait les opérateurs légaux et excluait de son domaine de compétence les activités illégales de déboisement et les changements d'affectation des terres. L'accord qui a été conclu ultérieurement pour incorporer toutes les questions relatives à l'utilisation des terres dans un nouveau mécanisme décentralisé suscite cependant de nouvelles inquiétudes à propos de la capacité des institutions et des coûts de mise en œuvre (Thiel, H., 2006, et al. mai).

\section{Ajustements au régime de mise en conformité}

Des ajustements pourraient être apportés à la mise en conformité pour contourner la difficulté liée à une norme de conformité interprétée au sens étroit du terme. Cette option suppose l'introduction progressive de la normalisation et/ou de donner plus d'importance au suivi des conditions dans lesquelles la mise en conformité est appliquée.

\section{Introduction progressive de la normalisation}

Comme indiqué dans l'encadré 19.1, un certain nombre d'ONG sont en faveur d'approches progressives qui tentent de réconcilier légitimité et viabilité. En principe, ce type d'approche peut aussi être adopté par les gouvernements lorsqu'ils fixent les normes à caractère obligatoire au niveau national. Cette façon de procéder implique néanmoins le recours à un système d'audit rigoureux pour veiller à ce que les améliorations s'appliquent à tous les échelons sans exception. Elle prête aussi à controverse en raison d'un certain assouplissement des conditions requises, notamment des délais, en matière d'applicabilité (par exemple en ce qui concerne « l'origine légale ») qui deviennent moins exigeantes que celles inscrites dans les lois et règlements des pays producteurs. 


\section{Donner plus d'importance au suivi de la mise en conformité}

Le respect des normes n'implique pas seulement leur entrée en vigueur mais aussi la nécessité de prendre les mesures qui permettront d'améliorer progressivement la performance de ceux qui sont chargés de les faire appliquer. A cet effet, il conviendra d'établir une distinction entre les infractions qui entraînent une mesure coercitive (par ex. des sanctions administratives et/ou des poursuites judiciaires), et celles qui donnent lieu à une mesure corrective (par ex., demande de réparation du dommage causé). L'Etat malaisien de Sabah a adopté cette approche pour rattraper progressivement son retard en matière de légalisation et a mis en place un programme régulier d'audit et de renforcement des capacités dans le cadre des permis d'exploitation accordés au titre de son plan pour "Cent années de gestion durable des forêts " [100-year Sustainable Forest Management License Agreements]. Un système de suivi efficace de la mise en conformité permettrait de renforcer la capacité de se conformer à la norme de légalité, sans devoir en restreindre le champ d'application.

\section{Réforme légale et institutionnelle}

Dans de nombreux cas, la facilité d'application dans la pratique et la légitimité des normes de légalité pourraient dépendre, en dernière analyse, d'une grande réforme institutionnelle et législative. Une telle réforme pourrait notamment porter sur : (i) la prise de mesures visant à assurer que l'Etat assume ses responsabilités dans le cadre de l'administration des terres et vis-à-vis des droits des peuples autochtones; et (ii) la réduction des coûts de transaction encourus au titre de la mise en conformité.

\section{Responsabiliser l'Etat}

Les tensions existant entre les tenants d'une plus ou moins grande rigidité ( "baisser » ou " élever » la barre) du degré de conformité reflètent en partie le manque de clarté concernant les parties tenues de respecter les clauses d'un système de vérification, autrement dit ces clauses sont elles uniquement applicables aux détenteurs de permis d'exploitation ou aussi à l'Etat ? Dans le cas de l'Indonésie, par exemple, c'est à l'Etat, en vertu d'une disposition statutaire inscrite dans la constitution - plutôt qu'aux concessionnaires - qu'incombe l'obligation de négocier l'affectation des terres et le bornage des domaines forestiers avec les communautés locales. Il en est de même pour les normes concernant la publication des limites des concessions au journal officiel et le consentement libre et préalable qui relèvent essentiellement des administrations forestières et non des détenteurs de permis d'exploitation.

Le fait qu'une petite partie seulement des terres forestières relevant du domaine forestier national de l'Etat indonésien ait été enregistrée suivant les voies légales témoigne d'un manquement aux règles de la part du gouvernement qui ne doit pas nécessairement en faire supporter les conséquences par les détenteurs de permis. Les mêmes arguments peuvent être invoqués en Malaisie où de récentes décisions de la Cour d'Appel affirment le droit fiduciaire du gouvernement à inscrire les terres aborigènes au cadastre/procéder à l'enregistrement des terres aborigènes et à dédommager les communautés par des 
paiements de compensation raisonnables chaque fois que leurs terres seraient affectées à d'autres utilisations (entre autres, implicitement, à l'abattage). La question n'est donc pas de savoir s'il faut ou non inscrire de telles conditions dans le texte d'une norme concernant la légalité des opérations. Il s'agit plutôt de veiller à ce que les conditions requises au titre d'une norme de légalité établissent une distinction claire entre les engagements pris par l'Etat et ceux pris par les détenteurs de permis d'exploitation.

De même, il ne sera pas possible qu'une norme soit appliquée à la fois par les administrations publiques et par les utilisateurs des forêts sans qu' existent des garanties concernant la responsabilité de l'Etat. Cela inclura les droits statutaires des services publics de réclamations (ou des médiateurs - un service efficace en Papouasie Nouvelle Guinée) sur l'accès à l'information et à la participation et la capacité d'ester en justice (en Malaisie, par exemple, le manque d'assistance juridique restreint la capacité potentielle des communautés locales à recourir devant les tribunaux pour résoudre des litiges fonciers liés au bornage des terres).

\section{Diminution des coûts de transaction relatifs à la mise en conformité}

Reconnaissant les coûts particulièrement élevés de la vérification pour les petits opérateurs, l'Equateur a pris un certain nombre de mesures clé pour réformer le système existant afin de diminuer les frais encourus au titre du respect des obligations contractées dans le cadre du SNTCF, notamment des mesures visant à :

- Faciliter, dans le cas des propriétaires fonciers faisant partie des couches les plus pauvres, les formalités qu'ils doivent accomplir pour prouver qu'ils sont propriétaires de leurs terres forestières, aux fins de l'obtention d'un permis d'exploitation ;

- Déplacer les services administratifs pour assurer un contact direct avec les gestionnaires de forêts communautaires ;

- Simplifier les règles de procédure applicables à la GDF ; et

- Instituer l'exonération des taxes pour les permis visant à extraire certaines essences des systèmes agro-forestiers et à réduire la bureaucratie pour toutes les opérations concernant l'abattage des forêts plantées.

Il y a cependant lieu de noter que les procédures liées à l'élaboration des normes de légalité pourraient aussi exacerber les coûts de mise en conformité en accordant une importance démesurée aux conditions administratives requises pour valider légalement les exportations. Dans des contextes de faible gouvernance, il faudra une supervision efficace de l'Etat au cas où les dispositions prévues dans la norme ne suffiraient pas à favoriser des opportunités de rentabilité (Bohanic, A. 2006 et al. avril).

\subsection{Elaboration de normes efficaces}

Une très large participation de toutes les parties prenantes est essentielle à la réussite de tout accord concernant l'élaboration de normes légitimes et faciles à appliquer dans la pratique. Au nombre de ces succès figure le cas des C\&I nationaux du Costa Rica pour la gestion durable des forêts qui sont ressortis d'une révision complète du secteur forestier. 
Il n'est cependant jamais simple d'aboutir à un tel résultat. En l'absence de plaintes effectivement déposées par le public et de mécanismes de supervision, les processus de normalisation nationaux sont souvent, pour les parties prenantes, l'occasion à saisir pour contester les normes applicables en matière de légalité comme l'illustre le cas de l'Indonésie. Il n'est pas d'accord non plus qui permette de garantir le maintien d'un consensus. Malgré le déroulement d'un processus de consultation à large base, le succès du SNTCF en Equateur s'est rapidement démenti sous l'influence de pressions politiques exercées par l'industrie. La difficulté d'aboutir à un accord a aussi été exacerbée par la diversité des demandes des consommateurs qui différaient toutes les unes des autres selon qu'elles relevaient de la législation sur les importations, des marchés publics ou des initiatives sur la certification du bois. D’après certains pays producteurs, de tels accords ne sont conclus que pour « changer les règles du jeu ». ${ }^{2}$

La clé du succès réside en fait dans la capacité du mécanisme institutionnel à élaborer des normes de légalité qui permettront de résoudre ces divergences politiques. A cet effet, il conviendra de prêter tout particulièrement attention aux points suivants :

- La mesure dans laquelle le processus est supervisé par le pouvoir politique (l'exécutif ou le législatif), et si l'organe de supervision est bien investi du pouvoir de décider de ce qui est légal et de ce qui ne l'est pas ; dans le cas de l'Indonésie, par exemple, ni l'institution chargée de la médiation (l'Institut indonésien d'éco-étiquetage) ni le ministère des forêts n'avaient été mandatés pour régler le point principal du contentieux juridique, à savoir celui du régime foncier (Contreras-Hermosilla et Fay 2005) ;

- La crédibilité qui est accordée aux institutions médiatrices la Commission nationale pour la certification des forêts au Costa Rica qui englobe des universités publiques, des ONG techniques et des centres de recherche ;

- La pertinence de l'utilisation d'autres instances (par exemple, les tribunaux) pour régler les différends.

Le temps nécessaire pour mettre en place un mécanisme approprié et aboutir à un accord pourrait bien entrer en collision avec la pression exercée par la demande pour l'établissement de normes de légalité . Il faudra veiller à ce que l'exercice d'une telle pression ne réduise pas la négociation des normes à un simple processus technocratique sans qu'une attention suffisante soit portée aux mandats et à la capacité nécessaire pour résoudre les conflits d'ordre juridique.

\subsection{Mise en œuvre de la normalisation}

Si l'objectif recherché est d'instituer une base de référence obligatoire pour la légalité, il faudra aussi réfléchir aux procédures d'officialisation de l'inscription au cadastre et d'attribution des pouvoirs nécessaires à la délivrance des certificats de conformité. Il

2 Les producteurs de bois en Malaisie se réfèrent ici à des efforts préalables qui avaient été déployés pour établir des normes relatives à la GDF au titre d'un programme entrepris en collaboration avec l'Institut de normalisation néerlandais Keurhout, normes qui n'avaient pas été acceptées par les ONG néerlandaises. 
pourrait être opportun, par exemple - compte dûment tenu du champ d'application convenu lors de l'élaboration d'une norme de légalité - de publier cette norme, soit par la voie d'un arrêté émanant du cabinet du Premier Ministre, soit par celle d'un décret présidentiel (différent d'un arrêté ministériel) - en particulier dans les cas où la norme en question empièterait sur des domaines comme la terre (le foncier), les droits du travail ou la fiscalité qui ne relèvent ni de la compétence des ministères des Forêts ni de celle des ministères de l'Environnement. Pour la même raison, il pourrait être plus judicieux de déléguer à un organisme national de normalisation ou de certification le pouvoir de délivrer des certificats de conformité plutôt que charger un seul ministère (par ex. des Forêts ou de l'Environnement) d'assumer cette tâche (Nguiffo, S. 2006 et al. avril).

\subsection{Résumé}

S'il est vrai que l'élaboration d'une norme de légalité puisse donner lieu à un débat entre parties prenantes, il n'en reste pas moins qu'elle ne peut garantir de solution aux différends que susciteront les questions liées à la légitimité du cadre juridique en vigueur et à la mise en conformité sur le plan pratique. Il pourrait être nécessaire de procéder à une réforme législative et institutionnelle (entre autres mesures) afin de veiller à ce que l'Etat assume ses obligations au titre de l'administration des terres et des droits des peuples autochtones, et à ce que les coûts de transaction liés aux formalités requises pour demeurer dans la légalité restent le moins élevés possible.

Les délais impartis dans ce contexte risquent d'être plus longs que ceux de processus pilotés par la demande comme le FLEGT - plus de cinq ans dans le cas de l'Indonésie. Des solutions intérimaires pourront être trouvées dans les ajustements apportés à la mise en conformité, comme notamment : l'introduction progressive des normes, et une plus grande importance accordée au suivi du respect de la législation pour aider les administrateurs/gestionnaires des forêts à appliquer les normes requises.

Dans le contexte des normes, la réussite du processus d'élaboration dépendra en dernière analyse des facteurs suivants : (i) une bonne facilitation effectuée par une organisation techniquement compétente et dotée d'une légitimité incontestable comme un organisme national de certification ; et (ii) une supervision assurée par des institutions ayant été mandatées pour relever les défis juridiques, régler les différends, statuer et prendre les décisions. Selon le degré d'importance du conflit juridique en cause, il pourrait être nécessaire de porter l'affaire devant des commissions interministérielles ou parlementaires, voire de recourir à une interprétation judiciaire ou à une décision de justice.

Au-delà de la procédure d'élaboration des normes, des choix devront être faits quant aux organismes qui seront mandatés pour publier les normes au journal officiel et délivrer les certificats de conformité. Quand les normes concernent la conformité avec des lois et réglementations de secteurs très divers, il pourrait n'être pas suffisant de ne charger qu'un seul ministère (par exemple, celui des Forêts ou de l'Environnement) de veiller à leur application. 



\section{Chapitre 20}

\section{Indépendance de la vérification}

Note : David Brown et Josephine Tucker sont les auteurs de ce chapitre basé sur l'article de Brown, D. et Tucker, J. 2006 On Independence in Verification Work. VERIFOR, Briefing Paper No. 2. ODI, Londres.

\subsection{Introduction}

La capacité de prendre en compte des points de vue différents est essentiel au bon fonctionnement de l'administration publique. Cette attitude renforce les droits du citoyen et donne des gages de confiance, de crédibilité, de transparence et de discipline nationale. Dans la mesure où un jugement indépendant est réputé pouvoir être exercé, il s’opposera (voire même, fera échec) à l'ingérence d'acteurs politiques dans les systèmes administratifs, permettra de maintenir l'application de normes professionnelles et diminuera la tendance à la corruption dans la vie publique. Le principe de l'indépendance est aussi au cœur de toute action de vérification et de tous les débats portant sur des enjeux critiques comme la bonne gouvernance, « l'appropriation au niveau national » et l'efficacité.

\section{Le cadre institutionnel}

S'agissant de gouvernance, les processus globaux qui se déroulent au niveau international présentent certains avantages par rapport aux processus nationaux (voir Chapitre 2). Ces avantages sont particulièrement évidents dans le cas de l'indépendance de la fonction de supervision. La plupart des conventions mondiales, accords et systèmes d'audit d'internationaux ont en commun un ensemble de règles qui définissent clairement un ensemble de règles relatives à l'indépendance des acteurs intervenant à certains stades clé du système de vérification. Des ressources substantielles ont été consacrées à l'établissement de ces normes et à leur application. Il s'agit cependant d'un principe beaucoup moins développé dans le secteur forestier que dans d'autres secteurs. En l'absence d'un accord internationalement contraignant, la tension entre la souveraineté nationale et les intérêts et responsabilités prévalant au niveau mondial tend à s'exprimer plutôt à travers des négociations informelles hautement politisées qu'à travers des prescriptions et des règles internationalement convenues. La question est donc de savoir comment introduire l'indépendance dans le processus de vérification selon des modes compatibles avec la souveraineté nationale tout en prenant - par rapport à celle-ci - la distance dont a besoin le secteur des forêts en tant que ressource relevant du patrimoine commun de l'humanité.

Dans le secteur forestier, l'attention s'est récemment portée plus particulièrement sur un stade du processus de vérification - celui du suivi forestier indépendant ou de l'observation indépendante des forêts (IFM). Cette attitude doit beaucoup à l'excellent travail réalisé 
par les observateurs indépendants des forêts dans des pays comme le Cambodge et le Cameroun. Dans ces deux pays, l'observation indépendante des forêts (IFM) a été utilisée comme un instrument visant à faire respecter les conditions imposées par les bailleurs dans des circonstances caractérisées par une nette perte de confiance entre l'Etat producteur et ses bailleurs officiels. Bien que les observateurs indépendants aient été des ONG, leur capacité à agir " indépendamment " en menant leur enquête/assumant leur rôle d'enquêteur était précisément (et paradoxalement) due à leurs liens étroits avec l'une des deux parties - la communauté des bailleurs de fonds.

La prédominance de ce type d'activité a sans doute focalisé toute la réflexion concernant la vérification sur un seul stade du processus de l'IFM, quasiment considéré aujourd'hui comme synonyme de vérification. Le petit nombre d'intervenants actifs dans ce domaine a encore plus contribué à fausser la situation. La jurisprudence n'est pas encore assez étoffée pour permettre de discerner des systèmes plus généralement applicables ou de proposer d'établir des normes professionnelles. Il est cependant évident qu'il faut analyser la notion "d'indépendance » en matière de vérification et en isoler les principaux éléments constitutifs. L'objectif central ne doit pas être de préserver des fonctions ou des intérêts spécifiques mais d'assurer la plus grande crédibilité possible à l'ensemble du système de vérification.

\subsection{Deux perspectives pour l'indépendance : celle de l'acteur et celle de la fonction}

Dans cette section, l'observation indépendante est replacée dans deux dimensions principales pour en permettre l'analyse : elle est d'abord examinée dans le contexte dans lequel la vérification doit être effectuée (celui de l'acteur - quels sont les acteurs les mieux placés pour garantir un suivi/une observation indépendante ?), et ensuite dans le contexte des qualités requises des acteurs qui participent au processus (la dimension fonctionnelle - quelles sont les fonctions qui ont besoin d'une garantie d'indépendance pour que le système de vérification puisse fonctionner convenablement ?).

\section{Examen de la première perspective : celle de l'acteur}

La pratique internationale extra-sectorielle aide à distinguer certaines des questions principales liées à la notion d'indépendance. Le terme " indépendance " est bien défini dans tous les textes relatifs à l'action humanitaire (notamment, par exemple, dans les principes directeurs de la Fédération internationale des Sociétés de la Croix-Rouge et du Croissant Rouge) et se distingue clairement de concepts similaires comme ceux " d'impartialité » et de «neutralité ». En conséquence :

- L'indépendance se définit par l'autonomie de comportement, dissocié de tout lien ou de toute influence d'autres parties, dans le déroulement du processus décisionnel ;

- L'impartialité s'entend de la cohérence d'application des jugements portés ; et la

- Neutralité s'entend de l'absence de tout esprit partisan (« ne pas prendre parti »). 
S'agissant des efforts déployés dans le secteur forestier pour traduire ces principes dans la pratique, plusieurs options de prestation se présentent au nombre desquelles figurent notamment les interrogations suivantes:

- Quel type d'organisation participe à ces efforts ? L'Etat ? Les ONG ? Le secteur privé ?

- Situation par rapport à la société hôte ? Acteur national ? Acteur international ?

- A quel facteur attribuer la recherche d'indépendance : à l'intégrité de l'acteur ou à l'architecture du système?

Ces variables sont examinées les unes après les autres dans les paragraphes qui suivent.

\section{Type d'organisation}

La notion d'indépendance tend aujourd'hui à être rattachée (dans tous les textes traitant du développement) aux interventions des acteurs de la société civile. Il s'agit d'une attitude commune à de nombreux secteurs et pas seulement propre au secteur forestier. Elle est associée à une mouvance que ses partisans pourraient qualifier de propagation de la responsabilité démocratique entendue au sens de processus de " trans-nationalisation ", mais que ses détracteurs considèrent comme un processus d'érosion de cette même responsabilité à travers l'extension d'un phénomène de " corporatisme envahissant ". Dans le secteur forestier, il semble que les organisations de la société civile (en particulier les $\mathrm{ONG}$ ) soient de plus en en plus considérées comme les gardiennes naturelles du maintien de normes indépendantes et, plus généralement, de la moralité publique. La communauté des bailleurs s'est montrée plutôt favorable à cette approche en tendant à considérer la société civile comme un important vecteur de transmission pour mettre les gouvernements producteurs face à leurs responsabilités en leur demandant de rendre des comptes sur l'utilisation des ressources publiques à des fins d'extraction minière. Certains ont pu alléguer y voir l'émergence d'un phénomène en voie d'expansion entre les bailleurs internationaux et les ONG - phénomène qui donne un " avantage à la confiance » et qui peut être considéré comme inquiétant (voir Ronit et Schneider 1999, p. 245).

Les ONG constituent-elles nécessairement des partenaires plus souhaitables que les institutions du secteur privé comme tendent à le tenir pour acquis certains milieux militants? Il est difficile d'évaluer le bien-fondé d'une telle affirmation. Seules quelques expériences ont été menées à ce jour et elles ne sont pas nécessairement comparables entre elles. Les études de cas conduites par VERIFOR comportent cependant quelques éléments de preuve utiles (bien que non concluants) pour essayer de répondre à la question. Au Cambodge (Chapitre 13), par exemple, la SGS (secteur privé) a succédé à l'ONG Global Witness. Au Cameroun (Chapitre 11), toute une série d'opérateurs ont joué le rôle d'observateurs indépendants, y compris Global Witness, la REM et Global Forest Watch (des ONG), la SGS et le Cabinet Behle (secteur privé). En Indonésie (chapitre15), l'EIA (en association avec des ONG et des entités du secteur privé) a assumé la fonction d'observateur externe. Au vu des cas cités, il ne semble pas qu'il y ait à première vue de raison pour considérer qu'une catégorie d'acteurs est supérieure à une autre du point de vue de son indépendance intrinsèque. Cela étant, il existe de bonnes raisons de penser 
que les ONG sont mieux placées pour influencer l'opinion publique internationale et aussi, probablement, pour promouvoir la transparence.

\section{Acteurs internationaux ou acteurs nationaux}

Le débat sur l'observation indépendante (IFM) tend aussi à nourrir la conviction que le changement, lorsqu'il survient dans les pays producteurs de bois, serait essentiellement dû à des sources exogènes. On peut extrapoler en ajoutant que les ressortissants de pays très divisés en factions rivales, ne peuvent agir indépendamment parce qu'ils ne pourront jamais se libérer d'une appartenance politique, qu'il s'agisse de l'adhésion à une association politique ou de l'absorption de perceptions externes et par défaut. Plus radicales encore, certaines critiques pourraient faire valoir que le principe de l'indépendance - appliqué dans des conditions bien particulières à connotation géo-éthique -ne sert qu'à renforcer la subordination des pays du tiers monde aux intérêts du monde développé et symbolise la dépendance idéologique des pays du Sud vis-à-vis des pays du Nord. Il en est qui pensent même que c'est un moyen fourbi par des groupes militants pour boycotter l'exportation de bois tropical vers les marchés du nord et imposer de nouvelles voies d'acheminement. Pour d'autres, il y aurait comme un parfum de complot et l'observation indépendante [Independent forest monitoring] (IFM), d'après eux, servirait à compléter l'action de certification en privilégiant les producteurs des pays tempérés du Nord dont les coûts de production sont bas au détriment des pays tropicaux dont les coûts de production sont élevés (voir Smouts 2002). Que de tels soupçons puissent exister est sans doute l'expression d'inquiétudes sous-jacentes concernant la sélection de vérificateurs réellement indépendants et la faible participation de groupes et ressortissants nationaux.

\section{Les institutions et l'architecture du système}

Les organisations ne sont pas seules à se préoccuper de l'indépendance de la fonction et il pourrait être utile d'axer la discussion sur des aspects du système de vérification plutôt que sur des mesures spécifiques ou certains attributs des institutions. Si l'observation indépendante conduite par les ONG environnementales permet d'aboutir à une forme de reddition des comptes responsable bénéficiant d'une bonne réputation sur le plan international, la question de la pérennité de cette façon de procéder n'en demeure pas moins un souci à plus long terme. Il pourrait être plus avantageux - pour arriver à une reddition de comptes démocratique à la fois plus grande et plus stable - de tabler sur l'architecture du système et des arrangements visant à garantir l'indépendance de la fonction à travers des mécanismes de vérification (checks and balances) adaptés.

Il existe deux variantes principales de l'approche architecturale : passive et active. Une bonne illustration de la variante passive est donnée par les comités philippins de protection multisectorielle des forêts [Philippines Multi-sectoral Forest Protection Committees] (MFPC) (voir chapitre14). Dans ce cas, l'indépendance est " passive " au sens où elle résulte de l'architecture du système et n'a pas pour origine des objectifs poursuivis par des acteurs spécifiques. Ce qui est une vertu tant qu'une grande diversité d'intérêts en font partie (au sens où il n'est pas besoin de prendre activement fait et cause pour 
l'indépendance pour la défendre contre des intérêts politiques), est cependant clairement une forme d'indépendance fragile pouvant aboutir à un système dont le fonctionnement sera faussé en cas de réduction du nombre d'acteurs concernés. Il semble que ce scénario se soit produit aux Philippines quand diverses formes de soutien ainsi que l'apport de ressources financières ont diminué et que les comités (MFPC) ont donc commencé à dépendre dans de très fortes proportions des contributions volontaires des ONG dont la plupart sont plus intéressées par des actions militantes en faveur de l'environnement que par le développement économique local ou national.

Comme nous l'avons signalé cependant, l'approche architecturale comporte aussi une variante " active ", au sens où des mécanismes sont délibérément incorporés dans la structure du système pour en promouvoir le fonctionnement indépendant. Au nombre des exemples classiques de ce type d'approche, figurent notamment les suivants :

- L'Equateur a externalisé son système de suivi en confiant les fonctions de suivi et d'observation à toute une série d'institutions différentes (ONG, administrations étatiques et secteur privé), l'indépendance résultant des divers systèmes de vérification et contrepoids (checks and balances) que ces institutions ont mis en place (voir Chapitre 10).

- La Colombie Britannique - dont certaines caractéristiques sont similaires même si le statutaire y est privilégié (voir Chapitre 5) - est un cas particulièrement intéressant en ce qu'il souligne l'importance qu'il convient d'attacher à la crédibilité du système tout entier plutôt qu'à l'un ou à l'autre de ses attributs.

- La Malaisie péninsulaire (voir Chapitre 16) s'est aussi orientée vers une approche de type 'architectural' en matière d'indépendance, mais plutôt que d'adopter un accord à multiples parties prenantes, elle a ciblé la mise en place d'un système de poids et contrepoids entre le secteur public et les auditeurs externes. Ce système comprend des audits à caractère obligatoire effectués par le Département fédéral de la Foresterie (Federal Forestry Department), des évaluations réalisées par des tierces parties sous le couvert du Conseil malaisien de certification du bois d'œuvre (Malaysian Timber Certification Council) ainsi que des audits conduits par les départements de foresterie des divers Etats de la Malaisie (State Forestry Departments) pour vérifier la conformité à la norme ISO 9001:2004 sur les normes de qualité en matière de gestion.

- Une autre solution pourrait être d'associer la fonction de suivi et d'observation aux activités d'un organisme statutaire mandaté pour enquêter sur les abus de pouvoir. Au Honduras, par exemple, un observateur indépendant a agi sous le contrôle de la Commission hondurienne des droits de l'Homme dont l'intention, toutefois, est de reprendre à son compte le rôle d'observateur indépendant des forêts (IFM) à une date ultérieure (voir Chapitre 7).

Ces solutions de type architectural sont celles qui offrent le meilleur potentiel de responsabilisation (et de fiabilité des opérations de reddition des comptes) à l'intérieur même des pays puisqu'elles sont proposées dans le cadre de fonctionnement des systèmes de gouvernance existants. En théorie du moins, le degré de responsabilité, de robustesse 
et de gestion durable en résultant au niveau national devrait être supérieur (et préférable pour le public) à celui des approches pilotées de l'extérieur par une seule institution.

\section{Indépendance de la fonction}

Une autre perspective consiste à s'intéresser aux différentes fonctions et à la façon dont elles s'exercent pendant le déroulement du processus de vérification plutôt qu'aux acteurs qui en assument la charge. Il est possible d'établir, parmi d'autres, la liste de fonctions suivante :

- Les audits de routine et les audits conduits à intervalles réguliers ;

- Les activités de certification ;

- Les prestations relatives à la délivrance des licences (permis);

- Le suivi assuré par des observateurs indépendants; et

- Les mécanismes externes mais chevauchants (comme les médiateurs ou ombudsmen).

Certaines de ces fonctions pourront vraisemblablement être remplies par des organisations de normalisation internationales, dûment accréditées et certifiées, et offrir (à certaines réserves près) des garanties suffisantes quant à la qualité de la prestation (voir Encadré 20.1). Toutefois, le stade considéré comme le plus sensible du processus dans le secteur forestier - l'observation indépendante - est encore à ce jour totalement dérèglementé. C'est une question qui suscite quelques inquiétudes, en particulier dans les milieux proches des gouvernements producteurs. Etant donné l'importance des intérêts économiques, sociaux et commerciaux en jeu, il semble indéniablement justifié d'envisager aussi une certification des activités d'observation indépendante conformément aux normes internationales en vigueur ainsi qu'un contrôle professionnel des opérations.

\subsection{La vérification dans la pratique}

Quelles conclusions peuvent être tirées de cette étude sur l'indépendance des fonctions liées à la vérification des activités du secteur forestier ? Il s'agit de déterminer si la vérification est effectuée suivant des méthodes qui sont non seulement crédibles pour toutes les parties légitimes mais aussi équitables et réalisables sur le plan pratique. Quatre aspects retiendront plus particulièrement notre attention : (i) le champ d'application du mandat des vérificateurs ; (ii) les contraintes liées à la confidentialité commerciale ; (iii) les questions liées au financement pour veiller au maintien de l'indépendance ; et (iv) les questions relevant des conflits d'intérêts.

\section{Champ d'application des mandats : réforme de gouvernance ou certification commerciale (étiquetage)}

Le premier point litigieux concerne le mandat du vérificateur. Une condition essentielle est d'énoncer clairement les objectifs de la vérification en procédant à une analyse aussi 
crédible qu'objective de façon à gagner aussi la confiance de toutes les parties prenantes légitimes. Les ouvrages spécialisés concernant les procédures d'audit ou les normes à appliquer en théorie recommanderaient de se limiter à un ensemble de fonctions aussi succinct que possible et à une définition objective des normes à évaluer. Des esprits critiques pourraient néanmoins faire observer que limiter le nombre de fonctions possibles risque d'aller à l'encontre du but recherché en réduisant la vérification à un exercice où on ne fait que cocher des cases dans des conditions excessivement contrôlées. L'impact est beaucoup plus grand, pourraient-ils ajouter, si les assesseurs sont libres de " poursuivre leur proie dans quelque fourré qu'elle les entraîne " pour terminer en citant à l'appui de leurs affirmations l'impact encore bien plus important des observateurs des droits environnementaux dans des pays comme le Cameroun et le Cambodge.

Il faut cependant noter un changement majeur d'orientation du travail de vérification. Comme dans le cas du débat sur les normes de légalité (Chapitre 19), la question qui se pose désormais devient celle de la légitimité de l'élargissement des objectifs jusqu'au point où l'objectif essentiel risque de ne plus être l'audit technique mais des questions plus vastes comme celles qui relèvent de l'économie politique. Ce changement peut avoir des retombées positives pour les agendas des réformes générales concernant la gouvernance avec une large participation nationale mais être moins adapté à des mesures liées au commerce comme les APV de l'UE. Des craintes spécifiques ont été exprimées à propos des observateurs des ONG qui ont adopté des positions environnementales antagonistes de celles de l'Etat et de l'industrie et justifié leurs positions par les éléments de preuve glanées au fil de leurs missions d'observation. Les critiques pourraient opposer à cela qu'il est tout à fait inapproprié pour des agents qui sont ostensiblement des " observateurs indépendants " d'adopter des positions hostiles sur des questions comme le système des concessions d'exploitation forestière. Les textes sur les normes (par ex. ISO 65/EN4501) insistent sur le fait que les observateurs ne doivent en aucun cas émarger à des intérêts commerciaux susceptibles d'influencer leur jugement. Certains estiment que la liberté devrait avoir un caractère plus général et n'inclure aucune forme d'association avec un groupe d'appartenance partisane, quel qu'il soit. Le Costa Rica pourrait être un modèle fort utile en l'occurrence, tous les agents participant aux fonctions de vérification étant tenus de réaffirmer leur neutralité sur tous les points précités.

Il pourrait aussi être justifié d'établir quelques principes relatifs à la supervision qui serviraient à la fois à valider les données générées par l'observation indépendante (IFM) et en faciliter la diffusion auprès du grand public, assurant par là même transparence et responsabilisation. Une condition fondamentale pourrait être celle de la reconnaissance du principe d'interdiction d'un droit de veto - autrement dit, de ne laisser à aucun individu ni à aucune organisation le pouvoir de décider des normes à appliquer ou de se prononcer unilatéralement sur la performance des systèmes de vérification ou des acteurs participant à l'opération (voir CPET 2006). L'approche suivie par le «Comité de lecture " au Cameroun devrait nous encourager dans la même voie malgré la possibilité d'avoir recours à d'autres arrangements qui pourraient contribuer au déroulement d'un meilleur processus d'appropriation au niveau national. 


\section{Encadré 20.1 Conditions générales requises par l'ISO au titre des organismes d'accréditation et des systèmes de certification}

L'ISO est le mieux connu des systèmes de normalisation, même si d'autres systèmes également applicables (et souvent similaires) existent comme le système de gestion environnementale et d'éco-audit européen [the European Eco-management and Audit Scheme](EMAS) qui est utilisé dans I'UE et la zone économique européenne. Les normes internationales présentent un certain nombre d'avantages dans le contexte actuel, notamment :

- Une reconnaissance internationale ;

- La non-association à des gouvernements ou intérêts spécifiques ;

- La clarté et la cohérence ;

- Un accès et un statut de base de référence universels ;

- Des méthodes participative ; et

- Une composition élargie mais des membres volontaires.

Certaines des normes ISO sont déjà largement appliquées dans le domaine de la vérification du bois comme, par exemple, la série de normes ISO 9000 (sur les systèmes de gestion de la qualité), ISO 14001 (sur les systèmes de gestion environnementale) et ISO 19000 (sur la supervision des systèmes de suivi, combinant ISO 9000 et ISO 14000). Nombreux sont les pays, entre autres les différents Etats composant la Fédération de Malaisie, qui font grand usage de ces normes et, notamment, dans le contexte dont il est question ici, des deux séries concernant les domaines de l'accréditation et de la vérification : ISO 17011 (« Exigences générales concernant les organismes d'accréditation procédant à l'évaluation et à l'accréditation des organismes d'évaluation de la conformité »), et ISO 65/EN45011 («Exigences générales relatives aux organismes procédant à la certification de produits $»)^{1}$

Il s'agit de normes de performance clairement définies qui visent à affermir l'assurance de I'opérateur et à renforcer sa prestation. A titre d'exemple de cette approche, la norme ISO 65/EN4501 stipule que l'organe de certification doit :

- Être constitué en tant qu'entité juridiquement identifiable, doté d'un pouvoir séparé l'autorisant à exercer une activité de certification ;

- Confiner ses opérations à des activités de certification spécifiques ;

- Définir clairement les liens tissés avec toute organisation plus importante ;

- Veiller à ce que ses activités et celles d'entités lui étant rattachées et d'employés non permanents ne portent pas préjudice à la confidentialité, à l'objectivité et à l'impartialité des activités de certification ;

A suivre

1 La norme ISO 65 est identique à la norme européenne EN 45011. La norme ISO 17011 comme la norme ISO 65/EN 45011 ont été notamment adoptées par le CPET (Central Point of Expertise on Timber Procurement), service consultatif pour les marchés du bois d'œuvre établi par le gouvernement du Royaume Uni. 


\section{Encadré 20.1 (suite)}

- Distinguer ses travaux de certification de toute activité de consultation ; et

- Assurer la confidentialité à tous les niveaux de ses activités.

Les conseils d'administration doivent représenter tous les intérêts impliqués dans le processus de certification, sans qu'aucun intérêt soit prédominant. Le but recherché est d'aboutir à « une structure qui préserve l'impartialité ».

S'il apparaît à première vue que des normes claires sont ainsi établies pour les opérateurs relevant du domaine public, force est de constater que des questions continuent à se poser quant aux niveaux d'indépendance requis et à la conversion des principes dans la pratique. Dans le premier cas cité par exemple, un point de controverse reste celui de la mesure dans laquelle d'éventuels conflits d'intérêt seraient tolérés. Dans le dernier cas cité, le volontarisme du système, l'obsession de la confidentialité et l'absence de recours à des fonctions de surveillance quasi-policière constituent autant de contraintes et ont contribué à la réputation de « sagesse » de l'approche ISO (voir Bass 1998). L'orientation est fermement donnée et c'est celle du consensus. La norme ISO 17011 par exemple n'interdit désormais plus à ses membres de prendre des mesures en cas de conflit d'intérêt ; elle précise seulement qu'il faut identifier les gérer les conflits potentiels (voir Proforest/ ISEAL 2005).

\section{Confidentialité commerciale}

Une question corollaire tient à la mesure dans laquelle les vérificateurs ont accès aux informations - classées comme commercialement confidentielles - détenues par les concessionnaires, et à l'usage qu'il leur serait permis d'en faire. Le danger est que le principe de confidentialité puisse être utilisé sans discrimination de façon à empêcher le public d'être informé de questions qu'il est en droit de connaitre (le versement de prébendes à des partis et des dirigeants politiques fait partie, par exemple, de ces informations). Toutefois, le respect de la confidentialité est aussi un principe important, aux nombreuses ramifications juridiques, et probablement essentiel pour que l'industrie y adhère.

\section{Financement d'un examen approfondi indépendant}

Les inspections techniques sont souvent financées par l'industrie ou imputées sur les recettes fiscales de l'Etat. Les points faibles des systèmes d'administration forestière sont souvent situés à la charnière entre deux types de pouvoirs dans des situations isolées et excentrées, celui qu'exercent de puissantes forces industrielles et celui qui est assumé au titre de responsabilités dévolues par l'Etat. Les " régents " (administrateurs) des forêts constituent au Costa Rica et en Equateur, par exemple, les nœuds ou points charnières vulnérables. Bien qu'ils soient censés être indépendants, le fait qu'ils soient le plus souvent payés par les propriétaires des forêts ou les concessionnaires d'exploitations forestières les 
laissent sans grande défense face à l'accusation portée contre eux, à savoir qu'ils sont redevables envers leurs clients.

Un autre sujet prêtant à controverse est celui de l'observation indépendante des forêts dont le financement a jusqu'ici dépendu dans de très fortes proportions des bailleurs internationaux institutionnels afin de préserver l'indépendance de la fonction, à l'écart d'intérêts politiques et industriels. Cette façon de procéder a peut-être permis de se démarquer du gouvernement et de l'industrie du pays producteur et d'être indépendant vis-à-vis de ces deux secteurs mais non de tous les intérêts relevant du secteur forestier.

Il existe de solides arguments pour appuyer un financement issu de sources nationales, sous réserve que les procédures de contrôle en soient démocratiques. Les mécanismes faisant intervenir des médiateurs (ombudsmen) pourraient constituer un modèle fort utile à cet égard (voir Chapitre 3). Si les fonds pouvaient être rassemblés et affectés par la voie législative, il en résulterait un renforcement de l'indépendance vis-à-vis non seulement des forces politiques partisanes mais aussi des bailleurs étrangers. Dans la pratique, cependant, l'appareil législatif pourrait bien être subordonné à l'appareil d'Etat, de sorte que le risque est d'aboutir à un renforcement et non à une diminution de « l'incertitude en matière de gouvernance " (c.-à-d. de la situation dans laquelle l'autorité revient à ceux que les étrangers considèrent comme les plus suspects). Même en Colombie britannique (caractérisée par une "forte gouvernance », au sens conventionnel du terme), le Conseil des pratiques forestières (Forest Practices Board) n'a pas de comptes à rendre au corps législatif - contrairement au médiateur national (BC Ombudsman) et au commissaire de la Cour des Comptes (Auditor-General) - mais est directement financé par le Trésor Public. Cette procédure le rend potentiellement vulnérable aux influences, aussi bien directes (du gouvernement) qu'indirectes, à travers les liens politiques qu'il entretient avec l'industrie du bois.

Certes, il existe aussi d'autres options (voir Brown et al. 2004). Certains systèmes fonctionnent, en partie du moins, en hypothéquant (affectant) amendes et sanctions financières - même si le principe en est considéré comme suspect au motif qu'il crée un intéressement du public à l'indiscipline. Autre possibilité encore : les parties prenantes peuvent être incitées à participer en s'autofinançant - ce que les comités chargés du projet multisectoriel de la protection des forêts aux Philippines ont fait lors de la deuxième phase du projet. Le risque en est toutefois que l'entité chargée de la vérification devienne la proie de groupes d'intérêts particuliers.

\section{L'approche issue du " conflit d'intérêt »}

L'inquiétude suscitée par d'éventuels conflits d'intérêts est toujours présente et revient constamment en filigrane du débat sur l'indépendance. Les textes concernant la certification sont intéressants à cet égard. S’agissant de certification éthique, par exemple, le code de bonnes pratiques pour la mise en place de normes sociales et environnementales (ISEAL) identifie une série de raisons (intérêts) pouvant donner lieu à des situations conflictuelles dont certaines peuvent aussi être rattachées à l'indépendance des prestations (Proforest/ ISEAL, 2005) et regroupées sous cinq grandes rubriques : 
- Pressions externes - qu'elles soient d'ordre politique ou financier. Les pressions financières peuvent concerner des sources directes de revenus ou des pressions commerciales indirectes (par exemple, le désir de ne pas porter atteinte à d'autres champs d'activité d'une entreprise) ;

- Conflitsentrefonctionsalliées, légitimes-par exemple, quand les activités de renforcement des capacités peuvent interférer avec l'indépendance, au point que cette ingérence est perçue comme un intérêt porté par l'organisation à ce que des évaluations positives résultent de l'examen approfondi de la situation d'un partenaire ;

- Problèmes liés au fait d'être à la fois 'juge et partié-dans le cas, par exemple, où la même organisation assume des tâches d'accréditation et de normalisation, ou de certification et d'élaboration de normes;

- Plaidoyer - dans les cas où la mission de l'organisation est considérée comme susceptible de compromettre son devoir d'objectivité ; et

- Considérations d'ordre pratique - dans le cas où un organisme (ou un individu) est reconnu comme ayant des intérêts dans le conflit mais qu'il est le seul acteur disponible ayant les compétences nécessaires.

Le rapport Proforest de l'ISEAL recommande l'adoption d'une approche axée sur les processus qui vise à l'obtention de résultats non conflictuels à travers l'identification systématique des conflits et la mise en œuvre d'une série de mécanismes pour pouvoir en assurer la gestion (ibid.). Au nombre de ces mécanismes figurent les suivants : mettre fin aux conflits à travers la division des activités ou l'interdiction de ces activités ; identifier les règles et les systèmes, comme par exemple, les directives de l'ISO (bien qu'elles ne permettent pas toujours de résoudre les conflits, comme noté plus haut); utiliser des systèmes de vérification ou de freins et contrepoids (checks and balances); et veiller à ce que les activités concernées soient soumises à des procédures de transparence et de rééquilibrage.

\subsection{Résumé}

Dans tous les ouvrages concernant la vérification, l'intérêt pour la notion d'indépendance s'est plutôt focalisé sur l'observation indépendante dans le secteur forestier [Independent Forest Monitoring] (IFM), la justification donnée étant que l'indépendance constituait le facteur le plus critique du secteur en question. Un bulletin de bonne santé signé par un observateur indépendant (IFM) sceptique quant au caractère légal de l'approvisionnement en bois d'un pays ou d'un producteur suffirait à lui seul à valider toute la filière. Il ne s'ensuit cependant pas nécessairement que la fonction de vérification doive incomber à une seule institution.

Deux autres préoccupations sont aussi apparues comme revêtant une importance critique : il s'agit de déterminer quelles mesures doivent être prises d'emblée par les acteurs pour préserver leur indépendance et quelles actions les gouvernements et leurs partenaires doivent engager pour assurer la transparence et la fiabilité de la reddition des comptes à long terme par rapport aux informations fournies par les vérificateurs indépendants. 



\section{Chapitre 21}

\section{Impact sur le développement des systèmes de vérification du secteur forestier}

Note : Kate Schreckenberg et Neil Bird sont les auteurs de ce chapitre, extrait de l'article de Schreckenberg, K. et Bird, N. 2006 Developmental Impacts of Verification Systems in the Forest Sector. VERIFOR, Briefing Paper No. 3. ODI, London.

\subsection{Introduction}

Ce chapitre passe en revue les impacts possibles des systèmes de vérification en se basant sur les enseignements tirés des études de cas rapportées dans le cadre de VERIFOR. Quelques rares évaluations formelles seulement ont été réalisées à ce jour sur l'impact des systèmes de vérification du secteur forestier. De tous les impacts éventuels, l'impact sur le développement semble avoir été celui qui a le moins retenu l'attention et a été aussi le moins bien considéré et le moins compris. Outre le problème récurrent qui consiste à en attribuer la cause aux interventions pour le développement, il semble que deux raisons principales peuvent expliquer cette situation. Premièrement, la plupart des systèmes de vérification n'ont été mis en place que depuis peu de temps et leur impact sur le développement reste encore indéterminé pour une large part. Deuxièmement, quand un système est imposé de l'extérieur, l'évaluation de son impact peut ne présenter que peu d'intérêt à l'échelle nationale. Les conclusions auront donc nécessairement un caractère provisoire à ce stade. Il est toutefois possible de dégager un certain nombre de points saillants sur la base des expériences globales qui ont été menées à l'échelle internationale dans le cadre de la vérification du caractère légal des activités menées dans le secteur forestier :

- Comment les intérêts de toutes les parties prenantes peuvent-ils être pris en compte de façon à permettre de fixer des objectifs clairs et sans ambiguïté pour le système de vérification?

- Quelle est la nature de l'impact éventuel d'un système de vérification sur le développement et comment en évaluer les résultats pour aboutir à un degré raisonnable de confiance en ce qui concerne leur répartition en impacts direct et indirects?

- Comment séparer les questions liées à la mise en œuvre de l'influence exercée par le cadre juridique sur lequel le système est basé ?

- Comment minimiser les impacts potentiellement négatifs de la vérification? 


\subsection{Parties prenantes, facteurs déterminants et objectifs des systèmes de vérification}

L'établissement des systèmes de vérification est déterminé par une série d'objectifs qui varient en fonction des différentes parties prenantes et de leur influence sur l'adoption des programmes. Les raisons pour lesquelles chacun de ces groupes veulent mettre en place un système de vérification peuvent être très différentes les unes des autres et tandis que certaines seront explicitement énoncées, d'autres (y compris, au niveau national, la question des droits fonciers sur la forêt et, au niveau international, celle des déséquilibres du commerce du bois d'œuvre) pourraient l'être moins. Comme indiqué au Chapitre 18, certains objectifs pourraient se renforcer mutuellement alors que d'autres pourraient être en conflit et nécessiter des compromis (concernant l'architecture du système, par exemple).

Pour de nombreuses parties prenantes, le but ultime est probablement celui qui consiste à " améliorer la croissance économique nationale de façon durable ». La possibilité que la vérification contribue à l'objectif de réduction de la pauvreté n'est pas souvent explicitée - reflétant peut-être le fait que les défenseurs de cet objectif, comme les sociétés civiles nationales, sont ceux qui sont le moins écoutés pendant la période d'élaboration des systèmes de vérification. Un débat est en cours pour déterminer si ces systèmes doivent ou non prendre en compte l'objectif de réduction de la pauvreté (rester neutres ou cibler des actions ayant des retombées positives pour les populations pauvres). Le Plan d'action de l'UE pour l'application des législations forestières, de la gouvernance et du commerce semble adopter une position de neutralité, se contentant de rechercher des solutions à l'abattage illégal qui n’aient "pas d'impact négatif sur les populations pauvres".

D'un point de vue plus limité, l'objectif primordial unique de tout système de vérification est de démontrer que le comportement observable sur le terrain est compatible avec la législation existante. Les entités individuelles composant un système de vérification peuvent cependant avoir leurs propres objectifs qui se réferent parfois spécifiquement aux objectifs de développement. En Equateur, par exemple, l'entité publique/privée Vigilancia Verde qui est l'une des trois composantes du système de vérification a inclus dans ses objectifs celui de faire participer la société civile à la vérification et de rendre la légalité plus accessible aux petits producteurs. Aux Philippines, les comités de protection multisectorielle des forêts (MFPC) sont non seulement chargés des tâches relatives à la vérification mais aussi des services de vulgarisation et sont invités à contribuer à la création de modes de vie alternatifs.

\subsection{Impacts directs et indirects des systèmes de vérification}

Les impacts d'un système de vérification se déroulant conformément à son objectif principal qui consiste à vérifier la conformité avec la législation existante sont multiples et peuvent être soit directs, soit indirects. 
Au nombre des impacts directs figurent, notamment, les suivants :

- Une amélioration de la conformité. Impact le plus évident d'un système de vérification, il a été observé dans plusieurs pays. En Equateur, par exemple, les saisies de bois illégalement transporté ont doublé les deux années qui ont suivi l'introduction du système de vérification, tandis que plus de 120 permis d'exploitation ont été retirés pour non-conformité.

- Une augmentation des recettes perçues par le gouvernement au titre des amendes pour infraction. Les sanctions imposées pour non conformité ont rapporté des gains substantiels aux services fiscaux de pays aussi éloignés les uns des autres que le Cambodge, le Cameroun et la Malaisie.

- Un nombre accru de dépôts de plaintes et autres litiges. La vérification peut être contestée. Par exemple, le Conseil chargé de l'examen des vérifications (Verification Review Council) en Indonésie a été institué pour connaître des plaintes concernant la certification des unités de gestion forestière.

- Une augmentation des coûts de fonctionnement pour l'industrie du bois, l'observance de la conformité risquant d'être plus onéreuse que les activités illégales. Bien que la différence puisse parfois n'être que marginale du point de vue financier (comme cela semble être le cas en Equateur), force est de constater que l'observance de la conformité dans un certain nombre de pays entraîne des frais considérables pour pouvoir satisfaire à des procédures bureaucratiques.

- Une meilleure disponibilité et une plus grande précision de l'information. La mise en place de nouveaux systèmes de vérification dépend énormément des technologies de l'information comme l'a montré l'expérience brésilienne. L'utilisation d'outils comme le GPS et les systèmes de traçabilité par satellite a amélioré le degré de précision des informations concernant les sources et les mouvements du bois d'œuvre dans des proportions jamais atteintes auparavant.

Les impacts indirects, induits par l'utilisation du processus de vérification comprennent notamment :

- Une concentration de l'industrie. A mesure que les conditions relatives à la vérification de la conformité deviennent plus strictes, certaines sociétés (en général, les plus petites) pourraient ne pas être en mesure d'appliquer les normes prescrites. En outre, les petits producteurs supportent souvent une part relativement plus élevée des sanctions parce qu'ils sont une proie plus facile pour les fonctionnaires du gouvernement qui manquent de ressources et qu'ils sont politiquement moins influents que les grosses entreprises industrielles. Au Costa Rica, par exemple, le fait qu'un délai d'une année soit nécessaire pour obtenir l'approbation des plans de gestion a abouti à un système discrétionnaire qui favorise ceux qui ont un pouvoir politique ou financier. Au fur et à mesure de la cessation des opérations des petits producteurs, l'activité du secteur se trouve de plus en plus concentrée entre quelques gros établissements.

- L'illégalité qui guette les petits opérateurs marginalisés. A mesure que les activités légales sont de plus en plus concentrées, les petits producteurs peuvent être poussés 
à l'illégalité. En Papouasie indonésienne, par exemple, les descentes de police ont été très efficaces pour mettre un terme aux transports de bois illégal. Il en est cependant résulté un impact très négatif sur la population pauvre en raison du retrait de tous les permis d'exploitation forestière délivrés aux communautés. Ces permis auraient été annulés au motif que les communautés étaient abusées par les syndicats désireux de s'assurer un accès à la ressource. La mesure a abouti à une situation de vide juridique pour les communautés avec des villages entiers prisonniers pendant que la police y menait ses enquêtes.

- Le déplacement des activités d'exploitation illégale du bois d'auvre dans les terres non forestières. Le fait que les systèmes de vérification mettent moins l'accent sur les terres forestières et la production de bois d'œuvre - ciblant plutôt l'exploitation illégale et le changement d'affectation des terres - a entrainé un déplacement des activités illégales vers d'autres utilisations des terres (agricoles), comme relevé au Costa Rica.

- Une amélioration de la planification. La collecte d'informations plus précises sur les volumes réels de production permet de mieux planifier les activités de l'industrie de transformation.

- Une plus grande transparence. Une plus large diffusion des informations entraîne une plus grande responsabilisation des communautés, comme dans le cas du Cameroun où les communautés reçoivent une partie des taxes forestières au cours d'une cérémonie publique. Il peut aussi en découler un engagement plus fort de la société civile et d'autres parties prenantes dans les processus politiques forestiers. La disponibilité des informations en ligne dans le cadre du système de fichiers brésilien a entrâné initialement de vives critiques de la population à l'égard de l'agence fédérale pour l'environnement, IBAMA.

- Un impact sur les processus politiques forestiers. Un taux élevé de litiges peut éventuellement entraîner le démantèlement du système de vérification comme cela s'est passé au Cambodge. L'antagonisme initial peut cependant avoir un résultat bénéfique à plus long terme en sensibilisant davantage le public aux questions relevant de la gouvernance forestière, comme cela a été le cas au Cameroun.

- Un effet de dissuasion sur la plantation d'arbres. Selon le barème des coûts appliqué aux transactions y afférentes, la vérification peut parfois involontairement avoir un effet dissuasif sur la plantation d'arbres. C'est le cas en Equateur, où la commercialisation des arbres plantés a désormais atteint des coûts de transaction plus élevés que celle des produits agricoles.

\subsection{Quels sont les facteurs déterminants de l'impact d'un système de vérification ?}

Comme il ressort clairement de la précédente section, l'impact de la vérification est à facettes multiples et peut être perçu comme négatif ou positif selon les différents groupes de parties prenantes. Afin de maximiser les chances de succès d'un système de vérification, il importe de comprendre quels sont les facteurs qui en détermineront l'impact. 


\section{Politique et législation}

Le but d'un système de vérification est de vérifier que la législation est bien appliquée. Ainsi, même un excellent système de vérification ne peut assurer plus de prestations que celles qui sont requises au titre de la loi. Dans le cas de la Colombie britannique, par exemple, les ONG et autres institutions restent préoccupées par l'absence de mise en œuvre d'une utilisation durable des terres sous la législation actuelle - pourtant relativement plus rigoureuse, toutes proportions gardées, que celle des autres pays - en dépit d'un système de vérification performant avec de très hauts niveaux de conformité. Selon le mouvement écologiste, s'il est souhaitable d'améliorer la vérification, il est incontestablement encore plus nécessaire d'améliorer la législation forestière de la province. Dans les pays tropicaux producteurs de bois d'œuvre, les mêmes préoccupations apparaissent à propos du manque d'intérêt porté aux besoins des communautés forestières dans le cadre politique et législatif existant. Dans une étude axée sur les législations forestières et leur application en Bolivie, au Cameroun, au Canada, au Honduras, en Indonésie et au Nicaragua, Colchester et al. (2006) ont conclu que les droits des pauvres n'étaient pas protégés de façon adéquate. Dans ces conditions, tout système de vérification favorisant une application plus stricte de la législation a vraisemblablement pour corollaire l'inconvénient majeur de désavantager les pauvres.

\section{Architecture du système de vérification}

La question visant à déterminer quelles sont les législations qu'il convient de vérifier (voir Chapitre 19) relève de l'architecture du système de vérification. Un élément important de l'architecture est celui du degré d'indépendance atteint. Comme indiqué au Chapitre 20, l'indépendance peut être le résultat de plusieurs façons de procéder, y compris à travers l'architecture du système (impliquant la participation d'un certain nombre d'acteurs différents qui peuvent se surveiller les uns les autres) et/ou en ciblant l'indépendance d'éléments constitutifs clé du système (par ex. l'observateur indépendant dans les cas du Cambodge et du Cameroun). Un système qui n'est pas indépendant mais redevable - en ce qu'il est rattaché à un groupe d'intérêts particuliers du secteur forestier - est probablement moins exposé et devrait donc ne pas ressentir les impacts qui auront un effet sur d'autres groupes d'intérêts.

\section{Mise en œuvre du système de vérification}

Un système de vérification peut être bien conçu mais gêné par des contraintes financières et/ou pâtir d'un manque de personnel. Comme indiqué aux Chapitres 9 et 23, le nouveau système de navigation par satellite établi par IBAMA au Brésil a permis de créer des cartes de situation spécifiques pour faire apparaître les zones d'activités légales et illégales de l'exploitation de bois d'œuvre. La robustesse du système est affaiblie par sa dépendance vis-à-vis d'un cycle de financement trimestriel pour acheter les images satellite (cartographiées) dont il a besoin pour permettre aux inspecteurs du gouvernement d'avoir une longueur d'avance sur les exploitants illégaux. IBAMA a un effectif réduit et manque de personnel pour conduire des inspections sur le terrain en nombre suffisant. Une autre contrainte est celle du financement dans presque tous les cas examinés, l'exemple extrême 
étant celui des Philippines où de nombreux comités MFPC ont cessé d'exister dès la fin de la période de financement du projet dont ils étaient chargés.

S'agissant de certaines fonctions (ou rôles), l'approche adoptée par des acteurs individuels au sein d'un système de vérification peut aussi avoir un effet sur le type d'impact produit. Ce fut le cas au Cambodge où le rôle d'observateur indépendant des forêts avait été initialement dévolu à une ONG internationale de défense des droits de l'Homme, Global Witness, dont les intentions de programme ont été fortement suspectées. En revanche, la société privée qui lui a succédé, la SGS, a été accusée d'interpréter son mandat de façon trop restrictive et de ne s'en tenir qu'à son domaine de compétence sans élargir son champ d'action. En Colombie britannique, le Conseil des pratiques forestières (Forest Practices Board) est devenu plus conciliant et constructif au fil des années ; en particulier, il a tenté d'apporter des améliorations à l'industrie du bois en se faisant le chantre des bonnes pratiques et des innovations et en fustigeant la non-conformité.

\subsection{Comment minimiser les impacts négatifs sur le développement?}

Un certain nombre des impacts indirects du système de vérification peuvent se révéler négatifs pour les plus démunis. Ces retombées négatives qui sont généralement involontaires et parfois inattendues peuvent résulter d'une compréhension et d'une prise en compte insuffisantes de plusieurs facteurs :

- Les intérêts des différentes parties prenantes ;

- Le pouvoir dont elles disposent pour imposer leurs intérêts ; et

- Les impacts, pris séparément, des différents éléments constitutifs du système de vérification.

Dans un contexte de développement, il est d'un intérêt capital de veiller à minimiser les retombées négatives sur les populations pauvres. Il faudrait au moins permettre aux plus démunis d'avoir accès aux mécanismes de règlement des différends (par ex. à travers un service d'assistance juridique) pour faire appel de tout impact négatif induit par un processus de vérification. Il y a cependant lieu de signaler que les études de cas VERIFOR menées dans différents pays et d'autres ouvrages thématiques relèvent un certain nombre d'options possibles pour minimiser les retombées négatives.

\section{Enoncé explicite des objectifs et des objections}

Un énoncé des objectifs clair et sans ambiguïté - accompagné d'objections éventuelles à ces objectifs - présenté pendant la phase d'élaboration de l'architecture du système de vérification pourrait contribuer à en minimiser les retombées négatives sur les plus démunis. Des préoccupations non résolues peuvent pousser des personnalités ou institutions clé à bloquer la mise en œuvre du système dans la pratique. En Equateur, par exemple, l'une des raisons non énoncées pour lesquelles le ministère de l'Environnement n'a pas apporté un soutien sans réserve à l'externalisation de certaines de ses fonctions 
administratives à un opérateur du secteur privé tient à la perte en résultant pour une activité clé génératrice de revenus. Cet exemple témoigne bien du précieux avantage qui consiste à incorporer le système de vérification dans un processus élargi de réforme politique (c.-à-d. le programme forestier national) qui, entre autres, favorise la détermination des objectifs d'une façon transparente par voie de consensus.

\section{Evaluation de l'impact sur la pauvreté et de l'impact social}

Dans un monde idéal, l'architecture d'un système de vérification doit bénéficier d'une évaluation "ex ante " de l'impact sur la pauvreté et sur la société (PSIA). Si les impacts probables de ce type peuvent être déterminés à l'avance, deux types d'actions pourraient en bénéficier : (i) de meilleures décisions pourraient être prises quant aux interventions à mener et la meilleure façon de procéder ; et (ii) les mesures d'atténuation ou de compensation des effets du système qui pourraient être mises en œuvre le cas échéant. Les évaluations d'impact sont aussi importantes en ce qu'elles apportent des éclaircissements sur la répartition des différents types d'impacts entre les différents groupes de la société et, en particulier, sur la charge relativement plus lourde qui pèse sur les groupes les plus vulnérables.

L'utilisation des PSIA ou d'une analyse des coûts et bénéfices "ex ante " pour toutes les parties prenantes pourrait aussi aider ces dernières à conclure un accord sur l'importance relative et le degré d'acceptabilité des différents impacts possibles. Certains des impacts inattendus ou indésirables pourraient être considérés comme suffisamment préjudiciables pour justifier une totale remise en question du système de vérification, voire même de son support juridique.

\section{Introduction d'un processus parallèle de réforme législative}

La vérification ne peut se substituer à une réforme législative. Par conséquent, si l'objectif recherché est un impact sur le développement, il faudra le spécifier dans la législation forestière dont le fonctionnement sera à son tour vérifié. Par exemple, quand les communautés rurales ne se voient pas reconnaître leurs droits les plus fondamentaux, il importera de trouver une façon de procéder pour changer la législation de sorte qu'une application rigoureuse de la loi ne les désavantage pas injustement. Les impacts de la vérification varieront en fonction de la mesure dans laquelle le cadre juridique et réglementaire est suffisamment souple pour s'adapter au changement.

\section{Reconnaissance légale des diverses utilisations du bois par les populations pauvres}

Pour reprendre le point soulevé dans le paragraphe précédent, il se pourrait que le seul changement requis soit simplement que le législateur se borne à reconnaître la réalité concernant la façon dont les plus démunis utilisent le bois d'œuvre. Au Costa Rica et en Equateur, des permis d'exploitation sont délivrés dans le cadre de différents régimes fonciers en reconnaissance du fait que, souvent, les petits producteurs n'ont pas la 
pleine jouissance de leur titre de propriété. De même, la loi brésilienne sur les forêts domaniales (Brazilian Law on Public Forest) a ouvert la voie à la légalisation des activités d'extraction du bois dans 45 pour cent de l'Amazonie brésilienne, là où l'exploitation était auparavant illégale dans les forêts domaniales. Dans le cadre du plan concernant les concessions d'exploitation forestière accordées pour une période de 100 ans dans l'Etat de Sabah, Malaisie, ce n'est pas seulement le droit d'usufruit des communautés installées à l'intérieur de ces concessions qui est reconnu, mais aussi leur droit à établir des forêts communautaires. Outre le fait de reconnaître ainsi le statut foncier incertain de nombreux habitants des forêts, le législateur a aussi veillé à établir, dans d'autres textes de loi, une distinction entre les arbres à l'état naturel dans les forêts et ceux qui sont plantés dans des systèmes agro-forestiers ou font partie de petites plantations. Ainsi le Costa Rica remet des bons de transport gratuits aux agriculteurs pour abattre jusqu'à trois arbres par an et par exploitation agricole. Les agriculteurs (producteurs) qui ont planté des arbres dans des systèmes agro-forestiers ou des forêts plantées n'ont pas besoin de permis ; ils doivent simplement obtenir un certificat auprès d'un « régent » (administrateur des forêts).

\section{Accès accru des populations pauvres aux voies administratives légales}

Des actions ont été menées dans le cadre de plusieurs systèmes de vérification examinés dans les études de cas par pays pour essayer de faciliter l'accès des petits producteurs à la légalisation de leurs activités. En Equateur, ces tentatives se sont soldées par l'introduction d'unités administratives itinérantes aux niveaux régional et local pour venir directement à la rencontre des communautés dans les lieux où elles sont installées. Au Brésil, les permis de transport du bois d'œuvre peuvent être obtenus par internet, même depuis des sites très éloignés et excentrés.

\section{Amélioration des flux d'informations et de la transparence}

Fournir des informations en plus grand nombre - sous une forme qui soit accessible aux petits producteurs - est un moyen important de faciliter leur engagement dans le processus de vérification. Au Cameroun, les communautés peuvent tenir pour responsables leurs conseils locaux et leur demander de rendre des comptes puisqu'elles ont désormais connaissance des montants des recettes fiscales qui leur sont versés par le Fonds central des forêts (Central Forest Fund). De meilleurs flux d'informations pourraient aussi élargir le débat sur la vérification aux zones les moins boisées dont les résidents pourraient élever des objections à l'encontre du versement des taxes forestières aux seules zones richement dotées en forêts.

\section{Suivi et évaluation}

Les systèmes de vérification ne doivent pas seulement faire l'objet d'évaluations d'impact "ex ante » mais aussi d'un processus de suivi et d'évaluation cohérent et transparent visant à mieux définir leurs impacts sur leurs différents groupes-cibles. Au nombre des indicateurs mesurables de ces impacts - cités dans les études de cas - figurent notamment : 
- L'accroissement des ressources financières mises à disposition par le gouvernement ;

- Le renforcement des capacités de mise en application des législations (personnel qualifié, nombre d'affaires instruites, etc.) ;

- La réduction des formalités administratives requises/procédures bureaucratiques (délais nécessaires pour obtenir un permis d'exploitation ; nombre de démarches administratives auxquelles doit se plier l'utilisateur de la ressource forestière) ;

- Le renforcement du système de freins et contrepoids (checks and balances) du système réglementaire, pour satisfaire toutes les parties ; et

- Le renforcement du processus d'appropriation au niveau national avec la participation du gouvernement et de la société civile au sens large.

Il importera également de mettre en place les processus nécessaires pour que les résultats des activités de suivi et d'évaluation fassent l'objet de rapports libres de toute influence et que les mesures correctrices nécessaires soient prises, le cas échéant.

\subsection{Résumé}

La complexité du processus menant à garantir la légalité des opérations dans un cadre international n'est nulle part plus apparente qu'au stade de l'évaluation des impacts. La grande diversité des parties prenantes avec des intérêts dans la ressource forestière est encore renforcée par l'entrée en jeu de nouveaux intervenants et l'émergence de nouvelles préoccupations quant à la conservation des biens publics relevant du patrimoine commun de l'humanité. Enfin, la durabilité à long terme d'un système de vérification ne saurait être garantie sans en aborder les questions clé - minimiser les impacts négatifs et veiller à ce que les amendements nécessaires soient apportés pour tenir compte des impacts imprévus - préalablement à sa mise en œuvre. 



\section{Chapitre 22}

\section{Convergence de la certification et de la vérification à travers la légalisation des activités forestières}

Note : David Brown et Neil Bird sont les auteurs de ce chapitre, extrait de l'article de Brown, D. et Bird, N. 2007 Convergence between certification and verification in the drive to legality assurance: assessing the pros and cons. VERIFOR, Briefing Paper No. 6. ODI, London.

\subsection{Introduction}

Les tentatives de légalisation du commerce international de bois d'œuvre ont accru l'intérêt porté au potentiel des projets de certification forestière du secteur privé en tant que moyen pour promouvoir la gestion durable des forêts et vérifier la légalité de la production forestière. Il existe des points de convergence manifestes entre les deux courants de pensée et de stratégies, plusieurs gouvernements producteurs étant même réputés disposés à traiter la certification comme une substitution de l'assurance formelle de légalité. Si une telle convergence offre de réels bénéfices en diminuant les coûts et la charge administrative pour les producteurs, elle n'est pas sans risques et les avantages et inconvénients en sont étudiés dans ce chapitre.

Depuis le début des années 1990 , la certification en matière forestière a été l'un des principaux moyens de promouvoir les pratiques de gestion durable et un certain nombre de projets de certification ont acquis une grande importance. Institué en 1993, le Conseil de supervision des forêts [Forest Stewardship Council (FSC)] a été pionnier en la matière et, pendant plusieurs années, est resté le seul organisme international d'accréditation. Doté de structures de prise de décision novatrices permettant de regrouper des intérêts divergents et à travers ses principes directeurs " Principles for Forest Stewardship ", le FSC a contribué à définir ce que devrait être une gestion durable des forêts. D'autres projets de certification ont suivi, initialement au niveau national, (celui de l'Association canadienne de normalisation (Canadian Standards Association scheme) et l'Initiative pour une foresterie durable (Sustainable Forestry Initiative) aux Etats-Unis). Le principal concurrent de la FSC est cependant le résultat d'une initiative régionale - le projet paneuropéen de certification pour les forêts (Pan-European Forest Certification scheme) ou PEFC - qui a été lancé en 1999. Les bons résultats obtenus par le PEFC en travaillant avec l'industrie forestière européenne et les petits propriétaires de forêts ont favorisé une reprise de ses activités en 2003, avec couverture mondiale cette fois, sous le nom de programme pour entériner les projets de certification forestière (Programme for the Endorsement of Forest Certification schemes). Le label de certification PEFC est le label 
qui prévaut désormais pour la plus grande partie, exprimée en superficie, des forêts du monde (Nussbaum et Simula 2005).

\subsection{La légalité dans les systèmes de certification}

Tous les principaux systèmes de certification forestière exigent que preuve soit apportée de la légalité des opérations en tant que condition préalable de la délivrance d'un certificat (voir Tableau 22.1).

Il est particulièrement intéressant dans ce contexte de relever la liberté accordée aux agents de certification pour se faire leur propre opinion quant au niveau de conformité avec les lois nationales. Quand les conditions juridiques ont été précisées par le gouvernement, l'agent chargé de la certification utilisera les normes ainsi fixées pour prendre une décision en matière de conformité. Les situations plus problématiques sont celles où la loi est contestée par d'importants groupes d'intérêts et le danger vient alors de l'hésitation des agents de certification à examiner de plus près des interprétations qui seraient en conflit (entre l'Etat et la société civile, par exemple).

Dans le cas du FSC, la conformité avec la loi est l'un des dix principes de gestion durable des forêts qu'il convient d'observer pour qu'une opération forestière puisse être certifiée. Ces principes sont exprimés en tant que critères et indicateurs (C\&I) génériques (globaux) du FSC, qui doivent ensuite être adaptés au contexte national. Cela se fait habituellement à travers l'élaboration de normes de certification nationales impliquant un processus de consultation des parties prenantes. En l'absence de consultation ou de directives, les agents

\section{Tableau 22.1 Conditions imposées en matière de légalité et de durabilité des principaux systèmes de certification forestière}

\begin{tabular}{lcc}
\hline Système de certification & $\begin{array}{l}\text { Conditions en matière } \\
\text { de légalité }\end{array}$ & $\begin{array}{l}\text { Conditions en matière de } \\
\text { durabilité }\end{array}$ \\
\hline Canadian Standards Association (CSA) & $\sqrt{ }$ & $\sqrt{ }$ \\
\hline Forest Stewardship Council (FSC) & $\sqrt{ }$ & $\sqrt{ }$ \\
\hline $\begin{array}{l}\text { Malaysian Timber Certification Council } \\
\text { (MTCC) }\end{array}$ & $\sqrt{ }$ & $\sqrt{ }$ \\
\hline $\begin{array}{l}\text { Programme for the Endorsement of } \\
\text { Forest Certification (PEFC) }\end{array}$ & $\sqrt{ }$ & $\begin{array}{l}\sqrt{ } \\
\text { Sustainable Forestry Initiative (SFI) }\end{array}$ \\
& $\sqrt{ }$ & $\begin{array}{l}\text { [sous réserve de l'utilisation } \\
\text { d'un système COC] }\end{array}$ \\
\hline
\end{tabular}

Note : Les cinq systèmes de certification ci-dessus sont ceux qui ont été acceptés par le Point central d'expertise du Royaume-Uni pour les achats de bois d'œuvre (Central Point of Expertise on Timber Procurement) (CPET), et les comparaisons sont extraites des évaluations spécifiques du CPET pour ces projets.

Source: www.proforest.net/cpet/cpet-s-assessment-of-evidence/assessment-of-certification-schemes-category-a/ 
de certification doivent interpréter eux-mêmes les C\&I génériques, et dresser une liste des points à certifier qui est ensuite mise à la disposition du public. Ils peuvent s'inspirer de normes consultatives internationales comme celles qui sont proposées par l'Organisation internationale des bois tropicaux (OIBT) et, dans le contexte sous-régional de l'Afrique centrale, par l'Organisation africaine du bois (OAB). L'agent de certification individuel pourra alors juger au cas par cas sur la base de ces principes directeurs et décider s'il y a ou non conformité avec la loi. Les délais très courts qui sont impartis à la plupart des missions d'évaluation de certification forestière signifient que la décision doit souvent être prise rapidement, sur la base d'éléments de preuve facilement disponibles. En effet, l'application de la législation devient un exercice d'audit. Il ne s'agit pas d'un processus garanti à toute épreuve mais les agents de certification sont protégés par la clause de non responsabilité qui figure dans l'introduction des principes et critères du FSC qui stipule : « ni le FSC ni les organismes de certification accrédités auprès du FSC n’insisteront sur la perfection en appliquant les $\mathrm{P} \& \mathrm{C}$ »(Paragraphe 4 de l'introduction). Cela ne ressemble en rien - et s'en écarte même très nettement - à une procédure judiciaire au sens où l'entend un tribunal.

La certification s'est avéré un instrument efficace dans le cas de certains types de forêts, surtout les forêts plantées et les forêts caractérisées par un haut niveau de normalisation et la sécurité des droits d'accès et de propriété foncière. Elle est ainsi bien utilisée dans les zones géographiques tempérées d'Europe occidentale et d'Amérique du Nord, et (avec plus de variations) en Amérique centrale et en Amérique du Sud. Elle s'est cependant montrée moins applicable dans d'autres contextes, en particulier dans les régions tropicales d'Afrique et d'Asie du Sud-est dont les forêts ont une structure beaucoup plus complexe (et, par conséquent, moins facile à reconstituer après une période d'exploitation) et dont la situation en matière de droits d'accès et de propriété foncière - pour diverses raisons tenant entre autres à l'héritage colonial - suscite bien plus de difficultés qu'ailleurs. Ces pays tendent aussi à devenir des pays qualifiés de pays "à hauts risques " dans l'industrie du bois, en raison d'un niveau de gouvernance forestière insuffisant.

\subsection{La vérification en tant que nouvelle stratégie}

C'est en partie en reconnaissance de tous ces problèmes que l'attention s'est portée, au début des années 2000, sur la vérification du caractère légal des activités forestières/de la légalité en tant que vecteur de développement d'une politique forestière internationale. Certains ont considéré qu'il s'agissait là d'une méthode plus abordable pour améliorer la qualité de la gestion des forêts, sans la nécessité d'avoir recours à des notions complexes de pérennité. La légalité leur est apparue comme un concept plus simple que les Etats auraient moins de difficultés à traduire dans les faits.

Cependant, au fil des progrès réalisés - sous l'impulsion, notamment, d'initiatives comme le programme FLEGT de l'UE - en matière de garanties concernant la légalité des activités forestières, la convergence entre les deux opérations - certification et vérification - est devenue de plus en plus manifeste. Il est aussi apparu de plus en plus clairement que la certification pouvait se substituer aux procédures visant à garantir la 
légalité des opérations - ce qui est assez paradoxal étant donné la plus grande complexité de ses associations - et plusieurs gouvernements producteurs sont réputés envisager la possibilité de traiter certains systèmes de certification du secteur privé comme équivalents à une procédure de vérification de la légalité, fournissant ainsi un moyen détourné aux concessions certifiées pour être en règle avec les formalités administratives qu'elles doivent remplir au titre des demandes de justification du caractère légal de leurs activités. La convergence entre les deux procédures de certification et de vérification est tout à fait distincte des initiatives pilotées par l'industrie qui visent à " vérifier » l'origine légale et la conformité du bois d'œuvre avec la législation (par exemple, la norme "bois contrôlé " du FSC, et le programme "Validation du bois légal » du SGS).

Comment évaluer la convergence entre certification et validation sous l'angle de la qualité de la gestion forestière compte tenu des enjeux de justice sociale et d'équité pour les populations dépendantes des forêts?

\subsection{Le FLEGT et l'assurance de la légalité}

Le concept de l'assurance de la légalité est un concept beaucoup moins large que celui de la durabilité et ne vise que la conformité des activités d'abattage et de transformation avec les législations locales, nationales et internationales; il ne prend pas en compte les questions plus complexes relatives à l'exploitation durable dans ses diverses dimensions relevant des domaines économique, social et environnemental. Cela dit, les procédures visant à garantir la légalité des activités forestières reste un enjeu important, en particulier dans les pays en développement comme en témoignent certaines des études de cas rapportées dans ce livre. Un cas extrême est celui de l'Indonésie (voir Chapitre 15) où coexistent environ 800 lois, règlements et décrets applicables au secteur des forêts. Dans de telles situations, il peut être tout bonnement impossible d'assurer la conformité avec toutes les lois locales et nationales. Comme indiqué au Chapitre 19, ce pourrait être tâche ardue que d'avoir à décider quelles lois sont déterminantes pour la reconnaissance de la légalité des activités forestières. L'industrie forestière sera probablement encline à retenir l'interprétation la plus étroite possible, se bornant aux normes prescrites pour le secteur forestier ; les ONG militantes seront probablement en faveur d'interprétations beaucoup plus larges qui tiennent compte de questions relatives aux droits fonciers et aux intérêts des peuples autochtones. Cela pourrait fort bien conduire tout droit à une impasse avec des gouvernements peu désireux d'arbitrer entre les deux parties.

Dans d'autres situations, les enjeux pourraient être plus simples et représentés par deux scénarios : dans l'un, il s'agit de trouver les moyens de mettre en échec la connivence entre acteurs de l'Etat et exploitants forestiers malhonnêtes ; dans l'autre, la technicité des conditions pourtant tout à fait valables exigées au titre de l'assurance de la légalité des activités en rend l'application difficile dans la pratique et compromet les efforts des opérateurs, voire des plus légitimes d'entre eux, qui cherchent à " travailler en toute légalité ». 


\subsection{Convergence : les avantages et les inconvénients}

La convergence existant entre l'assurance de la légalité et la certification est une nouvelle étape vers la légalisation des opérations et pourrait avoir des répercussions aussi bien pour les organismes que pour les agents de certification. D'un point de vue fonctionnel, elle présente de nombreux avantages. Toutefois, la certification et la vérification constituent deux instruments très contrastés et leurs visées sont notablement différentes (voir Tableau 22.2). ${ }^{1}$ Dans cette section, nous examinerons tout d'abord les arguments avancés en faveur d'une convergence de ces deux instruments, puis les arguments contraires.

\section{Arguments en faveur de la convergence}

Tout d'abord, le fait que les principaux projets de certification exigent tous l'adhésion aux lois nationales et locales comme condition préalable de la certification suppose qu'il y a fondamentalement un empiètement entre la certification et la vérification du caractère légal des opérations forestières. Si une superficie forestière est certifiée, il en découle que la production de bois de cette forêt doit être en conformité avec les lois et règlements applicables existants. Il est donc raisonnable d'un point de vue administratif et financier, aussi bien pour les producteurs que pour les gouvernements, de traiter la certification comme un instrument de remplacement de la vérification du caractère légal de la production pour éviter toute répétition inutile en matière de formalités et des coûts y afférents.

Deuxièmement, comme la certification dépend en dernière analyse de l'évaluation indépendante d'adhésion à la légalité effectuée par un agent de certification accrédité (habituellement un cabinet commercial), une assurance de légalité délivrée sous certification permet de ne pas mettre en cause la souveraineté nationale au cas où un débat opposerait des non nationaux (donateurs, ONG et autres) aux gouvernements producteurs sur une question d'interprétation des lois nationales.

\section{Tableau 22.2 Quelques différences essentielles entre certification et vérification dans le secteur forestier}

\begin{tabular}{ll}
\hline Certification & Vérification \\
\hline Initiative volontaire pilotée par le secteur privé & $\begin{array}{l}\text { Initiative statutaire pilotée par le } \\
\text { gouvernement }\end{array}$ \\
\hline $\begin{array}{l}\text { Portée globale appliquée globalement dans } \\
\text { la pratique, mais plus en avance dans les pays } \\
\text { du Nord }\end{array}$ & $\begin{array}{l}\text { Portée globale mais pas d'application globale } \\
\text { dans la pratique ; cible les pays à 'hauts risques' }\end{array}$ \\
\hline $\begin{array}{l}\text { Vise le concept élargi de gestion durable des } \\
\text { forêts }\end{array}$ & $\begin{array}{l}\text { Vise le concept plus restreint de conformité } \\
\text { légale }\end{array}$ \\
\hline $\begin{array}{l}\text { Implique les normes relatives aux processus et } \\
\text { à la performance }\end{array}$ & $\begin{array}{l}\text { Question non encore résolue, même si la } \\
\text { position de l'UE sur les APV est de privilégier } \\
\text { les normes de performance }\end{array}$ \\
\hline
\end{tabular}

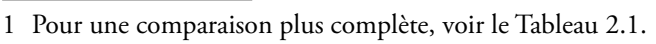


Troisièmement, les nouveaux projets existants du côté de la demande, comme les divers systèmes de marchés publics européens qui ont récemment été mis en place, sont considérés par leurs partisans comme fonctionnant bien et pourraient constituer un modèle à la fois pour les Etats producteurs et les Etats consommateurs. Des systèmes comme le CPET (Central Point of Expertise on Timber) du Royaume-Uni visent à fournir des renseignements et des conseils détaillés sur la façon dont les acheteurs du secteur public et leurs fournisseurs peuvent satisfaire à la condition exigée par le gouvernement du RU en matière de bois d'œuvre, à savoir que seul le bois en provenance de sources durables et légalement vérifiées sera utilisé par les entreprises avec lesquelles il a passé des contrats. Ces systèmes semblent répondre à un réel besoin de la part des acheteurs et détaillants de bois d'œuvre qui souhaitent travailler sur une base légale mais ont eu jusqu'ici des difficultés à évaluer leurs fournisseurs.

Le CPET identifie quatre dimensions clé de la légalité : droits d'usage, conformité avec la législation applicable, paiement des taxes gouvernementales et conformité avec les conditions exigées au titre de la Convention sur le commerce international des espèces de faune et de flore sauvages menacées d'extinction (CITES). Les droits d'usage sont ceux qui suscitent habituellement les plus grandes difficultés. Par exemple, le Conseil malaisien de certification du bois [Malaysian Timber Certification Council] (MTCC) a vu son action contestée par des ONG de défense des droits au motif que les droits coutumiers des populations autochtones pourraient être érodés en cas de formalisation des régimes fonciers des forêts (voir Chapitre 16 et Nussbaum et Simula, 2005).

Les capacités requises pour évaluer les fournisseurs individuels sont bien supérieures à celles dont disposent les systèmes d'approvisionnement/des marchés publics qui, dans la pratique, dépendent en fait des normes de certification pour faire des évaluations approximatives. Les gouvernements producteurs semblent faire appel aux systèmes de certification essentiellement pour les mêmes raisons. Ceux qui sont en faveur de la mise en place de systèmes d'approvisionnement pourraient soutenir que même s'ils ne sont pas parfaits pour garantir la légalité, ils sont suffisamment performants pour bien remplir la tâche qui leur est assignée et résoudre un réel dilemme pour le secteur forestier.

L'expérience glanée en dehors du secteur forestier montre aussi le bien fondé de cette évaluation positive. Par exemple, les autorités chargées d'élaborer les normes en matière de viande en Europe reconnaissent la valeur des systèmes de certification du secteur privé, et récompensent les entreprises qui se se donnent du mal pour se conformer aux normes en abaissant la barre des conditions réglementaires requises dans leur cas (voir Chapitre 3). Cela semble être une pratique de gestion éminemment frappée au coin du bon sens.

\section{Arguments critiquant la convergence}

Tous les commentateurs ne sont pas aussi favorablement disposés vis-à-vis de la convergence. Les arguments opposés à l'utilisation de la certification en tant que remplaçant de la procédure visant à garantir la légalité ont trait à des questions de principe et aussi à des aspects pratiques. 


\section{Questions de principe}

Pour les sceptiques, la question fondamentale est celle qui consiste à déterminer s'il est possible, en toute légitimité, de substituer des systèmes volontaires à la gouvernance nationale. Ils avancent que la certification est un outil du secteur commercial qui n'a été conçu ni pour servir l'intérêt public ni pour faire appliquer les dispositions réglementaires édictées par les Etats-nations. Les systèmes volontaires peuvent être une solution crédible basée sur l'économie de marché pour les exploitants forestiers qui souhaitent faire valoir la qualité de leur gestion auprès des acheteurs de produits ligneux. Toutefois les critiques relèveront que la certification ne doit pas se substituer au gouvernement en assumant un rôle qui revient légitimement à ce dernier, à savoir la réglementation de l'activité économique. Les organismes d'accréditation internationaux comme le FSC ou le PEFC sont responsables devant leurs électorats comme le sont les gouvernements démocratiques nationaux. Si ces organismes sont effectivement responsables, ils tendront à l'être à un échelon supra-national plutôt que national. On pourrait même ajouter que les systèmes de certification ont le potentiel de saper le développement des Etats en assumant en parallèle certaines responsabilités étatiques qui doivent impérativement relever des institutions nationales conformément aux dispositions de la Constitution. Cette éventualité semblerait plus particulièrement applicable aux Etats fragiles dans lesquels des efforts sont faits pour réhabiliter les institutions de l'Etat. De ce point de vue, la conformité avec la loi n'est pas un domaine dans lequel le secteur privé est investi de la légitimité nécessaire pour mener seul les procédures y afférentes.

Une deuxième critique concerne le fait que les systèmes de certification n'ont pas été conçus pour assurer la conformité avec la loi mais plutôt que la certification forestière a été établie afin d'aider les utilisateurs légitimes des forêts à faire la preuve de leur capacité à gérer les forêts de façon responsable (Colchester, 2006). Les systèmes de certification ont donc été conçus dans un but très général - celui de la gestion durable des forêts plutôt que dans le but plus limité de la conformité avec la loi. En outre, les systèmes de certification prévoient un groupe-cible d'utilisateurs spécifiques, composé de ceux qui souhaitent montrer de hauts niveaux de supervision forestière. La vérification de la légalité doit s'appliquer à tous les opérateurs du secteur forestier. Pour avoir un impact, la vérification doit permettre d'identifier ceux qui exercent leurs activités en dehors de la loi et être dotée des moyens suffisants pour mettre un terme à ces activités.

Comme le montrent les Figures 1.1 et 1.2 du Chapitre 1, la certification est peu avancée dans les pays où l'exploitation illégale du bois est monnaie courante. Elle atteint son plus haut degré de développement dans les pays où la conformité avec la loi n’est pas considérée comme un problème majeur pour l'élaboration des stratégies et reste largement confinée aux pays développés de l'hémisphère nord (même s'il existe une certification pour les forêts plantées dans le sud). Les raisons pour lesquelles la certification n'a pas progressé plus rapidement dans les forêts tropicales naturelles tiennent aux difficultés inhérentes à ce type d'environnement sur le plan des objectifs économiques, écologiques et sociaux de la gestion forestière. Comme nous l'a montré l'expérience acquise tout au long de la dernière décennie, aucune solution n'est facilement ni rapidement applicable. On peut également assumer dans ce genre de situation que le manque de certification constaté 
dans ces environnements forestiers ne résulte ni d'un défaut d'engagement de la part des organismes de certification ni de l'insuffisance de leurs efforts. Les sceptiques pourraient ajouter un corollaire, à savoir que tout rapide accroissement du nombre de forêts certifiées dans des régions non-certifiables auparavant ne pourrait résulter que d'un compromis sur les normes, plutôt que d'un changement soudain des niveaux de conformité à ces normes.

\section{Questions pratiques}

Les ONG de défense des droits environnementaux ont allégué qu'il y a des cas où les normes de certification ont été acceptées comme preuves de la légalité des activités dans des situations où il y a des signes manifestes de production illégale. Même dans les cas où de telles allégations sont justifiées, elles ne sapent pas complètement les fondements de l'entreprise ; il est inévitable que des erreurs soient commises. Elles pourraient toutefois renforcer les soupçons portant à croire que partout ou le processus est piloté par des intérêts commerciaux, ce sont les normes de performance qui en pâtissent.

\section{Autres domaines de préoccupation}

Il y a deux autres domaines principaux de préoccupation :

- Les conflits d'intérêts entre prestataires de services; et

- Les partis pris contre les petits producteurs et les pauvres.

Conflits d'intérêts. Une question qui mérite de retenir l'attention est celle du pouvoir détenu par les organismes d'accréditation pour prévenir les conflits d'intérêts survenant avec des agents de certification individuels. L'introduction de la vérification du caractère légal des opérations en tant que nouvelle initiative politique internationale de grande envergure a entraîné une réponse de l'industrie de certification désireuse de conquérir de nouveaux marchés. Le résultat en est que les agents de certification peuvent à la fois vérifier la conformité avec la loi au titre de systèmes d'assurance de la légalité du bois relevant du secteur public et certifier la conformité avec la loi au titre de systèmes de certification relevant du secteur privé. Cette situation porte en elle les germes d'un conflit d'intérêt et pourrait favoriser des allégations selon lesquelles les intérêts commerciaux influenceraient les décisions en matière de conformité. Elle rejoint les préoccupations exprimées à propos du personnel de certification qui pourrait parfois se retrouver de mèche avec les exploitants dont il certifie les activités. Malgré la réglementation mise en place par les organismes d'accréditation pour limiter ce type d'abus, force est de constater la permanence d'une zone grise en la matière et l'accentuation du problème à travers la pratique de certains organismes de certification qui engagent sous contrat - pour des missions de certification - des consultants freelance qui pourraient se retrouver à " travailler pour les deux côtés ".

Parti pris contre les petits producteurs et les pauvres. Un thème récurrent dans ce livre est celui des relations entre la politique et l'équité. De nombreux opérateurs qui se conforment à la législation (et, en particulier, les petits producteurs) n'ont reçu aucune incitation financière 
pour investir dans des systèmes de certification volontaire. Ces opérateurs pourraient être désavantagés au cas où la certification serait utilisée comme première approximation de la conformité avec la législation. La certification ayant déjà été adoptée, à travers le monde, par les exploitants forestiers caractérisés par une plus forte intensité de capital, il en résulte une menace pour les petits producteurs qui pourraient être écrasés par ces entreprises beaucoup plus importantes. Les exploitants certifiés existants qui captureront les marchés avant tous leurs concurrents seront ceux qui prendront l'avantage en sachant démontrer la légalité de leurs activités de façon crédible, en particulier dans le contexte de la commercialisation de produits à valeur d'exportation plus élevée. Cela pourrait accentuer la consolidation d'une industrie naissante (voir le Chapitre 26 pour un examen plus approfondi de la question).

\subsection{Perspectives d'avenir - la certification en tant que complément de la vérification?}

Les controverses entourant la question de la certification des forêts sont bien connues et font désormais partie du territoire de tous les principaux organismes de certification. Ce qui peut toutefois présenter un caractère de nouveauté est l'effet sur la certification de l'intérêt accru porté à la question de la légalité, suite à l'importance stratégique donnée à l'application des législations forestières, de la gouvernance et du commerce (FLEGT) et, en particulier à la mise en œuvre du Plan d'action de l'UE. De plus en plus pris en compte dans les politiques forestières, le thème de la légalité pourrait avoir une grande influence sur l'intérêt du public pour les questions liées à la légalité ainsi que les perceptions qu'il en a. Il pourrait y avoir des répercussions sur les marchés de consommation - répercussions qui seraient le plus souvent évidemment négatives et toucheraient l'extrémité à plus haut risque du spectre. Les organismes d'accréditation et de certification devront prendre acte de cette évolution et veiller encore plus à l'application des normes élaborées. Dans les pays dans lesquels des APV (accords de partenariat volontaires) avec l'UE sont en voie de préparation, les définitions concernant la légalité feront vraisemblablement l'objet d'un très vif intérêt de la part du public. Dans un contexte de certification, il pourrait être nécessaire de revoir les critères à l'aune desquels est évaluée - dans de telles situations - la conformité avec les normes nationales en s'efforçant à une plus grande rigueur et à une transparence accrue.

Une question quelque peu différente concerne la façon dont la convergence entre la certification et la vérification devrait être analysée du point de vue de la gouvernance et pas seulement par rapport à la conformité juridique. Il y a certainement de bonnes raisons a priori de se féliciter de toute mesure visant à promouvoir l'instauration possible d'une gestion durable et des mécanismes de consultation publique nécessaires pour atteindre cet objectif. De même, il semble raisonnable d'accorder un traitement préférentiel aux exploitants forestiers qui essaient déjà d'agir en toute légalité et de les exonérer de tous frais supplémentaires inutiles. Des esprits critiques pourraient cependant soutenir qu'utiliser la certification comme substitution de légalisation est un peu comme de " mettre la charrue avant les bœufs ". L'autorité de l'Etat est indispensable à l'efficacité du processus de vérification de la légalité dont les responsabilités devront aussi être clairement définies 
et ne pas être liées à des intérêts financiers. Si cette autorité était déléguée trop en aval et laissée aux forces du marché, la légalité dans l'intérêt du commerce risquerait d'être battue en brèche avant même que le programme FLEGT ait eu le temps de prendre son envol.

Il existe donc de solides arguments pour maintenir une séparation non seulement conceptuelle mais aussi institutionnelle entre la certification et l'assurance de la légalité. Cette séparation donnerait la priorité aux définitions de l'Etat en matière de normes de légalité et en encouragerait l'évaluation rigoureuse et sans ambiguïté.

Si cette interprétation est acceptée, deux séries de conditions pourraient alors être imposées aux agents chargés de la certification :

- Les agents de certification pourraient avoir besoin de se montrer bien plus rigoureux et d'appliquer des méthodes plus transparentes en rendant leurs jugements de conformité avec la législation s'ils veulent avoir quelque chance que leurs interprétations soient acceptées par les ONG et autres groupes de militants - puis en dernière analyse par le grand public - sans que des questions soient posées.

- Les organismes d'accréditation devront prendre des mesures pour veiller à que leurs objectifs ne soient pas compromis par des intérêts commerciaux liés :

- Aux évaluations de conformité avec les normes compte tenu, en particulier, du passage du temps ; il faudra suivre la situation de très près pour s'assurer qu'il n'y aura pas de tendance à la baisse du niveau de conformité.

- Aux individus et organisations auxquels ils (les organismes d'accréditation) font appel pour l'évaluation du degré de conformité ; il faudra veiller à éviter les conflits d'intérêts.

Il pourrait être nécessaire de disposer de codes de conduite clairs pour régir les deux courants d'opérations et maintenir leur séparation. Il pourrait aussi être utile d'envisager l'établissement d'un système autorisant les agents qui vérifient le caractère légal des opérations du secteur forestier à exercer leurs fonctions.

\subsection{Conclusion}

Des ambiguïtés demeurent dans le fonctionnement actuel des principaux systèmes de certification quant aux preuves de conformité avec la législation, et il est probable qu'elles seront mises à profit par les tenants de la nouvelle démarche - favorable à la vérification de la légalité - qui n'hésiteront pas à en grossir les inconséquences. En tout état de cause, il importe que les deux courants de réglementation, volontaire et statutaire, se développent simultanément. Il ne faut toutefois pas oublier leur raison d'être - la nécessaire amélioration d'une gouvernance généralement médiocre du secteur forestier. L'Etat a un rôle critique bien défini à jouer puisqu'il doit rendre des comptes aux citoyens (même de manière imparfaite) suivant des procédures qui ne sont absolument pas applicables aux institutions du secteur privé. 
Cette évaluation ne vaut pas seulement pour la certification. Le contexte dans lequel se déroule le processus de vérification de la légalité semble aussi se transformer et les mêmes effets pourraient donc également s'y faire sentir. En particulier dans le domaine des institutions, les autorités forestières pourraient tirer un grand parti des enseignements de ces dix dernières années en matière de certification et tenter d'en utiliser les points forts lorsqu'elles doivent vérifier le caractère légal des activités du secteur. Au nombre de ces points forts figurent tous ceux qui se rattachent à la gouvernance des systèmes et à l'accréditation des prestataires de services - deux domaines qui ne font jusqu'ici pas la part assez belle aux systèmes de vérification et d'assurance de la légalité et qui sont pourtant essentiels à la crédibilité du processus.

Si la convergence entre la certification et l'assurance de la légalité peut présenter un défi particulier pour le processus le mieux établi (celui de la certification), la certification pourrait cependant se révéler fort utile pour la nouvelle initiative. 



\section{Chapitre 23}

\section{Nouvelles technologies pour appuyer une gouvernance forestière améliorée}

Note : Neil Bird et Hans Thiel sont les auteurs de ce chapitre basé sur leur article : Bird, N. et Thiel, H. 2007 New technologies in support of improved forest governance: factors influencing success. VERIFOR, Briefing Paper No. 7. ODI, Londres.

\subsection{Introduction}

L'application de technologies novatrices ouvre de nouvelles perspectives en matière de contrôle réglementaire dans le secteur forestier. De nouvelles technologies sont introduites progressivement à mesure que les pays en reconnaissent les bénéfices potentiels et mettent en place les moyens nécessaires. Dans ce chapitre, nous examinons ces nouvelles technologies sous l'angle de la gouvernance forestière et formulons des suggestions sur la façon dont elles pourraient être appuyées par des arrangements institutionnels qui renforceraient la responsabilité et la transparence du secteur public et favoriserait l'indépendance des actions entreprises. Nous commençons par donner un aperçu général de ces technologies dans le contexte du secteur forestier avant d'examiner les faits en nous référant au cas du Brésil en particulier. Dans la dernière partie, nous regroupons toutes nos observations et identifions un certain nombre de questions clé susceptibles de favoriser la réussite de l'adoption de ces technologies dans les systèmes de gouvernance forestière.

\subsection{Quelles sont les technologies en cause?}

L'introduction au cours des quelques dernières années de données enregistrées à distance au moyen de satellites et d'avions volant à très haute altitude a constitué un progrès majeur dans la production d'images détaillées de paysages forestiers sur de vastes surfaces. Des images de forêts entières peuvent maintenant être facilement acquises et utilisées par les autorités forestières pour identifier toute une série de phénomènes allant de feux et d'incendies de forêts à des défrichages non autorisés en plein cœur des forêts.

Etant donné que ces informations se présentent sous forme numérique, elles peuvent être transformées et incorporées dans des systèmes d'information géographique (SIG) et combinées avec d'autres types de sources d'information géographique (par ex. des cartes topographiques) pour procéder à une analyse complète du couvert boisé et, sur une période donnée, à celle du changement d'affectation des terres (dont le déboisement qui présente évidemment un intérêt tout particulier). La superposition des cartes topographiques (établissant les limites officielles des forêts) et les images satellitaires récentes permet de mettre facilement en évidence les différences (ou les écarts) éventuels entre les limites enregistrées et le couvert boisé actuel. 
D'autres informations peuvent être ajoutées au SIG à travers les systèmes manuels de géolocalisation par satellite qui peuvent relever et enregistrer la position (exacte) des points géodésiques. Les données applicables au secteur forestier vont de la localisation des installations de transformation des bois à celle des aires d'empilement et de ramassage des grumes, voire à l'individualisation d'arbres de la forêt.

Les technologies basées sur internet (y compris les bases de données intégrées facilement accessibles en ligne) offrent de nouvelles opportunités de communiquer des informations qui avaient auparavant le papier pour seul support. Les formulaires administratifs et permis qui constituent l'essentiel des contrôles réglementaires appliqués pour les forêts au niveau national peuvent désormais être transmis par voie électronique entre l'administration forestière et les utilisateurs, permettant un gain de temps et une économie d'efforts considérables. La toile (le web) à l'échelle mondiale est un outil exceptionnel qui permet une diffusion beaucoup plus étendue et transparente des informations émanant des administrations forestières.

\subsection{Qu'attendre de ces nouvelles technologies en matière de gouvernance forestière?}

Les nouvelles technologies devraient pouvoir aider à combler un certain nombre de lacunes constatées dans le secteur forestier en matière de gouvernance. L'insuffisance de la coordination et des voies de transmission entre les services d'information gouvernementaux et les systèmes d'administration entraîne une très grande inefficacité. Il arrive souvent que les registres du cadastre, les procédures administratives relatives à l'environnement, le suivi des changements en matière d'affectation des terres et les données sur le produit des impôts et des recettes fiscales fassent défaut, soient incomplets ou totalement obsolètes. Dans les cas où les bureaux de l'administration sont installés dans des centres régionaux éloignés des zones forestières, les opérateurs commerciaux dans ces zones rurales ont souvent du mal à observer toutes les prescriptions administratives requises au titre de la conformité avec la loi.

La difficulté la plus communément rencontrée est liée au manque d'information, laquelle entraîne une médiocre performance de l'autorité forestière qui ne rend pas suffisamment compte aux contribuables. Le droit de regard du public sur les données concernant les permis d'exploitation forestière et les recettes fiscales n'est le plus souvent pas respecté ce qui rend le système vulnérable et sans défense par rapport à un usage abusif.

Il convient toutefois de faire apprécier par les différents groupes d'utilisateurs possibles le parti qui peut être tiré des nouvelles technologies pour en faciliter l'adoption à grande échelle dans le secteur forestier. S'agissant de l'Etat, trois grands bénéfices potentiels peuvent être relevés :

- Une plus grande transparence et une diminution de la probabilité de corruption les procédures de saisie automatisées de formulaires et permis normalisés rendent la falsification plus difficile que dans le cas du support papier; 
- De meilleures chances de publier en temps opportun les informations statistiques voulues (données géographiques, processus de délivrance de permis, sanctions encourues et résultats obtenus) - ce qui augmentera la transparence du système de réglementation dans son ensemble et incitera le public à faire davantage confiance à l'autorité chargée de l'application des règlements ;

- Éventuellement, une meilleure gestion du secteur public à travers les actions suivantes, par exemple :

- La simplification des tâches et formalités administratives ;

- L'amélioration de la perception des recettes fiscales ;

- La redistribution des maigres ressources existantes pour faire appliquer la loi afin de les optimiser en les réorientant de façon stratégique ;

- L'identification - en la rendant plus facile - des goulets d'étranglement du système administratif éventuellement responsables de retards d'acheminement et de traitement de dossiers et susceptibles d'être plus exposés à la corruption ; et

- L'amélioration de la communication entre les différents services de l'administration gouvernementale - aboutissant à une diffusion de l'information en temps opportun pour la prise de décisions politiques spécifiques.

Les opérateurs forestiers peuvent aussi s'attendre à retirer un bénéfice direct d'un climat d'investissements plus propice dans le secteur privé suite à l'introduction de procédures d'attribution de marchés et de ventes (de bois) plus concurrentielles et transparentes et à l'amélioration des outils électroniques existants sur le marché. Ce dernier aspect s'applique cependant dans une moindre mesure au cas de nombreux pays producteurs de bois tropicaux qui disposent de maigres ressources dans ce domaine. Il n'en demeure pas moins que l'amélioration de l'outil informatique permet une rapidité et une efficacité d'exécution que n'avaient pas les méthodes traditionnelles de commercialisation. Les nouvelles technologies devraient aussi faciliter la mise à disposition de services plus pratiques et plus abordables dans le secteur forestier. Destinés à toutes les parties prenantes, ces services seraient rendus à travers un système de prestations rationalisé en permettant aux utilisateurs privés, en particulier, même à ceux qui résident dans des zones éloignées, d'avoir accès aux procédures administratives par voie électronique.

\subsection{Quels sont les facteurs qui empêchent ces technologies de contribuer à l'amélioration de la gouvernance forestière?}

L'introduction de nouvelles technologies ne doit pas être considérée sous l'angle purement technique même si cet aspect a une grande importance. D'autres facteurs doivent être pris en compte dès lors qu'est évalué le rôle potentiel de la technologie dans un système réglementaire. Comme le montre l'étude de cas pour l'Equateur, les facteurs politiques peuvent constituer une contrainte importante, en particulier si une faible priorité est accordée au secteur forestier à cet égard. Les groupes d'intérêts bénéficiant du statu quo pourraient fort bien s'opposer à des améliorations du système réglementaire s'ils 
estiment qu'ils y perdront. Il se pourrait donc que l'administration publique, l'industrie ou la société civile (ou les trois ensemble, à des degrés divers) doivent intervenir avec détermination pour exercer une pression sur les milieux politiques afin que ceux-ci se prononcent en faveur du changement. L'introduction de nouvelles technologies pourrait aussi être compromise par une absence de politiques et de stratégies en recommandant l'adoption et en facilitant la gestion. Il s'agit souvent d'un problème de politique générale dont la solution ne relève pas de la compétence du secteur forestier.

En outre, l'inertie du public peut aussi constituer une contrainte. Quand leur participation au processus décisionnel est limitée, les citoyens sont moins enclins à prendre fait et cause pour les avancées technologiques susceptibles d'améliorer la qualité de ce processus. Ces attitudes peuvent trouver leur origine dans un sentiment de méfiance généralisée vis-à vis de la capacité des institutions publiques à mener à bien leurs fonctions statutaires. D'autres facteurs doivent aussi être pris en compte comme le cadre juridique dans lequel se déroulent des transactions électroniques qui ne seront pas reconnues parce que le système administratif ne reconnaît pas les identités qui sont déclinées par voie électronique ou que les données enregistrées à distance devront être révisées avant l'introduction de nouveaux contrôles réglementaires. Cela pourrait ralentir l'introduction de la technologie, en particulier dans le cas du suivi des obligations légales.

Les facteurs institutionnels (par ex., l'insuffisance de l'infrastructure administrative et du budget consacrés à l'administration des forêts et le manque de personnel qualifié en résultant pour faire face à l'évolution technologique) peuvent aussi faire obstacle à l'adoption des nouvelles technologies. La technologie reste onéreuse et inabordable au vu des maigres ressources de trésorerie d'un grand nombre de services publics. La concentration de toutes les activités dans une seule institution qui assume toutes les responsabilités - souvent le cas de l'administration nationale chargée des forêts - pourrait être un déni de l’opportunité de bénéficier de techniques plus spécialisées. La constitution d'un grand service regroupant les techniques de l'information n'est tout simplement pas possible pour de petites institutions (dont les ressources se limitent souvent à un effectif constitué d'un ou deux techniciens spécialisés).

L'efficacité de la mise en œuvre dépendra donc vraisemblablement d'une collaboration entre divers départements. Tout manque de coordination entre institutions quant à l'introduction et au développement de ces technologies et tout chevauchement et/ou conflit de fonctions et de compétences entre différents niveaux de gouvernement risquent toutefois de susciter des difficultés dont il faudra sans doute tenir compte.

Enfin, il faudra aussi surmonter des obstacles d'ordre technologique. L'accès à Internet reste peu fiable, voire inexistant, dans de nombreuses zones rurales. En outre, bien que le matériel informatique soit d'une qualité bien meilleure qu'auparavant, il risque d'être beaucoup moins fiable dans un environnement très poussiéreux ou un climat très humide. Le manque de logiciels spécialisés et de données satellites (télédétection) continue à gêner les efforts visant à développer l'utilisation de cette technologie dans le secteur forestier. Par exemple, la technique de l'imagerie visible par structures en bande n'est utilisable 
dans les régions tropicales humides que lorsque se dissipe la couverture nuageuse qui limite considérablement le nombre de jours dans l'année où ces données peuvent être collectées. Même dans les cas où des jeux de données assez complets existent, il peut être difficile de comparer ces images aux observations sur le terrain si la résolution n'est pas assez élevée - ce qui en limiterait l'usage au suivi de l'application des législations.

\subsection{Comment surmonter les contraintes existantes?}

Ces contraintes commencent à être prises en compte dans un certain nombre de pays. Ainsi en est-il du cas particulièrement instructif du Brésil qui a investi des montants substantiels dans ces nouvelles technologies pour répondre aux inquiétudes s'exprimant à la fois aux niveaux international et national sur la question du déboisement de l'Amazonie.

Trois types de réformes ont permis d'accélérer le rythme d'adoption de ces technologies dans le secteur forestier.

Tout d'abord, les rôles et les compétences des différents niveaux de gouvernement ont été précisés. C'est un problème habituel dans les pays qui ont connu une progression de la décentralisation ces dernières années sans que les mandats réglementaires aient été clairement définis et élucidés.

En deuxième lieu, le renforcement des compétences professionnelles dans l'administration des forêts a fait l'objet d'une attention spécifique. Ayant toujours traditionnellement privilégié le travail sur le terrain, les départements des forêts dans de nombreux pays ont une perspective limitée des avantages qu'ils pourraient retirer de l'adoption de systèmes technologiques modernes. La situation évolue lentement à mesure qu'une génération de jeunes techniciens, plus sensibilisés à l'importance de l'informatique, rejoint les rangs des services administratifs du gouvernement.

Troisièmement, de nouveaux systèmes ont vu le jour, financés par les investissements publics colossaux nécessaires pour les mettre en service. Il n’est possible de procéder à ce type de réforme qu'avec la volonté institutionnelle et politique suffisante pour la mener à bien et la mise à disposition, par le gouvernement, des ressources financières nécessaires qui doivent être renouvelées tous les ans à travers le processus d'affectation budgétaire annuel.

Ces trois types de réforme sont analysés ci-après.

\section{Clarification des fonctions et des compétences aux différents niveaux de gouvernement}

Comme noté au Chapitre 9, le Conseil national pour l'environnement du Brésil (CONAMA) a procédé à une réforme politique de grande ampleur fin 2006, et accordé un délai d'un an aux Etats pour assumer leurs compétences dans le cadre du Système 
national pour la gestion de l'environnement (SISNAMA). Tous les Etats étaient sommés de mettre en place, dans les délais ainsi impartis (une année), leurs propres systèmes d'administration et de contrôle forestiers, rattachés à la fois au Système national d'information pour les forêts (SISCOM) et au Système national d'information pour l'environnement (SISNIMA).

Au moment de la rédaction de ce chapitre, un protocole établissant un partage des rôles et des responsabilités dans un nouveau régime forestier décentralisé (MMA 2006c) est en cours de discussion au sein de la Commission inter-institutionnelle pour coordonner le Programme national des forêts (CONAFLOR). L'étape ainsi franchie est importante. Avant l'adoption de ces résolutions, l'Agence fédérale pour l'environnement (IBAMA) était dotée de compétences qui empiétaient sur celles des Etats provinciaux - ce qui entraînait inefficacité et double emploi et donnait même parfois lieu à des conflits importants. Une division des rôles et des compétences plutôt floue entre les différents niveaux de gouvernement empêchait totalement la mise en place d'un système cohérent d'administration des forêts et de délivrance de permis foncier, et limitait sérieusement les gains pouvant être retirés de l'adoption de la nouvelle technologie.

Bien que les Etats soient libres de mettre en œuvre leurs propres systèmes de contrôle, la coopération de l'agence fédérale leur était acquise à travers la conclusion d'un accord pour fournir ce type d'expertise (mise en place de systèmes électroniques) et la création d'une commission composée de multiples parties prenantes pour assurer le suivi de la mise en service. Les institutions opérant au niveau fédéral, IBAMA et le ministère de l'Environnement (MMA), ont mis gratuitement à la disposition des Etats un nouveau système de contrôle forestier électronique et assurent aussi la formation nécessaire à son utilisation (voir section 23.6 ci-après).

Le suivi des activités forestières illégales au niveau national continue à être assuré par les agences fédérales, la responsabilité de la coordination étant assumée par le Groupe de travail interministériel permanent pour réduire la déforestation dans l'Amazonie brésilienne (GTPDA). Le décret présidentiel ayant porté création du GTPDA a prévu la participation de dix ministères à ce groupe de façon à assurer une réponse collective au sein de la plus large représentation possible. Les institutions nationales à vocation technique, comme l'Institut national pour la recherche spatiale (INPE), sont également membres de ce groupe. La fourniture des jeux de données techniques (par ex. télédétection) a été essentielle au début pour avoir un aperçu général de la situation afin d'orienter les actions réglementaires. A présent, le système de suivi par satellite DETER, capable de détecter des activités illégales de déboisement en Amazonie quasiment " en temps réel ", est le système privilégié pour les observations.

\section{Renforcement de la compétence des administrations forestières étatiques}

Depuis 2003, l'Agence fédérale pour l'environnement (IBAMA) a recruté de nouveaux employés, ajoutant plus de 25 pour cent à ses effectifs précédents. En parallèle, l'Agence 
a pris une série de sanctions disciplinaires et licencié pour corruption des douzaines de membres de son personnel. Le résultat en est une équipe de jeunes professionnels dynamiques qui connaissent et utilisent les nouvelles technologies avec brio. Un processus similaire se déroule actuellement au niveau fédéral, étant donné que la décentralisation en cours (dévolution des compétences forestières aux Etats) n’inclut pas le transfert des ressources de l'Agence.

\section{Création statutaire de nouveaux systèmes d'information électronique}

La Résolution 379 du Conseil national pour l'environnement (CONAMA) porte création d'un système au titre duquel toutes les agences environnementales étatiques doivent mettre en place des systèmes d'information forestiers. Les normes fondamentales et critères minimaux que ces systèmes doivent adopter sont définis au niveau fédéral. L'accès aux systèmes mis en place au niveau des différents Etats doit être libre pour permettre leur transfert dans le système national d'information pour les forêts (SISCOM).

L'Instruction normative No. 5 (MMA IN No. 5/11.XII.2006) stipule que tous les plans de gestion forestière qui ont été approuvés doivent être enregistrés au cadastre national des plans pour une gestion durable des forêts (CNPM) auquel le public pourra accéder librement par internet. Des mises à jour des informations seront saisies au moins deux fois par an dans une base de données centralisée et un rapport annuel devra être transmis par chaque niveau de gouvernement au service forestier fédéral.

Il n'aurait tout simplement pas été possible d'atteindre un tel degré de sophistication en matière de gestion de l'information avant l'introduction des bases de données électroniques. Certains des tout premiers systèmes d'information mis en place l'ont été au niveau des Etats, en particulier dans l'Etat du Mato Grosso (voir Encadré 23.1).

\subsection{Remplacement d'un système de contrôle discrédité sur support papier par des systèmes électroniques}

L'Agence fédérale de l'environnement (IBAMA) du Brésil met en place progressivement depuis plusieurs années un nouveau système de contrôle et d'administration électronique des forêts du pays. Il s'agit du système DOF (Documento de Origen Florestal) dont l'introduction au niveau national remonte au mois de septembre 2006. Pour satisfaire aux dispositions du nouveau système, les exploitants forestiers sont tenus de s'inscrire auprès d'une base de données administrative, le CTF (Cadastro Técnico Federal). Le CTF est en service depuis 2001 et accessible en ligne. Contrairement à la procédure retenue pour la base de données CC-SEMA du Mato Grosso (Encadré 23.1), les utilisateurs peuvent s'inscrire eux-mêmes en ligne. Cependant, si l'authenticité des informations portées par les utilisateurs est mise en doute, l'administration forestière peut demander à l'utilisateur de soumettre une documentation à l'appui sous forme de documents imprimés (Chaves, J.H. 2006 et al. septembre). 


\section{Encadré 23.1 Programmes d'appui technologique à la réforme du secteur forestier dans le Mato Grosso, Brésil}

En 1999, la Fondation pour l'environnement du Mato Grosso [Fundación Estadual de Meio Ambiente] (FEMA), avec le concours du Programme-pilote (financé par des fonds internationaux) pour la conservation des forêts tropicales au Brésil (PPG-7), a mis en place le système de planification pour le zonage des terres [Sistema de Licenciamiento Ambiental em Propriedade Rural] (SLAPR)). Mis en service en 2000, le SLAPR dont l'objectif principal est la réduction de la déforestation illégale, est un système d'information pionnier pour le Brésil. Il s'agit d'une base de données centralisée qui combine les informations sur la délivrance de permis d'environnement aux propriétés rurales avec celles qui concernent le suivi et le contrôle des changements d'affectation des terres. Le système est applicable aux forêts privées et contient les informations suivantes : les limites (basées sur leurs coordonnées géographiques) de chaque propriété dûment autorisée ; les limites de la zone « réservée » (conformément aux dispositions de la loi) qui doit rester boisée à l'intérieur même de la propriété et les limites de toutes les «zones protégées », y compris les pentes raides et les zones tampon en bordure des cours d'eau. Dès qu'un permis d'environnement est émis pour une propriété donnée, toutes ces données sont saisies dans le système de façon à pouvoir surveiller les changements du couvert boisé à partir d'images satellite et, le cas échéant, à faire appliquer les mesures prévues par la législation.

Au début de sa mise en service, le système s'est révélé trop lent pour et n'a permis de détecter aucun impact notable résultant de l'application des législations en vigueur dans le secteur forestier. Les données recueillies en 2004 montrent que la déforestation illégale dans les zones agréées (au bénéfice d'un permis) n'était pas notablement de moindre ampleur que celle des zones non agréées - ce qui indiquait une persistance de l'inefficacité des mesures visant à faire appliquer la loi et à sanctionner la déforestation illégale.

Ces failles du système sont devenues apparentes en juin et août 2005 quand deux opérations anti-corruption spectaculaires appelées Operação Curupira 1 et 2 ont été lancées et menées en coopération par le ministère de l'Environnement, le Ministério Público et la Police fédérale. Ces opérations ont révélé que certains agents officiels de la FEMA avaient prêté main forte aux auteurs d'activités illégales de déforestation en trafiquant les images satellite et en fabriquant des faux (documents) pour blanchir des transactions de bois résultant d'une exploitation illégale de la ressource.

Peu de temps après ces deux opérations, il a été mis un terme aux activités de la FEMA (fondation de statut semi-autonome) qui, ultérieurement, a été remplacée par une institution étatique dépendant de l'administration publique, la SEMA (Secretaria de Estado de Meio Ambiente de Mato Grosso). Cette institution a ajouté deux nouvelles technologies à la panoplie du système SLAPR pour gérer les domaines de compétence applicables dans I'Etat du Mato Grosso : une base de données sur les producteurs et les consommateurs de produits forestiers (CC-SEMA) et un système de contrôle et d'administration en ligne des produits forestiers (SISFLORA). 


\section{Encadré 23.1 (suite)}

Pour extraire du bois ou défricher les forêts naturelles dans le Mato Grosso, il faut tout d'abord obtenir un permis d'environnement au titre du système SLAPR. En outre, tout plan de gestion des forêts comme tout permis de déforestation doit être approuvé par la SEMA qui accorde ensuite un « crédit » (sous forme de tonnages de bois timber-volume) au titre du système SISFLORA. Les producteurs de bois peuvent gérer ce « crédit-tonnage » de bois en ligne et imprimer eux-mêmes leurs permis de transport de bois. Pour ce faire, il est nécessaire que ces crédits et permis aient été préalablement inscrits et enregistrés dans la base de données CC-SEMA. Pour pouvoir être inscrits dans cette base, les utilisateurs devront apporter la preuve de leur statut juridique et de leur observation des dispositions applicables (en matière de régime foncier, de taxes et redevances, de permis d'environnement et d'approbation par les autorités municipales, etc.). Ces procédures ont été mises en œuvre afin que toute la filière de production du bois soit visée par le système de contrôle et aussi pour faire obstacle à l'utilisation de « sociétés bidon » pour blanchir du bois illégal (comme cela est arrivé dans le passé).

Dès qu'ils sont enregistrés dans la base de données CC-SEMA, les opérateurs forestiers peuvent avoir accès au système SISFLORA en ligne moyennant la saisie d'un mot de passe personnel. Les producteurs qui ont la possibilité d'accéder aux tonnages de bois peuvent les proposer à la vente en ligne à des consommateurs enregistrés dans cette même base. C'est seulement lorsque le consommateur accepte le bois proposé à la vente et que la transaction est saisie dans le système que les volumes de bois sont alors déduits automatiquement du " crédit-tonnage » (par essences) du plan de gestion et qu'un permis de transport individualisé sous forme de code-barres est imprimé. Une innovation intéressante de ce système est le lien établi entre les producteurs et les consommateurs à travers un processus administratif potentiellement porteur ( $d$ 'un marché) d'échanges électroniques de bois d'œuvre légalement exploité entre acteurs inscrits auprès de la CC-SEMA.

Dans le système DOF comme dans le système SISFLORA du Mato Grosso, le nouveau permis de transport du bois avec code-barres (DOF ou GF) émis par voie électronique remplace l'ancien permis de transport (discrédité) ATPF sur support papier. Les nouveaux permis comportent des informations (à l'intention des contrôles routiers qui sont effectués) sur l'origine et le tonnage des grumes transportées. A l'arrivée à l'usine de transformation du bois, les informations saisies dans le document avec code-barres sont lues en se branchant sur le système en ligne et permettent d'honorer le "crédit-tonnage » dont l'industrie a besoin pour émettre à son tour les permis de transport (DOF ou GF) des produits intermédiaires transformés. Si on compare ce système au précédent, on constate qu'il incombe désormais au producteur d'imprimer le permis DOF ou GF et de rendre compte à l'administration - ce qui réduit les coûts et la bureaucratie. Le codebarres individualise le permis de transport du bois et protège le système contre les fraudes. Le fait que le système soit en ligne permet aussi de le prémunir contre toute tentative 
arbitraire de la part des bureaucrates locaux et diminue le risque de corruption. En outre, il est désormais possible de relier entre eux les sites d'origine des coupes forestières et les sites où les grumes sont transportées pour être transformées, établissant ainsi une chaîne de traçabilité crédible qui peut être vérifiée par contrôles ponctuels effectués par les autorités. Tous les Etats brésiliens sont tenus, conformément aux dispositions en vigueur, de concéder à IBAMA le libre accès à ces informations de façon à les intégrer dans le système fédéral SISCOM.

Ces nouveaux systèmes peuvent cependant être gênés par un défaut de sensibilisation à l'utilisation d'internet et/ou par le niveau de connaissance en informatique de leurs utilisateurs potentiels. L'informatisation de la société et, par conséquent, l'accès à internet, progressent cependant rapidement au Brésil. Par exemple, le système de pensions public est désormais accessible en ligne, même depuis les régions éloignées, et permet aux ayant droits de percevoir leurs retraites. IBAMA envisage de fournir aux utilisateurs du système DOF un accès aux terminaux informatiques à travers ses bureaux régionaux disséminés dans tous les Etats en Amazonie.

Une autre contrainte sur le plan pratique est le manque actuel d'accès en ligne à la base de données depuis les aires de coupes forestières. Les informations concernant les volumes et les essences qui seront transportés devront donc être déterminés d'avance - ce qui implique une réduction de la marge de manœuvre au niveau de l'exécution puisque ces décisions ne pourront être prises sur les aires de coupe. Il en découle la nécessité de mieux planifier les opérations d'extraction et de transport du bois et d'accéder à un niveau d'efficacité et de consolidation de l'industrie qui est loin d'être universellement atteint.

\subsection{Comment réussir l'adoption des nouvelles technologies dans le contexte des systèmes de gouvernance forestière}

La situation décrite ci-dessus laisse entrevoir que le succès - en matière d'adoption des nouvelles technologies visant les activités de vérification et réglementation forestières dépendra des progrès qui seront accomplis dans six domaines clé. Une brève description en est donnée dans les paragraphes qui suivent.

Questions clé relevant du domaine politique. Une condition préalable essentielle semble bien être celle du soutien apporté par les plus hautes instances gouvernementales. En raison de leur nature même, les choix technologiques sont au centre du processus décisionnel et les décisions devront ensuite être systématiquement reprises à tous les niveaux de gouvernement. C'est un aspect important étant donné que l'utilisation des nouvelles technologies peut avoir un impact substantiel sur les dépenses publiques. Les coûts d'acquisition d'images satellite commerciales ou d'applications de logiciels commercial peuvent être colossaux et il est essentiel de sensibiliser le public aux retombées positives de ces technologies par des campagnes de communication bien orchestrées. Il sera tout particulièrement important de saisir le Parlement de cette question au moment des auditions budgétaires associées à la présentation annuelle du budget. Les investissements 
engagés doivent aussi être rendus publics à travers les instruments de planification financière intermédiaires comme les cadres de dépenses à mi-parcours [Medium-Term Expenditure Frameworks (MTEFs)], qui font partie du programme et budget du gouvernement. Il faut en effet que ces retombées bénéfiques soient mieux comprises non seulement par les décideurs (y compris le législateur) mais aussi par le grand public et par les média. La façon de procéder pourrait dépendre de la politique plus générale que le gouvernement adoptera aux divers échelons de l'administration quant à l'utilisation des systèmes technologiques en question.

Questions clé relevant du domaine juridique. Une autre condition essentielle est celle de l'existence d'un cadre juridique qui reconnaît l'utilisation des nouvelles technologies aux fins de l'application de la législation et qui autorise la conduite en ligne des procédures relatives à l'administration publique. De telles dispositions sont rarement inscrites dans les textes législatifs en vigueur et il importe donc que toute stratégie portant sur l'introduction de technologies dans le système de réglementation étatique prête attention à la nécessité de procéder à une réforme législative le plus tôt possible. Il est peu probable que ce type de législation ait un rang de priorité élevé dans le programme politique du gouvernement si elle reste confinée au secteur forestier. Dans le contexte de l'adoption des nouvelles technologies, une tactique efficace pour garantir un examen de ce type de législation en temps opportun par le législateur est de cibler une réforme plus vaste qui englobe des domaines de compétence plus étendus du gouvernement central.

Questions clé relevant du domaine institutionnel. Les rôles et compétences doivent être clairement définis dans le modèle des structures institutionnelles et sociétales qui sont à la base de tout système de gouvernance forestière. Les technologies de contrôle forestier devraient être intégrées aux technologies utilisées dans d'autres structures gouvernementales élargies (par ex. régime foncier, système judiciaire, impôts et recettes fiscales). Il n'est cependant pas facile d'administrer ce type d'actions intégrées et il pourrait être nécessaire de prévoir la création d'un organe de supervision comme une commission intersectorielle par exemple. Les autorités forestières doivent être capables d'utiliser ces technologies pour produire des informations stratégiques si elles veulent avoir un impact visible sur l'application des législations. Cela pourrait entraîner l'obligation de créer des unités de communication ou de renforcer celles qui existent déjà au sein de l'administration forestière. La participation du secteur privé à l'introduction et au maintien de nouvelles technologies pourrait être une option à envisager pour accélérer la mise en œuvre. Les technologies peuvent aussi promouvoir des actions décentralisées à travers l'amélioration radicale des communications entre l'Etat fédéral et les Etats. En outre, ces technologies peuvent constituer des outils efficaces pour permettre à l'administration centrale de suivre et de superviser la performance des autorités régionales ou locales dans leurs domaines de compétence décentralisés.

Questions clé relevant de la technologie. L'existence d'une infrastructure technique constitue bien évidemment une condition préalable incontournable afin que les utilisateurs puissent accéder à l'internet. Un problème spécifique sera probablement l'accès à internet depuis le site de coupe forestière. Les camps forestiers sont souvent trop 
éloignés pour être accessibles par les technologies sans fil (wi-fi) quoique cet obstacle soit actuellement contourné - grâce au rythme de progression de l'évolution technologique - par l'accès à distance aux bases de données depuis les réseaux de téléphonie cellulaires. La fiabilité de l'accès et la sécurité de gestion des données sont aussi des conditions préalables indispensables au bon fonctionnement de tout système de contrôle basé sur ce type de technologie. Dans ce contexte et afin de mieux préserver l'intégrité de ces nouveaux systèmes électroniques, il sera judicieux de faire bénéficier le secteur forestier de l'expérience d'autres secteurs (par ex. le secteur bancaire) pour assurer la protection et la sécurité des données électroniques.

Questions clé relevant de l'application de la législation. Les infractions à la loi détectées à travers l'utilisation des nouvelles ressources technologiques finissent souvent par être examinées (dans le cadre des sanctions appliquées) suivant des procédures bureaucratiques extrêmement lentes qui dépendent de l'administration ou des instances judiciaires. Si les délits ne sont pas sanctionnés efficacement en temps opportun, il en résultera une érosion de la confiance du public qui compromettra la crédibilité du système de gouvernance forestière. L'existence de services indépendants et efficaces pour veiller à l'imposition et à l'application des sanctions et la vigilance de la société civile sont donc des éléments d'importance cruciale pour favoriser l'application des législations forestières et faire en sorte que les nouvelles technologies aient l'impact souhaité.

Questions clé relevant du domaine culturel. Le faible niveau de l'éducation de base dans un grand nombre de communautés rurales augmente la dépendance de ces communautés vis-à-vis des intermédiaires, que l'administration forestière utilise des systèmes modernes (électroniques) ou traditionnels (support papier). Les risques de corruption s'en trouvent aussi accrus. Il est donc particulièrement important d'informer et d'éduquer ces utilisateurs pour leur faire connaître les règles et procédures requises pour extraire le bois en toute légalité ainsi que de mettre à leur disposition des systèmes simples et accessibles. Il y a lieu de signaler en même temps que le mythe selon lequel les communautés rurales seraient réticentes à l'utilisation des technologies modernes et d'internet a déjà volé en éclats comme le montre l'usage enthousiaste qu'elles font des téléphones portables et des messages SMS. Il importe que ce potentiel soit utilisé à bon escient pour l'introduction des nouvelles technologies dans le secteur forestier.

\subsection{Résumé}

Les nouvelles technologies peuvent présenter des opportunités supplémentaires de remédier à certaines insuffisances constatées en matière de gouvernance dans le secteur forestier, et générer des bénéfices substantiels pour les groupes d'utilisateurs principaux, notamment les Etats, les exploitants forestiers et d'autres parties prenantes. L'adoption de ces nouvelles technologies peut cependant être gênée par des facteurs politiques et institutionnels ainsi que par des contraintes d'ordre technologique. Un certain nombre de pays procèdent actuellement à un examen de ces obstacles et l'expérience du Brésil est fort instructive à cet égard en montrant la valeur d'une approche comportant les trois volets suivants : (i) clarification des rôles et des compétences des différents niveaux de 
gouvernement ; (ii) renforcement des compétences professionnelles dans l'administration forestière ; et (iii) création de nouvelles structures et investissements y afférents. Plus généralement, pour tirer pleinement parti des bénéfices potentiels des nouvelles technologies, les pays devront tenir compte de six domaines d'activités clé, portant sur les questions d'ordre politique, juridique, institutionnel, technologique et culturel sans oublier les aspects relatifs à l'application de la législation. 



\section{Chapitre 24}

\section{Processus à multiples parties prenantes : enseignements tirés du processus de vérification du bois}

Note : Cecilia Luttrell est l'auteure de ce chapitre, basé sur l'article de Luttrell, C. 2008 Multi-stakeholder processes: lessons for the process of timber verification. VERIFOR, Briefing Paper No. 8. ODI, Londres.

\subsection{Introduction}

Il y a eu profusion de délégations de pouvoirs en matière de gouvernance publique au cours des deux dernières décennies et cela a entraîné une grande diversité de partenariats entre l'Etat, le secteur des entreprises, les ONG et d'autres acteurs de la société civile ainsi que la mise en place de processus à multiples parties prenantes [multi-stakeholder processes] (MSP) pour tenter de concilier les points de vue divergents des partenaires. Les processus à multiples parties prenantes (MSP) ont aussi pu être considérés comme un moyen pour engager les parties dans la voie de l'application des législations, de la gouvernance et du commerce (FLEGT), sans oublier l'élaboration des politiques publiques. Les questions qui se posent au vu de la polarisation typique des intérêts et des opinions du secteur forestier concernent la façon de statuer et prendre des décisions à propos des processus politiques concernés.

\subsection{A quel moment convient-il d'élaborer un processus à multiples parties prenantes?}

La mise en place des MSP est très attendue mais les coûts de transaction élevés et l'éventualité que ces processus entrent en conflit avec les processus démocratiques imposent qu'ils se déroulent de façon à renforcer (et non à compromettre) la crédibilité des politiques publiques. Les réponses qui seront apportées à la question clé consistant à déterminer "dans quels contextes et à propos de quels enjeux il est utile (ou non) de mettre en place un processus MSP » dépendront d'une série de facteurs, notamment :

- De l'objectif du MSP ;

- Du choix du MSP en tant qu'outil de dialogue ou forum de prise de décision; et

- Du mandat exécutif et législatif du processus pour statuer sur les questions débattues et contribuer à la mise en œuvre des décisions prises.

\section{Préciser l'objectif du MSP (processus à multiples parties prenantes)}

Une première étape importante est d'être au clair sur les objectifs du processus MSP. L'une des raisons de la popularité du processus est qu'il est réputé promouvoir de meilleurs 
résultats qu'un processus n'impliquant qu'un individu ou une institution isolé(e). Cette efficacité accrue est attribuée au plus grand nombre de facteurs rendus disponibles à travers le processus ainsi qu'à l'ampleur des engagements en résultant. Les tenants du système soutiennent également que le caractère inclusif du processus peut réduire les coûts à travers des initiatives qui ciblent mieux les besoins des parties prenantes, réduisant par là même le temps passé à régler les conflits. S’agissant de mise en œuvre, les MSP peuvent jouer un rôle en comblant les lacunes d'un gouvernement qui n'aurait pas réussi à faire appliquer toutes les législations requises. Des groupes de citoyens, par exemple, pourraient suivre l'application des législations et identifier les violations qu'une institution qui n'a pas les ressources suffisantes pourrait ne pas avoir repérées.

Cependant, l'efficacité d'un processus à multiples parties prenantes n'est pas seulement déterminé par sa capacité à remplir des objectifs mais aussi par la façon dont il apporte crédibilité et légitimité au processus de décision. Vu sous l'angle du " droit », le processus fait valoir le droit des citoyens à participer et insiste sur la légitimité qui dérive de cette participation même, alors qu'une approche plus pragmatique (et moins dogmatique) tend à considérer les MSP comme un moyen pour les participants de faire entendre leur voix et d'œuvrer à une compréhension commune des questions débattues (van Bodegom et Hijweege 2006).

\section{Le processus à multiples parties prenantes en tant que mécanisme décisionnel}

La participation des parties prenantes au processus peut contribuer à promouvoir le dialogue et à accroître la transparence d'un processus de détermination d'une stratégie, mais l'aspiration à prendre les décisions qui prévaudront en dernière analyse n'est pas une caractéristique commune à tous les MSP. Une distinction devra donc être établie :

- Avec les contextes dans lesquels l'objectif du MSP est d'être une plateforme de dialogue et de consultation mais non un processus décisionnel (qui relève d'instances plus spécifiques comme une instance démocratiquement élue par exemple) ; et

- Avec les contextes dans lesquels le processus est considéré comme un véritable forum de prise de décision.

La consultation ou la participation à un processus n'impliquent pas nécessairement la capacité à mettre en œuvre les décisions qui ont été prises. Si les MSP ont été programmés en tant qu'instances de décision, il faudra les doter des pouvoirs nécessaires - ce qui risque de soulever un certain nombre de questions; il s'agira alors de déterminer notamment :

- Si le MSP considéré en tant qu'entité constituée a reçu un mandat clair et précis de l'exécutif et du législatif pour statuer et prendre des décisions ;

- Quelles limites ont été fixées en matière de pouvoirs de décision délégués au MSP ;

- Comment le MSP peut être défini par rapport aux institutions gouvernementales concernées ; et

- Les rapports du MSP avec les mécanismes démocratiques de reddition des comptes. 
L'étendue du mandat législatif et exécutif de l'entité composée de multiples parties prenantes pour statuer et prendre des décisions dépendra du contexte. Les pouvoirs ou mandats requis pour entériner une décision varient selon la nature de la question traitée ainsi qu'en fonction des diverses traditions des parties en matière administrative et juridique. Il appartiendra au législateur de chaque pays concerné de déterminer quelles sont les fonctions qui relèvent du domaine de compétence exclusif de l'Etat et quelles sont celles qui peuvent, au regard de la législation ou de la constitution, être externalisées et transférées à l'entité composée de multiples parties prenantes. Prenons le cas, par exemple, des normes de légalité ; certaines d'entre elles pourraient être assujetties à l'approbation d'instances législatives ou judiciaires et un forum composé de multiples parties prenantes ne semble donc pas être l'organe décisionnel adéquat.

Il importe que les limites du pouvoir de décision du MSP soient clairement et précisément définies et que ses compétences s'inscrivent dans un cadre mutuellement convenu entre l'entité MSP et les instances gouvernementales concernées. Dans le cas d'un MSP à caractère international, il s'agira d'examiner explicitement comment les actions engagées à ce titre se rattachent aux programmes officiels et d'en coordonner la mise en œuvre avec les politiques et processus existants aux niveaux international et national. Les actions de partenariat lancées par le Sommet mondial sur le développement durable (SMDD), par exemple, ont fait l'objet de critiques visant précisément leur manque de coordination et de synergie avec les programmes officiels de développement aux niveaux international et national (Malena 2004).

La participation d'acteurs non-étatiques aux MSP est souvent présentée comme nécessaire pour permettre aux citoyens d'être représentés dans le processus décisionnel. C'est un argument invoqué dans des contextes où les citoyens ne font pas confiance aux instances étatiques ou bien lorsque des acteurs importants estiment qu'il est nécessaire de contrebalancer le pouvoir de l'Etat. Dans ce genre de situations, les MSP sont considérés comme une étape sur la voie d'une plus grande démocratisation à travers leur mode de fonctionnement qui consiste à laisser s'exprimer un grand nombre d'opinions tout au long des processus de prise de décision. Le risque, néanmoins, pour les structures démocratiques qui sont en place même si elles fonctionnent de façon imparfaite, pourrait venir de MSP dont le fonctionnement saperait plutôt qu'il ne renforcerait les bases de la démocratie. Ribot (1999) s'est inquiété du fait que les MSP pourraient compromettre la fiabilité des mécanismes existants de reddition des comptes de l'Etat à la société par des prises de position aboutissant à contredire des lois politiques ou administratives ou à démanteler des mécanismes de représentation locaux plus responsables.

Il est donc nécessaire de structurer les MSP de façon à ce qu'ils contribuent à la démocratie et ne cherchent pas à en usurper les fonctions.

\section{Les MSP comme instrument de dialogue}

D'aucuns estiment que la valeur réelle des processus à multiples parties prenantes (MSP) tient plus à leur capacité d'instaurer un dialogue qu'à celle de prendre des décisions 
concrètes. Les MSP liés au Sommet mondial sur le développement durable (SMDD) étaient des processus "de recherche plutôt que de prise de décision " et restaient sous la coupe de la "règle d'airain " qui stipulait que seuls les gouvernements pouvaient négocier avec les gouvernements (Bäckstrand et Saward 2004, p. 16). L'absence d'une fonction formelle de prise de décision n'exclut cependant pas l'influence que peut exercer une partie prenante sur le processus. Même sans pouvoirs conférés ou délégués, certains MSP ont permis de faire avancer des négociations à travers le dialogue, l'obtention d'un consensus et le renforcement des réseaux, sans oublier leur rôle en tant que plateforme d'information sur les politiques publiques.

Il importe d'énoncer clairement le statut de chaque MSP et de préciser donc, dans chaque cas, la nature (formelle ou informelle) de la consultation engagée ainsi que les relations avec le gouvernement. Ces précisions (concernant l'étendue des pouvoirs des MSP) font souvent défaut - ce qui peut éroder la volonté des parties prenantes à honorer leurs engagements.

\subsection{Questions relatives à la participation}

Au nombre des facteurs critiques pour la participation, figurent les éléments suivants :

- Les catégories de parties prenantes qui seront incluses dans le processus ;

- Les mesures à prendre pour éviter l'exclusion de certains groupes ;

- La façon dont est traitée la dynamique du pouvoir ;

- Les mécanismes assurant l'indépendance ; et

- Les mécanismes de transparence et de responsabilisation.

\section{Qui doit être inclus dans le processus ?}

Faire participer à un MSP des groupes très divers de parties prenantes est l'un des moyens de renforcer la crédibilité du processus. Un défi clé sera de choisir les acteurs les plus qualifiés - choix qui se fondera sur un certain nombre de critères qui dépendront de l'objectif recherché - notamment :

- Ceux (les acteurs) dont l'appui est nécessaire pour mettre en œuvre les décisions ou dont le manque de soutien se traduirait par une érosion possible du processus ;

- Ceux qui peuvent contribuer par leurs connaissances spécialisées à la question étudiée ; et

- Ceux qui ont le « droit » d'être inclus dans le processus au motif qu'ils pourraient être affectés par les décisions prises.

La diversification des perspectives peut aussi contribuer à l'amélioration des connaissances et à une meilleure compréhension. Promouvoir le partage de connaissances spécialisées et de nouvelles opinions contribue à une meilleure information sur les processus politiques. L'inclusion d'acteurs importants et de ceux dont le soutien est nécessaire pour que les décisions politiques soient suivies d'effet peut être bénéfique pour l'appropriation et prévenir tout sabotage ultérieur. C'est ainsi, par exemple, que trois groupes de travail 
informels composés de représentants du secteur privé, des ONG et des pays concernés ont été institués dans le cadre du Protocole de Kimberley pour les diamants - procédure qui a contribué à étoffer le processus en incluant des avis de spécialistes et à accroître la transparence (Smillie 2005).

Les demandes des parties prenantes dans le secteur forestier sont particulièrement diversifiées et conflictuelles et une participation élargie pourrait aider à désamorçer d'éventuels conflits.

Un dilemme clé consiste à déterminer qui a un intérêt légitime. Les directives en matière de bonnes pratiques dans le contexte du MSP tendent à privilégier l'utilisation de critères clairement définis et d'un processus transparent pour identifier les acteurs à retenir (Malena 2004 ; Hemmati 2002). Ces directives concernent :

- La représentativité ;

- Le mandat organisationnel ;

- Les compétences spécifiques; et

- Le respect de valeurs spécifiques.

Le " droit à être inclus » est un critère important de sélection des parties prenantes et les MSP ont l'obligation d'inclure ceux qui sont affectés par des actions ou des décisions, en particulier quand les retombées risquent d'être négatives. Un processus de sélection basé sur des normes de représentativité et de légitimité articulées de façon transparente contribuera à éviter une domination par les groupes qui «crient le plus fort ». Il conviendra de s'assurer, toutefois, que ces accréditations n'entraînent pas d'effet de distorsion en créant des obstacles pouvant gêner la participation d'acteurs moins influents et de groupes minoritaires.

\section{Préserver l'indépendance du processus}

Un autre point critique est celui de la désignation (ou de la nomination) des représentants. Des processus légitimes visant à garantir la cohérence et la transparence du choix des représentants doivent être mis en place à cet effet. La question de savoir qui nomme les représentants revêt une importance critique et une sélection opérée par des électorats clé pourrait contribuer à la légitimité de la désignation des représentants. Dans la publication "Initiative pour la transparence des industries extractives " [Extractive Industries Transparency Initiative (Publish What You Pay and Revenue Watch 2006)], un rapport fait état d'une situation où l'indépendance du comité à multiples parties prenantes est compromise dans un grand nombre de pays par l'interdiction faite à la société civile de choisir ses représentants. L'auto-sélection peut être particulièrement importante dans un contexte politique très tendu avec de multiples enjeux et renforcer la responsabilité des représentants envers leurs électeurs.

Le mécanisme de financement est un autre facteur déterminant pour l'indépendance et la crédibilité. Un financement de source gouvernementale peut entraîner des distorsions du 
programme et un financement par le secteur privé est souvent perçu comme susceptible de compromettre la mission, les valeurs et la réputation du MSP. Ce n'est pas non plus une solution idéale que d'attendre des parties prenantes qu'elles s'autofinancent étant donné que les capacités de rémunération des organisations diffèrent les unes des autres et que se profile donc le risque que des groupes d'intérêts particuliers avec des moyens suffisants (à la fois en ressources financières et en temps) saisissent l'occasion (Bäckstrand et Saward 2004). Dans le cas de la Commission mondiale des barrages, des efforts ont été déployés pour diversifier les sources de financement afin de démontrer que la Commission n'était asservie à aucun jeu d'intérêts particuliers (Dubash et al. 2001).

\section{Uniformiser les règles du jeu : éviter l'exclusion}

De nombreuses parties prenantes ne sont pas en mesure de participer aux MSP parce qu'elles manquent de ressources ou n'ont pas les capacités suffisantes. L'absence de structures institutionnelles adéquates est souvent citée comme autre raison de la nonreprésentation de certains groupes dans ces processus. Des mesures spécifiques visant à favoriser l'instauration de règles du jeu plus équitables pourraient inclure notamment :

- La formalisation de règles de partage des pouvoirs ;

- Un taux de représentation plus élevé des groupes de parties prenantes "les plus faibles";

- Des politiques de rééquilibrage des ressources ;

- Des mesures pour renforcer les capacités ; et

- Des garanties d'accès à l'information.

Par exemple, les frais de voyage des représentants ayant besoin d'un concours financier sont pris en charge par la Commission mondiale des barrages - ce qui permet d'élargir la participation (Dubash et al. 2001). La tenue de consultations régionales permet aussi de favoriser la mobilisation de la base et sa leur participation au débat.

Il convient cependant de prendre garde aux risques induits par ces tentatives visant à canaliser la participation, notamment aux risques qui sont liés à :

- Une homogénéité apparente, autrement dit le risque de stéréotype et de faire rentrer les individus dans des " catégories " qui arrangent les décideurs mais ne reflètent pas la diversité des intérêts locaux ;

- Une participation nominale plutôt que significative : la présence physique de groupes marginalisés, voire leur prise de parole pendant les débats, ne leur garantit pas une écoute suffisante ni que leurs inquiétudes seront prises en compte. Il importe donc de veiller à ce que les MSP ne soient pas perçus comme « jouant le jeu » de la participation. On peut l'éviter en informant à l'avance les participants sur les actions spécifiques qui seront engagées et la façon dont ces questions seront abordées.

- Les processus symboliques : dans de nombreuses situations, les prises de position 'hors salle de conférence' peuvent avoir bien plus d'influence que celles qui sont adoptées en séance publique (Hemmati 2002). 


\section{Laisser la contestation s'exprimer et savoir reconnaître les différences}

Les opinions diffèrent en ce qui concerne le caractère nécessaire ou souhaitable d'une standardisation des valeurs, des intérêts et des objectifs. Un certain degré de désaccord manifeste n'est pas nécessairement indésirable ou inutile. Un MSP efficace sera celui qui, en effet, permettra à une saine contestation de s'exprimer et, de surcroît, la stimulera. Vaines sont les tentatives qui, adeptes avant tout de la recherche d'un consensus et pour gommer l'inégalité des pouvoirs, dissimulent les divergences d'opinion et ignorent les conflits potentiels; plutôt que de supposer nécessaire de jeter aux oubliettes les différences d'opinions politiques ou, en tous cas, de les neutraliser temporairement, les facilitateurs pourraient plus utilement chercher à renforcer les pouvoirs décisionnels des groupes désavantagés (Edmunds et Wollenberg 2002). Pour cela, il leur faudra reconnaître les disparités de pouvoir et aborder de front les questions sensibles. Peut être faudra-t-il aussi établir des paramètres acceptables pour mesurer ces divergences (et disparités) et encourager les partenaires à débattre de l'opportunité d'accepter de ne pas être d'accord. Un MSP qui tenterait de tenir compte des préoccupations de toutes les parties prenantes pourrait bien priver les acteurs individuels de la possibilité de se faire entendre. La création d'espaces de liberté d'expression pourrait contribuer à éviter de telles tensions. L'initiative multi-institutions Safe Motherhood Initiative par exemple, a pu régler un désaccord fondamental concernant la question de l'avortement en autorisant certains membres à choisir de se retirer de certaines fonctions tout en restant partenaires de l'initiative globale (Malena 2004).

\section{Clarté des procédures}

Une consultation efficace et élargie depuis les premiers stades de son déroulement tend à être associée à de robustes processus à multiples parties prenantes, de sorte que les parties intéressées peuvent influencer la formulation des objectifs principaux, les prestations et les indicateurs de performance (voir, par exemple, van Bodegom et Hijweege 2006). Un comité directeur bien équilibré peut aider à la planification technique et à l'élaboration du programme. Il faudra aussi convenir explicitement des tâches dévolues à chaque partenaire et veiller à ce que les responsabilités qui lui seront conférées correspondent à ses droits légitimes et soient adaptées à ses compétences et intérêts spécifiques. Enfin, une définition claire et précise des objectifs, des rôles et des résultats attendus est essentielle pour assurer une bonne reddition des comptes. De même et malgré l'importance qu'il convient d'attacher à la clarté des procédures, il importera également de faire preuve de souplesse du point de vue des objectifs et du contenu pour que la complexité sous-jacente des questions étudiées soit bien prise en compte et que cela ouvre la voie à de nouvelles réflexions (Hajer 2005).

Dans le cas des MSP dotés de pouvoirs décisionnels, un certain nombre de choix devront être faits, y compris sur la question de savoir s'il faut opter pour le consensus ou la procédure du vote à la majorité, ou encore sur la façon d'aborder les opinions exprimées par une minorité de participants. Nombreux sont les processus qui associent des structures internes (telles que conseils d'administration, comités et groupes consultatifs) à leurs propres pouvoirs et procédures en matière de prise de décisions. L'échantillonnage 
stratifié par groupes d'intérêts organisés en " sous-chambres " de délibération (subchambers, comme dans le cas du Forest Stewardship Council) peut permettre d'éviter qu'un groupe soit dominant. Dans le cas de l'initiative intitulée Children's Vaccine Initiative (alliance regroupant les Nations Unies, le secteur privé et les organisations non-gouvernementales), le secteur privé participe à la mise en ouvre mais est exclu des processus décisionnels élargis sur les orientations stratégiques afin d'éviter tout conflit d'intérêts (Malena 2004).

Avant tout, il faut être réaliste et conscient des longs délais impliqués dans tout processus à multiples parties prenantes qui se fonde sur le dialogue et prend donc du temps, sans oublier les délais imposés par les cycles budgétaires et autres. Ce dernier point a été source de difficultés dans le cas de la plateforme à multiples parties prenantes liée à l'APV entre le Ghana et l'UE dont les délais sont réputés avoir été fixés en fonction de conditions politiques externes et internationales et non selon les dispositions stipulées dans la plateforme (Beeko 2008).

\section{Renforcer les procédures de responsabilisation et de transparence}

Les processus à multiples parties prenantes sont confrontés à des problèmes particuliers en matière de responsabilisation en raison des multiples sources de délégation de pouvoir existantes, souvent peu claires de surcroît. Sans procédures de responsabilisation, les MSP perdent rapidement leur légitimité. Il importe donc de préciser quelles sont les autorités auxquelles les parties prenantes doivent rendre des comptes et d'établir les mécanismes chargés des évaluations et des rapports à cet égard. Un certain nombre de techniques peuvent être utilisées pour renforcer ces procédures (Benner et al. 2002), notamment :

- La « responsabilité interne" (conseils, comités de supervision);

- La « responsabilité professionnelle » (adhésion aux codes de conduite professionnelles);

- La « responsabilité en matière de réputation » (désigner et faire honte (naming and shaming)) ;

- La « responsabilité commerciale » (récompenses/sanctions attribuées par les consommateurs); et

- La « responsabilité financière » (normes comptables).

En général, la responsabilisation peut être renforcée en maintenant la transparence et en adoptant des stratégies de communication dynamiques. Les « Principes généraux et normes minimales pour la consultation » de la CE soulignent l'importance d'un contenu clair du processus de consultation et la nécessité d'une communication ciblée et prévisible (voir van Bodegom et Hijeweege 2006).

L'accessibilité à l'information est un aspect important et distinct de celui de la disponibilité des informations. L'article 6 de la Convention de l'OIT relative aux peuples indigènes et tribaux des pays indépendants stipule que les gouvernements « doivent consulter les peuples intéressés, par des procédures appropriées " et "sous une forme appropriée aux 
circonstances ». C'est une chose de divulguer des informations, mais c'en est une autre de veiller à ce que ces informations soient comprises par tous.

\subsection{Conclusion}

Un certain nombre de raisons militent en faveur de la prise en compte des processus à multiples parties prenantes dans l'architecture des systèmes de vérification, notamment celles qui sont liées aux besoins suivants :

- Accroître l'efficacité à travers le renforcement des capacités et des intrants techniques ;

- Étoffer le soutien politique en s'assurant de la participation des acteurs influents mais aussi de ceux qui pourraient compromettre le déroulement et les progrès du processus ; et

- Promouvoir la légitimité du processus en impliquant un large éventail de parties prenantes de tous horizons.

De telles différences de motivation ont des effets sur tous les niveaux de participation et la nature du processus qui sera retenu.

S'agissant des procédures relatives à l'assurance de la légalité, la contribution des parties prenantes pourra s'exercer à différents stades du processus de vérification et prendre la forme, soit d'un mécanisme de prise de décision, soit d'un forum pour renforcer le dialogue et la consultation. Chaque fois que le MSP sera une instance de dialogue, il importera de le qualifier de "forum de dialogue " depuis le début en énonçant clairement les limites du processus. Chaque fois que le MSP sera utilisé avec l'intention de lui confier un rôle de décideur, il conviendra de veiller aux dispositions pratiques suivantes, notamment :

- Un mandat précis concernant la prise de décisions et leur mise en œuvre ;

- La clarté concernant la nature de la question débattue afin de déterminer si un organe de type pluraliste est autorisé à prendre des décisions légitimes et à statuer ;

- L'inclusion d'organes décisionnels et de mise en œuvre parmi les parties prenantes représentées ;

- Des ressources adéquates pour pouvoir adhérer à certains principes clé (disposer de délais suffisants, maintenir la transparence et des financements indépendants) ; et

- Un soutien institutionnel ou politique suffisant pour mettre en œuvre les décisions qui auront été prises. 



\section{Partie D}

\section{Elaborer des systèmes de vérification des forêts efficaces et équitables}





\section{Chapitre 25}

\section{Principes et pratique : conception du système de vérification dans le secteur de la forêt}

\subsection{Introduction}

Ce chapitre reprend les dix principes généraux sous jacents à une vérification efficace identifiés au Chapitre 3 et basés sur une expérience extra sectorielle, afin de mieux éclairer les processus de vérification des forêts. Cette analyse s'inspire également du Chapitre 4 qui porte sur des études de cas de pays sur la vérification et des Chapitres 17 à 24 qui portent sur les questions transversales découlant de ces études.

"L'efficacité " dépend dans une grande mesure, de critères imposés sur le phénomène considéré par la partie intéressée. Partant des témoignages des études de cas précédentes, il semble que pour développer un régime de vérification efficace, il est essentiel d'identifier des principes et des systèmes qui jouiront d'une forte appropriation nationale et qui sont suffisamment robustes et flexibles pour supporter les défis permanents non seulement dans leur fonctionnement quotidien mais également supporter que leur existence même soit contestée. Ces défis sont presque inévitables dans un milieu aussi mouvementé que la gestion de la forêt (en particulier sous les tropiques où l'on voit s'installer des perspectives polarisées "conservation contre production»), comme l'attestent les nombreuses études de cas présentées dans ce livre. La robustesse et la flexibilité apparaissent comme des qualités importantes des systèmes déjà en place, et il existe de bonnes raisons de croire qu'elles constituent des qualités souhaitables pour tous les systèmes futurs, notamment dans des cadres où la gouvernance de la forêt reste en grande partie immuable (non touchée par la réforme). Dans de tels contextes, la volonté d'introduire des mesures d'assurance de légalité se heurtera à une forte opposition des forces qui défendent le statu quo.

Lors de l'évaluation de la pertinence des principes s'inspirant de la revue des études de cas extra sectoriels du Chapitre 3, il faut prendre en compte le fait que la plupart des processus étudiés étaient internationaux. Par contre, les processus du secteur forestier étudiés dans la Partie B sont essentiellement nationaux par nature, bien que contraignants, $\mathrm{du}$ moins dans le principe, pour l'ensemble des acteurs sur le territoire national. Ces processus n'ont de caractère international que dans le cadre des AVP (toujours en cours de négociation), essentiellement (du moins à ce stade) au niveau bilatéral, avec le pays producteur de l'UE. 


\subsection{Appliquer les principes de vérification au commerce du bois d'œuvre}

Dans les paragraphes qui suivent, les principes identifiés lors de la revue des modèles de vérification sont réexaminés à la lumière des études de cas du secteur forestier en tant que points qui pourraient être intégrés dans toutes les approches de la conception du système de vérification.

En cherchant à appliquer ces principes au secteur de la forêt, il faut prendre en compte les caractéristiques du bois d'œuvre en tant que produit de base commercialisé au niveau international, ainsi que l'absence d'un traité international pour contrôler ce secteur et ce commerce. Alors que le bois d'œuvre est un produit de base tangible (qui devrait le rendre plus facilement vérifiable que le carbone atmosphérique par exemple), il est également très variable dans ses caractéristiques (ce qui ajoute aux difficultés de vérification, en particulier au niveau des maillons vulnérables de la chaîne d'approvisionnement, comme les douanes et les autorités portuaires).

Une même image mitigée est donnée des marchés du bois d'œuvre. Comme le bois d'œuvre est un produit facilement identifiable et qu'il représente un volume important, il est plus facilement reconnu en transit que par exemple les diamants. Cependant, les marchés du bois d'œuvre sont décentralisés dans les pays producteurs et au-delà, sans intérêts dominants sur le marché qui pourrait faire preuve d'un solide intérêt propriétaire sur le produit de base et donc influencer les perceptions publiques (contrairement à l'entreprise principale De Beers sur le marché mondial du diamant). Le bois d'œuvre a également de multiples usages, certains dans le prestige et le luxe, d'autres plus mondains. Ainsi, les défis sur le marché du prestige n’affecteront pas nécessairement la propension à consommer. Là encore, ceci offre un contraste important avec des produits comme les diamants ayant un éventail d'usages prestigieux et des usages industriels restreints, et beaucoup plus vulnérables aux impacts de la perte de leur image internationale. Ces caractéristiques poseront probablement des difficultés particulières pour la vérification de la production et du commerce du bois d'œuvre, et augmenteront les difficultés à obtenir des impacts positifs avec les mesures de vérification.

\section{Principe 1 : Réciprocité}

La réciprocité entre les signataires, pour qu'ils soient tous tenus de la même façon par les conditions de l'accord, a été établie comme principe important dans l'efficacité des régimes internationaux de vérification. Les expériences du secteur forestier sont spécifiques à cet égard. Lorsque les accords du secteur de la forêt sont nationaux ou infranationaux par nature, alors un degré de réciprocité est implicite, du moins dans le principe, et la législation nationale prévoit un mécanisme pour encourager une large conformité. Les Comités multi sectoriels de la protection de la forêt aux Philippines et les Systèmes de pilotage sous traités de l'Equateur sont des exemples de cette approche. La principale préoccupation dans ces cas consiste à savoir si l'égalité nominale des normes favorise en fait des catégories particulières de producteurs et en désavantage d'autres, et 
dans le premier cas il s'agit plus probablement des petites et moyennes entreprises. Au niveau international, il n'existe pas encore d'accord dans le secteur forestier qui exige une telle réciprocité. L'expérience internationale récente a simplement confirmé qu’il est improbable d'instaurer un régime réciproque dans le secteur forestier, tout aussi contraignant pour ses signataires, même à moyen terme. Toute tentative d'un groupe de parties à faire passer en force un tel régime serait mal perçue par les autres. Ainsi, en élaborant un système de légalité à caractère bilatéral pour le secteur de la forêt, l'accent doit être mis sur les moyens de dépasser l'absence de réciprocité grâce à des moyens de substitution.

Certains enseignements peuvent être tirés de l'expérience extra sectorielle quant à la façon de dépasser les contraintes de souveraineté en l'absence d'un principe de réciprocité généralisé. Le mieux serait peut-être de capitaliser sur la revue par les pairs comme cela a été fait dans le cas de l'accord Kimberley sur les diamants. Cela a permis de créer une seule communauté de pays producteurs, liés par les mêmes règles et intérêts. Ceci aiderait à s'attaquer à la question de la comparabilité. Il se peut par exemple, que les consommateurs des pays importateurs ne souhaitent pas ou soient incapables de faire la distinction entre différentes normes de légalité concernant les AVP, et ceci peut entraîner des allégations d'injustice de la part des états producteurs pour lesquels la barre a été placée très haut. La régionalisation des normes serait une façon de gérer cette pression sans "baisser le niveau ", même si sa faisabilité est discutable. Van Midwoud (2006) donne des arguments convaincants pour l'application d'un mécanisme de revue par les pairs aux AVP du secteur forestier.

\section{Principe 2 : Migration vers les non parties}

L'approche AVP a une capacité limitée à sanctionner la migration vers les non parties comparée aux conventions extra sectorielles abordées dans le Chapitre 3, un fait qui est particulièrement pertinent pour les producteurs asiatiques qui bénéficient d'un accès facile au marché chinois florissant. Certains producteurs (comme ceux d'Afrique de l'ouest et d'Afrique centrale) sont déjà fortement dépendants des marchés verts, et la simplicité relative de leurs chaînes de produits accroît la probabilité de leur conformité. Par opposition, le marché chinois ne s'est pas montré particulièrement vert jusqu'ici, et des questions se posent déjà sur la capacité de l'Union Européenne à exercer une pression du côté de la demande sur les producteurs malaisiens et indonésiens auxquels s'offrent des opportunités importantes. L'accord de ces producteurs à signer des AVP pourrait avoir des implications, même s'il devrait s'accompagner d'autres mesures pour empêcher la migration vers les non parties afin que les impacts soient durables. Les deux mesures les plus évidentes seraient la diplomatie internationale et le plaidoyer des ONG. Cependant, l'accession de la Chine à l'OMC pourrait également avoir des effets positifs, notamment dans la mesure où elle renforce la dépendance croissante du pays aux marchés du détail, qui sont déjà écosensibles.

Le recours aux pressions du côté de la demande dans des sociétés de consommation (comme dans les AVP) pour détourner le commerce des fournisseurs non conformes 
vers les fournisseurs conformes, même en l'absence d'une série de règles de marché obligatoires, est une initiative audacieuse qui pourrait bien comporter des avantages, bien que le problème ( $\mathrm{du}$ moins au début) soit que l'avantage du premier utilisateur pourrait bien se réaliser au détriment d'un risque politique inhabituellement élevé. Les premiers utilisateurs doivent évaluer la sécurité de l'accès au marché qu'ils pourraient obtenir comparé au fait que leurs industries pourraient bien se trouver soumises à un niveau inhabituel de surveillance internationale, en particulier par les ONG. Certains états producteurs pourraient considérer qu'ils ont plus à perdre avec la conformité qu'avec la non-conformité, tant sur le plan financier que sur le plan réputation, en particulier du fait que les non signataires aux AVP ne se verront pas refuser l'accès au marché, du moins dans le court terme. Dans ces cas, la décision finale de savoir si un premier adoptant accepte un régime de vérification des forêts pourrait être influencée tant par les autres avantages qu'ils peuvent obtenir de l'UE, par nature liés au développement en général, que par les avantages immédiats de l'AVP.

Les AVP sont de toute évidence un compromis et leur caractère non réciproque restreint leur potentiel à transformer la gouvernance de la forêt. Des mesures incitatives positives financières du côté de la demande de la chaîne encourageraient sans nul doute fortement les partenaires tropicaux à se conformer, mais il semble improbable de pouvoir offrir ces incitations, du moins à court terme. L'expérience de certification met en garde contre l'optimisme sur ce point, et la situation est plus limitée encore par le fait qu'une prime sur le "bois d'œuvre légal » ne peut être offerte en guise d'alternative au «bois d'œuvre illégal ". La possibilité d'accroître la valeur marchande d'un produit en l'étiquetant "vérifié légal » est limitée par les opportunités extrêmement restreintes qui seraient probablement disponibles pour réduire l'opposition, une fois que le produit en question a obtenu l'accès au marché européen. Les publics nordiques supposeraient probablement que tout produit qui arrive sur leur marché doit être légal et que la distinction entre les produits légaux et illégaux a été faite avant que les produits n'arrivent chez eux. Les mesures supplémentaires envisagées actuellement par l'Union Européenne constituent une réponse à cette situation (voir par exemple, Brack 2006), même si on ne sait pas encore quelles seront les mesures, si mesures il y a, qui seront adoptées.

La nature volontaire des négociations AVP constitue également une contrainte d'un autre genre. Par exemple, une approche basée sur les risques présente également beaucoup d'avantages, tant au niveau logique que sur le plan du contrôle des coûts, comme le montre l'expérience des normes de transformation de la viande (voir l'encadré 3.5). Dans les territoires nationaux, cette stratégie peut (au moins dans certains cas) être valable pour le secteur forestier. Cependant, appliquée au niveau international, les coûts politiques sont importants dans une situation de non réciprocité. La propension à l'illégalité n'est pas également répartie dans les pays producteurs, et un nombre de facteurs interviennent, jouant sur le risque au niveau international, allant de la qualité générale de la gouvernance (incluant, mais ne se limitant pas à la gouvernance de la forêt) au caractère du domaine forestier (diverses forêts anciennes ou des peuplements simplifiés d'espèces de plantation). Les tentatives des consommateurs nordiques de réduire les efforts internationaux pour se concentrer sur les cas à haut risque, et une gouvernance faible, pourraient être contrecarrées 
par les pays cibles qui se plaindront de l'absence d'impartialité dans la stratégie. Tous les pays producteurs ont le même accès à l'atout que constitue la souveraineté nationale sur les domaines forestiers.

Une dimension de l'effet de la « migration vers les non parties " aux niveaux domestique et régional se retrouve dans le fait que les régimes de vérification du bois d'œuvre ne s'appliquent habituellement qu'aux zones de "forêt " et que de nombreuses activités de déforestation et d'activités illégales d'exploitation forestières se déroulent à l'extérieur des zones de forêts définies. Elles ne sont donc pas arrêtées - ou pire encore, elles sont même encouragées - en durcissant la gestion des forêts. Ces effets de suintement pourraient exiger l'implication et l'engagement de parties en dehors de l'administration nationale des forêts si l'on souhaite les éviter. Dans la mesure où ils impliquent le déplacement de l'illégalité hors du territoire national, ils fournissent un argument supplémentaire en faveur d'une approche régionale.

\section{Principe 3 : Définition claire des domaines de problème}

Une définition claire du (des) problème(s) que l'intervention devrait traiter est importante pour élaborer un système de vérification efficace. L'élément important ici est l'origine du doute. La vérification, telle qu'elle est abordée dans ce livre, répond à un doute sur le fonctionnement d'un système de contrôle de routine qui à son tour implique l'additionnalité des moyens appliqués pour s'attaquer à ce doute. Une approche logique de la définition du problème serait donc de se demander en premier lieu pourquoi un élément de doute s'est immiscé, et quelle situation finale indiquerait que ce doute a été en grande partie surmonté.

Les problèmes concernés varient à la fois selon les situations et dans le temps. Par exemple, une distinction pourrait être faite entre des contextes dans lesquels la demande de vérification découle des préoccupations relatives à la criminalité flagrante et les liens entre la mafia criminelle et l'ordre politique du parti (une indiscipline qui peut se retrouver dans tous les secteurs d'extraction de l'économie) et celles où l'illégalité est plus le fait des forces structurelles - soit à l'intérieur du secteur (par exemple, des politiques qui ont généré une capacité installée de l'industrie bien au-delà des coupes annuelles autorisées) soit au niveau extra sectoriel (par exemple, des politiques agricoles qui encouragent la conversion de la terre). Il existe un certain nombre de cas dans les études de cas où des questions ont été posées pour savoir si l'objectif ostensible du processus de changement répond aux véritables moteurs du changement dans l'utilisation de la forêt ; dans des cas comme le Brésil, l'Indonésie et le Nicaragua, le point en question est l'équilibre entre les forces qui sont internes au secteur et celles qui ont des origines extra sectorielles.

La conception du problème pourrait bien évoluer progressivement. Et donc, la première étape de nombreux processus de réforme est d'assurer les niveaux de base de la légalité dans le fonctionnement de l'industrie et le comportement des acteurs clés (comme les militaires) qui peuvent les saper. En Malaisie par exemple, la majorité de la production nationale de bois d'œuvre est maintenant légale sur le plan de l'autorisation et du 
transport, et les commentateurs et les partisans sont de plus en plus préoccupés par la nature des normes juridiques plus élevées, notamment celles concernant la tenure foncière, les droits indigènes et la gestion durable de la forêt par rapport auxquels évaluer la conformité, ainsi que les failles des systèmes de contrôle des importations de bois de coupe venues d'ailleurs dans la région de l'Asie.

L'idéal est un processus de dialogue national ouvert dans lequel les problèmes à résoudre seront clairement définis et leur séquence acceptée, préliminaire à une voie de réforme plus progressive. Une hiérarchie d'objectifs pourrait s'appliquer, en commençant par des phases à court terme spécifiques à l'industrie et en allant vers des questions socialement plus exigeantes dans un cadre temporel plus long, comme :

1. La conformité aux normes de gestion légale de la forêt (récolte légale du bois d'œuvre) ;

2. La conformité aux exigences d'une utilisation légale de la terre (déforestation réglementé ou réduite) ;

3. Le paiement de taxes pour augmenter les recettes publiques (pour garantir ou accroître les exportations vers des marchés exigeants, ou pour augmenter la part des recettes actuelles) ;

4. Le bien-fondé des processus administratifs d'octroi d'une licence forestière (bonne gouvernance); et

5. La conformité aux normes environnementales et sociales élargies et aux objectifs de développement.

Habituellement, ces objectifs sont liés. Le Ghana en est un exemple : les objectifs de vérification visent à améliorer le pilotage de l'utilisation des ressources forestières et les flux de revenus générés par la récolte du bois d'œuvre, tout en maintenant l'accès à une destination d'exportation importante (l'UE) et en faisant les premières démarches pour aller vers une durabilité à long terme de la gestion des ressources.

Comme le soulignent les Chapitres 18 et 21, une définition claire des domaines de problème exige une grande clarté sur la motivation. Quelquefois, le problème a déjà été identifié avec des moyens fiables et démocratiques - par exemple, un dialogue national sur les forêts, avec un processus multilatéral pour définir les politiques forestières et les plans d'action sur les forêts, souvent dans le cadre du Programme national sur les forêts (PNF). A travers ces processus, le problème que le système de vérification est supposé résoudre peut être défini, explicitement indiqué et agréé par les acteurs clés. Lorsqu’il n'existe pas de liens clairs avec les processus démocratiques nationaux, les défis sont encore plus importants.

Le caractère de biens publics des forêts tropicales renforce la complexité, car il permet à un plus grand nombre de voix extérieures s'intéressant au résulta au niveau national de s'élever (souvent des acteurs du nord dans les agences de développement et des ONG). Lorsque les conditionnalités des agences de bailleurs de fonds liées à d'autres secteurs sont impliquées, (par exemple, un financement avec les prêts d'ajustement structurel 
comme dans le cas du Cambodge et du Cameroun), la complexité se trouve multipliée. Enfin, il y a des situations dans lesquelles le processus du changement est une réponse à la pression de la communauté internationale dans les états dits " défaillants " dans lesquels les illégalités dans le secteur de la forêt étaient liées à la guerre et au non respect des droits de l'Homme (comme au Cambodge, au Libéria et en RDC). Dans ces cas, le secteur forestier peut être inhabituellement soumis à l'influence internationale, avec la possibilité d'une appropriation extrêmement limitée (comme dans le moratoire des Nations Unies sur l'exploitation forestière au Liberia de 2003 à 2006). Le principal défi dans ces situations porte sur la transition vers la démocratie, et sur la façon de créer des électorats nationaux pour le changement dans des conditions où les règles ont été fixées par des acteurs extérieurs.

\section{Principe 4 : Adoption d'une approche système et accent sur la distribution et l'équilibre des pouvoirs}

Une fois les premiers problèmes résolus, la prochaine étape pour les décideurs politiques consistera à évaluer la mesure dans laquelle le système de contrôle et d'administration de la forêt peut être amélioré. Si le pronostic est négatif, il peut être nécessaire d'effectuer une réforme complète. Une décision dans ce sens dépendrait fortement du niveau de la volonté politique dans la société - ceci étant considéré comme un engagement national de haut niveau pour une meilleure gouvernance du secteur de la forêt. Ces décisions doivent être étayées par une bonne analyse politique. Le scénario à éviter est celui qui oblige les acteurs ayant le moins de pouvoir à fournir les résultats souhaités. Comme l'attestent plusieurs de ces cas, (le Brésil, l'Equateur et les Philippines, par exemple), la volonté politique peut être inégale dans le temps, et il faut saisir les opportunités lorsqu'elles se présentent, en espérant qu'une fois le mouvement enclenché, l'élan pourra être maintenu.

Même lorsque des réformes plus limitées sont envisagées, la volonté politique pourrait continuer à s’avérer une préoccupation importante car il se peut qu'il soit nécessaire de prévoir un niveau très élevé de financement initial et d'investissement humain pour lancer le processus (comme dans le cas du Ghana). De la même façon (et plus couramment pour l'ensemble des cas examinés), l'absence de volonté politique est extrêmement problématique étant donné la suprématie de l'état souverain sur les ressources forestières. De nombreuses études de cas concernent des scénarios qui cherchent à s'en sortir avec l'absence de volonté politique, cherchant de nouveaux moyens de soutenir les processus de transformation et de générer la résilience à supporter des défis potentiels. La façon dont l'information est gérée pourrait dans ce cas être un élément central, car ceci est essentiel à la crédibilité du système de vérification.

La décentralisation politique pourrait également aider à générer la volonté politique, mais de façon perverse. En Indonésie, par exemple, les tensions entre les niveaux ministériels nationaux, provincial et de district ont servi à accroître l'intérêt des autorités centrales pour la vérification, qui autrement sont privées d'informations sur les activités au niveau régional. Cependant, la transparence est encore limitée pour les décisions de vérification, 
qui restent le privilège exclusif du Ministère des forêts, et donc la prolifération des acteurs politiques n’aboutit pas nécessairement à une réelle réforme de gouvernance.

Les systèmes de traitement de l'information sont solides dans le cas de l'Equateur et du Cameroun, et ce dernier est particulièrement intéressant en ce sens qu'il y a eu une évolution très positive à un stade précoce, passant d'un régime de suivi imposé de l'extérieur à un système de gestion et d'action sur l'information avec la possibilité d'une intégration à long terme dans le système de gouvernement national. Le comité de lecture du Cameroun (qui lit et se prononce sur l'information du moniteur indépendant) constituait une innovation institutionnelle importante, même si les pouvoirs excessifs du ministère de la forêt affaiblissent son objectif de contribuer à une transparence améliorée. Bien que ce potentiel positif n'ait pas été entièrement maintenu, le principe s'applique toujours.

L'administration, le suivi, l'audit, l'application et la surveillance externe sont des fonctions potentiellement conflictuelles. Certains des systèmes de vérification comportent (ou aspirent à développer) un niveau important de séparation des fonctions de sorte que les cubes sont un ensemble d'acteurs autonomes, chacun ayant un pouvoir sur différentes fonctions. Cependant, la majorité des systèmes concentrent toujours les fonctions dans les mains de quelques acteurs, avec en général des implications négatives sur la qualité de la gouvernance. Un principe général important dans la conception du système de vérification pourrait consister à réduire la concentration des fonctions et à essayer de les distribuer entre les instances autonomes qui ne relèvent pas de la même chaîne de contrôle et de commandement. Ce principe est clairement respecté dans la conception du nouveau système de validation du bois d' œuvre au Ghana, qui devrait attribuer les fonctions de base de l'administration, de la validation, de l'audit et de la surveillance externe à quatre institutions séparées, autonomes.

Une exigence importante est la séparation des pouvoirs entre la collecte de données, la gestion de l'information et les activités d'autorisation. Ceci garantit à son tour, une séparation claire des niveaux de décision - entre les niveaux politiques et techniques, et entre les domaines opérationnels de vérification et d'exécution. Le classement de Lang, indiqué au Chapitre 3, qui identifie trois éléments institutionnels comme exigences minimums pour un système satisfaisant de contrôle de la conformité, souligne ce point. Il indique (1996) la nécessité de :

- Une institution pour collecter des données ;

- Un «mécanisme d'examen " pour évaluer et interpréter des données et offrir des informations utiles; et

- Une fonction "prise de mesures ", réservée à une instance politique, "l'autorité suprême ", qui agit sur les recommandations du mécanisme d'examen.

L'accent est mis sur la vérification en tant que processus, et différentes fonctions sont exécutées séparément par des institutions discrètes. Un tel système de gestion d'information offre une fonction officielle de retour d'informations à travers laquelle les informations 
générées par les acteurs de la vérification (comme les moniteurs, les observateurs, les auditeurs et les avocats de l'environnement) peuvent être validées et par la suite renvoyées aux institutions afin de contribuer au processus de réforme.

Cependant, il y a trois exigences supplémentaires qui doivent également être réunies pour offrir la garantie que l'arrangement contribuera à améliorer la gouvernance. La première est un mécanisme d'appel réalisable pour assurer que les erreurs peuvent être corrigées et, plus généralement, pour garder à bord les principaux acteurs de la communauté des ONG et de l'industrie forestière. Le secteur forestier est connu pour l'imprécision de la base d'informations (les cartes sont souvent particulièrement peu fiables) et si ceci peut être sciemment utilisé par des éléments non scrupuleux de l'industrie, il existe toujours un besoin de s'assurer que les normes de base de l'impartialité sont appliquées des deux côtés.

La deuxième exigence supplémentaire est un mécanisme pour garantir que l'information, une fois validée, est mise à la disposition du public de façon transparente et responsable. Ceci est loin d'être la norme. Par exemple, la pratique générale en Malaisie ne met pas systématiquement les rapports d'audit publics à la disposition du public (même si ils sont disponibles à la demande) et a engendré une transparence limitée. De façon intéressante, à Sabah, l'industrie elle-même a demandé une plus grande transparence, aboutissant à la nomination d'une tierce partie en tant qu'auditeur ad hoc. La nécessité de transparence n'implique pas nécessairement que les groupes de plaidoyer ont la liberté totale de diffuser l'information de la manière qui semble leur convenir - le mécanisme doit également se conformer aux exigences de bonne gouvernance du mécanisme d'appel (il est beaucoup plus facile d'utiliser l'information pour détruire la crédibilité des acteurs dans le secteur de la forêt que de l'étoffer), et ceci peut exiger un certain élément de confidentialité, en particulier dans les premières étapes. En outre, se posent les questions de responsabilité juridique, à la fois pour les acteurs officiels (ministres de la forêt, par exemple) et les acteurs privés. Ceci étant dit, l'industrie de la forêt s'est en général révélée beaucoup moins litigieuse que, disons l'industrie des diamants, et ceci a offert un certain champ de liberté aux groupes de plaidoyer.

Aussi nécessaires que soient ces étapes, elles sont néanmoins jalonnées de difficultés et de possibilités de manipulation, ce qui souligne l'importance de la troisième exigence - que les procédures de gestion des informations soient elles-mêmes intégrées dans un système de gouvernance plus large et fonctionnant correctement. Un des principaux défis dans un certain nombres de cas examinés (le Cambodge souligne le point, même si la question est assez générale) est que la "fonction de prise de mesures " (ou " l'autorité suprême ") doit bénéficier de la confiance publique. Sinon, la frontière entre les niveaux techniques et politiques pourrait se trouver fortement compromise. Certaines mesures techniques peuvent être prises pour limiter les dégâts (par exemple, réunir régulièrement et automatiquement le comité de lecture, pour éviter que des personnes n'utilisent leurs pouvoirs pour bloquer le processus de prise de décision), mais une leçon qui en découle est que des tentatives isolées de réformer la gouvernance de la forêt auront du mal à transcender les problèmes dans l'environnement plus large de la gouvernance, et ceux- 
ci pourraient devoir être réglés de façon concomitante (de préférence avant) avec les préoccupations plus spécifiques au secteur de la forêt.

Dans certaines études de cas VERIFOR, le principe de séparation des pouvoirs est à peine évident. En général, l'agence publique concernée (à travers ses différents départements, unités, bureaux locaux ou d'autres acteurs) cherche à garder un rôle de supervision important et un pouvoir sur les foncions clés. Ceci pourrait s'avérer un compromis acceptable dans certains cas (par exemple, des petits pays avec des problèmes de gestion non controversés et un pouvoir de générer des revenus limité). Cependant, cette concentration des fonctions sera en conflit avec une bonne gouvernance. Le souci d'éviter un tel conflit sous-tend la décision prise de ne pas donner au British Columbia Forest Practices Board le droit de sanctionner et d'exécuter. Considérée par certains critiques comme une faiblesse, cette séparation des fonctions est considérée par d'autres comme une force, lorsque l'on adopte une vue plus large, mettant le Conseil en conformité avec les pratiques sectorielles bien établies concernant la séparation entre l'évaluation de la conformité et la mise en œuvre (voir le Chapitre 3).

Si la surconcentration de fonctions entre les mains de l'état peut s'avérer un problème, l'expérience met en garde contre les tentatives de déléguer des fonctions qui pourraient être considérées comme des compétences publiques. Les « fonctions régaliennes de l'état » (c.à-d., la réglementation, l'adjudication et l'autorisation) ne peuvent être déléguées qu'aux organisations privées si le gouvernement maintient une surveillance et un contrôle. Le Costa Rica et l'Equateur sont des exemples opposés de ces tentatives. Les défis politiques constants qui se sont posés au système équatorien sous traité soulignent l'importance de préciser préalablement les fonctions exclusives à l'état dans son exercice de l'autorité publique et qui ne peuvent donc pas être fournies par un acteur privé ou sociétal. Mais il est également nécessaire d'être clair sur la performance des rôles extérieurs. Les fonctions à déléguer aux acteurs privés et à d'autres acteurs non étatiques devraient être cohérentes avec leurs rôles spécifiques. Beaucoup se demanderaient si par exemple, les instances de surveillance environnementale des ONG sont des candidats idéals pour un travail de vérification basé sur le commerce, car leur évaluation de l'approche de la concession industrielle est biaisée ; ils y sont souvent implacablement opposés. De même, les rôles et responsabilités spécifiques de la police et du judiciaire dans le processus d'autorisation doivent être prudemment délimités. Etant donné l'intérêt commercial important pour le secteur de la forêt, le potentiel de l'ingérence politique est important.

On considère que les mécanismes de conformité (c.-à-d. les procédures pour faire face à une non conformité alléguée et réelle, incluant l'adjudication et la mise en ouvre) devraient rester entre les mains de l'état, même si en réalité aujourd'hui, l'activisme environnemental semble transférer certains de ces pouvoirs (notamment une certaine adjudication de facto) aux éléments des acteurs de la société civile sans nécessairement des responsabilités ou des mécanismes d'appel. Alors que ceci trouve une justification lorsque la légitimité de l'état est mise en doute, il n'apporte pas nécessairement lui-même " une bonne gouvernance ". C’est un des dilemmes de l'énigme de la " mauvaise gouvernance ». 


\section{Principe 5 : Large participation pour accroître l'efficacité des processus de vérification}

Inclure l'agenda de l'exploitation illégale dans les priorités d'un processus national comme le Programme national des forêts (PNF) montre que le système de vérification bénéficie d'un mandat politique solide, de l'appropriation de la société, et d'une certaine légitimité. Un mandat clair donné au gouvernement pour conduire et coordonner la mise en œuvre du PNF et entreprendre une action dans le sens de la mise en œuvre de tous les accords conclus, serait également très bénéfique. Cependant, il est probable que deux obstacles s'opposeront à un tel engagement national dans la vérification des forêts. D'abord, comme le montrent la majorité des études de cas actuelles, les processus PNF ont à ce jour rarement abordé les questions de conception relatives aux systèmes de vérification des forêts, en termes de rôles, d'architecture institutionnelle ou de normes de légalité. Un processus de dialogue supplémentaire, la confiance et le renforcement du consensus peuvent s'avérer nécessaires pour aborder ces préoccupations. Le deuxième point est plus essentiel. Il concerne la difficulté à générer une certaine forme de clôture politique (clôture des débats et prise de décisions) sur la gouvernance de la forêt, en particulier sous les tropiques où les intérêts des parties prenantes sont multiples et fortement polarisés, et certaines parties semblent préférer bloquer un processus plutôt que de rechercher un compromis.

Aucune des études de cas de la Partie B ni l'analyse détaillée des processus multilatéraux (PMP) du Chapitre 24 n'offre de formule magique permettant de résoudre ces problèmes. Les structures des classes sociales sont souvent complexes, et un large éventail de forces, pour beaucoup en dehors du secteur de la forêt, pourrait devoir se regrouper avant que la société civile n'obtienne un espace suffisant pour sérieusement influencer les processus politiques ou agir de façon indépendante par rapport à l'élite politique dominante. Cependant, on peut en tirer un certain nombre d'enseignements utiles.

Tout d'abord, il est important d'être clair par rapport au mécanisme participatif et de savoir s'il doit fonctionner en tant que processus de décision (dans lequel on peut mettre un terme à une question) ou en tant qu'opportunité de dialogue pour informer les décisions futures. L'utilisation de PMP en tant que mécanisme de prise de décision (par opposition à un forum de dialogue) est préférée dans certaines circonstances où l'instance multilatérale possède déjà un mandat clair pour la prise de décisions et la mise en œuvre des décisions qu'elle a prises. Ceci dépendra à la fois du sujet en discussion et du contexte, la source d'autorité nécessaire pour mettre un terme à une décision variant en fonction de la question et de la tradition juridique et administrative. Cette tradition déterminera les fonctions qui sont du domaine exclusif de l'état et qui peuvent, légalement ou constitutionnellement, être sous traitées à des instances pluralistes. Dans le cas des normes de légalité par exemple, il est en général nécessaire d'obtenir une approbation législative ou judiciaire du processus de définition, et donc le dernier mot revient au gouvernement.

Deuxièmement, comme le montre le cas de l'Equateur, un engagement dans un processus multilatéral impliquant diverses parties prenantes peut aboutir à une impasse 
politique. Lorsque cette impasse politique concerne des questions fondamentales avec des ramifications allant au-delà du secteur de la forêt, sur des questions que certaines ONG et la société civile considèrent non négociables (le plus évidemment, les droits des peuples autochtones) il sera difficile d'éviter de se retrouver dans une impasse. Lorsque les principaux acteurs le comprennent, y consentent et l'acceptent, si le processus consultatif n'aboutit à aucun accord, il incombe à l'autorité forestière de prendre la décision finale, il existe une pression intégrée pour accepter un compromis et l'énigme de la clôture des débats et de la prise de décisions peut être dépassée. Le Costa Rica constitue un modèle du genre, en ce sens qu'il est demandé aux parties prenantes de disposer d'un agenda ouvert avant de s'engager dans des délibérations, et l'autorité finale (le gouvernement national) a une légitimité démocratique considérable. Cependant, la situation de ce pays est exceptionnelle, tant en termes de solidité du fonctionnement démocratique que du large éventail de bénéficiaires. Avec la forte dépendance du Costa Rica à l'écotourisme et son statut de pays à revenu moyen, les avantages y sont appréciés bien plus largement que cela n'est souvent le cas ailleurs.

Troisièmement, la crédibilité d'un PMP peut être accrue en portant une attention particulière au type d'acteurs inclus dans le processus. Des PMP efficaces exigent un soutien institutionnel ou politique au niveau des décisions à mettre en œuvre. Le cas de l'Equateur montre que le processus doit être à la fois participatif et inclusif. Bien que participative, l'approche adoptée dans ce pays n'englobait pas les principaux acteurs qui auraient pu offrir un soutien utile lorsqu'elle s'est trouvée confrontée à l'opposition, soit du personnel du ministère soit des militants de la société civile. Les groupes de populations autochtones, les propriétaires de forêt et le personnel d'autres institutions du gouvernement (sans enjeu personnel dans le statu quo) auraient tous pu jouer ces rôles et prendre fait et cause.

Et enfin, l'exemple de l'Equateur souligne également la nécessité de savoir qui perd dans le processus de réforme, et de chercher à les retenir. C'est là une des limites importantes à l'utilisation des conditionnalités des bailleurs de fonds pour poursuivre une réforme de la politique, car ceci écarte la prise de décision du domaine politique national, laissant les divergences d'intérêt irrésolues.

En résumé, s'il faut être prudent en supposant que les conditions préalables à la vérification des forêts peuvent être facilement créées en partant de zéro, il est clair que la conception est importante, et qu'un processus participatif peut compenser dans une certaine mesure l'absence de volonté politique initiale. Ceci aura probablement des avantages positifs le long de la chaîne de prise de décisions, lorsqu'il faudra prendre des décisions de vérification controversées. Un dialogue ouvert et transparent pour une meilleure exécution de la loi forestière et de la gouvernance est un élément important, en particulier lorsque ceci fait partie d'un processus politique permanent et plus inclusif. Le dialogue peut aider à créer une meilleure communication, crédibilité et confiance entre toutes les parties prenantes impliquées dans la chaîne de production de bois d'œuvre. La crédibilité peut être améliorée dans un processus de confiance et d'espoir entre les acteurs de la société - il n'est pas toujours nécessaire d'impliquer de nouvelles institutions (ou 
des institutions externes). C'est une question qui risque d'être noyée dans la campagne pour imposer des accords déterminés de l'extérieur. Un système de vérification ne sert pas toujours ou uniquement les besoins d'un marché extérieur du bois d'œuvre, comme le montrent les cas de l'Amérique centrale et du sud, et la crédibilité et l'implication locales pourraient se révéler des atouts importants qui précèdent ces intérêts extérieurs, et devraient donc être encouragés en premier lorsque cela est possible.

\section{Principe 6 : Clarté des normes d'évaluation}

Les normes de légalité doivent être claires et simples et s'inscrire dans les capacités du système à fonctionner sans recours à l'expertise qui fait défaut dans la sphère officielle. Comme le montre le Chapitre 19, un effort de vérification efficace se base sur une norme claire et sans ambiguïté par rapport à laquelle la vérification se fait. Cependant, il existe de nombreux cas dans lesquels le cadre juridique sous jacent à la gestion de la forêt n'a pas progressé jusqu'à un point où une série de normes claires peut être formulée, et encore moins convertie en critères et indicateurs. Dans ces situations, un processus expressément dédié à la formulation d'une norme de légalité est nécessaire tant pour conditionner la viabilité et l'efficience du système de vérification, que pour garantir et renforcer la robustesse essentielle à sa survie future.

Dans les contextes examinés, les défis que cela représente semblent autant politiques que techniques. Même lorsque le problème est largement reconnu dans la société (comme par exemple en Indonésie où la législation concernant la forêt est abondante) il peut néanmoins être difficile de trouver des catalyseurs pour développer et affiner une norme nationale. Lorsque ces catalyseurs concernent des processus imposés de l'extérieur (par exemple les AVP), le défi politique peut être plus important. Dans le cas du Ghana, par exemple, il y a eu une résistance dés le début de la société civile à la notion de soustraction d'une norme de légalité dans la loi, essentiellement pour se conformer aux exigences d'un AVP initié à l'extérieur (même si la résistance a par la suite diminué alors que se développait un processus plus participatif). Dans l'idéal, un processus de réforme juridique précédera la clarification de la norme, comme dans le cas du Brésil. L'intégration dans un processus politique national facilitera également l'amélioration et la rationalisation qu'exige cette clarification, comme cela s'est produit en Equateur où les critères et les indicateurs nationaux sont démontés jusqu'au plus petit à des fins de vérification. En Colombie britannique au Canada, les normes de légalité existant étaient considérées trop rigides et prescriptibles - d'où le mouvement en direction d'un système plus basé sur les résultats. Une telle évolution n'est possible que lorsque la bonne volonté de l'industrie inspire confiance, et qu'il existe une volonté de lui céder la responsabilité des moyens de réaliser les résultats souhaités. Lorsque l'industrie inspire du scepticisme sur tous les fronts, un tel mouvement peut être mal avisé.

Un certain nombre de cas soulignent l'importance d'une clarté non seulement des normes de légalité claires mais également des sanctions qui peuvent être utilisées pour inciter à la conformité. Le cas du Costa Rica souligne ce point et indique aussi la nécessité de lier les sanctions aux incitations, point qui est examiné plus loin, dans le principe 7. 
Il faut garder à l'esprit un certain nombre de points pour que la norme de légalité ait une utilité pratique. Il est essentiel que la norme de légalité soit acceptée et comprise par les acteurs qui vont devoir s'y conformer. Un processus participatif avec diverses parties prenantes tend à disposer d'un espace limité pour traiter les questions techniques. Ceci exige des normes forestières en nombre limité et simples. Cependant, cet impératif pratique doit être comparé aux intérêts sous jacents que le processus de vérification est supposé traiter. Pour de nombreuses parties prenantes, la préoccupation principale porte sur les avantages sociaux, et il est donc essentiel de résoudre cette préoccupation pour que le processus politique se conclue positivement et pour que les impacts sur le développement soient positifs. La plus grande préoccupation concerne les pressions des activistes de la société civile pour s'intéresser à des problèmes sociaux importants comme les droits des populations indigènes, mais cette question n'est qu'une composante (même si importante) de la variété des questions environnementales et sociales qui pourraient se retrouver mises sur la touche si l'accent est excessivement mis sur le technique et le pratique.

L'expérience avec les normes pour une gestion durable de la forêt donne un aperçu utile de ces débats politiques. Elargir la sphère d'intérêt pour inclure les questions sociales et environnementales a constitué une grande avancée dans le traitement des normes de certification pour une GDF. Ceci était en grande partie le résultat des pressions de la société civile et on peut supposer que les mêmes pressions s'appliqueront à la vérification de la légalité.

Les faiblesses dans la pratique actuelle mettent l'accent par anticipation sur certains dangers. Par exemple, la législation environnementale dans un nombre de cas stipule que les concessions forestières devraient faire l'objet d'une évaluation sur l'impact environnemental (EIE). Ceci ne se produit souvent pas (comme par exemple au Ghana) et même là où l'échec est reconnu, il y a rarement de tentative de réconcilier les exigences réglementaires avec la législation forestière existante (par exemple, en décidant qu'un plan valable de gestion de la forêt serait une alternative acceptable). De même, les lois sociales sont souvent ignorées, par exemple, concernant la main d' œuvre et les pratiques d'emploi, notamment la conformité avec les régimes d'assurance et de rémunération. Ainsi, le problème peut ne pas être juste l'échec à s'engager dans des agendas sociaux de longue date comme les droits indigènes, mais à un niveau plus terre à terre, la conformité avec la législation existante.

Une des barrières à l'avancement ici est le fait que la législation nationale concernée est souvent extrêmement large. Une approche pratique de cette difficulté impliquerait la reconnaissance des objectifs économiques, environnementaux et sociaux de la gestion de la forêt comme dans tous les principaux traités internationaux liés à la forêt et les normes du secteur privé. Ceci signifierait que les activités de vérification devraient examiner plus que la simple législation forestière. Le suivi de la conformité avec les EIE, les normes existantes du travail et du bien-être et la législation de la santé et de la sécurité, serait entrepris dans le cadre des activités de vérification. Ceci ne serait pas nécessairement favorable aux préoccupations de multinationales plus importantes et bien financées. La 
législation environnementale et sociale prend souvent en compte la taille de l'entreprise lors de la fixation des normes, et il existe des expériences extra sectorielles intéressantes (par exemple, concernant la production artisanale de spécialités de viandes fumées en Europe du sud) qui indiquent également la capacité de la législation à satisfaire les besoins des petites et moyennes entreprises sans renoncer aux garanties exigées.

\section{Principe 7 : Créer des incitations pour se conformer et faire rapport}

Un dilemme sous jacent à la vérification de la forêt est l'accent qui tend à être mis sur les coupeurs de bois d'œuvre qui opèrent légalement, car la vérification n'offre que des possibilités limitées d'action pour contrôler la conversion de la forêt et le bois d'œuvre illégal clandestin. Il faut donc prendre grand soin à s'assurer que les opérateurs légaux sont encouragés à se conformer à la législation et à faire un compte rendu, et que leur transparence est récompensée positivement.

La question des incitations s'applique à deux niveaux : l'industrie (l'auditée) et le système. Les études de cas extra sectoriels soulignent la valeur stratégique de l'accent mis sur les incitations à la conformité, en gardant bien l'œil sur les facteurs qui pourraient induire la non-conformité et la migration vers les non parties à l'accord de vérification. Les incitations pour l'industrie doivent être claires. Les témoignages montrent que les éléments de l'industrie forestière qui sont bien intégrés dans les marchés internationaux sont soucieux de leur réputation, et voient cela comme un aspect essentiel de la gestion des risques. Ceci est peut-être moins pressant pour les éléments moins capitalisés de l'industrie locale, qui ont moins d'investissements dans le secteur et moins à gagner de la conformité aux normes internationales. Le danger est qu'une réglementation inappropriée aboutisse à une bifurcation du secteur, assainissant le commerce international mais ne contribuant pas nécessairement à la durabilité de l'environnement. Ceci a été un problème dans l'industrie de la viande, où un commerce d'exportation florissant, se conformant à des normes techniques peut exister en parallèle du marché local, qui accorde peu d'attention aux normes internationales de santé humaine et animale en vigueur. Dans cette situation, c'est le public national qui est perdant. Dans le secteur de la forêt, les effets peuvent être négatifs tant pour la population nationale que (en raison de l'érosion des stocks) pour les intérêts à long terme de l'industrie internationale. Le cas du Ghana est particulièrement instructif, en ce sens que la Commission forestière a très tôt adopté un principe d'action clair établissant que les besoins du secteur seraient traités dans leur totalité, sans discrimination en faveur du commerce d'exportation. En dehors des avantages nationaux immédiats, cette approche pourrait probablement favoriser une estimation réaliste des opportunités commerciales alternatives, intercontinentales (par exemple destinée aux marchés européens) ou régionales (destinée aux marchés comme le Nigeria).

Les responsables doivent également être encouragés. Dans un certain nombre de cas du secteur forestier (Cambodge et Cameroun par exemple), l'absence de volonté à se conformer de la part des fonctionnaires de niveaux peu élevés avait tendance à être 
interprétée par les manifestants des ONG comme une preuve de corruption, et ceci a tempéré la façon dont ces activistes ont approché la question de la réforme. La corruption est souvent répandue dans le secteur, mais l'attitude des bureaucrates pourrait être conditionnée autant par un désir d'auto préservation que de gain financier. Là encore, ceci encouragerait à mettre l'accent sur la réforme des structures de gouvernance de la forêt plutôt que sur des interventions à bas niveau qui pourraient bien aboutir à une forme de " reproche aux victimes".

Un dilemme plutôt différent porte sur la question des sanctions et pénalités. Des normes claires exigent des sanctions claires en cas de non-conformité. Même lorsque les normes sont acceptées, il peut y avoir des litiges sur la catégorisation juridique - en particulier sur le code juridique (administratif ou pénal) qui s'applique. L'inconvénient avec la voie administrative c'est que la décision reste entre les mains des institutions forestières. Lorsque (comme au Cameroun), c'est le fonctionnement de celles-ci qui est surtout mis en doute, la capacité de la vérification de contribuer à une réforme de la gouvernance peut être limitée. Un problème supplémentaire est que les pénalités sont rarement à la mesure de la valeur du bois d'œuvre, et ne découragent pas vraiment la non-conformité aux normes. L'énigme de la gouvernance s'applique à nouveau. Les pays qui font montre d'une gouvernance médiocre du secteur de la forêt sont ceux dans lesquels la dynamique croisée du secteur milite contre la réforme. Une industrie qui sait que quels que soient ses délits, les conséquences seront probablement dérisoires comparées aux gains illicites, subit peu de pressions pour modifier son comportement dans des directions progressistes. Le message qui en découle est que des mesures de vérification coûteuses et qui demandent du temps doivent s'inscrire dans de plus vastes programmes de réforme de la gouvernance de la forêt.

Concernant les mesures incitatives pour l'industrie afin de fournir des informations pour la vérification (elle-même un élément important de transparence et de " bonne gouvernance »), le secteur de la forêt est particulièrement mal placé car de nombreux opérateurs ne sont pas des entreprises cotées en bourse et ont tout intérêt à ne pas divulguer des informations qui pourraient compromettre leur avantage sur le marché. Il incombe clairement à ceux qui souhaitent discipliner l'industrie de montrer la valeur d'une culture basée sur les règles et la gestion prudente de l'information - des flux d'informations ouverts là où cela est approprié, mais également une garantie de confidentialité là où cela ne l'est pas. Le terme " limitation du mandat " dans son sens le plus restreint peut comporter des exigences, et le principal défi peut se poser aux activistes de la société civile qui souhaiteraient s'engager dans l'industrie de la vérification. Contrairement à la recherche sur la gouvernance, le travail de vérification n'offre pas nécessairement d'opportunités sans limites pour " ouvrir " les économies politiques des états qui échouent. Une approche plus restrictive et plus axée sur l'industrie serait probablement exigée. Le même type de raisonnement encouragerait l'accent sur " la gestion de la conformité " (dans le sens appliqué par exemple dans les cas de la Malaisie et de la Colombie britannique au Canada) plutôt que sur "l'exécution de la conformité ». Cette question est traitée plus en détail dans les paragraphes qui suivent. 


\section{Principe 8 : Surveillance indépendante}

Le principe de surveillance indépendante est valable à lui seul, mais complète également d'autres questions de principes déjà abordées, essentiellement la séparation des pouvoirs. Le fait que le secteur de la forêt soit souvent un monopole d'état, et marqué par une gouvernance médiocre en partie en conséquence de ceci, soulignel'importanceà introduire des acteurs et des points de vue indépendants dans la conception du système de vérification. Les origines particulières du mouvement de vérification ont eu tendance à encourager l'accent sur une étape de la séquence (suivi indépendant ou observation indépendante) et sur les acteurs qui pourraient le mieux sauvegarder cette étape. Cependant, les questions sont plus générales et se rapportent non seulement aux dernières étapes de la vérification, mais également à tous les acteurs et aux interventions en amont qui doivent être protégées des influences politiques et financières. Dans certaines situations, l'indépendance est compromise par le fait que l'industrie forestière conserve trop de responsabilités - par exemple, là où elle est responsable non seulement de ses propres activités, mais également de régler les salaires des superviseurs sur le terrain et des régents de la forêt en Equateur. Il est nécessaire de faire une distinction claire entre la gestion de la forêt et les activités réglementaires. Combiner les rôles de gestion (par exemple, l'élaboration de plans de gestion) et les rôles réglementaires (par exemple, inspecter et certifier la mise en ouvre correcte de ces plans) aboutit inévitablement à un conflit d'intérêt d'un type qui doit être activement évité plutôt que simplement " géré ».

Ainsi, sauvegarder l'indépendance est aussi importante avec les contrôleurs locaux sur le terrain en Amérique central et du sud qu'avec les observateurs indépendants qui pourraient se voir demander de signer des AVP. Toutes les indications laissent à penser que si ces rôles doivent être acceptés par tous ceux qui ont un enjeu légitime dans le secteur, alors il faut rechercher «l'indépendance " dans ses trois dimensions - neutralité et impartialité ainsi qu'indépendance (voir le Chapitre 20) - et l'indépendance des 'suspects habituels' (essentiellement le gouvernement producteur et l'industrie) n'est pas suffisante pour apporter la crédibilité. Ceci a des implications tant pour les gouvernements (intégrant la " vérification indépendante " des filiales publiques en Malaisie, par exemple) et pour les ONG (l'activisme anti-industrie semblerait incompatible avec les rôle officiels du FMI).

Là où l'état producteur a conservé un solide contrôle du processus de vérification, l'option préférée avait tendance à être proche du gouvernement, avec les limites que cela implique. Ainsi, les Instituts indonésiens indépendants de vérification (LPI) sont supposés être des entreprises d'évaluation indépendantes (accréditées par le Ministère), mais leur indépendance est douteuse parce que leurs activités sont financées par le Département de la Forêt et la décision définitive pour les permis d'extension et de révocation est toujours entre les mains du Ministère, sans garantie de transparence. Un cas plus urgent pourrait être celui de la Malaisie, où l'accent a été mis sur l'audit de routine, même si ceci est souvent confié à des entreprises liées à l'état, semi privées comme Harwood ou le Malaysian Timber Certification Council (Conseil malaisien de certification du bois d'œuvre) (MTCC). Ceci compromet l'indépendance et aboutit à se détourner pour aller vers un FSC tierce partie et les certificateurs et les vérificateurs tierce partie ad hoc. Simultanément, l'indépendance du MTCC est améliorée par l'établissement d'un fonds 
de dotation pour couvrir ses coûts opérationnels, même s'il restera probablement sous l'autorité du Ministère fédéral des industries de plantations et des produits de base.

Là où l'aide communautaire externe conduit le processus, l'accent avait tendance à être mis sur le contrôle indépendant des forêts (CIF) entrepris par des ONG axées sur le plaidoyer. C'est une façon d'offrir la crédibilité aux acteurs sceptiques et qui a une valeur particulière lorsqu'il existe des arguments légitimes pour " démarrer " un processus de réforme de la gouvernance, et sortir l'industrie et les autorités de l'auto satisfaction. Cependant, du moins à ce jour, le CIF entrepris par des ONG axées sur le plaidoyer n'a pas nécessairement été un moyen très efficace de créer une appropriation nationale dans les sociétés productrices, et s'est révélé un mécanisme fragile pour soutenir la réforme de la gouvernance à long terme. Ceci ne peut seulement (ou essentiellement) être placé à la porte des CIF - ils ont fonctionné dans des environnements hostiles et leurs objectifs ont souvent été des objectifs d'investigation, clandestins, décourageant le partage d'informations dans les premières étapes. Dans plusieurs cas, la teneur générale des interventions plus larges dans lesquelles ils fonctionnaient constituait également une base infertile pour réaliser des impacts d'appropriation ou de gouvernance, en raison de leur négativité inhérente. Au Cambodge par exemple, le CIF fonctionnait dans le cadre d'un "système de compte rendu sur les crimes de la forêt " qui influençait négativement l'ensemble du système. Cette discrimination intégrée a eu tendance à accroître la vulnérabilité politique de ces efforts de suivi indépendants. Cependant, même en tant que principe général, se reposer sur des acteurs individuels en tant que gardiens de la vertu morale semblerait naturellement déséquilibré, et dans l'ensemble trop enclin à opposer le vérificateur à l'état, dans une situation où la donne est largement défavorable aux premiers.

Sur les critères de durabilité entre autres, il est possible d'encourager une forme plus complexe de fourniture dans laquelle les acteurs individuels se renforcent et se contrôlent mutuellement - comme en Equateur, en Indonésie et aux Philippines. Le cas du Brésil est instructif car il englobe une série d'institutions publiques et fédérales et une série d'organismes publics, intégrés horizontalement et verticalement. Des opérations de contrôle conjointes encouragent son intégrité. Cependant, un tel système exige une forte volonté politique.

Là où une telle volonté fait défaut, d'autres approches composées peuvent s'avérer efficaces. Une implication multiple de la société civile a sans doute plus de potentiel d'émousser l'opposition d'éléments clés dans le gouvernement et de contourner une forte part de l'hostilité de l'industrie. Les institutions qui sont naturellement prédisposées à la transparence - comme les universités - n’ont pas été jusque là très impliquées dans la vérification (bien qu'il y ait des exceptions, comme pour le Costa Rica) mais l'intervention de tels acteurs aiderait non seulement à libérer la vérification d'une association excessive avec une distorsion 'anti-industrie' biaisée, mais pourrait aussi introduire un rythme et un cadre temporel plus adaptés aux longs cycles d'exploitation de la forêt. 
Le cas de la Colombie britannique est un exemple particulièrement intéressant d'indépendance consacré dans la législation. L'autorité se trouve à l'extérieur du secteur de la forêt et la responsabilité incombe au Cabinet, et non au Ministère de la forêt. Les membres du Forest Practices Board (FPB) agissent en leurs capacités personnelles et ne représentent pas les parties prenantes. Il existe une procédure d'appel confidentielle pour soutenir la confiance de l'industrie et tout l'arrangement est limité et très professionnel dans le ton. Le Conseil rend compte directement au public, et les acteurs politiques n'ont pas le pouvoir d'interrompre le flux d'information. Il livre ses résultats comme il le juge (quelquefois en faveur d'une partie, quelquefois en faveur d'une autre), et opère en conformité avec toutes les exigences d'indépendance (c.-à-d., indépendance, impartialité et neutralité). Le FPB ne fait pas de vérification de lui-même. Il fonctionne au sein d'un système de composantes agissant l'une sur l'autre, incluant d'autres acteurs potentiellement impartiaux comme le gouvernement, le secteur privé et les diverses composantes d'une société civile forte.

Mettre en place des arrangements financiers pour assurer que l'ensemble du système peut être suffisamment et durablement financé est une exigence importante pour réussir. Aligner les ressources et les capacités pendant la conception du système est essentiel, et peut aider à soutenir une répartition des pouvoirs appropriée et efficace. Le financement des opérations de vérification est souvent problématique et peut compromettre l'indépendance. Il peut s'agir quelquefois de choisir l'option financement qui est la moins mauvaise, car les solutions optimales sont rarement disponibles. En Equateur, le financement de Vigilancia Verde, la coalition public/privé chargée de surveiller le transport de produits de la forêt et de la faune, provenait en partie de subventions des donateurs et d'entreprises, ce qui a compromis l'indépendance de cette instance, et $50 \%$ de la vente du bois d'œuvre mis aux enchères, ce qui aurait pu avoir un effet incitatif perverse en faveur de l'illégalité (car la détection de bois d'œuvre illégal est devenue plus rentable que la prévention). Les membres du Conseil de la forêt étaient en général payés par les propriétaires de la forêt ou les commerçants de bois d'œuvre et étaient donc sensibles à l'idée qu'ils étaient trop proches de leurs clients. La société privée SGS chargée de l'administration et de la vérification était également payée par les utilisateurs et tout aussi vulnérable. Cependant, si ces acteurs avaient été payés par le Ministère de l'environnement, ils auraient été beaucoup plus vulnérables aux pressions politiques de haut niveau et à d'autres machinations comme les retards de paiement et/ou le refus de payer.

Les Comités multi sectoriels de protection de la forêt (Multi-Sectoral Forest Protection Committees (MFPC)) aux Philippines fournissent un exemple de la façon dont un processus indépendant multilatéral pourrait être conçu et utilisé en tant que système de vérification forestier autorisé par l'état bénéficiant d'un niveau d'appropriation élevé, de transparence et de crédibilité en raison du large éventail d'acteurs et d'intérêts concernés. Cependant, une fois que le financement généreux de la Banque Mondiale se terminait, les MFPC étaient facilement ouverts à une capture par des intérêts spécifiques et bien financés comme les ONG environnementales. Des mécanismes de financement public 
adéquats pourraient aider à soutenir le modèle et éviter des associations partisanes, même s'ils pourraient fausser la participation en faveur de l'état.

\section{Principe 9 : Inclusion de toutes les étapes dans la chaîne de garde et efforts spéciaux pour sécuriser les étapes les plus vulnérables}

Ce principe comporte deux aspects. D’abord, un accent mis sur la vulnérabilité aux niveaux opérationnels, à travers le développement d'un système ou de systèmes pour suivre le mouvement des produits le long de la chaîne de valeur en mettant un accent particulier sur des points faibles comme le site d'abattage des arbres ; et deuxièmement un accent sur la vulnérabilité institutionnelle et la nécessité de protéger les acteurs vulnérables dans la chaîne de supervision. Le premier peut être largement traité avec des moyens techniques (voir par exemple, Crossin et al. 2003). Le second aspect, cependant, est plus compliqué et soumis aux forces politiques.

Des innovations technologiques récentes ont considérablement accru les possibilités de contrôle réglementaire, même si comme le note le Chapitre 23, les avancées technologiques à elles seules ne permettront pas d'améliorer la gouvernance. L'information est un outil puissant dans les mauvaises mains comme dans les bonnes, et il y a des cas (le cas de l'état de Mato Grosso au Brésil, par exemple) où une mauvaise gouvernance a déjoué les intentions d'investissement dans la technologie, et accru plutôt que limité les opportunités en faveur d'un comportement axé sur la rente. Le potentiel de la nouvelle technologie à concentrer les informations dans quelques mains au lieu de les répandre plus largement, en fait une lame à double tranchant. La technologie ne fournit pas non plus un moyen garanti de maîtriser les complexités des systèmes modernes de production, là où par exemple, le bois d'œuvre d'origines multiples et multinationales est mélangé dans la scierie ou l'installation de transformation, comme cela est souvent le cas en Asie du sud-est. La diversité institutionnelle au sein des autorités réglementaires peut également constituer une barrière à la gestion de l'information. Ainsi, au Cameroun, il existe quatre systèmes d'information pour suivre le mouvement des rondins (bûches) et du bois de construction, impliquant les ministres de la forêt et des finances et le service des douanes, mais les systèmes n'ont toujours pas été unifiés en dépit des nombreuses promesses du gouvernement. De même, au Costa Rica, les différentes instances responsables de la vérification de la forêt ne disposent pas d'un système d'informations pour vérifier l'information qu'ils recueillent grâce aux permis de récolte de bois d'œuvre, rendant difficile et inefficace le contrôle effectif de la forêt. Chaque acteur dans le système de vérification et de contrôle n'affecte qu'un petit segment de la chaîne de production.

Le message qui en découle est que l'introduction de technologies dans le système réglementaire de l'état doit répondre à l'exigence de gestion efficace de l'information, et ceci soulève des questions de nature essentiellement politiques, concernant la précision des limites de l'autorité et l'harmonisation des objectifs entre les services du gouvernement. 


\section{Principe 10: Incorporation des approches en faveur des pauvres dans la conception des systèmes de vérification}

Parmi les innovations examinées, plusieurs étaient fortement motivées par les préoccupations environnementales des ONG de conservation sans intérêt particulier pour le développement, et ceci soulève la question de la notion selon laquelle, un secteur « moderne » de la société civile s'assurera nécessairement que le système de vérification offert est équitable et « favorable aux pauvres ». Plutôt que d'être intégrés dans la conception du système de vérification, les agendas en faveur des pauvres ont tendance à être soit ajoutés, soit pire, bloqués par l'énormité des changements législatifs qui seraient impliqués dans la réforme des droits autochtones (comme dans les cas de l'Asie du sud est). Une difficulté supplémentaire avec des moteurs de l'environnement forts est que l'économie de base tend à être un élément marginal parmi les préoccupations majeures sur la durabilité environnementale. Ceci peut engendrer des coûts de conformité beaucoup plus élevés pour le petit producteur que pour les grands producteurs, comme en Equateur.

Les systèmes de vérification peuvent également s'avérer anti-pauvres par défaut, car l'élan donné par l'application de la loi peut facilement évoluer et se transformer en reproche aux victimes. Les personnes chargées d'appliquer la loi pourraient trouver plus facile de prendre des mesures contre les petits producteurs et les groupes communautaires que contre des membres ayant des liens politiques importants et qui sont à même de les sortir des difficultés. C'était le problème avec certains MFPC aux Philippines, où les petits opérateurs et les communautés pauvres représentaient des cibles beaucoup plus faciles pour la mise en œuvre que les élites puissantes, et au Cameroun, où la vulnérabilité de la foresterie communautaire à une capture par l'industrie forestière a été interprétée davantage comme un problème pour l'effort communautaire que pour l'industrie. Cependant, le problème est un problème général, en particulier là où l'évolution de carrière des responsables du gouvernement est plus le fait des relations que du mérite, et là où le marché est si restreint que rares sont ceux qui veulent faire un pas de travers.

\subsection{En résumé}

Il n'émerge des études de cas, aucun modèle, approche ou composante qui soit essentiel à la conception de la vérification. Cependant, les diverses systèmes analysés dans les chapitres précédents sont à leur façon, des réponses comparables aux pressions que connaît le secteur, permettant (selon " la matrice conceptuelle " du Tableau 17.1) des variations dans l'environnement juridique et institutionnel, les mandats politiques, et les objectifs de développement poursuivis au niveau public et privé. Les principes considérés ci-dessus peuvent être anticipés pour améliorer le fonctionnement de tout système de vérification, indépendamment de l'interaction de chacun de ces facteurs. Cependant, ils ne garantissent pas nécessairement son fonctionnement efficace, notamment dans les cas où les problèmes sous jacents de la gouvernance ne sont pas traités. 
Le dernier chapitre de ce livre reprend l'énigme de la gouvernance et évalue ses implications. La conclusion que l'on peut en tirer est que divers arrangements sont appropriés dans différentes situations et périodes de l'évolution d'un secteur, mais que c'est la constellation des acteurs plutôt que les compétences individuelles de chacun qui au final détermine leur efficacité. Et enfin, l'annexe présente un certain nombre de messages politiques qui peuvent découler des principes sus mentionnés et des témoignages dans la Partie B ; autant de points de référence à garder à l'esprit au moment de la conception ou de la mise en œuvre des systèmes de vérification. 


\section{Chapitre 26}

\section{Conclusions : vérification de la légalité et de la réforme de la gouvernance de la forêt}

\subsection{Introduction}

L'étude VERIFOR couvre un domaine émergeant de la recherche sur la politique de la forêt qui mélange les préoccupations spécifiques à la forêt et techniques et, les questions plus importantes sur la gouvernance mondiale, nationale et infranationale, les mécanismes de responsabilité publique nationale et internationale, et l'équilibre entre le contrôle de l'état souverain et la gestion internationale sur les biens publics internationaux. Le mouvement de vérification est encore dans une étape formative et on ne peut avec certitude se prononcer sur la direction qu'il suivra - soit dans un contexte national soit plus généralement - et à ce stade, toute évaluation de son efficacité ne peut être que suggestive. Le dernier chapitre de l'étude, réfléchit sur la nature des défis que présente la vérification ainsi que la preuve disponible sur l'efficacité des diverses initiatives politiques à ce jour. Sous-jacents à ces préoccupations, on trouve un intérêt en faveur de la transformation à deux niveaux : d'abord, passer d'arrangements de gouvernance sur la forêt qui sont souvent cohérents et bien intégrés, même si fortement transactionnels et inéquitable, à des systèmes basés sur les règles qui pourraient mieux servir les objectifs des sociétés de producteurs et de consommateurs; et deuxièmement, réconcilier deux types de normes, l'intérêt de l'industrie pour les avantages financiers et la réduction des risques et l'intérêt du public en général pour la légitimité sociale et environnementale et la durabilité.

\subsection{Equilibre entre le contrôle de l'état souverain et la gestion internationale des biens publics internationaux}

Les forêts et les arbres sont un volet puissant au niveau international. Ils fournissent un indicateur émotionnel du bien-être environnemental à l'échelon mondial. Ils possèdent un important volet biens publics internationaux, qui deviendra probablement encore plus important au fur et à mesure que les préoccupations sur le changement climatique s'inscrivent au centre de la politique mondiale. Les forêts tropicales en particulier sont largement considérées comme insuffisamment réglementées, voire non réglementées. Dans ces circonstances, l'intérêt international pour le sort des forêts du monde s'explique facilement.

Cependant, l'intérêt externe accru pour la question de l'exploitation illégale des forêts tropicales exige quelques explications. Il illustre un mouvement plus large qui est un des éléments les plus distinctifs de l'orientation que prend la mondialisation. Une grande partie 
des débats sur l'interface entre le capital national et étranger était axée sur les façons dont les intérêts des premiers devaient être gérés, dans le cadre de la mondialisation et de ses forces ainsi que des lois et des règlements nationaux. Dans de telles situations, l'intrusion des intérêts étrangers dans les affaires souveraines des états était justifiée par la nécessité de protéger leurs consommateurs de problèmes de contamination ou d'autres problèmes avec les produits en question. Les problèmes de santé, par exemple, justifient des niveaux élevés d'intervention dans les affaires des producteurs étrangers de viande, pour s'assurer qu'ils respectent les niveaux qu'attendent les consommateurs, et les questions de santé et de sécurité justifient de même l'implication internationale dans les politiques nationales sur l'énergie nucléaire. Dans les deux cas, le volet transnational du commerce est plus réglementé par les normes consommateurs que les normes producteurs, et il existe une logique claire dans cette relation déséquilibrée. Par opposition, il existe une catégorie de produits qui va en s'élargissant - le bois d'œuvre en est une, même s'il y en a actuellement beaucoup d'autres (de la banane au baseball, des vêtements aux tapis, et des diamants aux vêtements de designer) - où l'intérêt externe n'est ni matériel ni égoïste mais exprime une préoccupation morale sur les origines des produits plutôt que sur les effets matériels de leur consommation. Alors qu'il serait une erreur de présenter le mouvement du commerce équitable comme motivé par la seule valeur et non par le bien-être (en ce sens que le bienêtre des personnes touchées est la première des préoccupations), c'est essentiellement le bien-être de ceux qui sont directement affectés par le processus de production et non la consommation qui est en jeu. Et pourtant ce sont ces derniers (c.-̀̀-d., ceux touchés par le processus de consommation) qui dans une grande mesure, se font les champions de la cause du plaidoyer en faveur du commerce équitable. Il existe là des dimensions internationales, mais elles ne sont pas de celles qui viennent le plus facilement à l'esprit.

De telles préoccupations expriment des valeurs fortes et positives, et impliquent une généralisation et une internationalisation des normes morales, ce qui est sans nul doute louable. Cependant, en tant que facteur de négociation politique dans le secteur de la forêt, elles sont un problème dans au moins deux domaines. Tout d'abord, la connexion entre d'une part le désir noble des consommateurs de garantir que les produits qu'ils consomment ne nuisent pas à ceux immédiatement affectés par leur production et, d'autre part, les modifications de la politique qui peuvent être le résultat de leurs préoccupations, n'est pas évidente. Aussi bonnes que soient les intentions de ces politiques, le risque est leurs effets qui pourraient ne pas s'avérer positifs. Dans le cas présent, par exemple, il n'existe pas de preuve que la politique de développement du sud riche en forêts soit motivée par les intérêts de l'industrie du bois d'œuvre du nord, mais ceci pourrait bien encore constituer un effet ex post. L'industrie pourrait à tout le moins utiliser son important bras financier et politique pour essayer de s'approprier et de canaliser l'orientation des politiques émergeantes. Une production tropicale à bas coûts est une menace évidente pour une industrie modérée où les coûts sont élevés et les normes de travail de haut niveau, et si l'avantage comparatif est le résultat de l'illégalité, l'intérêt commercial est alors clairement de l'éliminer. Deuxièmement, il existe des sensibilités compréhensibles dans les états producteurs, en particulier au sein des gouvernements qui agissent en tant que gardiens de la souveraineté nationale, à la perspective d'une intervention étrangère dans leurs affaires nationales, qui va au-delà des préoccupations 
matérielles et clairement légitimes des consommateurs étrangers. Négocier les nouvelles normes de légalité s'est révélé de façon inattendue, controversé dans un certain nombre de cas, et le fait que les états donateurs intéressés sont souvent les anciennes puissances coloniales n'a fait qu'accroître la sensibilité.

Le défi consiste donc à exploiter la forte préoccupation morale en faveur du commerce équitable du bois d'œuvre et de la mettre au service des intérêts réels des populations nationales qui sont le plus touchées par son absence et qui risqueraient le plus de souffrir si les orientations de la nouvelle politique venaient à faire défaut. Ces populations se trouvent dans les pays producteurs et non consommateurs.

\subsection{La vérification en tant que moteur de la réforme de la gouvernance}

Un deuxième domaine apparenté dans lequel le thème de cet ouvrage présente des défis pour la politique forestière, est le fait que l'action n'a été entamée de l'intérieur des sociétés de producteurs que dans une mesure limitée. Les principaux moteurs du changement sont essentiellement situés dans (mais pas uniquement) le domaine international. C'est une question complexe et elle n'implique pas une auto satisfaction locale sur l'illégalité. Les pratiques illégales dans l'industrie de la forêt ont longtemps été une source de préoccupations dans de nombreuses sociétés productrices. Cependant, à l'exception de certaines situations particulières (essentiellement en Amérique latine), les processus formels décrits dans ce livre avaient tendance à être lancés par des acteurs externes dans le contexte de réformes de la politique internationale. Savoir dans quelle mesure les interventions en question (par opposition aux intérêts sous-jacents) sont adéquatement intégrées dans le contexte local est donc un sujet de préoccupation. En outre, même s'il existe d'importants moteurs en faveur du changement, les problèmes que les gens souhaitent voir traités ne sont que partiellement liés aux pratiques illégales de l'industrie du bois d'œuvre. Il y a donc un problème concernant le point d'entrée qu'offre "l'illégalité » dans les préoccupations qui touchent la majorité des habitants des forêts tropicales.

Pour de nombreux activistes, tant locaux qu'internationaux, la production illégale n'est rien d'autre qu'un point d'entrée - même si elle jouit actuellement d'un profil élevé et commode - dans des débats et des préoccupations plus larges, et il est largement considéré comme un véhicule pour exercer une influencer sur d'autres avantages plus importants. En toute probabilité, un nombre relativement restreint de personnes concernées par le mouvement FLEGT dans les pays producteurs de bois d'œuvre considèrent la légalité du commerce international du bois d'œuvre comme leur seule ou principale préoccupation. Le mouvement aurait en fait été considéré comme un échec par de nombreux partisans si ses succès s'étaient limités à offrir un nouveau label vert aux producteurs de bois d'œuvre tropical, en tant que preuve de la légalité de leur production et en tant que forme innovante de certification de marché dans un cadre de référence essentiellement commercial. 
Ces préoccupations posent des difficultés particulières au niveau développement de la politique. La théorie de la conformité suggère que les principaux facteurs qui conduisent à une plus grande conformité seront probablement de fortes pressions externes à se conformer à la législation, une forte probabilité de détection de la non conformité, et une assurance de sanction importante en cas de non conformité (voir, par exemple, INECE 2005). Dans les pays de forêts tropicales, l'électorat local et national qui encourage la conformité est souvent assez limité. Nombre de ceux que l'on pourrait s'attendre à voir participer avec plus d'enthousiasme au contrôle et à la détection pourraient être réticents à intervenir. Ceci découle en partie du fait que dans les contextes de faible gouvernance caractérisés par des régimes juridiques complexes et impraticables, un grand nombre de citoyens autrement respectueux de la loi pourraient eux-mêmes agir illégalement ou être vulnérables à ces perceptions, et vivre dans des situations d'insécurité foncière importante, et seront donc moins susceptibles de " jeter la première pierre ". Il existe de plus en plus de travaux qui portent sur cette question (voir par exemple, Colchester et al. 2006).

Le commerce illégal de bois d'œuvre pose des interrogations intéressantes sur l'approche de la réforme de la gouvernance. Le commerce international (réciproque par nature) estil un véhicule solide pour les réformes de la gouvernance (qui ont tendance à présenter un caractère plus unidirectionnel) ? La vérification de la légalité s'avère-t-elle un bon point d'entrée pour la réforme de la gouvernance ? Plus généralement, peut-on utiliser des actions spécifiques au secteur pour lancer des processus plus généralisés de réforme de la gouvernance ? Le secteur forestier est sans doute un secteur particulièrement exigeant (beaucoup plus que disons les minéraux et l'énergie) en ce sens que les valeurs d'utilisation sont multiples, la production et les marchés sont fortement dispersés et décentralisés et ses produits (diverses espèces de bois d'œuvre d'environnements différents) sont facilement substituables.

Des questions intéressantes sont posées sur la stratégie et l'impact. L'approche de la théorie de la gouvernance pourrait suggérer, par exemple, que les gains les plus importants viendront des actions qui s'attaquent à la mauvaise gouvernance à la base plutôt qu'à travers ses effets. Sur ce point, on pourrait dire que le suivi indépendant des attributions de concessions forestières pourrait être plus efficace qu'une observation indépendante des opérations de forêts, et que par conséquent, il faudrait procéder à des investissements plus importants dans le premier que dans le second. Comme cela ressort clairement dans le cas du Cameroun, l'argument utilisé serait que l'attribution des concessions aille au cœur de la patrimonialité dans la gouvernance publique et donc, introduire un système plus axé sur les règles réduirait considérablement le potentiel d'une mauvaise gouvernance. Il faudrait donc concentrer les efforts pour s'assurer que les résultats de ce suivi sont intégrés dans les procédures futures d'allocations et aboutissent à des réformes plus systématiques. Par opposition, les observateurs indépendants des opérations forestières peuvent faire du bon travail, et bien plus visible au niveau international, mais pourraient trouver plus ardu de s'attaquer aux faiblesses sous-jacentes de la gouvernance. Comme dans le cas du Cameroun, ce que peut faire une influence extérieure pour s'attaquer à une gouvernance médiocre est limité. Le Comité de lecture du Cameroun a été une innovation positive, 
mais les acteurs externes n'ont pas pu remettre en cause l'autorité exclusive du ministre de la forêt sur l'application en aval et le contrôle de la conformité.

\subsection{Mécanismes de responsabilité publique nationale et internationale}

La sensibilité qui entoure la campagne en faveur de la légalité du commerce du bois d'œuvre découle finalement des tensions qui existent entre les volets souverain et international du secteur de la forêt, et les tentatives d'utiliser ce secteur comme véhicule menant à de plus amples réformes de la gouvernance. Cependant, l'expression la plus tangible de ces sensibilités a été la façon dont certains acteurs et programmes privés ont été introduits dans le domaine public. Dans l'ensemble, ces acteurs étaient des ONG et des activistes de la société civile. Les rôles des ONG sur les droits environnementaux sont ceux qui ont été les plus controversés, et qui ont fait l'objet des plus grandes revendications, arguant du fait qu'elles interviennent sur des questions ne relevant pas de leurs compétences. Il y a là une certaine ironie, en ce sens que les acteurs privés - au sens d'opérateurs industriels - ont longtemps bénéficié d'immenses privilèges dans l'exploitation des forêts nationales, et ont beaucoup prospéré ce faisant, même au détriment des populations habitant la forêt et qui ont des droits de revendication prioritaires sur les terres concernées. Ceux qui sont contre la " privatisation » de la responsabilité de l'état de la forêt (par rapport aux rôles des $\mathrm{ONG}$ ) feraient bien de se rappeler que la condition de la forêt est déjà dans une certaine mesure, subordonnée aux intérêts du secteur privé (par rapport à l'industrie du bois d'œuvre).

Donc, à un niveau, l'intrusion des ONG en tant qu'acteurs privés dans le domaine public n'a pas mérité l'accusation d'insulte à la souveraineté nationale faite de façon totalement nouvelle et posant de multiples problèmes. Cependant, le plus important est peut- être le fait que ces acteurs privés se sont mis à élaborer des normes. Que les acteurs nationaux le fassent n'est pas étonnant, ni particulièrement controversé. La même approche de la part d'acteurs internationaux est plus controversée, en raison de leur statut non national et du soutien disproportionné que leur apporte la communauté internationale. Les surveillants indépendants de la forêt dans des pays comme le Cambodge et le Cameroun sont les instances de plus haut niveau traitées dans ce livre, même si d'autres organisations non couvertes ici (du type Greenpeace, par exemple) ont joué des rôles quelque peu similaires dans ce secteur et ailleurs. Tout en ayant préparé des rapports de surveillance assez bien définis, à l'instar des instances de contrôle (sans doute de façon beaucoup plus approfondie), les surveillants de la forêt n'ont pas cherché à limiter leur verdict à ces questions, et ont clairement indiqué que leurs intérêts sous-jacents ne sont pas techniques mais normatifs et axés sur la valeur. Leurs positions sur l'exploitation industrielle des forêts sont souvent claires et hostiles, et ils n'ont pas hésité à identifier et à révéler publiquement les ramifications politiques du parti dans l'industrie de la forêt concernée. Ceci a en fait été une des révélations de l'étude recherche. 
Pour les observateurs occidentaux du secteur de la forêt, les surveillants indépendants ont été la caractéristique la plus distincte des systèmes de vérification, et le CIF a été l'élément privé le plus évident et le plus controversé de l'autorité dans FLEGT. L'importance historique du CIF dans le domaine émergeant de la vérification se reflète dans les préoccupations politiques actuelles, essentiellement les AVP et le parcours en dents de scie de la plupart des négociations AVP ne peut être compris que par rapport aux sensibilités envers le CIF.

Le risque existe que le mouvement FLEGT s'intéresse au CIF, et on pourrait en fait dire que dans certains cas importants, ceci est déjà le cas. En dépit du travail intéressant effectué par certains des surveillants de l'environnement, le CIF n'est qu'une étape dans l'élaboration d'un système de vérification et n'est pas considéré comme une étape essentielle par de nombreux groupes d'intérêt. Les études de cas pays abordées dans ce livre ont montré qu'il existe un certain nombre d'autres approches du FLEGT, moins dépendantes du CIF, en particulier dans les sociétés où la classe moyenne constitue un électorat important et qui fait entendre sa voix.

Pour éviter l'impasse dans les négociations des nouveaux systèmes de vérification (de toute évidence des AVP), il serait utile de commencer par reconnaitre que la préoccupation principale n'est pas les révélations à l'extérieur mais plutôt la réforme de la gouvernance, et que la responsabilité publique devrait être l'élément central d'une bonne gouvernance. La responsabilité est une dimension plus importante de la bonne gouvernance que la participation d'acteurs externes et prime sur les mécanismes qui la renforcent, notamment le CIF.

En prenant comme point de départ la richesse des études de cas abordées dans ce livre et en portant une attention particulière à la responsabilité et non aux révélations, il en ressort des points intéressants qui sont essentiels à la bonne gouvernance du secteur de la forêt, et à la bonne gouvernance au sens large dans des états jusque là fracturés et instables. Toutes les directions convergent vers les identités nationales et les intérêts des états producteurs, et la garantie que toutes les actions à l'interface des ressources nationales ainsi que les intérêts externes sont bien ancrés dans les valeurs et les impératifs nationaux et capables de tenir leurs engagements. La voie vers l'avant consisterait à renforcer les capacités nationales de la voix publique en guise de premier pas vers des actions internationales. Les rôles des donateurs ne se limitent peut-être pas au secteur de la forêt. Ils seraient peut-être mieux avisés d'encourager la collaboration des gouvernements producteurs et de la société civile sur un large front, en utilisant leur propre influence pour prendre fait et cause pour les acteurs et intérêts nationaux, avec le soutien des acteurs extérieurs.

\subsection{Conclusions}

Les expériences analysées dans ce livre prouvent que l'efficacité et la crédibilité dépendent essentiellement des perceptions, et seront souvent menacées. Pour qu'un système de vérification soit suffisamment robuste pour tirer des enseignements de ses erreurs, il devra générer un large éventail de champions nationaux prêts à le défendre contre toutes sortes 
de défis - et, si nécessaire, exercer une pression sur l'industrie et le gouvernement pour prendre des mesures fermes afin de rectifier leurs insuffisances, plutôt que simplement les couvrir. Cette perspective est une justification au renforcement des capacités nationales en tant que condition préalable nécessaire à un système de vérification. Ceci ouvrirait la voie à des approches plus créatives et plus " architecturales » de la conception du système de vérification que les modèles techniques et les approches dans lesquelles la crédibilité et la vertu morale résident essentiellement dans les actions de groupes individuels de plaidoyer. Une fonction de suivi indépendante pourrait bien encore s'avérer nécessaire. Il existe pour cela une variété d'options, en conformité avec l'engagement d'une évaluation objective des témoignages. Les études de cas présentent un certain nombre de ces options - il faut rappeler que presque toutes sont encore en cours d'élaboration.

L'approche adoptée dépend dans une grande mesure du cadre temporel associé à la fourniture d'un système de vérification. Si elle est considérée comme un objectif réalisable à court terme (comme cela peut être le cas avec certains AVP), cela encourage un suivi indépendant des forêts dans le sens traditionnel. Une telle approche pourrait fonctionner efficacement, mais elle risque de tomber à plat. Les « auditeurs professionnels » du secteur privé pourraient paraître trop sûrs aux yeux de la communauté des ONG internationales. Les ONG pourraient accepter davantage de surveillants activistes, mais cela pourrait contrarier les états souverains producteurs, et il pourrait y avoir des interrogations sur l'appropriation et la durabilité qu'ils génèrent. Même s'il ne faut pas préjuger de la performance, on peut facilement imaginer que cela puisse créer une situation où les activités de suivi externes servent à totalement discréditer un système de vérification, à un stade précoce, dans des circonstances où les éléments étaient réunis pour adopter une approche de renforcement des capacités plus prudente et plus axée sur le processus. Ceci pourrait bien avoir des effets pervers (et négatifs) sur la conservation de la ressource. Même s'il existe certainement des arguments en faveur d'un CIF pur, une perspective à plus long terme offre d'autres options de vérification de nature plus structurelle, et celles-ci pourraient s'avérer plus prometteuses sur le plan de l'appropriation et de la durabilité dans les pays producteurs. Le système d'assurance de légalité devra être adapté au contexte particulier de chaque pays partenaire. Divers instruments pourraient être applicables dans divers contextes (et dans différents pays), même si une participation nationale forte et diverse et une appropriation semblent essentielles dans tous les cas. L'importance croissante des marchés non européens souligne ce point.

Une conclusion importante à tirer est qu'une vérification efficace doit être envisagée dans le contexte d'un processus plus large de réforme de gouvernance de la forêt. Les tentatives d'utiliser les activités de vérification en tant que source indépendante et autonome de cette réforme ont peu de probabilité d'être efficaces en particulier à plus long terme. Ceci s'explique par le fait qu'elles conservent les principales faiblesses structurelles qui génèrent l'illégalité. Ces conclusions ne résolvent pas le problème de réforme entamée dans des situations de gouvernance médiocre, mais suggèrent qu'il n'existe pas de solutions faciles et que la voie vers la réforme dans ces contextes ne peut qu'être plus difficile et non plus facile que dans les contextes où les pressions indigènes existent déjà. Les témoignages affluent sur les pressions commerciales qui peuvent aider à exercer une influence sur les 
réformes de la gouvernance si elles sont exercées de façon flexible et réactive. Les approches sectorielles et extra sectorielles abordées dans cette étude suggèrent une variété d'options en matière de conception des systèmes de vérification qui pourraient contribuer à cette flexibilité. En fin de compte, le but devrait être de donner un pouvoir à des sociétés de producteurs pour gérer efficacement leurs ressources nationales tout en sauvegardant les intérêts des faibles et des vulnérables, et non uniquement d'exposer les limites des systèmes actuels de contrôle des ressources. 


\section{Annexe : messages politiques}

L'annexe présente une série de messages politiques tirés directement des résultats de l'étude VERIFOR et proposés ici en tant qu'indicateurs possibles du développement de systèmes de vérification efficaces et équitables. Ils sont répartis en quatre sections, répondant aux quatre thèmes mis en exergue dans la matrice conceptuelle (Tableau 17.1) - notamment : l'appropriation, les normes de légalité, l'indépendance et les impacts. Chaque message politique s'accompagne d'une stratégie de soutien qui offre des suggestions pour la mise en œuvre de la politique sur le terrain. Ces messages politiques et ces stratégies de soutien ne sont nullement considérés comme normatifs mais essayent plutôt de distiller certaines des expériences utiles acquises par des pays qui à ce jour se débattent encore avec la vérification du secteur de la forêt.

\section{Appropriation des systèmes de vérification}

\begin{tabular}{|c|c|}
\hline Message politique & Stratégie d’appui \\
\hline $\begin{array}{l}\text { 1. Les objectifs du } \\
\text { système de vérification } \\
\text { déterminent le degré } \\
\text { d'appropriation }\end{array}$ & $\begin{array}{l}\text { Les systèmes de vérification conçus pour porter sur les } \\
\text { priorités nationales et pour compléter les institutions } \\
\text { nationales existantes et les processus politiques } \\
\text { bénéficieront de niveaux d'appropriation élevés }\end{array}$ \\
\hline $\begin{array}{l}\text { 2. Les buts des réformes } \\
\text { doivent être agréés } \\
\text { et spécifiés avant } \\
\text { le processus de } \\
\text { conception des } \\
\text { systèmes }\end{array}$ & $\begin{array}{l}\text { Les responsabilités de mise en œuvre et les décisions quant } \\
\text { à qui devrait supporter les coûts devraient être réparties } \\
\text { en rapport avec les buts et les objectifs des réformes. La } \\
\text { conservation des biens publics internationaux devrait être } \\
\text { de la responsabilité de la communauté internationale, } \\
\text { alors que les biens nationaux sont de la responsabilité des } \\
\text { gouvernements nationaux. De même, les non bénéficiaires } \\
\text { ne devraient pas se voir demander de supporter les coûts }\end{array}$ \\
\hline $\begin{array}{l}\text { 3. L'appropriation par } \\
\text { le gouvernement } \\
\text { peut être accrue } \\
\text { en impliquant une } \\
\text { série d'agences et de } \\
\text { niveaux décentralisés }\end{array}$ & $\begin{array}{l}\text { Travailler avec des parties en dehors du secteur de } \\
\text { la forêt peut aider à assurer une large appropriation } \\
\text { par le gouvernement. Lorsque la décentralisation du } \\
\text { gouvernement est bien en place, ceci pourrait s'avérer } \\
\text { essentiel }\end{array}$ \\
\hline $\begin{array}{l}\text { 4. Accroître la } \\
\text { participation publique } \\
\text { et puiser dans la } \\
\text { diversité des points } \\
\text { de vue augmente } \\
\text { l'appropriation } \\
\text { publique }\end{array}$ & $\begin{array}{l}\text { L'appropriation par le gouvernement ne reflète pas } \\
\text { nécessairement une appropriation publique ou basée } \\
\text { sur les citoyens. Les deux peuvent être assimilés lorsqu'il } \\
\text { y a eu suffisamment de démarches, de consultations et } \\
\text { d'engagement efficace des publics concernés dans la prise } \\
\text { de décision, et lorsque des mécanismes sont en place pour } \\
\text { rapprocher les points de vue conflictuels }\end{array}$ \\
\hline $\begin{array}{l}\text { 5. Renforcer la } \\
\text { responsabilité et la } \\
\text { transparence améliore } \\
\text { l'appropriation } \\
\text { publique }\end{array}$ & $\begin{array}{l}\text { La conception des systèmes devrait refléter les critères } \\
\text { de bonne gouvernance en tenant compte des réalités } \\
\text { politiques. L'accès public à l'information sur le processus } \\
\text { est essentiel pour que le public s'implique activement et } \\
\text { demande aux institutions de rendre des comptes. Cette } \\
\text { implication devrait être encouragée davantage à travers la } \\
\text { fourniture de voies confidentielles de compte rendu et des } \\
\text { garanties de protection contre les sanctions }\end{array}$ \\
\hline
\end{tabular}




\begin{tabular}{ll}
\hline Message politique & Stratégie d'appui \\
\hline 6. Un examen détaillé et & L'appropriation n'est pas seulement réalisée à travers un \\
la communication sur & processus qui encourage la participation et l'implication, \\
la répartition des coûts & mais plutôt à travers des résultats clairs et bénéfiques et \\
et les avantages du & des certitudes quant au fardeau des coûts et à quel niveau \\
système de vérification & il serait supporté. Maintenir l'appropriation peut exiger \\
sont nécessaires & des spécificités telles que des tampons pour réduire les \\
pour maintenir cette & risques auxquels sont confrontés les producteurs, ou \\
appropriation dans le & une compréhension claire de la mesure dans laquelle les \\
temps & normes vont évoluer dans le temps \\
\hline
\end{tabular}

\section{Indépendance des systèmes de vérification}

\begin{tabular}{ll}
\hline Message politique & Stratégie d'appui \\
\hline 7. L'indépendance est & L'indépendance ne doit pas uniquement être considérée \\
un aspect de la bonne & comme « un plus » à confier à une seule agence (ceci \\
gouvernance, et & engendrera une plus grande vulnérabilité). Elle peut \\
devrait être intégrée & également découler des relations mutuelles entre les \\
dans la conception & acteurs, et les vérifications et équilibres dans la conception \\
des systèmes de & des systèmes. Les problèmes de gouvernance médiocre \\
vérification & devraient être traités à la source, avant la conception du \\
& système de vérification (par exemple, la surdépendance \\
envers des acteurs politiques individuels, intervenant & de façon non transparente ; des acteurs individuels \\
& ayant un droit de veto, n'encourageant pas le processus \\
démocratique)
\end{tabular}




\begin{tabular}{|c|c|}
\hline Message politique & Stratégie d'appui \\
\hline $\begin{array}{l}\text { 9. L'indépendance n'est } \\
\text { pas associée à une } \\
\text { classe particulière } \\
\text { d'acteurs (secteur } \\
\text { privé, ONG, etc.) } \\
\text { ou à des origines } \\
\text { particulières } \\
\text { (nationales par rapport } \\
\text { à internationales); } \\
\text { cependant, les acteurs } \\
\text { peuvent varier dans } \\
\text { leurs capacités à } \\
\text { partager l'information } \\
\text { et à exercer une } \\
\text { pression sur les } \\
\text { délinquants en la } \\
\text { matière }\end{array}$ & $\begin{array}{l}\text { L'accent devrait être mis sur la constellation d'acteurs } \\
\text { et sur leurs points faibles et forts respectifs, et non sur } \\
\text { la sauvegarde des vertus morales supposées d'acteurs } \\
\text { particuliers }\end{array}$ \\
\hline $\begin{array}{l}\text { 10. L'indépendance } \\
\text { s'intègre mieux dans } \\
\text { les systèmes de } \\
\text { vérification à travers } \\
\text { une conception } \\
\text { orientée }\end{array}$ & $\begin{array}{l}\text { Des mesures actives auraient probablement plus de } \\
\text { chances d'être efficaces et solides que des mesures } \\
\text { passives. Les forums institutionnels devraient surtout viser à } \\
\text { rassembler des acteurs choisis en fonction de règles claires } \\
\text { d'interaction ; la participation ne devrait pas seulement } \\
\text { être volontaire et flexible. Il est également nécessaire de } \\
\text { spécifier et d'adhérer aux politiques portant sur les conflits } \\
\text { d'intérêt }\end{array}$ \\
\hline $\begin{array}{l}\text { 11. L'adhésion aux normes } \\
\text { internationales fournit } \\
\text { une assurance crédible } \\
\text { d'indépendance }\end{array}$ & $\begin{array}{l}\text { Les vérificateurs devraient se conformer aux normes } \\
\text { internationales telles que les normes ISO, à la fois sur le } \\
\text { plan de l'accréditation (par exemple, ISO } 17001 \text { et ISO 65/ } \\
\text { EN 45011) et les normes de performance (par exemple ISO } \\
9000,1401,1900 \text { ) }\end{array}$ \\
\hline
\end{tabular}

\section{Normes de légalité}

\begin{tabular}{ll}
\hline Message politique & Stratégie d'appui \\
\hline $\begin{array}{l}\text { 12. Définir une norme } \\
\text { de légalité est }\end{array}$ & $\begin{array}{l}\text { Elle implique deux étapes : la priorisation des lois et } \\
\text { réglementations actuelles en fonction de l'objectif du } \\
\text { probablement une } \\
\text { condition préalable }\end{array}$ \\
$\begin{array}{l}\text { à une vérification } \\
\text { efficace, et plus le }\end{array}$ & $\begin{array}{l}\text { principale ; et l'élargissement des cadres réglementaires } \\
\text { pour y incorporer des normes de performance claires }\end{array}$ \\
cadre juridique est & \\
complexe, plus son & \\
abstraction dans la & \\
norme de légalité sera & \\
nécessaire & \\
\hline
\end{tabular}




\begin{tabular}{|c|c|}
\hline Message politique & Stratégie d'appui \\
\hline $\begin{array}{l}\text { 13. La clarté des objectifs } \\
\text { est essentielle }\end{array}$ & $\begin{array}{l}\text { Que la norme soit à la fois réalisable et légitime dépend } \\
\text { finalement fortement des capacités à s'accorder sur les } \\
\text { objectifs de la vérification. Ceci a engendré une pression } \\
\text { pour réduire les dits objectifs de vérification afin de } \\
\text { permettre d'arriver à un consensus sur une norme plus } \\
\text { spécifique dont les objectifs devront être examinés à une } \\
\text { date ultérieure. L'introduction progressive de normes, } \\
\text { le partage de la responsabilité avec l'état, les mesures } \\
\text { de conformité pertinentes et la maîtrise des coûts de } \\
\text { transaction de la conformité peuvent tous permettre } \\
\text { d'accroître la validité de l'application de la norme }\end{array}$ \\
\hline
\end{tabular}

14. Les normes de légalité doivent être réalisables
La validité de l'application implique l'adhésion aux principes de la légitimité (c.-à-d., une acceptation large des parties prenantes en tant que référence à la conformité juridique), la validité de l'application (c.-à-d., la norme doit être applicable sans imposer des coûts de transaction qui rendent la conformité non rentable) et la spécificité (elle doit être mesurable et comporter des normes de performance claires, autrement auditer la conformité juridique pourrait s'avérer difficile)

15. Les normes de légalité ont des composantes variables; les objectifs de vérification détermineront quelles sont ces composantes
Les composantes à prendre en considération incluent l'origine juridique (le droit légal à récolter, incluant peutêtre également la détermination et le règlement préalables des revendications foncières, la publication officielle des frontières des concessions, ainsi que le Consentement libre, préalable et informé (CLPI) des communautés locales concernant les opérations de coupe sur les terres communautaires) ; la récolte légale (notamment la conformité aux conditions d'octroi de permis, le versement de redevances, les règlements de la forêt incluant des critères et des indicateurs obligatoires pour une gestion durable de la forêt, ainsi que les lois sur l'environnement, la main d'œuvre, le bien-être et la santé et la sécurité) ; la transformation juridique (conformité aux quotas nationaux de transformation, garanties contre le mélange avec les sources non juridiques, et le versement de taxes sur la transformation) ; et le commerce et l'exportation légaux (autorisation d'exporter, règlement des droits de douane)

II pourrait s'avérer nécessaire de faire des distinctions entre les délits qui déclenchent l'application de la conformité (par exemple une sanction administrative et/ou des poursuites judiciaires), et ceux qui déclenchent la gestion de la conformité (par exemple, des demandes d'action rectificatrice)
16. Lors de la définition des normes de légalité, mettre l'accent sur la conformité des mesures peut être aussi important que de définir leur portée 


\begin{tabular}{ll}
\hline Message politique & Stratégie d'appui \\
\hline $\begin{array}{l}\text { 17. Les processus selon } \\
\text { lesquels les normes } \\
\text { sont définies peuvent } \\
\text { être aussi importants } \\
\text { que les normes elles- } \\
\text { mêmes }\end{array}$ & $\begin{array}{l}\text { Le mécanisme institutionnel pour développer une norme } \\
\text { de légalité doit être à même de résoudre les différences } \\
\text { entre les parties prenantes. L'exigence essentielle est la } \\
\text { surveillance par une institution qui a le mandat juridique et } \\
\text { politique de réaliser la « clôture politique » }\end{array}$ \\
\hline $\begin{array}{l}\text { 18. Le statut juridique de la } \\
\text { norme doit être clair }\end{array}$ & $\begin{array}{l}\text { Ceci implique de se concentrer sur quatre séries de } \\
\text { questions : (i) l'exigence de publier formellement la norme } \\
\text { de légalité ; (ii) déterminer si la norme choisie représente } \\
\text { des lois et des règlements applicables en accord avec } \\
\text { les objectifs de vérification déclarés; (iii) le mandat } \\
\text { d'émettre des certificats de conformité juridique ; et (iv) la } \\
\text { responsabilité lorsque les déterminations de conformité } \\
\text { s'avèrent fausses ou inadéquates. }\end{array}$ \\
\hline $\begin{array}{l}\text { 19. La définition d'une } \\
\text { norme de légalité } \\
\text { impose des devoirs à }\end{array}$ & $\begin{array}{l}\text { Pour qu'une norme garantisse la conformité des } \\
\text { l'état et aux utilisateurs } \\
\text { de la forêt }\end{array}$ \\
$\begin{array}{l}\text { ne peut fonctionner isolément par rapport à des garanties } \\
\text { plus larges relatives à la responsabilité publique; ceci } \\
\text { inclut les droits statutaires sur l'accès à l'information et à }\end{array}$ \\
la participation et les mécanismes publics de plainte, et \\
l'autonomisation juridique
\end{tabular}

\section{Impacts sur le développement}

\begin{tabular}{|c|c|}
\hline Message politique & Stratégie d'appui \\
\hline $\begin{array}{l}\text { 20. Les systèmes de } \\
\text { vérification doivent } \\
\text { reconnaître et } \\
\text { répondre aux objectifs } \\
\text { caractéristiques d'un } \\
\text { large éventail de } \\
\text { parties prenantes }\end{array}$ & $\begin{array}{l}\text { Les mécanismes multilatéraux de résolution doivent faire } \\
\text { partie de la conception du système de vérification. Le fait } \\
\text { d'être intégrés dans un processus de réforme plus large de } \\
\text { la politique peut être un avantage pour ces mécanismes } \\
\text { (par exemple, les programmes nationaux de la forêt) }\end{array}$ \\
\hline $\begin{array}{l}\text { 21. La vérification est } \\
\text { un processus qui } \\
\text { demande beaucoup } \\
\text { d'informations. } \\
\text { L'information } \\
\text { collationnée à travers } \\
\text { toutes les activités de } \\
\text { vérification demande } \\
\text { donc une gestion } \\
\text { active et transparente }\end{array}$ & $\begin{array}{l}\text { Une vérification efficace et efficiente peut bénéficier } \\
\text { de l'introduction et de la mise en œuvre de nouvelles } \\
\text { technologies, depuis les systèmes de positionnement } \\
\text { internationaux jusqu'aux bases de données en ligne. Les } \\
\text { politiques sur l'accès à l'information doivent être précisées } \\
\text { dès le début et devraient promouvoir une plus grande } \\
\text { transparence lorsque cela est possible }\end{array}$ \\
\hline
\end{tabular}




\begin{tabular}{|c|c|}
\hline Message politique & Stratégie d'appui \\
\hline $\begin{array}{l}\text { 22. Les systèmes de } \\
\text { vérification doivent } \\
\text { englober les questions } \\
\text { d'acquisition des } \\
\text { ressources afin de } \\
\text { s'attaquer aux racines } \\
\text { d'une grande partie du } \\
\text { conflit dans le secteur } \\
\text { de la forêt }\end{array}$ & $\begin{array}{l}\text { La conception d'un système national de vérification doit } \\
\text { voir plus loin que les flux de ressources (et l'accent sur la } \\
\text { "chaîne de garde ») et inclure l'examen de la conformité } \\
\text { juridique de l'acquisition des ressources }\end{array}$ \\
\hline $\begin{array}{l}\text { 23. Pour que les systèmes } \\
\text { de vérification ne } \\
\text { désavantagent pas les } \\
\text { pauvres, il faut élaborer } \\
\text { des mécanismes } \\
\text { qui garantissent } \\
\text { une conformité } \\
\text { réglementaire } \\
\text { accessible à tous les } \\
\text { groupes }\end{array}$ & $\begin{array}{l}\text { L'utilisation d'unités administratives mobiles de l'autorité } \\
\text { de la forêt, ainsi que l'accès internet dans les économies } \\
\text { plus développées, peut améliorer l'accès réglementaire } \\
\text { pour tous les groupes d'utilisateurs de la forêt. Ceci } \\
\text { exige un engagement total des services décentralisés } \\
\text { envers les objectifs de la réforme. Des mesures doivent } \\
\text { également être adoptées pour assurer que les procédures } \\
\text { administratives ne désavantagent pas les pauvres - par } \\
\text { exemple, les coûts de permis pour les petits propriétaires } \\
\text { fonciers et/ou ceux vendant de petits volumes, devraient } \\
\text { être proportionnels aux intérêts de ces catégories }\end{array}$ \\
\hline $\begin{array}{l}\text { 24. Un cadre explicite de } \\
\text { suivi et d'évaluation } \\
\text { est nécessaire pour } \\
\text { mesurer l'impact } \\
\text { général de tout } \\
\text { système national de } \\
\text { vérification }\end{array}$ & $\begin{array}{l}\text { Il faudrait développer un cadre conceptuel pendant } \\
\text { l'élaboration du système de vérification qui établit un } \\
\text { lien avec un certain nombre de critères et d'indicateurs } \\
\text { complémentaires pour faciliter un suivi ultérieur du système } \\
\text { de vérification. L'instance responsable d'entreprendre des } \\
\text { actions sur les résultats du suivi devrait être indiquée }\end{array}$ \\
\hline
\end{tabular}




\section{Références}

Acosta, R. (2008) 'Forest Law Enforcement and Governance Strategies in the Philippines', Présentation PowerPoint lors de la semaine de la foresterie Asie Pacifique, 22 avril, Hanoï, Vietnam.

ACP/EC (2000) 'Accord de partenariat entre les membres du Groupe des pays d'Afrique, des Caraïbes et du Pacifique d'une part, et la Communauté européenne et ses états membres d'autre part, signé à Cotonou le 23 juin 2000.' Cotonou, Luxembourg.

AFE-COHDEFOR (2006) 'Evaluación Nacional Forestal, Resultados del Inventario de Bosques y Árboles 2005-2006’. AFE-COHDEFOR, Tegucigalpa.

Álvaro, M. (2003) 'Ruling Undercuts Ecuador’s Forestry Reform', EcoAméricas 6(1).

Amaro, M.C. (2006) 'Philippines: Progress on National Forest Law Enforcement and Support to EA-FLEG', Réunion sur l'application de la loi forestière et la gouvernance en Asie et dans le Pacifique (Forest Law Enforcement and Governance) (FLEG), 7 mars, Manille, Philippines.

Arias, G. (2005) 'Identificación y priorización de los actores de la ilegalidad', Rapport de consultants PTC/COS/3003/FAO pour ECTI SINAC-MINAE, Costa Rica.

Auzel, P., Fomété, T., Odi, J. et Owada, J-C. (2002) 'Evolution de l'exploitation des forêts du Cameroun: production nationale, exploitation illégale, perspectives'. DFID-Yaoundé, Cameroun.

Bäckstrand, K. et Saward, M. (2004) 'Democratizing Global Environmental Governance? Stakeholder Democracy at the World Summit for Sustainable Development', Document présenté à la cinquième conférence paneuropéenne sur les relations internationales, 9-11 septembre, La Haye, Pays-Bas.

Baker, M., Clausen, R., Kanaan, R., N'Goma, M., Roule, T., et Thomson, J. (2004) 'Conflict timber: dimensions of the problem in Asia and Africa. Volume 3: African cases', Rapport final remis à l'USAID. Vermont: ARD.

Banque Mondiale (1999) 'Cambodia: A Vision for Forestry Sector Development'. Washington, DC: Banque mondiale.

Banque Mondiale (2006) Strengthening Forest Law Enforcement and Governance: Addressing a Systemic Constraint to Sustainable Development, Rapport No. 36638GLB des Départements de l'environnement et de l'agriculture et développement rural - Août 2006.

Banque Mondiale (2007) Ghana: country environmental analysis, Rapport No. 36985GH. Washington, DC: Banque mondiale.

Banque Mondiale (2008) 'Strengthening Forest Law Enforcement and Governance in the Philippines: Revival and Institutionalization of the Multisectoral Forest Protection Committees', Projet de mandat.

Barrett, S. (1999) 'Montreal versus Kyoto: International Cooperation and the Global Environment', dans I. Kaul, I. Grunberg and M. Stern (eds), Global Public Goods: International Cooperation in the 21st Century. Oxford: Oxford University Press. 
Bass, S. (1998) 'Forest Certification - The Debate about Standards', Rural Development Forestry Network Papers No. 23b. Londres: ODI.

BC Stats (2004) Quick facts about British Columbia. BC Stats, Ministère des services de gestion, $\mathrm{CB}$.

BCN (2006) 'Informe Anual 2005'. Banque Centrale du Nicaragua (BCN), Managua, Nicaragua.

Beeko, C. (2008) 'Ghana's VPA Multi-Stakeholder Platform: Opportunities and Challenges', présentation lors de la réunion sur l'actualisation sur l'exploitation forestière illégale et la consultation des parties prenantes, 17-18 janvier, Chatham House, Londres, Royaume Uni.

Benner, T., Witte, J.M. and Reinicke, W. (2002) 'Innovating Global Governance: Multisectoral Networks and Accountability', document préparé pour la Conférece sur la gouvernance mondiale Miliband Conference on Global Governance, 17-19 mai, Londres, Royaume Uni.

Bernsen, M. et Cochrane, L. (2005) 'SGS Report Praises Government Action on Forest Crimes'. Phnom Penh Post, 26 août-8 septembre.

Bird, N. et Cabral, L. (2007) 'Changing Aid Delivery and the Environment: Can General Budget Support Be Used To Meet Environmental Objectives?', Document d'information No. 17 de l'ODI. Londres : ODI.

Bird, N. et Thiel, H. (2007) 'New Technologies for Forest Governance: Factors Influencing Success', Document d'information VERIFOR No. 7. Londres : ODI.

Birikorang, G. et Rhein, M. (2005) 'Reforming Ghana's Forest Fiscal Regime. Phase 1 Draft Report.' FSDP, Accra.

Birikorang, G., Okai, R., Asenso-Okyere, K., Afrane, S. et Robinson, G. (2001) 'Ghana Wood Industry and Log Export Ban Study (final report)’. Londres : DFID.

van Bodegom, A. et Hijweege, W. (2006) 'Overview FLEGT related stakeholder processes and initiatives in the European Union and The Netherlands'. Wageningen: Wageningen International.

Booth, D. (2004) 'Budgets not projects: a new way of doing business for aid donors', ODI Opinions 9, Février 2004.

Brack, D. (2003) 'Monitoring the Montreal Protocol', Annuaire de la vérification, 2003. Londres : VERTIC.

Brack, D. (2006) 'Excluding illegal timber from EU markets: options for the EU and its Member States'. Londres : Chatham House. www.illegal-logging.info/uploads/ RIIA_EU_control_of_imports.doc

Brack, D., Gray, K. et Hayman, G. (2002) 'Controlling the International Trade in Illegally Logged Timber and Wood Products’. Londres : Programme sur le développement durable du Royal Institute of International Affairs.

Brosius, P. (1997) 'Endangered Forest, Endangered People: Environmentalist Representations of Indigenous Knowledge', Human Ecology, 25(1): 47-69. 
Brown, D. (2006) 'Designing Verification Systems for the Timber Trade: Learning from International Processes', Document d'information VERIFOR No. 1. Londres : ODI.

Brown, D. et Luttrell, C. avec des chercheurs associés au Cambodge, au Cameroun, en Indonésie et aux Philippines (2004) 'Review of Independent Forest Monitoring', for Policy Division, UK Department for International Development (DFID), août. Londres : ODI.

Brown, D. et Swails, E. (2006) 'CITES', Etude de cas comparative 3 de VERIFOR. Londres : ODI.

Brown, D. et van Midwoud, P. (2006) 'Verification for Food Safety: The Case of the Meat Industry', Etude de cas comparative 5. Londres: ODI.

Brown, D.W., Brown, T.H., Ediawan, A., Justianto, A. et Simangunsong, B. (2006) 'Returning Forest Areas to Community Management: What are the Welfare Gains?', Onzième conférence biennale, The International Association for the Study of Common Property (IASCP), 19 au 23 juin, Bali. 6 et 10.

Bruks Associates (2004) 'Sawn timber cost structure (final report)', rapport non publié pour la Ghana Timber Millers Organisation. Bruks Associates, Accra, Ghana.

C\&E (2004) 'Compliance and Enforcement Program Annual Report 2003-4'. CB : division conformité et mise en œuvre, Ministère des forêts, $\mathrm{CB}$, Canada.

Cameron, J., Werksman, J. et Roderick, P. (1996) Improving Compliance with International Environmental Law, Séries Law and Sustainable Development. Londres : Earthscan.

de Camino, R., Segura, O., Arias, L.G., Pérez, I. (2000) Costa Rica: Forest Strategy and Evolution of Land Use, Série Evaluation Country Case Study. Washington, DC: OED Banque mondiale.

Casley, D. et Kumar, K. (1987) Project Monitoring and Evaluation in Agriculture. Baltimore et Londres : The John Hopkins University Press.

Cashore, B., Auld, G. et Newsom, D. (2004) Governing Through Markets: Forest Certification and the Emergence of Non-State Authority. New Haven et Londres : Yale University Press.

Cashore, B., Gale, F., Meidinger, E. et Newsom, D. (eds) (2006) 'Confronting Sustainability: Forest Certification in Developing and Transitioning Countries', Yale School of Forestry and Environmental Studies, Rapport No.8, Juillet. New Haven: Université de Yale.

Cashore, B., McDermott, C. et Levin, K. (2006) 'The shaping and reshaping of British Columbia forest policy in the global era: a review of governmental and nongovernmental strategic initiatives', Discours- thème à la Conférence et réunion annuelle générale des professionnels du secteur forestier, 22-24 février Victoria, $\mathrm{CB}$, Canada.

CATIE (2001) 'La tala ilegal en Costa Rica. Un análisis para la discusión', CATIE/ Commision de suivi du Plan National de développement forestier. Turrialba, Costa Rica. 
CBFP (2006) 'Les Forêts du Bassin du Congo - Etat des Forêts 2006', Partenariat des forêts du bassin du Congo, Kinshasa, République Démocratique du Congo.

CE (2004) 'What are the WTO Implications?', Document d'information No. 8 FLEGT. Bruxelles: CE.

CE (2004-2008) Document d'information FLEGT (2004/5 series and 2007 series). Bruxelles: CE. http://ec.europa.eu/development/policies/9interventionareas/ environment/ forest/flegt_briefing_notes_en.cfm (16 Mai 2008).

CE (2005a) 'Council Regulation 2173/2005 concerning the establishment of a FLEGT licensing scheme for import of timber into the European Community'; 20 décembre 2005.

CE (2005b) 'A Timber Legality Assurance System', Document d'information FLEGT No. 9. Bruxelles: CE.

CE (2007) 'Guidelines for Independent Monitoring', Document d'information FLEGT No. 7. Bruxelles: CE.

Cerutti, P.O. et Assembe, S. (2005) 'Cameroon Forest Sector - Independent Observer - Global Witness End of Contract Project Review'. Department for International Development (DFID). Yaoundé, Cameroun.

Cerutti, P.O. et Tacconi, L. (2006) 'Forests, Illegality, and Livelihoods in Cameroon', Document de travail décentralisé No. 35 du CIFOR. Bogor, Indonésie.

CESS-ODI (2005) 'Multistakeholder Forestry Programme, Links between forests and poverty in Indonesia. What evidence? How can targeting of poverty in and near forests be improved?', Document d'information II pour le DFID.

CINCO et CIP (2006) 'Emergencia en el bosque. Resumen Ejecutivo', Centro de Investigación de la Comunicación (CINCO) et Center for International Policy (CIP). Managua, Nicaragua.

COFI (2005) Site internet du Council of Forest Industries. www.cofi.org (septembre 2005).

Colchester, M. (2006) 'Reflections on the Social Dimensions of Verification in FLEGT processes: Issues, Risks and Challenges', Point de vue de VERIFOR. Londres : ODI.

Colchester, M., Boscolo, M., Contreras-Hermosilla, A., Gatto, F.D., Dempsey, J., Lescuyer, G., Obidzinski, K., Pommier, D., Richards, M., Sembiring, S.S., Tacconi, L., Rios, M.T.S. et Wells, A. (2006) Justice in the forest: Rural livelihoods and forest law enforcement. Forest Perspectives 3, Bogor: CIFOR.

Commission des Communautés européennes (2003) 'Com. 2003/251 final. Communication de la Commission au Conseil et au Parlement européen 'Forest Law Enforcement, Governance and Trade (FLEGT) Proposal for an EU Action Plan'. Bruxelles, 21 mai 2003.

CONADEH et Global Witness (2005) 'Independent Forest Monitoring Pilot Project in Honduras', Premier rapport succinct, Mai-juillet 2005. Tegucigalpa et Londres. 
Contreras-Hermosilla, A. et Fay, C. (2005) Strengthening forest management in Indonesia through land tenure reform: Issues and framework action. Forest Trends, Washington DC.

Contreras-Hermosilla, A., Doornbosch, R. et Lodge, M. (2007) 'The Economics of Illegal Logging and Associated Trade', rapport préparé pour la Table ronde de l'OCDE sur le développement durable à Paris, 8-9 Janvier.

CPET (2006) UK Government Timber Procurement Policy: Definition of 'legal' and 'sustainable' for timber procurement, 2e édition, novembre 2006. Oxford: Central Point of Expertise on Timber Procurement.

Crossin, C., Hayman, G. et Taylor, S. (2003) 'Where Did It Come From? Commodity Tracking Systems' dans I. Bannon et P. Collier (eds), Natural Resources and Violent Conflict: Options and Actions. Washington, DC: Banque mondiale.

Cruz, R. et Pulhin, J. (2006) 'Review of Multi-stakeholder Forest Protection Committees in the Philippines', Etude de cas pays 7 de VERIFOR. Londres : ODI.

Cruz, R. et Tapia, M. (2005) 'A Review of the Multi-sectoral Forest Protection Committees in the Philippines', ODI Forestry Briefing 7, Mars. Londres : ODI.

Danso, E. et Opoku, K. (2005) Impacts and legality of forest utilisation permits in Ghana. Accra: Civic Response et Londres : IIED.

Del Gatto, F., Wells, A., Richards, M., Coindres, I. et Contreras-Hermosilla, A. (2004) 'The forestry sector in Honduras: the legal barriers', document d'information. www. talailegal-centroamerica.org/downloads/english/The \%20forestry $\% 20$ sector $\% 20$ in \%20Honduras_the \%20legal \%20barriers.pdf

DENR (1998) 'Manual of Procedures for DENR-DILG-LGU Partnership on Devolved and Other Forest Management Functions', Joint Memorandum Circular 98-01. Manille, République des Philippines.

Diaw, C. (2008) 'Ombudsmen', Etude de cas comparative 7 de VERIFOR. Londres : ODI.

Division des Statistiques des Nations Unies (2006) Indicateur des objectfs de développement du millénaire des Nations Unies (7.9.25). http://mdgs.un.org/unsd/ mdg/Data.aspx

Dubash, N., Dupar, M., Kothari, S. et Lissu, Tundu (2001) A Watershed in Global Governance: An Independent Assessment of the World Commission on Dams Executive Summary, World Resources Institute, Lokayan et Lawyers' Environmental Action Team. New York: Pantheon.

Eba'a Atyi, R. (1998) 'Cameroon's Logging Industry: Structure, Economic Importance and Effects of Devaluation', document hors série 14 du CIFOR. Bogor: CIFOR.

Echeverría, R.D. (2004) 'Análisis Económico-Financiero del Sector Forestal Ecuatoriano y del Sistema Nacional Tercerizado de Control Forestal'. Quito: Projet MAE/BID.

Edmunds, D. et Wollenberg, E. (2002) 'Disadvantaged groups in multi-stakeholder negotiations', Rapport de programme CIFOR. Bogor: CIFOR.

EIA (2005) 'La Crisis de la tala Ilegal en Honduras. De cómo la importación de madera ilegal hondureña por los Estados Unidos y la Unión Europea incrementa la pobreza, 
acelera la corrupción y destruye bosques y comunidades'. Washington, DC: Environmental Impact Agency.

FAO (2000) Global Forest Resources Assessment 2000. Rome: FAO.

FAO (2004) 'Trends and current status of the contribution of the forestry sector to national economies', document de travail de la FAO sur le financement de la gestion durable de la forêt: FSFM/ACC/07. Rome: FAO.

FAO (2005) 'Evaluación de los Recursos Forestales Mundiales - Informe Nacional Nicaragua'. Rome: FAO.

FAO (2006) Global Forest Resources Assessment 2005. Rome: FAO. www.fao.org/ forestry/site/32179/en/

FAO (2008) Environmental Science for Social Change, Forest Faces: Hopes and Regrets in Philippine Forestry. Bangkok: FAO.

Faurby, O. (2005) El Sector Forestal de Nicaragua - Un Potencial Para el Desarrollo. Managua, Nicaragua: IPADE.

FEHCAFOR, REMBLAH et NICAMBIENTAL (2003) 'La Producción Forestal no Controlada en Honduras y Nicaragua: los impactos sobre gobernabilidad y pobreza'. Un diagnostic avec de multiples acteurs pour l'identificacion des options polítiques et des actions. Avec l'appui de l'ODI, la FAO, Global Witness, l'ACDI, le DFID. Centre América.

Ferroukhi, L. et Aguilar Schramm, A. (2004) 'Progress and challenges of municipal forest management in Costa Rica’, dans L. Ferroukhi (ed.), Municipal forest management in latin America. CIFOR/IDRC.

Findlay, T. (2003) 'Multilateral verification in flux', Verification Yearbook. Londres : VERTIC.

Fischer, C., Aguilar, F., Jawahar, P. et Sedjo, R. (2005) 'Forest Certification: Toward Common Standards?' Document de discussion 05/10 élaboré pour le service consultatif des investissements étrangers du groupe de la Banque mondiale. Washington, DC: Resources for the Future.

Flores Rodas, J.G. et Eveline Hernández, L.R. (2004) 'Informe de Evaluación de la AFE-COHDEFOR', présenté à la Présidence de la Republique du Honduras, octobre 2004.

FMB-DENR (2005) Revised Master Plan for Forestry Development. Forest Management Bureau, Department of Environment and Natural Resources. Quezon City, Philippines.

Forest Certification Watch (2004) 'Third Party Forest Monitoring Report Attracts Criticisms'. www.certificationwatch.org/print.php3?id_article=1805 (25 juin 2004).

FPB (2004) 'BC’s Mountain Caribou: Last Chance for Conservation?' Rapport spécial 22. Victoria, CB : Forest Practices Board.

FPB (2005) ' 10 Years of promoting forest stewardship and values', Rapport annuel 2004/05. Victoria, CB: Forest Practices Board. 
FPB (2006a) 'Continuous Improvement at the Forest Practices Board', Report. Victoria, CB: Forest Practices Board.

FPB (2006b) 'Forest Stewardship Plan Review: A Public Responsibility'. Board Bulletin 7. Victoria, CB: Forest Practices Board.

FUNDECOR (2003) Mitos y Realidades de la Deforestación en Costa Rica. San José, Costa Rica: SINAC.

Ganapin, D. (2001) 'Regional Study on Forest Policy and Institutional Reform, Philippines Case Study'. Manille : Banque asiatique de développement.

Global Forest Watch (2000) An overview of logging in Cameroon. Washington, DC: WRI.

Global Forestry Services (2004) 'Review of Programmes on Traceability of Timber Material', projet de rapport pour le Workplan Legality, Asia Forest Partnership.

Global Timber (2007) Global timber country statistics. www.globaltimber.org.uk/ info.htm

Global Witness (2002) 'Reform of the Forest Crime Monitoring and Reporting Project', Rapport Global Witness, mars. Londres : Global Witness.

Global Witness (2003) 'Cambodian government terminates independent forest monitoring'. Communiqué de presse, 22 avril.

Global Witness (2004) 'See No Evil: the Government's Forest Crime Monitor', Phnom Penh Post Numéro 13/13, 18 Juin-1 Juillet.

Global Witness (2005a) A Guide to Independent Forest Monitoring. Londres : Global Witness.

Global Witness (2005b) IFM in Cameroon and Cambodia: Comparing Terms of Reference. Analyse de Global Witness Research.

Global Witness et CONADEH (2006) 'Independent Forest Monitoring in Honduras, Second Summary Report, May 2005-April 2006'. Londres et Tegucigalpa.

Goetz, A-M. et Jenkin, R. (2004) 'Voice and Accountability in Service Delivery', Development Outreach 6(1), mars.

GPTIRID (Grupo Permanente de Trabalho Interministerial para a Redução dos İndices de Desmatamento da Amazônia Legal) (2004) 'Plano de Ação para a Prevenção e Controle do Desmatamento na Amazônioa Legal'. Presidência Da República - Casa Civil, Brasília.

Greene, O. (1994) 'On Verifiability and How it could Matter for International Environmental Agreements', Document de travail de l'International Institute for Applied Systems Analysis WP-94-116.

de Guzman, M.V.P. (1993) 'A Review of the Applicability of Current DENR Tenurial Instruments to Issues Related to Ancestral Domains', Natural Resources Management Program Report 93-05. Louis Berger International, Inc.

Hajer, M.A. (2005) 'Setting the Stage: A Dramaturgy of Policy Deliberation', Administration and Society 36(6): 624-647. 
Haufler, V. (2001) A Public Role for the Private Sector: Industry Self-Regulation in a Global Economy. Washington, DC: Carnegie Endowment for International Peace.

Hemmati, M. (2002) Multi-stakeholder Processes for Governance and Sustainability: beyond Deadlock and Conflict. Londres: Earthscan.

Hoare, A., Macqueen, D., Kodi, M., Counsell, S., Long, C. et Hardcastle, P. (2007) 'Innovative Models for Forest Management and Financing in DRC', projet de document de référence, Chatham House, Londres.

Humphreys, D. (2006) Logjam: Deforestation and the Crisis of Global Governance. Londres: Earthscan.

IBAMA (2004) Manual do Vistoriador para o Acompanhamento Técnico de PMFS \& POA. Brasilia.

INAFOR et Global Witness (2006) 'Convenio para la implementación de un Proyecto Piloto de Monitoreo Forestal Independiente en Nicaragua', Instituto Nacional Forestal and Global Witness, Managua, Nicaragua.

Independent Forest Sector Review Team (2004) 'Independent Forest Sector Review', commandé par l'Administration forestière du gouvernement royal du Cambodge et le Groupe de travail sur la gestion des ressources naturelles. www.cambodia-forestsector.net/docs/summary.pdf

INPE (2007) www.obt.inpe.br/prodes/prodes_1988_2005.htm (Mars 2007).

INS (2006) 'Exportations par Produits'. Yaoundé, Cameroun: Institut National de la Statistique.

Inspection Panel (2006) 'Investigation Report: Cambodia Forest Management Concession and Control Management Project', 30 Mars 2006.

International Network for Environmental Compliance and Enforcement (2005) Making Law Work: Environmental Compliance \& Sustainable Development (eds D. Zaelke, D. Kaniaru et E. Kružíková), version abrégée. Washington, DC.

ITTO (2005) Annual review and assessment of the world timber situation 2005. www. itto.or.jp/live/PageDisplayHandler?pageId=199

Kaufmann, D., Kraay, A. et Mastruzzi, M. (2003) 'Governance Matters III: Governance Indicators for 1996-2002', Banque mondiale, Document de travail 3106 sur la recherche sur la politique.

Kaul, I., Conceicao, P., Le Goulven, K. et Mendoza, R.U. (eds) (2003) Providing Global Public Goods: Managing Globalization, publié pour le Programme de développement des Nations Unies, 2003. New York et Oxford: Oxford University Press.

Killick, T. (1998) Aid and the political economy of policy change. Londres et New York: Routledge.

Lang, W. (1996) 'Compliance Control in International Environmental Law: Institutional Necessities', Heidelberg Journal of International Law 56: 685-695.

Larson, A. et Ribot, J. (2007) 'The Poverty of Forest Policy: Double Standards on an Uneven Playing Field', Journal of Sustainability Science 2(2): 189-204. 
Le Billon, P. (2002) 'Logging in muddy waters- the politics of forest exploitation in Cambodia', Critical Asian Studies 34: 563-586.

Lebedys, A. (2004) 'Trends and current status of the contribution of the forestry sector to national economies', Document de travail FSFM/ACC/07 de la FAO. Rome: FAO.

Lipschultz, R. et Fogel, A. (2003) 'Regulation for the rest of us? Global civil society and the privatisation of transnational regulation', dans R. Hall et T. Bierstecker (eds) The Emergence of Private Authority in Global Governance. Cambridge, Royaune Uni: Cambridge University Press.

Luttrell. C. et Brown, D. (2006) 'The Experience of Independent Forestry Monitoring in Cambodia', Etude de cas pays 4 de VERIFOR. Londres : ODI.

Luttrell, C. et Nash, R. (2008) 'The experience of election observers: lessons for the process of timber verification, Etude de cas comparative 6 de VERIFOR. Londres : ODI.

Malayang, B.S., Srun, S.M. et Dominicus, B. (2002) 'Forest Crime Monitoring and Reporting Project: report of the evaluation mission'. RGC/UNDP/FAO.

Malena, C. (2004) 'Strategic Partnership: Challenges and Best Practices in the Management and Governance of Multi-Stakeholder Partnerships Involving UN and Civil Society Actors', Document d'information préparé pout l'atelier multi acteurs sur les partenariats et les relations Nations Unies-Société Civile, 10-12 février, New York. Collection of Materials from the Multi-Stakeholder Workshop on Partnerships and UN-Civil Society Relationships www.un.org/reform/ civilsociety/ pdfs/pocantico_booklet.pdf

de Maria, W. (2005) 'Whistleblower protection: is Africa ready?', Public Administration \& Development, Vol. 25: 217-226.

Matthews, P. (1996) 'Problems Related to the Convention on International Trade in Endangered Species', International and Comparative Law Quarterly 45: 421-431.

McKenny, B. (2001) 'Baseline Assessment of Cambodia's Forest Resources'. Cambodge: WWF.

MFR (2007) 'Annual Service Plan Report 2006/07'. CB: Ministère des Forêts et des parcours naturels.

van Midwoud, P. (2006a) 'Independent Monitoring of the FLEGT Timber Legality Assurance System: Thinking Outside the Box', Document d'information 4 de VERIFOR . Londres : ODI.

van Midwoud, P. (2006b) Independent monitoring in the FLEGT Timber Legality Assurance System: Lessons from other sectors. Wageningen: Département de la connaissance/Groupe de politique sur la conservation de la nature (Groupe des politiques sur la forêt et la conservation de la nature), Université de Wageningen.

Miller, F. (2004) 'Regulatory Mechanisms', IFSR, The Forest Sector in Cambodia (Part I: Policy Choices, Issues and Options). Independent Forest Sector Review. www. cambodia-forest-sector.net/index.htm 
Miller, F. et Boscolo, M. (2004) 'Post-harvest processing and the demand for wood products in Cambodia', IFSR, The Forest Sector in Cambodia (Partie I: Choix Politiques, Problèmes et Options). Independent Forest Sector Review. www. cambodia-forest-sector.net/index.htm

MINAE (2001) Plan Nacional de Desarrollo Forestal 2001-2010. Ministère de l'environnement et de l'énergie, Costa Rica.

MINEFI (2002) 'Conditions de vie des populations et profil de pauvreté au Cameroun en 2001'. Ministère de l'Économie et des Finances (MINEFI). Yaoundé, Cameroon.

MINEFI (2006) 'Audit économique et financier du secteur forestier au Cameroun Draft No. 1 - Août 2006'. Ministère de l'Économie et des Finances (MINEFI). Yaoundé, Cameroon.

MINFOF (2004) 'Statistiques d'abattage - DF10 enregistrées'. Ministère des Forêts et de la Faune (MINFOF). Yaoundé, Cameroon.

MINFOF (2005) 'Statistiques des produits forestières spécifiés au Port de Douala Exercice 2005'. Ministère des Forêts et de la Faune. Douala, Cameroun.

MINFOF (2007a) 'Bref aperçu du secteur forestier camerounais'. Ministère des Forêts et de la Faune (MINFOF). Yaoundé, Cameroun.

MINFOF (2007b) 'Rapport d'activités de spécification des produits forestiers au port de Douala'. Ministère des Forêts et de la Faune (MINFOF). Douala, Cameroun.

MINFOF et FAO (2005) 'Évaluation des ressources forestières nationales du Cameroun'. Ministère des Forêts et de la Faune (MINFOF) and FAO. Yaoundé, Cameroun.

MMA (2006a) Instrução Normativa MMA no 4, de 11 de Dezembro de 2006. 'Dispó sobre a Autorização Prévia à Análise Técnica de Plano de Manejo Florestal Sustentável-APAT, e dá outras providências'. Ministère de l'environnement. Cabinet du Ministre. Brésil.

MMA (2006b) Instrução Normativa MMA no 5, de 11 de Dezembro de 2006. 'Dispõe sobre procedimentos técnicos para elaboração, apresentação, execução e avaliação técnica de Planos de Manejo Florestal Sustentável-PMFSs nas florestas primitivas e suas formas desucessão na Amazônia Legal, e dá outras providências'. Ministère de l'environnement. Cabinet du Ministre. Brésil.

MMA (2006c) 'Descentralização da Gestão Florestal', Documento de Discussao, Versao 1.3. 30.03.06. Ministère de l'environnement. Secrétariat de la Biodiversité et des Florestas. Direction du Programme national de Florestas. Brésil.

MMA (2007) Nouvelles du ME du Brésil 02/03/2007. 'Operação tem 37 mandados de prisão contra quadrilha de madeireiros'. Ministère de l'environnement. Brésil. www. mma.gov.br/ascom/ultimas/index.cfm?id=3158

MdF (2003) BC Heartlands Economic Strategy. Forestry Revitalization Plan. BC: Ministère des Forêts.

MdF (2004) The State of British Columbia’s Forests 2004. BC: Ministère des Forêts.

Mountain Caribou Project (2005) 'Staring at extinction: Mountain Caribou in British Columbia. An Analysis of Planned Logging in BC's Inland Temperate Rainforest'rapport de projet. Projet Mountain Caribou Project, ForestEthics, Nelson, CB. 
Multi-stakeholder Forestry Programme (2006a), 'Community-based Forest Management in Indonesia. What is it and how can its potential be harnessed for economic growth and poverty reduction?'

Multi-stakeholder Forestry Programme (2006b), 'Whose rule of law? Forest law enforcement and community logging rights in Indonesian Papua'. Document d'information III du MFP.

Nussbaum, R. et Simula, M. (2005) The Forest Certification Handbook, 2e Edition. Londres: Earthscan.

Nussbaum, R., Gray, I. et Hickman, S. (2003) 'Modular Implementation and Verification System (MIV); a toolkit for phased application of forest management standards and certification'. Oxford: Proforest.

Oficina Nacional Forestal (2001) 'Manejo forestal y conservación de bosques en Costa Rica'. ONF/COSEFORMA/PNUD/CATIE. San José, Costa Rica.

Oficina Nacional Forestal (2004) 'La madera en Costa Rica'. ONF/ FONAFIFO/ SINAC. San José, Costa Rica.

Otoo, J.E. (2003) 'Chainsaw lumber production and sustainable forest management', in Chainsaw lumber production: a necessary evil? Tropenbos International Proceedings: 18. Tropenbos International - Ghana.

Oyono, P.R., Ribot, J. et Larson, A. (2006) 'Green and Black Gold in Rural Cameroon: Natural Resources for Local Governance, Justice and Sustainability.' Environmental Governance In Africa Document de travail No. 22.

PAS (2006) 'Plano Amazônia Sustentável, Versao Final para Consulta'. Casa Civil da Presidencia da República. Brésil.

Pastor, R.A. (1999) 'A Brief History of Electoral Commissions', dans A. Schedler, L. Diamond et M. Plattner (eds), The Self-Restraining State. Boulder et Londres: Lynne Rienner.

Pendleton, M. (1998) 'Taking the Forest: The Shared Meaning of Tree Theft', Society and Natural Resources 11(1): 35-46. New York: Taylor \& Francis Group.

Persbo, A., Mayo, B. et Peterson, M. (2005) 'An Overview of the Evolution, Operation and Status of Nuclear Safeguards', Etude de cas comparative 2 VERIFOR. Londres : ODI.

Persson, R. (2003) Assistance to forestry: experiencesand potential for improvement. Bogor: CIFOR. www.cifor.cgiar.org/publications/pdf_files/Books/AssistancetoForestry.pdf

Peskett, L. et Brown, D. (2006) 'The UN Framework Convention on Climate Change', Etude de cas comparative 4 VERIFOR. Londres : ODI.

PNUD (2005) 'Informe sobre Desarrollo Humano 2005 - La cooperación internacional ante una encrucijada'. New York: PNUD.

PNUD (2006a) Rapport 2006 sur le développement humain. Population Division Population des Nations Unies. http://hdr.undp.org/hdr2006/

PNUD (2006b) Informe Sobre Desarrollo Humano Honduras 2006, hacia la expansión de la ciudadanía. Tegucigalpa. 
Poku-Marboah, M., Juslin, H., Hansen, E. et Forsyth, K. (2003) 'Forest certification update for the UNECE region, 2003'. Geneva Timber and Forest Discussion Paper No. 39. Genève: Nations Unies.

Procuraduría Ambiental (2006) 'Informe Anual 2005'. Procuraduría General de la Republica (PGR). Managua, Nicaragua.

Proforest/ISEAL (2005) 'Managing conflict of interest in certification', Rapport de l'Alliance ISEAL, Bonn.

Publish What You Pay and Revenue Watch Institute (2006) 'Eye on EITI, Civil Society Perspectives and Recommendations on the Extractive Industries Transparency Initiative', octobre 2006, New York.

Rackham, O. (1986) The History of the Countryside. Londres : J.M. Dent.

Reeve, R. (2002) Policing International Trade in Endangered Species: The CITES Treaty and Compliance. The Royal Institute of International Affairs. Londres: Earthscan.

de Renzio, P. et Smith, S. (2005) 'Linking policies and budgets: implementing Medium Term Expenditure Frameworks in a PRSP context', Document d'information ODI, 2005.

de Renzio, P., Fritz, V., et Kizildash Agha, Z. avec Foresti, M., et O’Neil, T. (2006) 'Ownership: Illustration Paper on Human Rights and the Partnership Commitments of the Paris Declaration',rapport ODI commandé par le réseau sur la gouvernance OCDE - CAD.

République du Cameroun (2005) 'Third National Report to the CBD'. Yaoundé, Cameroun.

Resource Extraction Monitoring (2006) 'Independent Monitoring: Progress in tackling illegal logging in Cameroon. Project of Independent Forest Monitoring of Forest Law Enforcement and Governance', Rapport annuel- mars 2005 - février 2006. Cambridge, Royaume Uni : Resource Extraction Monitoring (REM).

Resource Extraction Monitoring (2007) 'Observation Indépendante - Evolution du contrôle et des sanctions de l'exploitation forestière illégale au Cameroun', Rapport annuel mars 2006-février 2007. Yaoundé, Cameroun: Resource Extraction Monitoring (REM).

Ribot, J.C. (1999) 'Decentralization, Participation, and Accountability in Sahelian Forestry: Legal Instruments of Political-Administrative Control', Afrique 69(1): 23-65.

Richards, M., Wells, A., Del Gatto, F., Contreras-Hermosilla, A. et Pommier, D. (2003) 'Impacts of illegality and barriers to legality: a diagnostic analysis of illegal logging in Honduras and Nicaragua', International Forestry Review 5(3): 282- 292.

Ronit, K. et Schneider, V. (1999) 'Global Governance through Private Organisations', Governance: An International Journal of Policy and Administration 12(3): 243-266.

Ross, M. (2001) Timber Booms and Institutional Breakdown in Southeast Asia: Cambridge, Royaume Uni: Cambridge University Press. 
Rudel, T.K. (1998) 'Is there a forest transition? Deforestation, reforestation, and development', Rural Sociology 63(4): 533-552.

Sabogal, C. et al. (2005) 'Manejo florestal empresarial na Amazonía Brasileira: restricioes e oportunidades para a adocao de boas prácticas de manejo’. Belém, Brésil: EMBRAPA.

SAMFU (2002) 'Plunder: The Silent Destruction of Liberia’s Rainforest'. Save My Future Foundation (SAMFU), Monrovie.

Seneca Creek Associates and Wood Resources International (2004) “Illegal' Logging and Global Wood Markets: The Competitive Impacts on the U.S. Wood Products Industry', rapport préparé pour l'American Forest \& Paper Association.

Seymour, F., et Dubash, N., (2000) The Right Conditions: The World Bank, Structural Adjustment and Forest Policy Reform. Washington, DC: WRI.

SLDF/FW (2002) 'Who's minding our forests? Deregulation of the forest industry in British Columbia'. Sierra Legal Defence Fund and Forest Watch of British Columbia. Vancouver, BC.

Smillie, I. (2005) 'The Kimberley Process Certification Scheme for Rough Diamonds', Etude de cas comparative 1 VERIFOR. Londres : ODI.

Smouts, M-C. (2002) 'Forest Certification and Timber Labelling: The Hidden Agenda', projet de document de discussion, dans 'Panel: The Moral Assessment of Global Trade', Convention de l'International Studies Association, mars.

Stokke, O.S., Hovi, J. et Ulfstein, G. (eds) (2005) Implementing the Climate Regime: International Compliance. Londres : Earthscan/James et James.

Sur, S. (ed.) (1991) Verification of Current Disarmament and Arms Limitation Agreements: Ways, Means and Practices. UNIDIR/Dartmouth Publishing/ University Press, Cambridge.

Tacconi, L. (ed.) (2007) Illegal Logging: Law enforcement, Livelihoods and the Timber Trade. Londres : Earthscan.

Tacconi, L., Obidzinski, K. Smith, J. et Suramengala, I. (2004) 'Can "Legalization” of Illegal Forest Activities Reduce Illegal Logging? Lessons from East Kalimantan', Journal of Sustainable Forestry 19(1/2/3).

The Economist (2003) 'Cut Down. The Courts Versus the Rainforest.' The Economist Vol. 369 No. 8348.

Thiel, H. (2004) 'El Inicio de una Reforma Política, Institucional y Normativa en el Sector Forestal Ecuatoriano y el Sistema Nacional Tercerizado de Control Forestal del Ecuador'. Rome: FAO.

Thiel, H. (2005) 'The Ecuadorian Outsourced Forest Control System,' Présentation PowerPoint. Quito, Equateur.

Thiel, H. et Viergever, M. (2006) 'Giants don't leap: verification in Brazil's process towards sustainable forestry', Etude de 5 cas pays de VERIFOR. Londres : ODI.

Transparency International (2006) Corruption perceptions index 2006. www. transparency.org/policy_research/surveys_indices/cpi/2006 
UN Population Division (2006c) National trends in population: country profiles. Division Population des Nations Unies. www.un.org/esa/population/publications/ countryprofile/profile.htm

Victor, D. (2001) The Collapse of the Kyoto Protocol and the Struggle to Slow Global Warming, édition révisé. Princeton: Princeton University Press.

Vogel, D. (1995) Trading Up: Consumer and environmental regulation in a global economy. Cambridge, MA: Harvard University Press.

Wells, A., Luttrell, C., Brown, D., et Bird, N. (2006) 'Public Goods and Private Rights: the Illegal Logging Debate and the Rights of the Poor'. ODI Forestry Briefing 9. Londres : ODI.

Wells, A., Nababan, A. et Poerba, B. (2006) 'Review of the UK - Indonesia Memorandum of Understanding on Illegal Logging'. Programme multilatéral sur le secteur de la forêt du DFID.

Wells, A., Ngadiono et Asycarya, D. (2006) 'Systems for verification of legality in the Indonesian forest sector', Etude de cas pays 9 VERIFOR. Londres: ODI.

Wells, A., Thang, H.C. et Chen, H.K. (2008) 'Systems for verification of legality in the forest sector, Malaysia: domestic timber production and timber imports', Etude de cas pays 8 VERIFOR. Londres: ODI.

WRI (2001) Canada's forests at a crossroads: An assessment in the year 2000. Washington, DC: WRI. 

Le bois légal étudie un sujet d'actualité de la politique forestière internationale : comment contrôler la légalité du bois vendu, de manière à satisfaire à la fois les intérêts commerciaux des pays producteurs et les préoccupations sociales et environnementales de la société civile et des consommateurs.

Cette question simple et technique d'apparence, se révèle à l'étude être complexe et politique. Elle fait appel à une interface critique dans les relations internationales, où les droits souverains des Etats producteurs s'opposent au rôle des forêts en tant que biens publics importants. Elle est liée à un thème, l'exploitation illégale de la forêt, qui se trouve au centre d'un mouvement où les acteurs privés jouent un rôle de plus en plus important dans la réforme du secteur forestier.

Le bois légal présente les résultats du projet VERIFOR, une association de recherche appliquée dont les partenaires viennent d'Europe, d'Afrique, d'Amérique latine et d'Asie. L'ouvrage s'appuie sur des études pratiques provenant de cinq continents; il analyse les façons selon lesquelles les problèmes de la gestion forestière font la lumière sur des processus plus larges de réforme de la gouvernance.

Ce livre ne manquera pas d'intéresser tous ceux qui travaillent dans la gouvernance forestière et la gestion des ressources extractives, la certification et l'étiquetage commerciaux, la défense de l'environnement et le développement participatif.
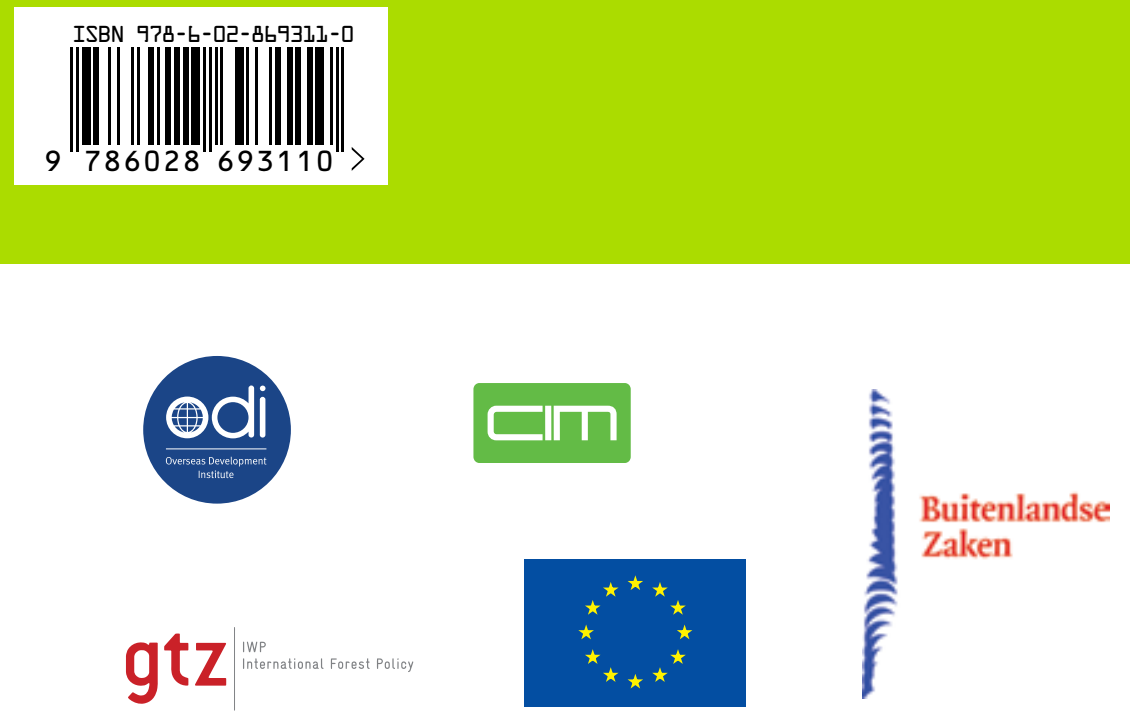

Centre de recherche forestière internationale

CIFOR défend le bien-être humain, la conservation de l'environnement et l'équité en menant une recherche pour éclairer les politiques et les pratiques qui affectent les forêts dans les pays en développement. CIFOR est l'un des 15 centres au sein du Groupe consultatif sur la recherche agricole internationale (CGIAR). Le siège du CIFOR est situé à Bogor, en Indonésie. CIFOR a également des bureaux en Asie, en Afrique et en Amérique du Sud. 